\title{
Copper-Catalyzed Vicinal Cyano-, Thiocyano-, and Chlorophosphorylation of Alkynes: A Phosphinoyl Radical-Initiated Approach for Difunctionalized Alkenes
}

\author{
Ze-Kun Tao, ${ }^{\dagger}$ Cheng-Kun Li, ${ }^{\dagger}$ Jian-an Li,${ }^{\dagger}$ Adedamola Shoberu, ${ }^{\dagger}$ Wei Zhang, ${ }^{*},{ }^{\ddagger}$ Jian-Ping Zou* ${ }^{, \dagger}$ \\ $\dagger$ Key Laboratory of Organic Synthesis of Jiangsu Province, College of Chemistry and Chemical Engineering, \\ Soochow University, Jiangsu 215123, China \\ ${ }^{\ddagger}$ Department of Chemistry, University of Massachusetts Boston, Boston, MA 02125, USA
}

\section{Table of Contents}

1. General Methods and Abbreviations

2. Cyanophosphorylation of Alkynes

3. Thiocyanophosphorylation of Alkynes

4. Chlorophosphorylation of Alkynes

5. Mechanistic Studies

6. Synthetic Applications of the Difunctionalization Products

7. Unsuccessful Substrates

8. Characterization Data of Compounds 4, 5, 7, 9, 10-15

S20

9. X-Ray Crystallization Data for Compounds $(E)-\mathbf{4 l},(Z)-7 \mathbf{a}$ and $(E)-\mathbf{9 p} \quad$ S49

10. ${ }^{1} \mathrm{H},{ }^{13} \mathrm{C}$ and ${ }^{31} \mathrm{P}$ NMR of Compounds $4,5,7, \mathbf{9}, \mathbf{1 0 - 1 5}$ 


\section{General Methods and Abbreviations}

General Methods: All reactions were performed under inert atmosphere (argon). ${ }^{1} \mathrm{H}$ NMR spectra were recorded using a Varian-Inova $400 \mathrm{MHz}$ instrument using $\mathrm{CDCl}_{3}$ or $\mathrm{DMSO}-d_{6}$ as solvent with tetramethylsilane (TMS) as an internal standard. ${ }^{13} \mathrm{C}$ NMR spectra were obtained at $100 \mathrm{MHz}$ and referenced to the internal solvent signals. ${ }^{19} \mathrm{~F}$ NMR spectra were obtained at $376 \mathrm{MHz}$ and referenced to the internal solvent signals. ${ }^{31} \mathrm{P}$ NMR spectra were obtained at $162 \mathrm{MHz}$ using $85 \% \mathrm{H}_{3} \mathrm{PO}_{4}$ as external standard. Chemical shifts were reported in parts per million (ppm) relative to TMS $(\delta$ ). Multiplicities were indicated by s (singlet), d (doublet), t (triplet), q (quartet), m (multiplet), coupling constant $J$ was reported in hertz $(\mathrm{Hz})$. High resolution mass spectra were recorded on a MicroMass-TOF machine (ESI). X-ray single crystal diffraction data were collected with a Rigaku Mercury CCD area detector in $\omega$ scan mode using Mo-K $\alpha$ radiation $(\lambda=0.71075 \AA)$. Column chromatography was performed with 300-400 mesh silica gel using flash column techniques. Unless stated otherwise, all reagents and alkynes $\mathbf{1}$ except $\mathbf{1 q}$ which was synthesized according to a previously described procedure, ${ }^{[1]}$ were obtained from commercial sources and used as received; di(hetero)arylphosphine oxides 2 except diphenylphosphine oxide were synthesized according to a previously described procedure. ${ }^{[2]}$

Abbreviations: $\mathrm{AIBN}=$ azobisisobutyronitrile; $\mathrm{CH}_{3} \mathrm{CN}=$ acetonitrile; $\mathrm{HOAc}=$ acetic acid; $\mathrm{BHT}=$ 2,6-di-tert-butyl-4-methylphenol; $\mathrm{Bu}=$ butyl; $\mathrm{CDX}=$ cyanobenziodoxolone; $\mathrm{DCE}=1$,2-dichloroethane; $\mathrm{DCM}=$ dichloromethane; $\mathrm{DMA}=N, N$-dimethylacetamide $; \mathrm{DMF}=N, N$-dimethylformamide $; \mathrm{DMSO}=$ dimethylsulfoxide $;$ $\mathrm{DPE}=1,2$-diphenylethylene $; \mathrm{DTBP}=$ di-tert-butyl peroxide $;$ EtOAc $=$ ethyl acetate $; \mathrm{EtOH}=$ ethanol; $\mathrm{Me}=$ methyl; NMP = N-methyl-2-pyrrolidinone $\mathrm{Ph}=$ phenyl $; \mathrm{TBAB}=$ tetrabutylammonium bromide; $\mathrm{TBACN}=$ tetrabutylammonium cyanide; TBHP = tert-butyl hydroperoxide; $\mathrm{TBN}=$ tert-butyl nitrite; $\mathrm{TBPB}=$ tert-butyl peroxybenzoate; TEMPO = 2,2,6,6-tetramethylpiperidine-1-oxyl; THF = tetrahydrofuran; $\mathrm{TMSCl}=$ trimethylsilyl chloride; TMSNCS = trimethylsilyl isothiocyanate Ts = tosyl . 


\section{Cyanophosphorylation of Alkynes}

\subsection{Optimization Studies for the Cyanophosphorylation of Phenylacetylene (1a)}

Table S1. Reaction Development ${ }^{[\mathrm{a}]}$

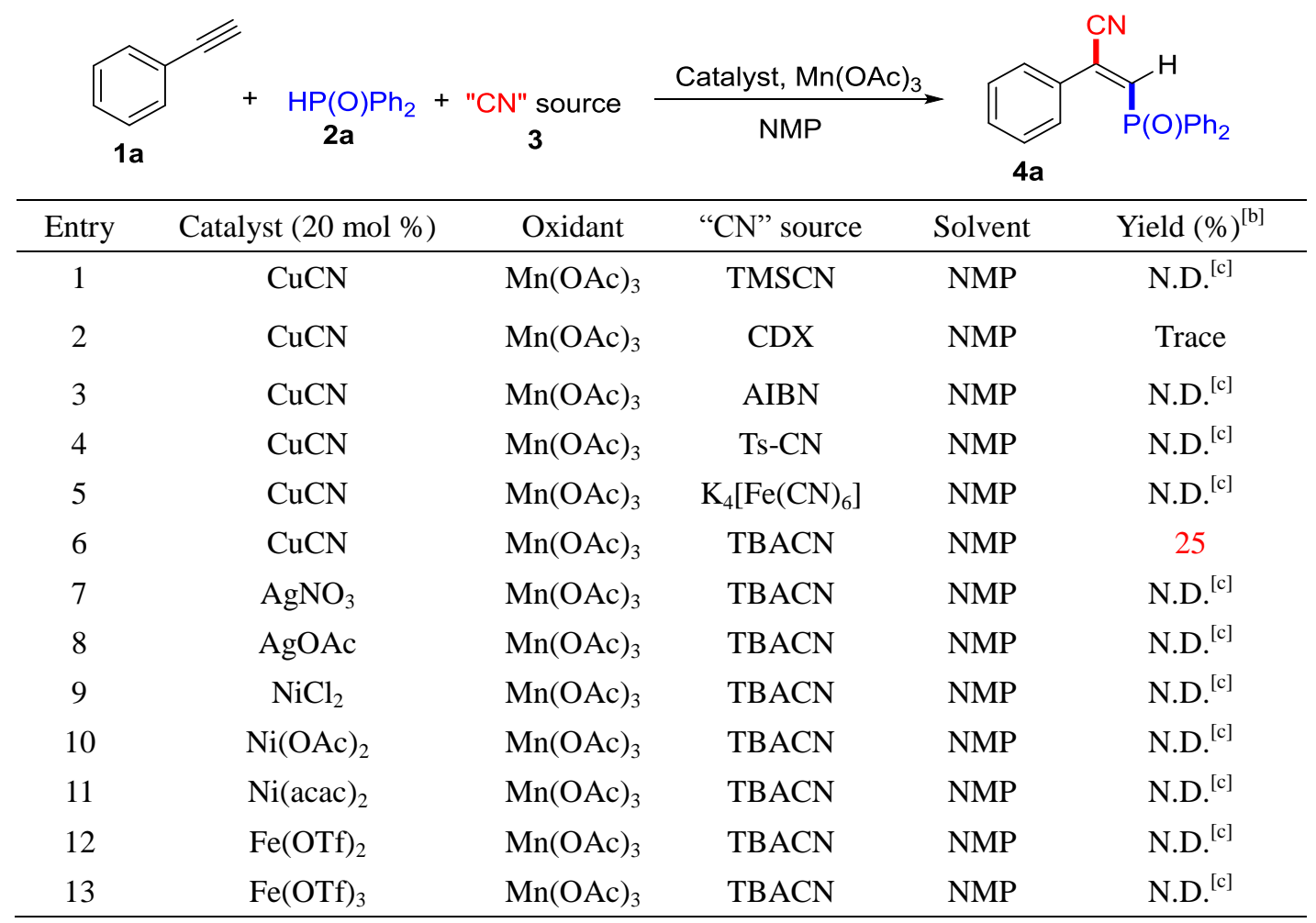

[a] Reaction conditions: Phenylacetylene 1a (0.25 mmol, 1.0 equiv), $\mathrm{HP}(\mathrm{O}) \mathrm{Ph}_{2} 2 \mathrm{2a}(0.375 \mathrm{mmol}, 1.5$ equiv), "CN" source ( $1 \mathrm{mmol}, 4$ equiv), catalyst $(20 \mathrm{~mol} \%), \mathrm{Mn}(\mathrm{OAc})_{3} \cdot 2 \mathrm{H}_{2} \mathrm{O}(1 \mathrm{mmol}, 4$ equiv), at room temperature for $0.5 \mathrm{~h}$ under argon atmosphere. [b] Isolated yield. [c] N.D. = Not detected.

Table S2. Screening of Oxidant ${ }^{[\mathrm{a}]}$

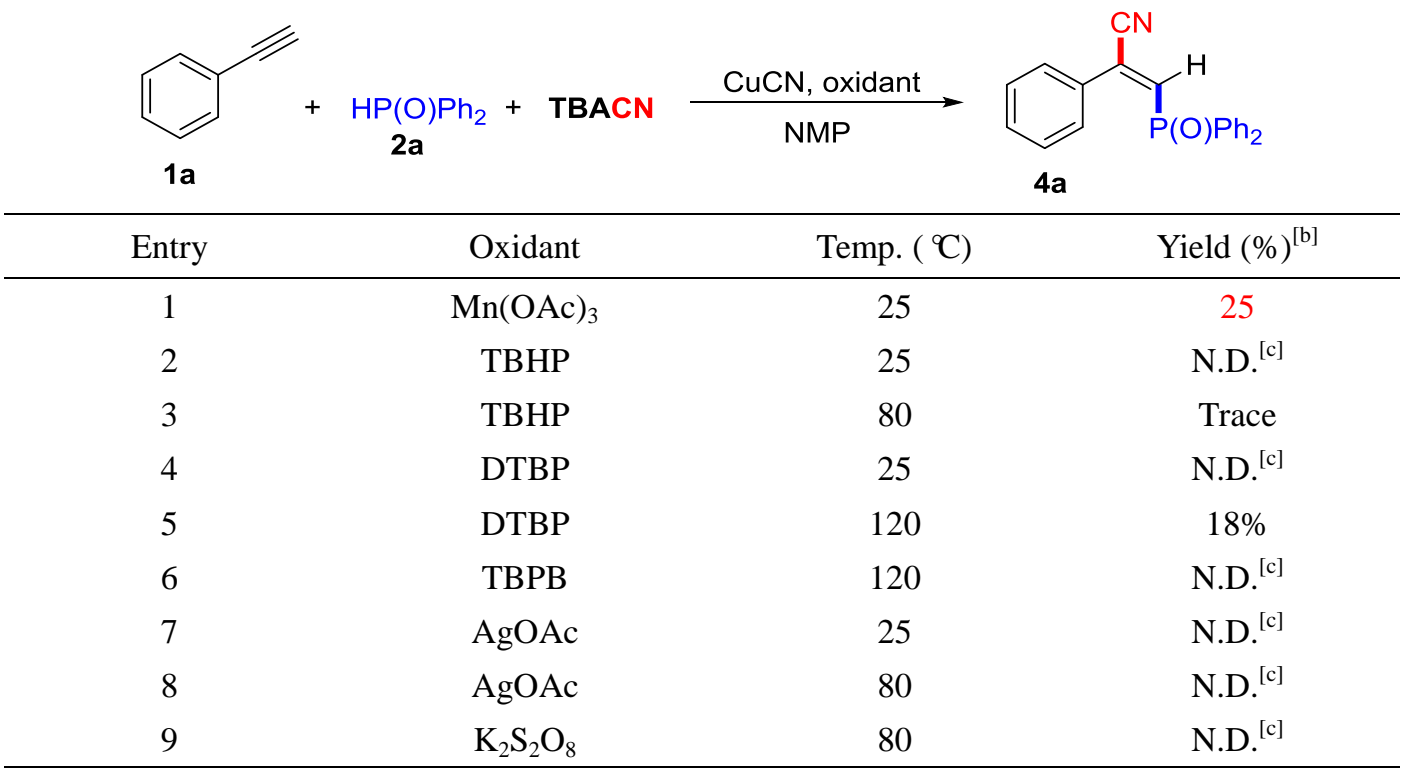

[a] Reaction conditions: Phenylacetylene 1a ( $0.25 \mathrm{mmol}, 1.0$ equiv), $\mathrm{HP}(\mathrm{O}) \mathrm{Ph}_{2} \mathbf{2 a}(0.375 \mathrm{mmol}, 1.5$ equiv), TBACN (1 mmol, 4 equiv), CuCN (20 mol \%), oxidant ( $1 \mathrm{mmol}, 4$ equiv), NMP (2.5 mL), at room temperature for $0.5 \mathrm{~h}$ under argon atmosphere. [b] Isolated yield. [c] N.D. $=$ Not detected. 
Table S3. Screening of Reaction Media ${ }^{[a]}$

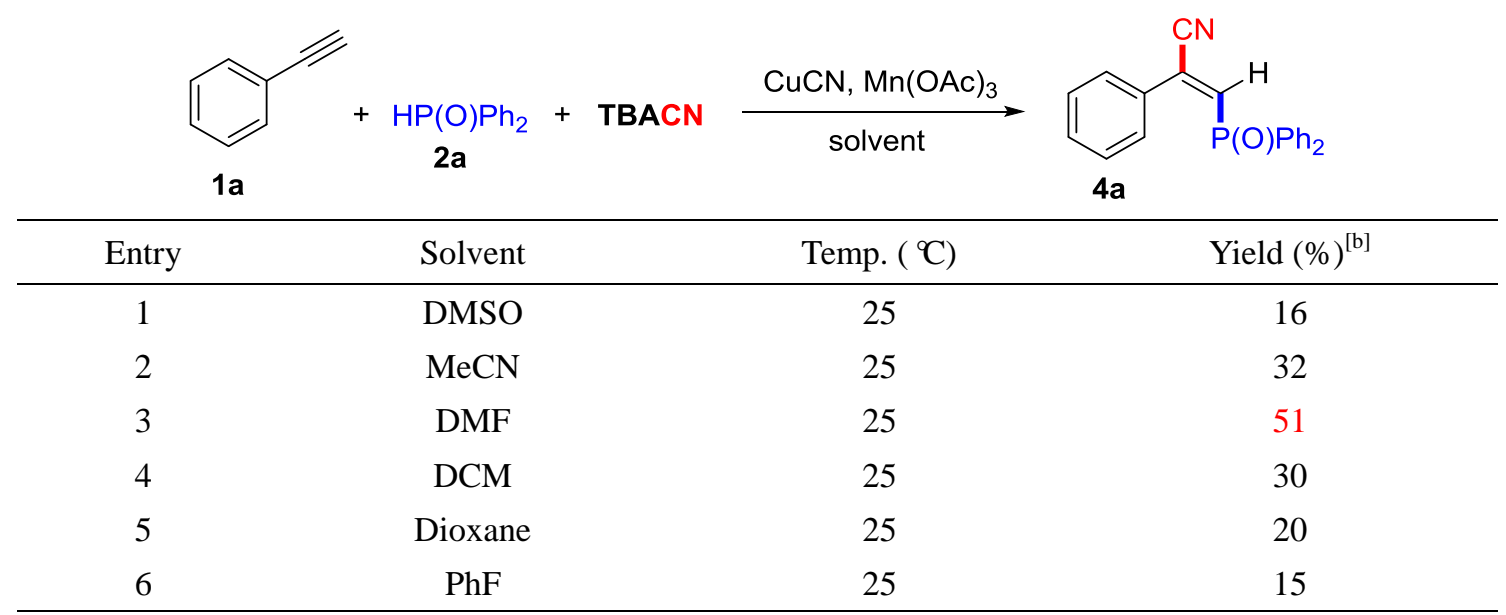

[a] Reaction conditions: Phenylacetylene 1a $\left(0.25 \mathrm{mmol}, 1.0\right.$ equiv), $\mathrm{HP}(\mathrm{O}) \mathrm{Ph}_{2}$ 2a (0.375 mmol, 1.5 equiv), TBACN ( $1 \mathrm{mmol}, 4$ equiv), $\mathrm{CuCN}(20 \mathrm{~mol} \%), \mathrm{Mn}(\mathrm{OAc})_{3} .2 \mathrm{H}_{2} \mathrm{O}(1 \mathrm{mmol}, 4$ equiv), solvent $(2.5 \mathrm{~mL})$, at room temperature for $0.5 \mathrm{~h}$ under argon atmosphere. [b] Isolated yield.

Table S4. Screening of Copper sources ${ }^{[a]}$

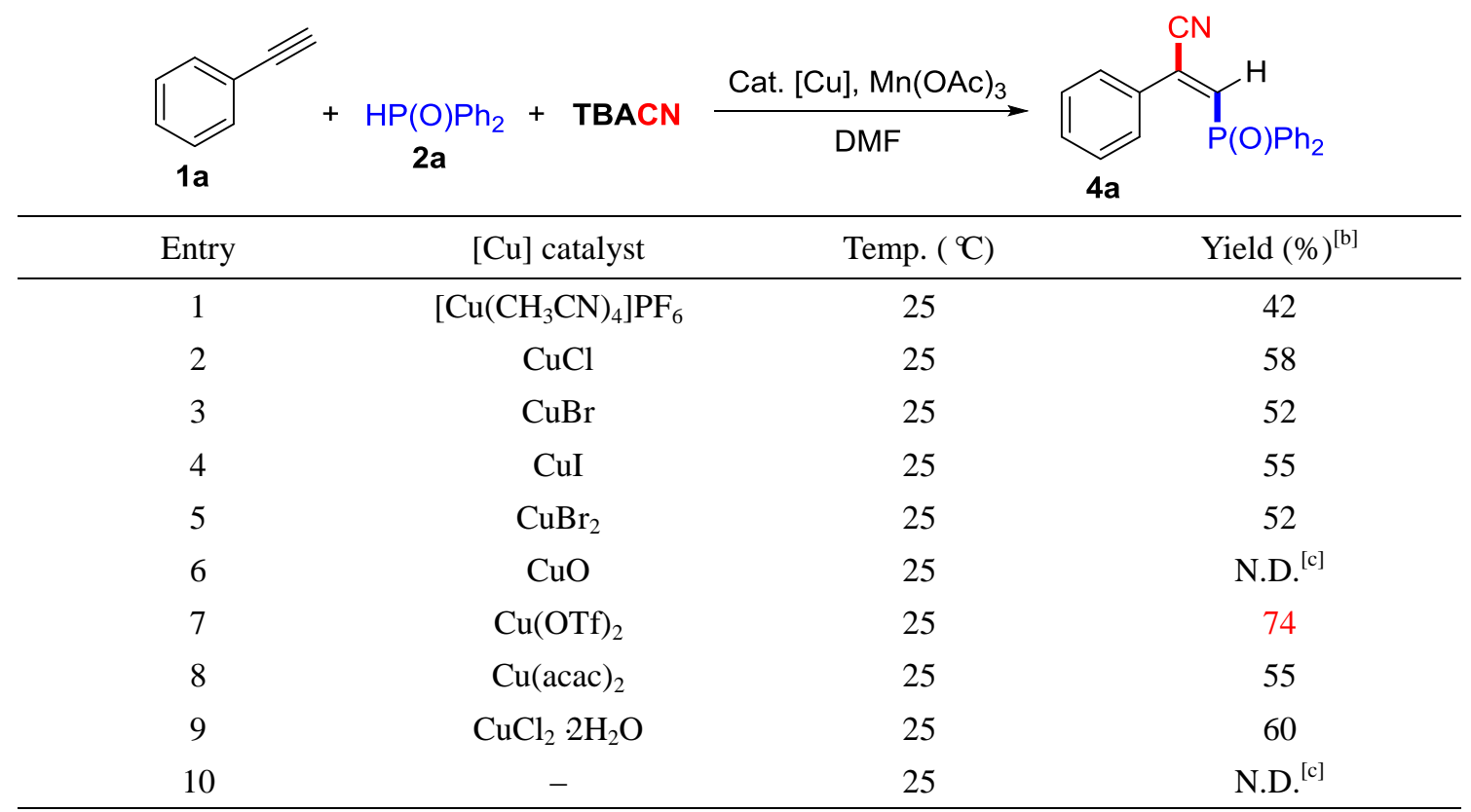

a] Reaction conditions: Phenylacetylene $\mathbf{1 a}\left(0.25 \mathrm{mmol}, 1.0\right.$ equiv), $\mathrm{HP}(\mathrm{O}) \mathrm{Ph}_{2} \mathbf{2 a}(0.375 \mathrm{mmol}, 1.5$ equiv), TBACN (: nmol, 4 equiv), Cu-catalyst (20 mol \%), $\mathrm{Mn}(\mathrm{OAc})_{3} \cdot 2 \mathrm{H}_{2} \mathrm{O}(1 \mathrm{mmol}, 4$ equiv), DMF $(1.5 \mathrm{~mL})$, at room temperature fo ).5 h under argon atmosphere. [b] Isolated yield. [c] N.D. = Not detected. 
Table S5. Screening of Relative Ratio of Reactants/Catalyst and Temperature ${ }^{[\mathrm{a}]}$

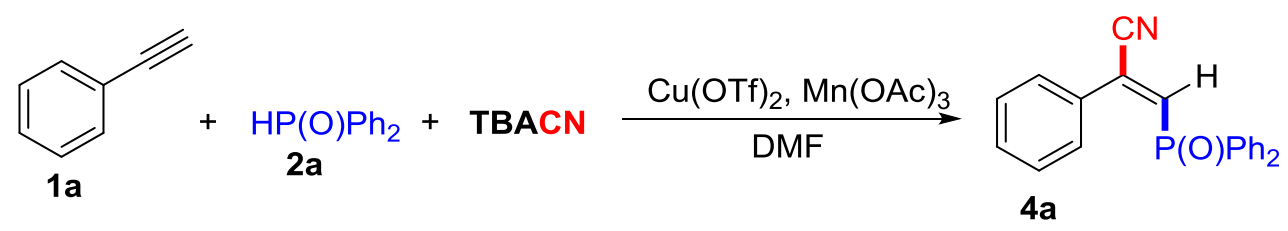

\begin{tabular}{ccccccc}
\hline Entry & $\begin{array}{c}\text { Amount of } \\
\mathrm{Cu}(\mathrm{OTf})_{2}\end{array}$ & $\begin{array}{c}\text { Amount of } \\
\text { Mn }(\mathrm{OAc})_{3}\end{array}$ & $\begin{array}{c}\text { Amount of } \\
\mathrm{HP}(\mathrm{O}) \mathrm{Ph}_{2}\end{array}$ & $\begin{array}{c}\text { Amount of } \\
\text { TBACN }\end{array}$ & $\begin{array}{c}\text { Time } \\
(\mathrm{min})\end{array}$ & $\begin{array}{c}\text { Yield } \\
(\%)^{[\mathrm{b}]}\end{array}$ \\
\hline $\mathbf{1}$ & $\mathbf{2 0 ~ m o l ~ \%}$ & $\mathbf{4}$ equiv & $\mathbf{1 . 5}$ equiv & $\mathbf{4}$ equiv & $\mathbf{3 0}$ & $\mathbf{7 4}$ \\
$2^{[\mathrm{c}]}$ & $20 \mathrm{~mol} \%$ & 4 equiv & 1.5 equiv & 4 equiv & 30 & 48 \\
$3^{[\mathrm{d}]}$ & $20 \mathrm{~mol} \%$ & 4 equiv & 1.5 equiv & 4 equiv & 30 & 66 \\
4 & $20 \mathrm{~mol} \%$ & 5 equiv & 1.5 equiv & 5 equiv & 30 & 72 \\
5 & $20 \mathrm{~mol} \%$ & 3 equiv & 1.5 equiv & 3 equiv & 30 & 66 \\
6 & $30 \mathrm{~mol} \%$ & 4 equiv & 1.5 equiv & 4 equiv & 30 & 74 \\
7 & $10 \mathrm{~mol} \%$ & 4 equiv & 1.5 equiv & 4 equiv & 30 & 60 \\
8 & $20 \mathrm{~mol} \%$ & 4 equiv & 1.0 equiv & 4 equiv & 30 & 62 \\
9 & $20 \mathrm{~mol} \%$ & 4 equiv & 2.0 equiv & 4 equiv & 30 & 72 \\
10 & $20 \mathrm{~mol} \%$ & 4 equiv & 1.5 equiv & 4 equiv & 15 & 50 \\
11 & $20 \mathrm{~mol} \%$ & 4 equiv & 1.5 equiv & 4 equiv & 45 & 66 \\
$12^{[\mathrm{e}]}$ & $20 \mathrm{~mol} \%$ & 4 equiv & 1.5 equiv & 4 equiv & 30 & 42 \\
\hline
\end{tabular}

[a] Reaction conditions: Phenylacetylene 1a $\left(0.25 \mathrm{mmol}, 1.0\right.$ equiv), $\mathrm{HP}(\mathrm{O}) \mathrm{Ph}_{2}$ 2a (x equiv), TBACN (x equiv), $\mathrm{Cu}(\mathrm{OTf})_{2}\left(\mathrm{x} \mathrm{mol} \mathrm{\% ),} \mathrm{Mn}(\mathrm{OAc})_{3} .2 \mathrm{H}_{2} \mathrm{O}\right.$ (x equiv), $\mathrm{DMF}(1.5 \mathrm{~mL})$, at $25{ }^{\circ} \mathrm{C}$ under argon atmosphere. [b] Isolated yield. [c] At $50{ }^{\circ} \mathrm{C}$. [d] At $10^{\circ} \mathrm{C}$. [e] In air.

\subsection{General Procedure for the Cyanophosphorylation of Alkynes (GP1)}

To a Schlenk tube equipped with a magnetic stir bar were added $\mathrm{HP}(\mathrm{O}) \mathrm{Ph}_{2}$ 2a $(75.7 \mathrm{mg}, 0.375 \mathrm{mmol})$, $\mathrm{Cu}(\mathrm{OTf})_{2}(18.1 \mathrm{mg}, 0.05 \mathrm{mmol})$, and $\mathrm{Mn}(\mathrm{OAc})_{3} .2 \mathrm{H}_{2} \mathrm{O}(268.0 \mathrm{mg}, 1 \mathrm{mmol})$. Then, the tube was flushed three times with argon gas. This was followed by the injection of a solution of the alkyne $\mathbf{1}(0.25 \mathrm{mmol})$ and TBACN (268.0 $\mathrm{mg}, 1 \mathrm{mmol})$ in DMF (1.5 mL) via syringe. The mixture was allowed to stir at room temperature for $0.5 \mathrm{~h}$. After the completion of reaction, water $(30 \mathrm{~mL})$ was added to the reaction mixture, and then extracted with ethyl acetate $(20 \mathrm{~mL} \times 3)$. The combined organic extract was washed with a saturated sodium bicarbonate solution $(20 \mathrm{~mL} \times 3)$, and dried with anhydrous $\mathrm{Na}_{2} \mathrm{SO}_{4}$, filtered, and concentrated in vacuo. The residue was purified by flash chromatography on silica gel using dichloromethane, ethyl acetate, and petroleum ether (4:1:1) as eluent to afford product $\mathbf{4}$ or $\mathbf{5}$.

Experimental procedure for synthesis of compound 4 a on 1 mmol scale: To a $25 \mathrm{~mL}$ tube equipped with a magnetic stir bar were added $\mathrm{HP}(\mathrm{O}) \mathrm{Ph}_{2}$ 2a $(303.3 \mathrm{mg}, 1.5 \mathrm{mmol}), \mathrm{Cu}(\mathrm{OTf})_{2}(72.3 \mathrm{mg}, 0.2 \mathrm{mmol})$, and $\mathrm{Mn}(\mathrm{OAc})_{3} \cdot 2 \mathrm{H}_{2} \mathrm{O}(1072.0 \mathrm{mg}, 4 \mathrm{mmol})$. This was followed by the injection of a solution of the phenylacetylene 1a (102.0 mg, $1 \mathrm{mmol}$ ) and TBACN (1072.0 mg, $4 \mathrm{mmol})$ in DMF (6 mL) via syringe. The mixture was allowed to 
stir at room temperature for $1 \mathrm{~h}$. After the completion of reaction, water $(80 \mathrm{~mL})$ was added to the reaction mixture, and then extracted with ethyl acetate $(30 \mathrm{~mL} \times 3)$. The combined organic extract was washed with a saturated sodium bicarbonate solution $(30 \mathrm{~mL} \times 3)$, and dried with anhydrous $\mathrm{Na}_{2} \mathrm{SO}_{4}$, filtered, and concentrated in vасио. The residue was purified by flash chromatography on silica gel using dichloromethane, petroleum ether and ethyl acetate $(v / v 4: 1: 1)$ as eluent to afford product $\mathbf{4 a}(230 \mathrm{mg}, 70 \%)$.

\section{Thiocyanophosphorylation of Alkynes}

3.1 Optimization Studies for the Thiocyanophosphorylation of Phenylacetylene (1a)

Table S6. Reaction Development ${ }^{[\mathrm{a}]}$

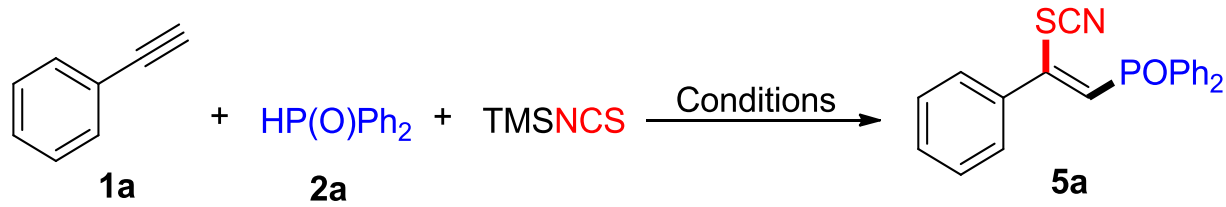

\begin{tabular}{|c|c|c|c|c|c|}
\hline Entry & Catalyst (mol \%) & Oxidant (equiv) & Solvent & Temp. $\left({ }^{\circ} \mathrm{C}\right)$ & Yield $(\%)^{[\mathrm{b}]}$ \\
\hline 1 & $\mathrm{Cu}(\mathrm{OTf})_{2}(20)$ & $\mathrm{Mn}(\mathrm{OAc})_{3}(4)$ & $\mathrm{DMF}$ & 25 & 25 \\
\hline 2 & CuSCN (20) & $\mathrm{Mn}(\mathrm{OAc})_{3}(4)$ & NMP & 25 & 10 \\
\hline 3 & CuSCN (20) & $\mathrm{Mn}(\mathrm{OAc})_{3}(2.5)$ & NMP & 25 & 10 \\
\hline 4 & CuSCN (20) & $\mathrm{AgNO}_{3}(2)$ & NMP & 50 & 34 \\
\hline 5 & CuSCN (20) & $\mathrm{AgNO}_{3}(0.05) / \mathrm{K}_{2} \mathrm{~S}_{2} \mathrm{O}_{8}(2)$ & NMP & 80 & 20 \\
\hline 6 & CuSCN (20) & $\mathrm{Ag}_{2} \mathrm{CO}_{3}(0.1) / \mathrm{Mg}\left(\mathrm{NO}_{3}\right)_{2}(1.5)$ & NMP & 80 & N.D..$^{[\mathrm{c}, \mathrm{d}]}$ \\
\hline 7 & CuSCN (20) & $\mathrm{AgNO}_{3}(0.1) / \mathrm{TBN}(2)$ & NMP & 25 & Trace \\
\hline 8 & CuSCN (20) & $\operatorname{DTBP}(2)$ & NMP & 80 & Trace \\
\hline 9 & CuSCN (20) & TBHP 70\% in $\mathrm{H}_{2} \mathrm{O}$ (4) & NMP & 50 & 45 \\
\hline 10 & CuSCN (20) & TBHP 70\% in $\mathrm{H}_{2} \mathrm{O}$ (4) & NMP & 25 & Trace \\
\hline 11 & CuSCN (20) & TBHP $70 \%$ in $\mathrm{H}_{2} \mathrm{O}$ (4) & NMP & 35 & Trace \\
\hline 12 & CuSCN (20) & TBHP $70 \%$ in $\mathrm{H}_{2} \mathrm{O}$ (4) & NMP & 80 & 74 \\
\hline 13 & CuSCN (20) & TBHP $70 \%$ in $\mathrm{H}_{2} \mathrm{O}$ (4) & NMP & 100 & 45 \\
\hline 14 & CuSCN (20) & TBHP $70 \%$ in $\mathrm{H}_{2} \mathrm{O}$ (2) & NMP & 80 & 45 \\
\hline 15 & CuSCN (20) & TBHP $70 \%$ in $\mathrm{H}_{2} \mathrm{O}(3)$ & NMP & 80 & 68 \\
\hline 16 & CuSCN (20) & TBHP $70 \%$ in $\mathrm{H}_{2} \mathrm{O}(5)$ & NMP & 80 & 72 \\
\hline
\end{tabular}

[a] Reaction conditions: Phenylacetylene 1a $(0.5 \mathrm{mmol}), \mathrm{HP}(\mathrm{O}) \mathrm{Ph}_{2} \mathbf{2 a}$ (2 equiv), TMSNCS 6 (4 equiv), solvent (2 $\mathrm{mL})$ under argon atmosphere for $5 \mathrm{~h}$. [b] Isolated yield. [c] $4 \AA$ A molecular sieve $(0.2 \mathrm{~g})$ was added. [d] N.D. = Not detected. $\mathrm{Mn}(\mathrm{OAc})_{3}$ is in dihydrate form. 
Table S7. Screening of Reaction Media ${ }^{[a]}$

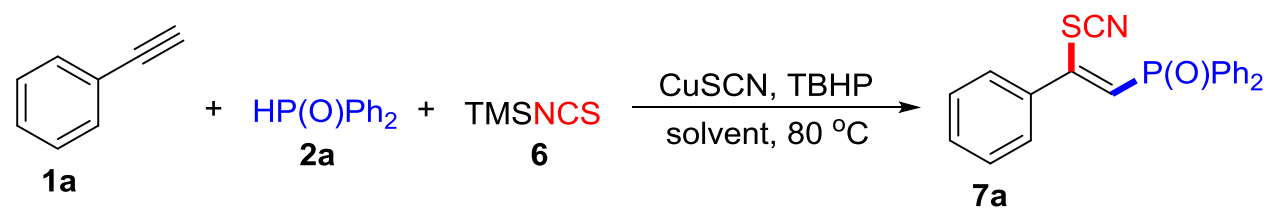

\begin{tabular}{cccc}
\hline Entry & Solvent & Temp. $\left({ }^{\circ} \mathrm{C}\right)$ & Yield $(\%)^{[\mathrm{b}]}$ \\
\hline 1 & DMF & 80 & 67 \\
2 & DMSO & 80 & N.D. ${ }^{[\mathrm{c}]}$ \\
3 & Toluene & 80 & Trace \\
4 & DCE & 80 & Trace \\
5 & $\mathrm{CH}_{3} \mathrm{CN}$ & 80 & 50 \\
6 & EtOH & 80 & Trace \\
7 & $\mathrm{HOAc}$ & 80 & N.D. ${ }^{[\mathrm{c}]}$ \\
8 & THF & 80 & N.D. ${ }^{[\mathrm{c}]}$ \\
9 & Dioxane & 80 & Trace \\
\hline
\end{tabular}

[a] Reaction conditions: Phenylacetylene 1a $(0.5 \mathrm{mmol}), \mathrm{HP}(\mathrm{O}) \mathrm{Ph}_{2} \mathbf{2 a}$ (2 equiv), TMSNCS 6 (4 equiv), TBHP (70\% in $\mathrm{H}_{2} \mathrm{O}, 4$ equiv), solvent $(2 \mathrm{~mL})$ at $80{ }^{\circ} \mathrm{C}$ under argon atmosphere for $5 \mathrm{~h}$. [b] Isolated yields. [c] N.D. = Not detected.

Table S8. Screening of Copper Catalyst ${ }^{[\mathrm{a}]}$

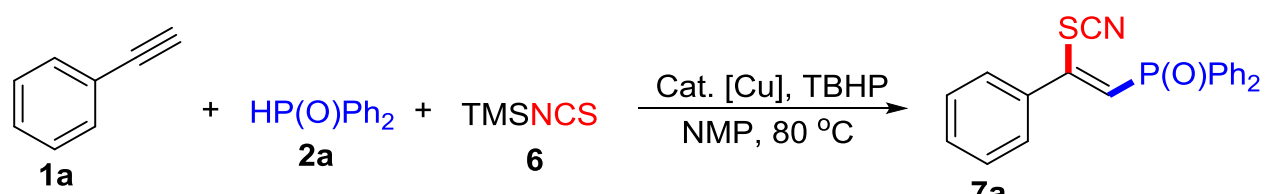

\begin{tabular}{cccc}
\hline Entry & {$[\mathrm{Cu}]$ Catalyst $(\mathrm{mol} \%)$} & Temp. $\left({ }^{\circ} \mathrm{C}\right)$ & Yield $(\%)^{[\mathrm{b}]}$ \\
\hline 1 & $\mathrm{CuSCN}(20)$ & 80 & 74 \\
2 & - & 80 & N.D. ${ }^{[\mathrm{c}]}$ \\
3 & $\mathrm{CuI}(20)$ & 80 & N.D. ${ }^{[\mathrm{c}]}$ \\
4 & $\mathrm{CuBr}(20)$ & 80 & 38 \\
5 & $\mathrm{CuCl}(20)$ & 80 & Trace \\
6 & {$\left[\mathrm{Cu}\left(\mathrm{CH}_{3} \mathrm{CN}\right)_{4}\right] \mathrm{PF}_{6}(20)$} & 80 & 71 \\
7 & $\mathrm{CuO}(20)$ & 80 & N.D. ${ }^{[\mathrm{c}]}$ \\
8 & $\mathrm{CuCl} \cdot 2 \mathrm{H}_{2} \mathrm{O}(20)$ & 80 & Trace \\
9 & $\mathrm{Cu}(\mathrm{OAc})_{2}(20)$ & 80 & 54 \\
10 & $\mathrm{Cu}(\mathrm{OTf})_{2}(20)$ & 80 & 59 \\
11 & $\mathrm{Cu}(\mathrm{acac})_{2}(20)$ & 80 & 68 \\
12 & $\mathrm{CuSO} \cdot 5 \mathrm{H}_{2} \mathrm{O}(20)$ & 80 & 32 \\
13 & $\mathrm{CuBr}_{2}(20)$ & 80 & 20 \\
\hline
\end{tabular}

[a] Reaction conditions: Phenylacetylene $\mathbf{1 a}(0.5 \mathrm{mmol}), \mathrm{HP}(\mathrm{O}) \mathrm{Ph}_{2} \mathbf{2 a}$ (2 equiv), TMSNCS 6 (4 equiv), TBHP (70\% in $\mathrm{H}_{2} \mathrm{O}, 4$ equiv), NMP $(2 \mathrm{~mL})$ at $80{ }^{\circ} \mathrm{C}$ under argon atmosphere for $5 \mathrm{~h}$. [b] Isolated yields. [c] N.D. $=$ Not detected. 
Table S9. Screening of Relative Ratio of Reactants/Catalyst and Temperature ${ }^{[\mathrm{a}]}$

\begin{tabular}{|c|c|c|c|c|c|c|}
\hline Entry & $\begin{array}{c}\text { Amount of } \\
\mathrm{CuSCN}\end{array}$ & $\begin{array}{c}\text { Amount of } \\
\text { TBHP }\end{array}$ & $\begin{array}{l}\text { Amount of } \\
\mathrm{HP}(\mathrm{O}) \mathrm{Ph}_{2}\end{array}$ & $\begin{array}{l}\text { Amount of } \\
\text { TMSNCS }\end{array}$ & Time (h) & $\begin{array}{l}\text { Yield } \\
(\%)^{[\mathrm{b}]}\end{array}$ \\
\hline 1 & $20 \mathrm{~mol} \%$ & 4 equiv & 2.0 equiv & 4 equiv & 5 & 74 \\
\hline $2^{[\mathrm{c}]}$ & $20 \mathrm{~mol} \%$ & 4 equiv & 2.0 equiv & 4 equiv & 5 & 62 \\
\hline $3^{[\mathrm{d}]}$ & $20 \mathrm{~mol} \mathrm{\%}$ & 4 equiv & 2.0 equiv & 4 equiv & 5 & 74 \\
\hline 4 & $20 \mathrm{~mol} \%$ & 5 equiv & 2.0 equiv & 5 equiv & 5 & 74 \\
\hline 5 & $20 \mathrm{~mol} \%$ & 3 equiv & 2.0 equiv & 3 equiv & 5 & 60 \\
\hline 6 & $10 \mathrm{~mol} \%$ & 4 equiv & 2.0 equiv & 4 equiv & 5 & 68 \\
\hline 7 & $20 \mathrm{~mol} \%$ & 4 equiv & 2.0 equiv & 4 equiv & 5 & 65 \\
\hline 8 & $20 \mathrm{~mol} \%$ & 4 equiv & 1.5 equiv & 4 equiv & 5 & 58 \\
\hline 9 & $20 \mathrm{~mol} \%$ & 4 equiv & 2.5 equiv & 4 equiv & 5 & 74 \\
\hline 10 & $20 \mathrm{~mol} \%$ & 4 equiv & 2.0 equiv & 3 equiv & 5 & 56 \\
\hline 11 & $20 \mathrm{~mol} \%$ & 4 equiv & 2.0 equiv & 2 equiv & 5 & 42 \\
\hline 12 & $20 \mathrm{~mol} \%$ & 4 equiv & 2.0 equiv & 4 equiv & 1 & 20 \\
\hline 13 & $20 \mathrm{~mol} \%$ & 4 equiv & 2.0 equiv & 4 equiv & 2 & 56 \\
\hline 14 & $20 \mathrm{~mol} \%$ & 4 equiv & 2.0 equiv & 4 equiv & 3 & 65 \\
\hline 15 & $20 \mathrm{~mol} \%$ & 4 equiv & 2.0 equiv & 4 equiv & 4 & 65 \\
\hline 16 & $20 \mathrm{~mol} \%$ & 4 equiv & 2.0 equiv & 4 equiv & 6 & 70 \\
\hline 17 & $20 \mathrm{~mol} \%$ & 4 equiv & 2.0 equiv & 4 equiv & 5 & $42^{[\mathrm{e}]}$ \\
\hline
\end{tabular}

[a] Reaction conditions: Phenylacetylene 1a $\left(0.5 \mathrm{mmol}, 1.0\right.$ equiv), $\mathrm{HP}(\mathrm{O}) \mathrm{Ph}_{2} 2 \mathrm{a}$ (x equiv), TMSNCS 6 (x equiv), $\mathrm{CuSCN}$ (x mol \%), TBHP 70\% in $\mathrm{H}_{2} \mathrm{O}$ (x equiv), NMP $\left(2 \mathrm{~mL}\right.$ ) at $80{ }^{\circ} \mathrm{C}$ under argon atmosphere. [b] Isolated yield. [c] At $70{ }^{\circ} \mathrm{C}$. [d] At $90^{\circ} \mathrm{C}$. [e] In air.

\subsection{General Procedure for the Thiocyanophosphorylation of Alkynes (GP2)}

To a Schlenk tube equipped with a magnetic stir bar were added $\mathrm{HP}(\mathrm{O}) \mathrm{Ph}_{2} \mathbf{2 a}(202.2 \mathrm{mg}, 1 \mathrm{mmol})$ and CuSCN (12.1 mg, $0.1 \mathrm{mmol})$. Then, the tube was flushed three times with argon gas. This was followed by the sequential addition of a solution of the alkyne $1(0.5 \mathrm{mmol})$ in NMP $(2 \mathrm{~mL})$, TBHP $70 \%$ in $\mathrm{H}_{2} \mathrm{O}(180 \mathrm{mg}, 2$ mmol), and TMSNCS $6(262.5 \mathrm{mg}, 2 \mathrm{mmol})$ via syringe. The mixture was allowed to stir at $80{ }^{\circ} \mathrm{C}$ in a heating mantle for $5 \mathrm{~h}$. After the completion of reaction, water $(60 \mathrm{~mL})$ was added to the reaction mixture, and then extracted with ethyl acetate $(20 \mathrm{~mL} \times 3)$. The combined organic extract was washed with a saturated sodium bicarbonate solution $(20 \mathrm{~mL} \times 3)$, and dried with anhydrous $\mathrm{Na}_{2} \mathrm{SO}_{4}$, filtered, and concentrated in vacuo. The residue was purified by flash chromatography on silica gel using ethyl acetate and petroleum ether $(v / v 1: 5)$ as eluent to afford product 7. 
Experimental procedure for synthesis of compound 7a on 1 mmol scale: To a $25 \mathrm{~mL}$ tube equipped with a magnetic stir bar were added $\mathrm{HP}(\mathrm{O}) \mathrm{Ph}_{2} \mathbf{2 a}(404.4 \mathrm{mg}, 2 \mathrm{mmol})$ and $\mathrm{CuSCN}(24.2 \mathrm{mg}, 0.2 \mathrm{mmol})$. Then, the tube was flushed three times with argon gas. This was followed by the sequential addition of a solution of phenylacetylene 1a (102 mg, $1 \mathrm{mmol})$ in NMP (4 mL), TBHP 70\% in $\mathrm{H}_{2} \mathrm{O}(360 \mathrm{mg}, 4 \mathrm{mmol})$, and TMSNCS 6 (525.0 mg, $4 \mathrm{mmol}$ ) via syringe. The mixture was allowed to stir at $80{ }^{\circ} \mathrm{C}$ in a heating mantle for $5 \mathrm{~h}$. After the completion of reaction, water $(100 \mathrm{~mL})$ was added to the reaction mixture, and then extracted with ethyl acetate $(30 \mathrm{~mL} \times 3)$. The combined organic extract was washed with a saturated sodium bicarbonate solution $(30 \mathrm{~mL} \times 3)$, and dried with anhydrous $\mathrm{Na}_{2} \mathrm{SO}_{4}$, filtered, and concentrated in vacuo. The residue was purified by flash chromatography on silica gel using ethyl acetate and petroleum ether $(v / v 1: 5)$ as eluent to afford product $7 \mathbf{a}$ (267 $\mathrm{mg}, 74 \%)$.

\section{Chlorophosphorylation of Alkynes}

\subsection{Optimization Studies for the Chlorophosphorylation of Phenylacetylene (1a)}

Table S10. Reaction Development ${ }^{[\mathrm{a}]}$

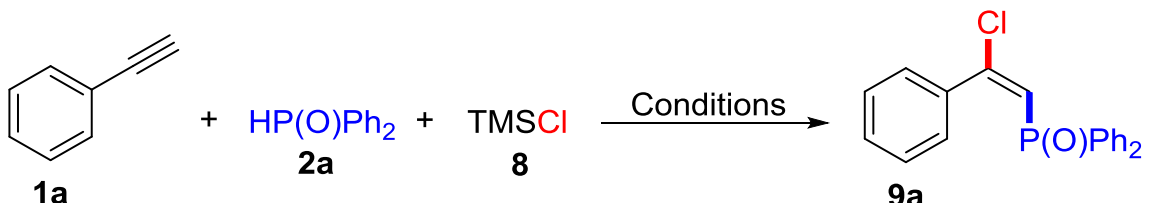

\begin{tabular}{|c|c|c|c|c|c|}
\hline Entry & Catalyst (mol \%) & Oxidant (equiv) & Solvent & Temp. $\left({ }^{\circ} \mathrm{C}\right)$ & Yield $(\%)^{[\mathrm{b}]}$ \\
\hline 1 & $\mathrm{Cu}(\mathrm{OTf})_{2}(20)$ & $\mathrm{Mn}(\mathrm{OAc})_{3}(4)$ & DMF & 25 & 62 \\
\hline 2 & CuSCN (20) & TBHP (4) & NMP & 80 & 20 \\
\hline 3 & $\mathrm{CuCl}(20)$ & $\mathrm{Mn}(\mathrm{OAc})_{3}(3)$ & NMP & 25 & 70 \\
\hline 4 & $\mathrm{CuCl}(20)$ & $\mathrm{AgNO}_{3}(0.1) / \mathrm{TBN}(2)$ & NMP & 25 & Trace \\
\hline 5 & $\mathrm{CuCl}(20)$ & $\mathrm{AgNO}_{3}(0.05) / \mathrm{K}_{2} \mathrm{~S}_{2} \mathrm{O}_{8}(2)$ & NMP & 25 & N.R. ${ }^{[\mathrm{c}]}$ \\
\hline 6 & $\mathrm{CuCl}(20)$ & $\mathrm{AgNO}_{3}(1)$ & NMP & 80 & 20 \\
\hline 7 & $\mathrm{CuCl}(20)$ & $\operatorname{AgOAc}(3)$ & NMP & 100 & N.R. ${ }^{[\mathrm{c}]}$ \\
\hline 8 & $\mathrm{CuCl}(20)$ & $\operatorname{DTBP}(1.5)$ & NMP & 80 & Trace \\
\hline 9 & $\mathrm{CuCl}(20)$ & TBHP $70 \%$ in $\mathrm{H}_{2} \mathrm{O}$ ( 4) & NMP & 60 & 26 \\
\hline 10 & $\mathrm{CuCl}(20)$ & $\mathrm{Ag}_{2} \mathrm{O}(3)$ & NMP & 120 & Trace \\
\hline 11 & $\mathrm{CuCl}(20)$ & $\mathrm{Ag}_{2} \mathrm{CO}_{3}(0.1) / \mathrm{Mg}\left(\mathrm{NO}_{3}\right)_{2}(0.3)$ & NMP & 90 & 48 \\
\hline 12 & $\mathrm{CuCl}(20)$ & $\mathrm{Ag}_{2} \mathrm{CO}_{3}(0.1)$ & NMP & 110 & N.R. ${ }^{[c]}$ \\
\hline 13 & $\mathrm{CuCl}(20)$ & $\mathrm{Ag}_{2} \mathrm{CO}_{3}(0.1) / \mathrm{Mg}\left(\mathrm{NO}_{3}\right)_{2}(0.1)$ & NMP & 110 & 16 \\
\hline 14 & $\mathrm{CuCl}(20)$ & $\mathrm{Ag}_{2} \mathrm{CO}_{3}(0.1) / \mathrm{Mg}\left(\mathrm{NO}_{3}\right)_{2}(0.3)$ & NMP & 110 & 68 \\
\hline 15 & $\mathrm{CuCl}(20)$ & $\mathrm{Ag}_{2} \mathrm{CO}_{3}(0.1) / \mathrm{Mg}\left(\mathrm{NO}_{3}\right)_{2}(0.5)$ & NMP & 110 & 58 \\
\hline 16 & $\mathrm{CuCl}(20)$ & $\mathrm{Ag}_{2} \mathrm{CO}_{3}(0.1) / \mathrm{Mg}\left(\mathrm{NO}_{3}\right)_{2}(0.8)$ & NMP & 110 & 46 \\
\hline
\end{tabular}

[a] Reaction conditions: Phenylacetylene 1a $(0.5 \mathrm{mmol}), \mathrm{HP}(\mathrm{O}) \mathrm{Ph}_{2}$ 2a (2 equiv), TMSCl 8 (3 equiv), solvent (2 mL) for $5 \mathrm{~h}$ under argon atmosphere. [b] Isolated yield. [c] N.R. = No reaction. $\mathrm{Mn}(\mathrm{OAc})_{3}$ is in dihydrate form. 
Table S11. Screening of Copper Sources ${ }^{[\mathrm{a}]}$

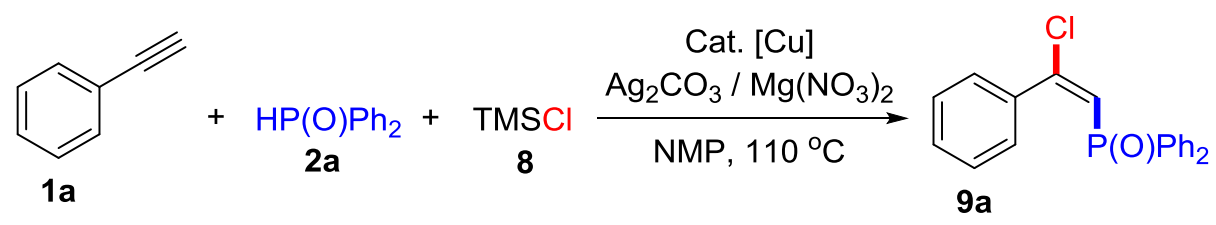

\begin{tabular}{cccc}
\hline Entry & {$[\mathrm{Cu}]$ Catalyst $(\mathrm{mol} \%)$} & Temp. $\left({ }^{\circ} \mathrm{C}\right)$ & ${\text { Yield }(\%)^{[\mathrm{b}]}}^{\circ}$ \\
\hline 1 & $\mathrm{CuCl}(20)$ & 110 & 68 \\
2 & {$\left[\mathrm{Cu}\left(\mathrm{CH}_{3} \mathrm{CN}\right)_{4}\right] \mathrm{PF}_{6}(20)$} & 110 & Trace \\
3 & $\mathrm{CuCl}_{2} .2 \mathrm{H}_{2} \mathrm{O}(20)$ & 110 & 35 \\
4 & $\mathrm{Cu}(\mathrm{OAc})_{2}(20)$ & 110 & 65 \\
5 & $\mathrm{Cu}(\mathrm{OTf})_{2}(20)$ & 110 & 67 \\
6 & $\mathrm{Cu}(\mathrm{acac})_{2}(20)$ & 110 & 42 \\
7 & - & 110 & N.D. ${ }^{[\mathrm{cc}]}$ \\
\hline
\end{tabular}

[a] Reaction conditions: Phenylacetylene 1a ( $0.5 \mathrm{mmol}), \mathrm{HP}(\mathrm{O}) \mathrm{Ph}_{2} \mathbf{2 a}$ (2 equiv), TMSCl 8 (3 equiv), $\mathrm{Ag}_{2} \mathrm{CO}_{3}(10$ mol \%), $\mathrm{Mg}\left(\mathrm{NO}_{3}\right)_{2}$ (0.3 equiv), [Cu] catalyst $(20 \mathrm{~mol} \%)$, NMP $(2 \mathrm{~mL})$ for $5 \mathrm{~h}$ under argon atmosphere. [b] Isolated yield. [c] N.D. $=$ Not detected.

Table S12. Screening of Reaction Media ${ }^{[a]}$

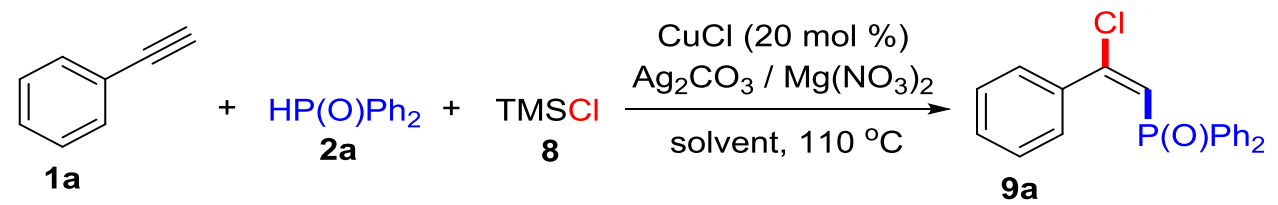

\begin{tabular}{cccc}
\hline Entry & Solvent & Temp. $\left({ }^{\circ} \mathrm{C}\right)$ & ${\text { Yield }(\%)^{[\mathrm{b}]}}^{-}$ \\
\hline 1 & $\mathrm{DMF}$ & 110 & 42 \\
2 & $\mathrm{DMSO}$ & 110 & 45 \\
3 & $\mathrm{H}_{2} \mathrm{O}$ & 110 & N.D. ${ }^{[\mathrm{cc}]}$ \\
4 & $\mathrm{DCE}$ & 110 & Trace \\
5 & $\mathrm{CH}_{3} \mathrm{CN}$ & 110 & 18 \\
6 & $\mathrm{EtOH}$ & 110 & Trace \\
7 & $\mathrm{HOAc}$ & 110 & Trace \\
8 & $\mathrm{THF}$ & 110 & N.D. \\
9 & Dioxane & 110 & Trace \\
\hline
\end{tabular}

[a] Reaction conditions: Phenylacetylene $1 \mathbf{a}(0.5 \mathrm{mmol}), \mathrm{HP}(\mathrm{O}) \mathrm{Ph}_{2}$ 2a (2 equiv), TMSCl 8 (3 equiv), $\mathrm{Ag}_{2} \mathrm{CO}_{3}(10$ mol \%), $\mathrm{Mg}\left(\mathrm{NO}_{3}\right)_{2}$ (0.3 equiv), $\mathrm{CuCl}(20 \mathrm{~mol} \%)$, solvent $(2 \mathrm{~mL})$ for $5 \mathrm{~h}$ under argon atmosphere. [b] Isolated yield. [c] N.D. $=$ Not detected. 
Table 13. Screening of Chlorination Source ${ }^{[a]}$

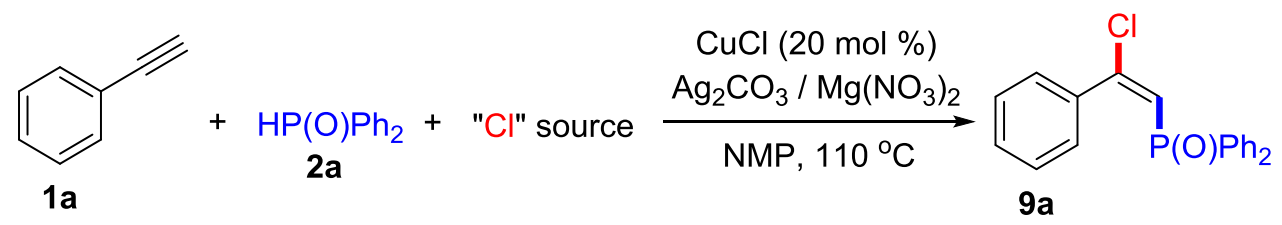

\begin{tabular}{cccc}
\hline Entry & "Cl” Source & Temp. $\left({ }^{\circ} \mathrm{C}\right)$ & Yield $(\%)^{[\mathrm{b}]}$ \\
\hline 1 & $\mathrm{KCl}$ & 110 & N.D. ${ }^{[\mathrm{c}]}$ \\
2 & $\mathrm{NaCl}$ & 110 & Trace \\
3 & $\mathrm{CuCl}$ & 110 & Trace \\
4 & $\mathrm{NCS}$ & 110 & N.R. ${ }^{[\mathrm{d}]}$ \\
\hline
\end{tabular}

[a] Reaction conditions: Phenylacetylene 1a $(0.5 \mathrm{mmol}), \mathrm{HP}(\mathrm{O}) \mathrm{Ph}_{2} \mathbf{2 a}$ (2 equiv), chlorination source (3 equiv), $\mathrm{Ag}_{2} \mathrm{CO}_{3}(10 \mathrm{~mol} \%), \mathrm{Mg}\left(\mathrm{NO}_{3}\right)_{2}$ (0.3 equiv), $\mathrm{CuCl}$ (20 mol \%), NMP (2 mL) for $5 \mathrm{~h}$ under argon atmosphere. [b] Isolated yield. [c] N.D. $=$ Not detected. $[\mathrm{d}]$ N.R. $=$ No reaction .

Table S14. Screening of Relative Ratios of Reactants/Catalyst and Temperature ${ }^{[\mathrm{a}]}$

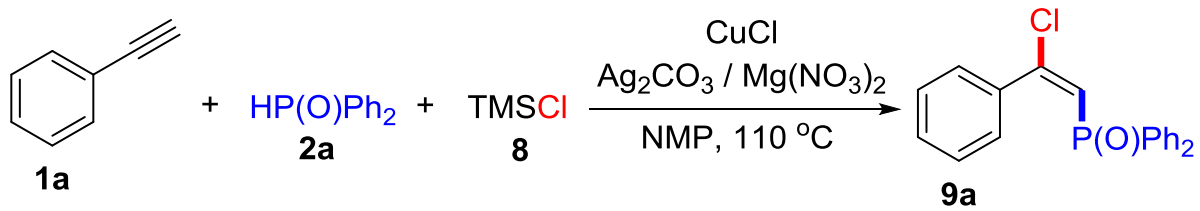

\begin{tabular}{ccccccc}
\hline Entry & $\begin{array}{c}\text { Amount of } \\
\mathrm{CuCl}\end{array}$ & $\begin{array}{c}\text { Amount of } \\
\mathrm{HP}(\mathrm{O}) \mathrm{Ph}_{2}\end{array}$ & $\begin{array}{c}\text { Amount of } \\
\text { TMSCl }\end{array}$ & Temp. $\left({ }^{\circ} \mathrm{C}\right)$ & Time $(\mathrm{h})$ & $\begin{array}{c}\text { Yield } \\
(\%)\end{array}$ \\
\hline $\mathbf{1}$ & $\mathbf{2 0}$ mol \% & $\mathbf{2 . 0}$ equiv & $\mathbf{3}$ equiv & $\mathbf{1 1 0}$ & $\mathbf{5}$ & $\mathbf{6 8}$ \\
2 & $20 \mathrm{~mol} \%$ & 2.0 equiv & 3 equiv & 25 & 5 & Trace \\
3 & $20 \mathrm{~mol} \%$ & 2.0 equiv & 3 equiv & 50 & 5 & 29 \\
4 & $20 \mathrm{~mol} \%$ & 2.0 equiv & 3 equiv & 70 & 5 & 39 \\
5 & $20 \mathrm{~mol} \%$ & 2.0 equiv & 3 equiv & 90 & 5 & 48 \\
6 & $20 \mathrm{~mol} \%$ & 2.0 equiv & 3 equiv & 130 & 5 & 68 \\
7 & $20 \mathrm{~mol} \%$ & 2.0 equiv & 2 equiv & 110 & 5 & 45 \\
8 & $20 \mathrm{~mol} \%$ & 2.0 equiv & 4 equiv & 110 & 5 & 68 \\
9 & $20 \mathrm{~mol} \%$ & 2.0 equiv & 3 equiv & 110 & 0.5 & 25 \\
10 & $20 \mathrm{~mol} \%$ & 2.0 equiv & 3 equiv & 110 & 1 & 46 \\
11 & $20 \mathrm{~mol} \%$ & 2.0 equiv & 3 equiv & 110 & 2 & 50 \\
12 & $20 \mathrm{~mol} \%$ & 2.0 equiv & 3 equiv & 110 & 3 & 58 \\
13 & $20 \mathrm{~mol} \%$ & 2.0 equiv & 3 equiv & 110 & 6 & 68 \\
14 & $10 \mathrm{~mol} \%$ & 2.0 equiv & 3 equiv & 110 & 5 & 52 \\
15 & $30 \mathrm{~mol} \%$ & 2.0 equiv & 3 equiv & 110 & 5 & 68 \\
16 & $20 \mathrm{~mol} \%$ & 1.5 equiv & 3 equiv & 110 & 5 & 56 \\
17 & $20 \mathrm{~mol} \%$ & 2.5 equiv & 3 equiv & 110 & 5 & 68 \\
18 & $20 \mathrm{~mol} \%$ & 2.0 equiv & 3 equiv & 110 & 5 & $60^{[\mathrm{c}]}$ \\
\hline
\end{tabular}

[a] Reaction conditions: Phenylacetylene $\mathbf{1 a}(0.5 \mathrm{mmol}), \mathrm{HP}(\mathrm{O}) \mathrm{Ph}_{2} \mathbf{2 a}$ (2 equiv), TMSCl 8 (3 equiv), $\mathrm{Ag}_{2} \mathrm{CO}_{3}$ $(10 \mathrm{~mol} \%), \mathrm{Mg}\left(\mathrm{NO}_{3}\right)_{2}$ (0.3 equiv), $\mathrm{CuCl}(20 \mathrm{~mol} \%), \mathrm{NMP}(2 \mathrm{~mL})$ for $5 \mathrm{~h}$ under argon atmosphere. [b] Isolated yield. [c] In air. 


\subsection{General Procedure for the Chlorophosphorylation of Alkynes (GP3)}

To a Schlenk tube equipped with a magnetic stir bar were added $\mathrm{HP}(\mathrm{O}) \mathrm{Ph}_{2} \mathbf{2 a}(202.2 \mathrm{mg}, 1 \mathrm{mmol}), \mathrm{Ag}_{2} \mathrm{CO}_{3}$ (13.8 $\mathrm{mg}, 0.05 \mathrm{mmol}), \mathrm{Mg}\left(\mathrm{NO}_{3}\right)_{2}(22.2 \mathrm{mg}, 0.15 \mathrm{mmol})$ and $\mathrm{CuCl}(9.9 \mathrm{mg}, 0.1 \mathrm{mmol})$. Then, the tube was flushed three times with argon gas. This was followed by the sequential addition of a solution of the alkyne $\mathbf{1}(0.5 \mathrm{mmol})$ in NMP (2 mL) and TMSCl $(162.9 \mathrm{mg}, 1.5 \mathrm{mmol})$ via syringe. The mixture was allowed to stir at $110{ }^{\circ} \mathrm{C}$ in a heating mantle for $5 \mathrm{~h}$. After the completion of reaction, water $(60 \mathrm{~mL})$ was added to the reaction mixture, and then extracted with ethyl acetate $(20 \mathrm{~mL} \times 3)$. The combined organic extract was washed with a saturated sodium bicarbonate solution $(20 \mathrm{~mL} \times 3)$, and dried with anhydrous $\mathrm{Na}_{2} \mathrm{SO}_{4}$, filtered, and concentrated in vacuo. The residue was purified by flash chromatography on silica gel using dichloromethane, petroleum ether and ethyl acetate $(4: 1: 1)$ as eluent to afford product 9.

Experimental procedure for synthesis of compound 9a on 1 mmol scale: To a $25 \mathrm{~mL}$ tube equipped with a magnetic stir bar was added $\mathrm{HP}(\mathrm{O}) \mathrm{Ph}_{2} \mathbf{2 a}(404.4 \mathrm{mg}, 2 \mathrm{mmol}), \mathrm{Ag}_{2} \mathrm{CO}_{3}(27.6 \mathrm{mg}, 0.1 \mathrm{mmol}), \mathrm{Mg}\left(\mathrm{NO}_{3}\right)_{2}(44.4 \mathrm{mg}$, $0.3 \mathrm{mmol})$ and $\mathrm{CuCl}(19.8 \mathrm{mg}, 0.2 \mathrm{mmol})$. Then, the tube was flushed three times with argon gas. This was followed by the sequential addition of a solution of the phenylacetylene 1a $(102.0 \mathrm{mg}, 1 \mathrm{mmol})$ in NMP (4 mL) and TMSCl (325.8 mg, $3 \mathrm{mmol}$ ) via syringe. The mixture was allowed to stir at $110^{\circ} \mathrm{C}$ in a heating mantle for $5 \mathrm{~h}$. After the completion of reaction, water $(100 \mathrm{~mL})$ was added to the reaction mixture, and then extracted with ethyl acetate $(30 \mathrm{~mL} \times 3)$. The combined organic extract was washed with a saturated sodium bicarbonate solution (30 $\mathrm{mL} \times 3$ ), and dried with anhydrous $\mathrm{Na}_{2} \mathrm{SO}_{4}$, filtered, and concentrated in vacuo. The residue was purified by flash chromatography on silica gel using dichloromethane, petroleum ether and ethyl acetate $(v / v 4: 1: 1)$ as eluent to afford product 9a $(219 \mathrm{mg}, 65 \%)$.

\section{Mechanistic Studies}

Some control experiments were carried out in order to understand the mechanistic pathway(s) involved in the cyano-, thiocyano-, and chloro-phosphorylation of alkynes.

\subsection{Cyanophosphorylation of Alkynes}

\section{Radical Trapping Experiments with TEMPO or BHT}

The experiments were carried out according to the general procedure (GP1) using phenylacetylene 1a (31.2 mg, $0.25 \mathrm{mmol}, 1.0$ equiv.), $\mathrm{HP}(\mathrm{O}) \mathrm{Ph}_{2} \mathbf{2 a}(75.75 \mathrm{mg}, 0.375 \mathrm{mmol}, 1.5$ equiv), TBACN (268 mg, $1 \mathrm{mmol})$, and radical trapping agent $(1.5 \mathrm{mmol}, 6$ equiv). TLC monitoring indicates that there was no product $4 \mathbf{a}$ formation in the presence of either TEMPO or BHT (Scheme S1a, b). The formation of compound $\mathbf{1 0}$ indicates the involvement of phosphorus-centered radical intermediate (Scheme S1b). 


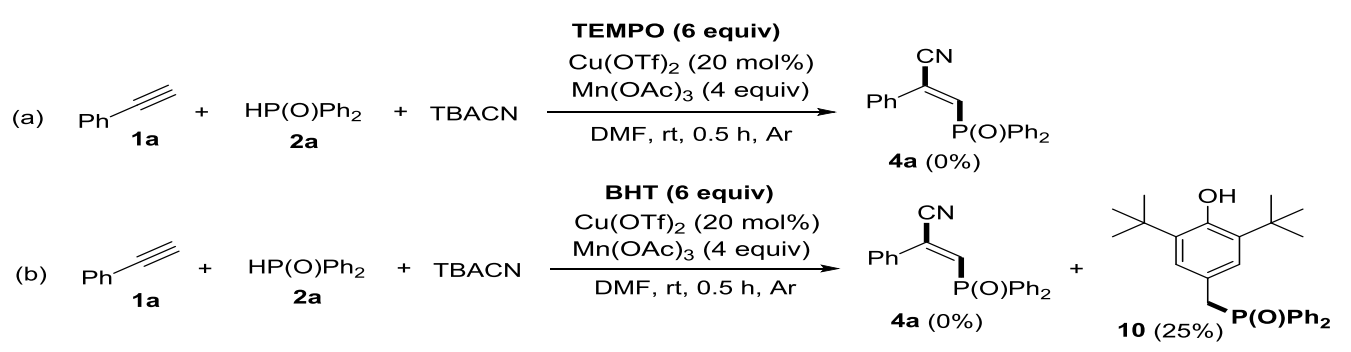

Scheme S1. Radical Trapping Experiments with TEMPO or BHT

\section{Experiments to probe the intermediacy of vinyl cation using TBAB:}

Some experiments were carried out to probe the presence of vinyl cation as putative reaction intermediate resulting from a $\mathrm{Mn}^{\mathrm{III}}$-mediated oxidation of vinyl radical intermediate. These experiments were carried out according to the general procedure (GP1) using phenylacetylene 1a (31.2 mg, $0.25 \mathrm{mmol}, 1.0$ equiv), $\mathrm{HP}(\mathrm{O}) \mathrm{Ph}_{2}$ 2a (76 mg, $0.375 \mathrm{mmol}, 1.5$ equiv), TBACN (268 mg, $1 \mathrm{mmol}$ ), and TBAB (322 mg, $1.0 \mathrm{mmol}, 4$ equiv). Variations were made to the conditions as noted in the reactions shown in Scheme S2 below.

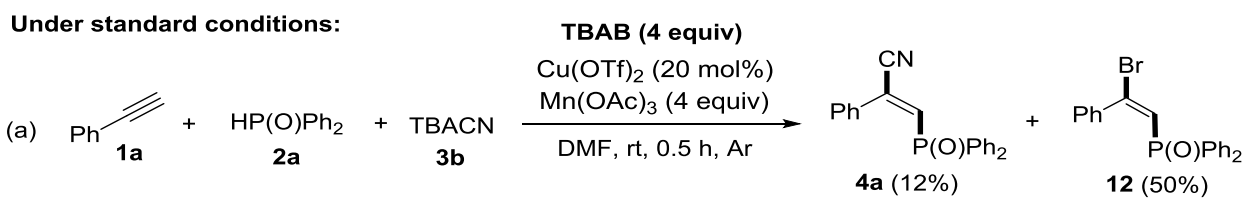

Without $\mathrm{Cu}(\mathrm{OTf})_{2}$ :

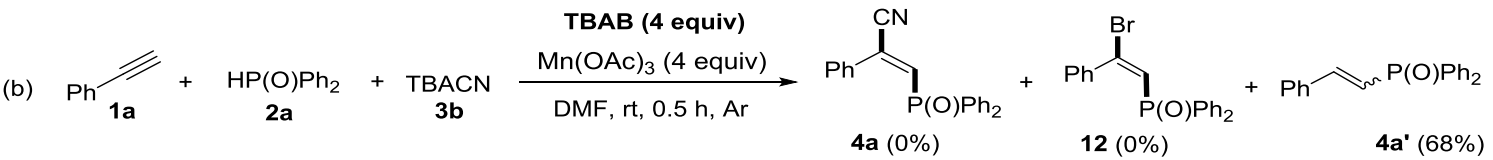

Without $\mathrm{Cu}(\mathrm{OTf})_{2}$ and TBACN:

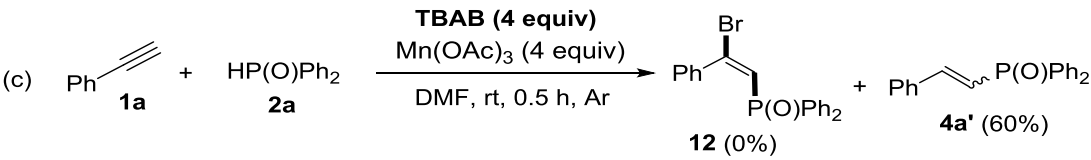

Without $\mathrm{Cu}(\mathrm{OTf})_{2}$ and TBAB:

(d) $\mathrm{Ph}_{\mathbf{1 a}}+\underset{\mathbf{2 a}}{\mathrm{HP}(\mathrm{O}) \mathrm{Ph}_{2}}+\underset{\mathbf{3 b}}{\mathrm{TBACN}} \underset{\mathrm{DMF}, \mathrm{rt}, 0.5 \mathrm{~h}, \mathrm{Ar}}{\stackrel{\mathrm{Mn}(\mathrm{OAc})_{3}(4 \text { equiv })}{\longrightarrow}}$

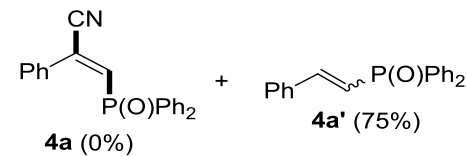

Scheme S2. Probing the intermediacy of vinyl cation

A mixture of $4 \mathbf{a}$ and bromophosphorylation product 12 were obtained from the reaction involving TBAB (Scheme S2a). However, other results show that the copper catalyst was necessary for the formation of $\mathbf{4 a}$ and $\mathbf{1 2}$ (Scheme S2b-d). Thus, in the absence of a copper catalyst, hydrophosphorylation product $\mathbf{4 a}$ ' was exclusively formed. These results clearly rule out any possibility of a $\mathrm{Mn}^{\mathrm{III}}$-mediated oxidation of vinyl radical to vinyl cation intermediate. 


\subsection{Thiocyanophosphorylation of Alkynes}

\section{Radical Trapping Experiments with TEMPO or BHT:}

The experiments were carried out according to the general procedure (GP2) using phenylacetylene 1a $(0.5$ mmol, 1.0 equiv.), $\mathrm{HP}(\mathrm{O}) \mathrm{Ph}_{2} \mathbf{2 a}(101 \mathrm{mg}, 1 \mathrm{mmol}, 2$ equiv), TMSNCS 6 (2 mmol, 4 equiv), and radical trapping agent ( 3 mmol, 6 equiv). TLC monitoring indicates that there was no formation of product $7 \mathbf{a}$ in the presence of either TEMPO or BHT (Scheme S3a, b). Moreover, the isolation of compound $\mathbf{1 0}$ implicates the involvement of phosphorus-centered radical intermediate (Scheme S3b).

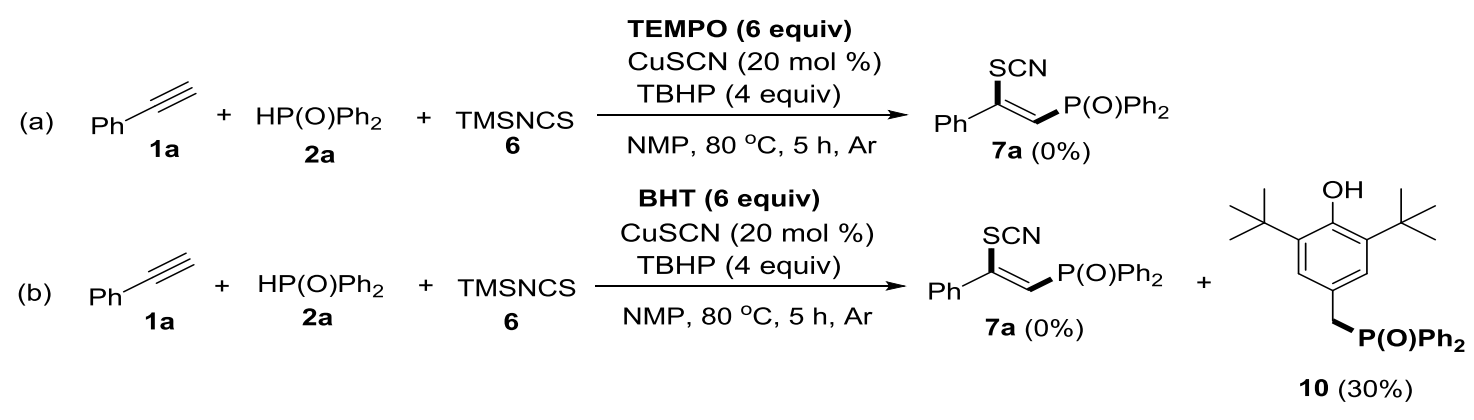

Scheme S3. Radical Trapping Experiments with TEMPO or BHT

\section{Experiments to probe the intermediacy of vinyl cation using TBAB:}

A series of control experiments were carried out to probe the formation of vinyl cation as putative reaction intermediate resulting from a TBHP-mediated oxidation of vinyl radical intermediate. These experiments were carried out according to the general procedure (GP2) using phenylacetylene 1a $\left(0.5 \mathrm{mmol}, 1.0\right.$ equiv), $\mathrm{HP}(\mathrm{O}) \mathrm{Ph}_{2}$ 2a (101 mg, 1 mmol, 2 equiv), TMSNCS 6 ( 2 mmol, 4 equiv), and TBAB (322 mg, 1.0 mmol, 4 equiv). Variations were made to the conditions as noted in the reactions shown in Scheme S4 below. 
Under standard conditions:

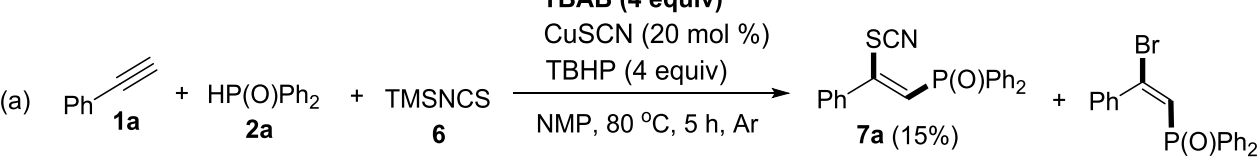

$12(60 \%)$

Without CuSCN:

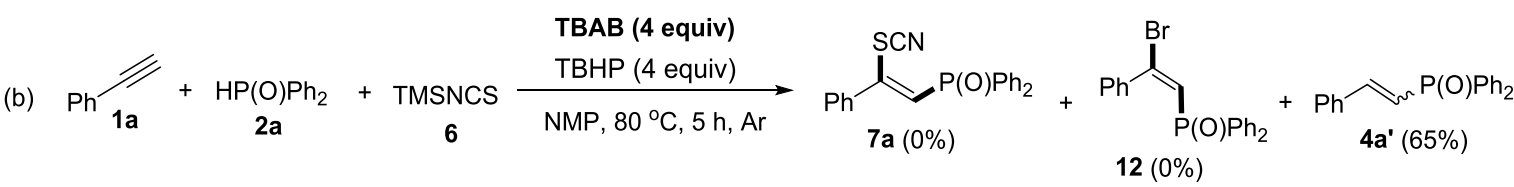

Without CuSCN and TMSNCS:

(c)

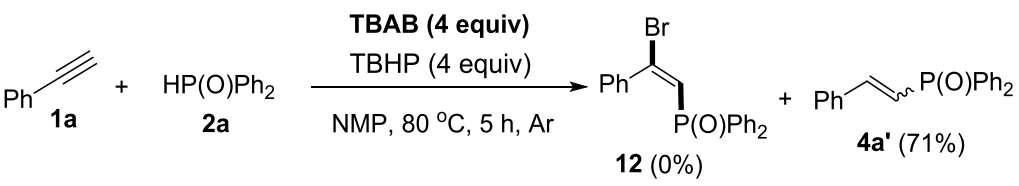

Without CuSCN and TBAB:

(d)

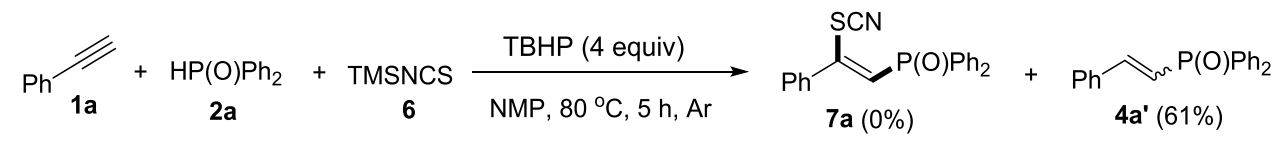

Scheme S4. Probing the intermediacy of vinyl cation

In the presence of $\mathrm{TBAB}$, a mixture of product $7 \mathbf{a}$ and compound $\mathbf{1 2}$ were obtained from the reaction (Scheme S4a). However, we found that the copper catalyst was necessary for the formation of compounds $7 \mathbf{a}$ and $\mathbf{1 2}$ (Scheme S4b-d). Thus, in the absence of a copper catalyst, hydrophosphorylation product 4a' was exclusively formed. These results rule out any possibility of a TBHP-mediated oxidation of vinyl radical to vinyl cation intermediate.

\subsection{Chlorophosphorylation of Alkynes}

\section{Radical Trapping Experiments:}

The experiments were carried out according to the general procedure (GP3) using phenylacetylene 1a $(0.5$ mmol, 1.0 equiv), $\mathrm{HP}(\mathrm{O}) \mathrm{Ph}_{2} \mathbf{2 a}(1 \mathrm{mmol}, 2$ equiv), TMSCl $\mathbf{8}$ (1.5 mmol, 3 equiv), and radical trapping agent (3 mmol, 6 equiv). TLC monitoring indicates that there was no product 9a formation in the presence of radical scavenger TEMPO, while the formation of 9a was greatly supressed in the presence of radical scavengers such as BHT and DPE(1,2-diphenylethylene) (Scheme S5a-c). The isolation of a DPE-adduct 11 confirms the involvement of phosphorus-centered radical intermediate (Scheme S5c). 


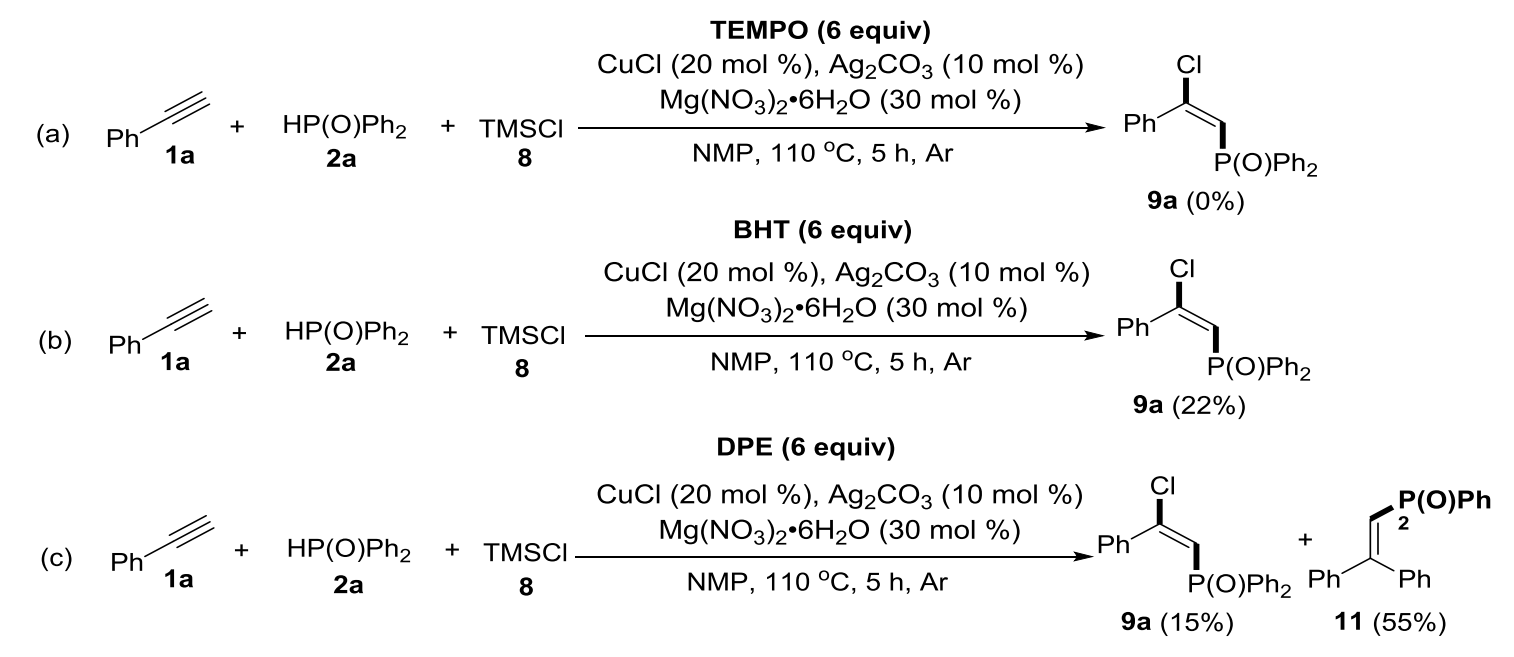

Scheme S5. Radical Trapping Experiments

\section{Experiments to probe the intermediacy of vinyl cation using TBAB:}

Some experiments were carried out to probe the presence of vinyl cation as putative reaction intermediate. These experiments were carried out according to the general procedure (GP3) using phenylacetylene $1 \mathbf{a}(0.5 \mathrm{mmol}, 1.0$ equiv), $\mathrm{HP}(\mathrm{O}) \mathrm{Ph}_{2} \mathbf{2 a}(1 \mathrm{mmol}, 2$ equiv), TMSCl 8 (1.5 mmol, 3 equiv), and TBAB (1.0 mmol, 4 equiv). Variations were made to the conditions as noted in the reactions shown in Scheme S6 below.

Under standard conditions:

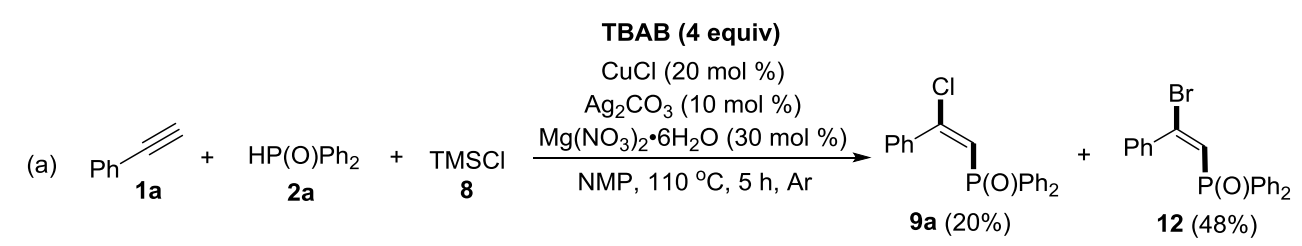

Without CuCl

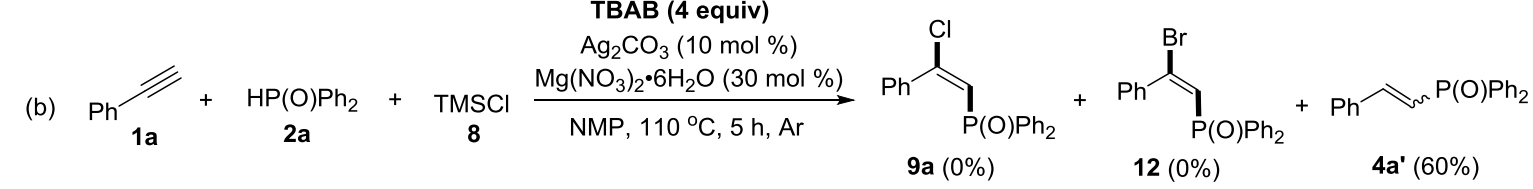

Without $\mathrm{CuCl}$ and $\mathrm{TMSCl}$ :

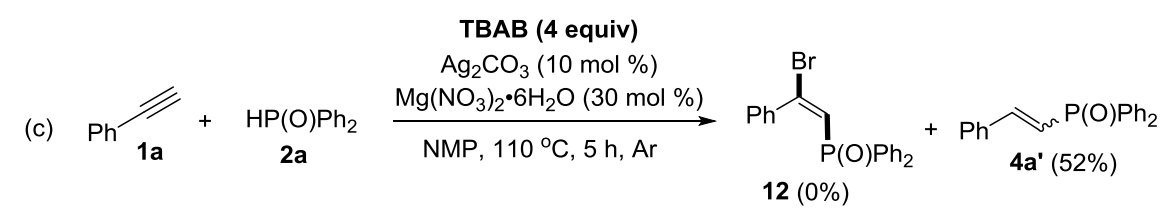

Without $\mathrm{CuCl}$ and TBAB:

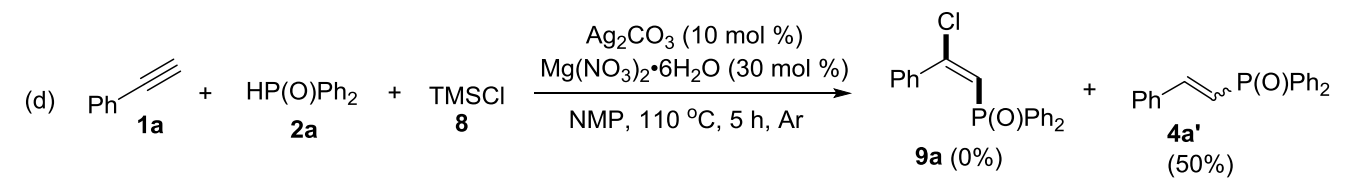

Scheme S6. Probing the intermediacy of vinyl cation 
A mixture of product 9a and compound $\mathbf{1 2}$ were obtained from the reaction involving TBAB (Scheme S6a). However, results show again that the copper catalyst was necessary for the formation of compounds $9 \mathbf{a}$ and $\mathbf{1 2}$ (Scheme S6b-d). On the basis of these results, the possibility of the oxidation of the vinyl radical intermediate to vinyl cation is highly unlikely.

\subsection{Proposed Mechanism for the Cyano-, Thiocyano- and Chlorophosphorylation of Alkynes}

On the basis of the above observations, a general mechanism for cyano-, thiocyano-, and chlorophosphorylation of alkynes is proposed (Scheme S7). The initial phosphinoyl radical A is generated in situ from $\mathrm{HP}(\mathrm{O}) \mathrm{Ph}_{2}$ (2a) in the presence of an oxidant such as $\mathrm{Mn}(\mathrm{OAc})_{3}$, or TBHP, or $\mathrm{Ag}_{2} \mathrm{CO}_{3} / \mathrm{Mg}\left(\mathrm{NO}_{3}\right)_{2}$. Meanwhile, $\mathrm{Cu}^{\mathrm{I}}$ or $\mathrm{Cu}^{\mathrm{II}}$ species $\mathbf{C}$ or $\mathbf{D}$ interacts with a $\mathbf{Y}$ source such as TBACN (3b), TMSNCS (6) or TMSCl $(\mathbf{8})$ to give $\mathrm{Cu}^{\mathrm{II}}$ species $\mathbf{E}$. The phosphinoyl radical $\mathbf{A}$ adds to phenylacetylene (1a) to deliver vinyl radical intermediate $\mathbf{B}$, which combines fast with the $\mathrm{Cu}^{\text {II }}$ species $\mathbf{E}$ to result $\mathrm{Cu}^{\text {III }}$ complex $\mathbf{F}$. $\mathbf{F}$ then undergoes reduction elimination to deliver the desired cyano-, thiocyano-, or chlorophosphorylation products $\mathbf{4 a}, \mathbf{7 a}$ or $\mathbf{9 a}$ and $\mathrm{Cu}^{\mathrm{I}}$ species $\mathbf{C}$, from which the $\mathrm{Cu}^{\mathrm{II}}$ species $\mathbf{E}$ is regenerated via oxidation and interaction with a $\mathbf{Y}$ source.

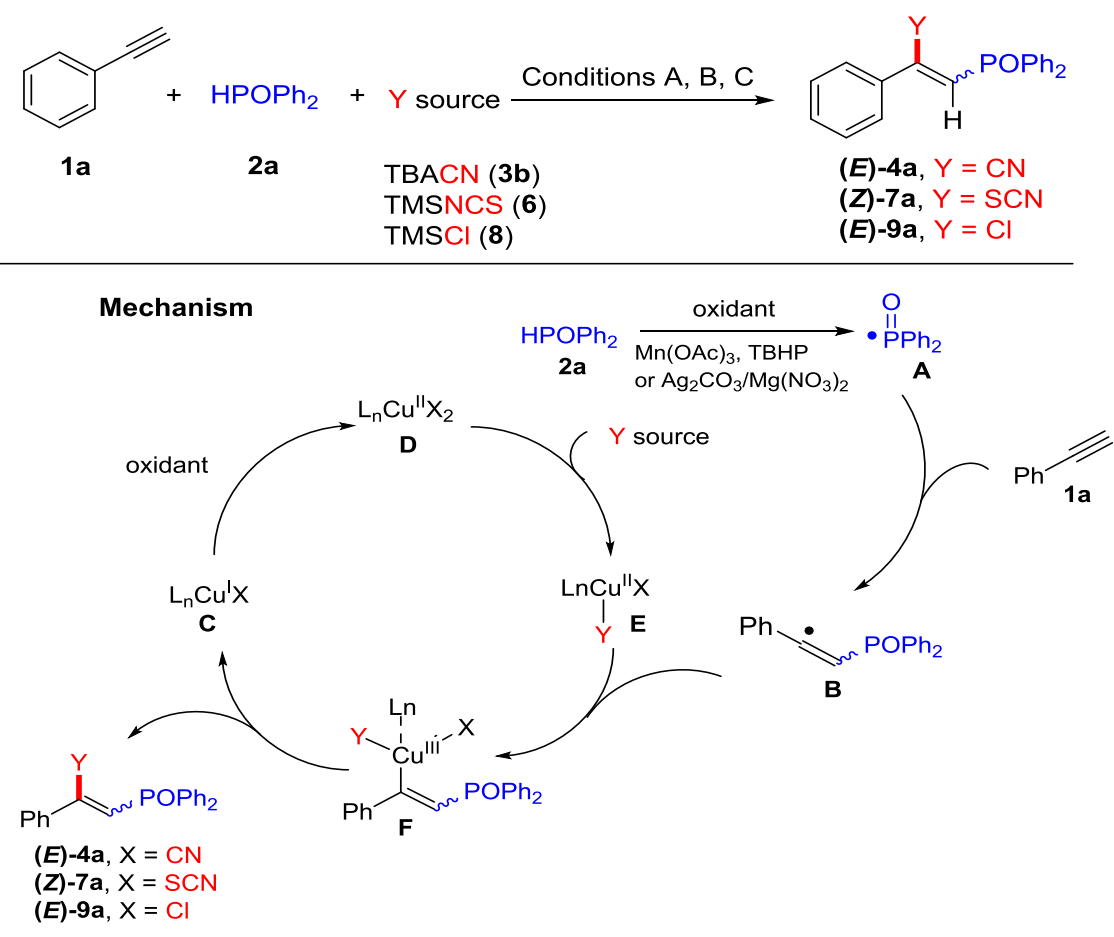

Scheme S7. Proposed mechanism for phosphono-functionalization of alkynes 


\section{Synthetic Applications of the Difunctionalization Products}

(a) Synthesis of Tetrazole $\mathbf{1 3}$ from Functionalized Alkene 7a

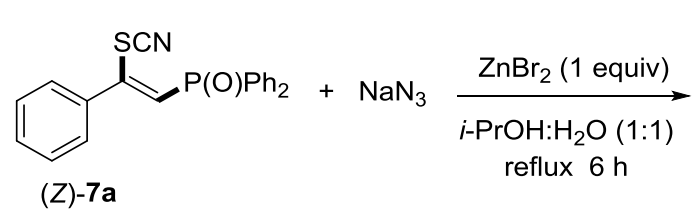

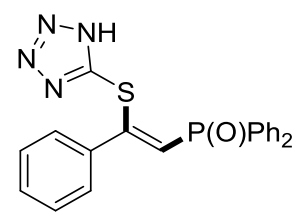

(Z)-13, (90\%)

Procedure: To a $25 \mathrm{~mL}$ reaction tube was added $(\boldsymbol{Z})-7 \mathbf{a}(72.2 \mathrm{mg}, 0.2 \mathrm{mmol}), \mathrm{ZnBr}_{2}(28.6 \mathrm{mg}, 0.2 \mathrm{mmol})$, $\mathrm{NaN}_{3}(26 \mathrm{mg}, 0.4 \mathrm{mmol})$ and a mixture of $i-\mathrm{PrOH} / \mathrm{H}_{2} \mathrm{O}(v / v 1: 1)$. The tube was capped and the mixture was refluxed in an oil bath $\left(100{ }^{\circ} \mathrm{C}\right)$ for 6 hours. At the end of the reaction, the mixture was allowed to cool, diluted with water $(60 \mathrm{~mL})$, and then extracted with ethyl acetate $(20 \mathrm{~mL} \times 3)$. The combined organic fractions were dried over anhydrous $\mathrm{Na}_{2} \mathrm{SO}_{4}$, and concentrated under vacuum to yield the crude product. This was purified by column chromatography on silica gel using ethyl acetate/petroleum ether (1:5) as eluent to give product $\mathbf{1 3}(75.2 \mathrm{mg}, 90 \%$ yield).

(b) Transformation of the thiocyanato group $(-\mathrm{SCN})$ of functionalized alkene 7a into trifluoromethylthio group $\left(-\mathrm{SCF}_{3}\right)$ of product 14 .

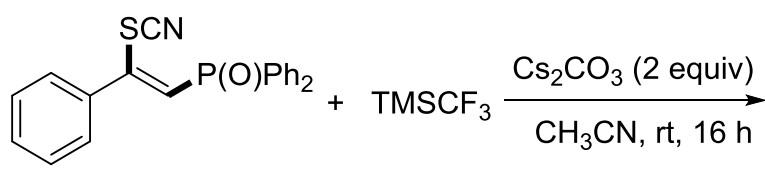

(Z)-7a<smiles>FC(F)(F)C=C(c1ccccc1)C(F)(F)F</smiles>

(Z)-14 (85\%)

Procedure: To a $25 \mathrm{~mL}$ tube was added (Z)-7a (72.2 mg, $0.2 \mathrm{mmol}), \mathrm{Cs}_{2} \mathrm{CO}_{3}(130.3 \mathrm{mg}, 0.4 \mathrm{mmol})$ and $\mathrm{CH}_{3} \mathrm{CN}(1.6 \mathrm{~mL})$. After cooling to $0{ }^{\circ} \mathrm{C}$, trifluoromethyltrimethylsilane $(56.8 \mathrm{mg}, 0.4 \mathrm{mmol})$ was added slowly over 2 hours, and the mixture was stirred at room temperature for 16 hours. The resulting mixture was filtered through a short pad of celite and extracted with ethyl acetate, then concentrated under vacuum. The residue was purified by column chromatography on silica gel using petroleum ether as eluent to give the corresponding thiotrifluoromethylation product $14(68.7 \mathrm{mg}, 85 \%$ yield $)$.

(c) Synthesis of Heterocycle-Fused Tetrazole 15 from Functionalized Alkene 7r

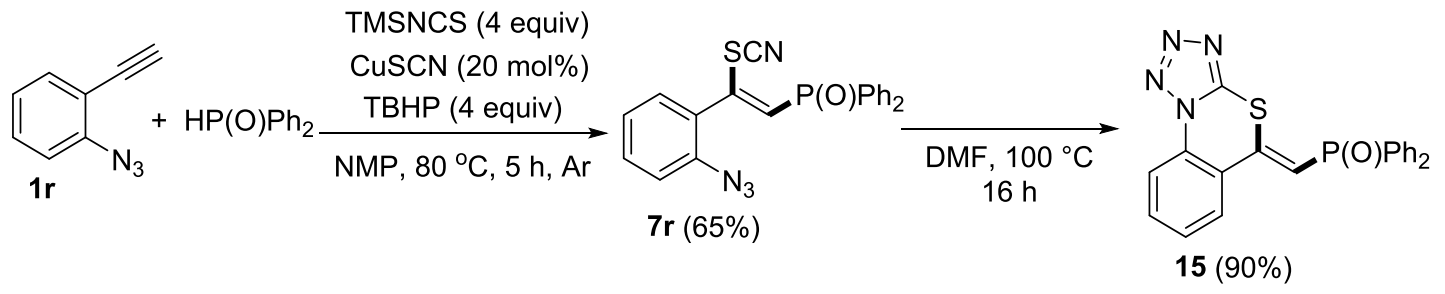


Procedure: To a Schlenk tube equipped with a magnetic stir bar were added $\mathrm{HP}(\mathrm{O}) \mathrm{Ph}_{2} \mathbf{2 a}(202 \mathrm{mg}, 1 \mathrm{mmol})$ and CuSCN (12.1 mg, $0.1 \mathrm{mmol})$. Then, the tube was flushed three times with argon gas. This was followed by the sequential addition of a solution of 1-azido-2-ethynylbenzene $1 \mathbf{r}(71.5 \mathrm{mg}, 0.5 \mathrm{mmol})$ in NMP (2 mL), TBHP $70 \%$ in $\mathrm{H}_{2} \mathrm{O}$ (4 equiv), and TMSNCS 8 (4 equiv) via syringe. The mixture was allowed to stir at $80{ }^{\circ} \mathrm{C}$ in an oil bath for $5 \mathrm{~h}$. After the completion of reaction, water $(60 \mathrm{~mL})$ was added to the reaction mixture, and then extracted with ethyl acetate $(20 \mathrm{~mL} \times 3)$. The combined organic extract was washed with a saturated sodium bicarbonate solution $(20 \mathrm{~mL} \times 3)$, and dried with anhydrous $\mathrm{Na}_{2} \mathrm{SO}_{4}$, filtered, and concentrated in vacuo. The residue was purified by flash chromatography on silica gel using ethyl acetate and petroleum ether (1:5) as eluent to afford product $7 \mathbf{r}$ (130.7 mg, 65\% yield).

Then, compound $7 \mathbf{r}$ obtained above $(40.2 \mathrm{mg}, 0.1 \mathrm{mmol})$ was dissolved in $3 \mathrm{~mL}$ of DMF, and the mixture was stirred at $100{ }^{\circ} \mathrm{C}$ in an oil bath. After 16 hours, the mixture was allowed to cool to room temperature, and then diluted with water $(60 \mathrm{~mL})$, and extracted with ethyl acetate $(20 \mathrm{~mL} \times 3)$. The combined organic fractions were dried over anhydrous $\mathrm{Na}_{2} \mathrm{SO}_{4}$, and concentrated under vacuum to yield the crude product. Purification by column chromatography on silica gel using ethyl acetate/petroleum ether (1:5) gave the pure compound $\mathbf{1 5}(36.2 \mathrm{mg}, 90 \%$ yield).

\section{Unsuccessful Substrates}

The difunctionalization protocols were not without limitations. Substrates that failed to either react or give the target products are shown below.

a) Cyanophosphorylation Reaction

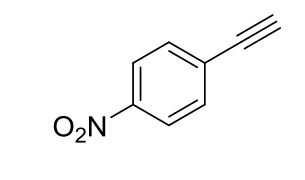

Alkynyl phosphine oxide 40' obtained exclusively

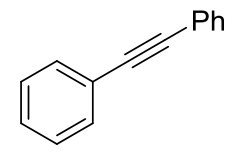

Product not detected

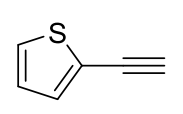
Complex mixture of unidentifiable products

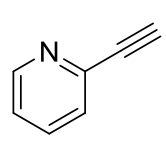

Product not detected

b) Thiocyanophosphorylation Reaction

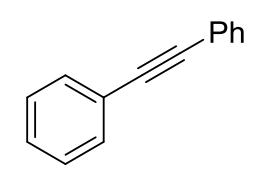

The expected product not detected

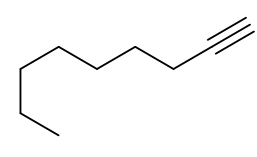

Hydrophosphorylation occurred exclusively

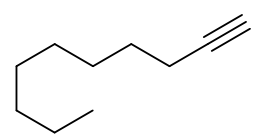

Hydrophosphorylation occurred exclusively

c) Chlorophosphorylation Reaction 
<smiles>C#Cc1ccccc1F</smiles>

Product not detected

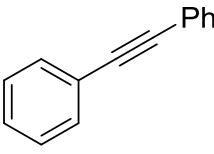

Product not detected<smiles>C#Cc1ccccn1</smiles>

Product not detected

\section{Characterization Data of Synthesized Compounds 4, 5, 7, 9, 10-15}

\subsection{Characterization Data for Cyanophosphorylation Products 4 and 5}

(E)-3-(Diphenylphosphoryl)-2-phenylacrylonitrile (4a)<smiles>N#C/C(=C/POc1ccccc1)c1ccccc1</smiles>

Following GP1, the residue was purified by flash chromatography on silica gel using dichloromethane/ petroleum ether/ethyl acetate $(v / v / v 4: 1: 1)$ as eluent to afford the pure product $\mathbf{4 a}\left(\mathbf{R}_{\mathrm{f}}=0.6\right)$ as yellow oil $(60 \mathrm{mg}, 74 \%$ yield $)$. ${ }^{1} \mathrm{H}$ NMR (400 MHz, $\left.\mathrm{CDCl}_{3}\right): \delta 7.77-7.67(\mathrm{~m}, 6 \mathrm{H}), 7.55-7.48(\mathrm{~m}, 2 \mathrm{H}), 7.47-7.40(\mathrm{~m}, 4 \mathrm{H}), 7.34-7.22(\mathrm{~m}, 4 \mathrm{H})$; ${ }^{13} \mathrm{C}$ NMR $\left(100 \mathrm{MHz}, \mathrm{CDCl}_{3}\right): \delta 137.4,136.5,131.9,131.8(\mathrm{~d}, J=2.9 \mathrm{~Hz}), 131.7,130.7(\mathrm{~d}, J=4.8 \mathrm{~Hz}), 130.6$, $130.5,130.4,130.3,129.0(\mathrm{~d}, J=0.7 \mathrm{~Hz}), 128.4,128.2,127.9,117.5(\mathrm{~d}, J=22.9 \mathrm{~Hz}){ }^{31} \mathrm{P}$ NMR $(162 \mathrm{MHz}$, $\mathrm{CDCl}_{3}$ ): $\delta$ 18.28. HRMS (ESI-TOF) $\mathrm{m} / \mathrm{z}:[\mathrm{M}+\mathrm{H}]^{+}$Calcd for $\mathrm{C}_{21} \mathrm{H}_{17} \mathrm{NOP} 330.1048$, found 330.1054.

\section{Diphenyl(styryl)phosphine oxide (4a') ${ }^{3}$}<smiles>[PbH]C=Cc1ccccc1</smiles>

Following GP1, the residue was purified by flash chromatography on silica gel using dichloromethane/ petroleum ether/ethyl acetate $(v / v / v 4: 1: 1)$ as eluent to afford the pure product $4 \mathbf{a}^{\prime}\left(\mathrm{R}_{\mathrm{f}}=0.5\right)$ as mixture of $E / Z$ isomers (0.6:1). ${ }^{1} \mathrm{H}$ NMR $\left(400 \mathrm{MHz}, \mathrm{CDCl}_{3}\right): \delta 7.79-7.64(\mathrm{~m}, 8 \mathrm{H}), 7.58-7.43(\mathrm{~m}, 7 \mathrm{H}), 7.41-7.28(\mathrm{~m}, 8 \mathrm{H}), 7.19-7.09$ $(\mathrm{m}, 3 \mathrm{H}), 6.93-6.73(\mathrm{~m}, 0.6 \mathrm{H}), 6.38-6.21(\mathrm{~m}, 1 \mathrm{H}) .{ }^{31} \mathrm{P}$ NMR $\left(162 \mathrm{MHz}, \mathrm{CDCl}_{3}\right): \delta 24.42,19.99$.

\section{(E)-3-(Diphenylphosphoryl)-2-(m-tolyl)acrylonitrile (4b)}<smiles>Cc1cccc(/C(C#N)=C\[PH2+]c2ccccc2)c1</smiles>

Following GP1, the residue was purified by flash chromatography on silica gel using dichloromethane/ petroleum ether/ethyl acetate $(v / v / v 4: 1: 1)$ as eluent to afford the pure product $\mathbf{4 b}\left(\mathrm{R}_{\mathrm{f}}=0.6\right)$ as yellow oil $(60 \mathrm{mg}, 70 \%$ yield $)$. ${ }^{1} \mathrm{H}$ NMR $\left(400 \mathrm{MHz}, \mathrm{CDCl}_{3}\right): \delta 7.70-7.62(\mathrm{~m}, 4 \mathrm{H}), 7.49-7.43(\mathrm{~m}, 4 \mathrm{H}), 7.41-7.36(\mathrm{~m}, 4 \mathrm{H}), 7.14(\mathrm{~d}, J=14.5$ $\mathrm{Hz}, 1 \mathrm{H}), 7.11-7.02(\mathrm{~m}, 2 \mathrm{H}), 2.22(\mathrm{~s}, 3 \mathrm{H}) ;{ }^{13} \mathrm{C} \mathrm{NMR}\left(100 \mathrm{MHz}, \mathrm{CDCl}_{3}\right): \delta 138.1,137.4,136.5,132.5(\mathrm{~d}, J=5.0$ 
$\mathrm{Hz}), 132.2(\mathrm{~d}, J=2.9 \mathrm{~Hz}), 132.2,131.7,131.0(\mathrm{~d}, J=1.1 \mathrm{~Hz}), 131.0,130.8,130.7,130.0(\mathrm{~d}, J=0.6 \mathrm{~Hz}), 128.8$, 128.7, 128.3, $126.5(\mathrm{~d}, J=0.8 \mathrm{~Hz}), 118.0(\mathrm{~d}, J=23.0 \mathrm{~Hz}), 21.1 ;{ }^{31} \mathrm{P}$ NMR $\left(162 \mathrm{MHz}, \mathrm{CDCl}_{3}\right): \delta 18.37 . \mathrm{HRMS}$ (ESI-TOF) $m / z:[\mathrm{M}+\mathrm{Na}]^{+}$Calcd for $\mathrm{C}_{22} \mathrm{H}_{18} \mathrm{NNaOP} 366.1024$, found 366.1021.

\section{(E)-3-(Diphenylphosphoryl)-2-(p-tolyl)acrylonitrile (4c)}<smiles>Cc1ccc(/C(C#N)=C\P(=O)(O)c2ccccc2)cc1</smiles>

Following GP1, the residue was purified by flash chromatography on silica gel using dichloromethane/ petroleum ether/ethyl acetate $\left(v / v / v\right.$ 4:1:1) as eluent to afford the pure product $\mathbf{4 c}\left(\mathrm{R}_{\mathrm{f}}=0.7\right)$ as yellow oil $(60 \mathrm{mg}, 70 \%$ yield $)$. ${ }^{1} \mathrm{H} \mathrm{NMR}\left(400 \mathrm{MHz}, \mathrm{CDCl}_{3}\right): \delta 7.71-7.63(\mathrm{~m}, 4 \mathrm{H}), 7.58(\mathrm{~d}, J=8.2 \mathrm{~Hz}, 2 \mathrm{H}), 7.50-7.44(\mathrm{~m}, 2 \mathrm{H}), 7.43-7.36(\mathrm{~m}$, 4H), $7.08(\mathrm{~d}, J=14.9 \mathrm{~Hz}, 1 \mathrm{H}), 7.02(\mathrm{~d}, J=7.9 \mathrm{~Hz}, 2 \mathrm{H}), 2.26(\mathrm{~s}, 3 \mathrm{H}) ;{ }^{13} \mathrm{C} \mathrm{NMR}\left(100 \mathrm{MHz}, \mathrm{CDCl}_{3}\right): \delta 141.7$, 136.2, 135.3, $132.4(\mathrm{~d}, J=4.8 \mathrm{~Hz}), 132.3(\mathrm{~d}, J=2.8 \mathrm{~Hz}), 131.2,130.9,130.8,129.5,129.1,128.9,128.8,128.5$, $128.4(\mathrm{~d}, J=1.9 \mathrm{~Hz}), 118.1(\mathrm{~d}, J=23.0 \mathrm{~Hz}), 21.4 ;{ }^{31} \mathrm{P}$ NMR $\left(162 \mathrm{MHz}, \mathrm{CDCl}_{3}\right): \delta 18.82 . \mathrm{HRMS}(\mathrm{ESI}-\mathrm{TOF}) m / z$ : $[\mathrm{M}+\mathrm{Na}]^{+}$Calcd for $\mathrm{C}_{22} \mathrm{H}_{18} \mathrm{NNaOP} 366.1024$, found 366.1022.

\section{(E)-3-(Diphenylphosphoryl)-2-(4-isopropylphenyl)acrylonitrile (4d)}<smiles>CC(C)(C)c1ccc(/C(C#N)=C/POc2ccccc2)cc1</smiles>

Following GP1, the residue was purified by flash chromatography on silica gel using dichloromethane/ petroleum ether/ethyl acetate $(v / v / v 4: 1: 1)$ as eluent to afford the pure product $\mathbf{4 d}\left(\mathbf{R}_{\mathrm{f}}=0.6\right)$ as yellow oil $(64 \mathrm{mg}, 70 \%) .{ }^{1} \mathrm{H}$ NMR (400 MHz, $\left.\mathrm{CDCl}_{3}\right): \delta 7.71-7.61(\mathrm{~m}, 4 \mathrm{H}), 7.61-7.56(\mathrm{~m}, 2 \mathrm{H}), 7.46-7.42(\mathrm{~m}, 2 \mathrm{H}), 7.40-7.35(\mathrm{~m}, 4 \mathrm{H})$, $7.23-7.18(\mathrm{~m}, 2 \mathrm{H}), 7.10(\mathrm{~d}, J=14.5 \mathrm{~Hz}, 1 \mathrm{H}), 1.21(\mathrm{~s}, 6 \mathrm{H}) ;{ }^{13} \mathrm{C} \mathrm{NMR}\left(100 \mathrm{MHz}, \mathrm{CDCl}_{3}\right): \delta 154.6,136.6,135.7$, $132.3,132.22(\mathrm{~d}, J=1.2 \mathrm{~Hz}), 132.17(\mathrm{~d}, J=2.8 \mathrm{~Hz}), 131.1,130.9,130.8,129.4,128.8,128.7,128.4(\mathrm{~d}, J=4.9$ $\mathrm{Hz}), 125.3,118.1(\mathrm{~d}, J=23.1 \mathrm{~Hz}), 34.8,31.0 ;{ }^{31} \mathrm{P}$ NMR $\left(162 \mathrm{MHz}, \mathrm{CDCl}_{3}\right): \delta 18.22 . \mathrm{HRMS}(\mathrm{ESI}-\mathrm{TOF}) m / z$ : $[\mathrm{M}+\mathrm{H}]^{+}$Calcd for $\mathrm{C}_{25} \mathrm{H}_{25} \mathrm{NOP} 386.1674$, found 386.1684 .

\section{(E)-3-(Diphenylphosphoryl)-2-(3-methoxyphenyl)acrylonitrile (4e)}<smiles>COc1cccc(/C(C#N)=C\POc2ccccc2)c1</smiles>

Following GP1, the residue was purified by flash chromatography on silica gel using dichloromethane/ petroleum 
ether/ethyl acetate $(v / v / v 4: 1: 1)$ as eluent to afford the pure product $4 \mathbf{e}\left(\mathrm{R}_{\mathrm{f}}=0.5\right)$ as yellow oil $(64 \mathrm{mg}, 72 \%) .{ }^{1} \mathrm{H}$ NMR (400 MHz, $\left.\mathrm{CDCl}_{3}\right): \delta 7.69-7.63(\mathrm{~m}, 4 \mathrm{H}), 7.49-7.43(\mathrm{~m}, 2 \mathrm{H}), 7.42-7.35(\mathrm{~m}, 5 \mathrm{H}), 7.23-7.09(\mathrm{~m}, 3 \mathrm{H})$, $6.82-6.76(\mathrm{~m}, 1 \mathrm{H}), 3.74(\mathrm{~s}, 3 \mathrm{H}) ;{ }^{13} \mathrm{C} \mathrm{NMR}\left(100 \mathrm{MHz}, \mathrm{CDCl}_{3}\right): \delta 159.2,137.8,136.9,132.4(\mathrm{~d}, J=4.7 \mathrm{~Hz})$, $132.3(\mathrm{~d}, J=2.8 \mathrm{~Hz}), 132.1,131.0,130.8,130.7,129.5,128.9,128.7,121.7(\mathrm{~d}, J=0.7 \mathrm{~Hz}), 118.0,114.0,55.4 ;{ }^{31} \mathrm{P}$ NMR (162 MHz, $\left.\mathrm{CDCl}_{3}\right): \delta$ 18.79. HRMS (ESI-TOF) $m / z:[\mathrm{M}+\mathrm{H}]^{+}$Calcd for $\mathrm{C}_{22} \mathrm{H}_{19} \mathrm{NO}_{2} \mathrm{P}$ 360.1153, found 360.1156 .

(E)-3-(Diphenylphosphoryl)-2-(4-methoxyphenyl)acrylonitrile (4f)<smiles>COc1ccc(/C(C#N)=C\POc2ccccc2)cc1</smiles>

Following GP1, the residue was purified by flash chromatography on silica gel using dichloromethane/ petroleum ether/ethyl acetate $(v / v / v 4: 1: 1)$ as eluent to afford the pure product $\mathbf{4 f}\left(\mathrm{R}_{\mathrm{f}}=0.6\right)$ as yellow oil $(64 \mathrm{mg}, 72 \%) .{ }^{1} \mathrm{H}$ $\operatorname{NMR}\left(400 \mathrm{MHz}, \mathrm{CDCl}_{3}\right) \delta: 7.75-7.66(\mathrm{~m}, 6 \mathrm{H}), 7.50-7.44(\mathrm{~m}, 2 \mathrm{H}), 7.43-7.36(\mathrm{~m}, 4 \mathrm{H}), 6.98(\mathrm{~d}, J=14.6 \mathrm{~Hz}$, $1 \mathrm{H}), 6.74-6.67(\mathrm{~m}, 2 \mathrm{H}), 3.74(\mathrm{~s}, 3 \mathrm{H}) ;{ }^{13} \mathrm{C} \mathrm{NMR}\left(100 \mathrm{MHz}, \mathrm{CDCl}_{3}\right): \delta 161.8,134.4,133.5,132.5,132.2(\mathrm{~d}, J=$ $2.8 \mathrm{~Hz}), 131.9(\mathrm{~d}, J=4.8 \mathrm{~Hz}), 131.5,131.4,130.9,130.8,128.8,128.7,123.8(\mathrm{~d}, J=4.8 \mathrm{~Hz}), 118.3(\mathrm{~d}, J=23.2$ $\mathrm{Hz}), \quad 113.8, \quad 55.4 ;{ }^{31} \mathrm{P} \mathrm{NMR}\left(162 \mathrm{MHz}, \mathrm{CDCl}_{3}\right): \delta$ 18.81. HRMS (ESI-TOF) $m / z:[\mathrm{M}+\mathrm{Na}]^{+} \mathrm{Calcd}$ for $\mathrm{C}_{22} \mathrm{H}_{18} \mathrm{NNaO}_{2} \mathrm{P} 382.0973$, found 382.0964.

(E)-3-(Diphenylphosphoryl)-2-(3-fluorophenyl)acrylonitrile (4g)<smiles>N#C/C(=C/POc1ccccc1)c1cccc(F)c1</smiles>

Following GP1, the residue was purified by flash chromatography on silica gel using dichloromethane/ petroleum ether/ethyl acetate $(v / v / v 4: 1: 1)$ as eluent to afford the pure product $\mathbf{4 g}\left(\mathrm{R}_{\mathrm{f}}=0.6\right)$ as yellow oil $(50 \mathrm{mg}, 60 \%) .{ }^{1} \mathrm{H}$ NMR (400 MHz, $\left.\mathrm{CDCl}_{3}\right): \delta 7.74-7.62(\mathrm{~m}, 4 \mathrm{H}), 7.55-7.40(\mathrm{~m}, 8 \mathrm{H}), 7.30-7.17(\mathrm{~m}, 2 \mathrm{H}), 7.04-6.94(\mathrm{~m}, 1 \mathrm{H})$; ${ }^{13} \mathrm{C}$ NMR $\left(100 \mathrm{MHz}, \mathrm{CDCl}_{3}\right): \delta 162.0(\mathrm{~d}, J=248.3 \mathrm{~Hz}), 139.6,138.7,132.5(\mathrm{~d}, J=2.8 \mathrm{~Hz}), 131.8,130.9,130.8$, $130.1(\mathrm{~d}, J=8.2 \mathrm{~Hz}), 128.9,128.8,125.4(\mathrm{~d}, J=2.3 \mathrm{~Hz}), 117.9(\mathrm{~d}, J=21.1 \mathrm{~Hz}), 117.6(\mathrm{~d}, J=22.4 \mathrm{~Hz}), 116.5(\mathrm{~d}$, $J=23.8 \mathrm{~Hz}) ;{ }^{31} \mathrm{P}$ NMR $\left(162 \mathrm{MHz}, \mathrm{CDCl}_{3}\right): \delta$ 18.06. HRMS (ESI-TOF) $m / z:[\mathrm{M}+\mathrm{H}]^{+}$Calcd for $\mathrm{C}_{21} \mathrm{H}_{16} \mathrm{FNOP}$ 348.0954, found 348.0947.

\section{(E)-3-(Diphenylphosphoryl)-2-(4-fluorophenyl)acrylonitrile (4h)}




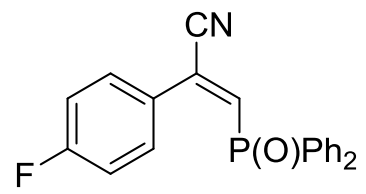

Following GP1, the residue was purified by flash chromatography on silica gel using dichloromethane/ petroleum ether/ethyl acetate $(v / v / v 4: 1: 1)$ as eluent to afford the pure product $\mathbf{4 h}\left(\mathrm{R}_{\mathrm{f}}=0.6\right)$ as yellow oil $(57 \mathrm{mg}, 66 \%) .{ }^{1} \mathrm{H}$ NMR $\left(400 \mathrm{MHz}, \mathrm{CDCl}_{3}\right): \delta 7.70-7.61(\mathrm{~m}, 6 \mathrm{H}), 7.53-7.46(\mathrm{~m}, 2 \mathrm{H}), 7.44-7.39(\mathrm{~m}, 4 \mathrm{H}), 7.16(\mathrm{~d}, J=14.7 \mathrm{~Hz}$, $1 \mathrm{H}), 6.94-6.88(\mathrm{~m}, 2 \mathrm{H}) .{ }^{13} \mathrm{C} \mathrm{NMR}\left(100 \mathrm{MHz}, \mathrm{CDCl}_{3}\right): \delta 164.1(\mathrm{~d}, J=253.5 \mathrm{~Hz}), 144.4,137.5,136.6,132.6(\mathrm{~d}, J$ $=2.8 \mathrm{~Hz}), 131.8(\mathrm{~d}, J=9.0 \mathrm{~Hz}), 131.6,131.2(\mathrm{~d}, J=4.7 \mathrm{~Hz}), 130.8(\mathrm{~d}, J=10.1 \mathrm{~Hz}), 130.5,128.9(\mathrm{~d}, J=12.6 \mathrm{~Hz})$, $127.3(\mathrm{dd}, J=4.9,3.4 \mathrm{~Hz}), 117.8(\mathrm{~d}, J=22.8 \mathrm{~Hz}), 115.6(\mathrm{~d}, J=22.2 \mathrm{~Hz}), 111.6 ;{ }^{31} \mathrm{P} \mathrm{NMR}\left(162 \mathrm{MHz}, \mathrm{CDCl}_{3}\right): \delta$ 19.15. HRMS (ESI-TOF) $m / z:[\mathrm{M}+\mathrm{H}]^{+}$Calcd for $\mathrm{C}_{21} \mathrm{H}_{16}$ FNOP 348.0954, found 348.0948.

\section{(E)-2-(2-Chlorophenyl)-3-(diphenylphosphoryl)acrylonitrile (4i)}<smiles>N#C/C(=C/POc1ccccc1)c1ccccc1Cl</smiles>

Following GP1, the residue was purified by flash chromatography on silica gel using dichloromethane/ petroleum ether/ethyl acetate $(v / v / v 4: 1: 1)$ as eluent to afford the pure product $\mathbf{4 i}\left(\mathrm{R}_{\mathrm{f}}=0.5\right)$ as yellow oil $(61 \mathrm{mg}, 68 \%$ yield $)$. ${ }^{1} \mathrm{H}$ NMR $\left(400 \mathrm{MHz}, \mathrm{CDCl}_{3}\right): \delta 7.62-7.54(\mathrm{~m}, 4 \mathrm{H}), 7.51-7.41(\mathrm{~m}, 4 \mathrm{H}), 7.40-7.32(\mathrm{~m}, 4 \mathrm{H}), 7.19-7.11(\mathrm{~m}, 2 \mathrm{H})$, $7.10-7.05(\mathrm{~m}, 1 \mathrm{H}) ;{ }^{13} \mathrm{C}$ NMR $\left(100 \mathrm{MHz}, \mathrm{CDCl}_{3}\right): \delta 142.6,141.7,132.50,132.45(\mathrm{~d}, J=1.0 \mathrm{~Hz}), 132.4(\mathrm{~d}, J=$ $2.8 \mathrm{~Hz}), 131.6,131.4,130.8,130.7,130.3,129.9(\mathrm{~d}, J=5.5 \mathrm{~Hz}), 129.6$ (d, $J=5.5 \mathrm{~Hz}), 129.4,128.8,128.7,126.7$, $116.2(\mathrm{~d}, J=22.1 \mathrm{~Hz}) ;{ }^{31} \mathrm{P}$ NMR $\left(162 \mathrm{MHz}, \mathrm{CDCl}_{3}\right): \delta 17.59$. HRMS (ESI-TOF) $\mathrm{m} / z:[\mathrm{M}+\mathrm{Na}]^{+} \mathrm{Calcd}$ for $\mathrm{C}_{21} \mathrm{H}_{15} \mathrm{ClNNaOP} 386.0477$, found 386.0478.

\section{(E)-2-(3-Chlorophenyl)-3-(diphenylphosphoryl)acrylonitrile (4j)}<smiles>N#C/C(=C/POc1ccccc1)c1cccc(Cl)c1</smiles>

Following GP1, the residue was purified by flash chromatography on silica gel using dichloromethane/ petroleum ether/ethyl acetate $(v / v / v 4: 1: 1)$ as eluent to afford the pure product $\mathbf{4} \mathbf{j}\left(\mathrm{R}_{\mathrm{f}}=0.6\right)$ as yellow oil $(61 \mathrm{mg}, 68 \%) .{ }^{1} \mathrm{H}$ NMR (400 MHz, $\left.\mathrm{CDCl}_{3}\right): \delta 7.71-7.64(\mathrm{~m}, 4 \mathrm{H}), 7.63-7.61(\mathrm{~m}, 1 \mathrm{H}), 7.56-7.53(\mathrm{~m}, 1 \mathrm{H}), 7.52-7.47(\mathrm{~m}, 2 \mathrm{H})$, $7.45-7.39(\mathrm{~m}, 4 \mathrm{H}), 7.30-7.22(\mathrm{~m}, 2 \mathrm{H}), 7.16(\mathrm{t}, J=7.9 \mathrm{~Hz}, 1 \mathrm{H}) ;{ }^{13} \mathrm{C} \mathrm{NMR}\left(100 \mathrm{MHz}, \mathrm{CDCl}_{3}\right): \delta 139.7,138.8$, 134.4, $132.6(\mathrm{~d}, J=5.0 \mathrm{~Hz}), 132.5(\mathrm{~d}, J=2.7 \mathrm{~Hz}), 131.6,130.92,130.87,130.8,130.6(\mathrm{~d}, J=4.8 \mathrm{~Hz}), 130.6$, 129.7, 129.4, 129.0, 128.8, 127.6, 117.5 (d, $J=22.4 \mathrm{~Hz}) ;{ }^{31} \mathrm{P}$ NMR $\left(162 \mathrm{MHz}, \mathrm{CDCl}_{3}\right): \delta 18.17 . \mathrm{HRMS}$ (ESI-TOF) 
$m / z:[\mathrm{M}+\mathrm{Na}]^{+}$Calcd for $\mathrm{C}_{21} \mathrm{H}_{15} \mathrm{ClNNaOP} 386.0477$, found 386.0480.

(E)-2-(4-Chlorophenyl)-3-(diphenylphosphoryl)acrylonitrile (4k)<smiles>N#C/C(=C/POc1ccccc1)c1ccc(Cl)cc1</smiles>

Following GP1, the residue was purified by flash chromatography on silica gel using dichloromethane/ petroleum ether/ethyl acetate $\left(v / v / v\right.$ 4:1:1) as eluent to afford the pure product $4 \mathbf{k}\left(\mathrm{R}_{\mathrm{f}}=0.6\right)$ as yellow oil $(67 \mathrm{mg}, 74 \%) .{ }^{1} \mathrm{H}$ NMR (400 MHz, $\left.\mathrm{CDCl}_{3}\right): \delta 7.70-7.61(\mathrm{~m}, 6 \mathrm{H}), 7.53-7.45(\mathrm{~m}, 2 \mathrm{H}), 7.43-7.39(\mathrm{~m}, 4 \mathrm{H}), 7.21-7.16(\mathrm{~m}, 3 \mathrm{H})$;

${ }^{13} \mathrm{C}$ NMR $\left(100 \mathrm{MHz}, \mathrm{CDCl}_{3}\right): \delta 138.6,137.7,137.3,132.48,132.45,131.9,130.89,130.87,130.8,129.6,129.5$, 129.0, 128.8, 128.6, $117.7(\mathrm{~d}, J=22.6 \mathrm{~Hz}) ;{ }^{31} \mathrm{P}$ NMR $\left(162 \mathrm{MHz}, \mathrm{CDCl}_{3}\right): \delta$ 18.39. HRMS (ESI-TOF) $m / z:$ $[\mathrm{M}+\mathrm{Na}]^{+}$Calcd for $\mathrm{C}_{21} \mathrm{H}_{15} \mathrm{ClNNaOP} 386.0477$, found 386.0464.

(E)-2-(3-Bromophenyl)-3-(diphenylphosphoryl)acrylonitrile (4I)<smiles>N#C/C(=C/POc1ccccc1)c1cccc(Br)c1</smiles>

Following GP1, the residue was purified by flash chromatography on silica gel using dichloromethane/ petroleum ether/ethyl acetate $(v / v / v 4: 1: 1)$ as eluent to afford the pure product $\mathbf{4 l}\left(\mathrm{R}_{\mathrm{f}}=0.5\right)$ as yellow oil $(66 \mathrm{mg}, 65 \%$ yield $)$. ${ }^{1} \mathrm{H}$ NMR $\left(400 \mathrm{MHz}, \mathrm{CDCl}_{3}\right): \delta 7.78-7.75(\mathrm{~m}, 1 \mathrm{H}), 7.71-7.63(\mathrm{~m}, 4 \mathrm{H}), 7.60-7.56(\mathrm{~m}, 1 \mathrm{H}), 7.50-7.43(\mathrm{~m}, 2 \mathrm{H})$, $7.43-7.35(\mathrm{~m}, 5 \mathrm{H}), 7.25(\mathrm{~d}, J=14.2 \mathrm{~Hz}, 1 \mathrm{H}), 7.10-7.04(\mathrm{~m}, 1 \mathrm{H}) ;{ }^{13} \mathrm{C} \mathrm{NMR}\left(100 \mathrm{MHz}, \mathrm{CDCl}_{3}\right): \delta 139.9,139.0$, $133.8,132.9(\mathrm{~d}, J=5.0 \mathrm{~Hz}), 132.5(\mathrm{~d}, J=2.8 \mathrm{~Hz}), 132.2(\mathrm{~d}, J=0.7 \mathrm{~Hz}), 131.7,130.9,130.8,130.6,130.4(\mathrm{~d}, J=$ $4.7 \mathrm{~Hz}), 129.8,128.9,128.8,128.0(\mathrm{~d}, J=0.8 \mathrm{~Hz}), 122.4,117.5(\mathrm{~d}, J=22.3 \mathrm{~Hz}) ;{ }^{31} \mathrm{P} \mathrm{NMR}\left(162 \mathrm{MHz}, \mathrm{CDCl}_{3}\right): \delta$ 17.91. HRMS (ESI-TOF) $m / z:[\mathrm{M}+\mathrm{Na}]^{+} \mathrm{Calcd}$ for $\mathrm{C}_{21} \mathrm{H}_{15} \mathrm{BrNNaOP} 429.9972$, found 429.9979 .

(E)-2-(4-Bromophenyl)-3-(diphenylphosphoryl)acrylonitrile (4m)<smiles>N#C/C(=C/POCc1ccccc1)c1ccc(Br)cc1</smiles>

Following GP1, the residue was purified by flash chromatography on silica gel using dichloromethane/ petroleum ether/ethyl acetate $(v / v / v 4: 1: 1)$ as eluent to afford the pure product $\mathbf{4 m}\left(\mathrm{R}_{\mathrm{f}}=0.5\right)$ as yellow oil $(66 \mathrm{mg}, 65 \%) .{ }^{1} \mathrm{H}$ NMR (400 MHz, $\left.\mathrm{CDCl}_{3}\right): \delta 7.69-7.63(\mathrm{~m}, 4 \mathrm{H}), 7.57-7.53(\mathrm{~m}, 2 \mathrm{H}), 7.52-7.46(\mathrm{~m}, 2 \mathrm{H}), 7.45-7.38(\mathrm{~m}, 4 \mathrm{H})$, $7.37-7.33(\mathrm{~m}, 2 \mathrm{H}), 7.20(\mathrm{~d}, J=14.5 \mathrm{~Hz}, 1 \mathrm{H}) ;{ }^{13} \mathrm{C} \mathrm{NMR}\left(100 \mathrm{MHz}, \mathrm{CDCl}_{3}\right): \delta 138.7,137.8,132.5(\mathrm{~d}, J=2.8 \mathrm{~Hz})$, $131.9,131.6,131.0(\mathrm{~d}, J=4.6 \mathrm{~Hz}), 130.9,130.8,130.0(\mathrm{~d}, J=5.0 \mathrm{~Hz}), 129.0,128.8,125.8,117.6(\mathrm{~d}, J=22.5 \mathrm{~Hz})$; 
${ }^{31} \mathrm{P}$ NMR $\left(162 \mathrm{MHz}, \mathrm{CDCl}_{3}\right): \delta$ 18.33. HRMS (ESI-TOF) $\mathrm{m} / z:[\mathrm{M}+\mathrm{Na}]^{+}$Calcd for $\mathrm{C}_{21} \mathrm{H}_{15} \mathrm{BrNNaOP} 429.9972$, found 429.9979 .

\section{Methyl (E)-4-(1-cyano-2-(diphenylphosphoryl)vinyl)benzoate (4n)}<smiles>COC(=O)c1ccc(/C(C#N)=C\Pc2ccccc2)cc1</smiles>

Following GP1, the residue was purified by flash chromatography on silica gel using dichloromethane/ petroleum ether/ethyl acetate $(v / v / v 4: 1: 1)$ as eluent to afford the pure product $4 \mathbf{n}\left(\mathrm{R}_{\mathrm{f}}=0.6\right)$ as yellow oil $(73 \mathrm{mg}, 76 \%$ yield $)$. ${ }^{1} \mathrm{H}$ NMR $\left(400 \mathrm{MHz}, \mathrm{CDCl}_{3}\right): \delta 7.87(\mathrm{~d}, J=8.6 \mathrm{~Hz}, 2 \mathrm{H}), 7.73(d, J=8.6 \mathrm{~Hz}, 2 \mathrm{H}), 7.70-7.63(\mathrm{~m}, 4 \mathrm{H}), 7.50-7.45$ $(\mathrm{m}, 2 \mathrm{H}), 7.43-7.37(\mathrm{~m}, 4 \mathrm{H}), 7.29(\mathrm{~d}, J=14.5 \mathrm{~Hz}, 1 \mathrm{H}), 3.89(\mathrm{~s}, 3 \mathrm{H}) .{ }^{13} \mathrm{C} \mathrm{NMR}\left(100 \mathrm{MHz}, \mathrm{CDCl}_{3}\right): \delta 166.0,140.1$, 139.2, $135.2(\mathrm{~d}, J=4.9 \mathrm{~Hz}), 132.5(\mathrm{~d}, J=2.8 \mathrm{~Hz}), 132.0,131.8,131.1(\mathrm{~d}, J=4.5 \mathrm{~Hz}), 130.9,130.8,130.7,129.6$ $(\mathrm{d}, J=0.5 \mathrm{~Hz}), 129.5,129.0,128.9,117.6(\mathrm{~d}, J=22.4 \mathrm{~Hz}), 52.4 ;{ }^{31} \mathrm{P} \mathrm{NMR}\left(162 \mathrm{MHz}, \mathrm{CDCl}_{3}\right): \delta 18.86 . \mathrm{HRMS}$ (ESI-TOF) $m / z:[\mathrm{M}+\mathrm{Na}]^{+}$Calcd for $\mathrm{C}_{23} \mathrm{H}_{18} \mathrm{NNaO}_{3} \mathrm{P} 410.0922$, found 410.0925 .

((4-Nitrophenyl)ethynyl)diphenylphosphine oxide $\left(4 o^{\prime}\right)^{4}$<smiles>O=[N+]([O-])c1ccc(C#CP(OCc2ccccc2)c2ccccc2)cc1</smiles>

Following GP1, the residue was purified by flash chromatography on silica gel using dichloromethane/ petroleum ether/ethyl acetate $(v / v / v 4: 1: 1)$ as eluent to afford the pure product $\mathbf{4 o}^{\prime}\left(\mathrm{R}_{\mathrm{f}}=0.4\right)$ as yellow oil $(56 \mathrm{mg}, 65 \%$ yield $)$. ${ }^{1} \mathrm{H}$ NMR $\left(400 \mathrm{MHz}, \mathrm{CDCl}_{3}\right) \delta 8.24(\mathrm{~d}, J=7.7 \mathrm{~Hz}, 2 \mathrm{H}), 7.95-7.85(\mathrm{~m}, 4 \mathrm{H}), 7.75(\mathrm{~d}, J=7.9 \mathrm{~Hz}, 2 \mathrm{H}), 7.61-7.56$ (m, 2H), $7.54-7.49(\mathrm{~m}, 4 \mathrm{H}) .{ }^{31} \mathrm{P}$ NMR $\left(162 \mathrm{MHz}, \mathrm{CDCl}_{3}\right): \delta 8.54$. HRMS (ESI-TOF) $m / z:[\mathrm{M}+\mathrm{Na}]^{+} \mathrm{Calcd}$ for $\mathrm{C}_{20} \mathrm{H}_{14} \mathrm{NNaO}_{3} \mathrm{P} 370.0609$, found 370.0617 .

\section{(E)-3-(Diphenylphosphoryl)-2-phenylbut-2-enenitrile (4p)}<smiles>C/C([PH2+]O)=C(/C#N)c1ccccc1</smiles>

Following GP1, the residue was purified by flash chromatography on silica gel using dichloromethane/ petroleum ether/ethyl acetate $(v / v / v 4: 1: 1)$ as eluent to afford the pure product $\mathbf{4 p}\left(\mathrm{R}_{\mathrm{f}}=0.7\right)$ as yellow oil $(55 \mathrm{mg}, 65 \% \mathrm{yield})$. ${ }^{1} \mathrm{H}$ NMR $\left(400 \mathrm{MHz}, \mathrm{CDCl}_{3}\right): \delta 7.63-7.54(\mathrm{~m}, 4 \mathrm{H}), 7.43-7.37(\mathrm{~m}, 2 \mathrm{H}), 7.37-7.29(\mathrm{~m}, 6 \mathrm{H}), 7.14-7.00(\mathrm{~m}, 3 \mathrm{H})$, $2.30(\mathrm{~d}, J=12.4 \mathrm{~Hz}, 3 \mathrm{H}) ;{ }^{13} \mathrm{C} \mathrm{NMR}\left(100 \mathrm{MHz}, \mathrm{CDCl}_{3}\right): \delta 148.6,147.7,132.6(\mathrm{~d}, J=5.4 \mathrm{~Hz}), 131.97,131.95$, $131.9,131.1,131.0,130.8,129.6,128.6,128.5,128.1,127.6(\mathrm{~d}, J=14.7 \mathrm{~Hz}), 117.1(\mathrm{~d}, J=21.4 \mathrm{~Hz}), 23.5(\mathrm{~d}, J=$ 
10.1 Hz); ${ }^{31} \mathrm{P}$ NMR $\left(162 \mathrm{MHz}, \mathrm{CDCl}_{3}\right): \delta 26.06$. HRMS (ESI-TOF) $m / z:[\mathrm{M}+\mathrm{H}]^{+}$Calcd for $\mathrm{C}_{22} \mathrm{H}_{19} \mathrm{NOP} 344.1204$, found 344.1200 .

(E)-3-(Diphenylphosphoryl)-2-((8R,9S,13S,14S)-13-methyl-17-oxo-7,8,9,11,12,13,14,15,16,17-decahydro-6Hcyclopenta[a]phenanthren-3-yl)acrylonitrile (4q)<smiles>C[C@]12CC[C@H]3c4ccc(/C=C\[PH](=O)[OH+])cc4CC[C@H]3[C@H]1CCC2=O</smiles>

Following GP1, the residue was purified by flash chromatography on silica gel using dichloromethane/ petroleum ether/ethyl acetate $(v / v / v 4: 1: 1)$ as eluent to afford the pure product $\mathbf{4 q}\left(\mathrm{R}_{\mathrm{f}}=0.5\right)$ as yellow oil $(80 \mathrm{mg}, 60 \%$ yield $)$. ${ }^{1} \mathrm{H}$ NMR $\left(400 \mathrm{MHz}, \mathrm{CDCl}_{3}\right): \delta 7.79-7.56(\mathrm{~m}, 4 \mathrm{H}), 7.53-7.42(\mathrm{~m}, 2 \mathrm{H}), 7.41-7.32(\mathrm{~m}, 6 \mathrm{H}), 7.12(\mathrm{~d}, J=8.0 \mathrm{~Hz}$, $1 \mathrm{H}), 7.08(\mathrm{~d}, J=14.7 \mathrm{~Hz}, 1 \mathrm{H}), 2.80-2.75(\mathrm{~m}, 2 \mathrm{H}), 2.59-2.47(\mathrm{~m}, 1 \mathrm{H}), 2.34-2.24(\mathrm{~m}, 1 \mathrm{H}), 2.18-2.12(\mathrm{~m}, 2 \mathrm{H})$, $2.10-1.87(\mathrm{~m}, 4 \mathrm{H}), 1.66-1.57(\mathrm{~m}, 1 \mathrm{H}), 1.52-1.42(\mathrm{~m}, 4 \mathrm{H}), 0.89(\mathrm{~s}, 3 \mathrm{H}) ;{ }^{13} \mathrm{C} \mathrm{NMR}\left(100 \mathrm{MHz}, \mathrm{CDCl}_{3}\right): \delta 143.2$, 136.7, 136.6, 135.7, $132.5(\mathrm{~d}, J=16.2 \mathrm{~Hz}), 132.2(\mathrm{~d}, J=4.9 \mathrm{~Hz}), 132.1(\mathrm{~d}, J=2.3 \mathrm{~Hz}), 131.4(\mathrm{~d}, J=16.1 \mathrm{~Hz})$, $130.8(\mathrm{~d}, J=4.0 \mathrm{~Hz}), 130.7(\mathrm{~d}, J=4.1 \mathrm{~Hz}), 130.2,128.8(\mathrm{~d}, J=5.1 \mathrm{~Hz}), 128.7(\mathrm{~d}, J=5.1 \mathrm{~Hz}), 126.6,125.4,118.1$ $(\mathrm{d}, J=22.9 \mathrm{~Hz}), 50.5,47.9,44.4,37.8,35.8,31.5,29.1,26.2,25.5,21.6,13.8 ;{ }^{31} \mathrm{P} \mathrm{NMR}\left(162 \mathrm{MHz}^{\mathrm{N}} \mathrm{CDCl}_{3}\right): \delta$ 18.24. HRMS (ESI-TOF) $m / z:[\mathrm{M}+\mathrm{H}]^{+}$Calcd for $\mathrm{C}_{33} \mathrm{H}_{33} \mathrm{NO}_{2} \mathrm{P} 506.2249$, found 506.2240.

\section{(E)-2-((Diphenylphosphoryl)methylene)-4-phenylbutanenitrile (4r)}<smiles>N#C/C(=C/[PH2+]([OH2+])c1ccccc1)CCc1ccccc1</smiles>

Following GP1, the residue was purified by flash chromatography on silica gel using dichloromethane/ petroleum ether/ethyl acetate $(v / v / v 4: 1: 1)$ as eluent to afford the pure product $4 \mathbf{r}\left(\mathrm{R}_{\mathrm{f}}=0.6\right)$ as yellow oil $(50 \mathrm{mg}, 56 \%$ yield $)$. ${ }^{1} \mathrm{H}$ NMR $\left(400 \mathrm{MHz}, \mathrm{CDCl}_{3}\right): \delta 7.62-7.55(\mathrm{~m}, 6 \mathrm{H}), 7.51-7.45(\mathrm{~m}, 4 \mathrm{H}), 7.27-7.14(\mathrm{~m}, 5 \mathrm{H}), 6.82(\mathrm{~d}, J=20.4 \mathrm{~Hz}$, $1 \mathrm{H}), 3.21(\mathrm{t}, J=7.5 \mathrm{~Hz}, 2 \mathrm{H}), 2.92(\mathrm{t}, J=7.1 \mathrm{~Hz}, 2 \mathrm{H}) ;{ }^{13} \mathrm{C} \mathrm{NMR}\left(100 \mathrm{MHz}, \mathrm{CDCl}_{3}\right): \delta 139.2,138.2,137.3,133.8$ $(\mathrm{d}, J=6.0 \mathrm{~Hz}), 132.6(\mathrm{~d}, J=2.6 \mathrm{~Hz}), 132.2,131.1,131.0,130.9,129.1,129.0,128.7,128.6,126.6,117.7(\mathrm{~d}, J=$ 24.0 Hz), $34.0(\mathrm{~d}, J=1.0 \mathrm{~Hz}), 32.8(\mathrm{~d}, J=4.6 \mathrm{~Hz}) ;{ }^{31} \mathrm{P}$ NMR $\left(162 \mathrm{MHz}, \mathrm{CDCl}_{3}\right): \delta 20.90(\mathrm{~d}, J=7.1 \mathrm{~Hz}) . \mathrm{HRMS}$ (ESI-TOF) $m / z:[\mathrm{M}+\mathrm{H}]^{+}$Calcd for $\mathrm{C}_{23} \mathrm{H}_{21} \mathrm{NOP} 358.1361$, found 358.1367.

(E)-2-((Diphenylphosphoryl)methylene)heptanenitrile (4s) 
<smiles>CCCCC/C(C#N)=C\PO</smiles>

Following GP1, the residue was purified by flash chromatography on silica gel using dichloromethane/ petroleum ether/ethyl acetate $(v / v / v 4: 1: 1)$ as eluent to afford the pure product $\mathbf{4 s}\left(\mathrm{R}_{\mathrm{f}}=0.7\right)$ as yellow oil $(36 \mathrm{mg}, 45 \%$ yield $)$. ${ }^{1} \mathrm{H}$ NMR $\left(400 \mathrm{MHz}, \mathrm{CDCl}_{3}\right): \delta 7.78-7.61(\mathrm{~m}, 4 \mathrm{H}), 7.61-7.54(\mathrm{~m}, 2 \mathrm{H}), 7.52-7.46(\mathrm{~m}, 4 \mathrm{H}), 6.86(\mathrm{~d}, J=20.3$ $\mathrm{Hz}, 1 \mathrm{H}), 2.86-2.77(\mathrm{~m}, 2 \mathrm{H}), 1.60-1.50(\mathrm{~m}, 2 \mathrm{H}), 1.27-1.18(\mathrm{~m}, 4 \mathrm{H}), 0.81(\mathrm{t}, J=6.9 \mathrm{~Hz}, 3 \mathrm{H}) ;{ }^{13} \mathrm{C} \mathrm{NMR}(100$ $\left.\mathrm{MHz}, \mathrm{CDCl}_{3}\right): \delta 137.5,136.6,135.2(\mathrm{~d}, J=6.5 \mathrm{~Hz}), 132.7,132.5,131.6,131.0,130.9,129.1,128.9,117.9(\mathrm{~d}, J=$ $24.1 \mathrm{~Hz}), 31.4(\mathrm{~d}, J=4.9 \mathrm{~Hz}), 31.0,27.7(\mathrm{~d}, J=1.3 \mathrm{~Hz}), 22.2,13.8 ;{ }^{31} \mathrm{P} \mathrm{NMR}\left(162 \mathrm{MHz}, \mathrm{CDCl}_{3}\right): \delta 19.74 . \mathrm{HRMS}$ (ESI-TOF) $m / z:[\mathrm{M}+\mathrm{H}]^{+}$Calcd for Calcd for $\mathrm{C}_{20} \mathrm{H}_{23} \mathrm{NOP} 324.1517$, found 324.1510.

\section{(E)-2-((Diphenylphosphoryl)methylene)octanenitrile (4t)}<smiles>CCCCCC/C(C#N)=C\PO</smiles>

Following GP1, the residue was purified by flash chromatography on silica gel using dichloromethane/ petroleum ether/ethyl acetate $(v / v / v 4: 1: 1)$ as eluent to afford the pure product $\mathbf{4 t}\left(\mathrm{R}_{\mathrm{f}}=0.7\right)$ as yellow oil $(43 \mathrm{mg}, 52 \%$ yield $)$. ${ }^{1} \mathrm{H}$ NMR $\left(400 \mathrm{MHz}, \mathrm{CDCl}_{3}\right): \delta 7.73-7.67(\mathrm{~m}, 4 \mathrm{H}), 7.60-7.55(\mathrm{~m}, 2 \mathrm{H}), 7.52-7.47(\mathrm{~m}, 4 \mathrm{H}), 6.86(\mathrm{~d}, J=20.3$ $\mathrm{Hz}, 1 \mathrm{H}), 2.91-2.68(\mathrm{~m}, 2 \mathrm{H}), 1.66-1.48(\mathrm{~m}, 2 \mathrm{H}), 1.24-1.16(\mathrm{~m}, 6 \mathrm{H}), 0.83(\mathrm{t}, J=6.8 \mathrm{~Hz}, 3 \mathrm{H}) ;{ }^{13} \mathrm{C} \mathrm{NMR}(100$ $\left.\mathrm{MHz}, \mathrm{CDCl}_{3}\right): \delta 137.5,136.6,135.2(\mathrm{~d}, J=6.5 \mathrm{~Hz}), 132.7,132.5(\mathrm{~d}, J=2.8 \mathrm{~Hz}), 131.6,131.0,130.9,129.1$, 128.9, $117.9(\mathrm{~d}, J=24.1 \mathrm{~Hz}), 31.4(\mathrm{~d}, J=5.0 \mathrm{~Hz}), 31.3,28.5,28.0(\mathrm{~d}, J=1.3 \mathrm{~Hz}), 22.4,14.0 ;{ }^{31} \mathrm{P} \mathrm{NMR}(162 \mathrm{MHz}$, $\mathrm{CDCl}_{3}$ ): $\delta$ 19.77. HRMS (ESI-TOF) $\mathrm{m} / z:[\mathrm{M}+\mathrm{H}]^{+}$Calcd for $\mathrm{C}_{21} \mathrm{H}_{25} \mathrm{NOP} 338.1674$, found 338.1672.

\section{(E)-3-(Di-p-tolylphosphoryl)-2-phenylacrylonitrile (5a)}<smiles>Cc1ccc(P(=O)(/C=C(\C#N)c2ccccc2)c2ccc(C)cc2)cc1</smiles>

Following GP1, the residue was purified by flash chromatography on silica gel using dichloromethane/ petroleum ether/ethyl acetate $(v / v / v 4: 1: 1)$ as eluent to afford the pure product $\mathbf{5 a}\left(\mathrm{R}_{\mathrm{f}}=0.6\right)$ as yellow oil $(64 \mathrm{mg}, 72 \%$ yield $)$. ${ }^{1} \mathrm{H}$ NMR $\left(400 \mathrm{MHz}, \mathrm{CDCl}_{3}\right): \delta 7.70-7.64(\mathrm{~m}, 2 \mathrm{H}), 7.56-7.49(\mathrm{~m}, 4 \mathrm{H}), 7.29-7.15(\mathrm{~m}, 8 \mathrm{H}), 2.33(\mathrm{~s}, 6 \mathrm{H}) ;{ }^{13} \mathrm{C}$ NMR (100 MHz, $\left.\mathrm{CDCl}_{3}\right): \delta 142.8(\mathrm{~d}, J=2.9 \mathrm{~Hz}), 138.4,137.5,131.7(\mathrm{~d}, J=4.7 \mathrm{~Hz}), 131.2(\mathrm{~d}, J=4.8 \mathrm{~Hz}), 130.9$, 130.8, 130.8, 129.6, 129.5, 129.4, 129.1, 128.3, 128.0, $118.2(\mathrm{~d}, J=22.7 \mathrm{~Hz}), 21.6(\mathrm{~d}, J=1.1 \mathrm{~Hz}){ }^{31} \mathrm{P}$ NMR $(162$ 
$\mathrm{MHz}, \mathrm{CDCl}_{3}$ ): $\delta$ 18.91. HRMS (ESI-TOF) $m / z:[\mathrm{M}+\mathrm{Na}]^{+}$Calcd for $\mathrm{C}_{23} \mathrm{H}_{20} \mathrm{NNaOP} 380.1180$, found 380.1177 .

(E)-3-(Bis(4-methoxyphenyl)phosphoryl)-2-phenylacrylonitrile (5b)<smiles>COc1ccc(P(=O)(/C=C(\C#N)c2ccccc2)c2ccc(OC)cc2)cc1</smiles>

Following GP1, the residue was purified by flash chromatography on silica gel using dichloromethane/ petroleum ether/ethyl acetate $(v / v / v 4: 1: 1)$ as eluent to afford the pure product $\mathbf{5} \mathbf{b}\left(\mathrm{R}_{\mathrm{f}}=0.5\right)$ as yellow oil $(73 \mathrm{mg}, 75 \%$ yield $)$. ${ }^{1} \mathrm{H}$ NMR (400 MHz, $\left.\mathrm{CDCl}_{3}\right): \delta 7.65-7.56(\mathrm{~m}, 2 \mathrm{H}), 7.51-7.43(\mathrm{~m}, 4 \mathrm{H}), 7.25-7.11(\mathrm{~m}, 3 \mathrm{H}), 7.07(\mathrm{~d}, J=14.3$ $\mathrm{Hz}, 1 \mathrm{H}), 6.83-6.77(\mathrm{~m}, 4 \mathrm{H}), 3.71(\mathrm{~d}, J=2.1 \mathrm{~Hz}, 6 \mathrm{H}) ;{ }^{13} \mathrm{C} \mathrm{NMR}\left(100 \mathrm{MHz}, \mathrm{CDCl}_{3}\right): \delta 162.7(\mathrm{~d}, J=2.9 \mathrm{~Hz})$, 138.8, 137.9, 132.8, 132.7, $131.3(\mathrm{~d}, J=4.8 \mathrm{~Hz}), 131.2(\mathrm{~d}, J=4.8 \mathrm{~Hz}), 130.8,129.5,128.3,123.5,122.3,118.2(\mathrm{~d}$, $J=22.7 \mathrm{~Hz}), 114.4,114.3,55.4 ;{ }^{31} \mathrm{P}$ NMR $\left(162 \mathrm{MHz}, \mathrm{CDCl}_{3}\right): \delta 18.81$. HRMS (ESI-TOF) $m / z:[\mathrm{M}+\mathrm{H}]^{+} \mathrm{Calcd}$ for $\mathrm{C}_{23} \mathrm{H}_{21} \mathrm{NO}_{3} \mathrm{P} 390.1259$, found 390.1251 .

\section{(E)-3-(Bis(4-chlorophenyl)phosphoryl)-2-phenylacrylonitrile (5c)}<smiles>N#C/C(=C/P(=O)(c1ccc(Cl)cc1)c1ccc(Cl)cc1)c1ccccc1</smiles>

Following GP1, the residue was purified by flash chromatography on silica gel using dichloromethane/ petroleum ether/ethyl acetate $(v / v / v 4: 1: 1)$ as eluent to afford the pure product $\mathbf{5} \mathbf{c}\left(\mathrm{R}_{\mathrm{f}}=0.6\right)$ as yellow oil $(65 \mathrm{mg}, 66 \%$ yield $)$. ${ }^{1} \mathrm{H}$ NMR $\left(400 \mathrm{MHz}, \mathrm{CDCl}_{3}\right): \delta 7.68-7.63(\mathrm{~m}, 2 \mathrm{H}), 7.61-7.54(\mathrm{~m}, 4 \mathrm{H}), 7.42-7.33(\mathrm{~m}, 4 \mathrm{H}), 7.32-7.28(\mathrm{~m}, 1 \mathrm{H})$, $7.28-7.21(\mathrm{~m}, 2 \mathrm{H}), 7.10(\mathrm{~d}, J=14.9 \mathrm{~Hz}, 1 \mathrm{H}) ;{ }^{13} \mathrm{C} \mathrm{NMR}\left(100 \mathrm{MHz}, \mathrm{CDCl}_{3}\right): \delta 139.2(\mathrm{~d}, J=3.3 \mathrm{~Hz}), 136.6,135.7$, $133.2(\mathrm{~d}, J=5.4 \mathrm{~Hz}), 132.2,132.1,131.3,131.0(\mathrm{~d}, J=5.0 \mathrm{~Hz}), 130.3,129.4,129.3,129.2,128.5,117.7(\mathrm{~d}, J=$ $23.3 \mathrm{~Hz}) ;{ }^{31} \mathrm{P}$ NMR $\left(162 \mathrm{MHz}, \mathrm{CDCl}_{3}\right): \delta 16.92$. HRMS (ESI-TOF) $\mathrm{m} / z:[\mathrm{M}+\mathrm{H}]^{+}$Calcd for $\mathrm{C}_{21} \mathrm{H}_{15} \mathrm{Cl}_{2} \mathrm{NOP}$ 398.0268, found 398.0260.

(E)-3-(Di(naphthalen-1-yl)phosphoryl)-2-phenylacrylonitrile (5d) 


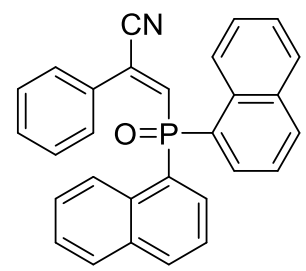

Following GP1, the residue was purified by flash chromatography on silica gel using dichloromethane/ petroleum ether/ethyl acetate $(v / v / v 4: 1: 1)$ as eluent to afford the pure product $\mathbf{5 d}\left(\mathrm{R}_{\mathrm{f}}=0.5\right)$ as yellow oil $(62 \mathrm{mg}, 58 \%$ yield $)$. ${ }^{1} \mathrm{H}$ NMR $\left(400 \mathrm{MHz}, \mathrm{CDCl}_{3}\right): \delta 8.50-8.28(\mathrm{~m}, 2 \mathrm{H}), 8.02-7.96(\mathrm{~m}, 2 \mathrm{H}), 7.95-7.89(\mathrm{~m}, 2 \mathrm{H}), 7.88-7.80(\mathrm{~m}, 2 \mathrm{H})$, $7.63-7.54(\mathrm{~m}, 4 \mathrm{H}), 7.52-7.35(\mathrm{~m}, 5 \mathrm{H}), 7.18-7.06(\mathrm{~m}, 1 \mathrm{H}), 7.08-6.97(\mathrm{~m}, 2 \mathrm{H}) ;{ }^{13} \mathrm{C} \mathrm{NMR}\left(100 \mathrm{MHz} \mathrm{CDCl}_{3}\right)$ : $\delta 139.2,138.3,134.2(\mathrm{~d}, J=11.1 \mathrm{~Hz}), 133.9(\mathrm{~d}, J=3.0 \mathrm{~Hz}), 133.8(\mathrm{~d}, J=9.5 \mathrm{~Hz}), 132.7(\mathrm{~d}, J=8.8 \mathrm{~Hz}), 131.4(\mathrm{~d}$, $J=5.1 \mathrm{~Hz}), 130.9(\mathrm{~d}, J=5.0 \mathrm{~Hz}), 130.5,129.3,128.9,127.9,127.8,127.4,126.64,126.55(\mathrm{~d}, J=5.4 \mathrm{~Hz}), 126.3$, $124.7(\mathrm{~d}, J=14.6 \mathrm{~Hz}), 118.2(\mathrm{~d}, J=23.1 \mathrm{~Hz}) ;{ }^{31} \mathrm{P} \mathrm{NMR}\left(162 \mathrm{MHz}, \mathrm{CDCl}_{3}\right): \delta 22.07 . \mathrm{HRMS}(\mathrm{ESI}-\mathrm{TOF}) m / z$ : $[\mathrm{M}+\mathrm{H}]^{+}$Calcd for $\mathrm{C}_{29} \mathrm{H}_{21} \mathrm{NOP} 430.1361$, found 430.1362 .

\section{(E)-3-(Di(thiophen-2-yl)phosphoryl)-2-phenylacrylonitrile (5e)}<smiles>N#C/C(=C/P(=O)(c1cccs1)c1cccs1)c1ccccc1</smiles>

Following GP1, the residue was purified by flash chromatography on silica gel using dichloromethane/ petroleum ether/ethyl acetate $\left(v / v / v\right.$ 4:1:1) as eluent to afford the pure product $\mathbf{5 e}\left(\mathrm{R}_{\mathrm{f}}=0.7\right)$ as yellow oil $(47 \mathrm{mg}, 55 \%$ yield $)$. ${ }^{1} \mathrm{H}$ NMR $\left(400 \mathrm{MHz}, \mathrm{CDCl}_{3}\right): \delta 7.77-7.71(\mathrm{~m}, 2 \mathrm{H}), 7.70-7.60(\mathrm{~m}, 2 \mathrm{H}), 7.55-7.50(\mathrm{~m}, 2 \mathrm{H}), 7.38-7.27(\mathrm{~m}, 3 \mathrm{H})$, $7.18(\mathrm{~d}, J=16.4 \mathrm{~Hz}, 1 \mathrm{H}), 7.11-7.08(\mathrm{~m}, 2 \mathrm{H}) ;{ }^{13} \mathrm{C} \mathrm{NMR}\left(100 \mathrm{MHz}, \mathrm{CDCl}_{3}\right): \delta 138.3,137.3,136.6(\mathrm{~d}, J=10.9$ Hz), $134.4(\mathrm{~d}, J=5.7 \mathrm{~Hz}), 133.2,132.0,131.9,131.14,131.09(\mathrm{~d}, J=5.6 \mathrm{~Hz}), 129.6(\mathrm{~d}, J=1.1 \mathrm{~Hz}), 128.6,128.5$, 128.4, $117.9(\mathrm{~d}, J=25.1 \mathrm{~Hz}){ }^{31} \mathrm{P}$ NMR $\left(162 \mathrm{MHz}, \mathrm{CDCl}_{3}\right): \delta$ 5.39. HRMS (ESI-TOF) $m / z:[\mathrm{M}+\mathrm{Na}]^{+} \mathrm{Calcd}$ for $\mathrm{C}_{17} \mathrm{H}_{12} \mathrm{NNaOPS}_{2} 363.9996$, found 363.9997.

Diethyl (E)-(2-cyano-2-phenylvinyl)phosphonate (5g)<smiles>CCOP(=O)(/C=C(/C#N)c1ccccc1)OCC</smiles>

Following GP1, the residue was purified by flash chromatography on silica gel using dichloromethane/ petroleum ether/ethyl acetate $\left(v / v / v\right.$ 4:1:1) as eluent to afford the pure product $\mathbf{5 g}\left(\mathrm{R}_{\mathrm{f}}=0.7\right)$ as yellow oil $(17 \mathrm{mg}, 30 \%$ yield $)$. ${ }^{1} \mathrm{H}$ NMR $\left(400 \mathrm{MHz}, \mathrm{CDCl}_{3}\right): \delta 7.80-7.54(\mathrm{~m}, 2 \mathrm{H}), 7.56-7.31(\mathrm{~m}, 3 \mathrm{H}), 6.20(\mathrm{~d}, J=9.1 \mathrm{~Hz}, 1 \mathrm{H}), 4.03-3.70(\mathrm{~m}$, 
4H), $1.12(\mathrm{t}, J=7.1 \mathrm{~Hz}, 6 \mathrm{H}) ;{ }^{13} \mathrm{C} \mathrm{NMR}\left(100 \mathrm{MHz}, \mathrm{CDCl}_{3}\right): \delta 150.9(\mathrm{~d}, J=17.5 \mathrm{~Hz}), 136.9,130.3,128.7(\mathrm{~d}, J=$ $1.3 \mathrm{~Hz}), 128.0,118.2,116.4,62.1(\mathrm{~d}, J=5.9 \mathrm{~Hz}), 16.1(\mathrm{~d}, J=6.7 \mathrm{~Hz}) ;{ }^{31} \mathrm{P}$ NMR $\left(162 \mathrm{MHz}, \mathrm{CDCl}_{3}\right): \delta 11.83$. HRMS (ESI-TOF) $m / z$ : [M+Na $]^{+}$Calcd for $\mathrm{C}_{13} \mathrm{H}_{16} \mathrm{NNaO}_{3} \mathrm{P} 288.0765$, found 288.0769.

\section{Diisopropyl (E)-(2-cyano-2-phenylvinyl)phosphonate (5h)}<smiles>CC(C)OP(=O)(/C=C(/C#N)c1ccccc1)OC(C)C</smiles>

Following GP1, the residue was purified by flash chromatography on silica gel using dichloromethane/ petroleum ether/ethyl acetate $(v / v / v 4: 1: 1)$ as eluent to afford the pure product $\mathbf{5 h}\left(\mathrm{R}_{\mathrm{f}}=0.7\right)$ as yellow oil $(29 \mathrm{mg}, 40 \%$ yield $)$. ${ }^{1} \mathrm{H}$ NMR $\left(400 \mathrm{MHz}, \mathrm{CDCl}_{3}\right): \delta 7.78-7.50(\mathrm{~m}, 2 \mathrm{H}), 7.40-7.35(\mathrm{~m}, 3 \mathrm{H}), 6.19(\mathrm{~d}, J=9.1 \mathrm{~Hz}, 1 \mathrm{H}), 4.64-4.37(\mathrm{~m}$, 2H), $1.20(\mathrm{~d}, J=6.2 \mathrm{~Hz}, 6 \mathrm{H}), 1.09(\mathrm{~d}, J=6.2 \mathrm{~Hz}, 6 \mathrm{H}) ;{ }^{13} \mathrm{C} \mathrm{NMR}\left(100 \mathrm{MHz}, \mathrm{CDCl}_{3}\right): \delta 150.3(\mathrm{~d}, J=17.0 \mathrm{~Hz})$, $137.0(\mathrm{~d}, J=4.1 \mathrm{~Hz}), 130.2,128.9(\mathrm{~d}, J=1.5 \mathrm{~Hz}), 127.9,119.7,117.9,71.1(\mathrm{~d}, J=6.3 \mathrm{~Hz}), 23.9(\mathrm{~d}, J=4.0 \mathrm{~Hz})$, $23.6(\mathrm{~d}, J=5.2 \mathrm{~Hz}) ;{ }^{31} \mathrm{P}$ NMR $\left(162 \mathrm{MHz}, \mathrm{CDCl}_{3}\right): \delta$ 9.62. HRMS (ESI-TOF) $m / z:[\mathrm{M}+\mathrm{Na}]^{+} \mathrm{Calcd}$ for $\mathrm{C}_{15} \mathrm{H}_{20} \mathrm{NNaO}_{3} \mathrm{P} 316.1078$, found 316.1071.

(Z)-Diphenyl(2-(trimethylsilyl)vinyl)phosphine oxide (5i')<smiles>C[Si](C)(C)/C=C\[Po](=O)O</smiles>

Following GP1, the residue was purified by flash chromatography on silica gel using petroleum ether/ethyl acetate $(v / v 2: 1)$ as eluent to afford the pure product $\mathbf{5 i} \mathbf{i}^{\prime}(\mathrm{Rf}=0.7)$ as white solid $(42 \mathrm{mg}, 55 \%$ yield $) .{ }^{1} \mathrm{H} \mathrm{NMR}(400 \mathrm{MHz}$, $\left.\mathrm{CDCl}_{3}\right): \delta 7.73-7.65(\mathrm{~m}, 4 \mathrm{H}), 7.54-7.36(\mathrm{~m}, 6 \mathrm{H}), 7.16-7.02(\mathrm{~m}, 1 \mathrm{H}), 7.00-6.96(\mathrm{~m}, 1 \mathrm{H}), 0.25(\mathrm{~s}, 9 \mathrm{H}) .{ }^{13} \mathrm{C}$ NMR (101 MHz, $\left.\mathrm{CDCl}_{3}\right): \delta 156.8(\mathrm{~d}, J=7.7 \mathrm{~Hz}), 138.7(\mathrm{~d}, J=101.0 \mathrm{~Hz}), 134.3(\mathrm{~d}, J=102.0 \mathrm{~Hz}), 131.5(\mathrm{~d}, J=$ $2.7 \mathrm{~Hz}), 131.1(\mathrm{~d}, J=9.6 \mathrm{~Hz}), 128.5(\mathrm{~d}, J=11.8 \mathrm{~Hz}), 0.4 .{ }^{31} \mathrm{P}$ NMR $\left(162 \mathrm{MHz}, \mathrm{CDCl}_{3}\right): \delta 20.43$. (Reference: Huang, T. Z.; Saga. Y.; Guo. H. Q.; Yoshimura, A.; Ogawa, A.; Han, L. B. Radical Hydrophosphorylation of Alkynes with $\mathrm{R}_{2} \mathrm{P}(\mathrm{O}) \mathrm{H}$ Generating Alkenylphosphine Oxides: Scope and Limitations. J. Org. Chem. 2018, 83, 8743-8749).

\section{Methyl (E)-3-cyano-2-(diphenylphosphoryl)-3-phenylacrylate (5j)}<smiles>COC(=O)/C(=C(\C#N)c1ccccc1)[PH+]([OH+])c1ccccc1</smiles> 
Following GP1, the residue was purified by flash chromatography on silica gel using dichloromethane/petroleum ether/ethyl acetate $(v / v / v 4: 1: 1)$ as eluent to afford the pure product $\mathbf{5 j}(\mathrm{Rf}=0.5)$ as yellow oil $(29 \mathrm{mg}, 30 \%$ yield). ${ }^{1} \mathrm{H}$ NMR $\left(400 \mathrm{MHz}, \mathrm{CDCl}_{3}\right): \delta 7.68-7.56(\mathrm{~m}, 4 \mathrm{H}), 7.52-7.45(\mathrm{~m}, 2 \mathrm{H}), 7.40-7.32(\mathrm{~m}, 2 \mathrm{H}), 7.33-7.24(\mathrm{~m}, 4 \mathrm{H})$, $7.21-7.14(\mathrm{~m}, 1 \mathrm{H}), 7.13-7.03(\mathrm{~m}, 2 \mathrm{H}), 3.37(\mathrm{~s}, 3 \mathrm{H}) .{ }^{13} \mathrm{C} \mathrm{NMR}\left(101 \mathrm{MHz}, \mathrm{CDCl}_{3}\right): \delta 165.5(\mathrm{~d}, J=9.7 \mathrm{~Hz})$, $145.7,144.9,132.4(\mathrm{~d}, J=2.9 \mathrm{~Hz}), 131.2,131.1,131.1,130.9,129.8,129.7,129.5,129.4,128.6,128.5,128.4$, $116.0(\mathrm{~d}, J=18.6 \mathrm{~Hz}), 53.2 .{ }^{31} \mathrm{P} \mathrm{NMR}\left(162 \mathrm{MHz}, \mathrm{CDCl}_{3}\right): \delta 20.02 . \mathrm{HRMS}(\mathrm{ESI}-\mathrm{TOF}) \mathrm{m} / z:[\mathrm{M}+\mathrm{Na}]^{+} \mathrm{Calcd}$ for $\mathrm{C}_{23} \mathrm{H}_{18} \mathrm{NNaO}_{3} \mathrm{P} 410.0922$, found 410.0910 .

\section{1,2,3-Triphenylphosphindole 1-oxide (5k')}<smiles>O=P1(c2ccccc2)C(c2ccccc2)=C(c2ccccc2)c2ccccc21</smiles>

Following GP1, compound 5k' was obtained as white solid (61 mg, 65\% yield). ${ }^{1} \mathrm{H}$ NMR (400 $\left.\mathrm{MHz} \mathrm{CDCl}_{3}\right): \delta$ $7.77(\mathrm{dd}, J=12.5,7.1 \mathrm{~Hz}, 2 \mathrm{H}), 7.74-7.65(\mathrm{~m}, 1 \mathrm{H}), 7.51-7.30(\mathrm{~m}, 10 \mathrm{H}), 7.24-7.18(\mathrm{~m}, 3 \mathrm{H}), 7.10-7.09(\mathrm{~m}$, 3H). ${ }^{31} \mathrm{P}$ NMR (162 MHz, $\left.\mathrm{CDCl}_{3}\right): \delta 39.15$ (Reference: Chen, Y. R.; Duan, W. L. Silver-Mediated Oxidative C-H/P-H Functionalization: An Efficient Route for the Synthesis of Benzo[b]phosphole Oxides. J. Am. Chem. Soc. 2013, 135, 16754-16757).

\subsection{Characterization Data for Thiocyanophosphorylation Products 7}

\section{(Z)-Diphenyl(2-phenyl-2-thiocyanatovinyl)phosphine oxide (7a)}<smiles>N#C/C(=C\P(c1ccccc1)c1ccccc1)c1ccccc1</smiles>

Following GP2, the residue was purified by flash chromatography on silica gel using ethyl acetate/ petroleum ether $(v / v 1: 5)$ as eluent to afford the pure product $7 \mathbf{a}\left(\mathrm{R}_{\mathrm{f}}=0.3\right)$ as yellow oil $(146.1 \mathrm{mg}, 74 \%$ yield $) .{ }^{1} \mathrm{H} \mathrm{NMR}$ $\left(400 \mathrm{MHz}, \mathrm{CDCl}_{3}\right): \delta 7.85-7.71(\mathrm{~m}, 4 \mathrm{H}), 7.67-7.39(\mathrm{~m}, 11 \mathrm{H}), 6.62(\mathrm{~d}, J=19.1 \mathrm{~Hz}, 1 \mathrm{H}) ;{ }^{13} \mathrm{C} \mathrm{NMR}(100 \mathrm{MHz}$, $\left.\mathrm{CDCl}_{3}\right): \delta 150.2(\mathrm{~d}, J=1.4 \mathrm{~Hz}), 137.5(\mathrm{~d}, J=13.4 \mathrm{~Hz}), 132.09,132.06,132.0,130.9,130.6,130.54,130.46,128.6$

128.5, 128.5, 127.5, $124.5(\mathrm{~d}, J=97.0 \mathrm{~Hz}), 109.0 ;{ }^{31} \mathrm{P}$ NMR $\left(162 \mathrm{MHz}, \mathrm{CDCl}_{3}\right): \delta 22.17 . \mathrm{IR}\left(\mathrm{cm}^{-1}\right) 2152.99$. HRMS (ESI-TOF) $m / z$ : $[\mathrm{M}+\mathrm{H}]^{+}$Calcd for $\mathrm{C}_{21} \mathrm{H}_{17}$ NOPS 362.0768, found 362.0771.

(Z)-Diphenyl(2-thiocyanato-2-(o-tolyl)vinyl)phosphine oxide (7b) 
P(O)Ph

Following GP2, the residue was purified by flash chromatography on silica gel using ethyl acetate/ petroleum ether $(v / v 1: 5)$ as eluent to afford the pure product $7 \mathbf{b}\left(\mathrm{R}_{\mathrm{f}}=0.3\right)$ as yellow oil $(125.6 \mathrm{mg}, 67 \%$ yield $) .{ }^{1} \mathrm{H}$ NMR $\left(400 \mathrm{MHz}, \mathrm{CDCl}_{3}\right): \delta 7.81-7.71(\mathrm{~m}, 4 \mathrm{H}), 7.61-7.49(\mathrm{~m}, 6 \mathrm{H}), 7.44-7.40(\mathrm{~d}, J=8.1 \mathrm{~Hz}, 2 \mathrm{H}), 7.24(\mathrm{~s}, 2 \mathrm{H}), 6.60$ (d, $J=19.1 \mathrm{~Hz}, 1 \mathrm{H}), 2.39$ (s, 3H); ${ }^{13} \mathrm{C}$ NMR $\left(100 \mathrm{MHz}, \mathrm{CDCl}_{3}\right): \delta 150.8,141.5(\mathrm{~d}, J=2.0 \mathrm{~Hz}), 132.5,131.14$, 131.12, 131.04, 131.02, 129.6, 129.0, 128.93, 128.91, 127.9, 124.8, 123.8, 109.6, 21.5; ${ }^{31} \mathrm{P}$ NMR (162 MHz, $\mathrm{CDCl}_{3}$ ): $\delta$ 22.04. IR $\left(\mathrm{cm}^{-1}\right)$ 2153.50. HRMS (ESI-TOF) $\mathrm{m} / z:[\mathrm{M}+\mathrm{Na}]^{+} \mathrm{Calcd}$ for $\mathrm{C}_{22} \mathrm{H}_{18} \mathrm{NNaOPS} 398.0744$, found 398.0747.

(Z)-Diphenyl(2-thiocyanato-2-(p-tolyl)vinyl)phosphine oxide (7c)<smiles>Cc1ccc(/C(=C/[Pb])[Se]P)cc1</smiles>

Following GP2, the residue was purified by flash chromatography on silica gel using ethyl acetate/ petroleum ether $(v / v 1: 5)$ as eluent to afford the pure product $7 \mathbf{c}\left(\mathrm{R}_{\mathrm{f}}=0.4\right)$ as yellow oil $(123.8 \mathrm{mg}, 66 \%$ yield $) .{ }^{1} \mathrm{H}$ NMR $\left(400 \mathrm{MHz}, \mathrm{CDCl}_{3}\right): \delta 7.75-7.63(\mathrm{~m}, 4 \mathrm{H}), 7.52-7.48(\mathrm{~m}, 2 \mathrm{H}), 7.46-7.41(\mathrm{~m}, 4 \mathrm{H}), 7.36-7.32(\mathrm{~m}, 2 \mathrm{H}), 7.20-$ $7.16(\mathrm{~m}, 2 \mathrm{H}), 6.52(\mathrm{~d}, J=19.1 \mathrm{~Hz}, 1 \mathrm{H}), 2.31(\mathrm{~s}, 3 \mathrm{H}) .{ }^{13} \mathrm{C} \mathrm{NMR}\left(101 \mathrm{MHz}, \mathrm{CDCl}_{3}\right): \delta 150.74(\mathrm{~d}, J=1.6 \mathrm{~Hz})$, $141.5,135.1(\mathrm{~d}, J=13.4 \mathrm{~Hz}), 132.6,132.5(\mathrm{~d}, J=2.8 \mathrm{~Hz}), 131.5,131.1,131.0,129.6,129.0,128.9,127.9,124.8$, 123.8, 109.6, 21.5. ${ }^{31} \mathrm{P}$ NMR $\left(162 \mathrm{MHz}, \mathrm{CDCl}_{3}\right): \delta 22.09$. IR $\left(\mathrm{cm}^{-1}\right)$ 2153.50. HRMS (ESI-TOF) $m / z:[\mathrm{M}+\mathrm{Na}]^{+}$ Calcd for $\mathrm{C}_{22} \mathrm{H}_{18} \mathrm{NNaOPS} 398.0744$, found 398.0747.

\section{(Z)-Diphenyl(2-thiocyanato-2-(m-tolyl)vinyl)phosphine oxide (7d)}

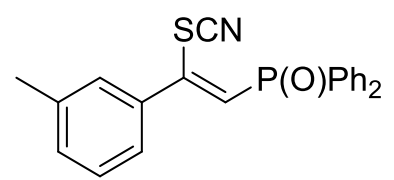

Following GP2, the residue was purified by flash chromatography on silica gel using ethyl acetate/ petroleum ether $(v / v 1: 5)$ as eluent to afford the pure product $7 \mathbf{d}\left(\mathrm{R}_{\mathrm{f}}=0.3\right)$ as yellow oil $\left(129.4 \mathrm{mg}, 69 \%\right.$ yield). ${ }^{1} \mathrm{H}$ NMR $\left(400 \mathrm{MHz}, \mathrm{DMSO}-d_{6}\right): \delta 7.88-7.79(\mathrm{~m}, 4 \mathrm{H}), 7.66-7.54(\mathrm{~m}, 6 \mathrm{H}), 7.48-7.30(\mathrm{~m}, 4 \mathrm{H}), 7.24(\mathrm{~d}, J=19.0 \mathrm{~Hz}, 1 \mathrm{H})$, 2.37 (s, 3H); ${ }^{13} \mathrm{C}$ NMR (100 MHz, DMSO- $\left.d_{6}\right): \delta 148.3(\mathrm{~d}, J=1.8 \mathrm{~Hz}), 138.2,137.6(\mathrm{~d}, J=13.5 \mathrm{~Hz}), 133.2,132.3$ (d, $J=2.4 \mathrm{~Hz}), 132.2,131.3,130.7,130.6,129.0,128.9,128.6,128.5,126.4,125.4,125.3,110.1,20.8 ;{ }^{31} \mathrm{P}$ NMR (162 MHz, DMSO- $\left.d_{6}\right): \delta 20.40$. IR $\left(\mathrm{cm}^{-1}\right)$ 2160.45. HRMS (ESI-TOF) $m / z:[\mathrm{M}+\mathrm{Na}]^{+}$Calcd for $\mathrm{C}_{22} \mathrm{H}_{18} \mathrm{NNaOPS}$ 398.0744, found 398.0746. 
<smiles>COc1ccccc1/C([Se])=C/[PbH]P</smiles>

Following GP2, the residue was purified by flash chromatography on silica gel using ethyl acetate/ petroleum ether $(v / v 1: 5)$ as eluent to afford the pure product $7 \mathbf{e}\left(\mathrm{R}_{\mathrm{f}}=0.2\right)$ as yellow oil $(132.9 \mathrm{mg}, 68 \%$ yield $) .{ }^{1} \mathrm{H}$ NMR $\left(400 \mathrm{MHz}, \mathrm{CDCl}_{3}\right): \delta 7.79-7.71(\mathrm{~m}, 4 \mathrm{H}), 7.61-7.48(\mathrm{~m}, 6 \mathrm{H}), 7.45-7.39(\mathrm{~m}, 1 \mathrm{H}), 7.24(\mathrm{dd}, J=7.6,1.7 \mathrm{~Hz}$, 1H), $7.03-6.95(\mathrm{~m}, 2 \mathrm{H}), 6.45(\mathrm{~d}, J=20.4 \mathrm{~Hz}, 1 \mathrm{H}), 3.93(\mathrm{~s}, 3 \mathrm{H}) ;{ }^{13} \mathrm{C}$ NMR $\left(150 \mathrm{MHz}, \mathrm{CDCl}_{3}\right): \delta 156.1,149.1$, $132.5,132.4,132.3,131.9,131.8,131.14,131.08,129.5,128.9,128.8,127.3(\mathrm{~d}, J=13.9 \mathrm{~Hz}), 122.9(\mathrm{~d}, J=96.4$ $\mathrm{Hz}), 120.7,111.4,110.1,55.8 ;{ }^{31} \mathrm{P}$ NMR $\left(162 \mathrm{MHz}, \mathrm{CDCl}_{3}\right): \delta 23.62 . \mathrm{IR}\left(\mathrm{cm}^{-1}\right) 2156.50 . \mathrm{HRMS}(\mathrm{ESI}-\mathrm{TOF}) \mathrm{m} / z$ : $[\mathrm{M}+\mathrm{H}]^{+}$Calcd for $\mathrm{C}_{22} \mathrm{H}_{19} \mathrm{NO}_{2} \mathrm{PS} 392.0874$, found 392.0864 .

(Z)-(2-(3-Methoxyphenyl)-2-thiocyanatovinyl)diphenylphosphine oxide (7f)

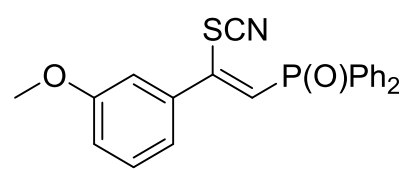

Following GP2, the residue was purified by flash chromatography on silica gel using ethyl acetate/ petroleum ether $(v / v 1: 5)$ as eluent to afford the pure product $7 \mathbf{f}\left(\mathrm{R}_{\mathrm{f}}=0.3\right)$ as yellow oil $(134.9 \mathrm{mg}, 69 \%$ yield $) .{ }^{1} \mathrm{H}$ NMR $(400$ $\left.\mathrm{MHz}, \mathrm{CDCl}_{3}\right): \delta 7.72-7.63(\mathrm{~m}, 4 \mathrm{H}), 7.53-7.48(\mathrm{~m}, 2 \mathrm{H}), 7.46-7.41(\mathrm{~m}, 4 \mathrm{H}), 7.31-7.25(\mathrm{~m}, 1 \mathrm{H}), 7.04-7.01$ $(\mathrm{m}, 1 \mathrm{H}), 6.95-6.89(\mathrm{~m}, 2 \mathrm{H}), 6.56(\mathrm{~d}, J=19.2 \mathrm{~Hz}, 1 \mathrm{H}), 3.76(\mathrm{~s}, 3 \mathrm{H}) .{ }^{13} \mathrm{C} \mathrm{NMR}\left(101 \mathrm{MHz}, \mathrm{CDCl}_{3}\right): \delta 159.7,150.4$ $(\mathrm{d}, J=1.7 \mathrm{~Hz}), 139.2(\mathrm{~d}, J=13.4 \mathrm{~Hz}), 132.5(\mathrm{~d}, J=2.8 \mathrm{~Hz}), 132.4,131.4,131.1,131.0,130.0,129.1,128.9$, 125.5, 124.5, 120.3, 116.3, 113.7, 109.4, 55.5. ${ }^{31} \mathrm{P}$ NMR (162 MHz, $\left.\mathrm{CDCl}_{3}\right): \delta 22.20 . \mathrm{IR}\left(\mathrm{cm}^{-1}\right) 2158.01 . \mathrm{HRMS}$ (ESI-TOF) $m / z:[\mathrm{M}+\mathrm{Na}]^{+}$Calcd for $\mathrm{C}_{22} \mathrm{H}_{18} \mathrm{NNaO}_{2} \mathrm{PS} 414.0694$, found 414.0684 .

(Z)-(2-(4-Methoxyphenyl)-2-thiocyanatovinyl)diphenylphosphine oxide (7g)<smiles>COc1ccc(/C(=C/[Pb](=O)c2ccccc2)[Si]#N)cc1</smiles>

Following GP2, the residue was purified by flash chromatography on silica gel using ethyl acetate/ petroleum ether $(v / v 1: 5)$ as eluent to afford the pure product $7 \mathrm{~g}\left(\mathrm{R}_{\mathrm{f}}=0.3\right)$ as yellow oil $(148.6 \mathrm{mg}, 76 \%$ yield $) .{ }^{1} \mathrm{H} \mathrm{NMR}$ $\left(400 \mathrm{MHz}, \mathrm{CDCl}_{3}\right): \delta 7.80-7.72(\mathrm{~m}, 4 \mathrm{H}), 7.60-7.48(\mathrm{~m}, 8 \mathrm{H}), 6.95(\mathrm{~d}, J=8.8 \mathrm{~Hz}, 2 \mathrm{H}), 6.58(\mathrm{~d}, J=18.9 \mathrm{~Hz}, 1 \mathrm{H})$, $3.84(\mathrm{~s}, 3 \mathrm{H}) ;{ }^{13} \mathrm{C}$ NMR $\left(100 \mathrm{MHz}, \mathrm{CDCl}_{3}\right): \delta 161.3,149.8(\mathrm{~d}, J=1.3 \mathrm{~Hz}), 132.3,132.0,131.9,131.2,130.6,130.5$, $129.6(\mathrm{~d}, J=13.6 \mathrm{~Hz}), 129.1,128.5,128.4,123.3(\mathrm{~d}, J=98.3 \mathrm{~Hz}), 113.8,109.2,55.0 ;{ }^{31} \mathrm{P} \mathrm{NMR}(162 \mathrm{MHz}$, $\left.\mathrm{CDCl}_{3}\right): \delta$ 21.83. IR $\left(\mathrm{cm}^{-1}\right)$ 2152.22. HRMS (ESI-TOF) $\mathrm{m} / z:[\mathrm{M}+\mathrm{Na}]^{+}$Calcd for $\mathrm{C}_{22} \mathrm{H}_{18} \mathrm{NaO}_{2} \mathrm{PS} 414.0694$, found 
414.0696.

(Z)-(2-(2-Chlorophenyl)-2-thiocyanatovinyl)diphenylphosphine oxide (7h)<smiles>N#C/C(=C\P(=O)(O)c1ccccc1)c1ccccc1Cl</smiles>

Following GP2, the residue was purified by flash chromatography on silica gel using ethyl acetate/ petroleum ether $(v / v 1: 5)$ as eluent to afford the pure product $7 \mathbf{h}\left(\mathrm{R}_{\mathrm{f}}=0.2\right)$ as yellow oil $(130.4 \mathrm{mg}, 66 \%$ yield $) .{ }^{1} \mathrm{H}$ NMR $\left(400 \mathrm{MHz}, \mathrm{CDCl}_{3}\right): \delta 7.81-7.70(\mathrm{~m}, 4 \mathrm{H}), 7.62-7.56(\mathrm{~m}, 2 \mathrm{H}), 7.55-7.48(\mathrm{~m}, 5 \mathrm{H}), 7.41-7.34(\mathrm{~m}, 3 \mathrm{H}), 6.49(\mathrm{~d}$, $J=19.8 \mathrm{~Hz}, 1 \mathrm{H}) ;{ }^{13} \mathrm{C} \mathrm{NMR}\left(100 \mathrm{MHz}, \mathrm{CDCl}_{3}\right): \delta 147.9(\mathrm{~d}, J=1.4 \mathrm{~Hz}), 136.8(\mathrm{~d}, J=14.0 \mathrm{~Hz}), 132.18,132.15$, 131.6, 130.9, 130.7, 130.6, 130.5, 129.6, 129.4, 128.6, 128.4, 126.8, 124.5, 123.5, 108.7; ${ }^{31} \mathrm{P}$ NMR (162 MHz, $\left.\mathrm{CDCl}_{3}\right): \delta$ 24.18. IR $\left(\mathrm{cm}^{-1}\right)$ 2158.32. HRMS (ESI-TOF) $\mathrm{m} / z:[\mathrm{M}+\mathrm{H}]^{+}$Calcd for $\mathrm{C}_{21} \mathrm{H}_{16} \mathrm{ClNOPS} 396.0379$, found 396.0364.

(Z)-(2-(3-Chlorophenyl)-2-thiocyanatovinyl)diphenylphosphine oxide (7i)<smiles>N#C/C(=C\[PH](=O)c1ccccc1)c1cccc(Cl)c1</smiles>

Following GP2, the residue was purified by flash chromatography on silica gel using ethyl acetate/ petroleum ether $(v / v 1: 5)$ as eluent to afford the pure product $7 \mathbf{i}\left(\mathrm{R}_{\mathrm{f}}=0.2\right)$ as yellow oil $(122.5 \mathrm{mg}, 62 \%$ yield $) .{ }^{1} \mathrm{H}$ NMR $(400$ $\left.\mathrm{MHz}, \mathrm{DMSO}-d_{6}\right): \delta 7.90-7.79(\mathrm{~m}, 4 \mathrm{H}), 7.77(\mathrm{~s}, 1 \mathrm{H}), 7.66-7.52(\mathrm{~m}, 9 \mathrm{H}), 7.38(\mathrm{~d}, J=18.6 \mathrm{~Hz}, 1 \mathrm{H}) ;{ }^{13} \mathrm{C} \mathrm{NMR}$ $\left(100 \mathrm{MHz}, \mathrm{DMSO}-d_{6}\right): \delta 146.3(\mathrm{~d}, J=1.6 \mathrm{~Hz}), 139.5(\mathrm{~d}, J=14.2 \mathrm{~Hz}), 133.4,133.0,132.4,132.3,131.9,130.8$, 130.7, 130.6, 130.4, 129.6, 129.0, 128.9, 127.9, 127.0, 110.1; ${ }^{31} \mathrm{P}$ NMR (162 MHz, DMSO-d $): \delta 22.35$. IR $\left(\mathrm{cm}^{-1}\right)$ 2154.43. HRMS (ESI-TOF) $\mathrm{m} / z$ : $[\mathrm{M}+\mathrm{Na}]^{+}$Calcd for $\mathrm{C}_{21} \mathrm{H}_{15} \mathrm{ClNNaOPS} 418.0198$, found 418.0181.

\section{(2-(4-Chlorophenyl)-2-thiocyanatovinyl)diphenylphosphine oxide (7j)}<smiles>N#[Si]/C(=C\[Pb]([OH+])c1ccccc1)c1ccc(Cl)cc1</smiles>

Following GP2, the residue was purified by flash chromatography on silica gel using ethyl acetate/ petroleum ether $(v / v 1: 5)$ as eluent to afford the pure product $7 \mathbf{j}\left(\mathrm{R}_{\mathrm{f}}=0.3\right)$ as yellow oil $\left(146.1 \mathrm{mg}, 74 \%\right.$ yield). ${ }^{1} \mathrm{H} \mathrm{NMR}(400$ $\left.\mathrm{MHz}, \mathrm{CDCl}_{3}\right): \delta 7.79-7.70(\mathrm{~m}, 4 \mathrm{H}), 7.64-7.57(\mathrm{~m}, 2 \mathrm{H}), 7.55-7.49(\mathrm{~m}, 4 \mathrm{H}), 7.46-7.40(\mathrm{~m}, 4 \mathrm{H}), 6.62(\mathrm{~d}, J=$ $18.8 \mathrm{~Hz}, 1 \mathrm{H}) .{ }^{13} \mathrm{C} \mathrm{NMR}\left(101 \mathrm{MHz}, \mathrm{CDCl}_{3}\right): \delta 149.4,137.2,136.4,136.2,132.7(\mathrm{~d}, J=2.8 \mathrm{~Hz}), 132.2,131.1$, 
131.0, 129.3, 129.2, 129.1, 129.0, 126.2, 125.2, 109.3. ${ }^{31} \mathrm{P}$ NMR (162 $\left.\mathrm{MHz}, \mathrm{CDCl}_{3}\right): \delta 22.34 . \mathrm{HRMS}$ (ESI-TOF) $m / z:[\mathrm{M}+\mathrm{Na}]^{+}$Calcd for $\mathrm{C}_{21} \mathrm{H}_{15} \mathrm{ClNNaOPS} 418.0198$, found 418.0183.

(Z)- (2-(4-Bromophenyl)-2-thiocyanatovinyl)diphenylphosphine oxide (7k)<smiles>N#CC(=C[PbH]c1ccccc1)c1ccc(Br)cc1</smiles>

Following GP2, the residue was purified by flash chromatography on silica gel using ethyl acetate/ petroleum ether $(v / v 1: 5)$ as eluent to afford the pure product $7 \mathbf{k}\left(\mathrm{R}_{\mathrm{f}}=0.3\right)$ as yellow oil $\left(114.1 \mathrm{mg}, 52 \%\right.$ yield). ${ }^{1} \mathrm{H}$ NMR $\left(400 \mathrm{MHz}, \mathrm{CDCl}_{3}\right): \delta 7.86-7.76(\mathrm{~m}, 4 \mathrm{H}), 7.68-7.63(\mathrm{~m}, 4 \mathrm{H}), 7.60-7.54(\mathrm{~m}, 4 \mathrm{H}), 7.46-7.42(\mathrm{~m}, 2 \mathrm{H}), 6.67(\mathrm{~d}$, $J=18.8 \mathrm{~Hz}, 1 \mathrm{H}) ;{ }^{13} \mathrm{C} \mathrm{NMR}\left(100 \mathrm{MHz}, \mathrm{CDCl}_{3}\right): \delta 150.6(\mathrm{~d}, J=8.1 \mathrm{~Hz}), 135.0(\mathrm{~d}, J=3.8 \mathrm{~Hz}), 133.3,132.2$, $131.78,131.75,130.93,130.91,130.8,130.6,128.6,128.5,124.8,123.9,122.9 ;{ }^{31} \mathrm{P} \mathrm{NMR}\left(162 \mathrm{MHz} \mathrm{CDCl}_{3}\right): \delta$ 20.61. IR $\left(\mathrm{cm}^{-1}\right)$ 2157.96. HRMS (ESI-TOF) $\mathrm{m} / \mathrm{z}$ : $[\mathrm{M}+\mathrm{Na}]^{+}$Calcd for $\mathrm{C}_{21} \mathrm{H}_{15} \mathrm{BrNNaOPS} 461.9693$, found 461.9689

(Z)- (2-(2-Fluorophenyl)-2-thiocyanatovinyl)diphenylphosphine oxide (7l)<smiles>N#C/C(=C\Pc1ccccc1)c1ccccc1F</smiles>

Following GP2, the residue was purified by flash chromatography on silica gel using ethyl acetate/ petroleum ether $(v / v 1: 5)$ as eluent to afford the pure product $7 \mathbf{l}\left(\mathrm{R}_{\mathrm{f}}=0.3\right)$ as yellow oil $(100.2 \mathrm{mg}, 53 \%$ yield $) .{ }^{1} \mathrm{H}$ NMR $(400$ $\left.\mathrm{MHz}, \mathrm{CDCl}_{3}\right): \delta 7.82-7.71(\mathrm{~m}, 4 \mathrm{H}), 7.62-7.57(\mathrm{~m}, 2 \mathrm{H}), 7.56-7.49(\mathrm{~m}, 4 \mathrm{H}), 7.48-7.38(\mathrm{~m}, 2 \mathrm{H}), 7.26-7.15$ $(\mathrm{m}, 2 \mathrm{H}), 6.60(\mathrm{~d}, J=19.4 \mathrm{~Hz}, 1 \mathrm{H}) ;{ }^{13} \mathrm{C} \mathrm{NMR}\left(100 \mathrm{MHz}, \mathrm{CDCl}_{3}\right): \delta 144.2,132.1(\mathrm{~d}, J=2.7 \mathrm{~Hz}), 132.0,131.9$, $131.7,130.7,130.64,130.57,129.6,128.6,128.5,126.3,125.4,124.1(\mathrm{~d}, J=3.6 \mathrm{~Hz}), 116.0,115.8,108.9 ;{ }^{31} \mathrm{P}$ NMR (162 MHz, $\left.\mathrm{CDCl}_{3}\right): \delta$ 23.48. IR $\left(\mathrm{cm}^{-1}\right)$ 2157.66. HRMS (ESI-TOF) $m / z:[\mathrm{M}+\mathrm{H}]^{+}$Calcd for $\mathrm{C}_{21} \mathrm{H}_{16} \mathrm{FNOPS}$ 380.0674 , found 380.0668 .

\section{(Z)-(2-(4-Fluorophenyl)-2-thiocyanatovinyl)diphenylphosphine oxide (7m)}<smiles>N#C/C(=C\P(=O)(O)c1ccccc1)c1ccc(F)cc1</smiles>

Following GP2, the residue was purified by flash chromatography on silica gel using ethyl acetate/ petroleum ether $\left(v / v\right.$ 1:5) as eluent to afford the pure product $7 \mathbf{m}\left(\mathrm{R}_{\mathrm{f}}=0.4\right)$ as yellow oil $(100.4 \mathrm{mg}, 53 \%$ yield $) .{ }^{1} \mathrm{H} \mathrm{NMR}$ 
$\left(400 \mathrm{MHz}, \mathrm{CDCl}_{3}\right): \delta 7.82-7.71(\mathrm{~m}, 4 \mathrm{H}), 7.64-7.45(\mathrm{~m}, 8 \mathrm{H}), 7.19-7.07(\mathrm{~m}, 2 \mathrm{H}), 6.60(\mathrm{~d}, J=18.9 \mathrm{~Hz}, 1 \mathrm{H})$; ${ }^{13} \mathrm{C}$ NMR $\left(100 \mathrm{MHz}, \mathrm{CDCl}_{3}\right): \delta 149.1(\mathrm{~d}, J=1.5 \mathrm{~Hz}), 132.1(\mathrm{~d}, J=2.6 \mathrm{~Hz}), 131.9,130.8,130.6,130.5,129.64$, 129.55, 128.6, 128.5, 125.3, 124.3, $115.7(\mathrm{~d}, J=22.2 \mathrm{~Hz}), 108.9,99.5 ;{ }^{31} \mathrm{P}$ NMR $\left(162 \mathrm{MHz}, \mathrm{CDCl}_{3}\right): \delta 22.14 . \mathrm{IR}$ $\left(\mathrm{cm}^{-1}\right)$ 2157.99. HRMS (ESI-TOF) $\mathrm{m} / z:[\mathrm{M}+\mathrm{Na}]^{+}$Calcd for $\mathrm{C}_{21} \mathrm{H}_{15} \mathrm{FNNaOPS} 402.0494$, found 402.0487.

\section{(Z)-Diphenyl(2-thiocyanato-2-(2-(trifluoromethyl)phenyl)vinyl)phosphine oxide (7n)}<smiles>N#C/C(=C\Pc1ccccc1)c1ccccc1C(F)(F)F</smiles>

Following GP2, the residue was purified by flash chromatography on silica gel using ethyl acetate/ petroleum ether $(v / v 1: 5)$ as eluent to afford the pure product $\mathbf{7 n}\left(\mathrm{R}_{\mathrm{f}}=0.4\right)$ as yellow oil $(120.1 \mathrm{mg}, 56 \%$ yield $) .{ }^{1} \mathrm{H}$ NMR $\left(400 \mathrm{MHz}, \mathrm{CDCl}_{3}\right): \delta 7.83-7.69(\mathrm{~m}, 5 \mathrm{H}), 7.67-7.42(\mathrm{~m}, 9 \mathrm{H}), 6.50(\mathrm{~d}, J=19.5 \mathrm{~Hz}, 1 \mathrm{H}) ;{ }^{13} \mathrm{C} \mathrm{NMR}(100 \mathrm{MHz}$, $\left.\mathrm{CDCl}_{3}\right): \delta 147.3(\mathrm{~d}, J=1.3 \mathrm{~Hz}), 136.5(\mathrm{~d}, J=12.5 \mathrm{~Hz}), 132.7(\mathrm{~d}, J=2.8 \mathrm{~Hz}), 132.3,131.9,131.1(\mathrm{~d}, J=10.4 \mathrm{~Hz})$, 130.8, 130.3, 130.1, $128.9(\mathrm{~d}, J=12.5 \mathrm{~Hz}), 127.9(\mathrm{q}, J=30.9 \mathrm{~Hz}), 126.6(\mathrm{q}, J=5.0 \mathrm{~Hz}), 125.2,124.9,124.3$, 122.2, 109.3; ${ }^{31} \mathrm{P}$ NMR $\left(162 \mathrm{MHz}, \mathrm{CDCl}_{3}\right): \delta 24.25$. IR $\left(\mathrm{cm}^{-1}\right)$ 2157.26. HRMS (ESI-TOF) $m / z:[\mathrm{M}+\mathrm{H}]^{+} \mathrm{Calcd}$ for $\mathrm{C}_{22} \mathrm{H}_{16} \mathrm{~F}_{3} \mathrm{NOPS} 430.0642$, found 430.0636 .

\section{(Z)-Diphenyl(2-thiocyanato-2-(4-(trifluoromethyl)phenyl)vinyl)phosphine oxide (7o)}<smiles>N#C/C(=C\P(c1ccccc1)c1ccccc1)c1ccc(C(F)(F)F)cc1</smiles>

Following GP2, the residue was purified by flash chromatography on silica gel using ethyl acetate/ petroleum ether $(v / v 1: 5)$ as eluent to afford the pure product $7 \mathrm{o}\left(\mathrm{R}_{\mathrm{f}}=0.4\right)$ as yellow oil $(128.7 \mathrm{mg}, 60 \%$ yield $) .{ }^{1} \mathrm{H} \mathrm{NMR}$ $\left(400 \mathrm{MHz}, \mathrm{CDCl}_{3}\right): \delta 7.82-7.71(\mathrm{~m}, 6 \mathrm{H}), 7.70-7.61(\mathrm{~m}, 4 \mathrm{H}), 7.60-7.53(\mathrm{~m}, 3 \mathrm{H}), 7.51-7.36(\mathrm{~m}, 1 \mathrm{H}), 6.69(\mathrm{~d}$, $J=18.6 \mathrm{~Hz}, 1 \mathrm{H}) ;{ }^{13} \mathrm{C} \mathrm{NMR}\left(100 \mathrm{MHz}, \mathrm{CDCl}_{3}\right): \delta 149.0,141.2(\mathrm{~d}, J=13.8 \mathrm{~Hz}), 132.7(\mathrm{~d}, J=2.7 \mathrm{~Hz}), 132.0$, $131.1(\mathrm{~d}, J=10.2 \mathrm{~Hz}), 130.8(\mathrm{~d}, J=10.0 \mathrm{~Hz}), 129.9,129.1(\mathrm{~d}, J=12.5 \mathrm{~Hz}), 128.7(\mathrm{~d}, J=12.4 \mathrm{~Hz}), 128.4,127.2$, 126.3, $126.0(\mathrm{q}, J=3.6 \mathrm{~Hz}), 124.8,109.2 ;{ }^{31} \mathrm{P} \mathrm{NMR}\left(162 \mathrm{MHz}, \mathrm{CDCl}_{3}\right): \delta 22.42 . \mathrm{IR}\left(\mathrm{cm}^{-1}\right)$ 2154.69; HRMS (ESI-TOF) $m / z:[\mathrm{M}]^{+}$Calcd for $\mathrm{C}_{22} \mathrm{H}_{15} \mathrm{~F}_{3}$ NOPS 429.0564, found 429.0561.

(Z)-(2-(4-Nitrophenyl)-2-thiocyanatovinyl)diphenylphosphine oxide (7p)<smiles>N#C/C(=C\Pc1ccccc1)c1ccc([N+](=O)[O-])cc1</smiles> 
Following GP2, the residue was purified by flash chromatography on silica gel using ethyl acetate/ petroleum ether $(v / v 1: 5)$ as eluent to afford the pure product $7 \mathbf{p}\left(\mathrm{R}_{\mathrm{f}}=0.4\right)$ as yellow oil $\left(101.5 \mathrm{mg}, 50 \%\right.$ yield). ${ }^{1} \mathrm{H}$ NMR $\left(400 \mathrm{MHz}, \mathrm{CDCl}_{3}\right): \delta 8.32(\mathrm{~d}, J=8.8 \mathrm{~Hz}, 2 \mathrm{H}), 7.81-7.72(\mathrm{~m}, 4 \mathrm{H}), 7.70-7.60(\mathrm{~m}, 4 \mathrm{H}), 7.59-7.49(\mathrm{~m}, 4 \mathrm{H})$, $6.71(\mathrm{~d}, J=18.4 \mathrm{~Hz}, 1 \mathrm{H}) ;{ }^{13} \mathrm{C} \mathrm{NMR}\left(100 \mathrm{MHz}, \mathrm{CDCl}_{3}\right): \delta 150.5,137.9(\mathrm{~d}, J=13.5 \mathrm{~Hz}), 133.9,133.2(\mathrm{~d}, J=11.1$ Hz), $133.0(\mathrm{~d}, J=8.7 \mathrm{~Hz}), 130.8,129.3,128.8,127.9,127.7,126.7,126.4,126.2,124.8(\mathrm{~d}, J=14.4 \mathrm{~Hz}), 109.5$; ${ }^{31} \mathrm{P}$ NMR $\left(162 \mathrm{MHz}, \mathrm{CDCl}_{3}\right): \delta$ 23.73. IR $\left(\mathrm{cm}^{-1}\right)$ 2154.39. HRMS (ESI-TOF) $\mathrm{m} / \mathrm{z}:[\mathrm{M}+\mathrm{Na}]^{+} \mathrm{Calcd}$ for $\mathrm{C}_{21} \mathrm{H}_{15} \mathrm{~N}_{2} \mathrm{NaO}_{3} \mathrm{PS} 429.0439$, found 429.0431 .

(Z)-Diphenyl(2-(4-methoxycarbonylphenyl)-2-thiocyanatovinyl)phosphine oxide (7q)<smiles>COC(=O)c1ccc(/C(=C/POc2ccccc2)[Se]#N)cc1</smiles>

Following GP2, the residue was purified by flash chromatography on silica gel using ethyl acetate/ petroleum ether $(v / v 1: 5)$ as eluent to afford the pure product $7 \mathbf{q}\left(\mathrm{R}_{\mathrm{f}}=0.3\right)$ as yellow oil $(129.9 \mathrm{mg}, 62 \%$ yield $) .{ }^{1} \mathrm{H}$ NMR $\left(400 \mathrm{MHz}, \mathrm{CDCl}_{3}\right): \delta 8.11(\mathrm{~d}, J=8.5 \mathrm{~Hz}, 2 \mathrm{H}), 7.79-7.73(\mathrm{~m}, 4 \mathrm{H}), 7.61-7.50(\mathrm{~m}, 8 \mathrm{H}), 6.68(\mathrm{~d}, J=18.8 \mathrm{~Hz}, 1 \mathrm{H})$, 3.93 (s, 3H); ${ }^{13} \mathrm{C}$ NMR (100 MHz, $\left.\mathrm{CDCl}_{3}\right): \delta 165.5,149.0,141.4$ (d, $\left.J=13.5 \mathrm{~Hz}\right), 132.24,132.22,131.7,131.6$, $130.6,130.54,130.51,129.7,128.7,128.6,127.6,125.8(\mathrm{~d}, J=95.6 \mathrm{~Hz}), 108.7,52.0 ;{ }^{31} \mathrm{P}$ NMR $(162 \mathrm{MHz}$, $\left.\mathrm{CDCl}_{3}\right): \delta 22.56 . \mathrm{IR}\left(\mathrm{cm}^{-1}\right)$ 2155.39; HRMS (ESI-TOF) $\mathrm{m} / \mathrm{z}:[\mathrm{M}+\mathrm{Na}]^{+}$Calcd for $\mathrm{C}_{23} \mathrm{H}_{18} \mathrm{NNaO}_{3} \mathrm{PS} 442.0643$, found 442.0645 .

(Z)-(2-(2-Azidophenyl)-2-thiocyanatovinyl)diphenylphosphine oxide (7r)<smiles>N#CC(=Cc1ccccc1)c1ccccc1N</smiles>

Following GP2, the residue was purified by flash chromatography on silica gel using ethyl acetate/ petroleum ether $(v / v 1: 5)$ as eluent to afford the pure product $7 \mathbf{r}\left(\mathrm{R}_{\mathrm{f}}=0.3\right)$ as yellow oil $(130.7 \mathrm{mg}, 65 \%) .{ }^{1} \mathrm{H}$ NMR (400 $\left.\mathrm{MHz}, \mathrm{CDCl}_{3}\right): \delta 7.85-7.79(\mathrm{~m}, 4 \mathrm{H}), 7.67-7.62(\mathrm{~m}, 2 \mathrm{H}), 7.59-7.54(\mathrm{~m}, 4 \mathrm{H}), 7.53-7.50(\mathrm{~m}, 1 \mathrm{H}), 7.36(\mathrm{dd}, J=$ 7.7, $1.5 \mathrm{~Hz}, 1 \mathrm{H}), 7.31(\mathrm{dd}, J=6.4,1.8 \mathrm{~Hz}, 1 \mathrm{H}), 7.26(\mathrm{td}, J=7.6,1.0 \mathrm{~Hz}, 1 \mathrm{H}), 6.49(\mathrm{~d}, J=19.9 \mathrm{~Hz}, 1 \mathrm{H}) ;{ }^{13} \mathrm{C} \mathrm{NMR}$ $\left(100 \mathrm{MHz}, \mathrm{CDCl}_{3}\right): \delta 147.0(\mathrm{~d}, J=1.6 \mathrm{~Hz}), 137.4(\mathrm{~d}, J=0.5 \mathrm{~Hz}), 132.13,132.10,131.7,131.2,130.73,130.66$, $130.6,129.5(\mathrm{~d}, J=0.9 \mathrm{~Hz}), 128.6,128.4,124.62,124.56,123.7,118.3,109.2 ;{ }^{31} \mathrm{P}$ NMR $\left(162 \mathrm{MHz}, \mathrm{CDCl}_{3}\right): \delta$ 23.95. HRMS (ESI-TOF) $m / z:[\mathrm{M}+\mathrm{H}]^{+}$Calcd for $\mathrm{C}_{21} \mathrm{H}_{16} \mathrm{~N}_{4} \mathrm{OPS} 403.0782$, found 403.0788 .

(Z)-Diphenyl(2-thiocyanato-2-(thiophen-2-yl)vinyl)phosphine oxide (7s) 


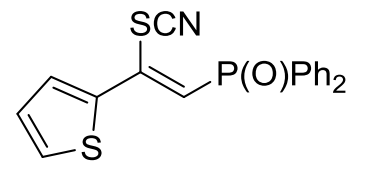

Following GP2, the residue was purified by flash chromatography on silica gel using ethyl acetate/ petroleum ether $(v / v 1: 5)$ as eluent to afford the pure product $7 \mathbf{s}\left(\mathrm{R}_{\mathrm{f}}=0.3\right)$ as yellow oil $(91.8 \mathrm{mg}, 50 \%$ yield $) .{ }^{1} \mathrm{H}$ NMR $(400$ $\left.\mathrm{MHz}, \mathrm{CDCl}_{3}\right): \delta 7.82-7.74(\mathrm{~m}, 4 \mathrm{H}), 7.59-7.46(\mathrm{~m}, 8 \mathrm{H}), 7.12(\mathrm{dd}, J=5.0,3.9 \mathrm{~Hz}, 1 \mathrm{H}), 6.82(\mathrm{~d}, J=17.1 \mathrm{~Hz}$, $1 \mathrm{H}) ;{ }^{13} \mathrm{C}$ NMR $\left(100 \mathrm{MHz}, \mathrm{CDCl}_{3}\right): \delta 140.3,139.2(\mathrm{~d}, J=14.8 \mathrm{~Hz}), 132.2,132.02,131.99,131.1,130.7,130.6$, 129.9, $129.2,128.6,128.5,127.9,125.6(\mathrm{~d}, J=98.8 \mathrm{~Hz}), 108.6 ;{ }^{31} \mathrm{P}$ NMR $\left(162 \mathrm{MHz}, \mathrm{CDCl}_{3}\right): \delta 21.15 . \mathrm{HRMS}$ (ESI-TOF) $m / z:[\mathrm{M}+\mathrm{Na}]^{+}$Calcd for $\mathrm{C}_{19} \mathrm{H}_{14} \mathrm{NNaOPS}_{2} 390.0152$, found 390.0149.

\section{(Z)-Diphenyl(1-phenyl-1-thiocyanatoprop-1-en-2-yl)phosphine oxide (7t)}<smiles>C/C(=C(\[Si])c1ccccc1)c1ccccc1</smiles>

Following GP2, the residue was purified by flash chromatography on silica gel using ethyl acetate/ petroleum ether $(v / v 1: 5)$ as eluent to afford the pure product $7 \mathbf{t}\left(\mathrm{R}_{\mathrm{f}}=0.4\right)$ as yellow oil $(84.4 \mathrm{mg}, 45 \%$ yield $) .{ }^{1} \mathrm{H}$ NMR $(400$ $\left.\mathrm{MHz}, \mathrm{DMSO}-d_{6}\right): \delta 7.78-7.67(\mathrm{~m}, 6 \mathrm{H}), 7.65-7.60(\mathrm{~m}, 4 \mathrm{H}), 7.55-7.50(\mathrm{~m}, 2 \mathrm{H}), 7.49-7.44(\mathrm{~m}, 3 \mathrm{H}), 1.57(\mathrm{~d}, J$ $=13.1 \mathrm{~Hz}, 3 \mathrm{H}) ;{ }^{13} \mathrm{C}$ NMR $\left(100 \mathrm{MHz}, \mathrm{DMSO}-d_{6}\right): \delta 142.6(\mathrm{~d}, J=2.8 \mathrm{~Hz}), 137.8(\mathrm{~d}, J=12.3 \mathrm{~Hz}), 133.07,133.06$, $131.9,131.8,131.5,131.3,130.8,130.0,129.54,129.46,129.4,128.9,110.9,21.4(\mathrm{~d}, J=11.0 \mathrm{~Hz}) ;{ }^{31} \mathrm{P}$ NMR $(162$ MHz, DMSO- $\left.d_{6}\right): \delta$ 31.13. IR $\left(\mathrm{cm}^{-1}\right)$ 2155.15. HRMS (ESI-TOF) $m / z:[\mathrm{M}+\mathrm{Na}]^{+}$Calcd for $\mathrm{C}_{22} \mathrm{H}_{18} \mathrm{NNaOPS}$ 398.0744, found 398.0747.

\section{(Z)-3-(Diphenylphosphoryl)-4-phenyl-4-thiocyanatobut-3-en-2-one (7u)}<smiles>CC(=O)/C([Pb]c1ccccc1)=C(\[Se])c1ccccc1</smiles>

Following GP2, the residue was purified by flash chromatography on silica gel using ethyl acetate/ petroleum ether $(v / v 1: 5)$ as eluent to afford the pure product $7 \mathbf{u}\left(\mathrm{R}_{\mathrm{f}}=0.2\right)$ as yellow oil $(76.6 \mathrm{mg}, 38 \%$ yield $) .{ }^{1} \mathrm{H}$ NMR $(400$ $\left.\mathrm{MHz}, \mathrm{CDCl}_{3}\right): \delta 7.91-7.82(\mathrm{~m}, 4 \mathrm{H}), 7.64-7.58(\mathrm{~m}, 2 \mathrm{H}), 7.56-7.46(\mathrm{~m}, 7 \mathrm{H}), 7.43-7.39(\mathrm{~m}, 2 \mathrm{H}), 1.38(\mathrm{~s}, 3 \mathrm{H})$;

${ }^{13} \mathrm{C} \mathrm{NMR}\left(100 \mathrm{MHz}, \mathrm{CDCl}_{3}\right): \delta 200.54,150.47,132.92,132.89,132.27,132.16,131.45,130.10,130.05,129.33$, 128.98, 128.90, 128.85, 128.72, 109.64, $30.86(\mathrm{~d}, J=1.4 \mathrm{~Hz}) ;{ }^{31} \mathrm{P}$ NMR $\left(162 \mathrm{MHz}, \mathrm{CDCl}_{3}\right): \delta 27.80 . \mathrm{IR}^{\left(\mathrm{cm}^{-1}\right)}$ 2155.99. HRMS (ESI-TOF) $m / z:[\mathrm{M}+\mathrm{Na}]^{+}$Calcd for $\mathrm{C}_{23} \mathrm{H}_{18} \mathrm{NNaO}_{2} \mathrm{PS} 426.0694$, found 426.0684 . 


\section{(Z)-Diphenyl(4-phenyl-2-thiocyanatobut-1-en-1-yl)phosphine oxide (7v)}<smiles>N#C/C(=C\[PH](=O)c1ccccc1)CCc1ccccc1</smiles>

Following GP2, the residue was purified by flash chromatography on silica gel using ethyl acetate/ petroleum ether $(v / v 1: 5)$ as eluent to afford the pure product $7 \mathbf{v}\left(R_{\mathrm{f}}=0.3\right)$ as yellow oil $\left(68.1 \mathrm{mg}, 35 \%\right.$ yield). ${ }^{1} \mathrm{H}$ NMR (400 $\left.\mathrm{MHz}, \mathrm{CDCl}_{3}\right): \delta 7.72-7.65(\mathrm{~m}, 4 \mathrm{H}), 7.58-7.53(\mathrm{~m}, 2 \mathrm{H}), 7.50-7.46(\mathrm{~m}, 4 \mathrm{H}), 7.25-7.15(\mathrm{~m}, 5 \mathrm{H}), 6.50(\mathrm{~d}, J=$ $17.7 \mathrm{~Hz}, 1 \mathrm{H}), 3.40-3.17(\mathrm{~m}, 2 \mathrm{H}), 2.93-2.75(\mathrm{~m}, 2 \mathrm{H}) ;{ }^{13} \mathrm{C} \mathrm{NMR}\left(100 \mathrm{MHz}, \mathrm{CDCl}_{3}\right): \delta 149.7(\mathrm{~d}, J=6.2 \mathrm{~Hz})$, 139.2, 133.5, 132.4, $132.2(\mathrm{~d}, J=2.7 \mathrm{~Hz}), 131.8(\mathrm{~d}, J=10.4 \mathrm{~Hz}), 130.9$ (d, $J=10.1 \mathrm{~Hz}), 129.7,128.9$ (d, $J=12.4$ Hz), $128.6(\mathrm{~d}, J=13.1 \mathrm{~Hz}), 126.6,121.3,120.7(\mathrm{~d}, J=4.8 \mathrm{~Hz}), 120.3,108.4,35.3(\mathrm{~d}, J=4.9 \mathrm{~Hz}), 35.1(\mathrm{~d}, J=1.3$ $\mathrm{Hz}) ;{ }^{31} \mathrm{P}$ NMR $\left(162 \mathrm{MHz}, \mathrm{CDCl}_{3}\right): \delta$ 20.27. IR $\left(\mathrm{cm}^{-1}\right)$ 2156.09. HRMS (ESI-TOF) $\mathrm{m} / z:[\mathrm{M}+\mathrm{Na}]^{+} \mathrm{Calcd}$ for $\mathrm{C}_{23} \mathrm{H}_{20} \mathrm{NNaOPS}$ 412.0901, found 412.0895.

\section{(Z)- (2-Cyclopropyl-2-thiocyanatovinyl)diphenylphosphine oxide (7w)}<smiles>N#CC(=CP)C1CC1</smiles>

Following GP2, the residue was purified by flash chromatography on silica gel using ethyl acetate/ petroleum ether $(v / v 1: 5)$ as eluent to afford the pure product $7 \mathbf{w}\left(\mathrm{R}_{\mathrm{f}}=0.3\right)$ as yellow oil $\left(78.0 \mathrm{mg}, 48 \%\right.$ yield). ${ }^{1} \mathrm{H}$ NMR (400 $\left.\mathrm{MHz}, \mathrm{CDCl}_{3}\right): \delta 7.68-7.60(\mathrm{~m}, 4 \mathrm{H}), 7.60-7.54(\mathrm{~m}, 2 \mathrm{H}), 7.53-7.45(\mathrm{~m}, 4 \mathrm{H}), 6.12(\mathrm{~d}, J=19.1 \mathrm{~Hz}, 1 \mathrm{H}), 2.15-$ $2.03(\mathrm{~m}, 1 \mathrm{H}), 1.17-1.08(\mathrm{~m}, 2 \mathrm{H}), 0.89-0.82(\mathrm{~m}, 2 \mathrm{H}) .{ }^{13} \mathrm{C} \mathrm{NMR}\left(150 \mathrm{MHz}, \mathrm{CDCl}_{3}\right): \delta 153.8(\mathrm{~d}, J=1.3 \mathrm{~Hz})$, 132.6, 132.41, 132.38, 131.5, 131.0, 130.9, 129.0, 128.8, 117.2, 116.2, 109.9, 21.9 (d, $J=14.6 \mathrm{~Hz}$ ), 9.6. ${ }^{31} \mathrm{P}$ NMR (162 MHz, $\left.\mathrm{CDCl}_{3}\right): \delta$ 23.56. IR $\left(\mathrm{cm}^{-1}\right)$ 2157.72. HRMS (ESI-TOF) $\mathrm{m} / \mathrm{z}:[\mathrm{M}+\mathrm{Na}]^{+}$Calcd for $\mathrm{C}_{18} \mathrm{H}_{16} \mathrm{NNaOPS}$ 348.0588, found 348.0581 .

\section{(Z)-Non-1-en-1-yldiphenylphosphine oxide $\left(7 x^{\prime}\right)$}

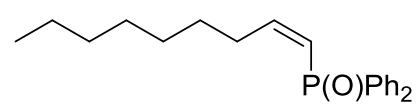

Following GP2, the residue was purified by flash chromatography on silica gel using ethyl acetate/ petroleum ether $(v / v 1: 5)$ as eluent to afford the pure product $7 \mathbf{x}^{\prime}\left(\mathrm{R}_{\mathrm{f}}=0.4\right)$ as yellow oil $\left(84.8 \mathrm{mg}, 52 \%\right.$ yield). ${ }^{1} \mathrm{H}$ NMR (400 $\left.\mathrm{MHz}, \mathrm{CDCl}_{3}\right): \delta 7.80-7.67(\mathrm{~m}, 4 \mathrm{H}), 7.54-7.40(\mathrm{~m}, 6 \mathrm{H}), 6.77-6.58(\mathrm{~m}, 1 \mathrm{H}), 6.10(\mathrm{dd}, J=25.4,12.7 \mathrm{~Hz}, 1 \mathrm{H})$, $2.55-2.48(\mathrm{~m}, 2 \mathrm{H}), 1.88-1.84(\mathrm{~m}, 2 \mathrm{H}), 1.26-1.21(\mathrm{~m}, 2 \mathrm{H}), 1.20-1.16(\mathrm{~m}, 6 \mathrm{H}), 0.84(\mathrm{t}, J=5.8 \mathrm{~Hz}, 3 \mathrm{H}) ;{ }^{13} \mathrm{C}$ $\operatorname{NMR}\left(100 \mathrm{MHz}, \mathrm{CDCl}_{3}\right): \delta 154.7,133.6,131.02,131.00,130.5,130.4,128.1,128.0,121.3,120.3,31.2,30.5$ (d, $J=8.1 \mathrm{~Hz}), 28.64,28.55,28.4(\mathrm{~d}, J=1.4 \mathrm{~Hz}), 22.1,13.6 ;{ }^{31} \mathrm{P}$ NMR $\left(162 \mathrm{MHz}, \mathrm{CDCl}_{3}\right): \delta 21.09 . \mathrm{HRMS}$ 
(ESI-TOF) $m / z:[\mathrm{M}+\mathrm{H}]^{+}$Calcd for $\mathrm{C}_{21} \mathrm{H}_{28} \mathrm{OP} 327.1878$, found 327.1865 .

(Z)-Dec-1-en-1-yldiphenylphosphine oxide $\left(7 \mathbf{y}^{\prime}\right)$<smiles>CCCCCCCC/C=C\POc1ccccc1</smiles>

Following GP2, the residue was purified by flash chromatography on silica gel using ethyl acetate/ petroleum ether $(v / v 1: 5)$ as eluent to afford the pure product $7 \mathbf{y}^{\prime}\left(\mathrm{R}_{\mathrm{f}}=0.4\right)$ as yellow oil $(86.7 \mathrm{mg}, 51 \%$ yield $) .{ }^{1} \mathrm{H}$ NMR $(400$ $\left.\mathrm{MHz}, \mathrm{CDCl}_{3}\right): \delta 7.84-7.63(\mathrm{~m}, 4 \mathrm{H}), 7.59-7.35(\mathrm{~m}, 6 \mathrm{H}), 6.77-6.59(\mathrm{~m}, 1 \mathrm{H}), 6.10(\mathrm{dd}, J=25.6,12.9 \mathrm{~Hz}, 1 \mathrm{H})$, $2.58-2.48(\mathrm{~m}, 2 \mathrm{H}), 1.62-1.57(\mathrm{~m}, 4 \mathrm{H}), 1.37-1.30(\mathrm{~m}, 2 \mathrm{H}), 1.15-1.12(\mathrm{~m}, 6 \mathrm{H}), 0.86(\mathrm{t}, J=6.9 \mathrm{~Hz}, 3 \mathrm{H}) ;{ }^{13} \mathrm{C}$ NMR (100 MHz, $\left.\mathrm{CDCl}_{3}\right): \delta 154.7,134.1(\mathrm{~d}, J=103.7 \mathrm{~Hz}), 131.0,131.0,130.5,130.4,128.1,128.0,121.3,120.3$, 31.2, 30.5, 30.4, 28.6, 28.5, $28.3(\mathrm{~d}, J=1.4 \mathrm{~Hz}), 22.1,13.6 ;{ }^{31} \mathrm{P}$ NMR $\left(162 \mathrm{MHz}, \mathrm{CDCl}_{3}\right): \delta 20.98 . \mathrm{HRMS}$ (ESI-TOF) $m / z:[\mathrm{M}+\mathrm{H}]^{+}$Calcd for $\mathrm{C}_{22} \mathrm{H}_{30} \mathrm{OP} 341.2034$, found 341.2031.

\subsection{Characterization Data for Chlorophosphorylation Product 9}

(E)-(2-Chloro-2-phenylvinyl)diphenylphosphine oxide (9a)<smiles>Cl/C(=C/Pc1ccccc1)c1ccccc1</smiles>

Following GP3, the residue was purified by flash chromatography on silica gel using dichloromethane/ petroleum ether/ethyl acetate $(v / v / v 4: 1: 1)$ as eluent to afford the pure product $9 \mathbf{a}\left(\mathrm{R}_{\mathrm{f}}=0.7\right)$ as yellow oil $(114.9 \mathrm{mg}, 68 \%)$. ${ }^{1} \mathrm{H}$ NMR $\left(400 \mathrm{MHz}, \mathrm{CDCl}_{3}\right): \delta 7.68-7.60(\mathrm{~m}, 4 \mathrm{H}), 7.55-7.51(\mathrm{~m}, 2 \mathrm{H}), 7.41-7.36(\mathrm{~m}, 2 \mathrm{H}), 7.34-7.28(\mathrm{~m}, 4 \mathrm{H})$, $7.19-7.09(\mathrm{~m}, 3 \mathrm{H}) 6.78(\mathrm{~d}, J=13.3 \mathrm{~Hz}, 1 \mathrm{H}) ;{ }^{13} \mathrm{C} \mathrm{NMR}\left(100 \mathrm{MHz}, \mathrm{CDCl}_{3}\right): \delta 151.7(\mathrm{~d}, J=8.5 \mathrm{~Hz}), 135.7(\mathrm{~d}, J=$ $3.7 \mathrm{~Hz}), 133.0,131.9,131.12,131.09,130.4,130.3,129.8,128.6,128.0,127.9,127.3,122.0(\mathrm{~d}, J=95.7 \mathrm{~Hz}) ;{ }^{31} \mathrm{P}$ NMR (162 MHz, $\mathrm{CDCl}_{3}$ ): $\delta$ 17.61. HRMS (ESI-TOF) $\mathrm{m} / \mathrm{z}:[\mathrm{M}+\mathrm{H}]^{+}$Calcd for $\mathrm{C}_{20} \mathrm{H}_{17} \mathrm{ClOP} 339.0706$, found 339.0718 .

(E)-(2-Chloro-2-(o-tolyl)vinyl)diphenylphosphine oxide (9b)<smiles>Cc1ccccc1/C(Cl)=C\P(=O)(O)c1ccccc1</smiles>

Following GP3, the residue was purified by flash chromatography on silica gel using dichloromethane/ petroleum ether/ethyl acetate $(v / v / v 4: 1: 1)$ as eluent to afford the pure product $\mathbf{9 b}\left(\mathrm{R}_{\mathrm{f}}=0.7\right)$ as yellow oil $(123.2 \mathrm{mg}, 70 \%$ yield). ${ }^{1} \mathrm{H}$ NMR $\left(400 \mathrm{MHz}, \mathrm{CDCl}_{3}\right): \delta 7.66-7.49(\mathrm{~m}, 4 \mathrm{H}), 7.46-7.28(\mathrm{~m}, 6 \mathrm{H}), 7.22-7.18(\mathrm{~m}, 1 \mathrm{H}), 7.14-7.07$ $(\mathrm{m}, 1 \mathrm{H}), 7.04-6.97(\mathrm{~m}, 1 \mathrm{H}), 6.97-6.84(\mathrm{~m}, 2 \mathrm{H}), 2.13(\mathrm{~s}, 3 \mathrm{H}) ;{ }^{13} \mathrm{C} \mathrm{NMR}\left(100 \mathrm{MHz}, \mathrm{CDCl}_{3}\right): \delta 152.0(\mathrm{~d}, J=10.0$ 
Hz), 135.9, 135.8, $135.6(\mathrm{~d}, J=0.5 \mathrm{~Hz}), 131.58,131.56,130.8,130.7,129.9,129.8,129.43,129.41,128.4,128.3$, 125.3, $124.6(\mathrm{~d}, J=96.0 \mathrm{~Hz}), 19.5 ;{ }^{31} \mathrm{P} \mathrm{NMR}\left(162 \mathrm{MHz}, \mathrm{CDCl}_{3}\right): \delta 16.47 . \mathrm{HRMS}(\mathrm{ESI}-\mathrm{TOF}) \mathrm{m} / z:[\mathrm{M}+\mathrm{H}]^{+} \mathrm{Calcd}$ for $\mathrm{C}_{21} \mathrm{H}_{19} \mathrm{ClOP} 353.0862$, found 353.0859.

(E)-(2-Chloro-2-(m-tolyl)vinyl)diphenylphosphine oxide (9c)<smiles>Cc1cccc(/C(Cl)=C\Pc2ccccc2)c1</smiles>

Following GP3, the residue was purified by flash chromatography on silica gel using dichloromethane/ petroleum ether/ethyl acetate $(v / v / v 4: 1: 1)$ as eluent to afford the pure product $9 \mathbf{c}\left(\mathrm{R}_{\mathrm{f}}=0.7\right)$ as yellow oil $(123.4 \mathrm{mg}, 70 \%$ yield). ${ }^{1} \mathrm{H}$ NMR $\left(400 \mathrm{MHz}, \mathrm{CDCl}_{3}\right): \delta 7.71-7.60(\mathrm{~m}, 4 \mathrm{H}), 7.41-7.28(\mathrm{~m}, 8 \mathrm{H}), 7.04-6.94(\mathrm{~m}, 2 \mathrm{H}), 6.76(\mathrm{~d}, J=$ $13.1 \mathrm{~Hz}, 1 \mathrm{H}), 2.18(\mathrm{~s}, 3 \mathrm{H}) ;{ }^{13} \mathrm{C} \mathrm{NMR}\left(100 \mathrm{MHz}, \mathrm{CDCl}_{3}\right): \delta 152.5(\mathrm{~d}, J=4.1 \mathrm{~Hz}), 137.4,136.0(\mathrm{~d}, J=3.8 \mathrm{~Hz})$, $133.4,132.3,131.52,131.50,131.1,130.9,130.8,129.6,128.4,128.3,127.7,126.1,122.2(\mathrm{~d}, J=96.7 \mathrm{~Hz}), 21.1$; ${ }^{31} \mathrm{P}$ NMR (162 MHz, $\mathrm{CDCl}_{3}$ ): $\delta$ 20.40. HRMS (ESI-TOF) $m / z:[\mathrm{M}+\mathrm{H}]^{+}$Calcd for $\mathrm{C}_{21} \mathrm{H}_{19} \mathrm{ClOP} 353.0862$, found 353.0852 .

(E)-(2-Chloro-2-(p-tolyl)vinyl)diphenylphosphine oxide (9d)<smiles>Cc1ccc(/C(Cl)=C\Pc2ccccc2)cc1</smiles>

Following GP3, the residue was purified by flash chromatography on silica gel using dichloromethane/ petroleum ether/ethyl acetate $(v / v / v 4: 1: 1)$ as eluent to afford the pure product $9 d\left(R_{\mathrm{f}}=0.8\right)$ as yellow oil $(126.7 \mathrm{mg}, 72 \%$ yield). ${ }^{1} \mathrm{H}$ NMR $\left(400 \mathrm{MHz}, \mathrm{CDCl}_{3}\right): \delta 7.72-7.60(\mathrm{~m}, 4 \mathrm{H}), 7.47-7.29(\mathrm{~m}, 8 \mathrm{H}), 6.92(\mathrm{~d}, J=8.0 \mathrm{~Hz}, 2 \mathrm{H}), 6.71(\mathrm{~d}$, $J=13.6 \mathrm{~Hz}, 1 \mathrm{H}), 2.22(\mathrm{~s}, 3 \mathrm{H}) .{ }^{13} \mathrm{C} \mathrm{NMR}\left(100 \mathrm{MHz}, \mathrm{CDCl}_{3}\right): \delta 152.4(\mathrm{~d}, J=8.4 \mathrm{~Hz}), 140.7,133.7,133.4(\mathrm{~d}, J=$ $3.8 \mathrm{~Hz}), 132.6,131.48,131.45,130.9,130.8,129.1,128.44,128.37,128.3,121.6(\mathrm{~d}, J=96.1 \mathrm{~Hz}), 21.3 .{ }^{31} \mathrm{P} \mathrm{NMR}$ $\left(162 \mathrm{MHz}, \mathrm{CDCl}_{3}\right): \delta$ 17.80. HRMS (ESI-TOF) $\mathrm{m} / z:[\mathrm{M}+\mathrm{H}]^{+}$Calcd for $\mathrm{C}_{21} \mathrm{H}_{19} \mathrm{ClOP} 353.0862$, found 353.0855 .

Mixture of (E)-(2-Chloro-2-(4-methoxyphenyl)vinyl)diphenylphosphine $\quad$ oxide $\quad(9 \mathrm{e}) \quad$ and diphenyl(phenylethynyl)phosphine oxide (9e')<smiles>COc1ccc(/C(Cl)=C\P(O)c2ccc(OC)cc2)cc1</smiles>

Following GP3, the residue was purified by flash chromatography on silica gel using dichloromethane/ petroleum ether/ethyl acetate $(v / v / v 4: 1: 1)$ as eluent to afford the mixture of $9 \mathbf{e}$ and $9 \mathbf{e}^{\prime}\left(\mathrm{R}_{\mathrm{f}}=0.6\right)$ as yellow oil $(93.84 \mathrm{mg}, 51 \%$ 
yield). ${ }^{1} \mathrm{H}$ NMR (400 MHz, $\left.\mathrm{CDCl}_{3}\right): \delta 7.93-7.86(\mathrm{~m}, 2 \mathrm{H}), 7.69-7.63(\mathrm{~m}, 4 \mathrm{H}), 7.57-7.53(\mathrm{~m}, 2 \mathrm{H}), 7.51-7.45$ (m, 2H), $7.42-7.38(\mathrm{~m}, 2 \mathrm{H}), 7.36-7.30(\mathrm{~m}, 4 \mathrm{H}), 6.88(\mathrm{~d}, J=8.8 \mathrm{~Hz}, 1 \mathrm{H}), 6.69-6.59(\mathrm{~m}, 3 \mathrm{H}), 3.83(\mathrm{~s}, 1 \mathrm{H})$, $3.71(\mathrm{~s}, 3 \mathrm{H}) ;{ }^{13} \mathrm{C} \mathrm{NMR}\left(100 \mathrm{MHz}, \mathrm{CDCl}_{3}\right): \delta 161.5,161.2,152.2(\mathrm{~d}, J=8.5 \mathrm{~Hz}), 134.3(\mathrm{~d}, J=1.8 \mathrm{~Hz}), 133.7$, $132.6,132.2,132.1,131.54,131.51,131.03,130.99,130.9,130.8,128.7,128.63,128.59,128.55,128.5,128.4$, $120.6(\mathrm{~d}, J=96.7 \mathrm{~Hz}), 114.1,113.1,55.4,55.3 ;{ }^{31} \mathrm{P} \mathrm{NMR}\left(162 \mathrm{MHz}, \mathrm{CDCl}_{3}\right): \delta 18.11,8.29$. HRMS (ESI-TOF) $m / z:[\mathrm{M}+\mathrm{H}]^{+}$Calcd for $\mathrm{C}_{21} \mathrm{H}_{19} \mathrm{ClO}_{2} \mathrm{P} 369.0811$, found 369.0808.

(E)-(2-Chloro-2-(3-methoxyphenyl)vinyl)diphenylphosphine oxide (9f)<smiles>COc1cccc(/C(Cl)=C\POc2ccccc2)c1</smiles>

Following GP3, the residue was purified by flash chromatography on silica gel using dichloromethane/ petroleum ether/ethyl acetate $(v / v / v 4: 1: 1)$ as eluent to afford the pure product $9 \mathbf{f}\left(\mathrm{R}_{\mathrm{f}}=0.6\right)$ as yellow oil $(130.0 \mathrm{mg}, 69 \%$ yield). ${ }^{1} \mathrm{H}$ NMR $\left(400 \mathrm{MHz}, \mathrm{CDCl}_{3}\right): \delta 7.69-7.60(\mathrm{~m}, 4 \mathrm{H}), 7.41-7.30(\mathrm{~m}, 6 \mathrm{H}), 7.20-7.16(\mathrm{~m}, 1 \mathrm{H}), 7.12-7.08$ (m, 1H), $7.05-7.00(\mathrm{~m}, 1 \mathrm{H}), 6.76(\mathrm{~d}, J=13.3 \mathrm{~Hz}, 1 \mathrm{H}), 6.73-6.68(\mathrm{~m}, 1 \mathrm{H}), 3.74(\mathrm{~s}, 3 \mathrm{H}) ;{ }^{13} \mathrm{C} \mathrm{NMR}(100 \mathrm{MHz}$, $\left.\mathrm{CDCl}_{3}\right): \delta 158.6,152.1(\mathrm{~d}, J=8.3 \mathrm{~Hz}), 137.3(\mathrm{~d}, J=3.8 \mathrm{~Hz}), 132.9(\mathrm{~d}, J=108.4 \mathrm{~Hz}), 131.6,131.5,130.8,130.7$, 129.0, 128.5, 128.3, 123.1, 122.2, 121.4, 117.3, 113.7, 55.3; ${ }^{31} \mathrm{P}$ NMR (162 $\left.\mathrm{MHz}, \mathrm{CDCl}_{3}\right): \delta$ 17.91. HRMS (ESI-TOF) $m / z:[\mathrm{M}+\mathrm{H}]^{+}$Calcd for $\mathrm{C}_{21} \mathrm{H}_{19} \mathrm{ClO}_{2} \mathrm{P} 369.0811$, found 369.0796.

\section{(E)-(2-Chloro-2-(2-methoxyphenyl)vinyl)diphenylphosphine oxide (9g)}<smiles>COc1ccccc1/C(Cl)=C\PO</smiles>

Following GP3, the residue was purified by flash chromatography on silica gel using dichloromethane/ petroleum ether/ethyl acetate $(v / v / v 4: 1: 1)$ as eluent to afford the pure product $\mathbf{9 g}\left(\mathrm{R}_{\mathrm{f}}=0.6\right)$ as yellow oil $(92.0 \mathrm{mg}, 50 \%$ yield). ${ }^{1} \mathrm{H}$ NMR (400 MHz, $\left.\mathrm{CDCl}_{3}\right): \delta 7.67-7.57(\mathrm{~m}, 4 \mathrm{H}), 7.41-7.36(\mathrm{~m}, 3 \mathrm{H}), 7.34-7.28(\mathrm{~m}, 4 \mathrm{H}), 7.17-7.11$ $(\mathrm{m}, 1 \mathrm{H}), 6.87-6.79(\mathrm{~m}, 2 \mathrm{H}), 6.47(\mathrm{~d}, J=8.3 \mathrm{~Hz}, 1 \mathrm{H}), 3.57(\mathrm{~s}, 3 \mathrm{H}) ;{ }^{13} \mathrm{C} \mathrm{NMR}\left(100 \mathrm{MHz}, \mathrm{CDCl}_{3}\right): \delta 155.2,148.9$ $(\mathrm{d}, J=9.4), 133.1,132.0,131.0,130.9,130.8,130.49,130.48,130.4,130.3,127.7,127.6,124.9(\mathrm{~d}, J=4.1 \mathrm{~Hz})$, $124.2(\mathrm{~d}, J=97.1 \mathrm{~Hz}), 114.5(\mathrm{~d}, J=980.6 \mathrm{~Hz}), 54.4 ;{ }^{31} \mathrm{P}$ NMR $\left(162 \mathrm{MHz}, \mathrm{CDCl}_{3}\right): \delta 17.01 . \mathrm{HRMS}(\mathrm{ESI}-\mathrm{TOF})$ $m / z:[\mathrm{M}+\mathrm{H}]^{+}$Calcd for $\mathrm{C}_{21} \mathrm{H}_{19} \mathrm{ClO}_{2} \mathrm{P} 369.0811$, found 369.0812.

(E)-(2-Chloro-2-(2-chlorophenyl)vinyl)diphenylphosphine oxide (9h) 
<smiles>Cl/C(=C/POc1ccccc1)c1ccccc1Cl</smiles>

Following GP3, the residue was purified by flash chromatography on silica gel using dichloromethane/ petroleum ether/ethyl acetate $(v / v / v 4: 1: 1)$ as eluent to afford the pure product $\mathbf{9 h}\left(\mathrm{R}_{\mathrm{f}}=0.6\right)$ as yellow oil $(126.5 \mathrm{mg}, 68 \%$ yield). ${ }^{1} \mathrm{H}$ NMR $\left(400 \mathrm{MHz}, \mathrm{CDCl}_{3}\right): \delta 7.91-7.70(\mathrm{~m}, 4 \mathrm{H}), 7.62-7.48(\mathrm{~m}, 7 \mathrm{H}), 7.41-7.31(\mathrm{~m}, 2 \mathrm{H}), 7.19-7.01$ $(\mathrm{m}, 1 \mathrm{H}), 6.93(\mathrm{~d}, J=19.8 \mathrm{~Hz}, 1 \mathrm{H}) ;{ }^{13} \mathrm{C} \mathrm{NMR}\left(100 \mathrm{MHz}, \mathrm{CDCl}_{3}\right): \delta 148.4(\mathrm{~d}, J=8.5 \mathrm{~Hz}), 135.1(\mathrm{~d}, J=4.1 \mathrm{~Hz})$, $132.2(\mathrm{~d}, J=0.9 \mathrm{~Hz}), 131.31,131.30,130.90,130.88,130.83,130.80,130.7,129.4,128.4,128.3,126.5,126.3$, 125.6; ${ }^{31} \mathrm{P}$ NMR (162 MHz, $\mathrm{CDCl}_{3}$ ): $\delta$ 17.15. HRMS (ESI-TOF) $m / z:[\mathrm{M}+\mathrm{H}]^{+}$Calcd for $\mathrm{C}_{20} \mathrm{H}_{16} \mathrm{Cl}_{2} \mathrm{OP} 373.0316$, found 373.0318 .

\section{(E)-(2-Chloro-2-(3-chlorophenyl)vinyl)diphenylphosphine oxide (9i)}<smiles>Cl/C(=C/Pc1ccccc1)c1cccc(Cl)c1</smiles>

Following GP3, the residue was purified by flash chromatography on silica gel using dichloromethane/ petroleum ether/ethyl acetate $(v / v / v 4: 1: 1)$ as eluent to afford the pure product $\mathbf{9 i}\left(\mathrm{R}_{\mathrm{f}}=0.6\right)$ as yellow oil $(117.2 \mathrm{mg}, 63 \%$ yield). ${ }^{1} \mathrm{H}$ NMR $\left(400 \mathrm{MHz}, \mathrm{CDCl}_{3}\right): \delta 7.71-7.61(\mathrm{~m}, 4 \mathrm{H}), 7.48-7.39(\mathrm{~m}, 4 \mathrm{H}), 7.38-7.31(\mathrm{~m}, 4 \mathrm{H}), 7.16-7.11$ $(\mathrm{m}, 1 \mathrm{H}), 7.09-7.03(\mathrm{~m}, 1 \mathrm{H}), 6.83(\mathrm{~d}, J=13.0 \mathrm{~Hz}, 1 \mathrm{H}) ;{ }^{13} \mathrm{C} \mathrm{NMR}\left(100 \mathrm{MHz}, \mathrm{CDCl}_{3}\right): \delta 149.5(\mathrm{~d}, J=8.1 \mathrm{~Hz})$, $137.2(\mathrm{~d}, J=3.8 \mathrm{~Hz}), 133.2,132.6,131.6,131.31,131.28,130.4,130.3,129.8,128.6,128.4,128.1,128.0,126.9$, $123.6(\mathrm{~d}, J=94.3 \mathrm{~Hz}) ;{ }^{31} \mathrm{P}$ NMR $\left(162 \mathrm{MHz}, \mathrm{CDCl}_{3}\right): \delta 17.35$. HRMS (ESI-TOF) $m / z:[\mathrm{M}+\mathrm{H}]^{+} \mathrm{Calcd}$ for $\mathrm{C}_{20} \mathrm{H}_{16} \mathrm{Cl}_{2} \mathrm{OP}$ 373.0316, found 373.0304.

\section{(E)-(2-Chloro-2-(4-chlorophenyl)vinyl)diphenylphosphine oxide (9j)}<smiles>OPC=C(Cl)c1ccc(Cl)cc1</smiles>

Following GP3, the residue was purified by flash chromatography on silica gel using dichloromethane/ petroleum ether/ethyl acetate $\left(v / v / v\right.$ 4:1:1) as eluent to afford the pure product $\mathbf{9 j}\left(\mathrm{R}_{\mathrm{f}}=0.6\right)$ as yellow oil $(111.6 \mathrm{mg}, 60 \%$ yield). ${ }^{1} \mathrm{H}$ NMR $\left(400 \mathrm{MHz}, \mathrm{CDCl}_{3}\right): \delta 7.70-7.60(\mathrm{~m}, 4 \mathrm{H}), 7.46-7.32(\mathrm{~m}, 8 \mathrm{H}), 7.17-7.11(\mathrm{~m}, 1 \mathrm{H}), 7.09-7.03$ $(\mathrm{m}, 1 \mathrm{H}), 6.83(\mathrm{~d}, J=13.0 \mathrm{~Hz}, 1 \mathrm{H}) ;{ }^{13} \mathrm{C} \mathrm{NMR}\left(100 \mathrm{MHz}, \mathrm{CDCl}_{3}\right): \delta 150.0(\mathrm{~d}, J=8.1 \mathrm{~Hz}), 137.7(\mathrm{~d}, J=3.9 \mathrm{~Hz})$, $133.4(\mathrm{~d}, J=61.4 \mathrm{~Hz}), 132.0,131.8(\mathrm{~d}, J=2.8 \mathrm{~Hz}), 130.9,130.8,130.3,129.1,128.9,128.6,128.4,127.3,124.1$ $(\mathrm{d}, J=94.3 \mathrm{~Hz}) ;{ }^{31} \mathrm{P}$ NMR $\left(162 \mathrm{MHz}, \mathrm{CDCl}_{3}\right): \delta$ 17.34. HRMS (ESI-TOF) $m / z:[\mathrm{M}+\mathrm{H}]^{+}$Calcd for $\mathrm{C}_{20} \mathrm{H}_{16} \mathrm{Cl}_{2} \mathrm{OP}$ 


\section{(E)-(2-Chloro-2-(2-(trifluoromethyl)phenyl)vinyl)diphenylphosphine oxide (9k)}<smiles>OP(/C=C(/Cl)c1ccccc1C(F)(F)F)c1ccccc1</smiles>

Following GP3, the residue was purified by flash chromatography on silica gel using dichloromethane/ petroleum ether/ethyl acetate $(v / v / v 4: 1: 1)$ as eluent to afford the pure product $9 \mathbf{k}\left(\mathbf{R}_{\mathrm{f}}=0.6\right)$ as yellow oil $(111.6 \mathrm{mg}, 55 \%$ yield). ${ }^{1} \mathrm{H}$ NMR $\left(400 \mathrm{MHz}, \mathrm{CDCl}_{3}\right): \delta 7.76-7.62(\mathrm{~m}, 3 \mathrm{H}), 7.53-7.29(\mathrm{~m}, 11 \mathrm{H}), 6.89(\mathrm{~d}, J=14.4 \mathrm{~Hz}, 1 \mathrm{H}) ;{ }^{13} \mathrm{C}$ NMR (100 MHz, $\left.\mathrm{CDCl}_{3}\right): \delta 146.6(\mathrm{~d}, J=8.1 \mathrm{~Hz}), 134.3(\mathrm{~d}, J=3.8 \mathrm{~Hz}), 130.3,129.7,128.7,128.41,128.38$, $127.5,127.4,126.9,125.7,125.5,125.2,125.1,124.0,120.7(\mathrm{~d}, J=94.3 \mathrm{~Hz}) ;{ }^{31} \mathrm{P} \mathrm{NMR}\left(162 \mathrm{MHz}, \mathrm{CDCl}_{3}\right): \delta$ 17.09. HRMS (ESI-TOF) $\mathrm{m} / z$ : $[\mathrm{M}+\mathrm{H}]^{+}$Calcd for $\mathrm{C}_{21} \mathrm{H}_{16} \mathrm{ClF}_{3} \mathrm{OP} 407.0579$, found 407.0585 .

(E)-(2-Chloro-2-(4-(trifluoromethyl)phenyl)vinyl)diphenylphosphine oxide (9l)<smiles>FC(F)(F)c1ccc(/C(Cl)=C\POc2ccccc2)cc1</smiles>

Following GP3, the residue was purified by flash chromatography on silica gel using dichloromethane/ petroleum ether/ethyl acetate $(v / v / v 4: 1: 1)$ as eluent to afford the pure product $91\left(\mathrm{R}_{\mathrm{f}}=0.7\right)$ as yellow oil $(103.5 \mathrm{mg}, 51 \%$ yield). ${ }^{1} \mathrm{H}$ NMR (400 MHz, $\left.\mathrm{CDCl}_{3}\right): \delta 7.68-7.56(\mathrm{~m}, 6 \mathrm{H}), 7.45-7.29(\mathrm{~m}, 8 \mathrm{H}), 6.90(\mathrm{~d}, J=13.1 \mathrm{~Hz}, 1 \mathrm{H}) ;{ }^{13} \mathrm{C}$ NMR (100 MHz, $\left.\mathrm{CDCl}_{3}\right): \delta 149.9(\mathrm{~d}, J=8.1 \mathrm{~Hz}), 139.5(\mathrm{~d}, J=2.4 \mathrm{~Hz}), 133.0,131.89,131.86,130.9,130.8$, 129.43, 129.42, 128.6, 128.5, 125.2, 124.7 (q, $3.8 \mathrm{~Hz}), 124.2,122.2 ;{ }^{31} \mathrm{P}$ NMR (162 MHz, $\left.\mathrm{CDCl}_{3}\right): \delta 17.50$. HRMS (ESI-TOF) $m / z:[\mathrm{M}+\mathrm{H}]^{+}$Calcd for $\mathrm{C}_{21} \mathrm{H}_{16} \mathrm{ClF}_{3} \mathrm{OP} 407.0579$, found 407.0572.

(E)-(2-(4-Bromophenyl)-2-chlorovinyl)diphenylphosphine oxide (9m)<smiles>Cl/C(=C/POc1ccccc1)c1ccc(Br)cc1</smiles>

Following GP3, the residue was purified by flash chromatography on silica gel using dichloromethane/ petroleum ether/ethyl acetate $(v / v / v 4: 1: 1)$ as eluent to afford the pure product $\mathbf{9 m}\left(\mathrm{R}_{\mathrm{f}}=0.6\right)$ as yellow oil $(118.3 \mathrm{mg}, 57 \%$ yield). ${ }^{1} \mathrm{H}$ NMR $\left(400 \mathrm{MHz}, \mathrm{CDCl}_{3}\right): \delta 7.68-7.60(\mathrm{~m}, 4 \mathrm{H}), 7.45-7.32(\mathrm{~m}, 8 \mathrm{H}), 7.27-7.23(\mathrm{~m}, 2 \mathrm{H}), 6.79(\mathrm{~d}, J=$ $13.4 \mathrm{~Hz}, 1 \mathrm{H}) ;{ }^{13} \mathrm{C}$ NMR $\left(100 \mathrm{MHz}, \mathrm{CDCl}_{3}\right): \delta 150.6(\mathrm{~d}, J=8.1 \mathrm{~Hz}), 135.0(\mathrm{~d}, J=3.8 \mathrm{~Hz}), 133.3,132.2,131.78$, $131.75,130.93,130.91,130.8,130.6,128.6,128.5,124.9,123.4(\mathrm{~d}, J=94.6 \mathrm{~Hz}) ;{ }^{31} \mathrm{P} \mathrm{NMR}\left(162 \mathrm{MHz}, \mathrm{CDCl}_{3}\right): \delta$ 17.67. HRMS (ESI-TOF) $m / z:[\mathrm{M}+\mathrm{H}]^{+}$Calcd for $\mathrm{C}_{20} \mathrm{H}_{16} \mathrm{BrClOP} 416.9811$, found 416.9803 . 
<smiles>Fc1ccc(/C(Cl)=C\POc2ccccc2)cc1</smiles>

Following GP3, the residue was purified by flash chromatography on silica gel using dichloromethane/ petroleum ether/ethyl acetate $(v / v / v 4: 1: 1)$ as eluent to afford the pure product $\mathbf{9 n}\left(\mathrm{R}_{\mathrm{f}}=0.7\right)$ as yellow oil $(87.0 \mathrm{mg}, 50 \%$ yield). ${ }^{1} \mathrm{H}$ NMR $\left(400 \mathrm{MHz}, \mathrm{CDCl}_{3}\right): \delta 7.69-7.61(\mathrm{~m}, 4 \mathrm{H}), 7.57-7.52(\mathrm{~m}, 2 \mathrm{H}), 7.44-7.39(\mathrm{~m}, 2 \mathrm{H}), 7.38-7.32$ $(\mathrm{m}, 4 \mathrm{H}), 6.85-6.79(\mathrm{~m}, 2 \mathrm{H}), 6.77(\mathrm{~d}, J=11.1 \mathrm{~Hz}, 1 \mathrm{H}) ;{ }^{13} \mathrm{C} \mathrm{NMR}\left(100 \mathrm{MHz}, \mathrm{CDCl}_{3}\right): \delta 164.9,162.4,151.0(\mathrm{~d}, J$ $=8.1 \mathrm{~Hz}), 133.3,132.3(\mathrm{~d}, J=3.6 \mathrm{~Hz}), 131.7(\mathrm{~d}, J=2.2 \mathrm{~Hz}), 131.4,131.3,130.9,130.8,128.6,128.4,122.8(\mathrm{~d}, J$ $=95.3 \mathrm{~Hz}), 114.8(\mathrm{~d}, J=22.1 \mathrm{~Hz}) ;{ }^{31} \mathrm{P} \mathrm{NMR}\left(162 \mathrm{MHz}, \mathrm{CDCl}_{3}\right): \delta 17.76 . \mathrm{HRMS}(\mathrm{ESI}-\mathrm{TOF}) \mathrm{m} / z:[\mathrm{M}+\mathrm{H}]^{+} \mathrm{Calcd}$ for $\mathrm{C}_{20} \mathrm{H}_{16} \mathrm{ClFOP}$ 357.0611, found 357.0609.

\section{(E)-(2-Chloro-2-(4-nitrophenyl)vinyl)diphenylphosphine oxide (9o)}<smiles>O=[N+]([O-])c1ccc(/C(Cl)=C\[PbH](c2ccccc2)c2ccccc2)cc1</smiles>

Following GP3, the residue was purified by flash chromatography on silica gel using dichloromethane/ petroleum ether/ethyl acetate $(v / v / v 4: 1: 1)$ as eluent to afford the pure product $90\left(\mathrm{R}_{\mathrm{f}}=0.5\right)$ as yellow oil $(134.0 \mathrm{mg}, 70 \%$ yield). ${ }^{1} \mathrm{H}$ NMR $\left(400 \mathrm{MHz}, \mathrm{CDCl}_{3}\right): \delta 8.07-7.91(\mathrm{~m}, 2 \mathrm{H}), 7.75-7.57(\mathrm{~m}, 6 \mathrm{H}), 7.50-7.32(\mathrm{~m}, 6 \mathrm{H}), 6.94(\mathrm{~d}, J=$ $13.5 \mathrm{~Hz}, 1 \mathrm{H}) ;{ }^{13} \mathrm{C}$ NMR $\left(100 \mathrm{MHz}, \mathrm{CDCl}_{3}\right): \delta 148.6(\mathrm{~d}, J=7.5 \mathrm{~Hz}), 148.3,142.1(\mathrm{~d}, J=3.9 \mathrm{~Hz}), 132.9,132.12$, $132.09,131.8,130.9,130.8,130.2,128.8,128.6,125.6(\mathrm{~d}, J=92.5 \mathrm{~Hz}), 122.9 ;{ }^{31} \mathrm{P} \mathrm{NMR}\left(162 \mathrm{MHz}, \mathrm{CDCl}_{3}\right): \delta$ 17.58. HRMS (ESI-TOF) $m / z:[\mathrm{M}+\mathrm{H}]^{+}$Calcd for $\mathrm{C}_{20} \mathrm{H}_{16} \mathrm{ClNO}_{3} \mathrm{P} 384.0556$, found 384.0543 .

\section{(E)-Methyl-4-(1-chloro-2-(diphenylphosphoryl)vinyl)benzoate (9p)}<smiles>COC(=O)c1ccc(/C(Cl)=C\[PbH]c2ccccc2)cc1</smiles>

Following GP3, the residue was purified by flash chromatography on silica gel using dichloromethane/ petroleum ether/ethyl acetate $(v / v / v 4: 1: 1)$ as eluent to afford the pure product $\mathbf{9 p}\left(\mathrm{R}_{\mathrm{f}}=0.6\right)$ as yellow oil $(130.7 \mathrm{mg}, 66 \%$ yield). ${ }^{1} \mathrm{H}$ NMR $\left(400 \mathrm{MHz}, \mathrm{CDCl}_{3}\right): \delta 7.82-7.75(\mathrm{~m}, 2 \mathrm{H}), 7.68-7.55(\mathrm{~m}, 6 \mathrm{H}), 7.45-7.28(\mathrm{~m}, 6 \mathrm{H}), 6.86(\mathrm{~d}, J=$ $13.3 \mathrm{~Hz}, 1 \mathrm{H}), 3.88(\mathrm{~s}, 3 \mathrm{H}) ;{ }^{13} \mathrm{C} \mathrm{NMR}\left(100 \mathrm{MHz}, \mathrm{CDCl}_{3}\right): \delta 165.7,150.0(\mathrm{~d}, J=8.0 \mathrm{~Hz}), 139.8(\mathrm{~d}, J=3.8 \mathrm{~Hz})$, $132.2(\mathrm{~d}, J=108.4 \mathrm{~Hz}), 131.4,131.3,130.8,130.4,130.3,128.6,128.5,128.1,128.0,123.7(\mathrm{~d}, J=94.1 \mathrm{~Hz}), 51.8$; ${ }^{31} \mathrm{P}$ NMR $\left(162 \mathrm{MHz}, \mathrm{CDCl}_{3}\right.$ ): $\delta$ 17.53. HRMS (ESI-TOF) $\mathrm{m} / z:[\mathrm{M}+\mathrm{H}]^{+}$Calcd for $\mathrm{C}_{22} \mathrm{H}_{19} \mathrm{ClO}_{3} \mathrm{P} 397.0760$, found 
397.0745 .

(E)-(2-Chloro-2-(thiophen-2-yl)vinyl)diphenylphosphine oxide (9q)<smiles>OP/C=C(/Cl)c1cccs1</smiles>

Following GP3, the residue was purified by flash chromatography on silica gel using dichloromethane/ petroleum ether/ethyl acetate $(v / v / v 4: 1: 1)$ as eluent to afford the pure product $\mathbf{9 q}\left(\mathrm{R}_{\mathrm{f}}=0.7\right)$ as yellow oil $(113.5 \mathrm{mg}, 66 \%$ yield). ${ }^{1} \mathrm{H}$ NMR (400 MHz, $\left.\mathrm{CDCl}_{3}\right): \delta 7.88-7.75(\mathrm{~m}, 4 \mathrm{H}), 7.58-7.45(\mathrm{~m}, 7 \mathrm{H}), 7.42(\mathrm{~d}, J=5.0 \mathrm{~Hz}, 1 \mathrm{H}), 7.05$ (dd, $J=4.9,4.0 \mathrm{~Hz}, 1 \mathrm{H}), 6.74(\mathrm{~d}, J=12.9 \mathrm{~Hz}, 1 \mathrm{H}) ;{ }^{13} \mathrm{C} \mathrm{NMR}\left(100 \mathrm{MHz}, \mathrm{CDCl}_{3}\right): \delta 142.9(\mathrm{~d}, J=2.4 \mathrm{~Hz}), 140.6(\mathrm{~d}, J$ = 13.2 Hz), 133.2, 132.2, 131.5, 131.4, 130.7, 130.6, 129.2, 129.0, 128.3, 128.2, 127.7, 115.4 (d, J = 106.3 Hz);

${ }^{31} \mathrm{P}$ NMR $\left(162 \mathrm{MHz}, \mathrm{CDCl}_{3}\right.$ ): $\delta 20.36$. HRMS (ESI-TOF) $m / z:[\mathrm{M}+\mathrm{H}]^{+}$Calcd for $\mathrm{C}_{18} \mathrm{H}_{15} \mathrm{ClOPS} 345.0270$, found 345.0265 .

(E)-(1-Chloro-1-phenylprop-1-en-2-yl)diphenylphosphine oxide (9r)<smiles>CCP/C(C)=C(\Cl)c1ccccc1</smiles>

Following GP3, the residue was purified by flash chromatography on silica gel using dichloromethane/ petroleum ether/ethyl acetate $(v / v / v 4: 1: 1)$ as eluent to afford the pure product $9 \mathbf{r}\left(\mathrm{R}_{\mathrm{f}}=0.7\right)$ as yellow oil $(102.1 \mathrm{mg}, 58 \%$ yield). ${ }^{1} \mathrm{H}$ NMR (400 MHz, $\left.\mathrm{CDCl}_{3}\right): \delta 7.64-7.54(\mathrm{~m}, 4 \mathrm{H}), 7.41-7.33(\mathrm{~m}, 3 \mathrm{H}), 7.32-7.27(\mathrm{~m}, 4 \mathrm{H}), 7.25(\mathrm{~d}, J=$ $1.6 \mathrm{~Hz}, 1 \mathrm{H}), 7.06-6.97(\mathrm{~m}, 3 \mathrm{H}), 2.11(\mathrm{~d}, J=12.0 \mathrm{~Hz}, 3 \mathrm{H}) ;{ }^{13} \mathrm{C} \mathrm{NMR}\left(100 \mathrm{MHz}, \mathrm{CDCl}_{3}\right): \delta 147.0(\mathrm{~d}, J=19.8$ Hz), $138.1(\mathrm{~d}, J=4.3 \mathrm{~Hz}), 133.3,132.3,131.34,131.31,131.25,131.2,129.5,129.5,129.1,128.4,128.2,127.6$, $21.1(\mathrm{~d}, J=10.0 \mathrm{~Hz}) ;{ }^{31} \mathrm{P}$ NMR $\left(162 \mathrm{MHz}, \mathrm{CDCl}_{3}\right): \delta 26.74$. HRMS (ESI-TOF) $\mathrm{m} / z:[\mathrm{M}+\mathrm{H}]^{+}$Calcd for $\mathrm{C}_{21} \mathrm{H}_{19} \mathrm{ClOP} 353.0862$, found 353.0862.

(E)-4-Chloro-3-(diphenylphosphoryl)-4-phenylbut-3-en-2-one (9s)<smiles>CC(=O)C(=PO)c1ccccc1</smiles>

Following GP3, the residue was purified by flash chromatography on silica gel using dichloromethane/ petroleum ether/ethyl acetate $(v / v / v 4: 1: 1)$ as eluent to afford the pure product $9 \mathbf{s}\left(\mathrm{R}_{\mathrm{f}}=0.6\right)$ as yellow oil $(106.4 \mathrm{mg}, 56 \%$ yield). ${ }^{1} \mathrm{H}$ NMR (400 MHz, $\left.\mathrm{CDCl}_{3}\right): \delta 7.76-7.65(\mathrm{~m}, 4 \mathrm{H}), 7.47-7.41(\mathrm{~m}, 2 \mathrm{H}), 7.39-7.30(\mathrm{~m}, 6 \mathrm{H}), 7.20-7.15$ (m, 1H), $7.13-7.05(\mathrm{~m}, 2 \mathrm{H}), 2.19(\mathrm{~s}, 3 \mathrm{H}) ;{ }^{13} \mathrm{C}$ NMR $\left(100 \mathrm{MHz}, \mathrm{CDCl}_{3}\right): \delta 200.4(\mathrm{~d}, J=8.9 \mathrm{~Hz}), 146.1(\mathrm{~d}, J=$ $10.7 \mathrm{~Hz}), 140.0,136.1$ (d, $J=4.0 \mathrm{~Hz}), 132.1,132.0,131.9,131.7,131.6,130.8,130.0,128.9,128.5,128.4,127.8$, 31.5; ${ }^{31} \mathrm{P}$ NMR (162 MHz, $\mathrm{CDCl}_{3}$ ): $\delta$ 18.28. HRMS (ESI-TOF) $m / z:[\mathrm{M}+\mathrm{H}]^{+}$Calcd for $\mathrm{C}_{22} \mathrm{H}_{19} \mathrm{ClO}_{2} \mathrm{P} 381.0811$, 
found 381.0823 .

\section{(3,5-Di-tert-butyl-4-hydroxybenzyl)diphenylphosphine oxide (10)}

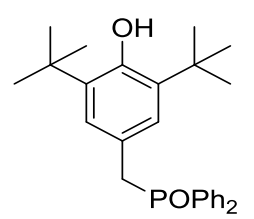

Following the radical trapping experiment with BHT, the residue was purified by flash chromatography on silica gel using dichloromethane/petroleum ether/ethyl acetate $(v / v / v 4: 1: 1)$ as eluent to afford the pure product $\mathbf{1 0}\left(\mathrm{R}_{\mathrm{f}}=\right.$ $0.5)$ as white solid $(26 \mathrm{mg}, 25 \%) .{ }^{1} \mathrm{H}$ NMR $\left(400 \mathrm{MHz}, \mathrm{CDCl}_{3}\right): \delta 7.71-7.61(\mathrm{~m}, 4 \mathrm{H}), 7.50-7.48(\mathrm{~m}, 2 \mathrm{H}), 7.45-$ $7.40(\mathrm{~m}, 4 \mathrm{H}), 6.73(\mathrm{~d}, J=2.1 \mathrm{~Hz}, 2 \mathrm{H}), 5.07(\mathrm{~s}, 1 \mathrm{H}), 3.57(\mathrm{~d}, J=13.8 \mathrm{~Hz}, 2 \mathrm{H}), 1.28(\mathrm{~s}, 18 \mathrm{H}) ;{ }^{13} \mathrm{C} \mathrm{NMR}(100 \mathrm{MHz}$, $\left.\mathrm{CDCl}_{3}\right): \delta 152.8(\mathrm{~d}, J=3.4 \mathrm{~Hz}), 135.7(\mathrm{~d}, J=2.7 \mathrm{~Hz}), 131.7(\mathrm{~d}, J=2.6 \mathrm{~Hz}), 131.4(\mathrm{~d}, J=9.0 \mathrm{~Hz}), 128.4(\mathrm{~d}, J=$ $11.5 \mathrm{~Hz}), 127.0(\mathrm{~d}, J=5.0 \mathrm{~Hz}), 121.1(\mathrm{~d}, J=7.9 \mathrm{~Hz}), 38.1(\mathrm{~d}, J=67.3 \mathrm{~Hz}), 34.1,30.1 ;{ }^{31} \mathrm{P} \mathrm{NMR}(162 \mathrm{MHz}$, $\mathrm{CDCl}_{3}$ ): $\delta$ 30.29. HRMS (ESI-TOF) $m / z:[\mathrm{M}+\mathrm{H}]^{+}$Calcd for $\mathrm{C}_{27} \mathrm{H}_{34} \mathrm{O}_{2} \mathrm{P} 421.2296$, found 421.2284.

\section{(2,2-Diphenylvinyl)diphenylphosphine oxide (11)}<smiles>CCOc1ccccc1</smiles>

Following the radical trapping experiment with DPE, the residue was purified by flash chromatography on silica gel using dichloromethane/petroleum ether/ethyl acetate $(v / v / v 4: 1: 1)$ as eluent to afford the pure product $\mathbf{1 1}\left(\mathbf{R}_{\mathrm{f}}=\right.$ $0.4)$ as white solid (52 mg, 55\% yield). ${ }^{1} \mathrm{H}$ NMR (400 MHz, $\left.\mathrm{CDCl}_{3}\right) \delta 7.71-7.64(\mathrm{~m}, 4 \mathrm{H}), 7.42-7.29(\mathrm{~m}, 11 \mathrm{H})$, $7.26-7.19(\mathrm{~m}, 2 \mathrm{H}), 7.17-7.05(\mathrm{~m}, 3 \mathrm{H}), 6.79(\mathrm{~d}, J=18.2 \mathrm{~Hz}, 1 \mathrm{H}) ;{ }^{13} \mathrm{C} \mathrm{NMR}\left(100 \mathrm{MHz}, \mathrm{CDCl}_{3}\right): \delta 162.0(\mathrm{~d}, J=$ $2.3 \mathrm{~Hz}), 141.9(\mathrm{~d}, J=16.2 \mathrm{~Hz}), 138.0(\mathrm{~d}, J=6.7 \mathrm{~Hz}), 134.9,133.9,131.1(\mathrm{~d}, J=2.7 \mathrm{~Hz}), 130.9,130.8,130.3(\mathrm{~d}, J$ $=1.0 \mathrm{~Hz}), 129.5,128.6,128.4,128.33,128.29,128.2,127.6,121.0,120.0 ;{ }^{31} \mathrm{P} \mathrm{NMR}\left(162 \mathrm{MHz}, \mathrm{CDCl}_{3}\right): \delta 18.77$. HRMS (ESI-TOF) $m / z$ : [M+Na $]^{+}$Calcd for $\mathrm{C}_{26} \mathrm{H}_{21} \mathrm{NaOP} 403.1228$, found 403.1225 .

\section{(E)-(2-Bromo-2-phenylvinyl)diphenylphosphine oxide (12)}<smiles>POC=C(Br)c1ccccc1</smiles>

Following the experiment with TBAB, the residue was purified by flash chromatography on silica gel using dichloromethane/petroleum ether/ethyl acetate $\left(v / v / v\right.$ 4:1:1) as eluent to afford the pure product $\mathbf{1 2}\left(\mathbf{R}_{\mathrm{f}}=0.6\right)$ as colorless oil (47 mg, 50\%). ${ }^{1} \mathrm{H}$ NMR $\left(400 \mathrm{MHz}, \mathrm{CDCl}_{3}\right): \delta 7.66-7.58(\mathrm{~m}, 4 \mathrm{H}), 7.49-7.43(\mathrm{~m}, 2 \mathrm{H}), 7.41-7.35$ (m, 2H), $7.33-7.28(\mathrm{~m}, 4 \mathrm{H}), 7.15-7.03(\mathrm{~m}, 4 \mathrm{H}) ;{ }^{13} \mathrm{C} \mathrm{NMR}\left(100 \mathrm{MHz}, \mathrm{CDCl}_{3}\right): \delta 143.0(\mathrm{~d}, J=5.4 \mathrm{~Hz}), 138.1(\mathrm{~d}$, $J=4.6 \mathrm{~Hz}), 133.4,132.3,131.6(\mathrm{~d}, J=2.8 \mathrm{~Hz}), 130.9,130.8,130.1,129.1(\mathrm{~d}, J=0.8 \mathrm{~Hz}), 128.5,128.4,127.8$, 127.7, 126.9; ${ }^{31} \mathrm{P}$ NMR $\left(162 \mathrm{MHz}, \mathrm{CDCl}_{3}\right): \delta$ 18.02. HRMS (ESI-TOF) $\mathrm{m} / z:[\mathrm{M}+\mathrm{H}]^{+}$Calcd for $\mathrm{C}_{20} \mathrm{H}_{17} \mathrm{BrOP}$ 
383.0200, found 383.0203.

(Z)-(2-((1H-tetrazol-5-yl)thio)-2-phenylvinyl)diphenylphosphine oxide (13)

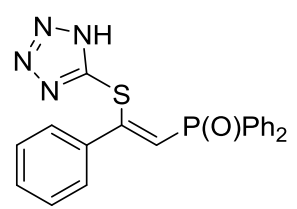

The crude product was purified by flash chromatography on silica gel using ethyl acetate/petroleum ether $(v / v 1: 5)$ as eluent to afford the pure product $13\left(\mathrm{R}_{\mathrm{f}}=0.2\right)$ as yellow solid $(75.2 \mathrm{mg}, 90 \%)$. Melting point: $298-300{ }^{\circ} \mathrm{C} .{ }^{1} \mathrm{H}$ NMR (400 MHz, DMSO- $\left.d_{6}\right): \delta 7.94-7.83(\mathrm{~m}, 4 \mathrm{H}), 7.61-7.49(\mathrm{~m}, 6 \mathrm{H}), 7.48-7.42(\mathrm{~m}, 2 \mathrm{H}), 7.23-7.13(\mathrm{~m}$, $3 \mathrm{H}), 6.51(\mathrm{~d}, J=20.1 \mathrm{~Hz}, 1 \mathrm{H}), 3.17(\mathrm{~s}, 1 \mathrm{H}) ;{ }^{13} \mathrm{C}$ NMR $\left(100 \mathrm{MHz}, \mathrm{DMSO}-d_{6}\right): \delta 159.4,151.7,139.4(\mathrm{~d}, J=14.5$ $\mathrm{Hz}), 135.6,134.5,131.9(\mathrm{~d}, J=2.4 \mathrm{~Hz}), 131.2,131.1,129.5,129.1,129.0,128.5,128.2,120.6(\mathrm{~d}, J=104.1 \mathrm{~Hz})$, 49.1; ${ }^{31} \mathrm{P}$ NMR (162 MHz, DMSO- $\left.d_{6}\right): \delta 22.27$. HRMS (ESI-TOF) $m / z:[\mathrm{M}+\mathrm{H}]^{+}$Calcd for $\mathrm{C}_{21} \mathrm{H}_{18} \mathrm{~N}_{4} \mathrm{OPS} 405.0939$, found 405.0949 .

\section{(Z)-Diphenyl(2-phenyl-2-((trifluoromethyl)thio)vinyl)phosphine oxide (14)}<smiles>FC(F)(F)C(=C[PbH](c1ccccc1)c1ccccc1)C(F)(F)F</smiles>

The crude product was purified by flash chromatography on silica gel using ethyl acetate/petroleum ether $(v / v 1: 5)$ as eluent to afford the pure product $14\left(\mathrm{R}_{\mathrm{f}}=0.4\right)$ as yellow oil $(68.7 \mathrm{mg}, 85 \%$ yield $) .{ }^{1} \mathrm{H} \mathrm{NMR}\left(400 \mathrm{MHz} \mathrm{CDCl}_{3}\right)$ : $\delta 7.98-7.79(\mathrm{~m}, 4 \mathrm{H}), 7.66-7.22(\mathrm{~m}, 12 \mathrm{H}) ;{ }^{13} \mathrm{C}$ NMR $\left(100 \mathrm{MHz}, \mathrm{CDCl}_{3}\right): 133.7,132.6,132.5,132.4,132.3$, 132.2, 131.1, 130.9, 130.7, 128.8, $128.6(\mathrm{~d}, J=1.0 \mathrm{~Hz}), 119.9(\mathrm{~d}, J=4.1 \mathrm{~Hz}), 105.5(\mathrm{~d}, J=30.0 \mathrm{~Hz}), 82.9(\mathrm{~d}, J=$ 169.6 Hz); ${ }^{31} \mathrm{P}$ NMR $\left(162 \mathrm{MHz}, \mathrm{CDCl}_{3}\right): \delta 8.26 ;{ }^{19} \mathrm{~F}$ NMR $\left(376 \mathrm{MHz}, \mathrm{CDCl}_{3}\right): \delta-38.00 . \mathrm{HRMS}$ (ESI-TOF) $m / z$ : $[\mathrm{M}+\mathrm{H}]^{+}$Calcd for $\mathrm{C}_{21} \mathrm{H}_{17} \mathrm{~F}_{3} \mathrm{OPS}$ 405.0690, found 405.0689.

\section{(Z)-((5H-Benzo[d]tetrazolo[5,1-b][1,3]thiazin-5-ylidene)methyl)diphenylphosphine oxide (15)}<smiles>C(=Pc1ccccc1)c1ccccc1</smiles>

The crude product was purified by flash chromatography on silica gel using ethyl acetate/petroleum ether $(v / v 1: 5)$ as eluent to afford the pure product $\mathbf{1 5}\left(\mathrm{R}_{\mathrm{f}}=0.2\right)$ as brown oil $(36.2 \mathrm{mg}, 90 \%$ yield $) .{ }^{1} \mathrm{H} \mathrm{NMR}\left(400 \mathrm{MHz}, \mathrm{CDCl}_{3}\right)$ : $\delta 8.23(\mathrm{~d}, J=8.1 \mathrm{~Hz}, 1 \mathrm{H}), 7.85-7.75(\mathrm{~m}, 5 \mathrm{H}), 7.66(\mathrm{t}, J=7.8 \mathrm{~Hz}, 1 \mathrm{H}), 7.60-7.54(\mathrm{~m}, 2 \mathrm{H}), 7.53-7.47(\mathrm{~m}, 5 \mathrm{H})$, $6.98(\mathrm{~d}, J=15.9 \mathrm{~Hz}, 1 \mathrm{H}) ;{ }^{13} \mathrm{C} \mathrm{NMR}\left(100 \mathrm{MHz}, \mathrm{CDCl}_{3}\right): \delta 147.9,142.1,132.9,132.6,132.5,132.4,131.3,131.2$, 
$131.1,130.5,129.5,129.1,129.0,126.4,121.3(\mathrm{~d}, J=14.5 \mathrm{~Hz}), 120.4(\mathrm{~d}, J=100.2 \mathrm{~Hz}), 118.7 ;{ }^{31} \mathrm{P}$ NMR $(162$ $\mathrm{MHz}, \mathrm{CDCl}_{3}$ ): $\delta$ 22.28. HRMS (ESI-TOF) $m / z:[\mathrm{M}+\mathrm{H}]^{+}$Calcd for $\mathrm{C}_{21} \mathrm{H}_{16} \mathrm{~N}_{4} \mathrm{OPS}$ 403.0782, found 403.0774.

\section{X-Ray Crystallization Data for Compounds $(E)-4 l,(Z)-7$ a and $(E)-9 p$}

Experimental procedure for preparation of single crystals of compounds 41, 7a and 9p): A pure sample (0.2 $\mathrm{mmol})$ was dissolved in dichloromethane $(0.4 \mathrm{~mL})$ in a vial at room temperature, and $n$-hexane $(3.2 \mathrm{~mL})$ was added. The vial was properly sealed with parafilm and kept at room temperature to allow the slow evaporation of the solvent until a single crystal was obtained. Intensity data were collected with a Rigaku Mercury CCD area detector in $\omega$ scan mode using Mo-K $\alpha$ radiation $(\lambda=0.71075 \AA)$. 
9-1. X-ray crystallographic data for (E)-2-(3-bromophenyl)-3-(diphenylphosphoryl) acrylonitrile (4I)

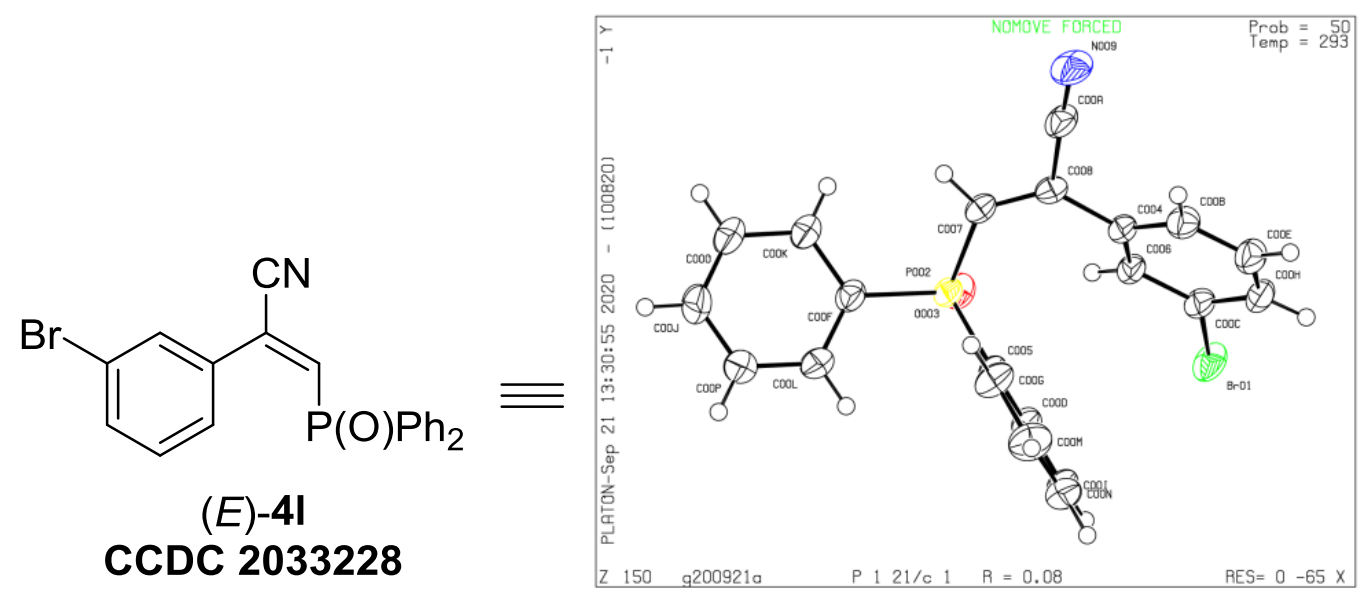

ORTEP diagram of 4l. The thermal ellipsoids are drawn at the $50 \%$ probability level.

\begin{tabular}{|c|c|}
\hline Compound & $(E)-\mathbf{4 l}$ \\
\hline formula & $\mathrm{C}_{21} \mathrm{H}_{15} \mathrm{BrNOP}$ \\
\hline $\mathrm{fw}$ & 408.22 \\
\hline crystal system & monoclinic \\
\hline$T / \mathrm{K}$ & 293(2) \\
\hline$a / \AA$ & $11.6535(6)$ \\
\hline$b / \AA$ & $15.6918(9)$ \\
\hline$c / \AA ̊ \AA$ & $10.2191(5)$ \\
\hline $1^{\circ}$ & 90 \\
\hline$\rho^{\circ}$ & $106.097(5)$ \\
\hline$\rho^{\circ}$ & 90.00 \\
\hline$V / \AA^{3}$ & $1795.44(17)$ \\
\hline Z & 4 \\
\hline$D_{C} / \mathrm{g} \mathrm{cm}^{-3}$ & 1.510 \\
\hline $\mathrm{F}(000)$ & 824 \\
\hline collected & 3797 \\
\hline GOF & 1.045 \\
\hline Final $\mathrm{R}$ indices [I>2 sigma (I)] & $\mathrm{R} 1=0.0830 \quad \mathrm{wR} 2=0.2214$ \\
\hline$R$ indices (all data) & $\mathrm{R} 1=0.0894 \quad \mathrm{wR} 2=0.2046$ \\
\hline Largest diff. peak, hole/e. $\AA^{-3}$ & $1.753,-1.015$ \\
\hline
\end{tabular}




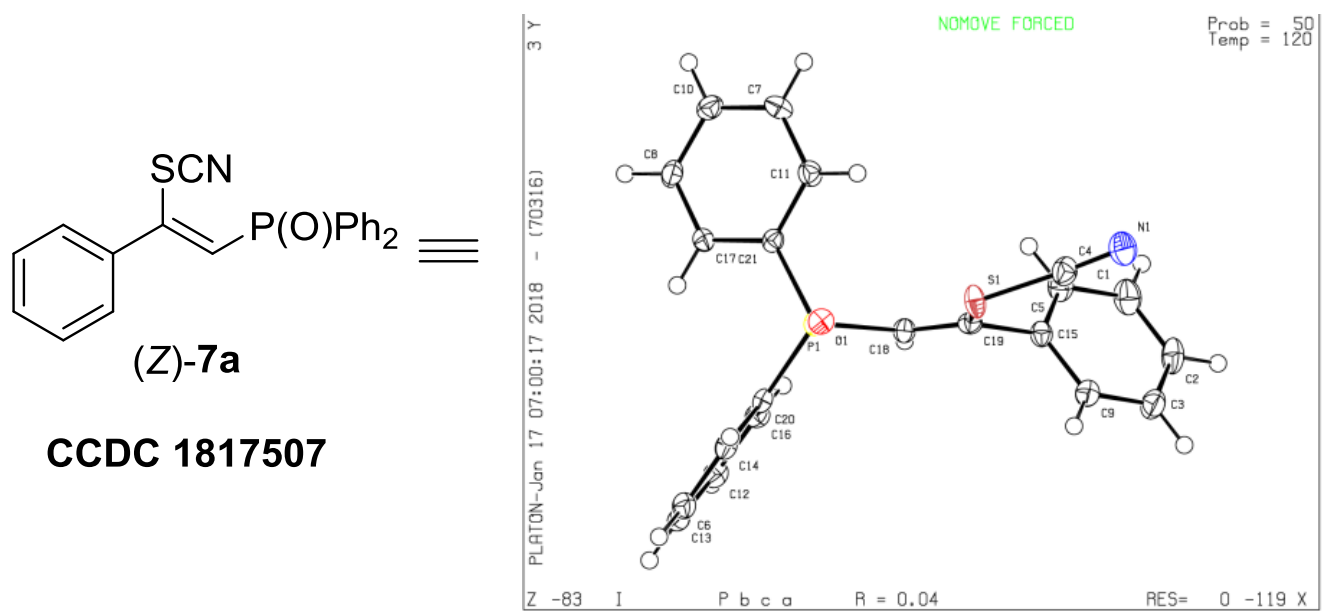

ORTEP diagram of $\mathbf{7 a}$. The thermal ellipsoids are drawn at the $50 \%$ probability level.

\begin{tabular}{|c|c|}
\hline Compound & $(Z)-7 \mathbf{a}$ \\
\hline formula & $\mathrm{C}_{21} \mathrm{H}_{16} \mathrm{NOPS}$ \\
\hline $\mathrm{fw}$ & 361.38 \\
\hline crystal system & orthorhombic \\
\hline$T / \mathrm{K}$ & $120(2) 6$ \\
\hline$a / \AA$ & $11.4391(9)$ \\
\hline$b / \AA$ & $12.3029(8)$ \\
\hline$c / \AA$ & $26.3082(17)$ \\
\hline$\rho^{\circ}$ & 90 \\
\hline$\rho^{\circ}$ & 90 \\
\hline$\rho^{\circ}$ & 90 \\
\hline$V / \AA^{3}$ & $3702.5(4)$ \\
\hline$Z$ & 8 \\
\hline$D_{C} / \mathrm{g} \mathrm{cm}^{-3}$ & 1.297 \\
\hline $\mathrm{F}(000)$ & 1504 \\
\hline collected & 4259 \\
\hline GOF & 1.067 \\
\hline Final $R$ indices $[I>2 \operatorname{sigma}(I)]$ & $\mathrm{R} 1=0.0422 \quad \mathrm{wR} 2=0.1482$ \\
\hline$R$ indices (all data) & $\mathrm{R} 1=0.0735 \quad \mathrm{wR} 2=0.1247$ \\
\hline Largest diff. peak, hole/e. $\AA^{-3}$ & $0.450,-0.747$ \\
\hline
\end{tabular}




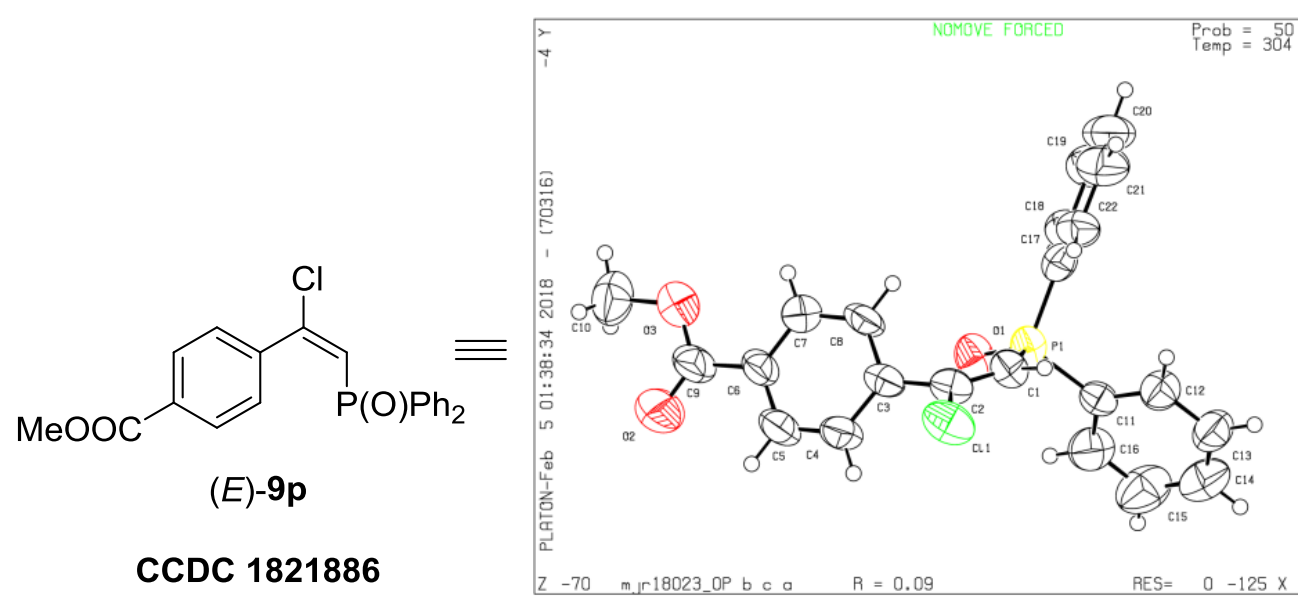

ORTEP diagram of 9p. The thermal ellipsoids are drawn at the 50\% probability level.

\begin{tabular}{|c|c|}
\hline Compound & $(E)-\mathbf{9 p}$ \\
\hline formula & $\mathrm{C}_{22} \mathrm{H}_{18} \mathrm{ClO}_{3} \mathrm{P}$ \\
\hline fw & 396.78 \\
\hline crystal system & orthorhombic \\
\hline$T / \mathrm{K}$ & 304 \\
\hline$a / \AA$ & $9.3285(7)$ \\
\hline$b / \AA$ & $10.6719(10)$ \\
\hline$c / \AA$ & $40.372(4)$ \\
\hline$\rho^{\circ}$ & 90 \\
\hline$\rho^{\circ}$ & 90 \\
\hline$\rho^{\circ}$ & 90 \\
\hline$V / \AA^{3}$ & $4019.2(6)$ \\
\hline$Z$ & 8 \\
\hline$D_{C} / \mathrm{g} \mathrm{cm}^{-3}$ & 1.311 \\
\hline $\mathrm{F}(000)$ & 1648.0 \\
\hline collected & 3799 \\
\hline GOF & 1.027 \\
\hline Final $R$ indices [I>2 sigma (I)] & $\mathrm{R} 1=0.0863 \quad \mathrm{wR} 2=0.2385$ \\
\hline$R$ indices (all data) & $\mathrm{R} 1=0.1911 \quad \mathrm{wR} 2=0.1900$ \\
\hline Largest diff. peak, hole/e. $\AA^{-3}$ & $0.323,-0.344$ \\
\hline
\end{tabular}




\section{References}

1. Su, L. B.; Ren, T. B.; Dong, J. Y.; Liu, L, X.; Xie, S. M.; Yuan, L.; Zhou, Y. B.; Yin, S, F. Cu(I)-Catalyzed 6-endo-dig Cyclization of Terminal Alkynes, 2-Bromoaryl Ketones, and Amides toward 1-Naphthylamines:Applications and Photophysical Properties. J. Am. Chem. Soc. 2019, 141, 2535-2544.

2. (a) Yang, G. H.; Zheng, H. L.; Li, X.; Cheng, J. P. Asymmetric Synthesis of Axially Chiral Phosphamides via Atroposelective N-Allylic Alkylation. ACS Catal. 2020, 10, 2324-2333. (b) Huang, H.; Zhu, H. J.; Kang, Y, J. Regio- and Stereoselective Hydrophosphorylation of Ynamides for the Synthesis of $\beta$-Aminovinylphosphine Oxides. Org. Lett. 2018, 20, 2778-2781. (c) Liu, W. Q.; Lei, T.; Zhou, S.; Yang, L. X.; Li, J.; Chen, B.; Sivaguru, J.; Tung, C. H.; Wu, L. Z. Cobaloxime Catalysis: Selective Synthesis of Alkenylphosphine Oxides under Visible Light. J. Am. Chem. Soc. 2019, 141, 13941-13947.

3. (a) Hu, G. B.; Gao, Y. X.; Zhao, Y. F. Copper-Catalyzed Decarboxylative C-P Cross-Coupling of Alkynyl Acids with $\mathrm{H}^{-}$Phosphine Oxides: A Facile and Selective Synthesis of (E)-1-Alkenylphosphine Oxides. Org. Lett. 2014, 16, 4464-4467. (b) Wang, H. M.; Li, Y. L.; Tang, Z. L.; Wang, S. C.; Zhang, H.; Cong, H. J.; Lei, A. W. Z-Selective Addition of Diaryl Phosphine Oxides to Alkynes via Photoredox Catalysis. ACS Catal. 2018, 8, 10599-10605.

4. Zhang, J. Q.; Chen, T. Q.; Zhang, J. S.; Han, L. B. Silver-Free Direct Synthesis of Alkynylphosphine Oxides via $s p \mathrm{C}-\mathrm{H} / \mathrm{P}(\mathrm{O})-\mathrm{H}$ Dehydrogenative Coupling Catalyzed by Palladium. Org. Lett. 2017, 19, 4692-4695. 
10. ${ }^{1} \mathrm{H},{ }^{13} \mathrm{C}$ and ${ }^{31} \mathrm{P}$ NMR of Compounds $4,5,7,9,10-15$

10-1. NMR spectra for cyanophosphorylation products 4 and 5

${ }^{1} \mathrm{H}$ NMR Spectrum of $4 \mathrm{a}\left(\mathrm{CDCl}_{3}, 400 \mathrm{MHz}\right)$

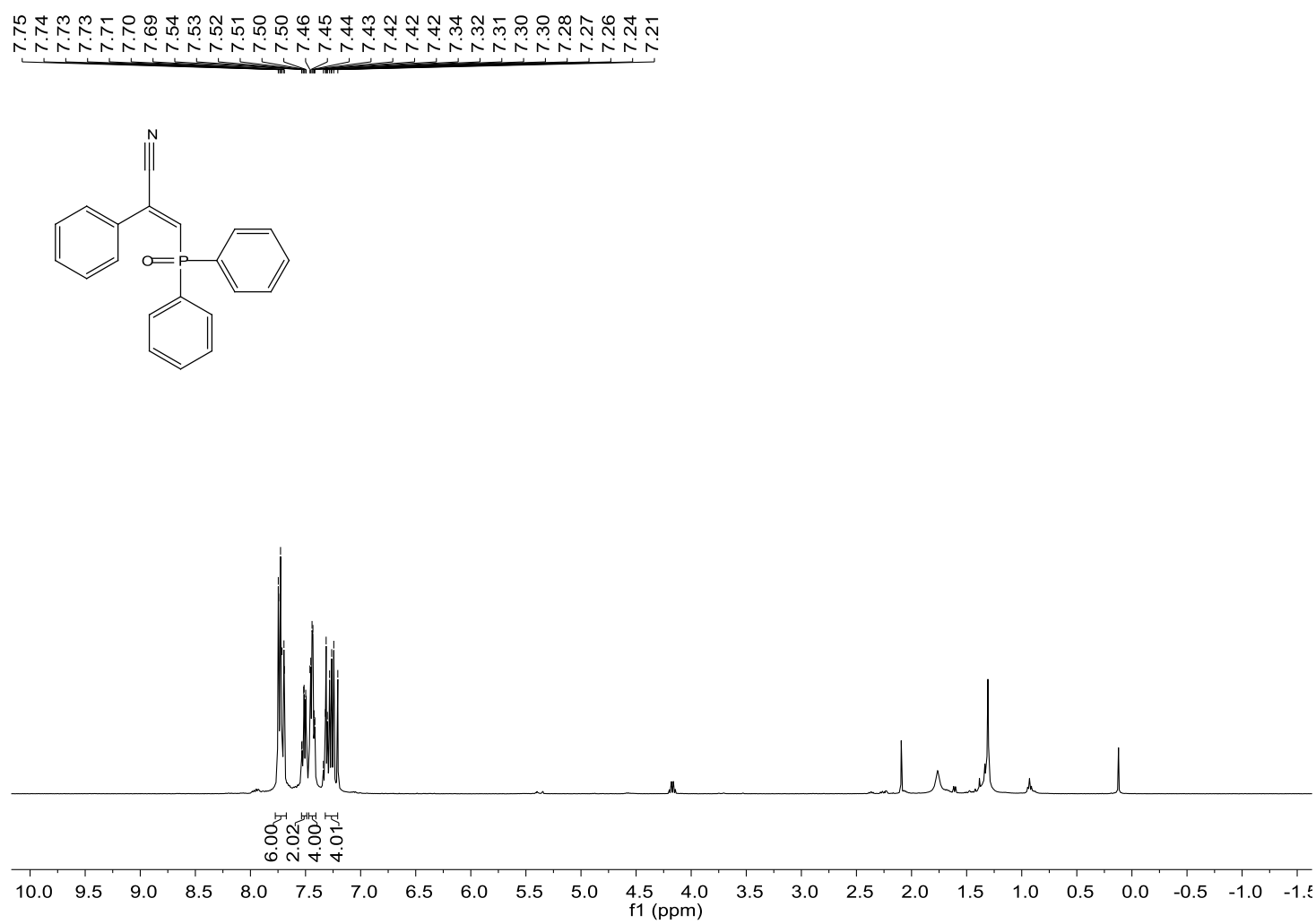

${ }^{31}$ P NMR Spectrum of $4 a\left(\mathrm{CDCl}_{3}, 162 \mathrm{MHz}\right)$

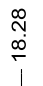<smiles>N#C/C(=C/P(=O)(c1ccccc1)c1ccccc1)c1ccccc1</smiles>

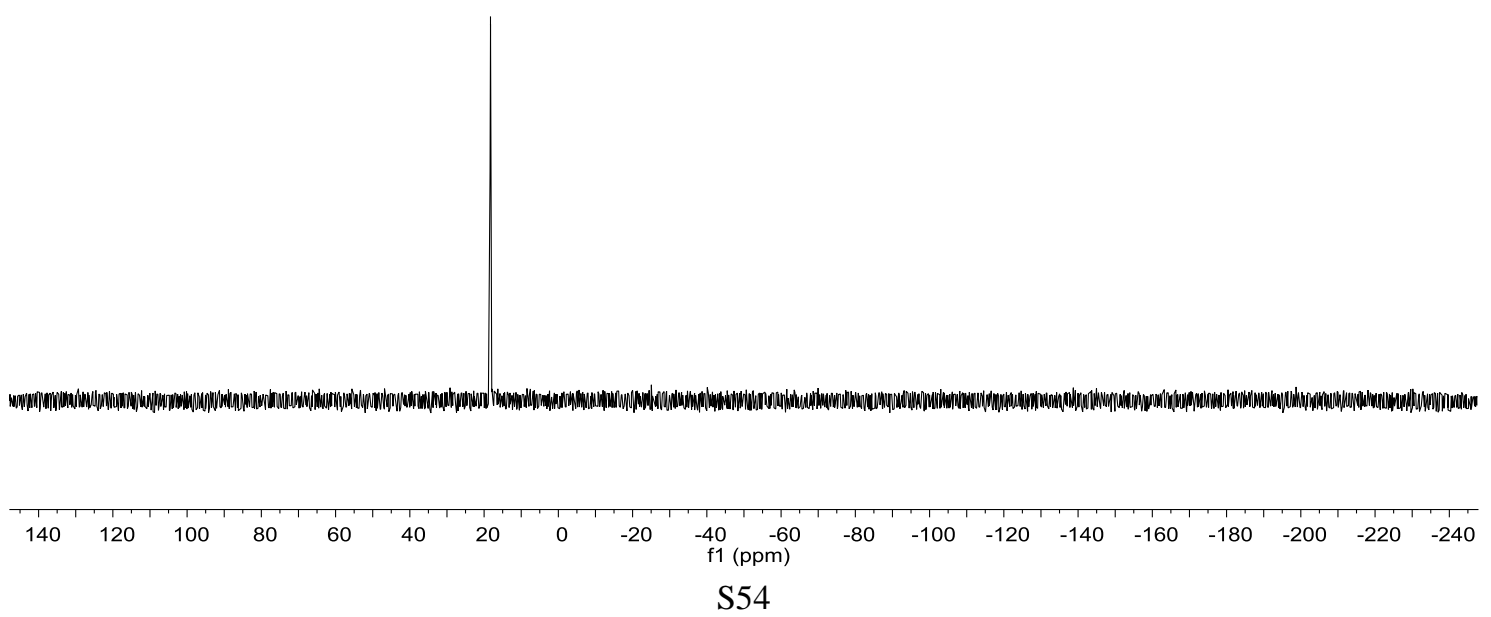


${ }^{13} \mathrm{C}$ NMR Spectrum of $4 \mathrm{a}\left(\mathrm{CDCl}_{3}, 100 \mathrm{MHz}\right)$

m d

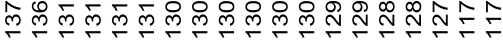<smiles>N#C/C(=C/C(=O)c1ccccc1)c1ccccc1</smiles>

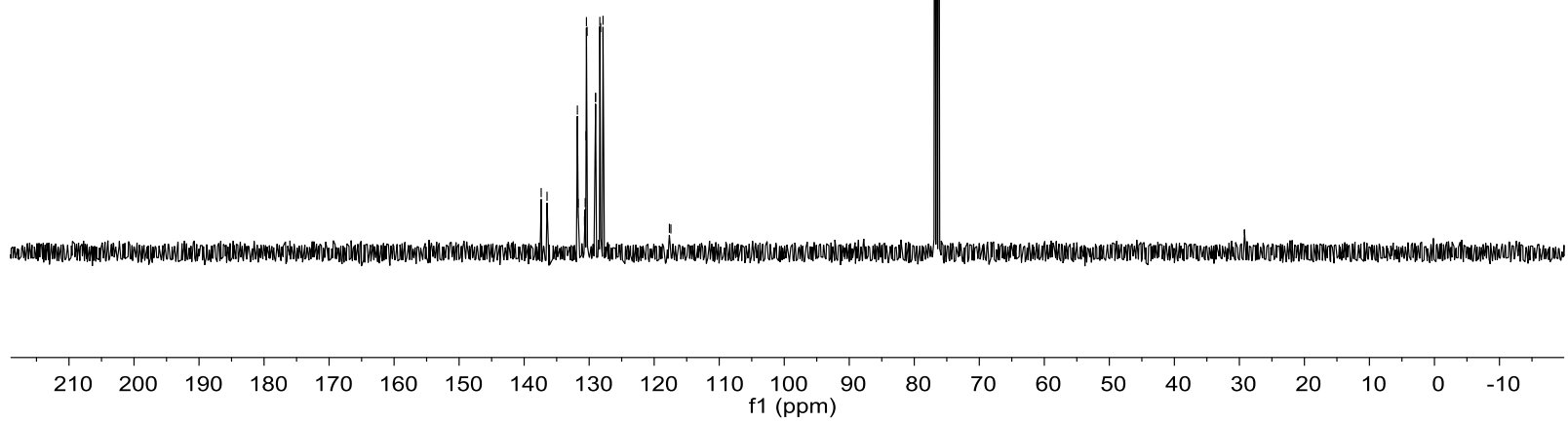

${ }^{1} \mathrm{H}$ NMR Spectrum of $4 \mathrm{a}^{\prime}\left(\mathrm{CDCl}_{3}, 400 \mathrm{MHz}\right)$

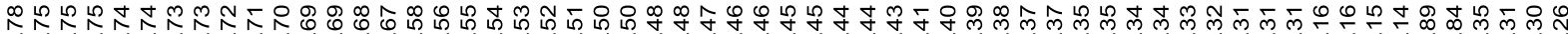

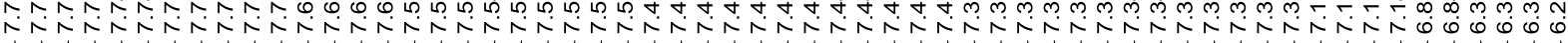<smiles>O=P(/C=C/c1ccccc1)(c1ccccc1)c1ccccc1</smiles>

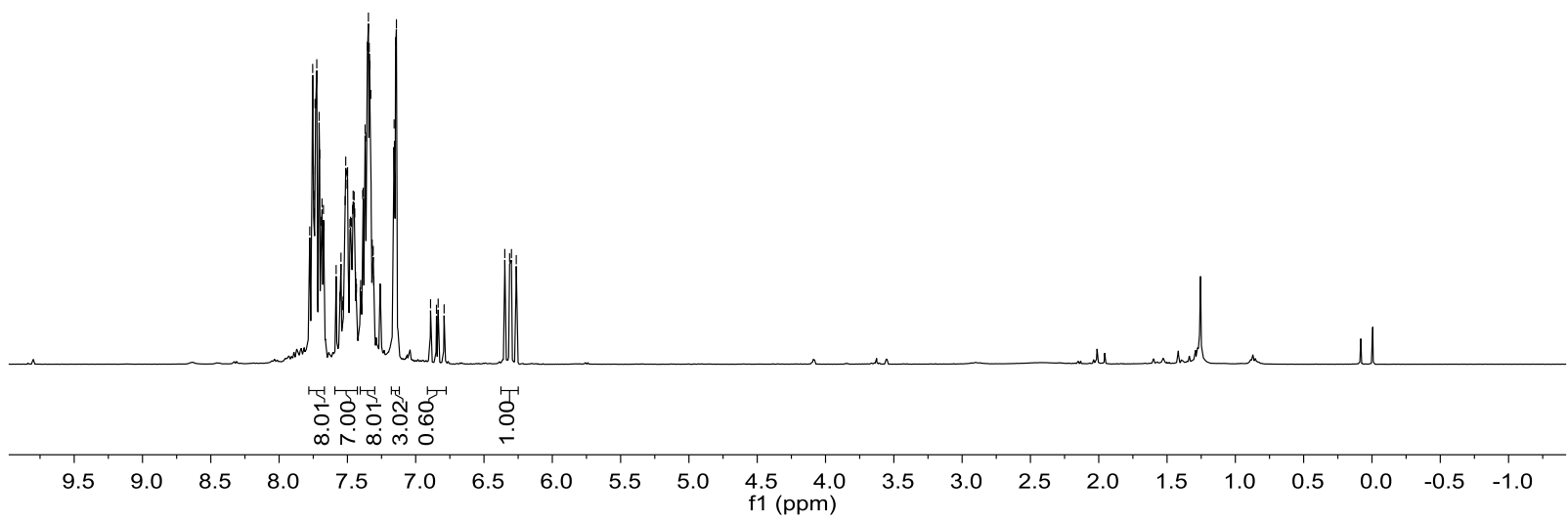



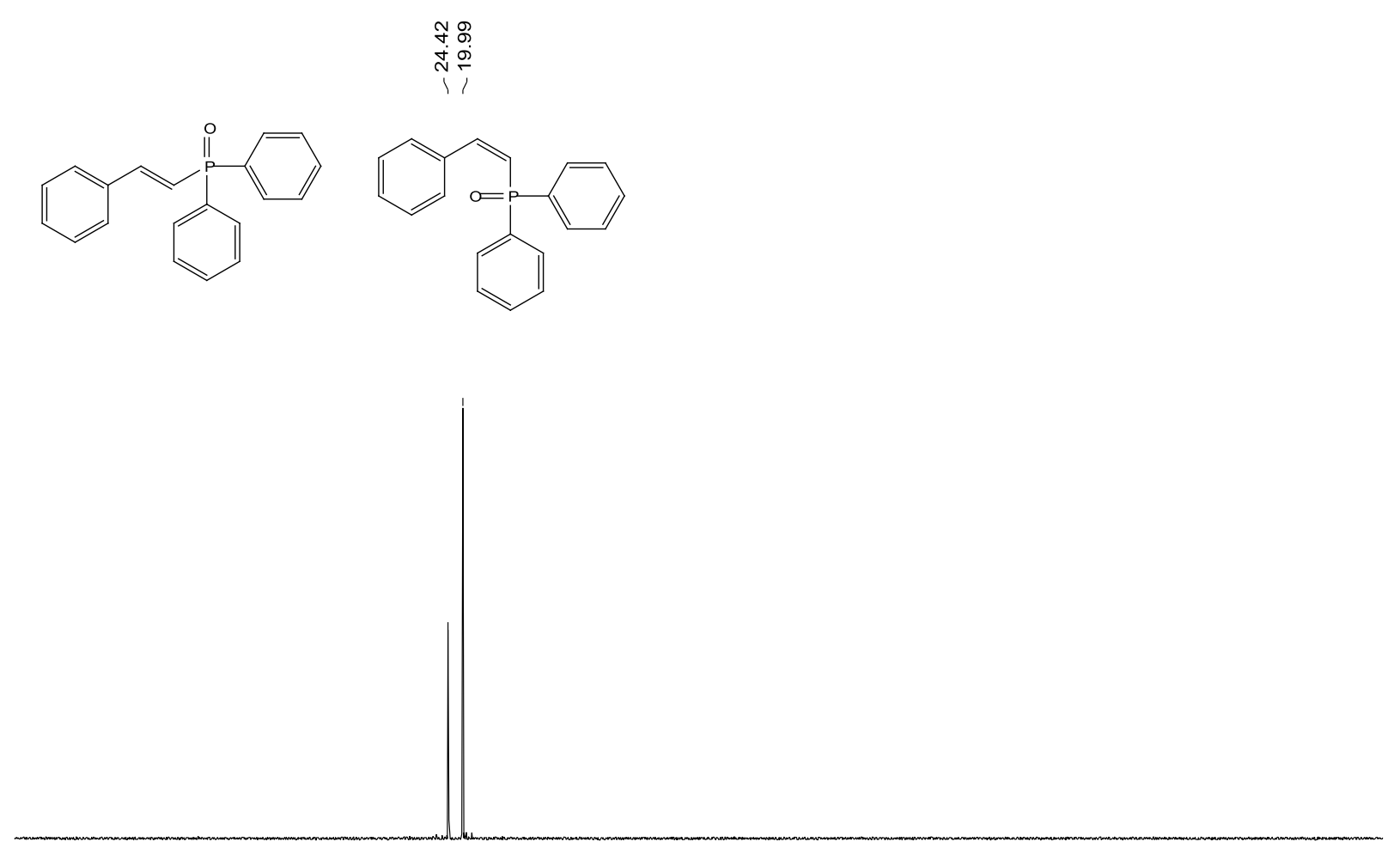

$\begin{array}{llllllllllllllllllllllll}150 & 130 & 110 & 90 & 70 & 50 & 30 & 10 & -10 & -30 & -50 & -70 & -90 & -110 & -130 & -150 & -170 & -190 & -210 & -230 & -25\end{array}$

${ }^{1} \mathrm{H}$ NMR Spectrum of $4 \mathrm{~b}\left(\mathrm{CDCl}_{3}, 400 \mathrm{MHz}\right)$

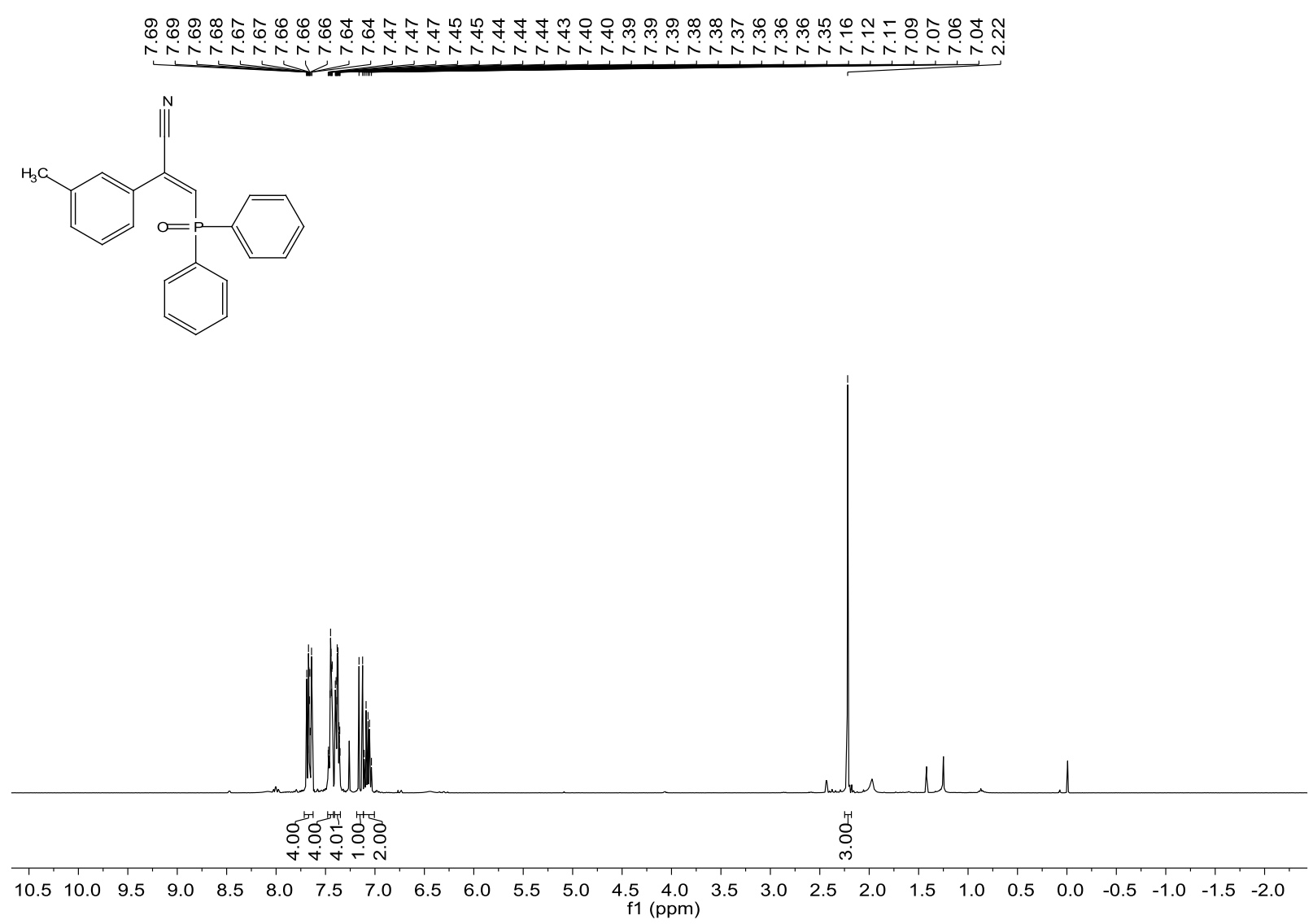


${ }^{31} \mathrm{P}$ NMR Spectrum of $4 \mathrm{~b}\left(\mathrm{CDCl}_{3}, 162 \mathrm{MHz}\right)$

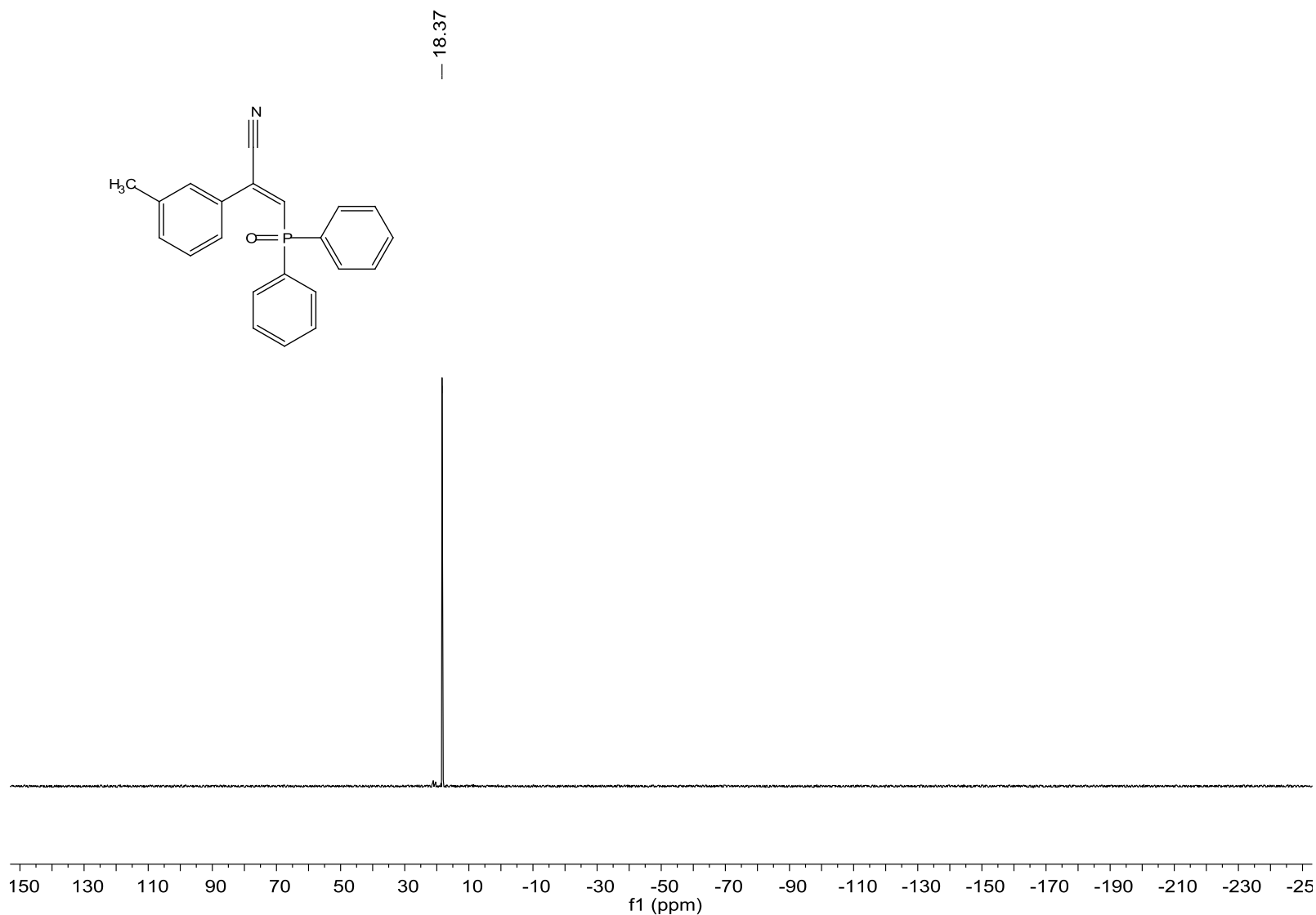

\section{${ }^{13} \mathrm{C}$ NMR Spectrum of $4 \mathrm{~b}\left(\mathrm{CDCl}_{3}, 100 \mathrm{MHz}\right)$}

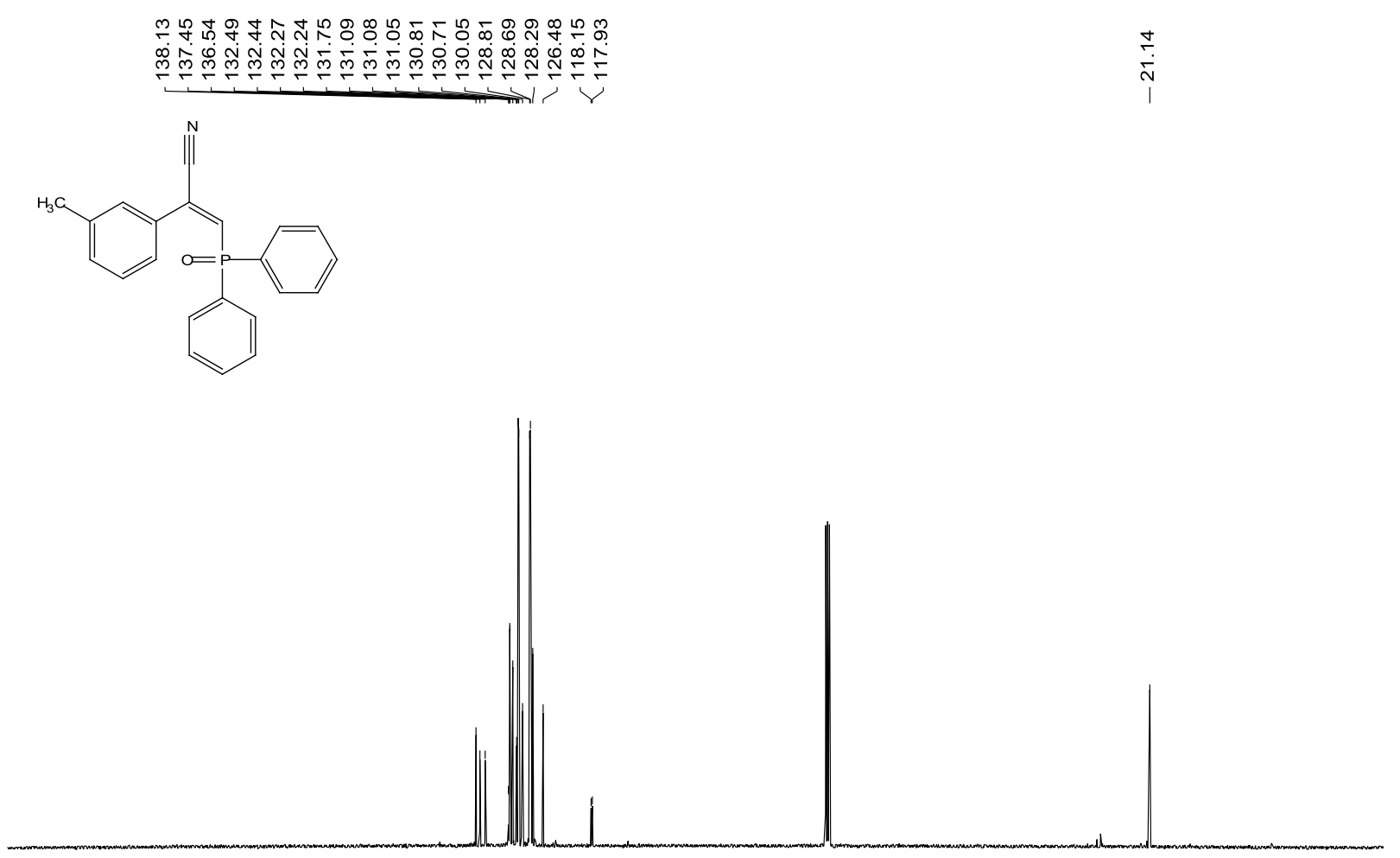

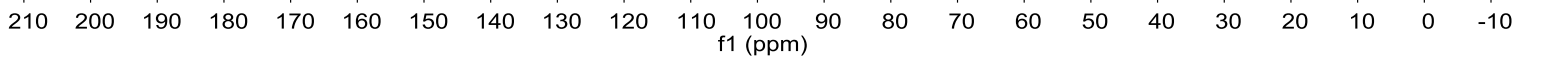




\section{${ }^{1} \mathrm{H}$ NMR Spectrum of $4 \mathrm{c}\left(\mathrm{CDCl}_{3}, 400 \mathrm{MHz}\right)$}

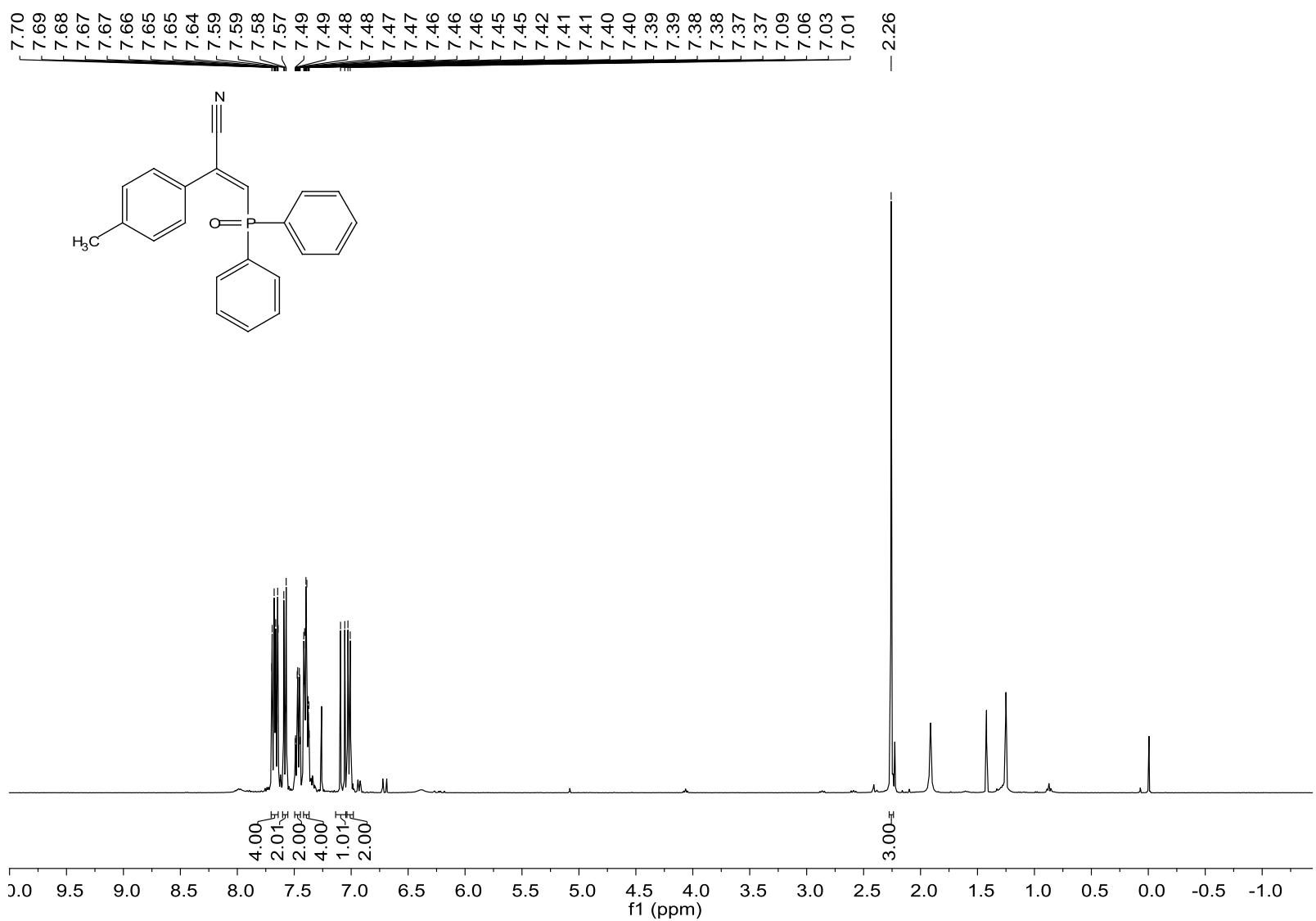

${ }^{31} \mathrm{P}$ NMR Spectrum of $4 \mathrm{c}\left(\mathrm{CDCl}_{3}, 162 \mathrm{MHz}\right)$
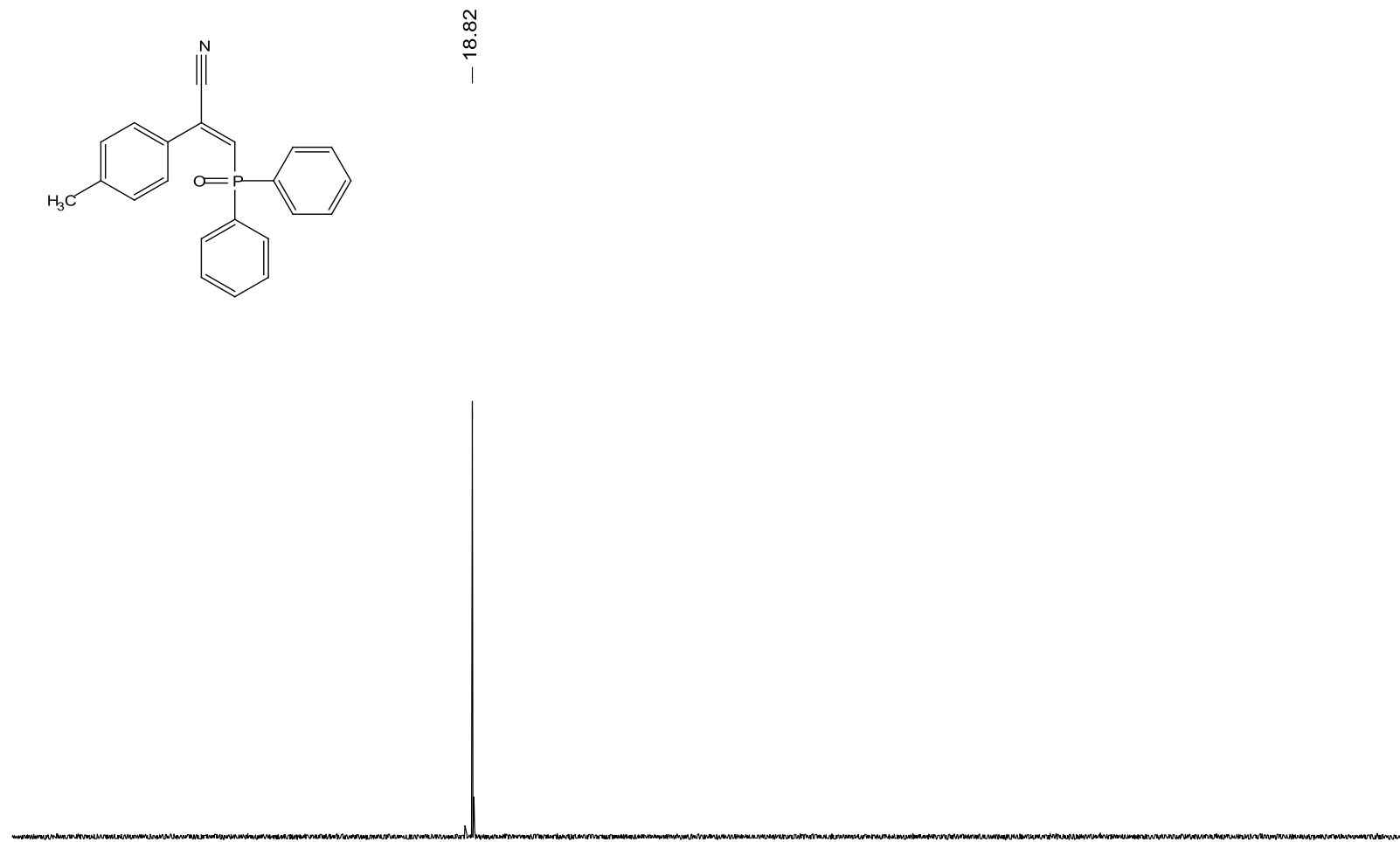

$\begin{array}{llllllllllllllllllllllllll}150 & 130 & 110 & 90 & 70 & 50 & 30 & 10 & -10 & -30 & -50 & -70 & -90 & -110 & -130 & -150 & -170 & -190 & -210 & -230 & -25\end{array}$ 
${ }^{13} \mathrm{C}$ NMR Spectrum of $4 \mathrm{c}\left(\mathrm{CDCl}_{3}, 100 \mathrm{MHz}\right)$

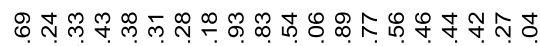

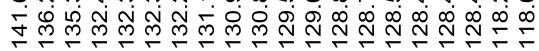<smiles>Cc1ccc(C(C#N)=Cc2ccccc2)cc1</smiles>

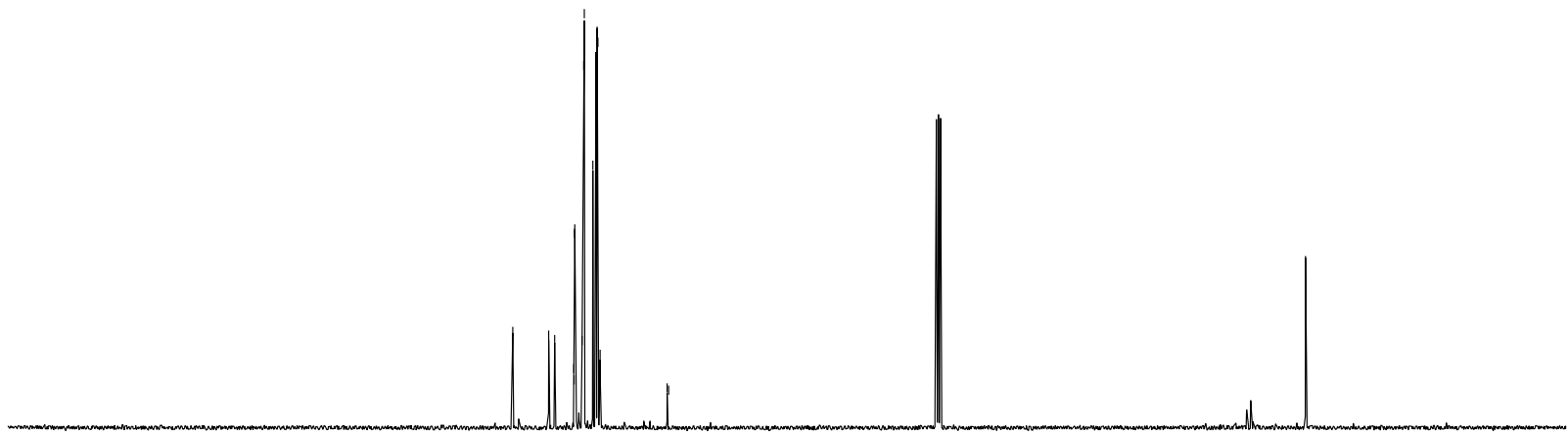

$\begin{array}{lllllllllllllllllllllll}210 & 200 & 190 & 180 & 170 & 160 & 150 & 140 & 130 & 120 & 110 & 100 & 90 & 80 & 70 & 60 & 50 & 40 & 30 & 20 & 10 & 0 & -10\end{array}$

${ }^{1} \mathrm{H}$ NMR Spectrum of $4 \mathrm{~d}\left(\mathrm{CDCl}_{3}, 400 \mathrm{MHz}\right)$

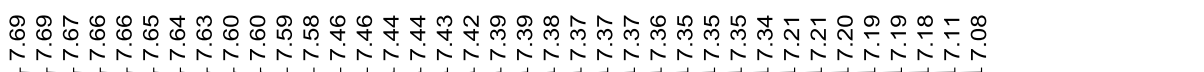<smiles>CC(C)(C)c1ccc(/C(C#N)=C\P(=O)(c2ccccc2)c2ccccc2)cc1</smiles>

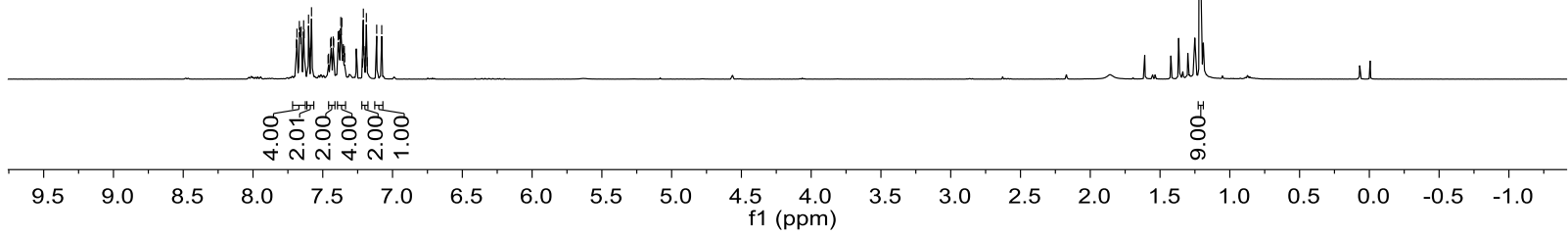


${ }^{31} \mathrm{P}$ NMR Spectrum of $4 \mathrm{~d}\left(\mathrm{CDCl}_{3}, 162 \mathrm{MHz}\right)$<smiles></smiles>

$\stackrel{\substack{\infty \\ \infty}}{+\infty}$

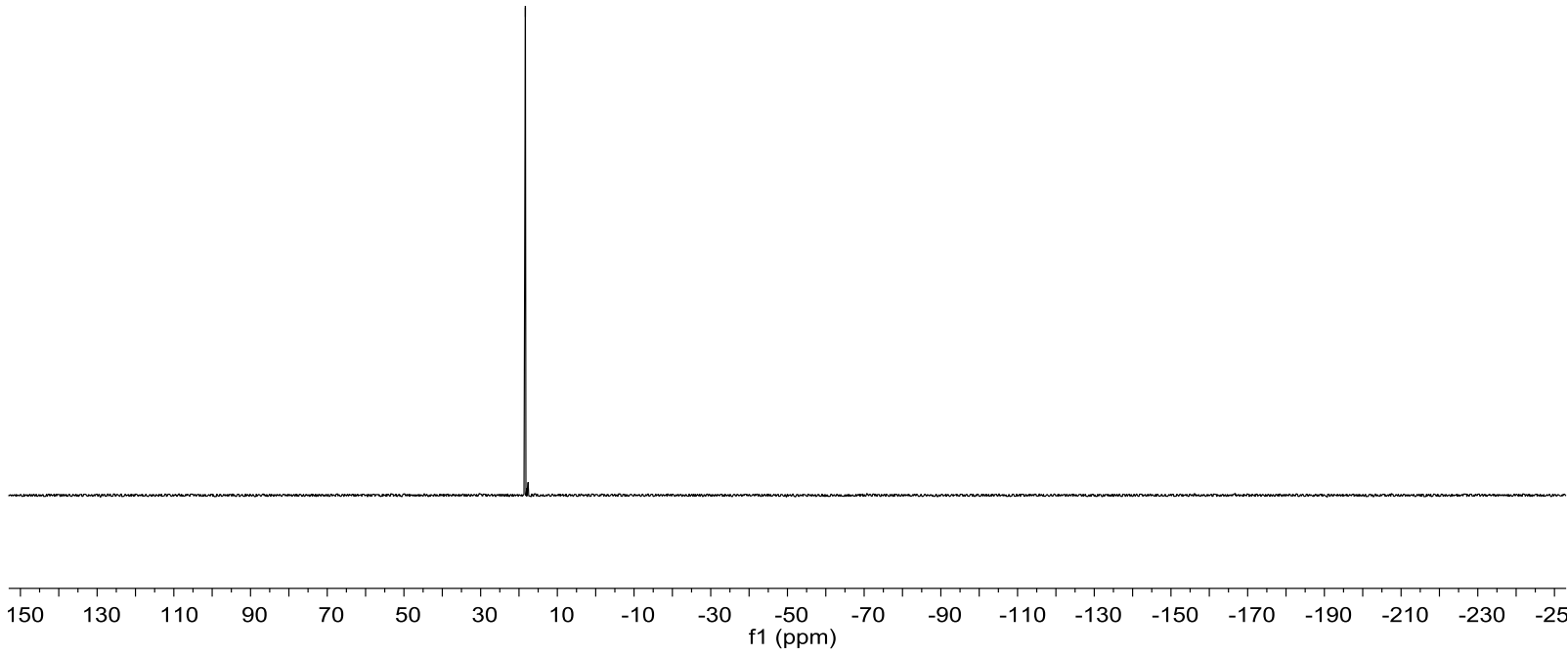

${ }^{13} \mathrm{C}$ NMR Spectrum of $4 \mathrm{~d}\left(\mathrm{CDCl}_{3}, 100 \mathrm{MHz}\right)$

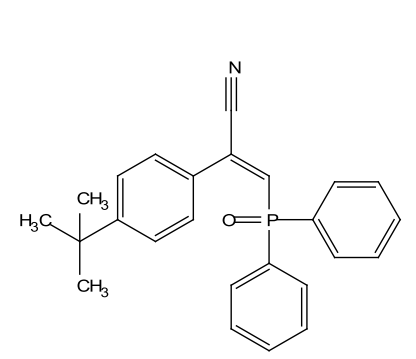

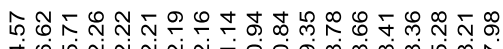

实官官宂

ㅇำ

में

11

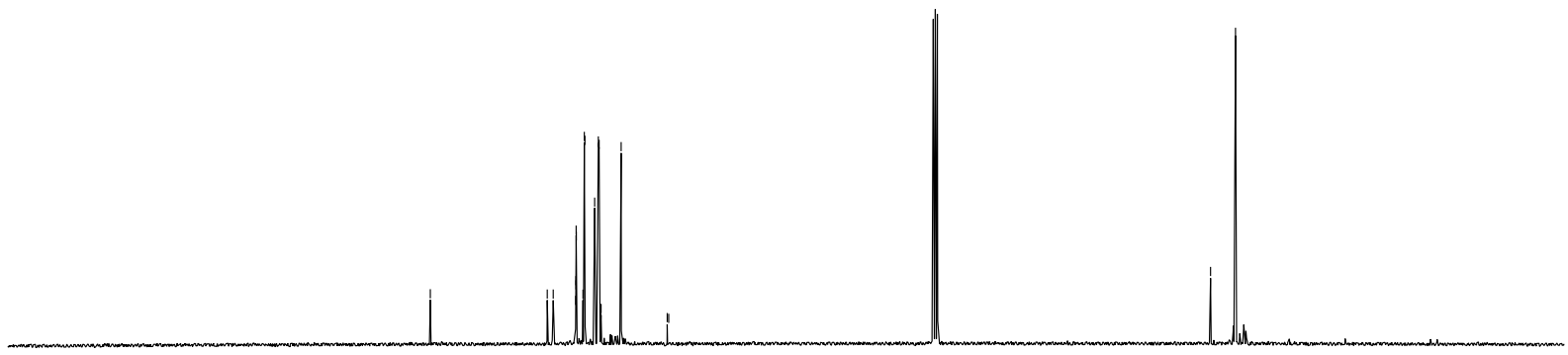

$\begin{array}{lllllllllllllllllllllll}210 & 200 & 190 & 180 & 170 & 160 & 150 & 140 & 130 & 120 & 110 & 100 & 90 & 80 & 70 & 60 & 50 & 40 & 30 & 20 & 10 & 0 & -10\end{array}$ 
${ }^{1} \mathrm{H}$ NMR Spectrum of $4 \mathrm{e}\left(\mathrm{CDCl}_{3}, 400 \mathrm{MHz}\right)$

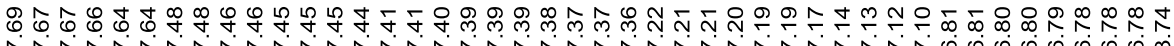

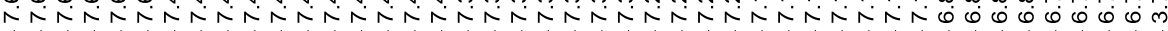

(1)

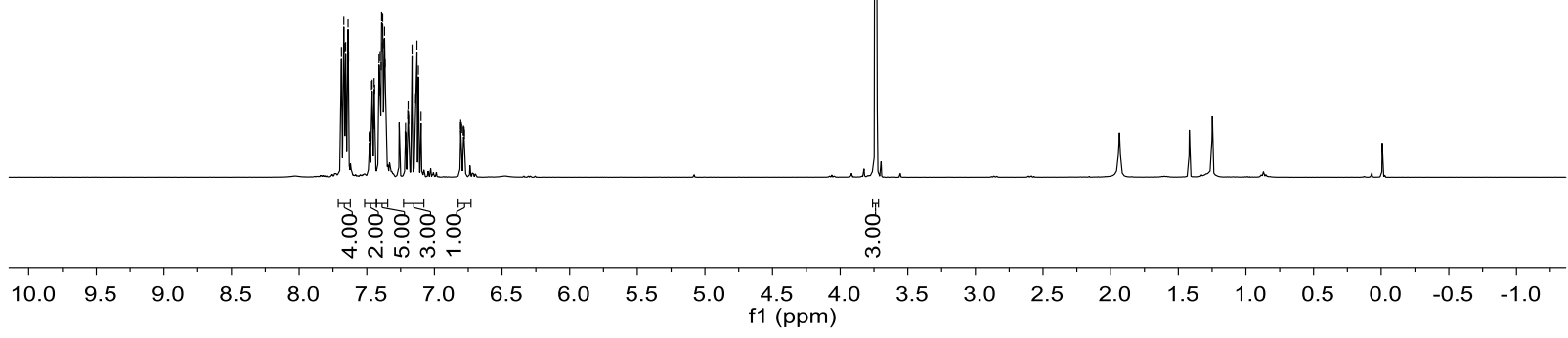

${ }^{31}$ P NMR Spectrum of $4 \mathrm{e}\left(\mathrm{CDCl}_{3}, 162 \mathrm{MHz}\right)$
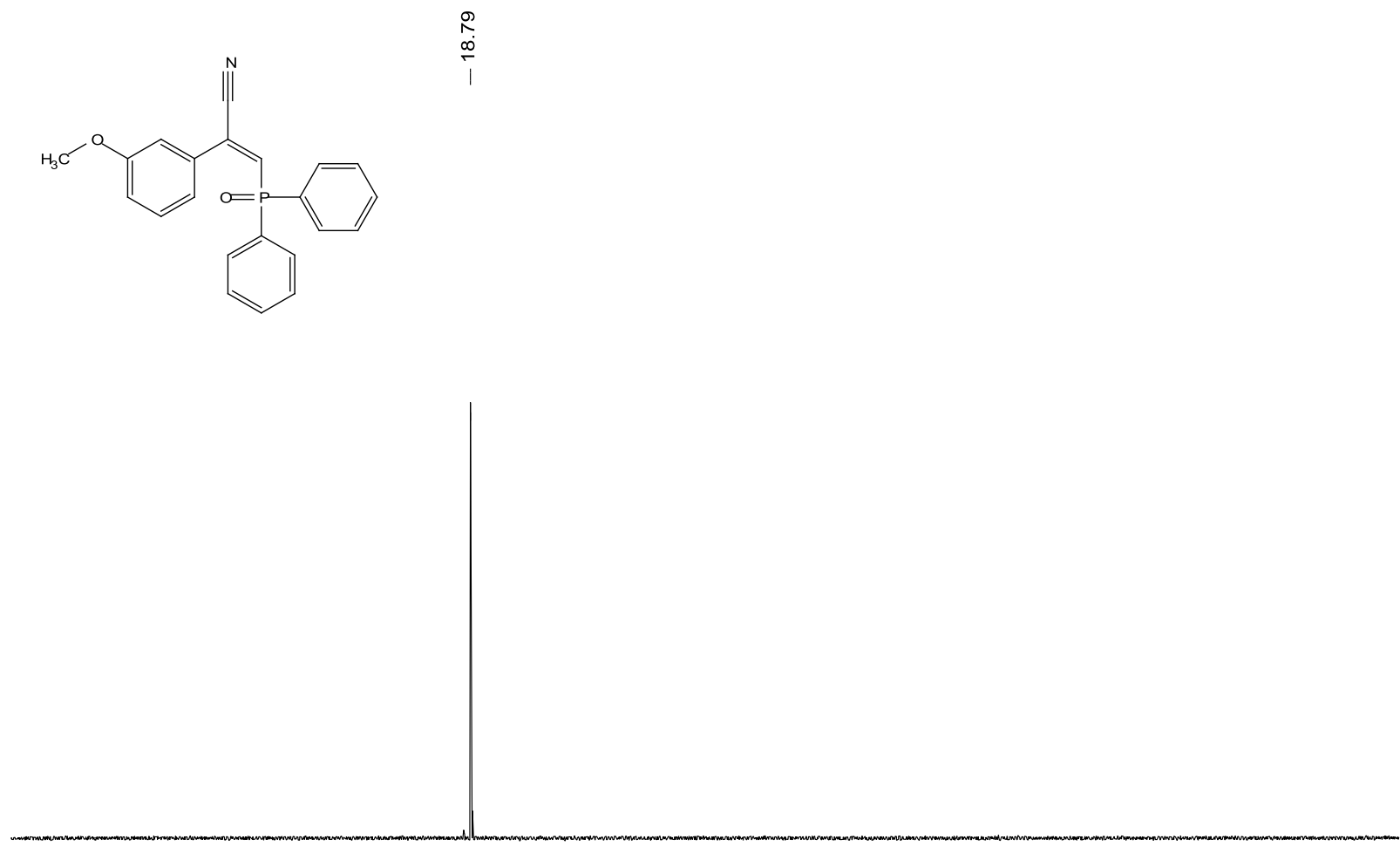

$\begin{array}{lllllllllllllllllllllllllllll}150 & 130 & 110 & 90 & 70 & 50 & 30 & 10 & -10 & -30 & -50 & -70 & -90 & -110 & -130 & -150 & -170 & -190 & -210 & -230 & -25\end{array}$ 
${ }^{13} \mathrm{C}$ NMR Spectrum of $4 \mathrm{e}\left(\mathrm{CDCl}_{3}, 100 \mathrm{MHz}\right)$
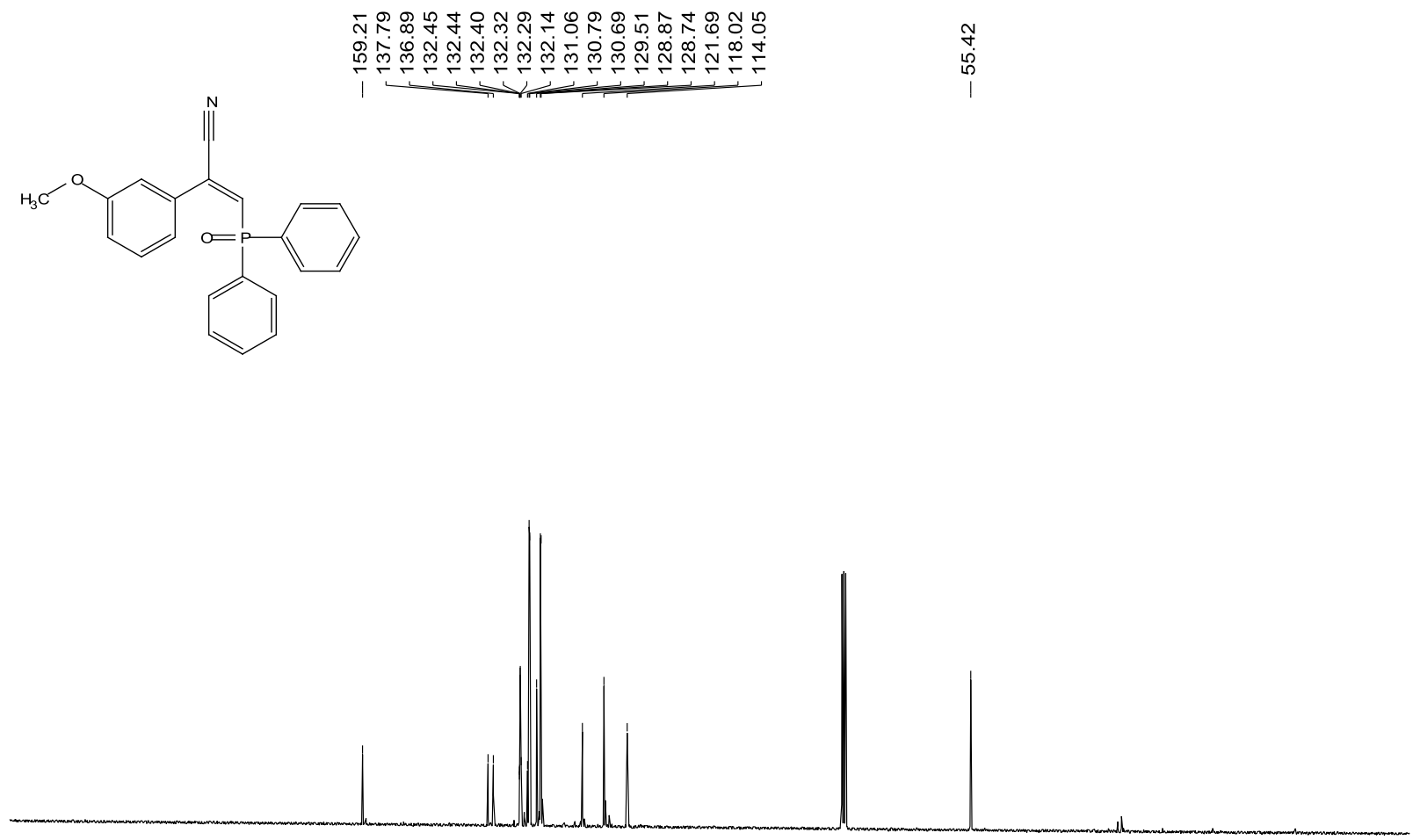

$\begin{array}{lllllllllllllllllllllll}210 & 200 & 190 & 180 & 170 & 160 & 150 & 140 & 130 & 120 & 110 & 100 & 90 & 80 & 70 & 60 & 50 & 40 & 30 & 20 & 10 & 0 & -10\end{array}$

${ }^{1} \mathrm{H}$ NMR Spectrum of $4 \mathrm{f}\left(\mathrm{CDCl}_{3}, 400 \mathrm{MHz}\right)$

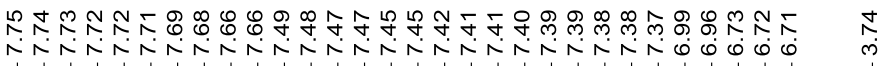<smiles>N#CC(=Cc1ccccc1)c1ccccc1</smiles>

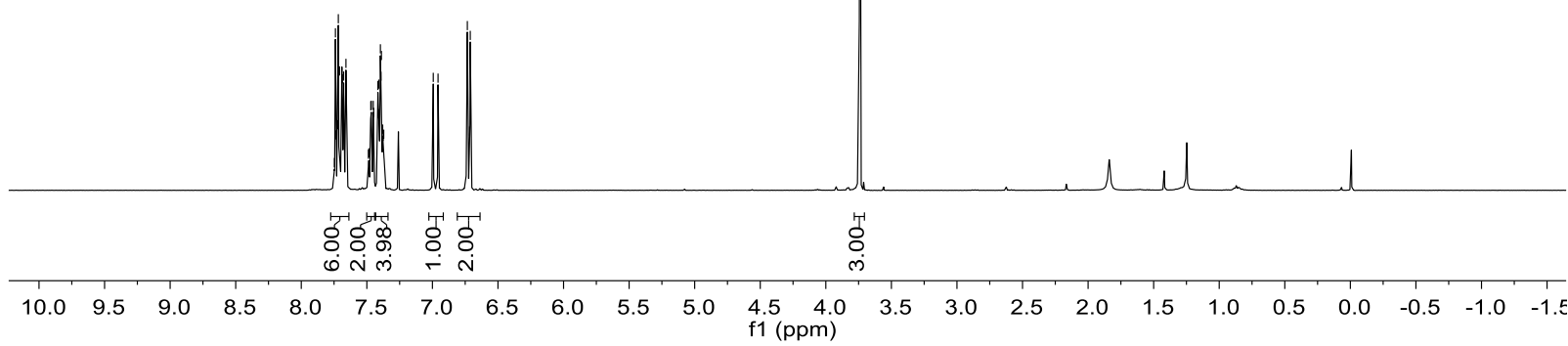


${ }^{31} \mathrm{P}$ NMR Spectrum of $4 \mathrm{f}\left(\mathrm{CDCl}_{3}, 162 \mathrm{MHz}\right)$
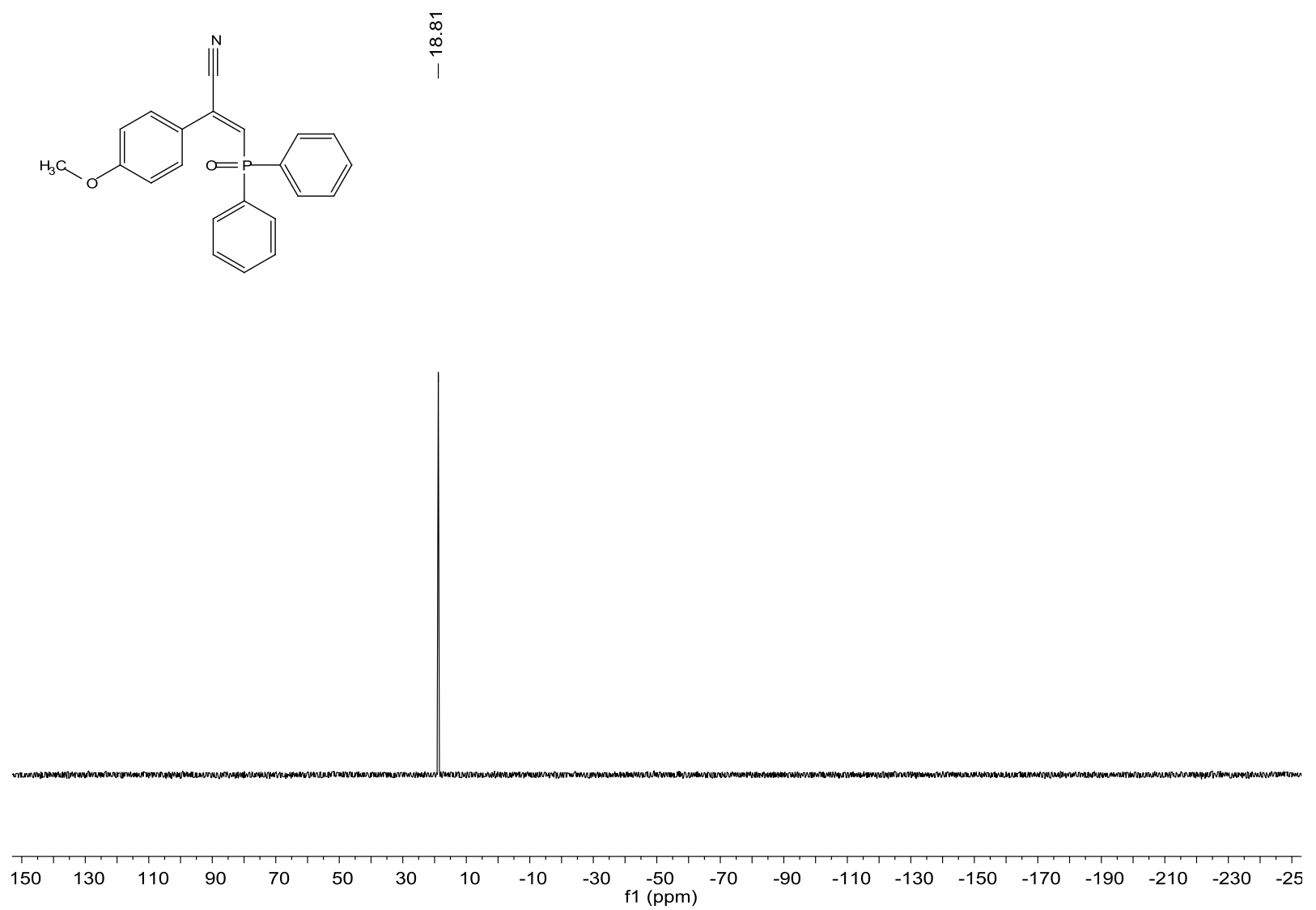

${ }^{13} \mathrm{C}$ NMR Spectrum of $4 \mathrm{f}\left(\mathrm{CDCl}_{3}, 100 \mathrm{MHz}\right)$
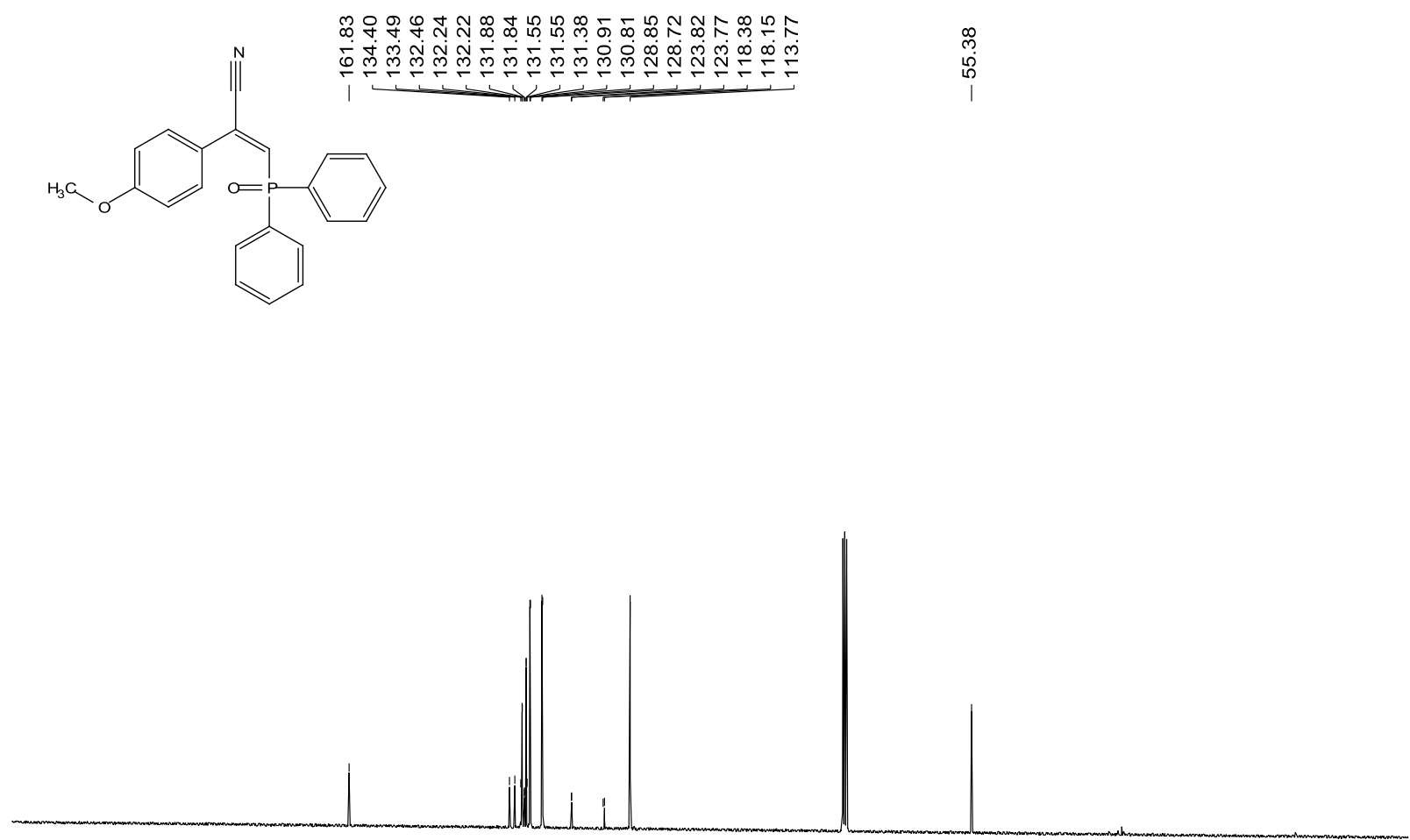

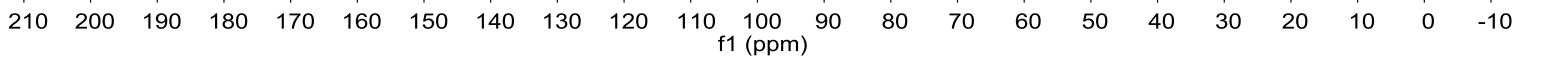


${ }^{1} \mathrm{H}$ NMR Spectrum of $4 \mathrm{~g}\left(\mathrm{CDCl}_{3}, 400 \mathrm{MHz}\right)$
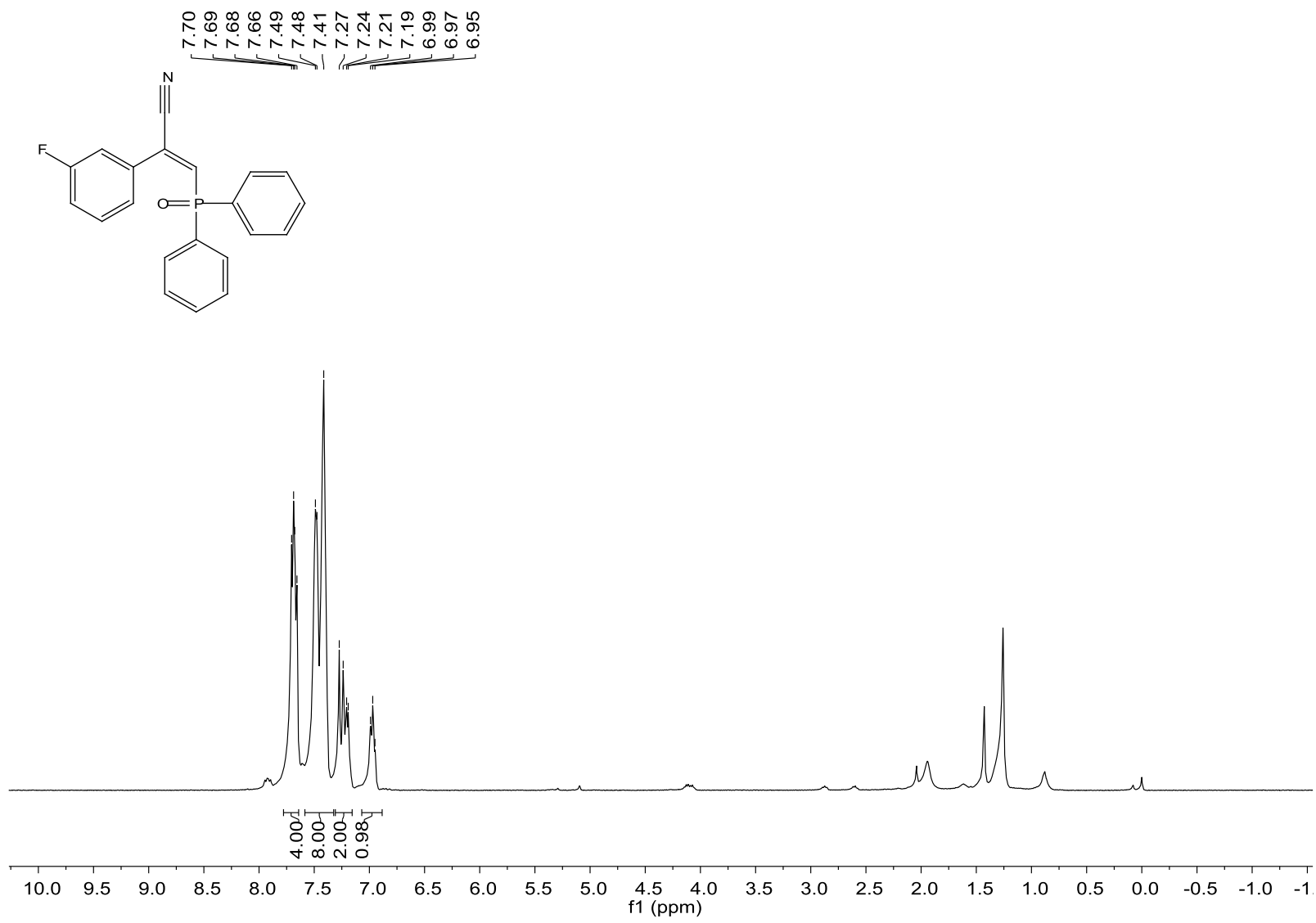

${ }^{31}$ P NMR Spectrum of $4 \mathrm{~g}\left(\mathrm{CDCl}_{3}, 162 \mathrm{MHz}\right)$
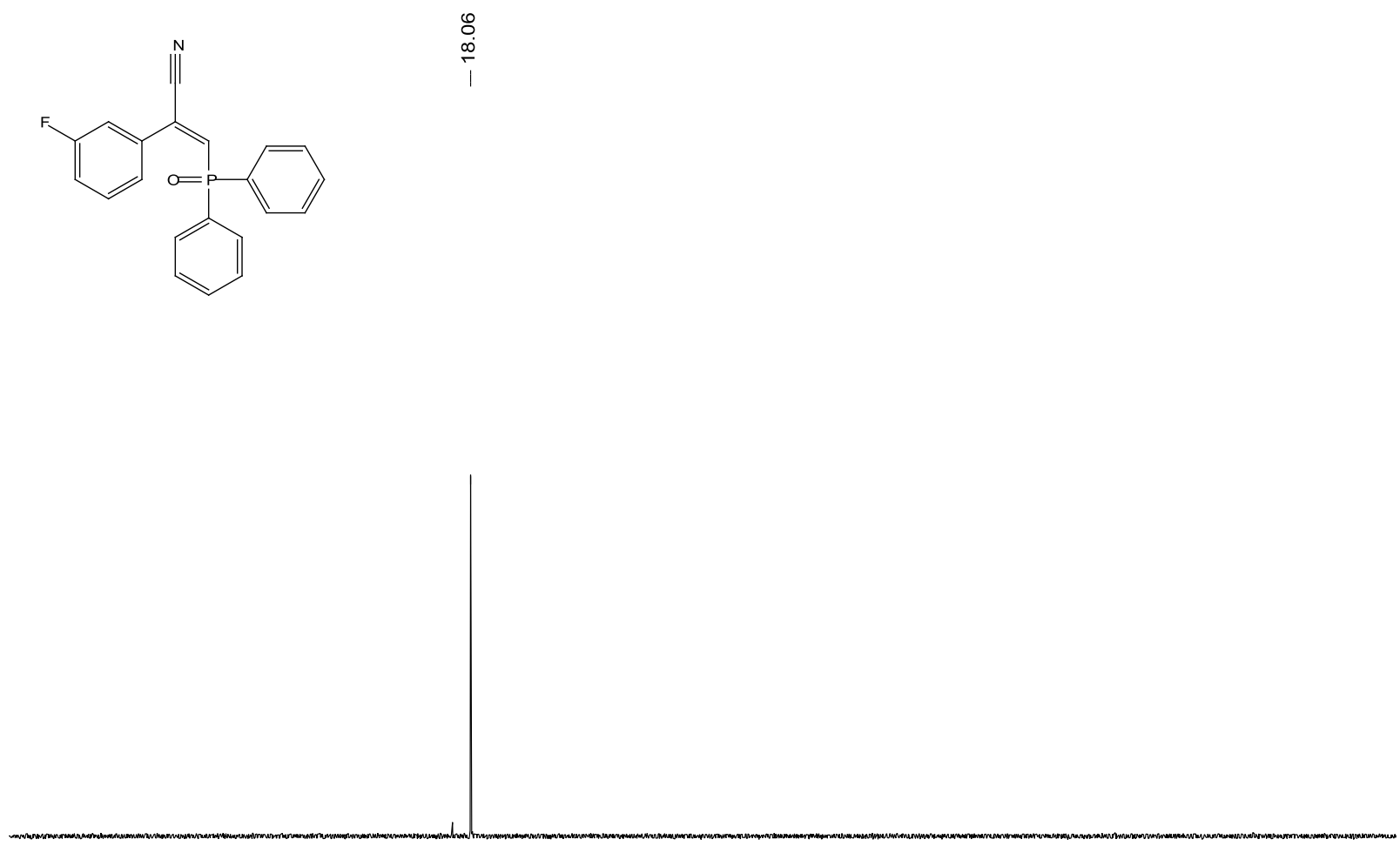

$\begin{array}{lllllllllllllllllllllll}150 & 130 & 110 & 90 & 70 & 50 & 30 & 10 & -10 & -30 & -50 & -70 & -90 & -110 & -130 & -150 & -170 & -190 & -210 & -230 & -25\end{array}$ 
${ }^{13} \mathrm{C}$ NMR Spectrum of $4 \mathrm{~g}\left(\mathrm{CDCl}_{3}, 100 \mathrm{MHz}\right)$

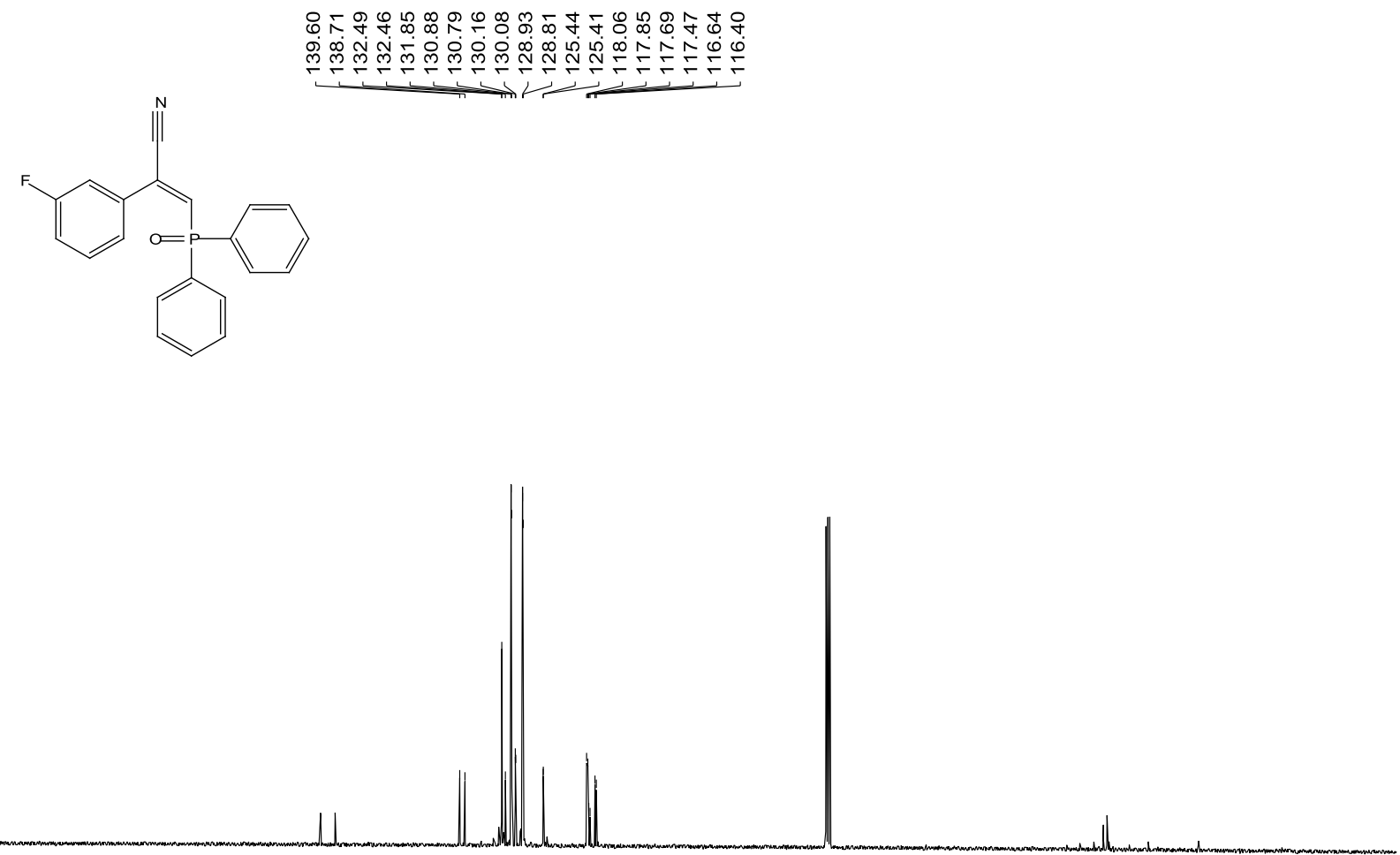

$\begin{array}{llllllllllllllllllllllll}210 & 200 & 190 & 180 & 170 & 160 & 150 & 140 & 130 & 120 & 110 & 100 & 90 & 80 & 70 & 60 & 50 & 40 & 30 & 20 & 10 & 0 & -10\end{array}$

${ }^{1} \mathrm{H}$ NMR Spectrum of $4 \mathrm{~h}\left(\mathrm{CDCl}_{3}, 400 \mathrm{MHz}\right)$

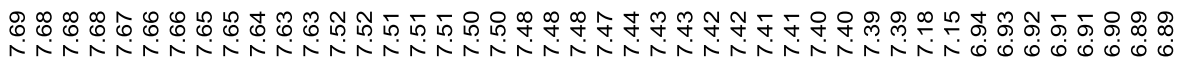<smiles>N#CC(=Cc1ccc(F)cc1)c1ccccc1</smiles>

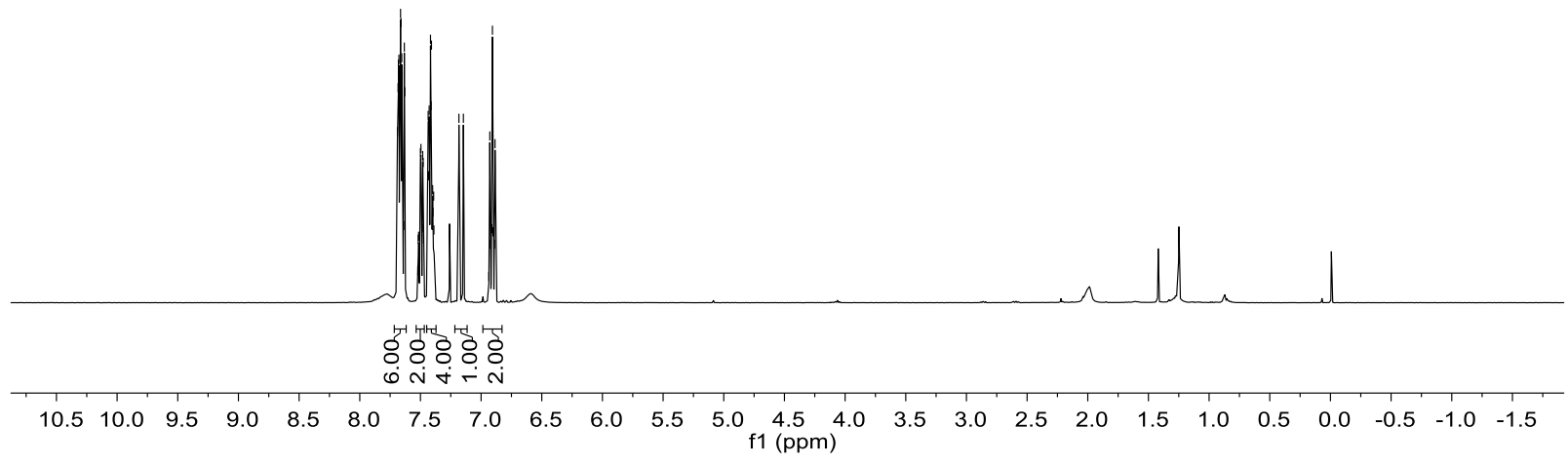


${ }^{31} \mathrm{P}$ NMR Spectrum of $4 \mathrm{~h}\left(\mathrm{CDCl}_{3}, 162 \mathrm{MHz}\right)$<smiles>N#C/C(=C/P(=O)(c1ccccc1)c1ccccc1)c1ccc(F)cc1</smiles>

$\stackrel{\frac{6}{6}}{\frac{1}{6}}$

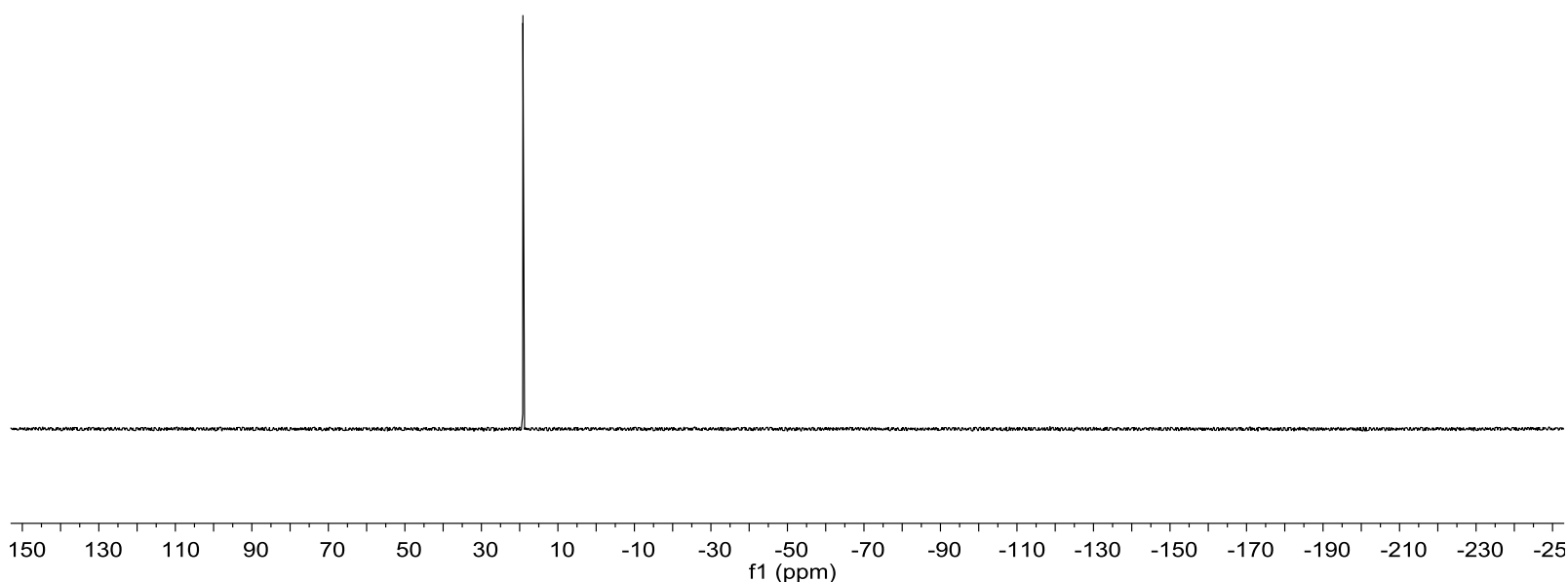

${ }^{13} \mathrm{C}$ NMR Spectrum of $4 \mathrm{~h}\left(\mathrm{CDCl}_{3}, 100 \mathrm{MHz}\right)$

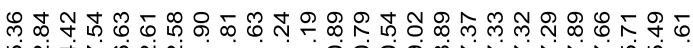

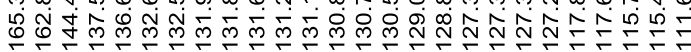
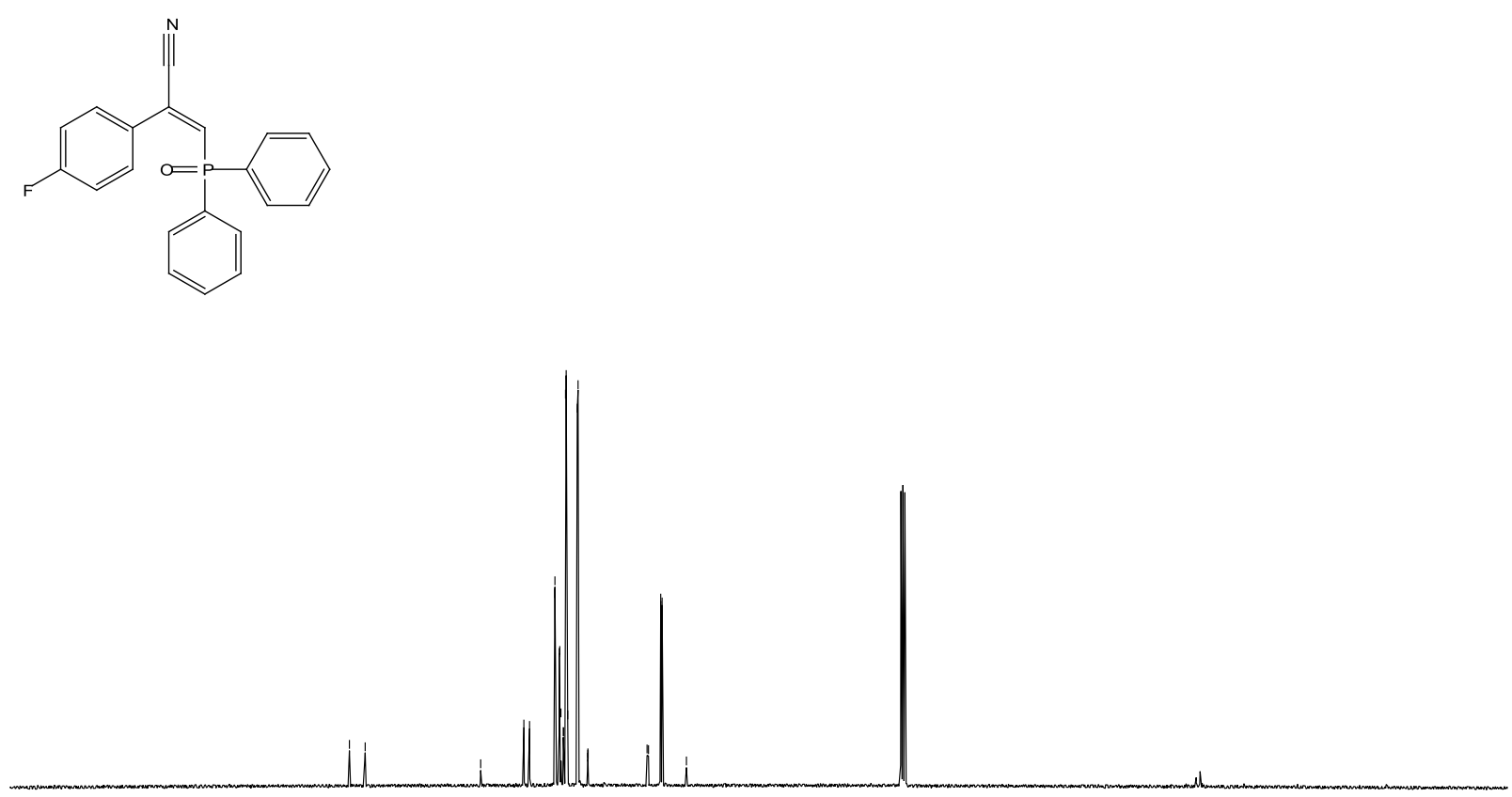

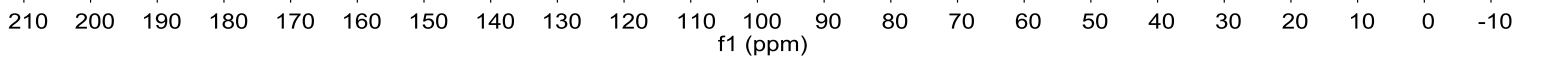


${ }^{1} \mathrm{H}$ NMR Spectrum of $4 \mathrm{i}\left(\mathrm{CDCl}_{3}, 400 \mathrm{MHz}\right)$

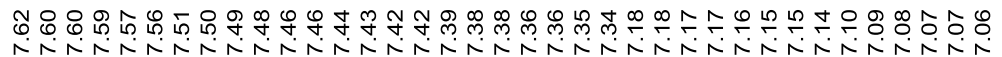

(1)

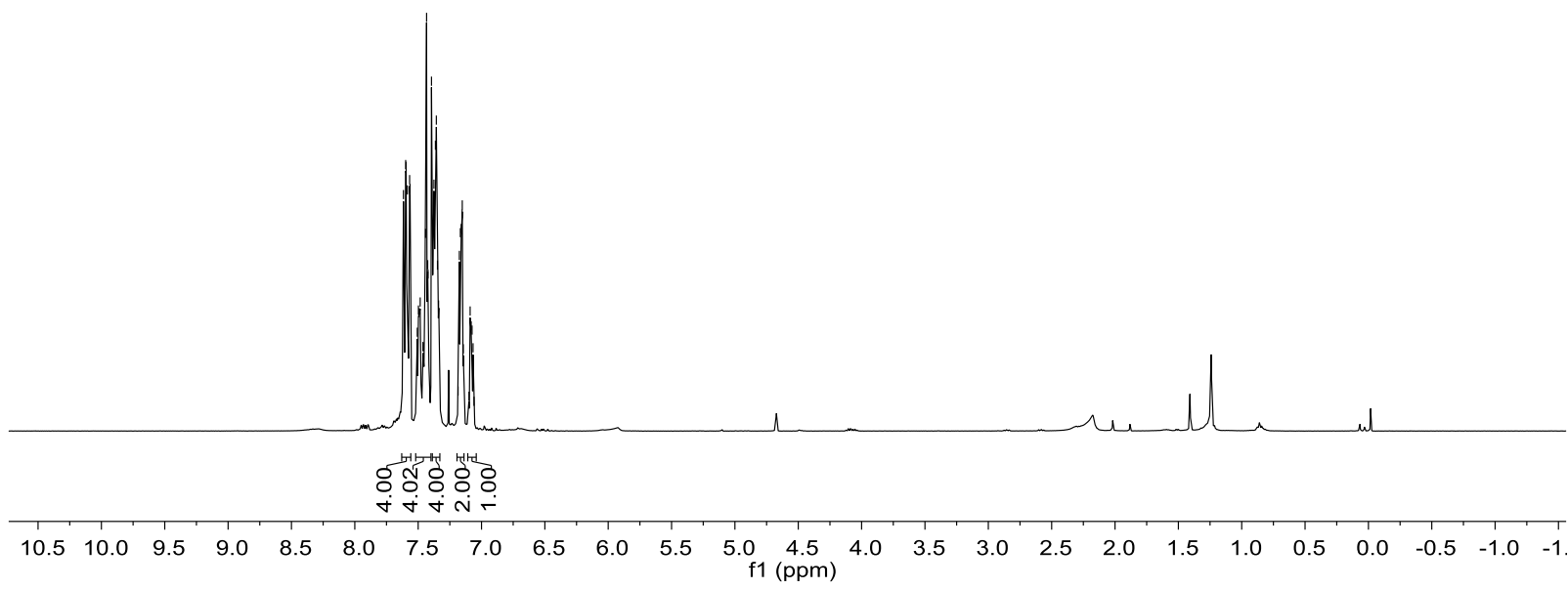

${ }^{31} \mathrm{P}$ NMR Spectrum of $4 \mathrm{i}\left(\mathrm{CDCl}_{3}, 162 \mathrm{MHz}\right)$

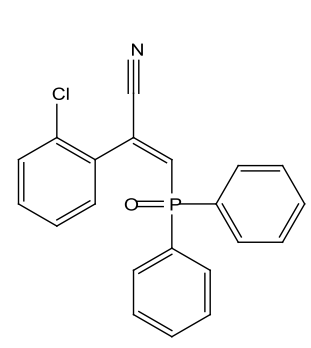

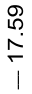

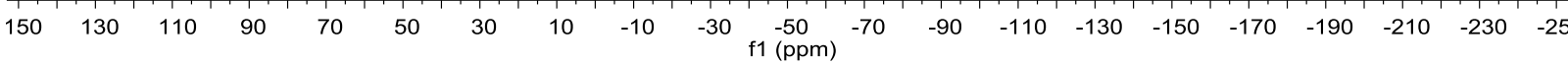




\section{${ }^{13} \mathrm{C}$ NMR Spectrum of $4 \mathrm{i}\left(\mathrm{CDCl}_{3}, 100 \mathrm{MHz}\right)$}

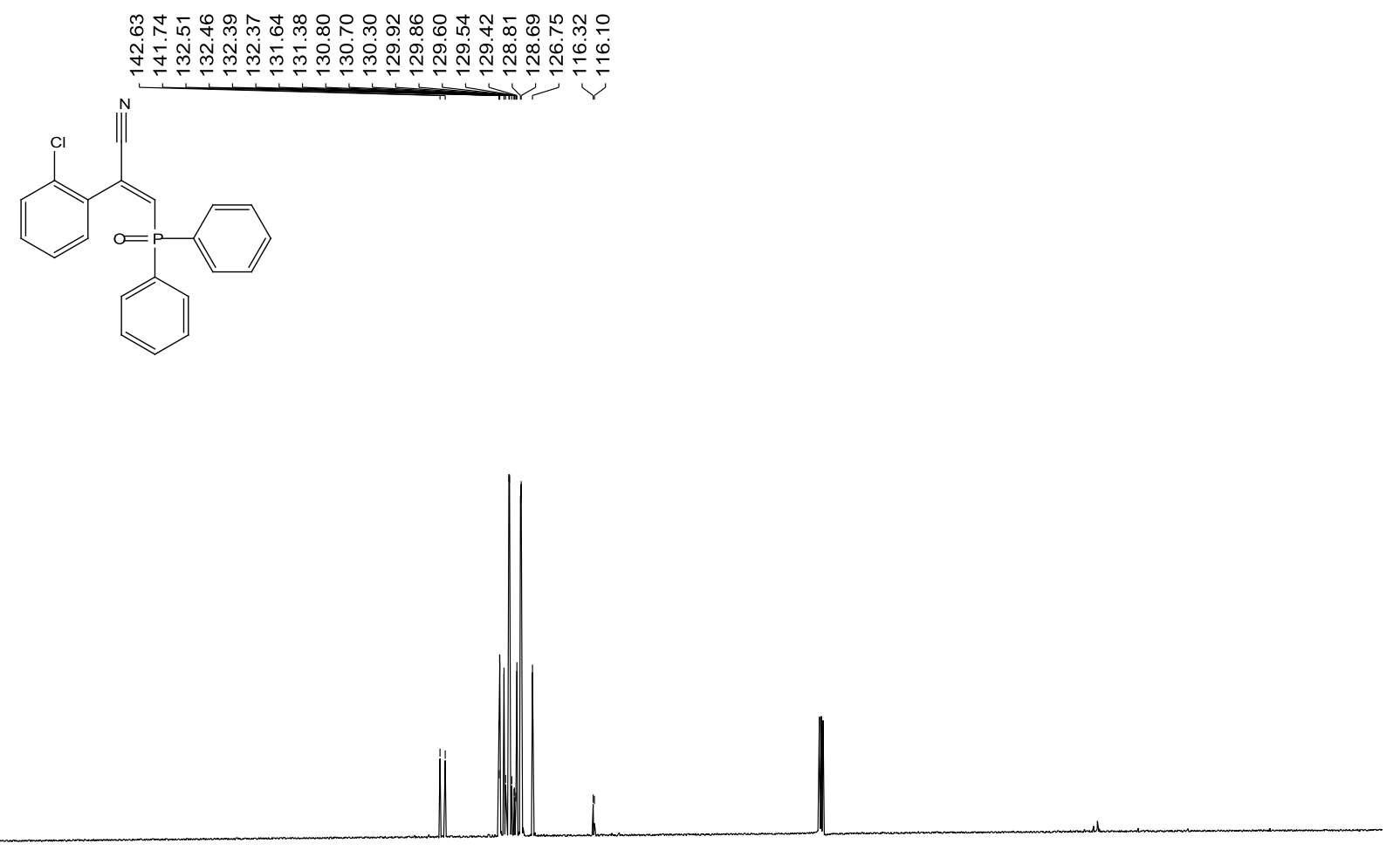

$\begin{array}{lllllllllllllllllllllllll}210 & 200 & 190 & 180 & 170 & 160 & 150 & 140 & 130 & 120 & 110 & 100 & 90 & 80 & 70 & 60 & 50 & 40 & 30 & 20 & 10 & 0 & -10\end{array}$

${ }^{1} \mathrm{H}$ NMR Spectrum of $4 \mathbf{j}\left(\mathrm{CDCl}_{3}, 400 \mathrm{MHz}\right)$

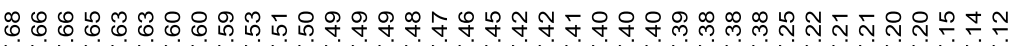

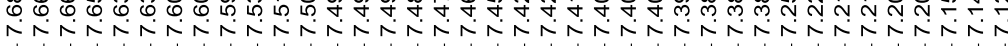
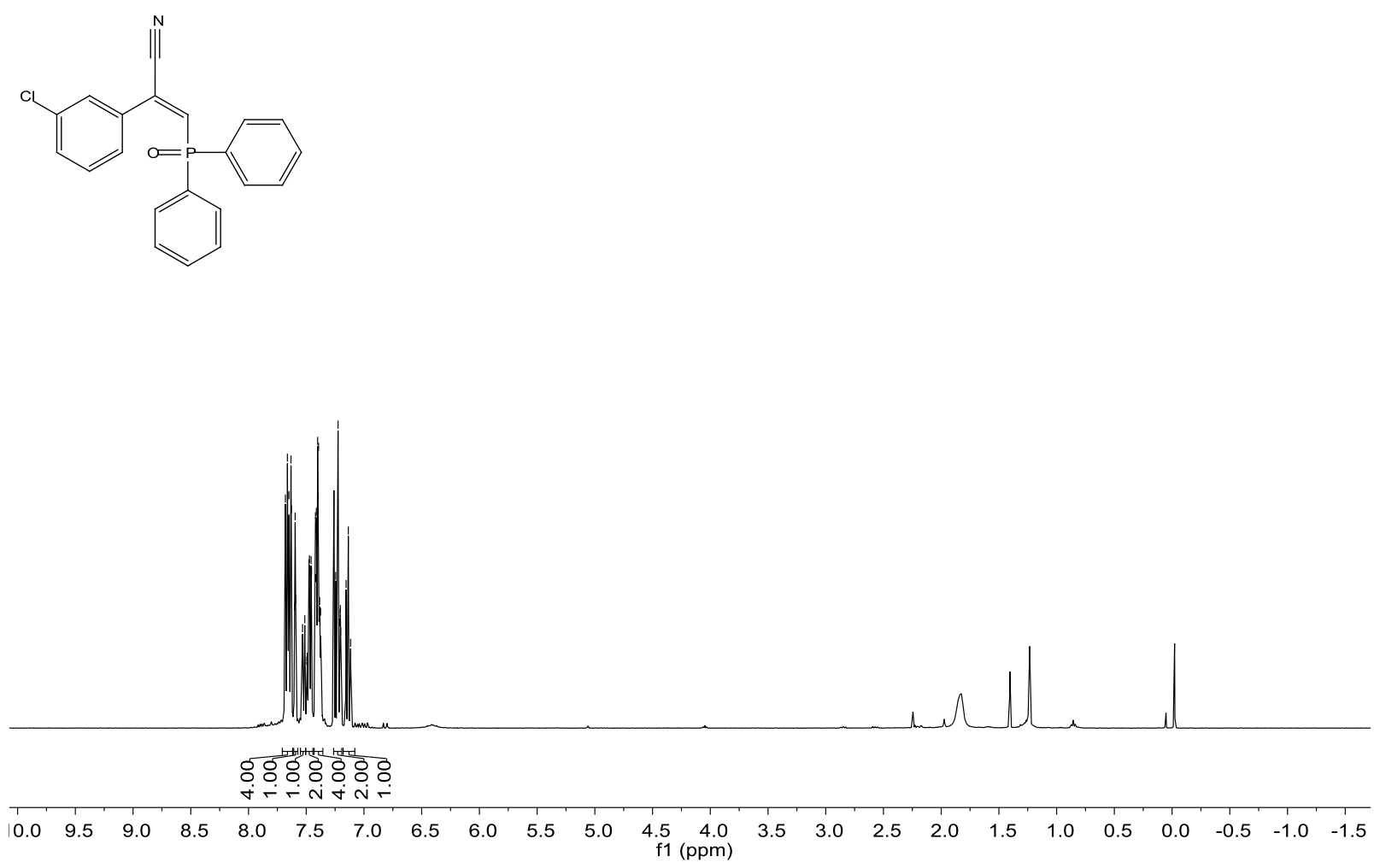
${ }^{31} \mathrm{P}$ NMR Spectrum of $4 \mathrm{j}\left(\mathrm{CDCl}_{3}, 162 \mathrm{MHz}\right)$

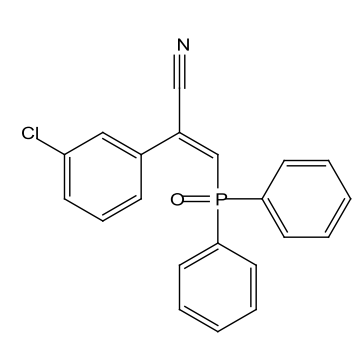

$\stackrel{\frac{\hbar}{\infty}}{\stackrel{\infty}{\prime}}$

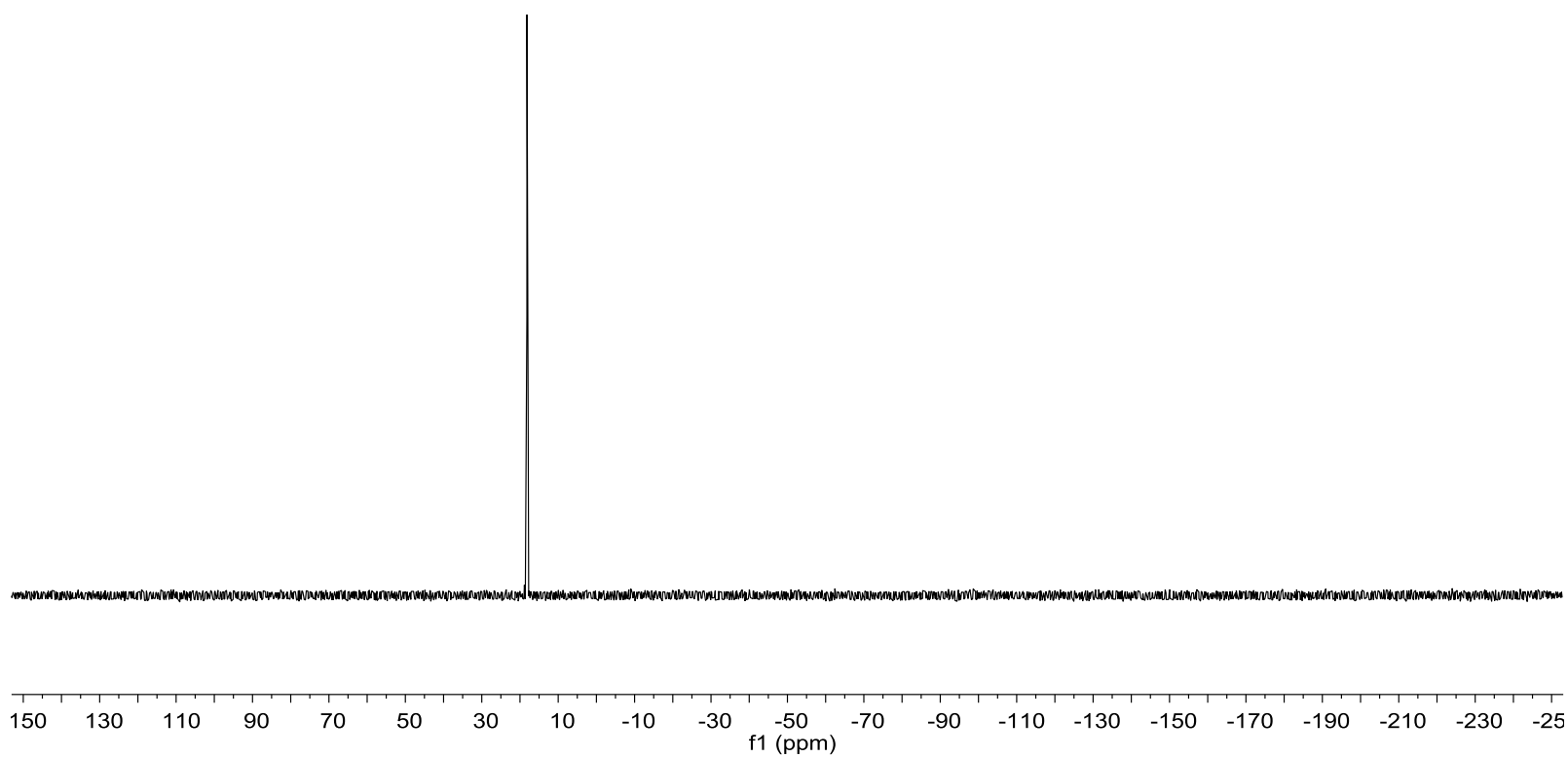

${ }^{13} \mathrm{C}$ NMR Spectrum of $4 \mathrm{j}\left(\mathrm{CDCl}_{3}, 100 \mathrm{MHz}\right)$

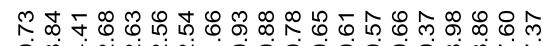

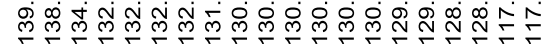

(1)

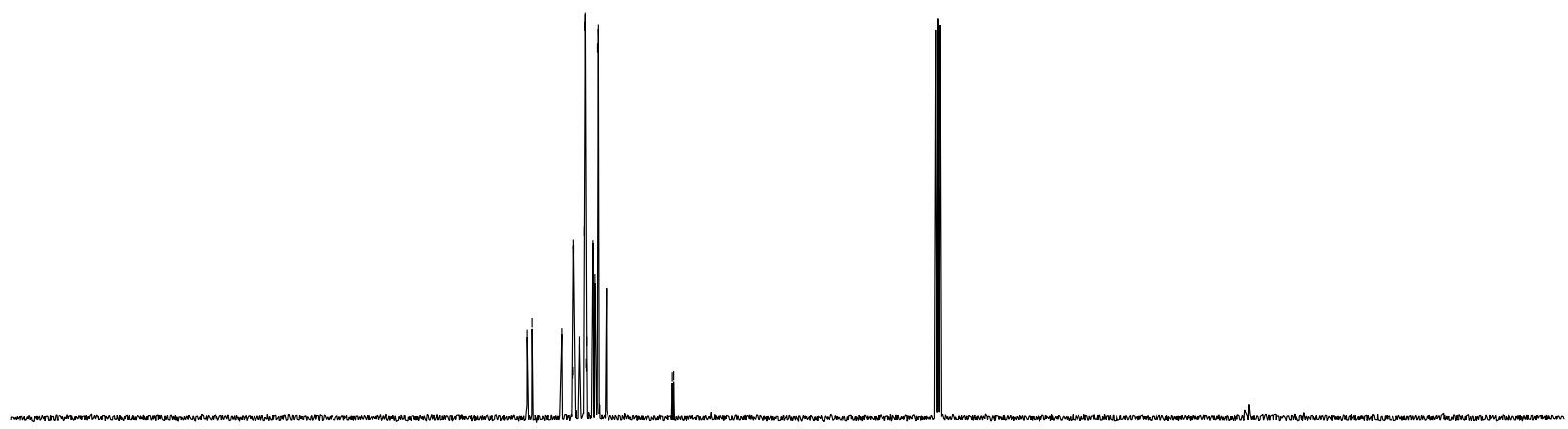

$\begin{array}{llllllllllllllllllllllll}210 & 200 & 190 & 180 & 170 & 160 & 150 & 140 & 130 & 120 & 110 & 100 & 90 & 80 & 70 & 60 & 50 & 40 & 30 & 20 & 10 & 0 & -10\end{array}$ 
${ }^{1} \mathrm{H}$ NMR Spectrum of $4 \mathrm{k}\left(\mathrm{CDCl}_{3}, 400 \mathrm{MHz}\right)$

ஓㅇำ

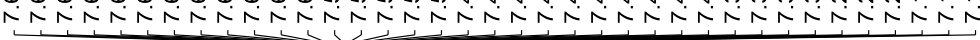<smiles>N#C/C(=C/P(=O)(c1ccccc1)c1ccccc1)c1ccc(Cl)cc1</smiles>

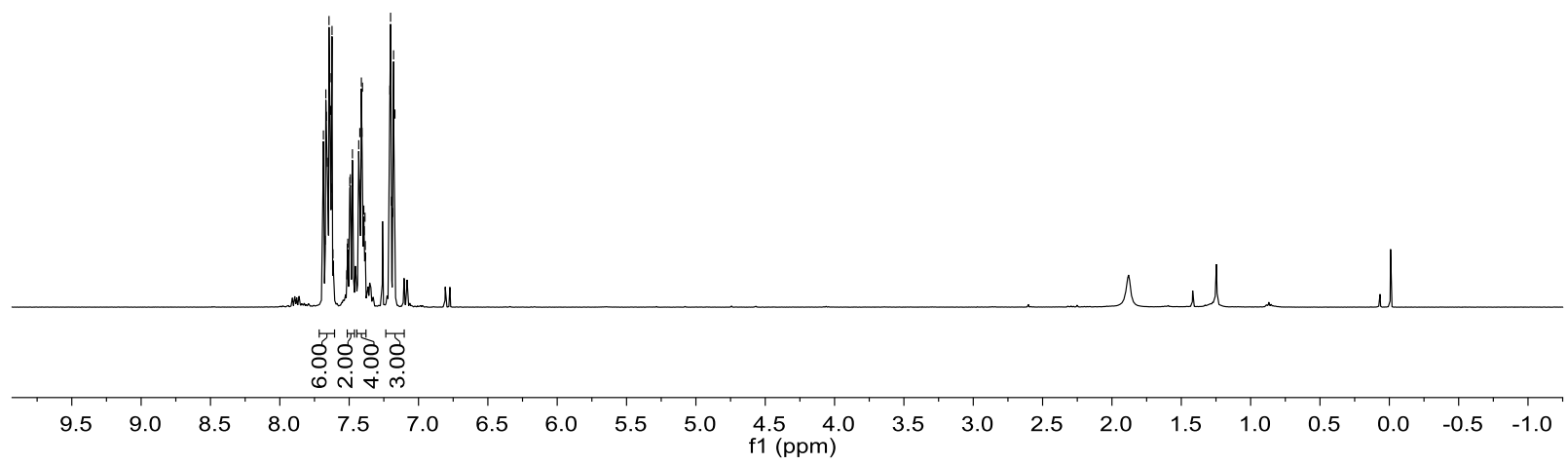

${ }^{31} \mathrm{P}$ NMR Spectrum of $4 \mathrm{k}\left(\mathrm{CDCl}_{3}, 162 \mathrm{MHz}\right)$

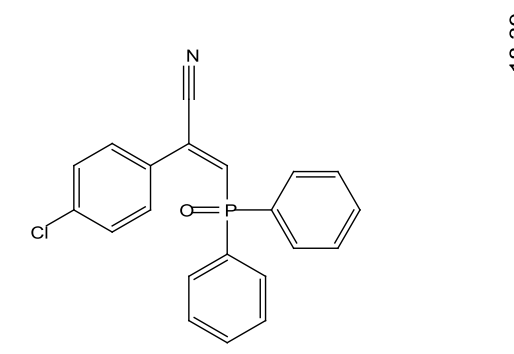

$\underset{\substack{\infty \\ \infty}}{\stackrel{\infty}{\perp}}$

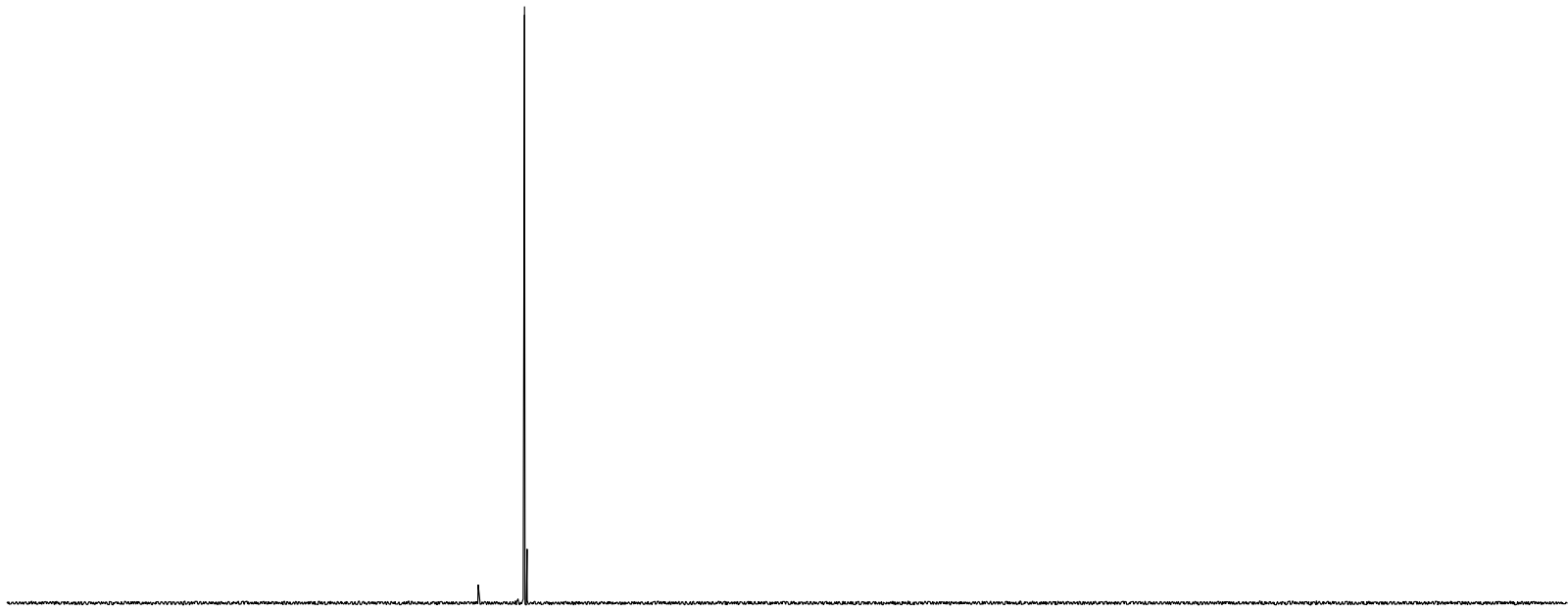

$\begin{array}{lllllllllllllllllllllllllllll}150 & 130 & 110 & 90 & 70 & 50 & 30 & 10 & -10 & -30 & -50 & -70 & -90 & -110 & -130 & -150 & -170 & -190 & -210 & -230 & -25\end{array}$ 
${ }^{13} \mathrm{C}$ NMR Spectrum of $4 \mathrm{k}\left(\mathrm{CDCl}_{3}, 100 \mathrm{MHz}\right)$
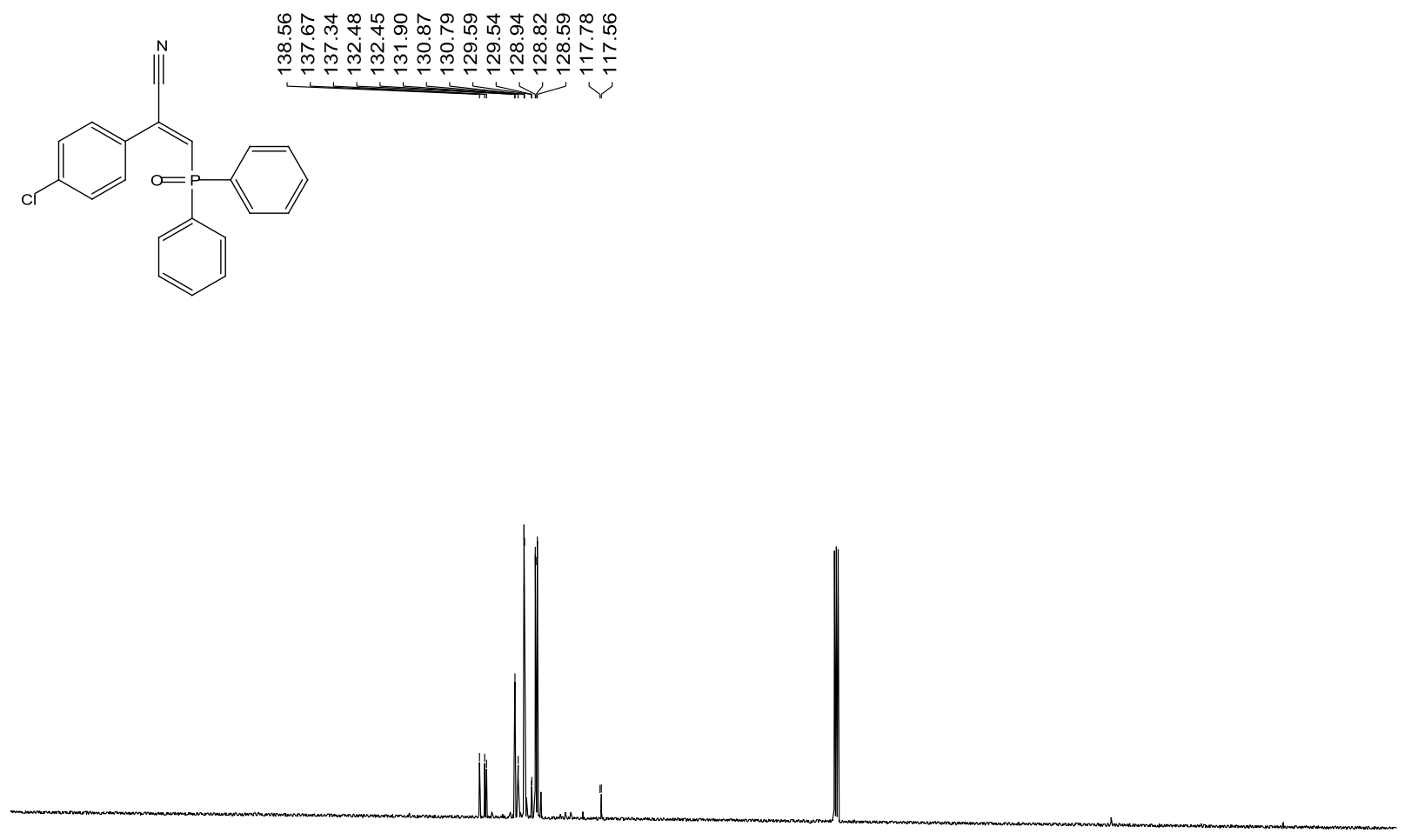

$\begin{array}{lllllllllllllllllllllll}210 & 200 & 190 & 180 & 170 & 160 & 150 & 140 & 130 & 120 & 110 & 100 & 90 & 80 & 70 & 60 & 50 & 40 & 30 & 20 & 10 & 0 & -10\end{array}$

${ }^{1} \mathrm{H}$ NMR Spectrum of $4 \mathrm{l}\left(\mathrm{CDCl}_{3}, 400 \mathrm{MHz}\right)$

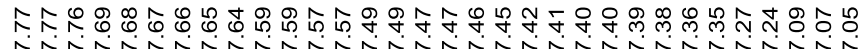

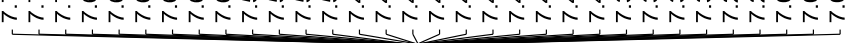<smiles>N#C/C(=C/c1ccccc1)c1ccccc1</smiles>

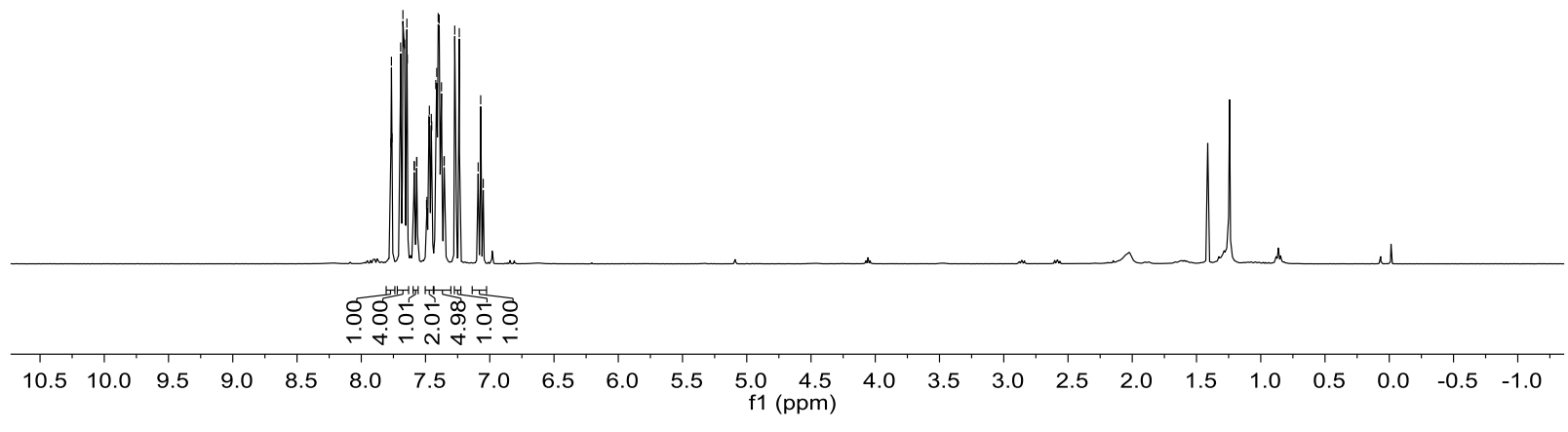




\section{${ }^{31} \mathrm{P}$ NMR Spectrum of $4 \mathrm{l}\left(\mathrm{CDCl}_{3}, 162 \mathrm{MHz}\right)$}<smiles>N#C/C(=C\C(=O)c1ccccc1)c1cccc(Br)c1</smiles>

$$
\stackrel{\bar{s}}{\stackrel{5}{\Gamma}}
$$
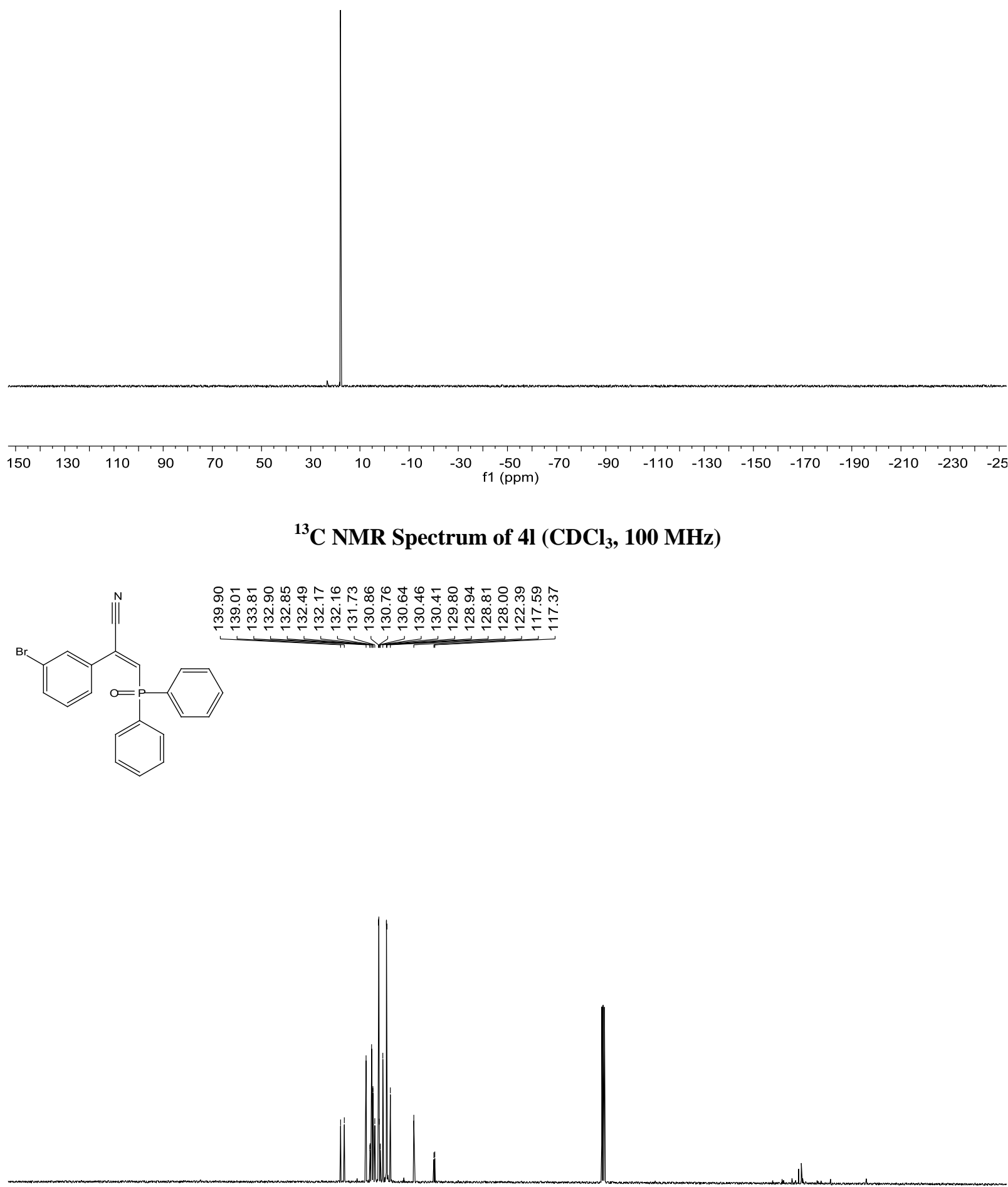

$\begin{array}{lllllllllllllllllllllll}210 & 200 & 190 & 180 & 170 & 160 & 150 & 140 & 130 & 120 & 110 \begin{array}{c}100 \\ \mathrm{f} 1(\mathrm{ppm})\end{array} & 90 & 80 & 70 & 60 & 50 & 40 & 30 & 20 & 10 & 0 & -10\end{array}$ 


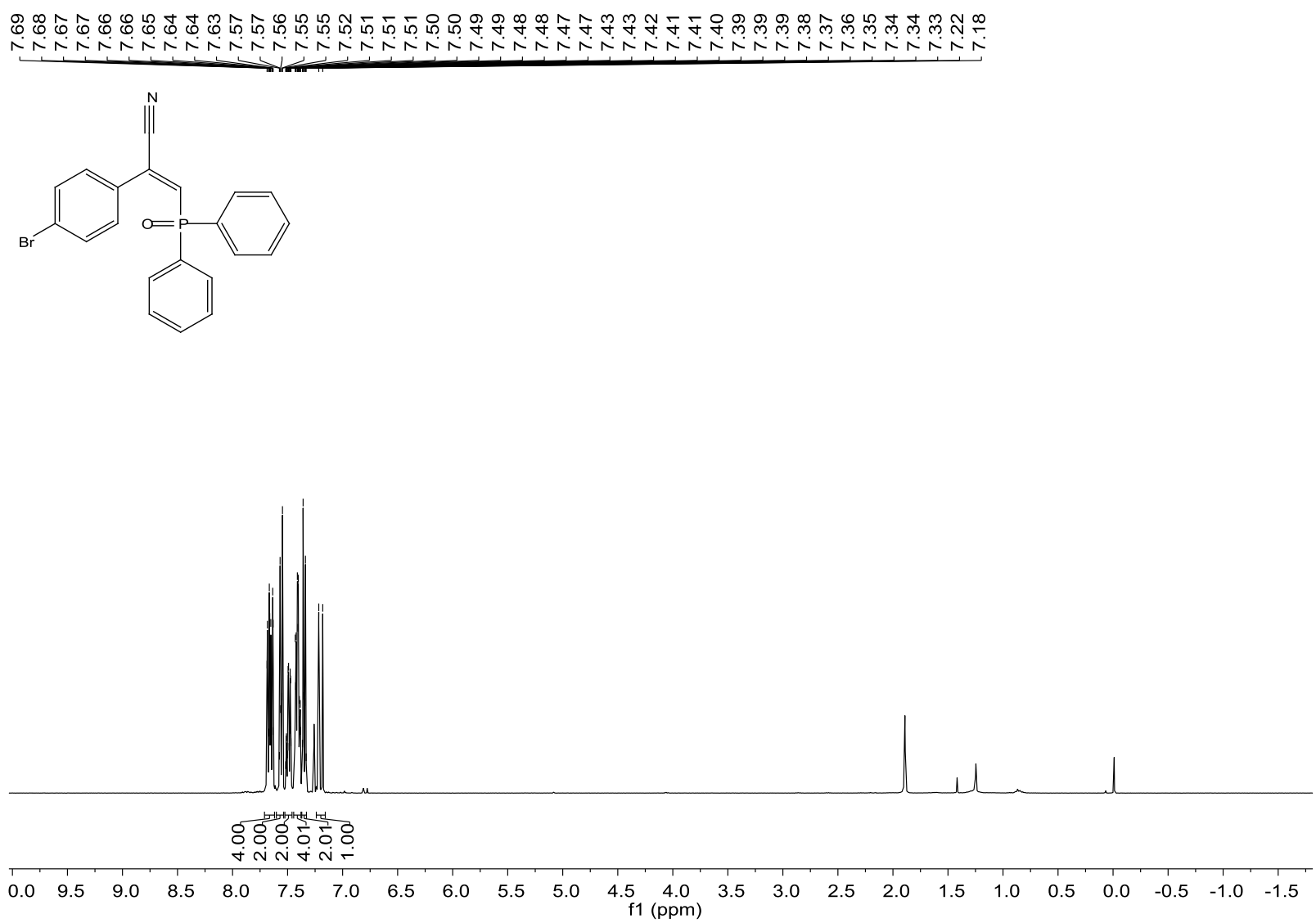

${ }^{31} \mathrm{P}$ NMR Spectrum of $4 \mathrm{~m}\left(\mathrm{CDCl}_{3}, 162 \mathrm{MHz}\right)$

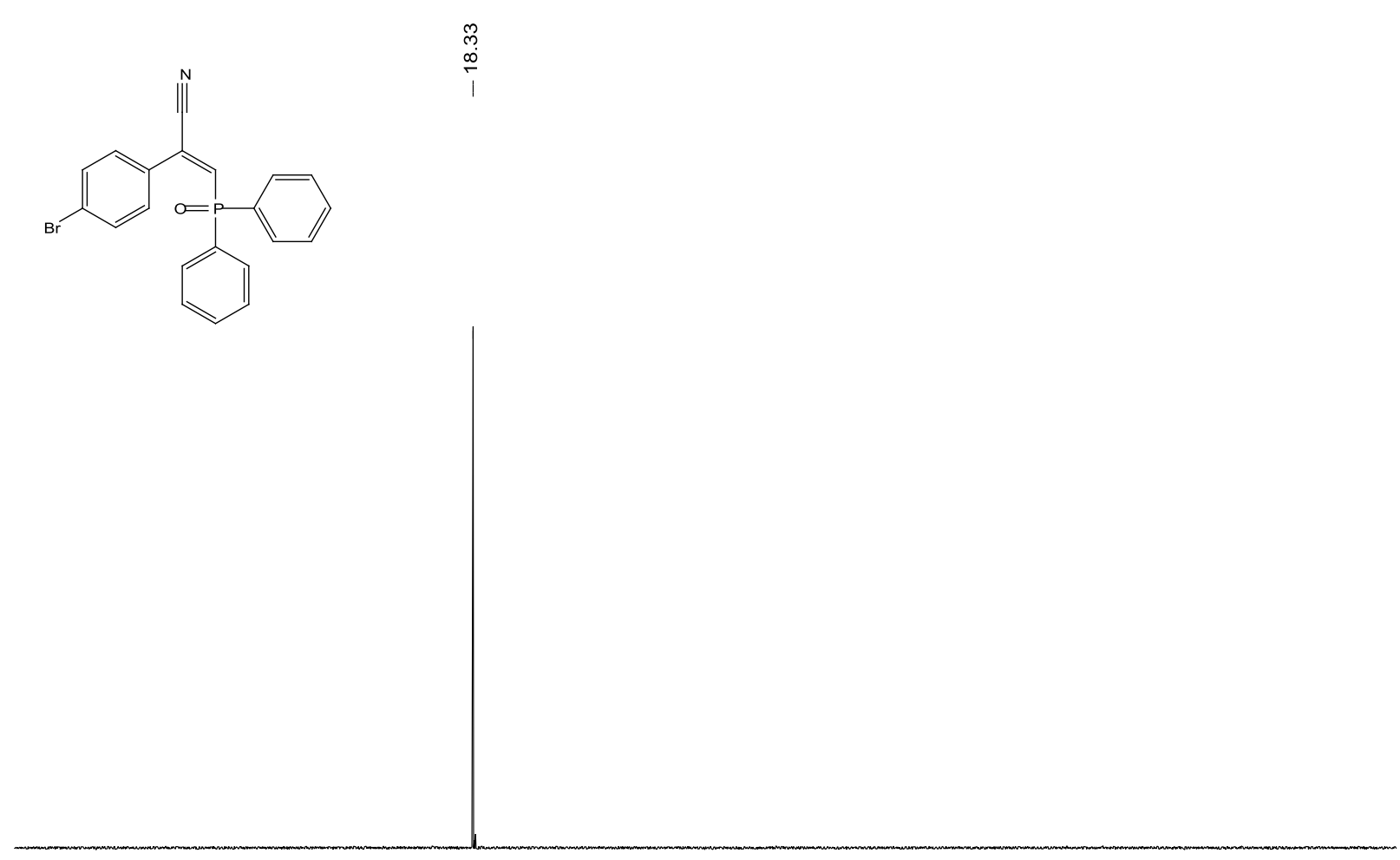

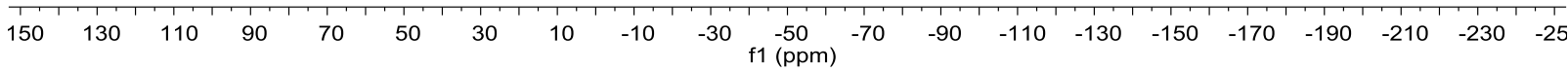




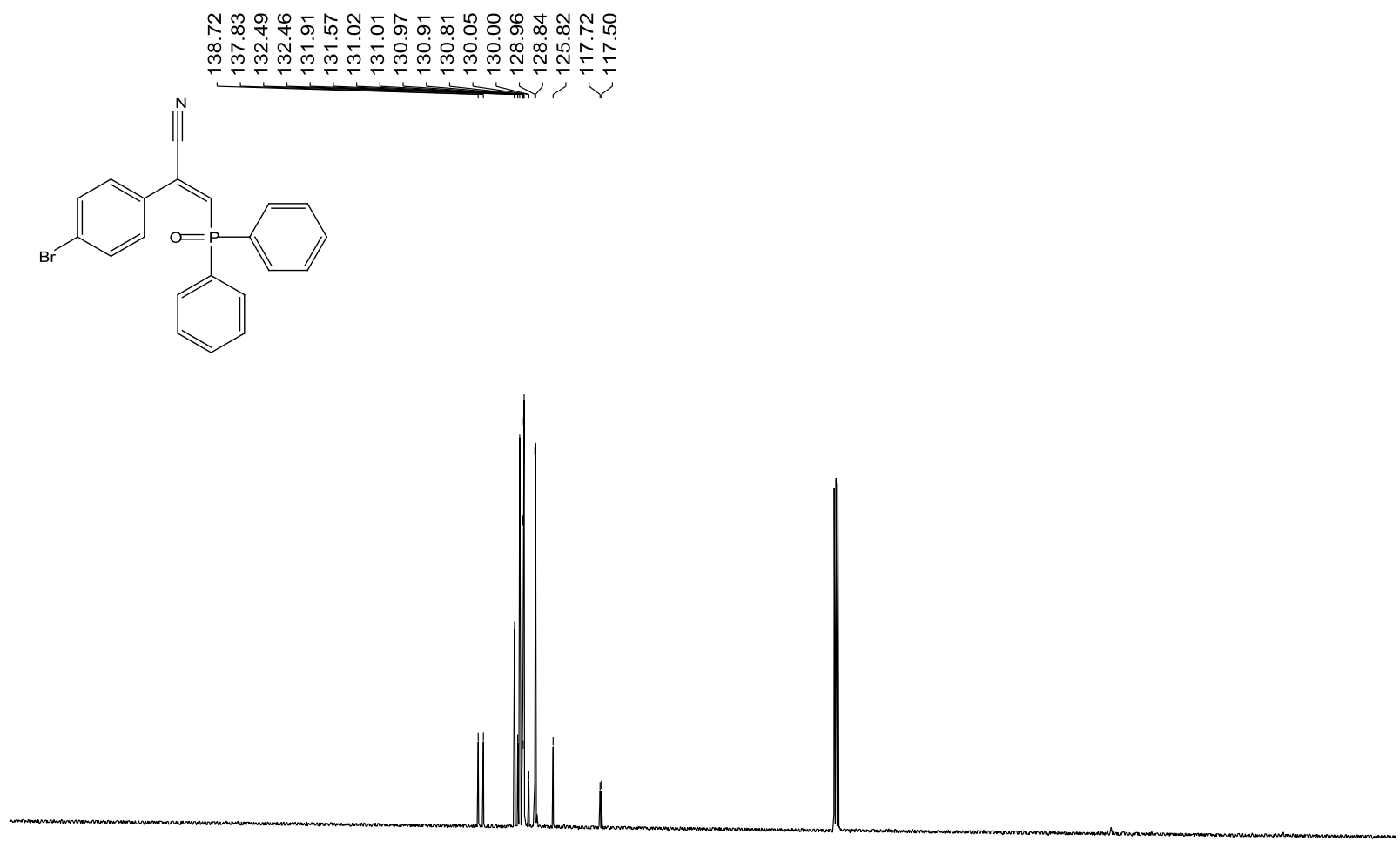

$\begin{array}{llllllllllllllllllllllllllllllll}210 & 200 & 190 & 180 & 170 & 160 & 150 & 140 & 130 & 120 & 110 & 100 & 90 & 80 & 70 & 60 & 50 & 40 & 30 & 20 & 10 & 0 & -10\end{array}$

${ }^{1} \mathrm{H}$ NMR Spectrum of $4 \mathrm{n}\left(\mathrm{CDCl}_{3}, 400 \mathrm{MHz}\right)$

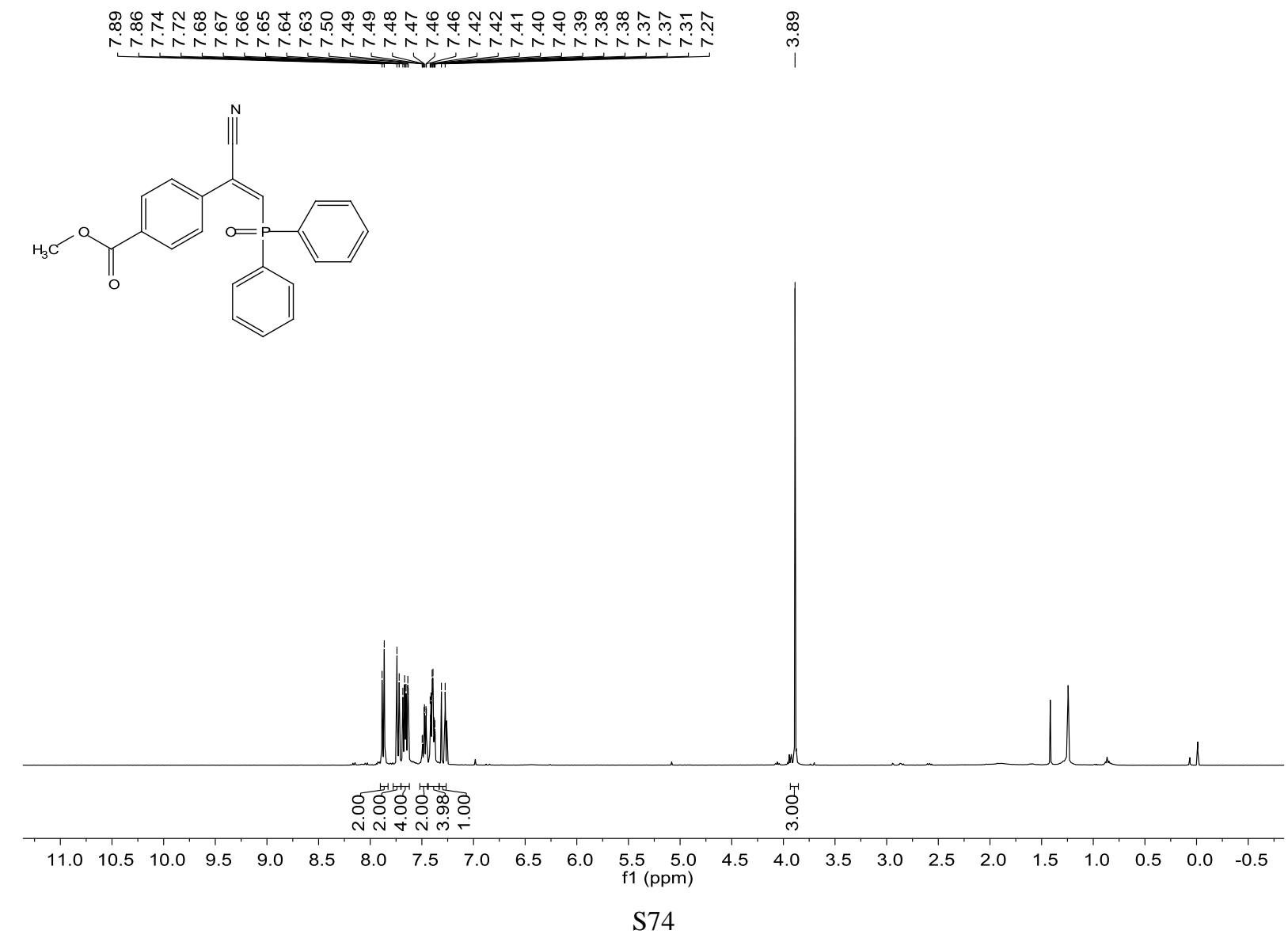


${ }^{31} \mathrm{P}$ NMR Spectrum of $4 \mathrm{n}\left(\mathrm{CDCl}_{3}, 162 \mathrm{MHz}\right)$
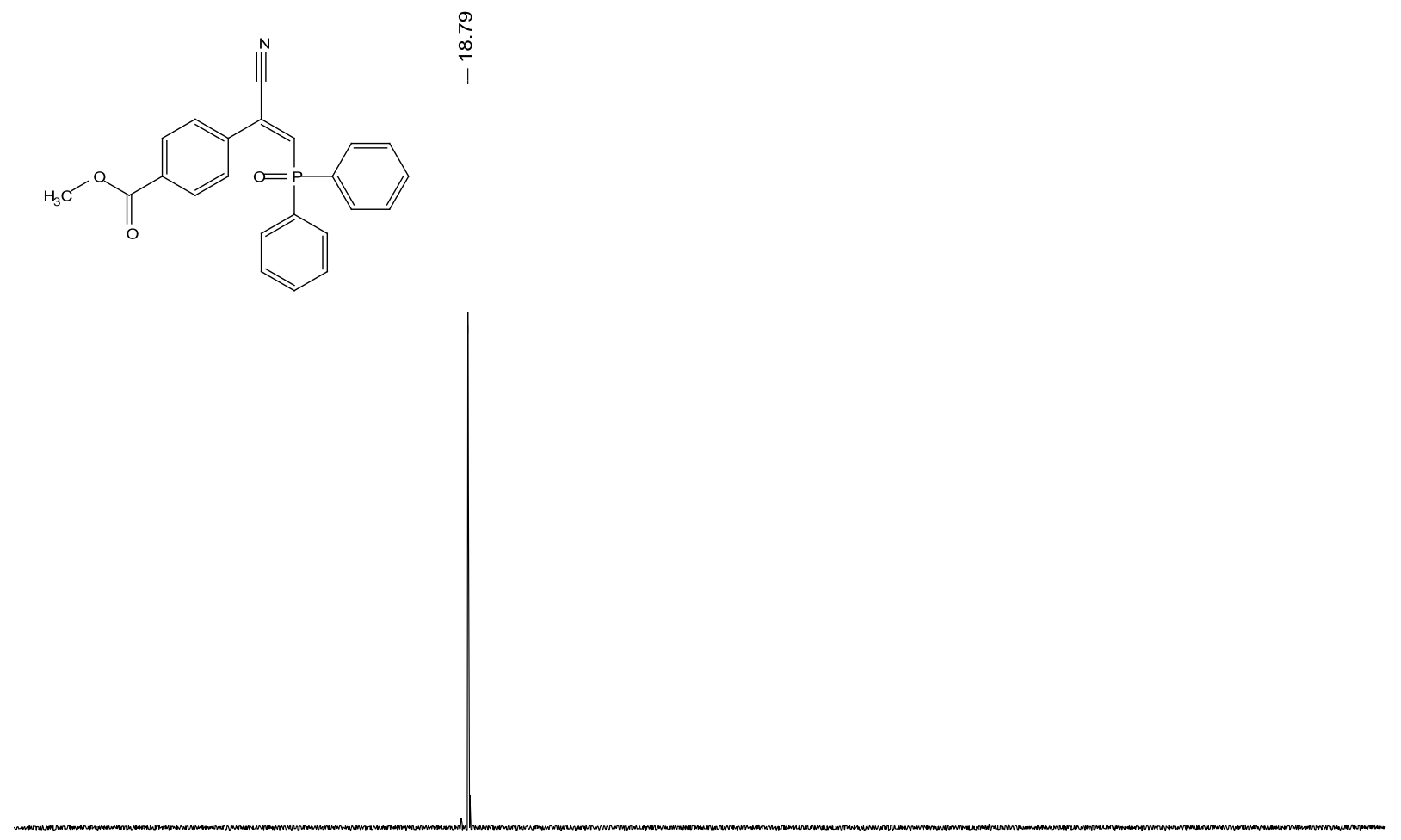

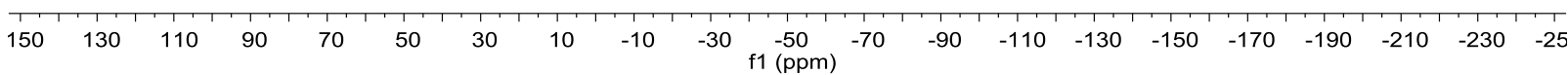

\section{${ }^{13} \mathrm{C}$ NMR Spectrum of $4 \mathrm{n}\left(\mathrm{CDCl}_{3}, 100 \mathrm{MHz}\right)$}

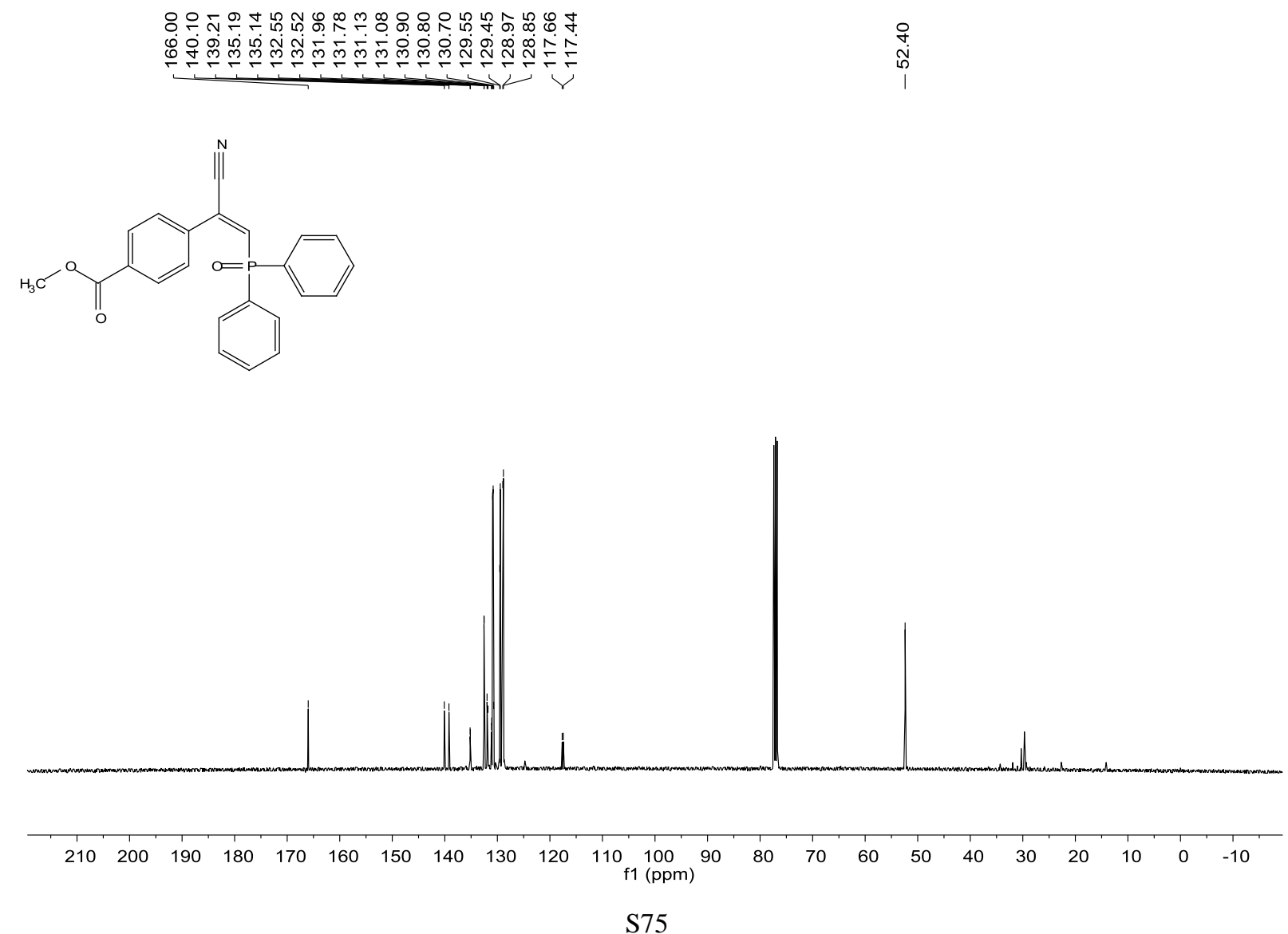


${ }^{1} \mathrm{H}$ NMR Spectrum of $40^{\prime}\left(\mathrm{CDCl}_{3}, 400 \mathrm{MHz}\right)$

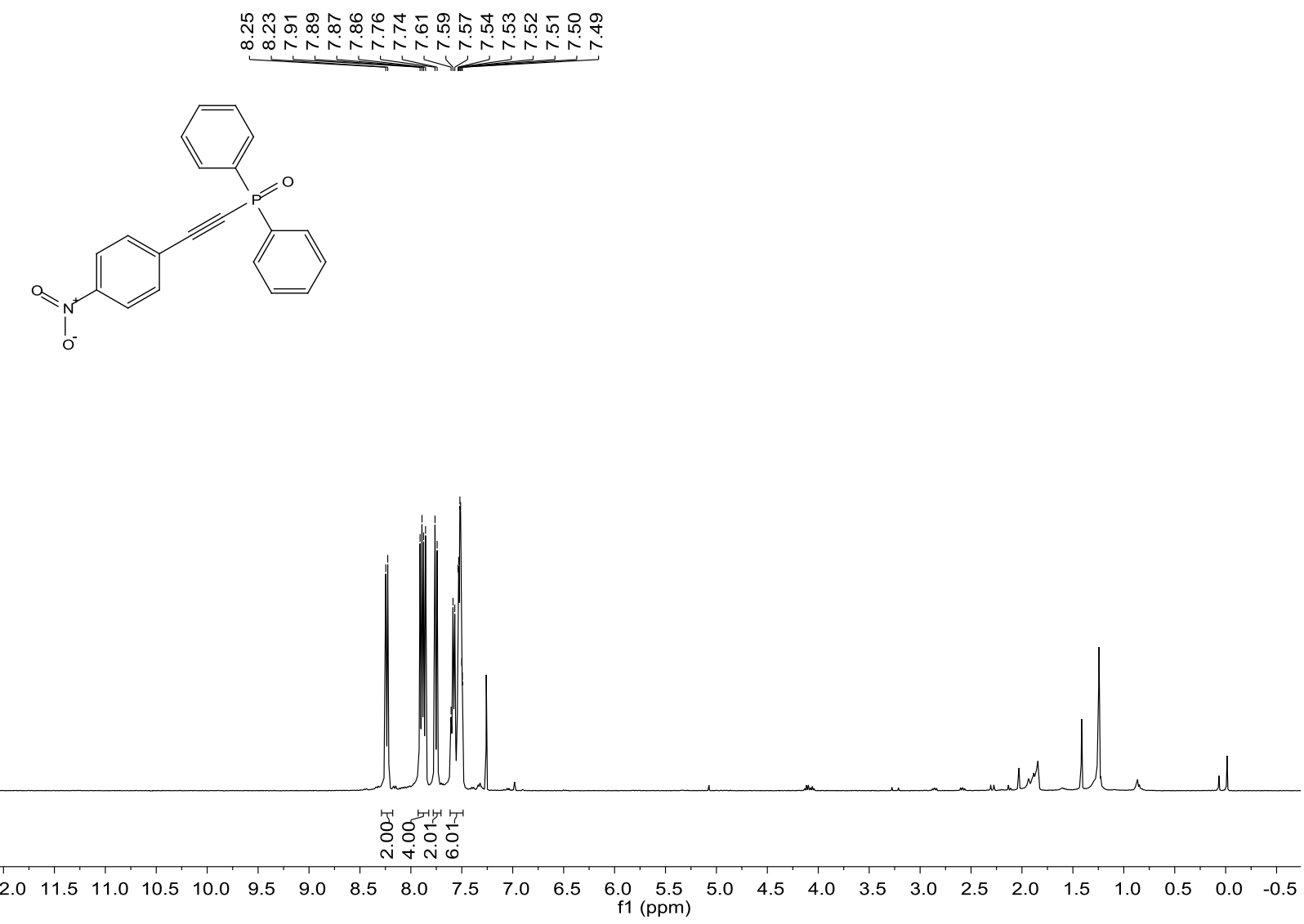

${ }^{31} \mathrm{P}$ NMR Spectrum of 4o' $\left(\mathrm{CDCl}_{3}, 162 \mathrm{MHz}\right)$

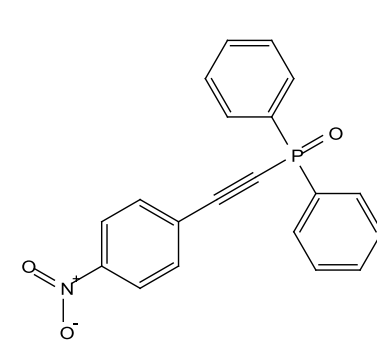

$\underset{\substack{0 \\ \infty}}{\infty}$

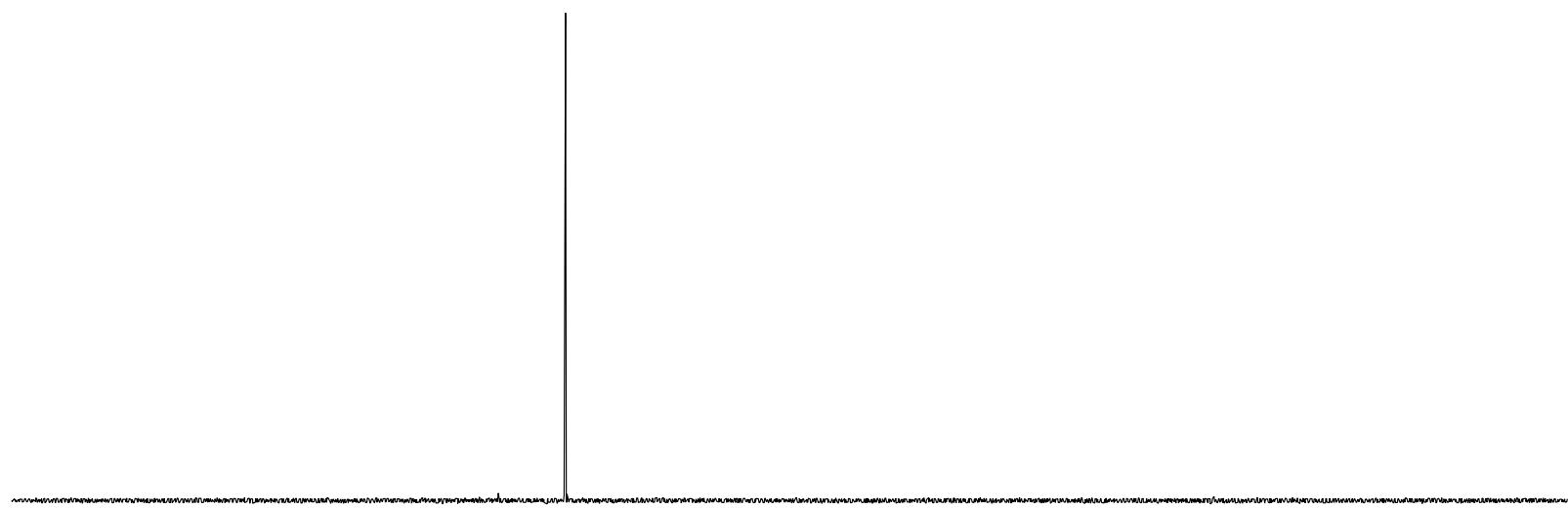

$\begin{array}{lllllllllllllllllllllllllllll}150 & 130 & 110 & 90 & 70 & 50 & 30 & 10 & -10 & -30 & -50 & -70 & -90 & -110 & -130 & -150 & -170 & -190 & -210 & -230 & -25\end{array}$ 
${ }^{1} \mathrm{H}$ NMR Spectrum of $4 \mathrm{p}\left(\mathrm{CDCl}_{3}, 400 \mathrm{MHz}\right)$

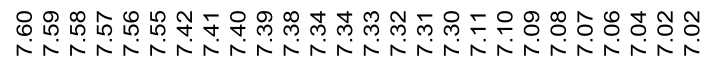<smiles>N#C/C(=C/C(=O)Oc1ccccc1)c1ccccc1</smiles>

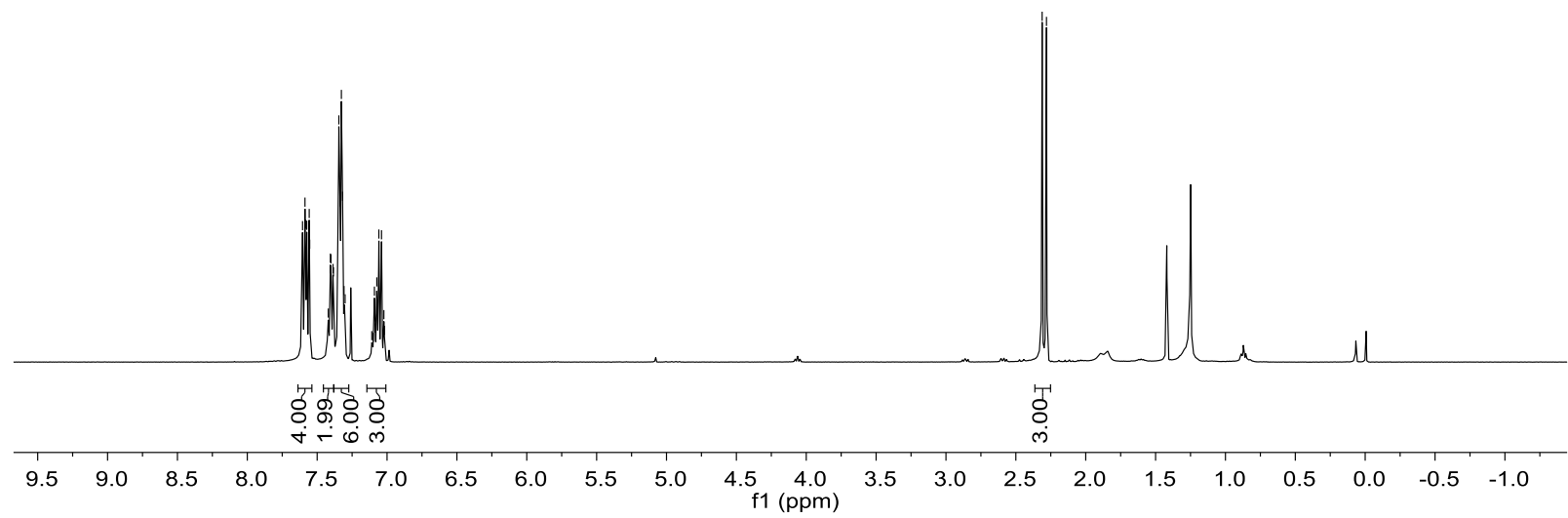

${ }^{31} \mathrm{P}$ NMR Spectrum of $4 \mathrm{p}\left(\mathrm{CDCl}_{3}, 162 \mathrm{MHz}\right)$

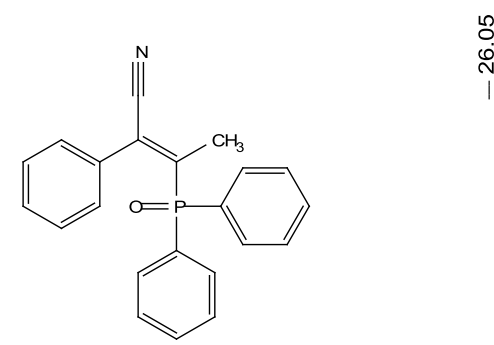

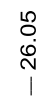

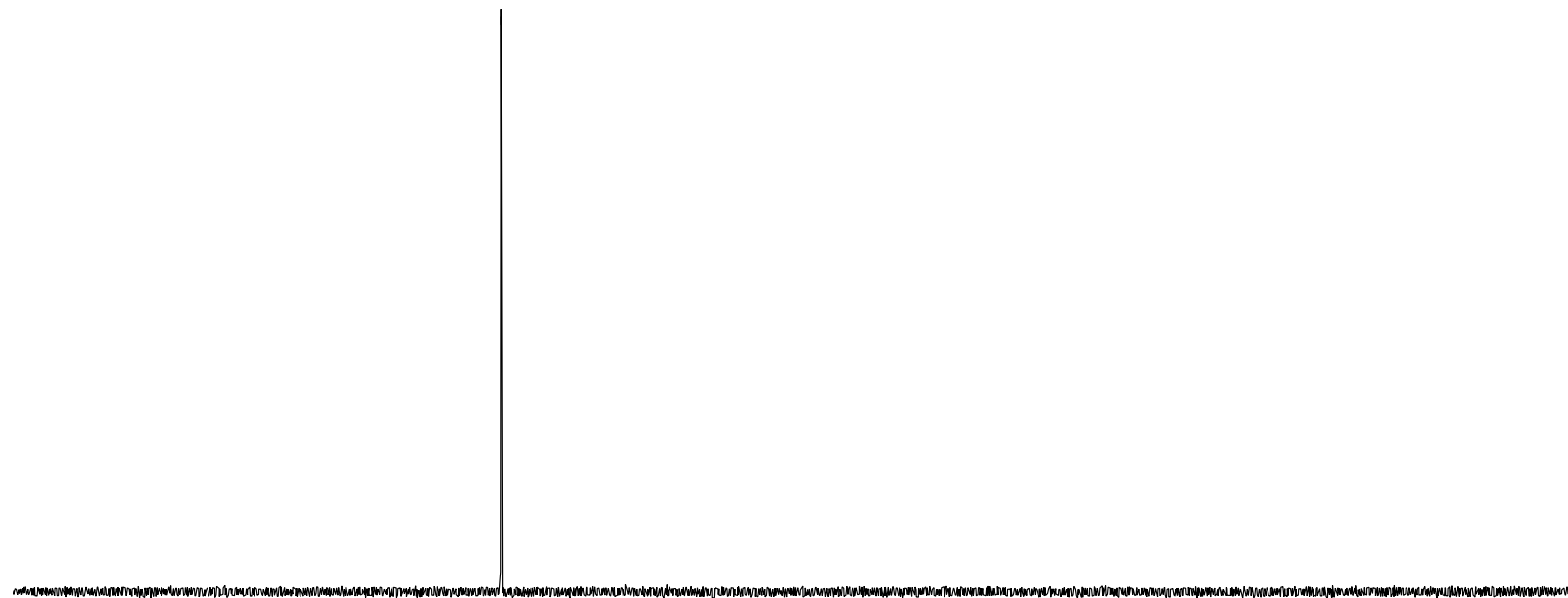

$\begin{array}{lllllllllllllllllllllll}150 & 130 & 110 & 90 & 70 & 50 & 30 & 10 & -10 & -30 & \begin{array}{c}-50 \\ \mathrm{f} 1(\mathrm{ppm})\end{array} & -70 & -90 & -110 & -130 & -150 & -170 & -190 & -210 & -230 & -25\end{array}$ 
${ }^{13} \mathrm{C}$ NMR Spectrum of $4 \mathrm{p}\left(\mathrm{CDCl}_{3}, 100 \mathrm{MHz}\right)$

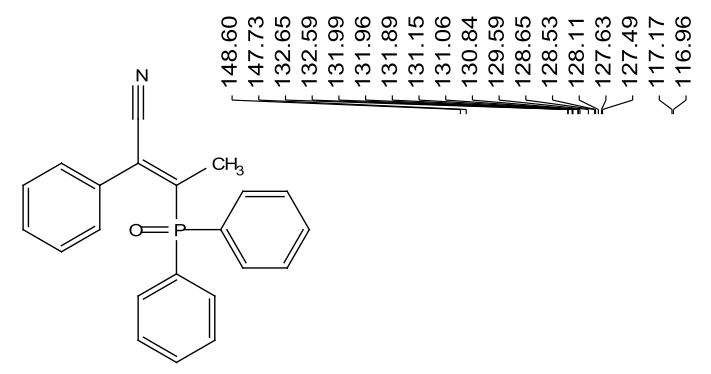

ind

$\stackrel{\text { in }}{\stackrel{\text { v }}{*}}$

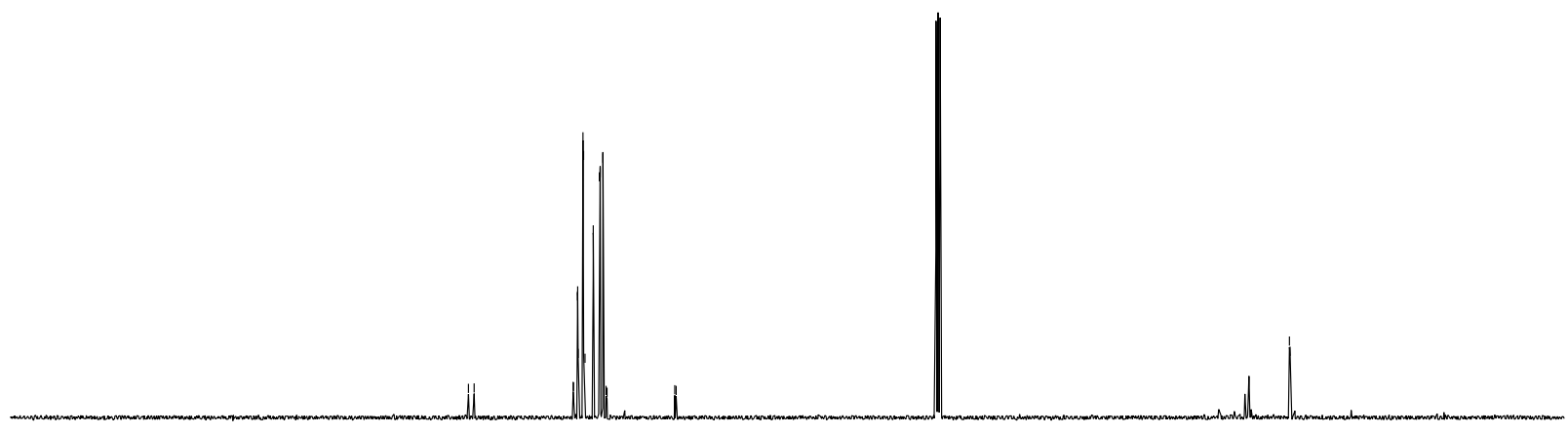

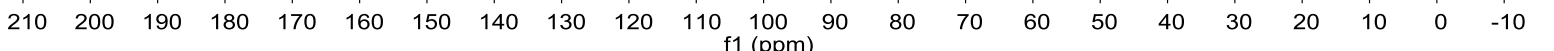

${ }^{1} \mathrm{H}$ NMR Spectrum of $4 \mathrm{q}\left(\mathrm{CDCl}_{3}, 400 \mathrm{MHz}\right)$

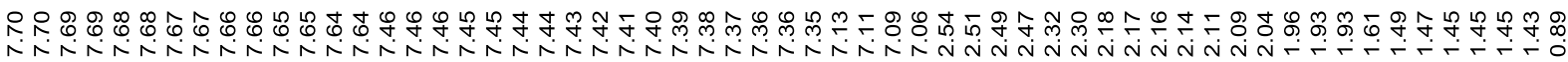<smiles>N#C/C(=C/P(=O)(c1ccccc1)c1ccccc1)c1ccc2c(c1)CC[C@H]1[C@@H]2CC[C@]2(O)C(=O)CC[C@@H]12</smiles>

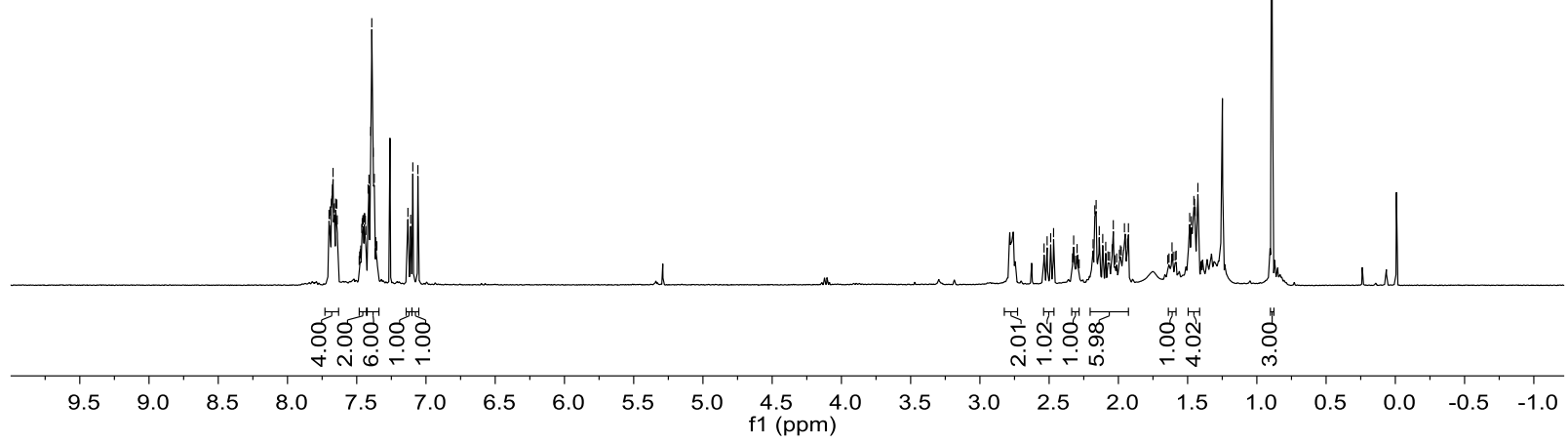



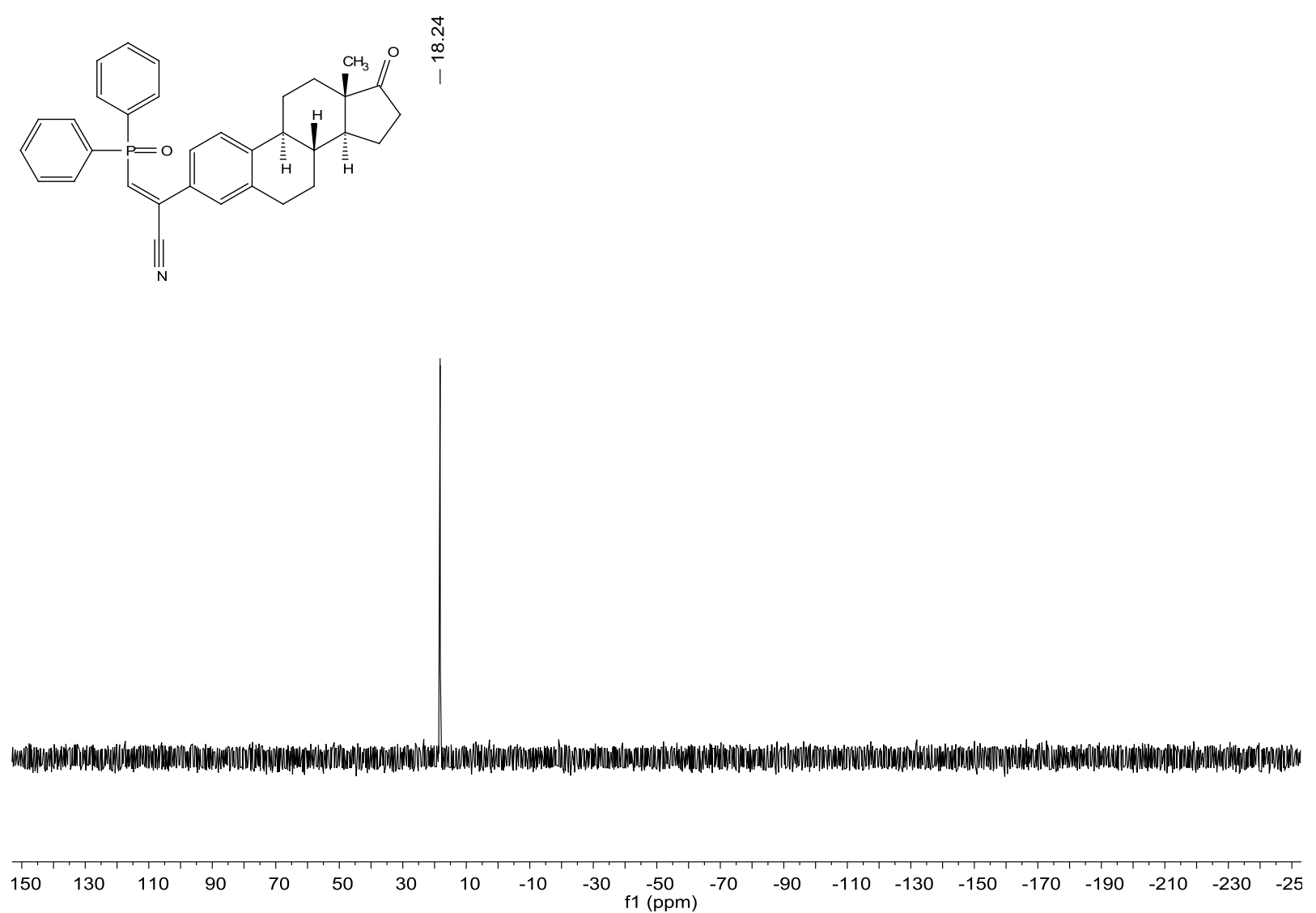

${ }^{13} \mathrm{C}$ NMR Spectrum of $4 \mathrm{q}\left(\mathrm{CDCl}_{3}, 100 \mathrm{MHz}\right)$
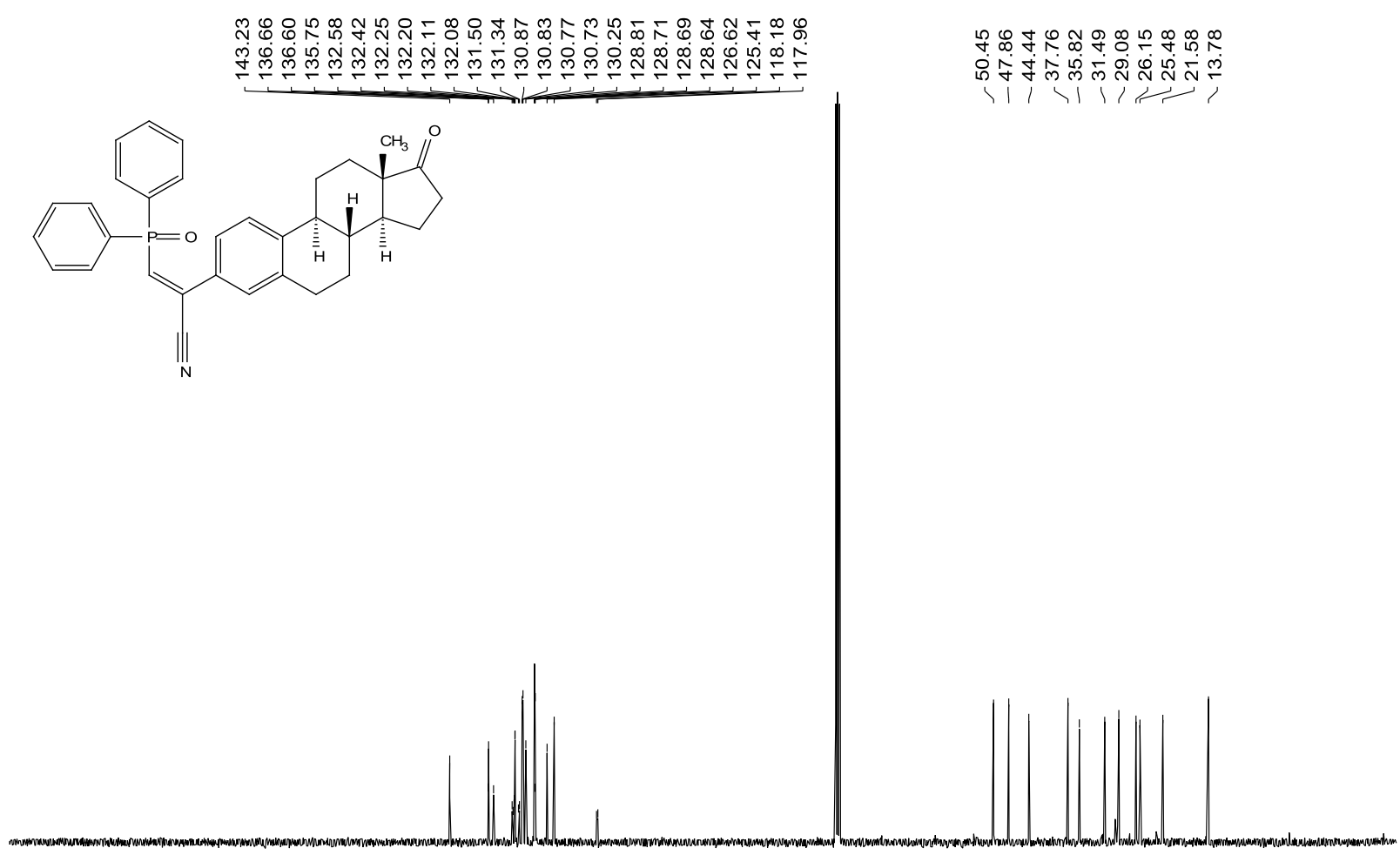

$\begin{array}{llllllllllllllllllllll}210 & 200 & 190 & 180 & 170 & 160 & 150 & 140 & 130 & 120 & \begin{array}{c}110 \\ \mathrm{f} 1\end{array} \begin{array}{l}100 \\ (\mathrm{ppm})\end{array} & 90 & 80 & 70 & 60 & 50 & 40 & 30 & 20 & 10 & 0 & -10\end{array}$ 
${ }^{1} \mathrm{H}$ NMR Spectrum of $4 \mathrm{r}\left(\mathrm{CDCl}_{3}, 400 \mathrm{MHz}\right)$

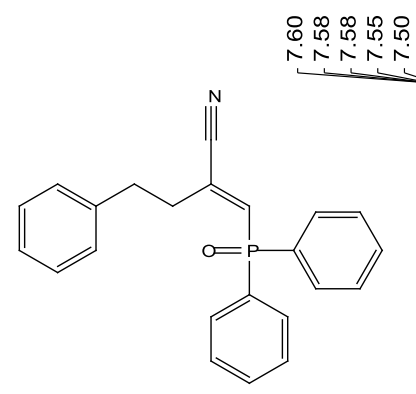

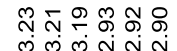

miñ

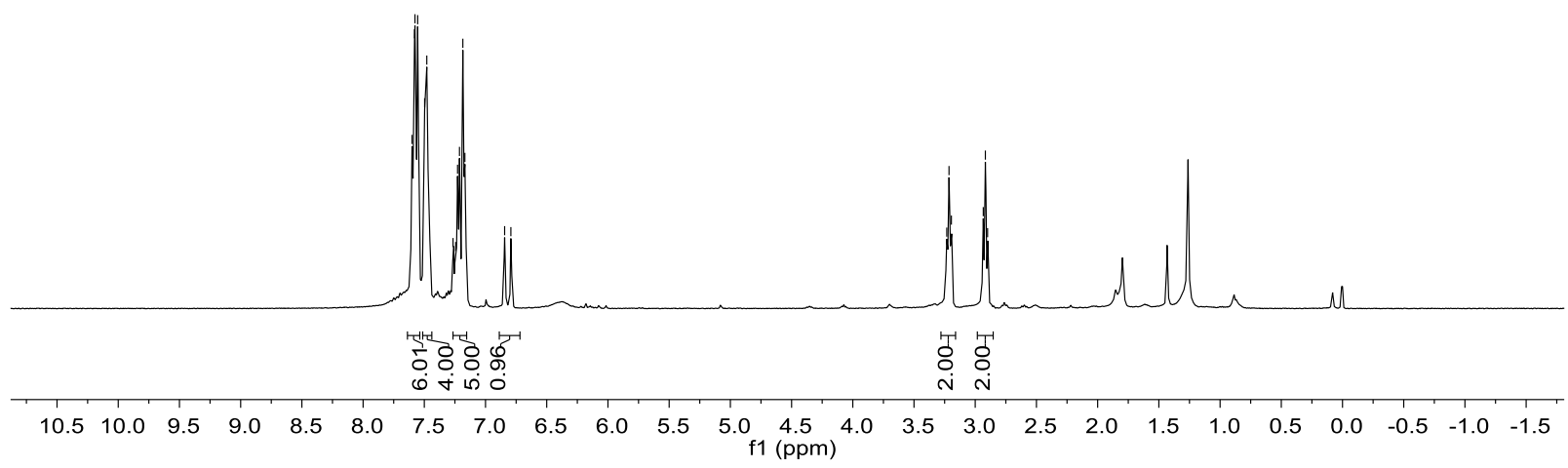

${ }^{31} \mathrm{P}$ NMR Spectrum of $4 \mathrm{r}\left(\mathrm{CDCl}_{3}, 162 \mathrm{MHz}\right)$

$\mathfrak{n}_{\substack{\infty \\ \infty}}^{\infty}$

$\sqrt[\text { กิ }]{\mathrm{N}}$<smiles>N#CC(=CC(=O)c1ccccc1)c1ccccc1</smiles>

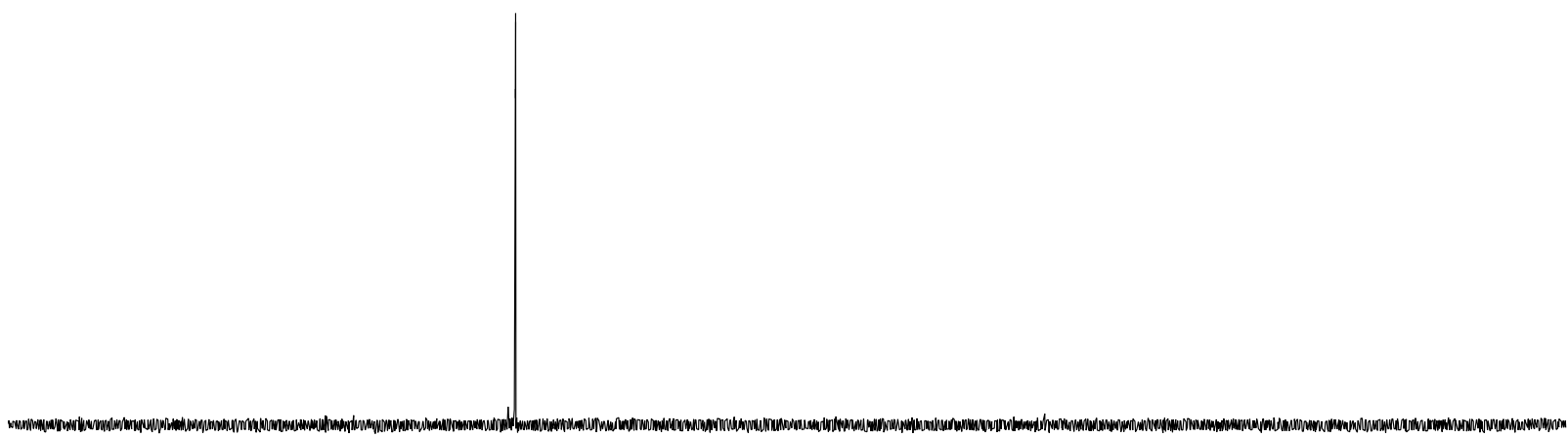

$\begin{array}{lllllllllllllllllllllllll}150 & 130 & 110 & 90 & 70 & 50 & 30 & 10 & -10 & -30 & \begin{array}{c}-50 \\ \mathrm{f} 1\end{array}(\mathrm{ppm}) & -70 & -90 & -110 & -130 & -150 & -170 & -190 & -210 & -230 & -25\end{array}$ 


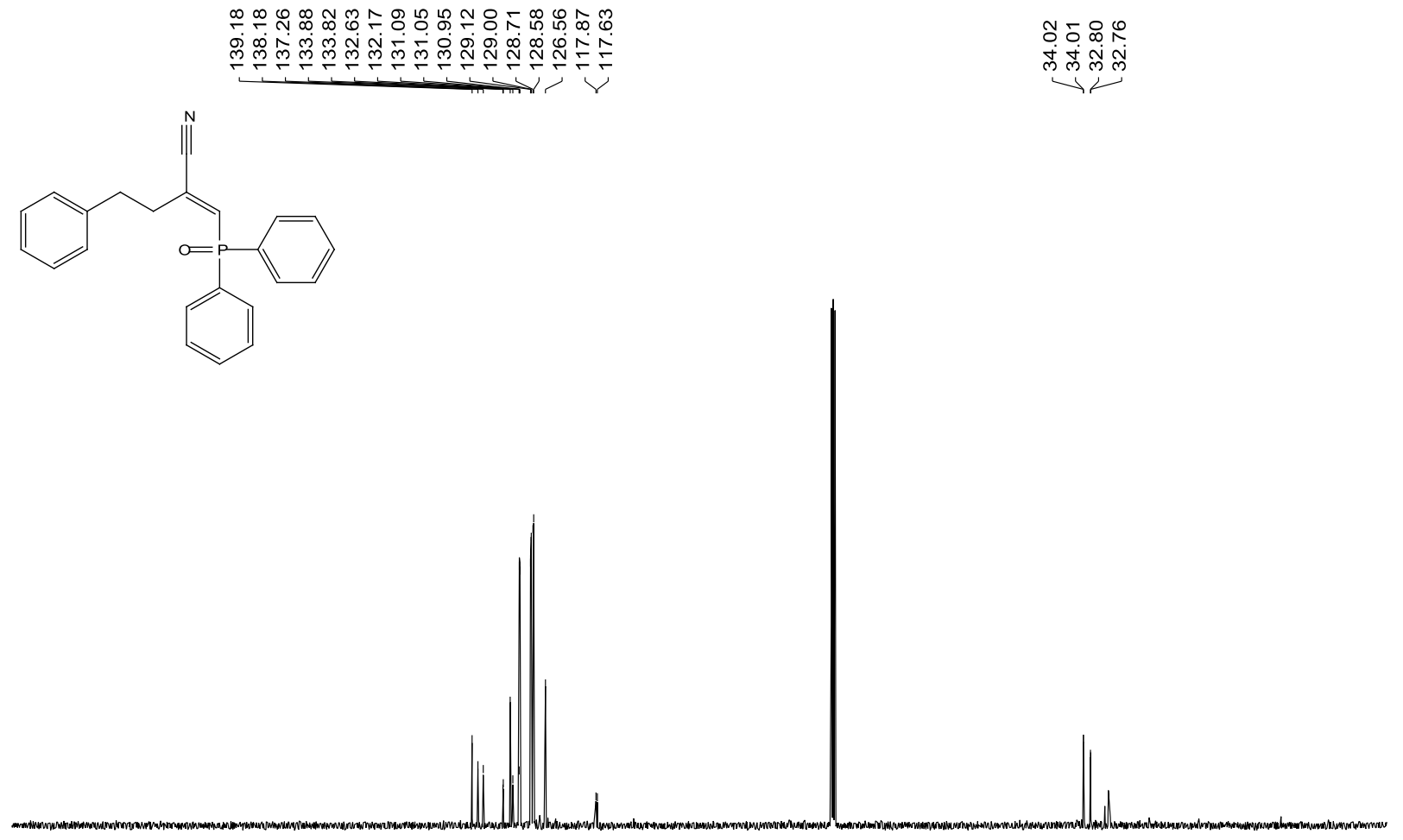

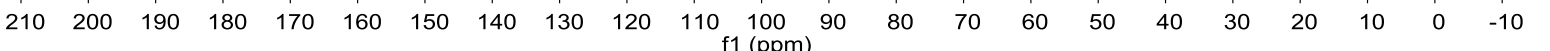

${ }^{1} \mathrm{H}$ NMR Spectrum of $4 \mathrm{~s}\left(\mathrm{CDCl}_{3}, 400 \mathrm{MHz}\right)$<smiles>CCCCC/C(C#N)=C\P(=O)(c1ccccc1)c1ccccc1</smiles>

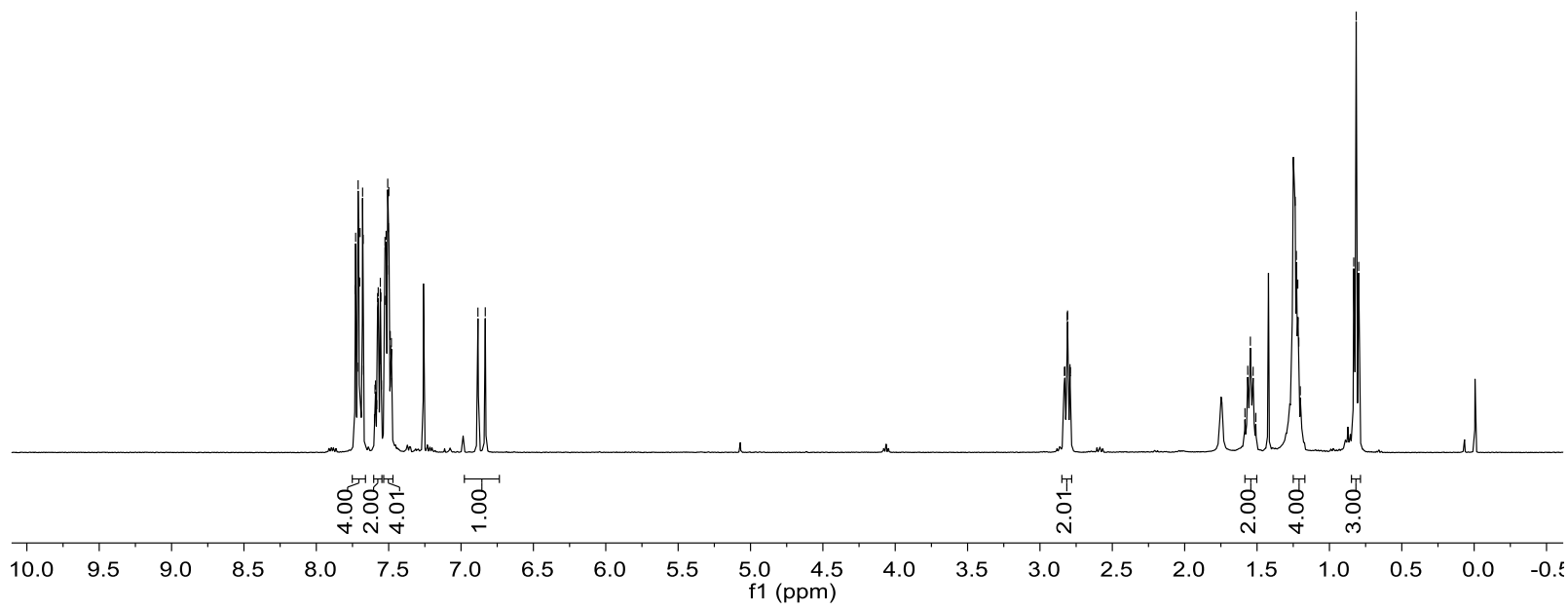


${ }^{31} \mathrm{P}$ NMR Spectrum of $4 \mathrm{~s}\left(\mathrm{CDCl}_{3}, 162 \mathrm{MHz}\right)$

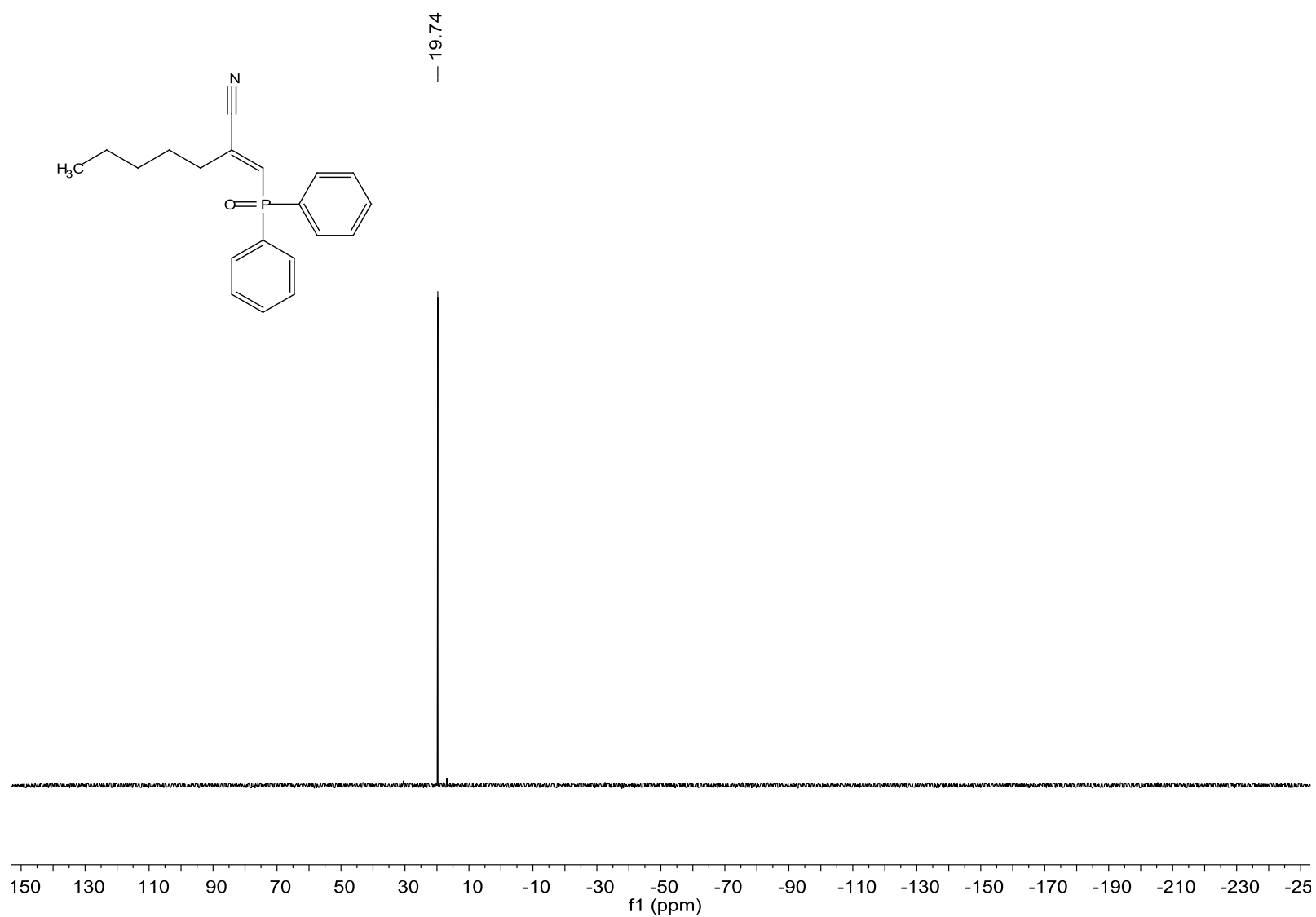

${ }^{13} \mathrm{C}$ NMR Spectrum of $4 \mathrm{~s}\left(\mathrm{CDCl}_{3}, 100 \mathrm{MHz}\right)$

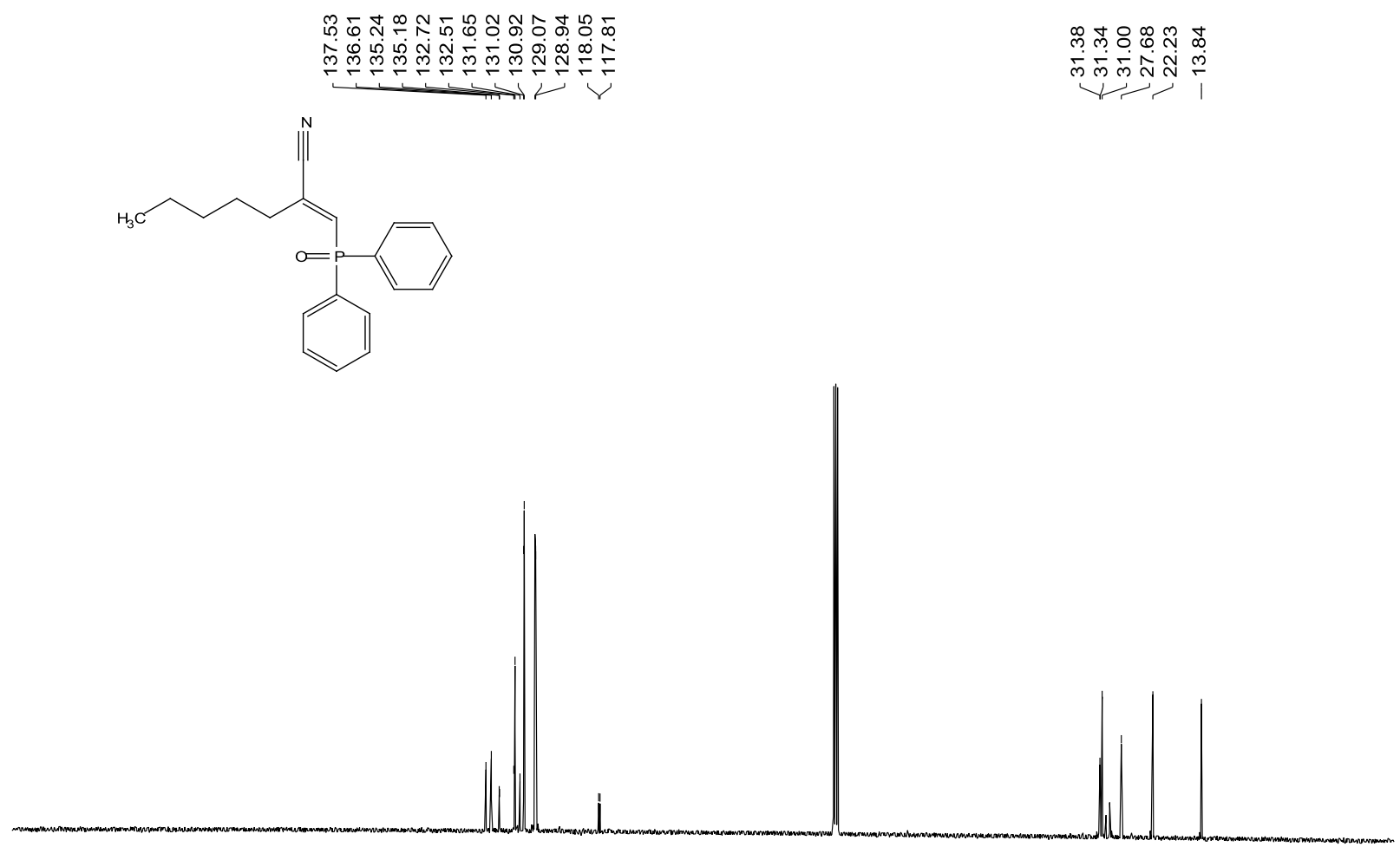

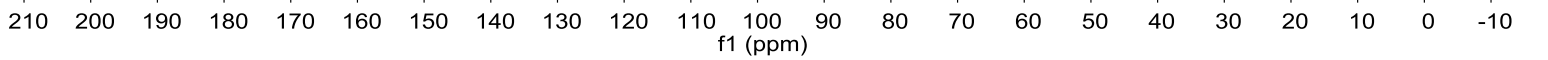


${ }^{1} \mathrm{H}$ NMR Spectrum of $4 \mathrm{t}\left(\mathrm{CDCl}_{3}, 400 \mathrm{MHz}\right)$
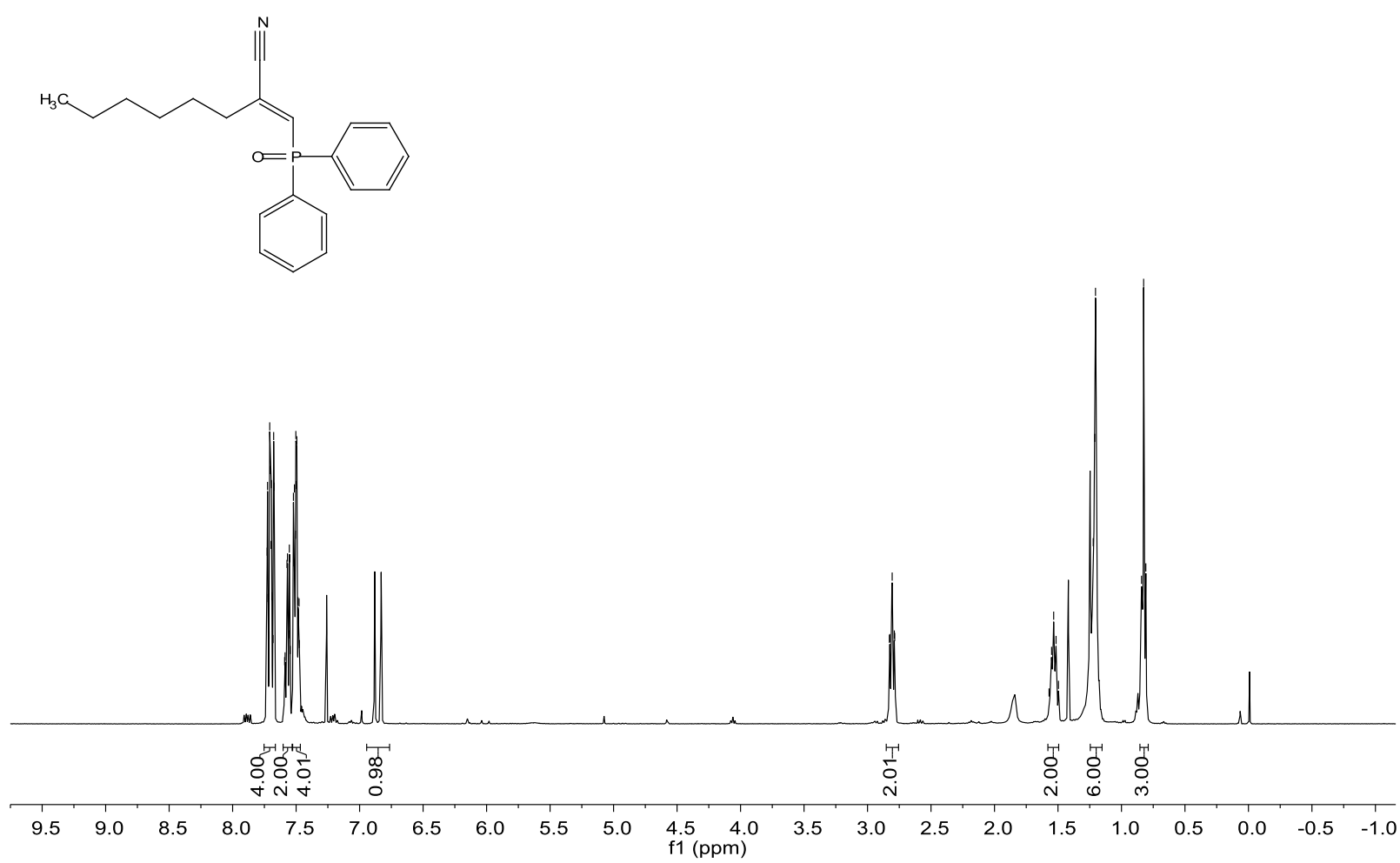

${ }^{31}$ P NMR Spectrum of $4 \mathrm{t}\left(\mathrm{CDCl}_{3}, 162 \mathrm{MHz}\right)$

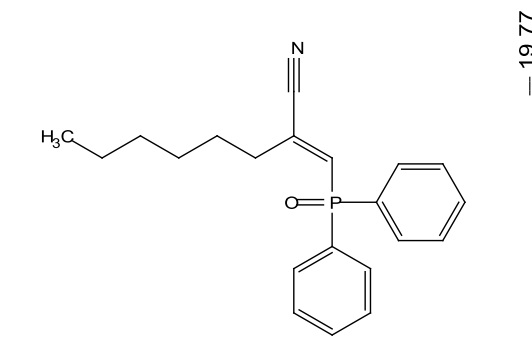

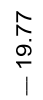


${ }^{13} \mathrm{C}$ NMR Spectrum of $4 \mathrm{t}\left(\mathrm{CDCl}_{3}, 100 \mathrm{MHz}\right)$

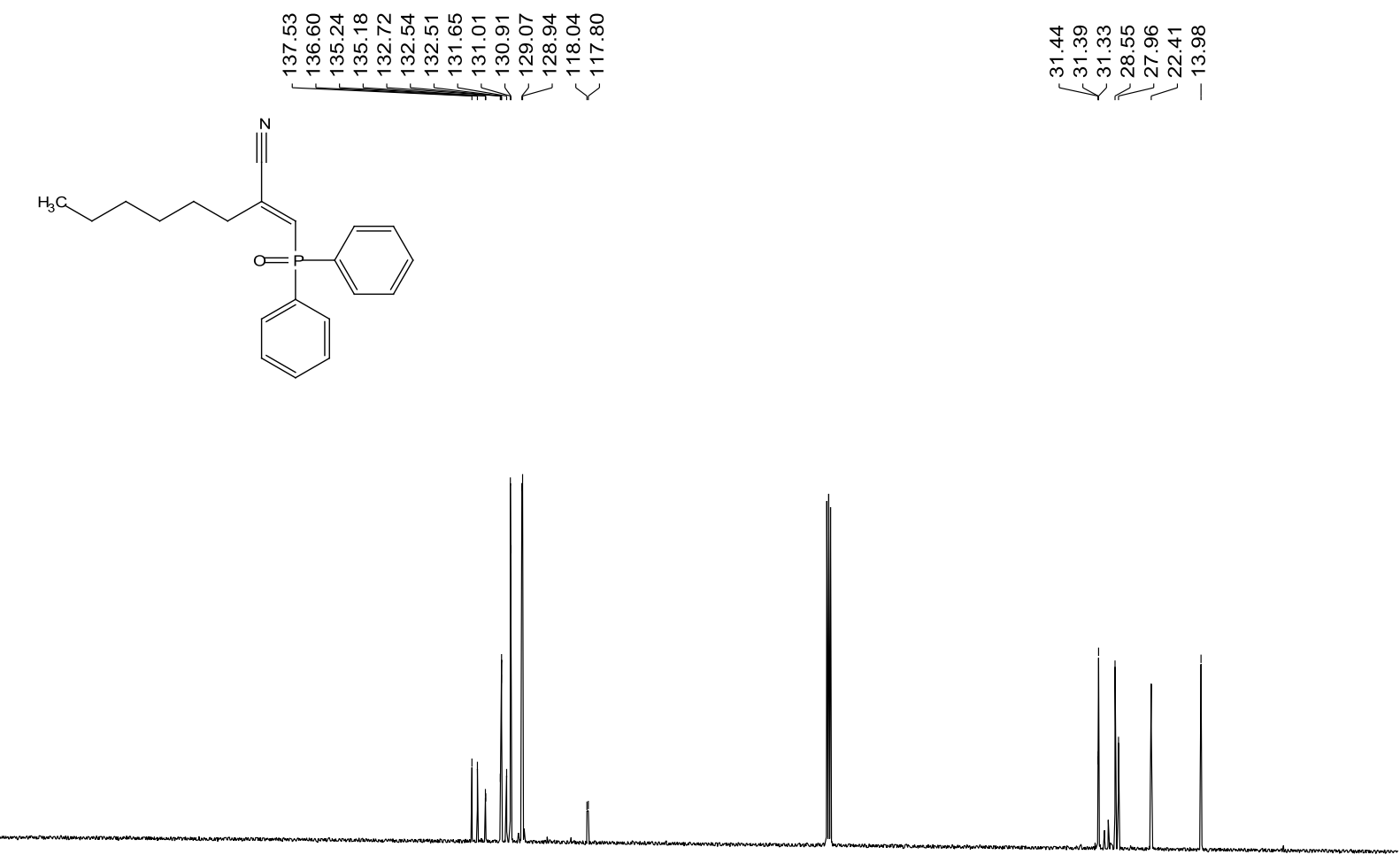

$\begin{array}{lllllllllllllllllllllllllllllll}210 & 200 & 190 & 180 & 170 & 160 & 150 & 140 & 130 & 120 & 110 & 100 & 90 & 80 & 70 & 60 & 50 & 40 & 30 & 20 & 10 & 0 & -10\end{array}$

${ }^{1} \mathrm{H}$ NMR Spectrum of $5 \mathrm{a}\left(\mathrm{CDCl}_{3}, 400 \mathrm{MHz}\right)$

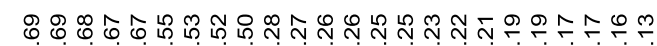

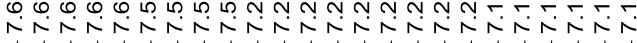
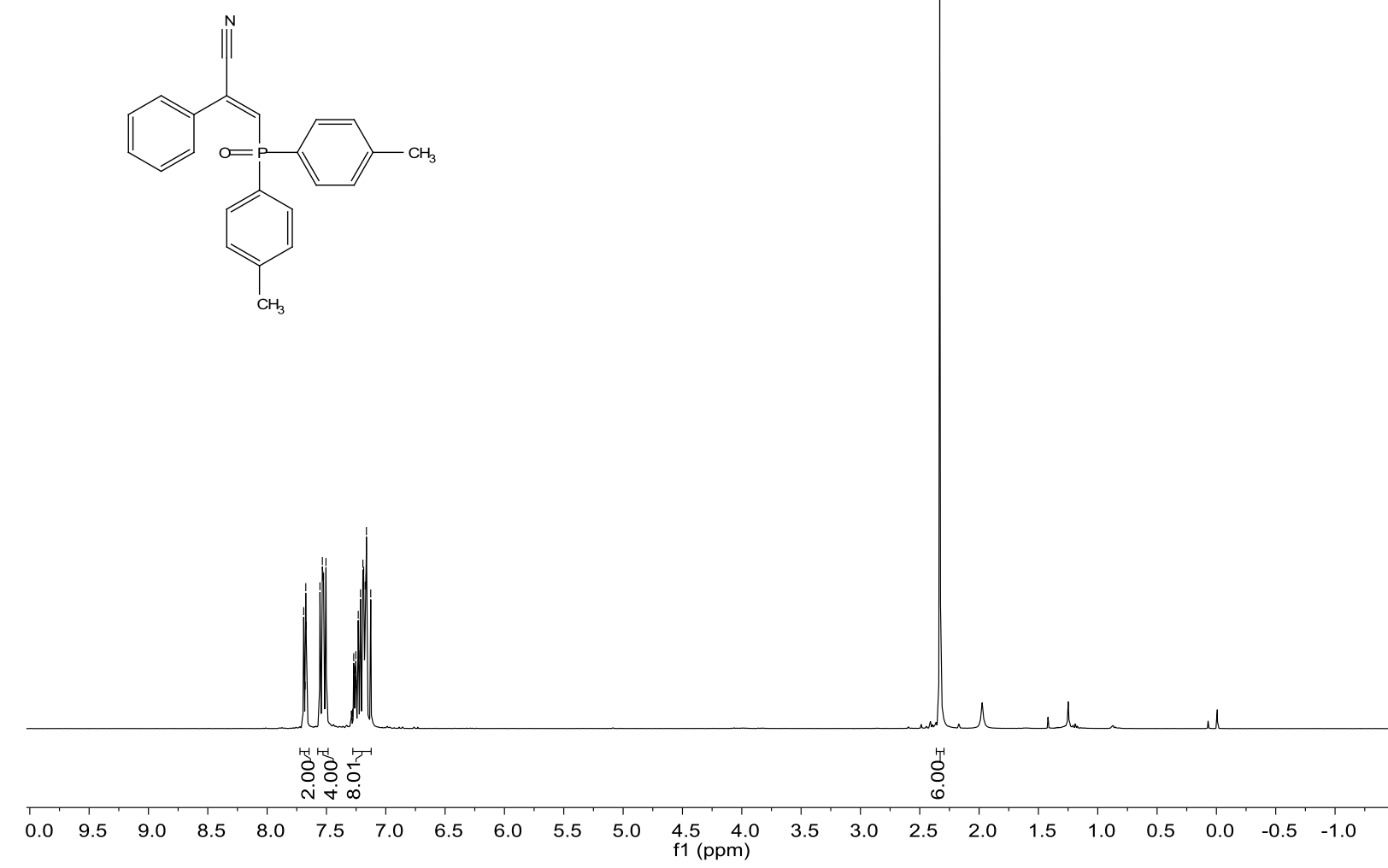
${ }^{31} \mathrm{P}$ NMR Spectrum of $5 \mathrm{a}\left(\mathrm{CDCl}_{3}, 162 \mathrm{MHz}\right)$

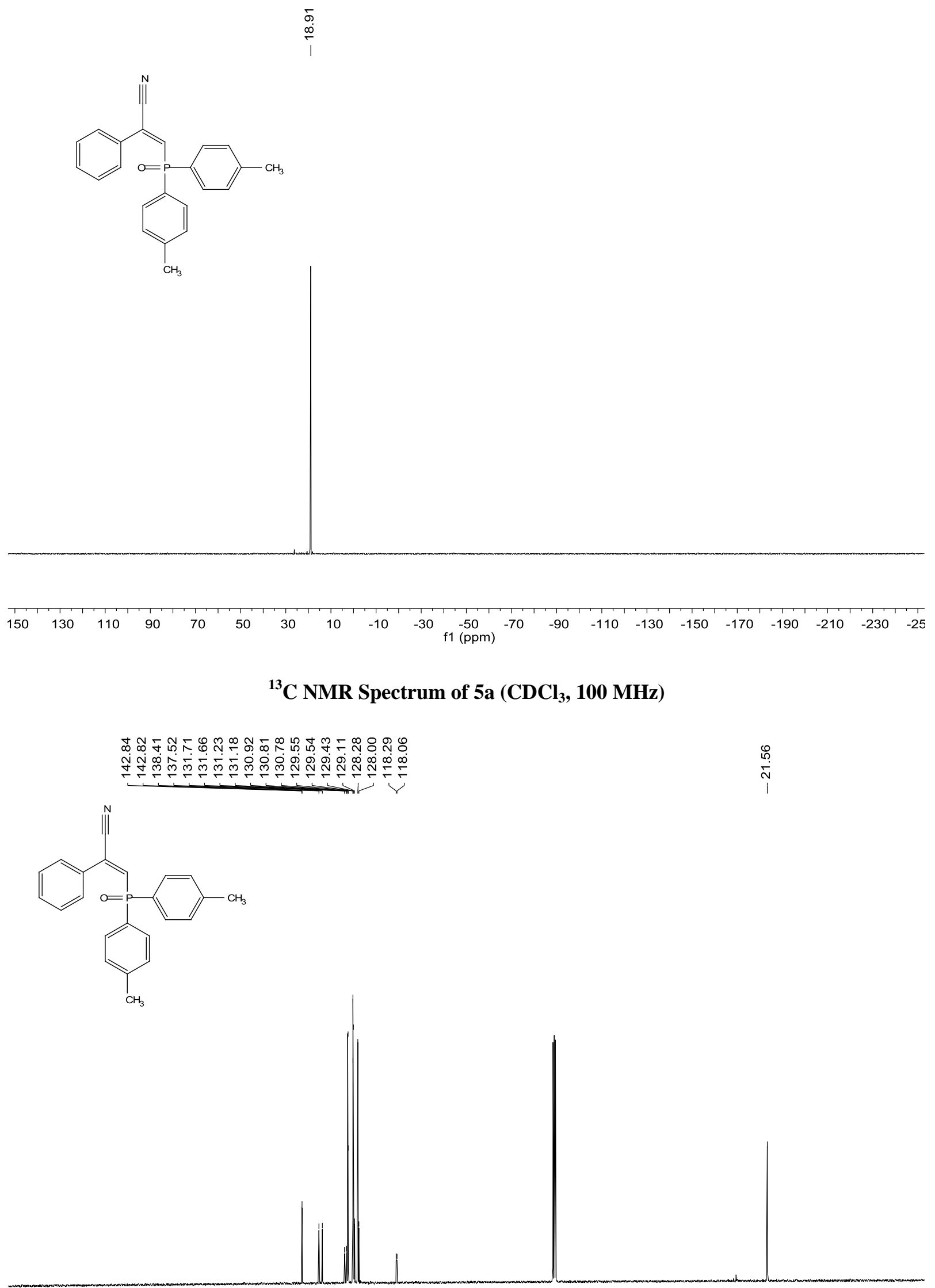

$\begin{array}{lllllllllllllllllllllll}210 & 200 & 190 & 180 & 170 & 160 & 150 & 140 & 130 & 120 & 110 \begin{array}{c}100 \\ \mathrm{f} 1(\mathrm{ppm})\end{array} & 90 & 80 & 70 & 60 & 50 & 40 & 30 & 20 & 10 & 0 & -10\end{array}$ 
${ }^{1} \mathrm{H}$ NMR Spectrum of $5 \mathrm{~b}\left(\mathrm{CDCl}_{3}, 400 \mathrm{MHz}\right)$

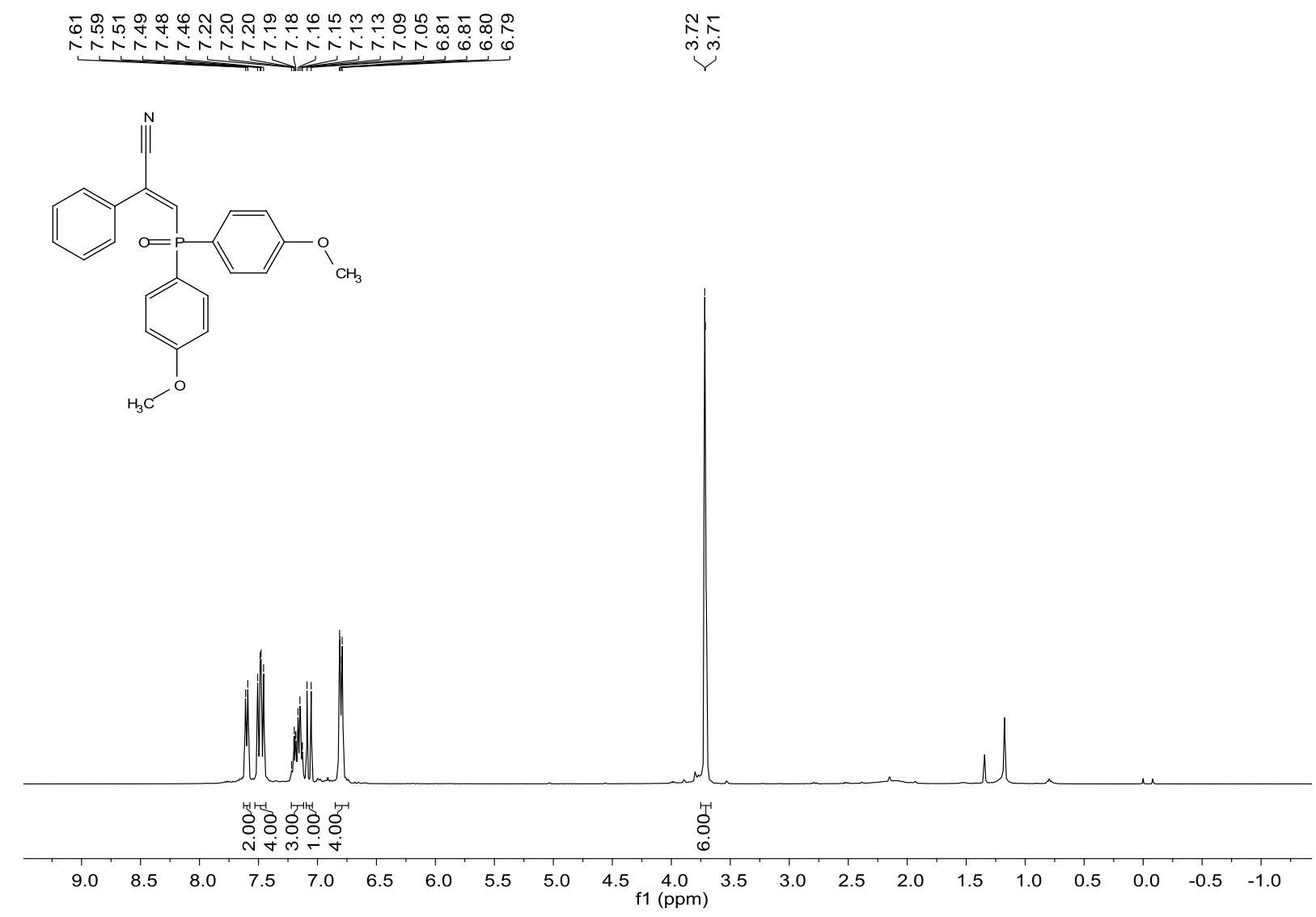

${ }^{31} \mathrm{P}$ NMR Spectrum of $5 \mathrm{~b}\left(\mathrm{CDCl}_{3}, 162 \mathrm{MHz}\right)$

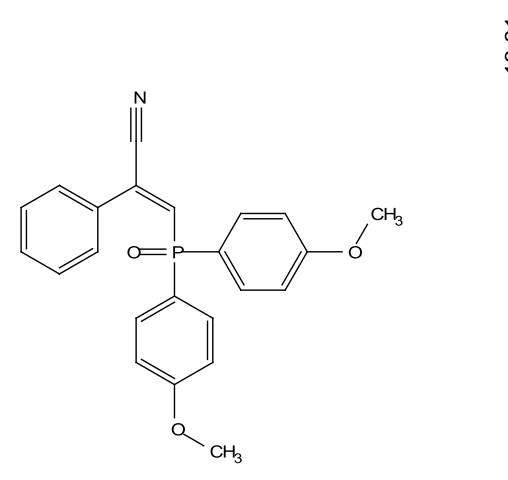

$\underset{\substack{\infty \\ \infty}}{\stackrel{\infty}{\infty}}$

$\begin{array}{lllllllllllllllllllllll}150 & 130 & 110 & 90 & 70 & 50 & 30 & 10 & -10 & -30 & -50 & -70 & -90 & -110 & -130 & -150 & -170 & -190 & -210 & -230 & -25\end{array}$ 
${ }^{13} \mathrm{C}$ NMR Spectrum of $5 \mathrm{~b}\left(\mathrm{CDCl}_{3}, 100 \mathrm{MHz}\right)$
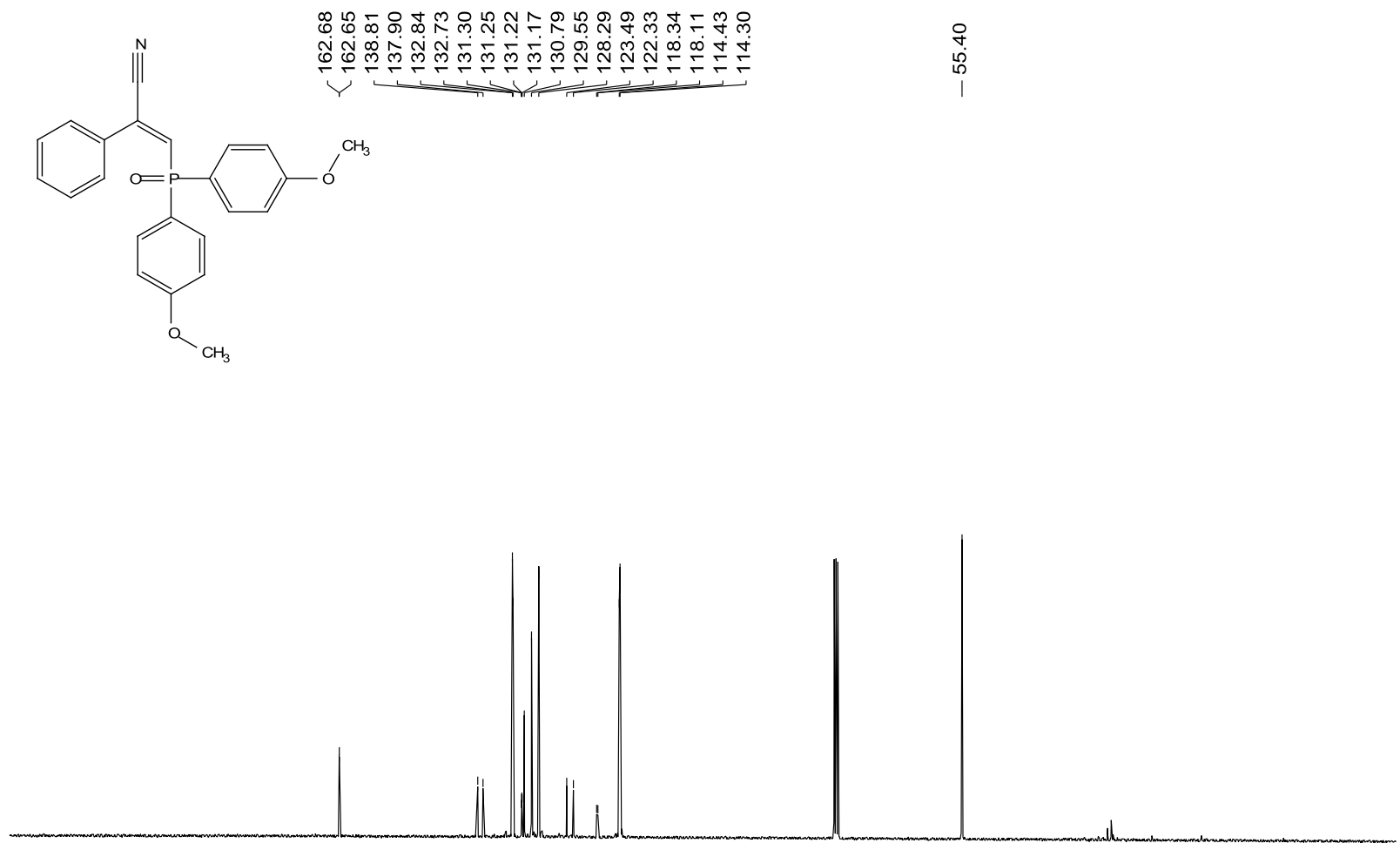

$\begin{array}{llllllllllllllllllllllll}210 & 200 & 190 & 180 & 170 & 160 & 150 & 140 & 130 & 120 & 110 & 100 & 90 & 80 & 70 & 60 & 50 & 40 & 30 & 20 & 10 & 0 & -10\end{array}$

${ }^{1} \mathrm{H}$ NMR Spectrum of $5 \mathrm{c}\left(\mathrm{CDCl}_{3}, 400 \mathrm{MHz}\right)$

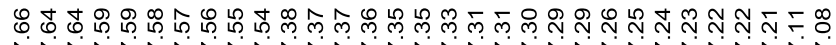

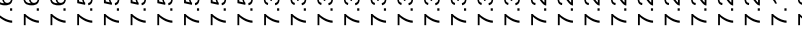
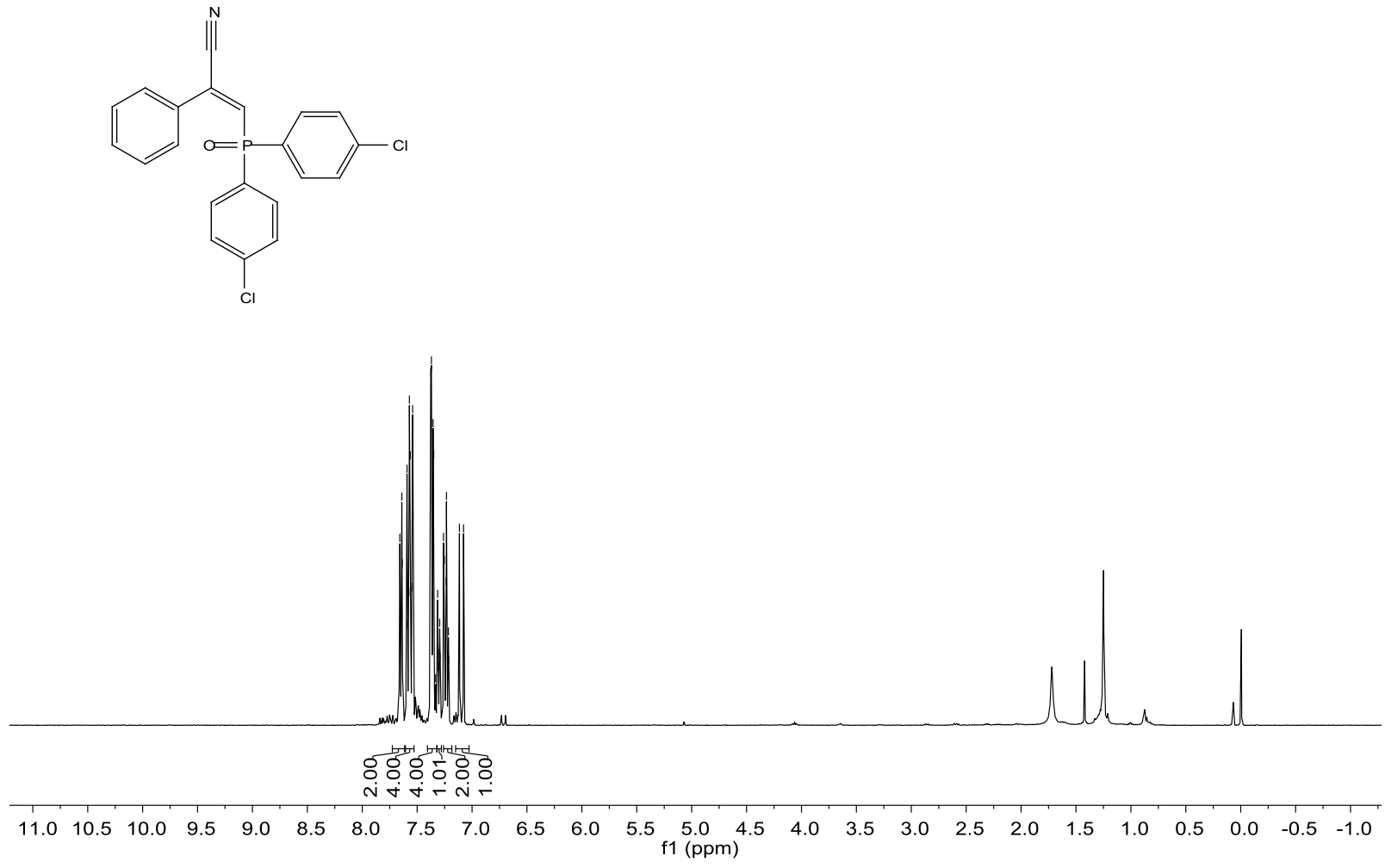
${ }^{31} \mathrm{P}$ NMR Spectrum of $5 \mathrm{c}\left(\mathrm{CDCl}_{3}, 162 \mathrm{MHz}\right)$

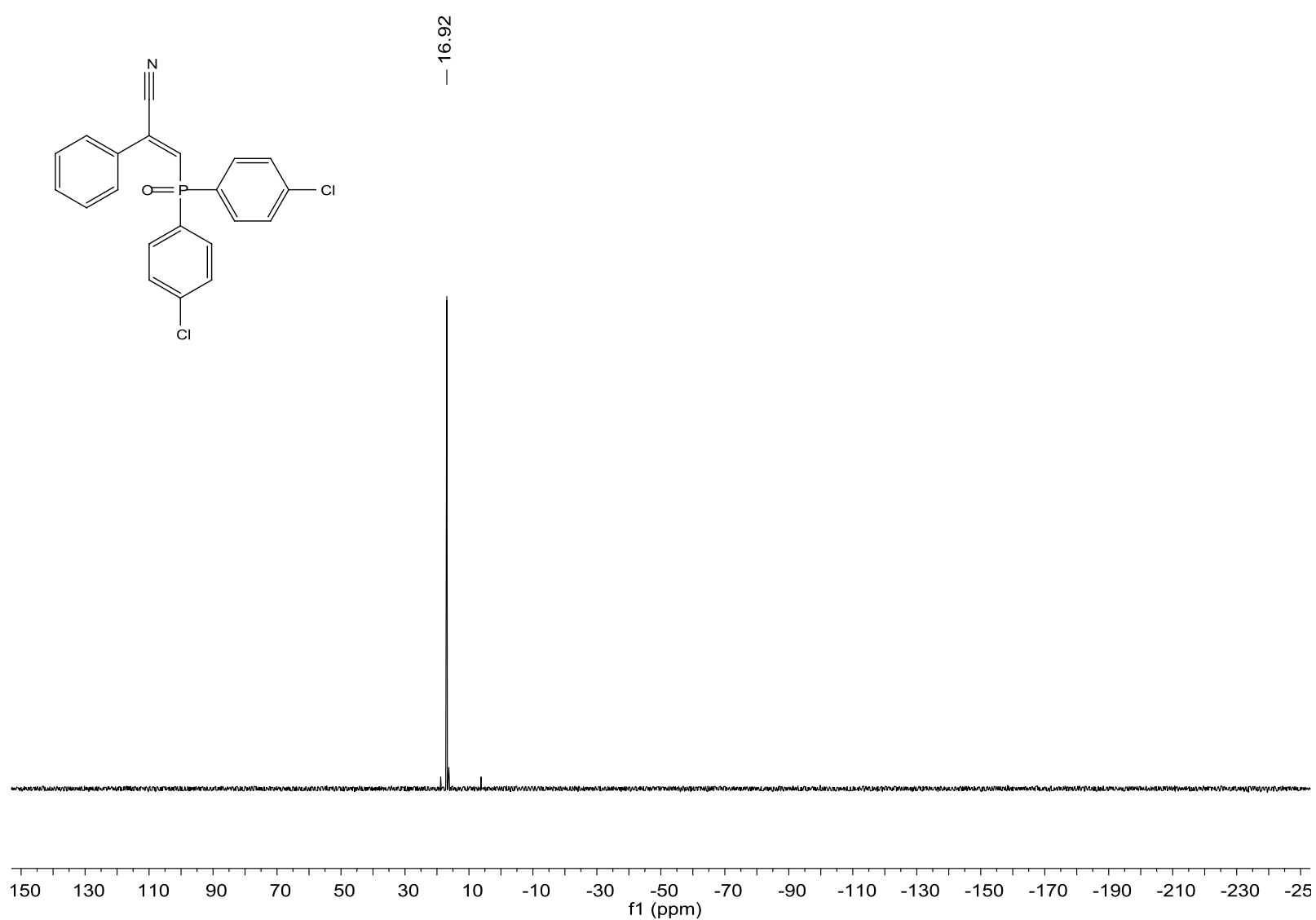

${ }^{13} \mathrm{C}$ NMR Spectrum of $5 \mathrm{c}\left(\mathrm{CDCl}_{3}, 100 \mathrm{MHz}\right)$

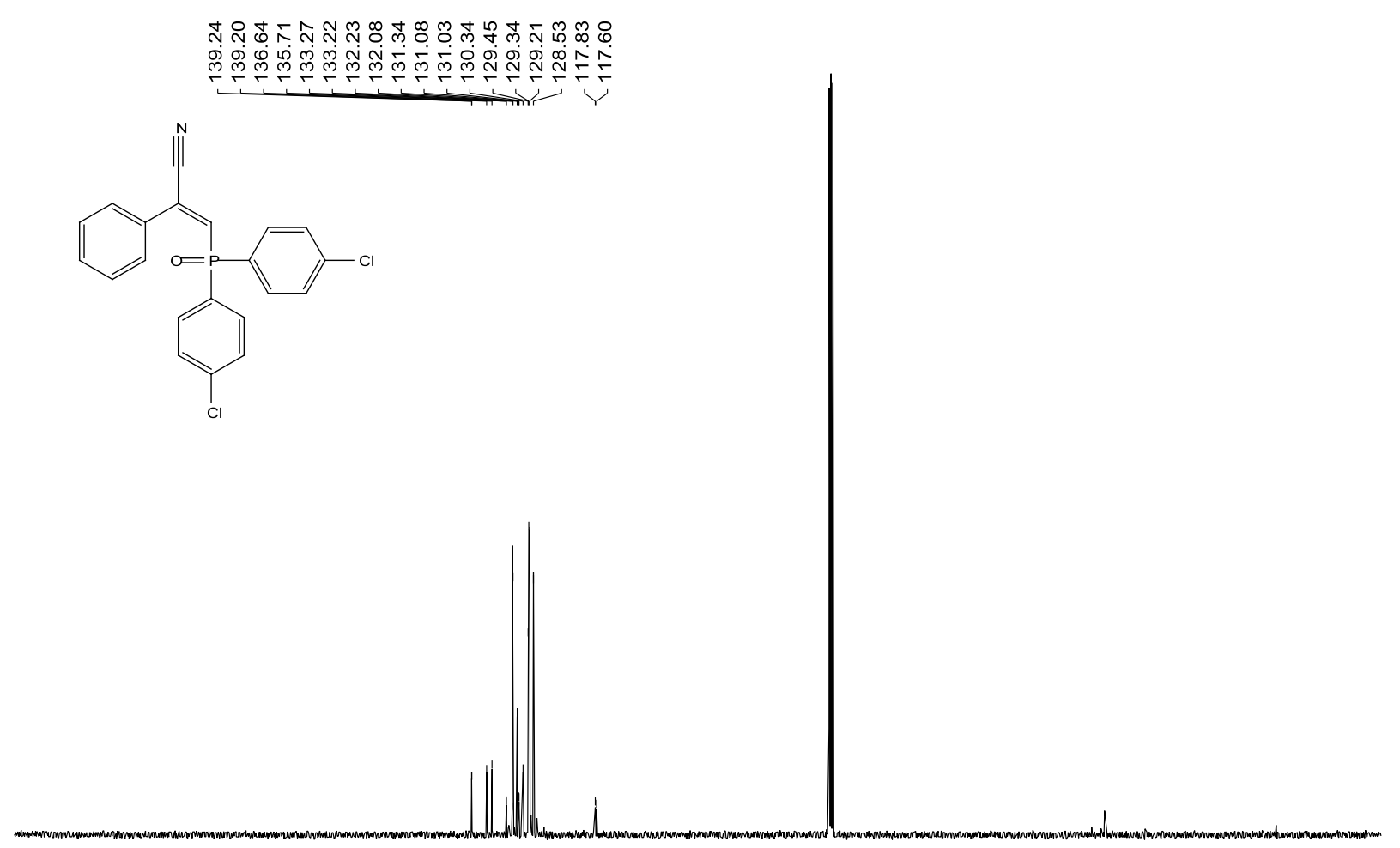

$\begin{array}{lllllllllllllllllllllllll}210 & 200 & 190 & 180 & 170 & 160 & 150 & 140 & 130 & 120 & 110 & 100 & 90 & 80 & 70 & 60 & 50 & 40 & 30 & 20 & 10 & 0 & -10\end{array}$ 
<smiles>N#C/C(=C/C(=O)c1cccc2ccccc12)c1cccc2ccccc12</smiles>

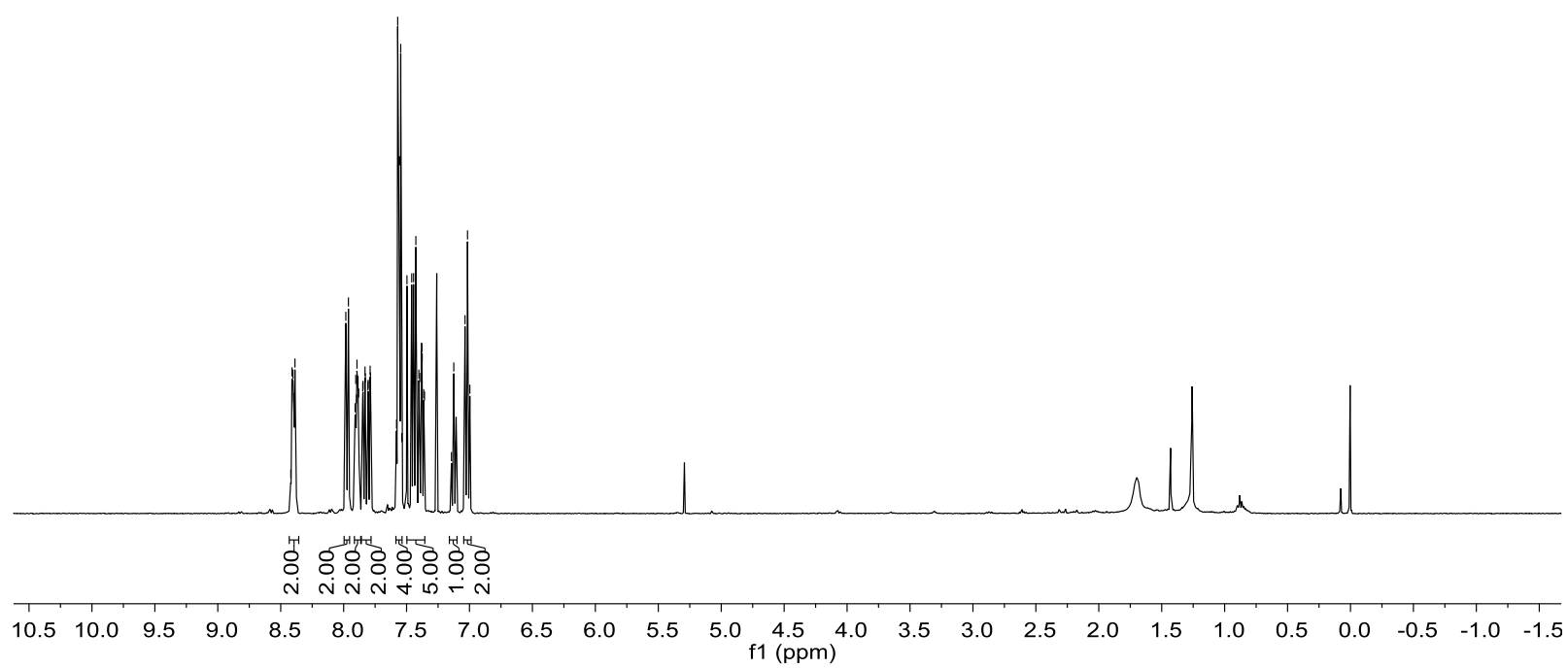

${ }^{31} \mathrm{P}$ NMR Spectrum of $5 \mathrm{~d}\left(\mathrm{CDCl}_{3}, 162 \mathrm{MHz}\right)$

ลิ

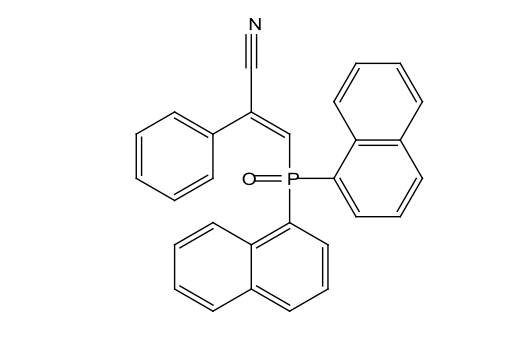

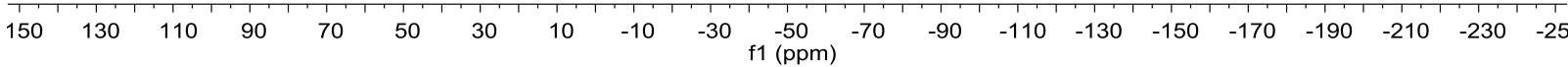


${ }^{13} \mathrm{C}$ NMR Spectrum of $5 \mathrm{~d}\left(\mathrm{CDCl}_{3}, 100 \mathrm{MHz}\right)$

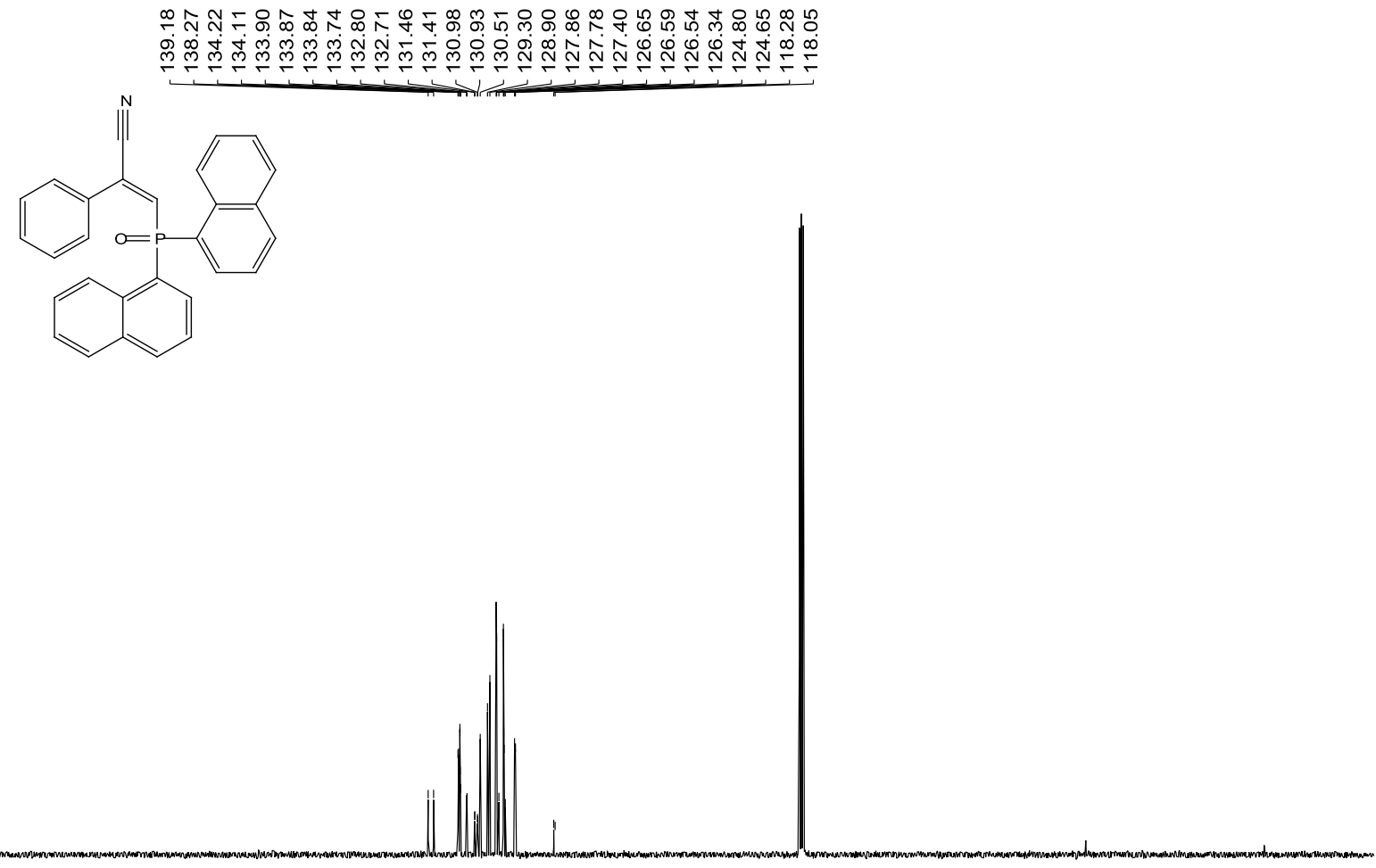

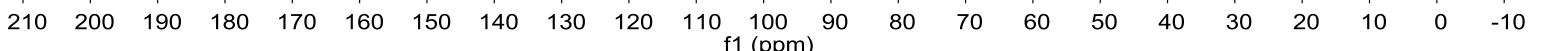

${ }^{1} \mathrm{H}$ NMR Spectrum of $5 \mathrm{e}\left(\mathrm{CDCl}_{3}, 400 \mathrm{MHz}\right)$

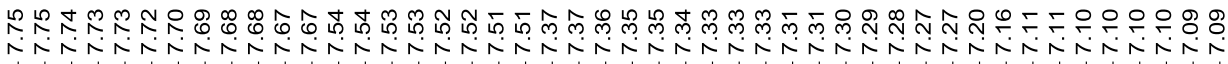
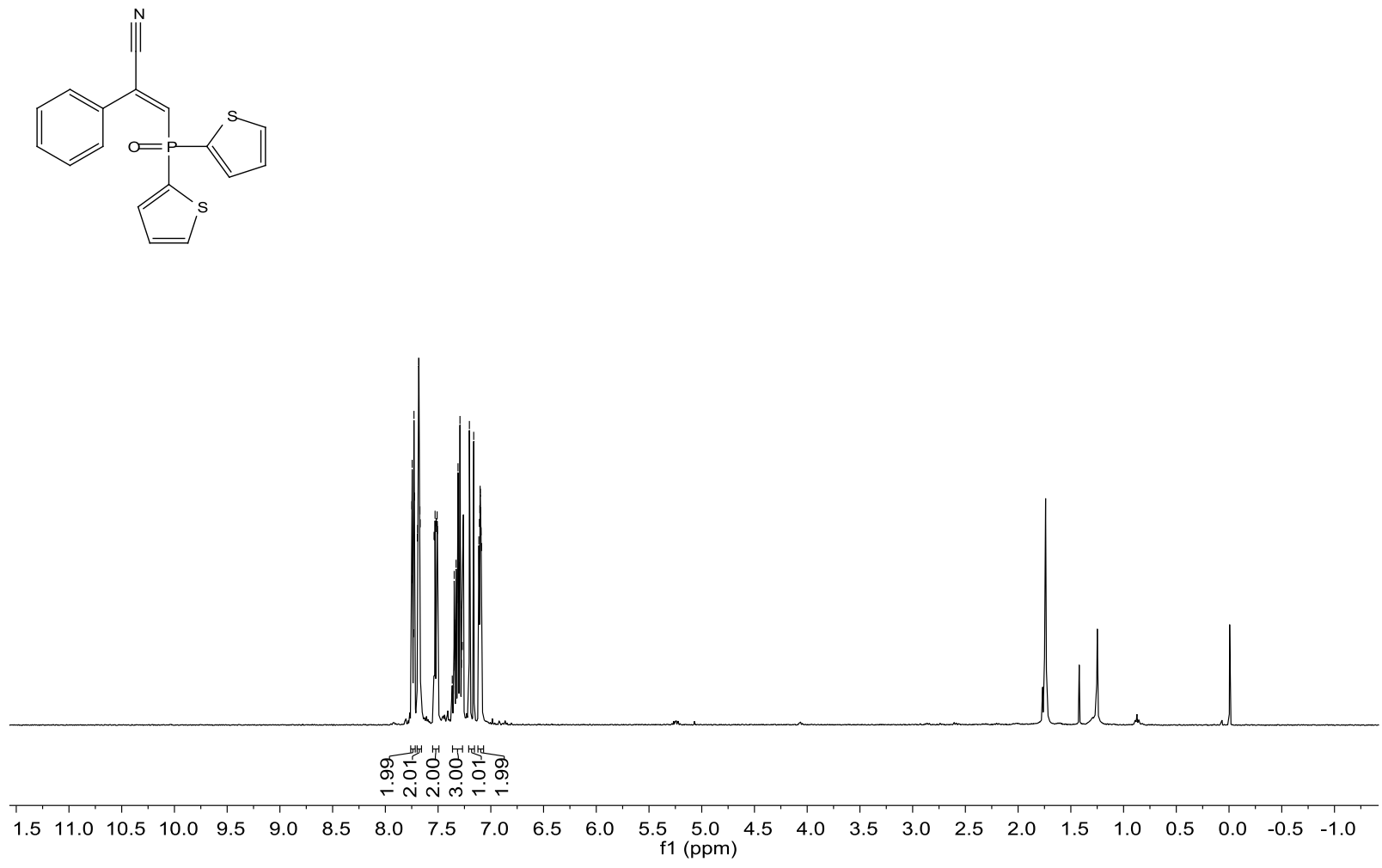
${ }^{31} \mathrm{P}$ NMR Spectrum of $5 \mathrm{e}\left(\mathrm{CDCl}_{3}, 162 \mathrm{MHz}\right)$

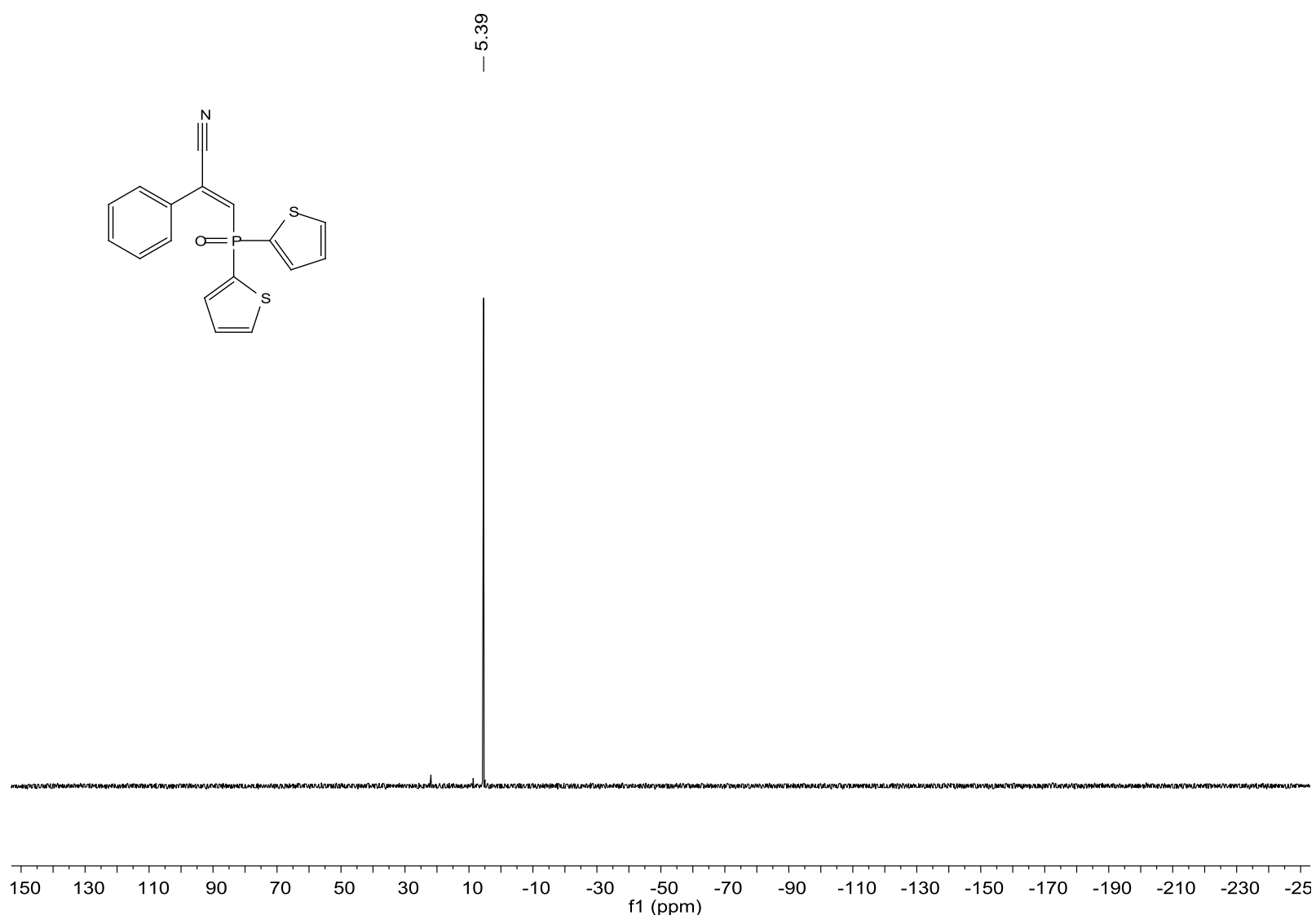

${ }^{13} \mathrm{C}$ NMR Spectrum of $5 \mathrm{e}\left(\mathrm{CDCl}_{3}, 100 \mathrm{MHz}\right)$

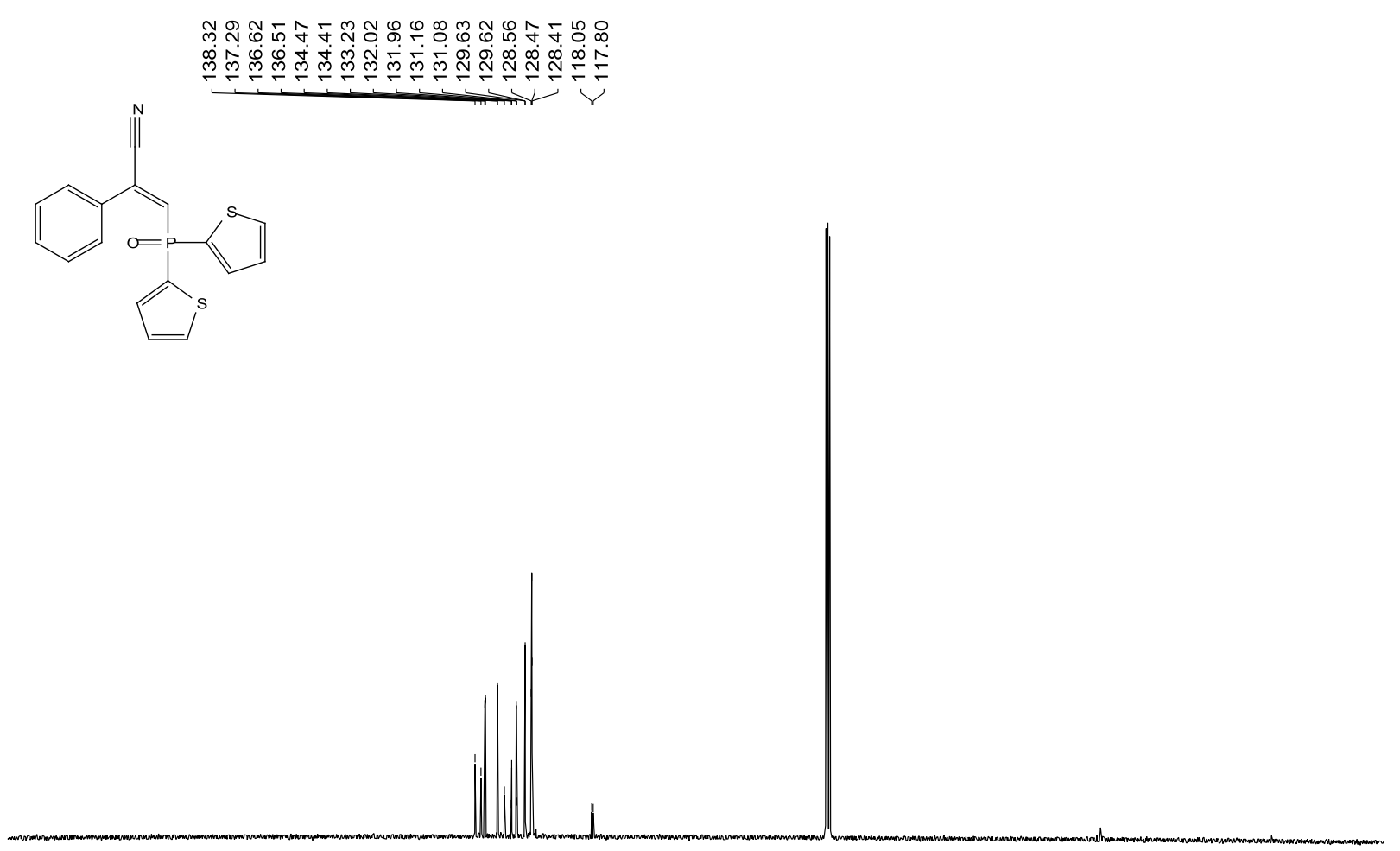

$\begin{array}{llllllllllllllllllllllllll}210 & 200 & 190 & 180 & 170 & 160 & 150 & 140 & 130 & 120 & 110 & 100 & 90 & 80 & 70 & 60 & 50 & 40 & 30 & 20 & 10 & 0 & -10\end{array}$ 
${ }^{1} \mathrm{H}$ NMR Spectrum of $5 \mathrm{~g}\left(\mathrm{CDCl}_{3}, 400 \mathrm{MHz}\right)$

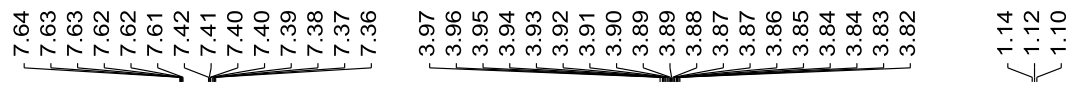<smiles>CCOP(=O)(/C=C(/C#N)c1ccccc1)OCC</smiles>

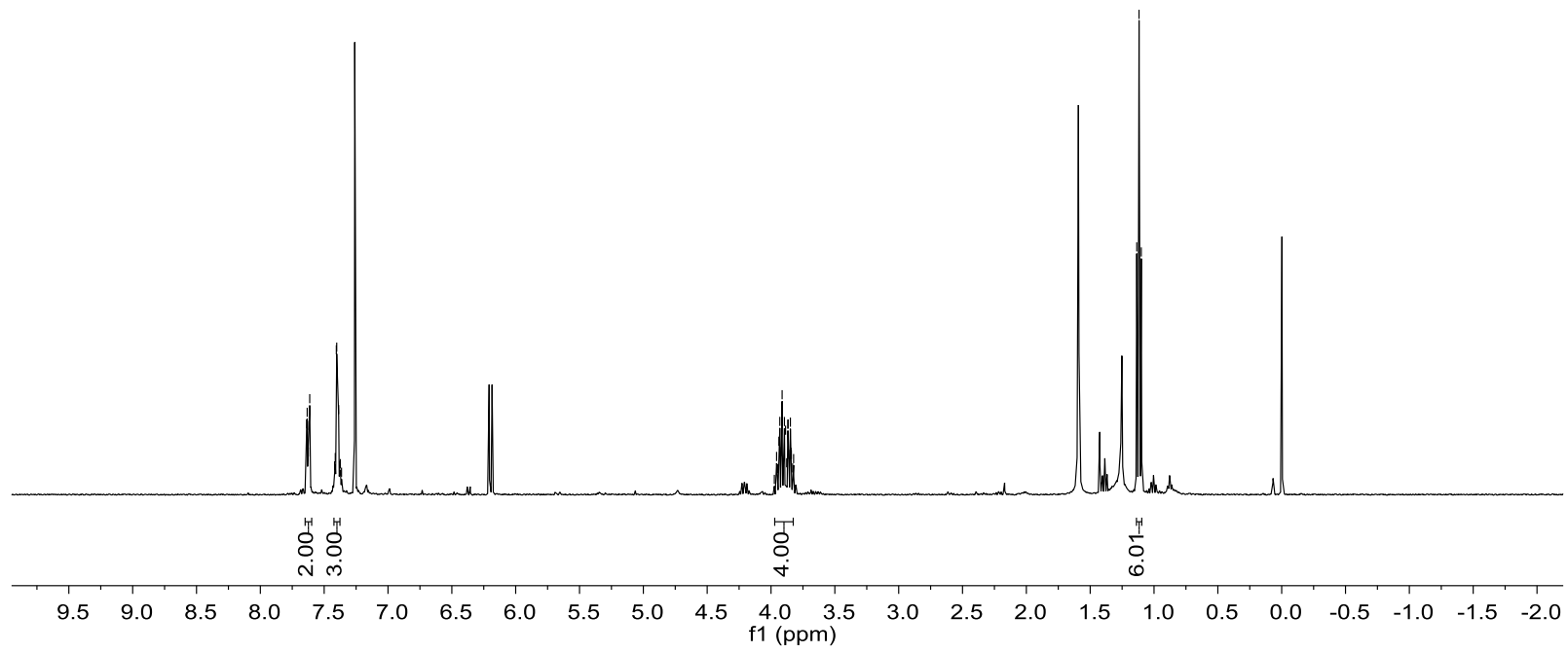

${ }^{31} \mathrm{P}$ NMR Spectrum of $5 \mathrm{~g}\left(\mathrm{CDCl}_{3}, 162 \mathrm{MHz}\right)$

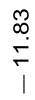<smiles></smiles>

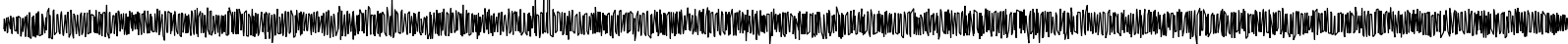

$\begin{array}{llllllllllllllllllllll}150 & 130 & 110 & 90 & 70 & 50 & 30 & 10 & -10 & -30 & \begin{array}{c}-50 \\ \mathrm{fppm})\end{array} & -70 & -90 & -110 & -130 & -150 & -170 & -190 & -210 & -230 & -25\end{array}$ 
${ }^{13} \mathrm{C}$ NMR Spectrum of $5 \mathrm{~g}\left(\mathrm{CDCl}_{3}, 100 \mathrm{MHz}\right)$

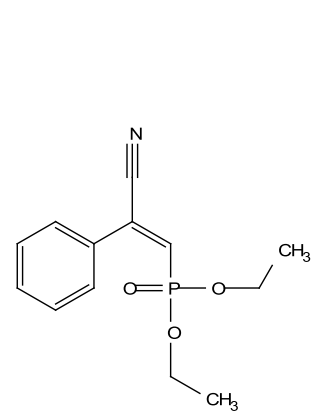

${ }^{1} \mathrm{H}$ NMR Spectrum of $5 \mathrm{~h}\left(\mathrm{CDCl}_{3}, 400 \mathrm{MHz}\right)$

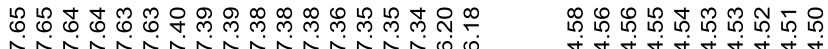

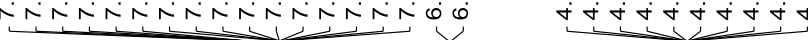<smiles>C=C(C=P(OC(C)C)(OC(C)C)OC(C)O)c1ccccc1</smiles>

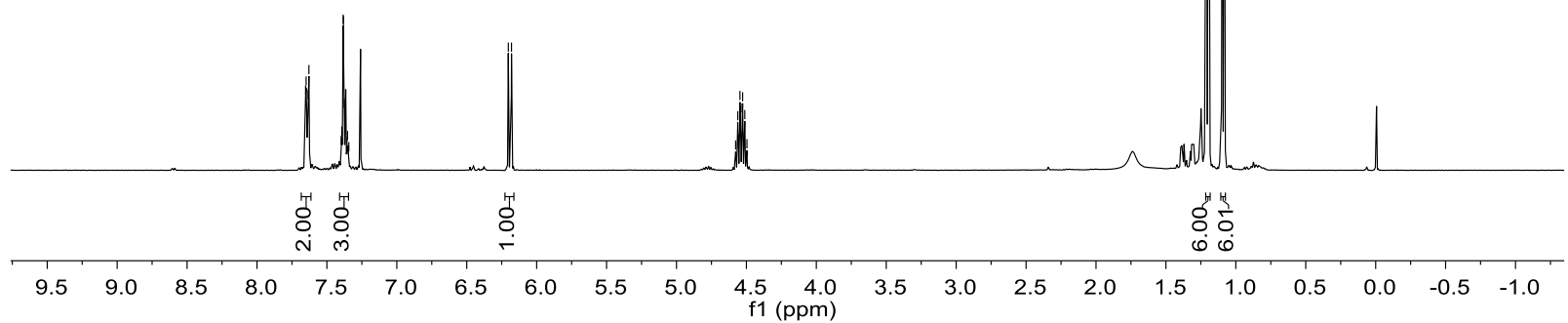


${ }^{31} \mathrm{P}$ NMR Spectrum of $5 \mathrm{~h}\left(\mathrm{CDCl}_{3}, 162 \mathrm{MHz}\right)$

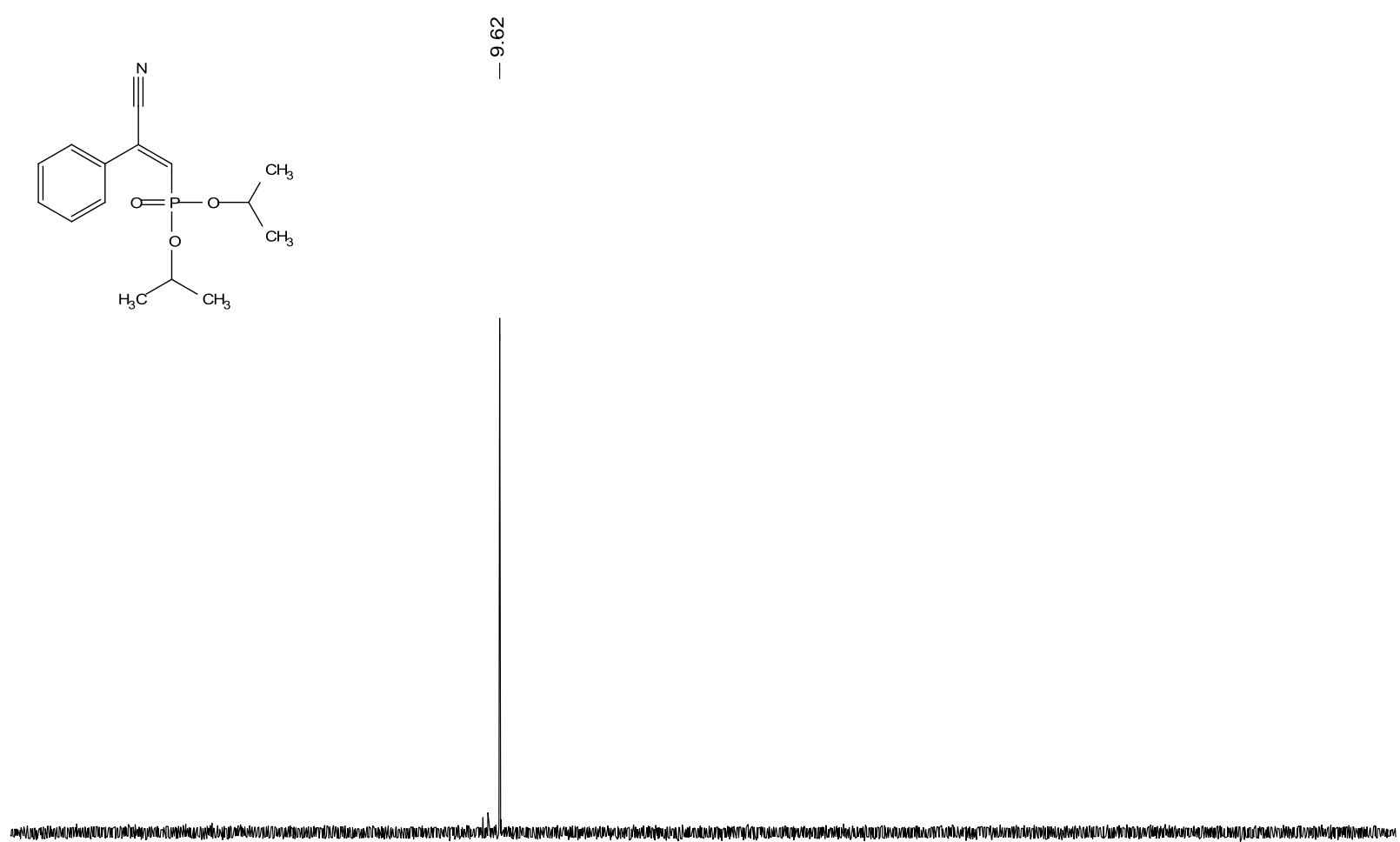

${ }^{13} \mathrm{C}$ NMR Spectrum of $5 \mathrm{~h}\left(\mathrm{CDCl}_{3}, 100 \mathrm{MHz}\right)$
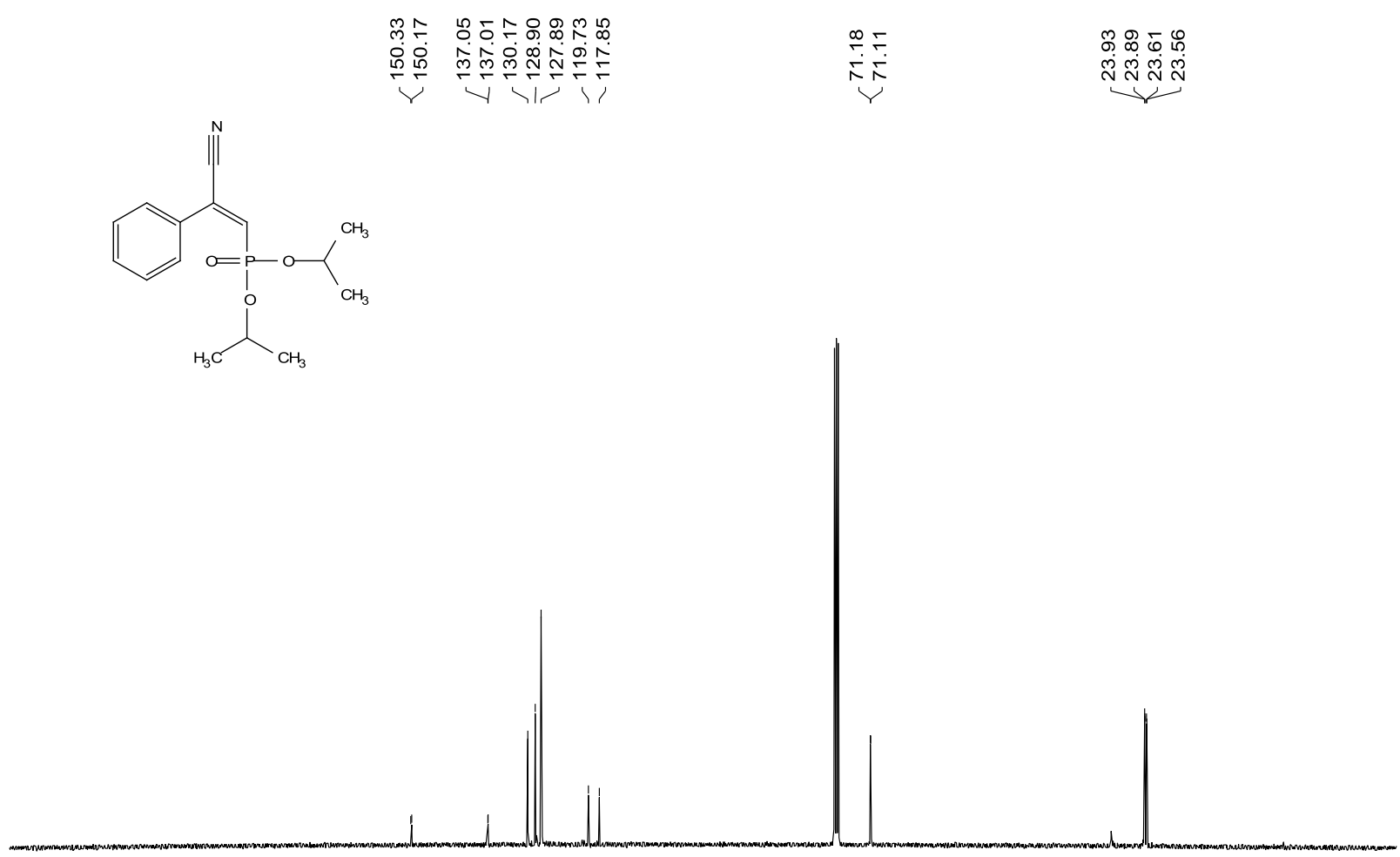

$\begin{array}{llllllllllllllllllllllll}210 & 200 & 190 & 180 & 170 & 160 & 150 & 140 & 130 & 120 & 110 & \begin{array}{l}100 \\ \mathrm{f} 1\end{array}(\mathrm{ppm}) & 90 & 80 & 70 & 60 & 50 & 40 & 30 & 20 & 10 & 0 & -10\end{array}$ 


\section{${ }^{1} \mathrm{H}$ NMR Spectrum of $5 \mathrm{i}{ }^{\prime}\left(\mathrm{CDCl}_{3}, 400 \mathrm{MHz}\right)$}

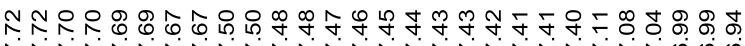

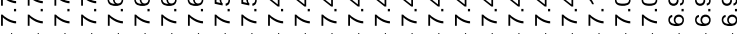

$\stackrel{\stackrel{n}{0}}{0}$
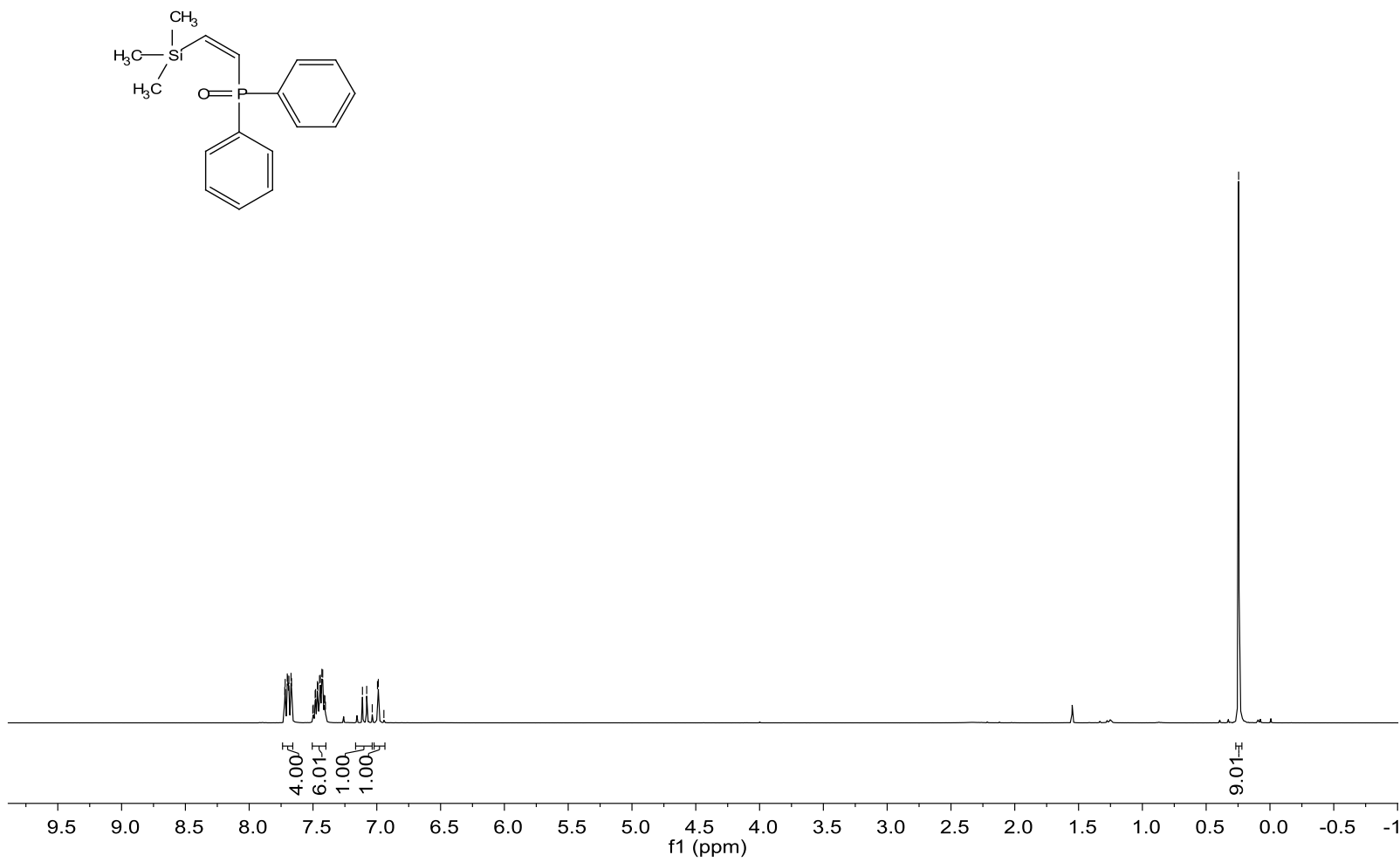

${ }^{31}$ P NMR Spectrum of 5i' $\left(\mathrm{CDCl}_{3}, 162 \mathrm{MHz}\right)$

$\stackrel{\text { ? }}{\stackrel{\text { s }}{*}}$

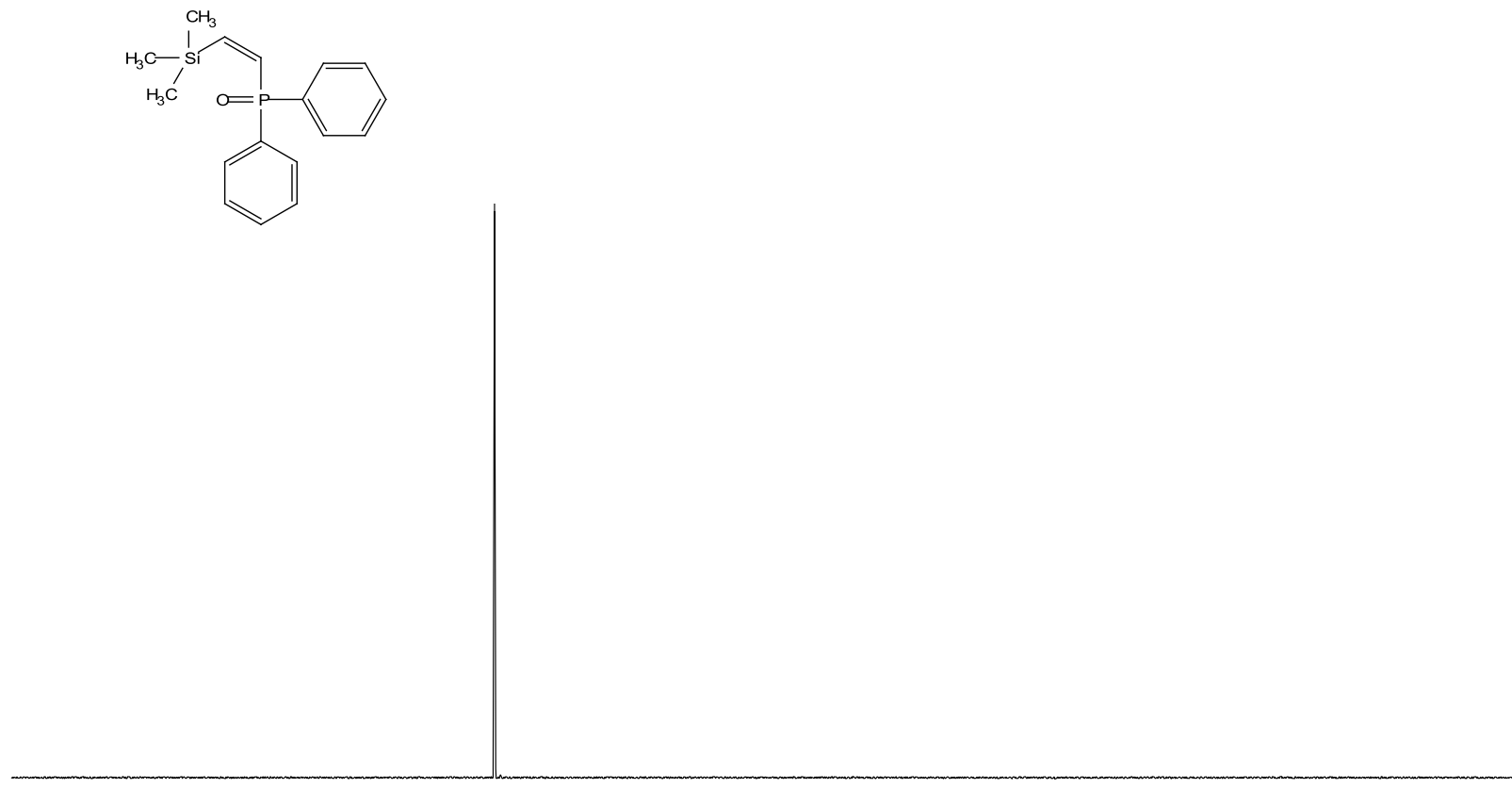

$\begin{array}{llllllllllllllllllll}140 & 120 & 100 & 80 & 60 & 40 & 20 & 0 & -20 & -40 & -60 & -80 & -100 & -120 & -140 & -160 & -180 & -200 & -220 & -240\end{array}$ 
${ }^{13} \mathrm{C}$ NMR Spectrum of $5 \mathrm{i}$ ' $\left(\mathrm{CDCl}_{3}, 100 \mathrm{MHz}\right)$

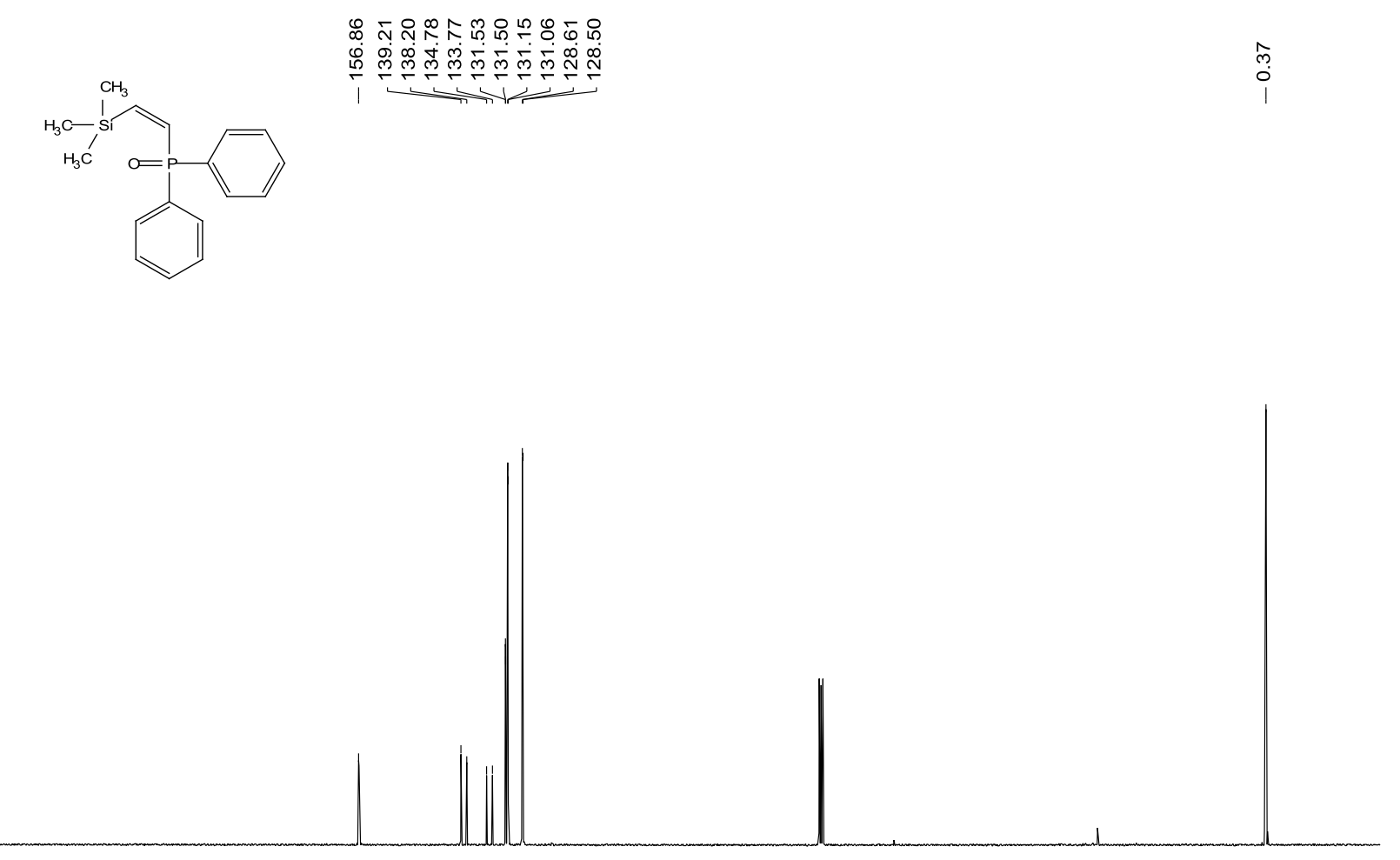

$\begin{array}{llllllllllllllllllllllllll}210 & 200 & 190 & 180 & 170 & 160 & 150 & 140 & 130 & 120 & 110 & 100 & 90 & 80 & 70 & 60 & 50 & 40 & 30 & 20 & 10 & 0 & -10\end{array}$

${ }^{1} \mathrm{H}$ NMR Spectrum of $5 \mathbf{j}\left(\mathrm{CDCl}_{3}, 400 \mathrm{MHz}\right)$

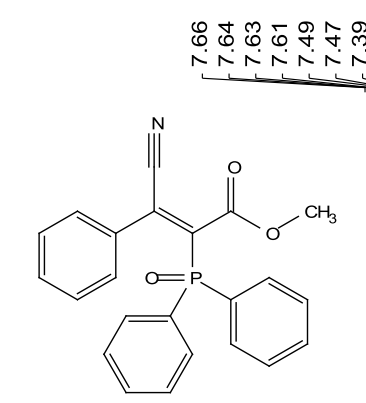

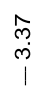

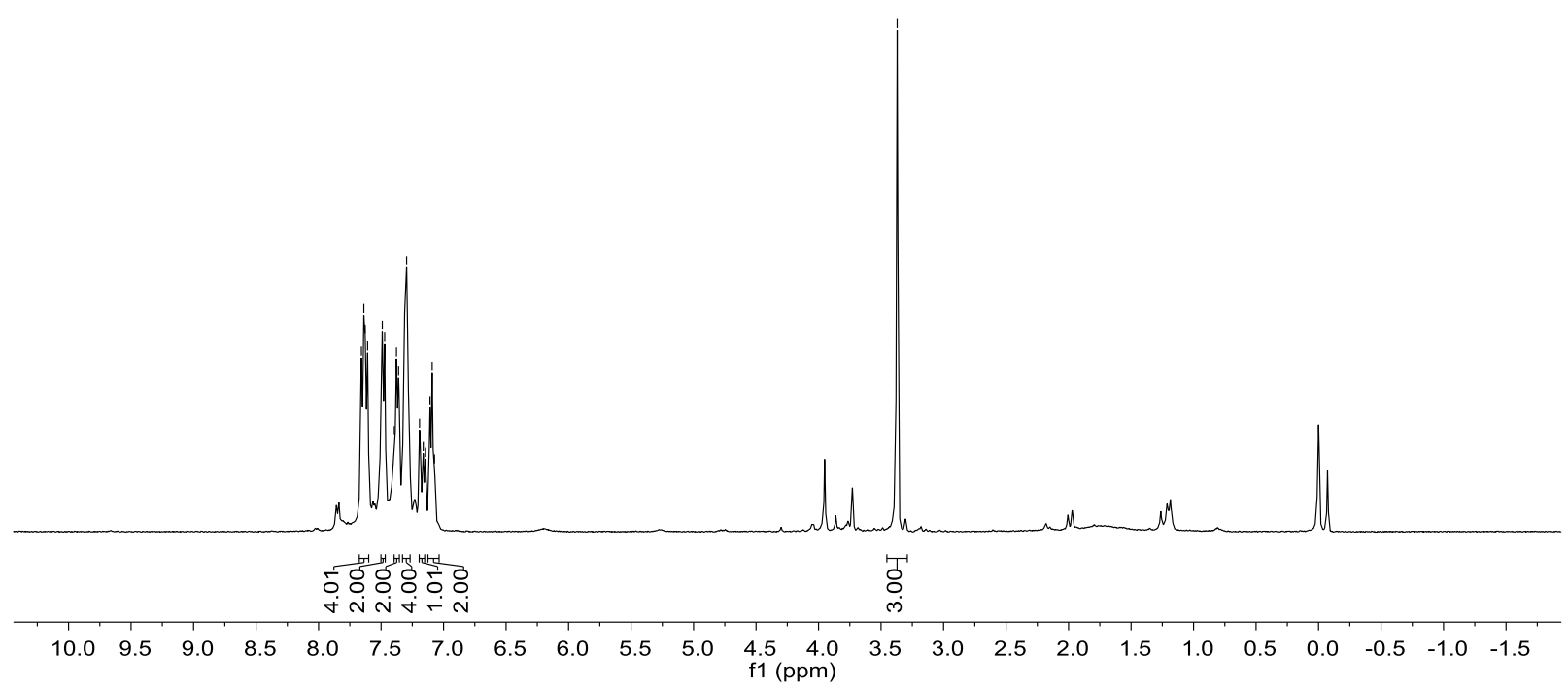


${ }^{31}$ P NMR Spectrum of $5 \mathbf{j}\left(\mathrm{CDCl}_{3}, 162 \mathrm{MHz}\right)$

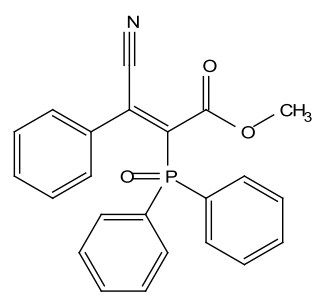

in

and.

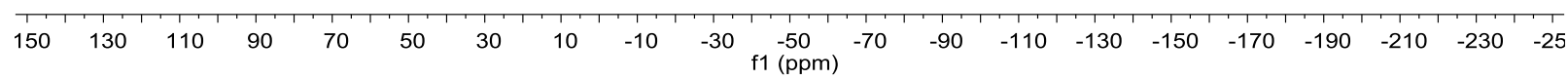

${ }^{13} \mathrm{C}$ NMR Spectrum of $5 \mathrm{j}\left(\mathrm{CDCl}_{3}, 100 \mathrm{MHz}\right)$
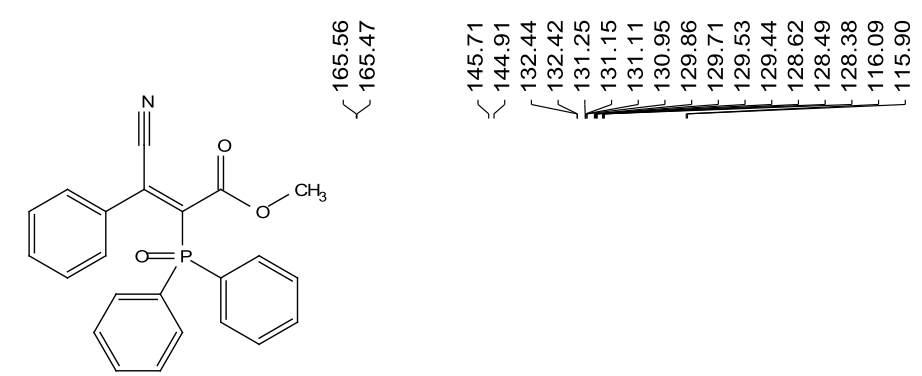

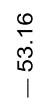

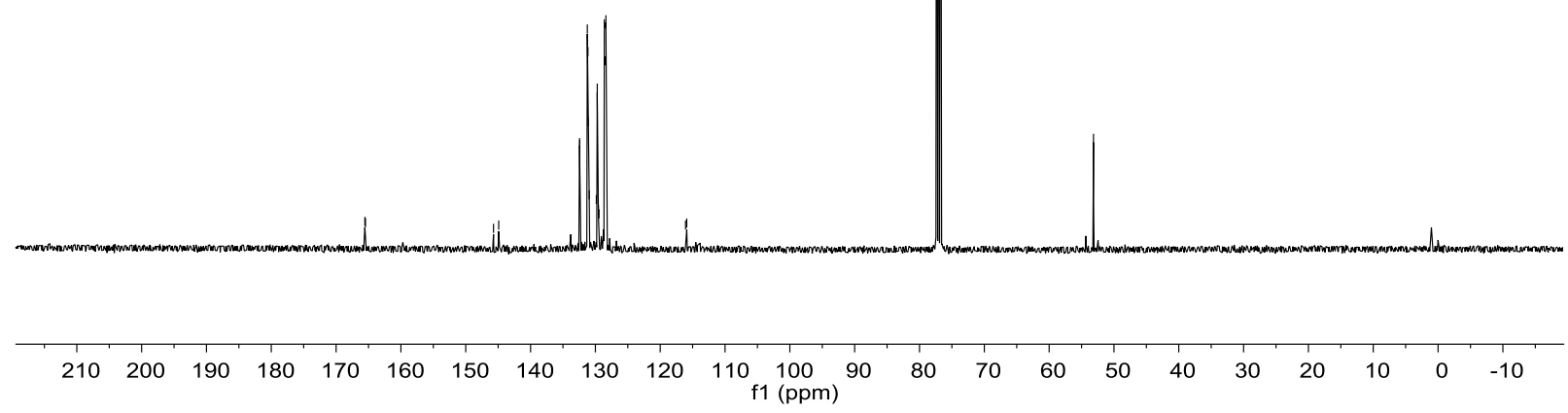

S97 
${ }^{1} \mathrm{H}$ NMR Spectrum of $5 \mathrm{k}^{\prime}\left(\mathrm{CDCl}_{3}, 400 \mathrm{MHz}\right)$

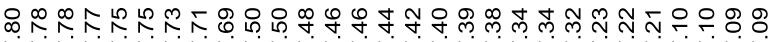

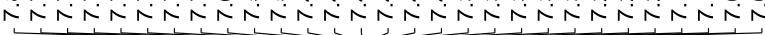
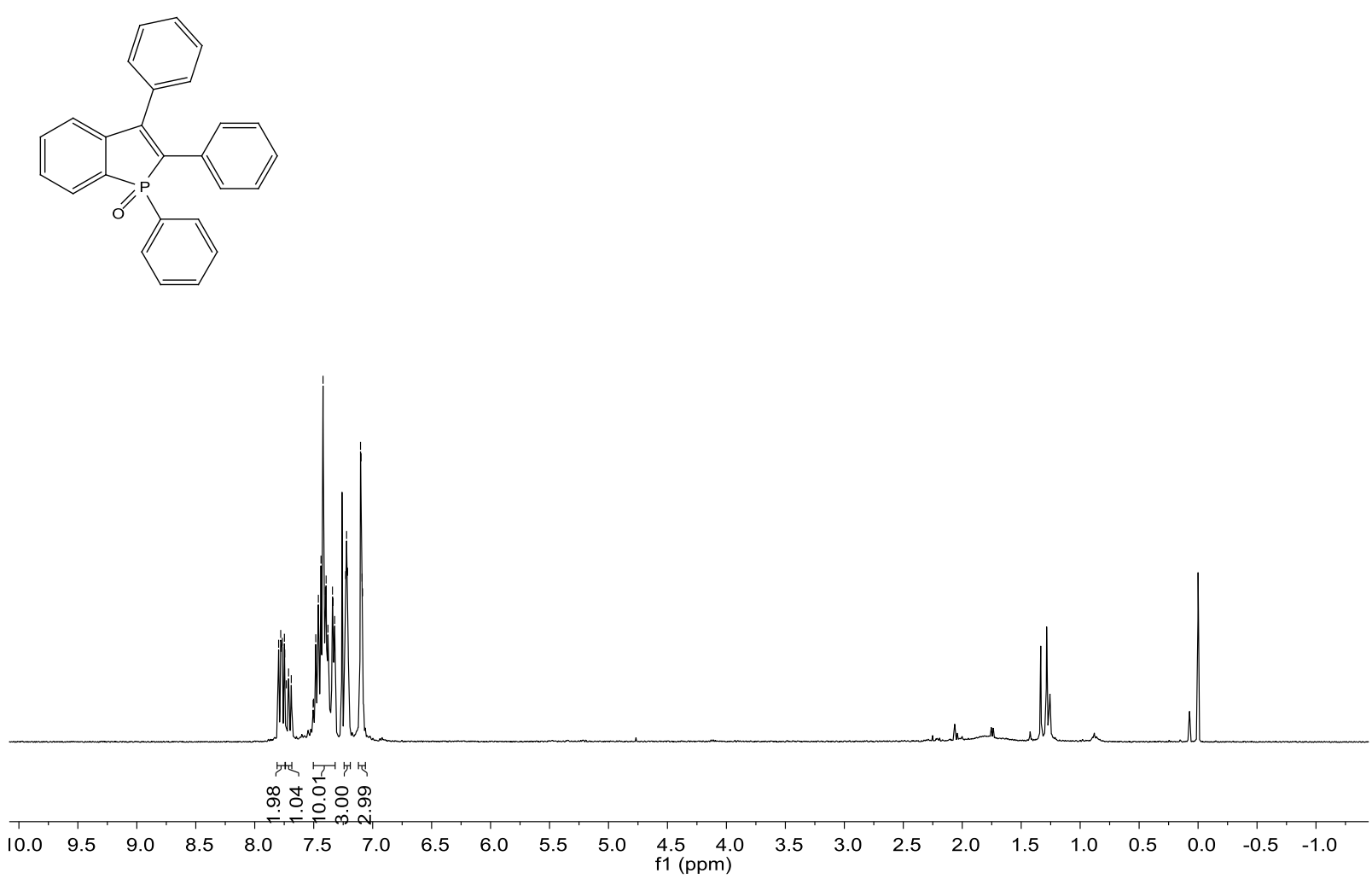

${ }^{31} \mathrm{P}$ NMR Spectrum of $5 \mathrm{k}^{\prime}\left(\mathrm{CDCl}_{3}, 162 \mathrm{MHz}\right)$
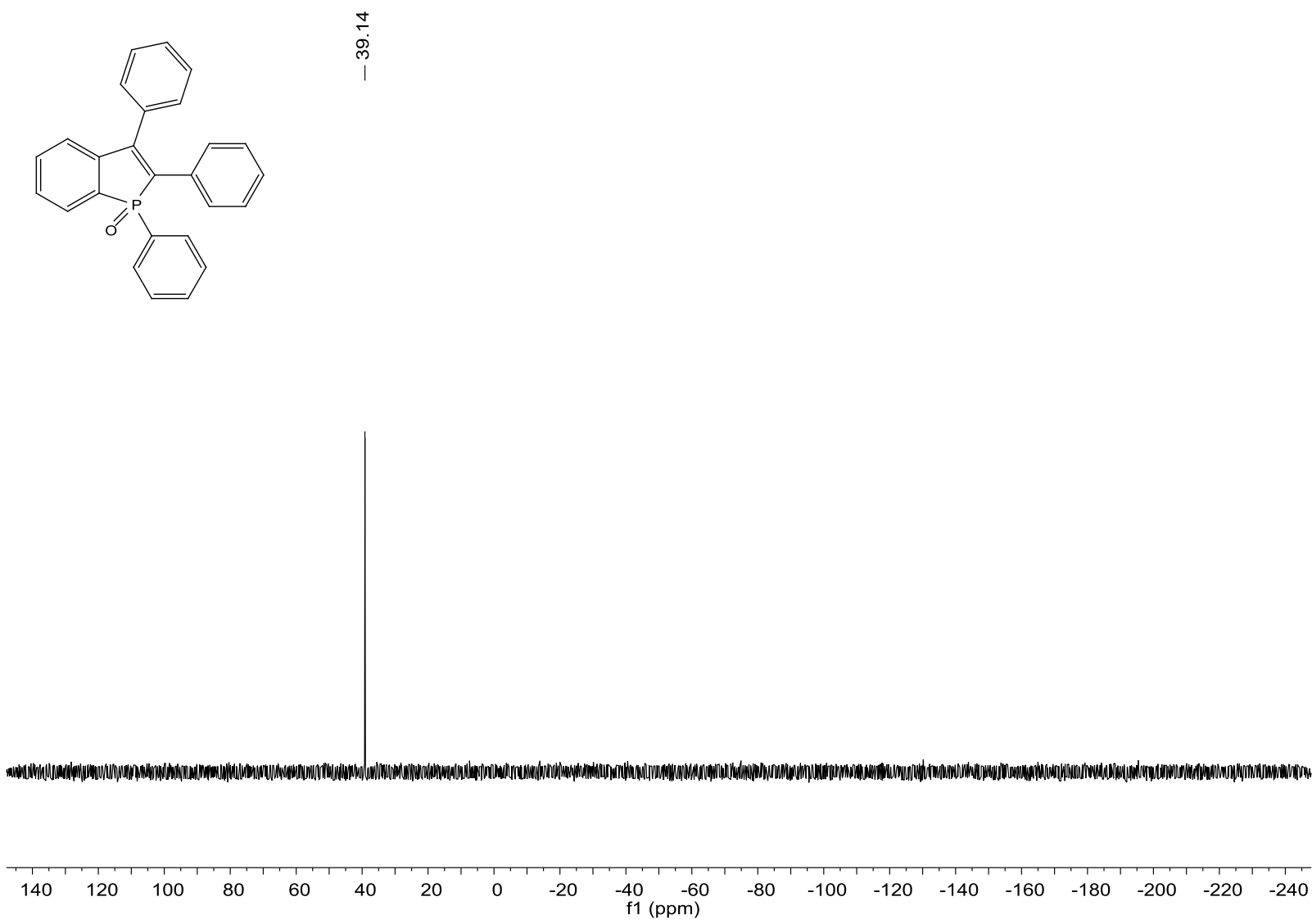
10-2 NMR spectra for thiocyanophosphorylation products 7

\section{${ }^{1} \mathrm{H}$ NMR Spectrum of $7 \mathrm{a}\left(\mathrm{CDCl}_{3}, 400 \mathrm{MHz}\right)$}
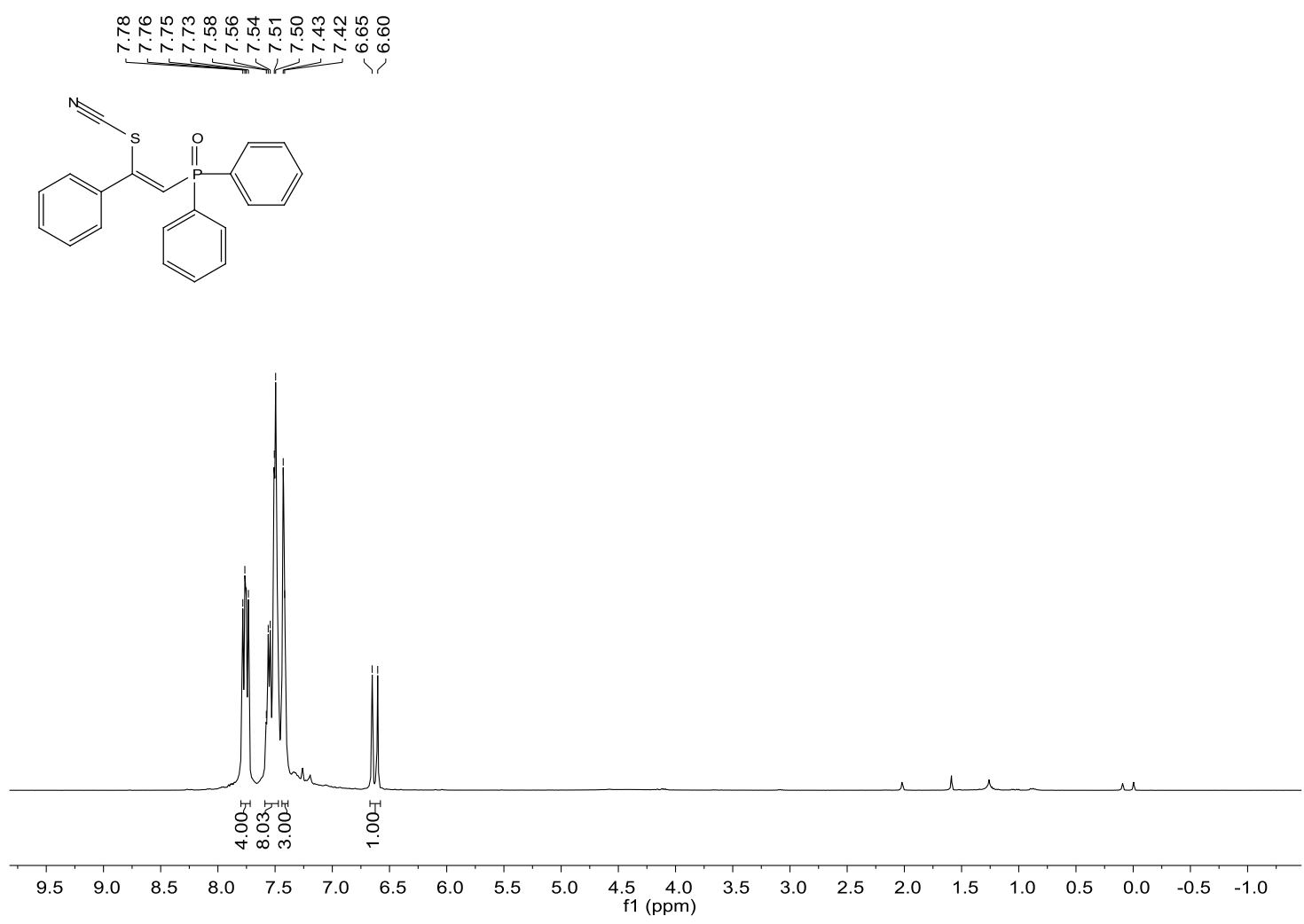

${ }^{31} \mathrm{P}$ NMR Spectrum of $7 \mathrm{a}\left(\mathrm{CDCl}_{3}, 162 \mathrm{MHz}\right)$

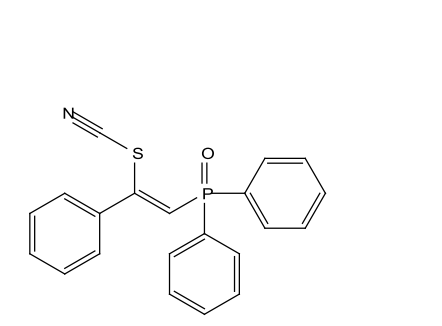

$\stackrel{\substack{n \\ \text { N }}}{1}$

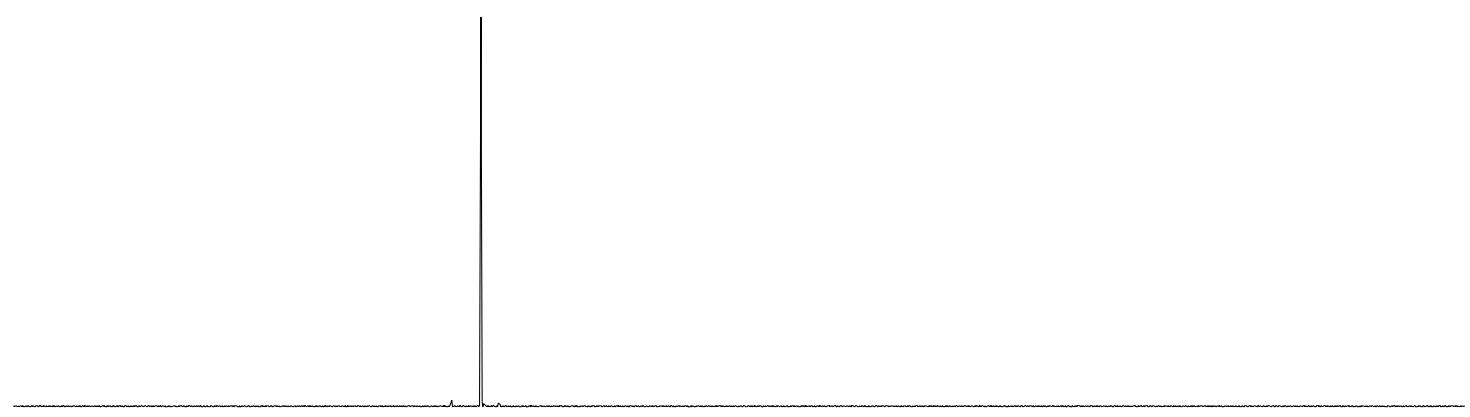

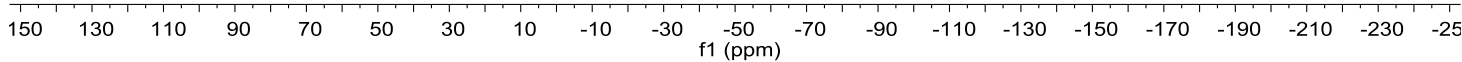



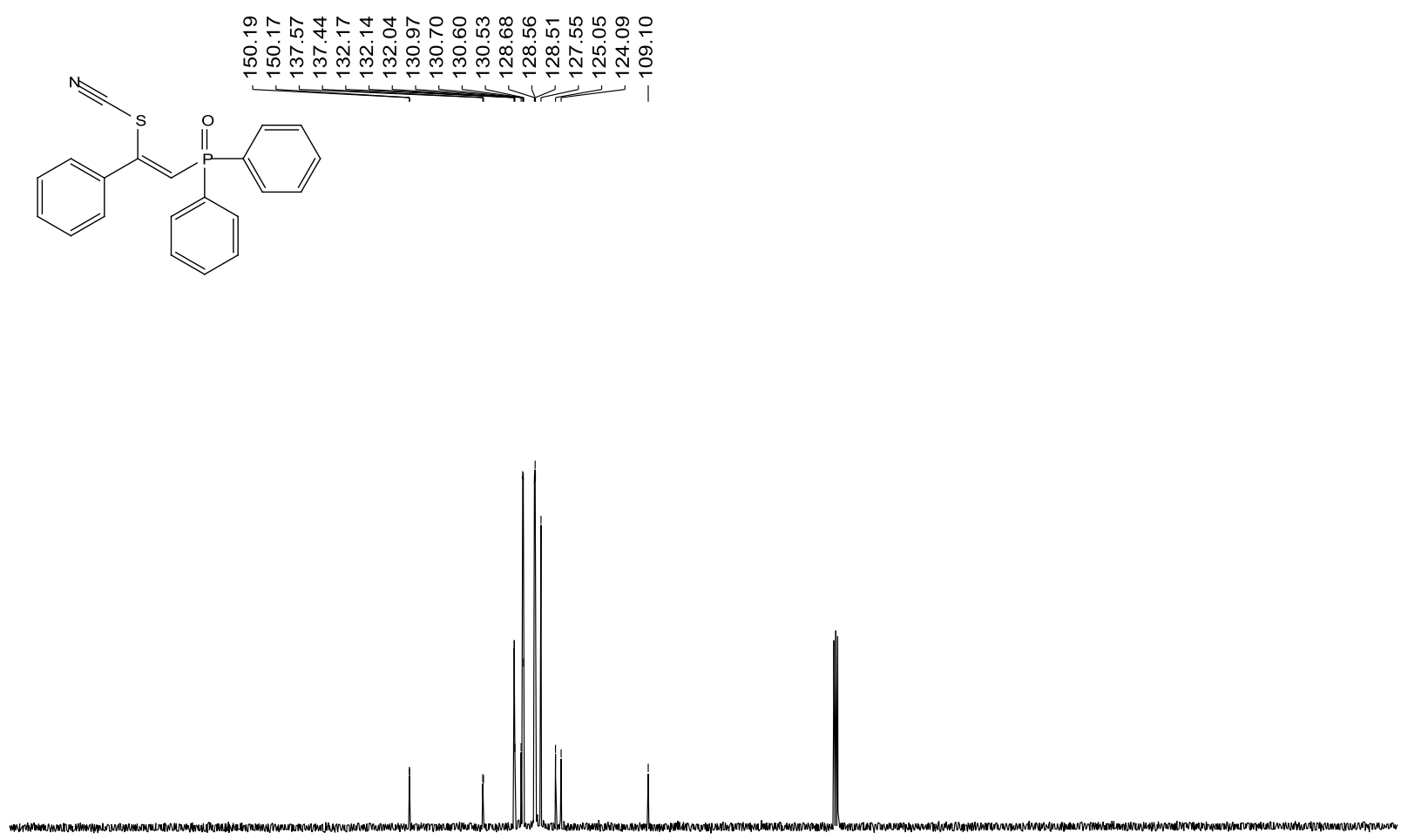

$\begin{array}{lllllllllllllllllllllll}210 & 200 & 190 & 180 & 170 & 160 & 150 & 140 & 130 & 120 & 110 & 100 & 90 & 80 & 70 & 60 & 50 & 40 & 30 & 20 & 10 & 0 & -10\end{array}$

\section{${ }^{1} \mathrm{H}$ NMR Spectrum of $7 \mathrm{~b}\left(\mathrm{CDCl}_{3}, 400 \mathrm{MHz}\right)$}

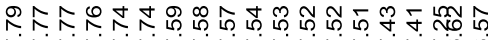

Nico
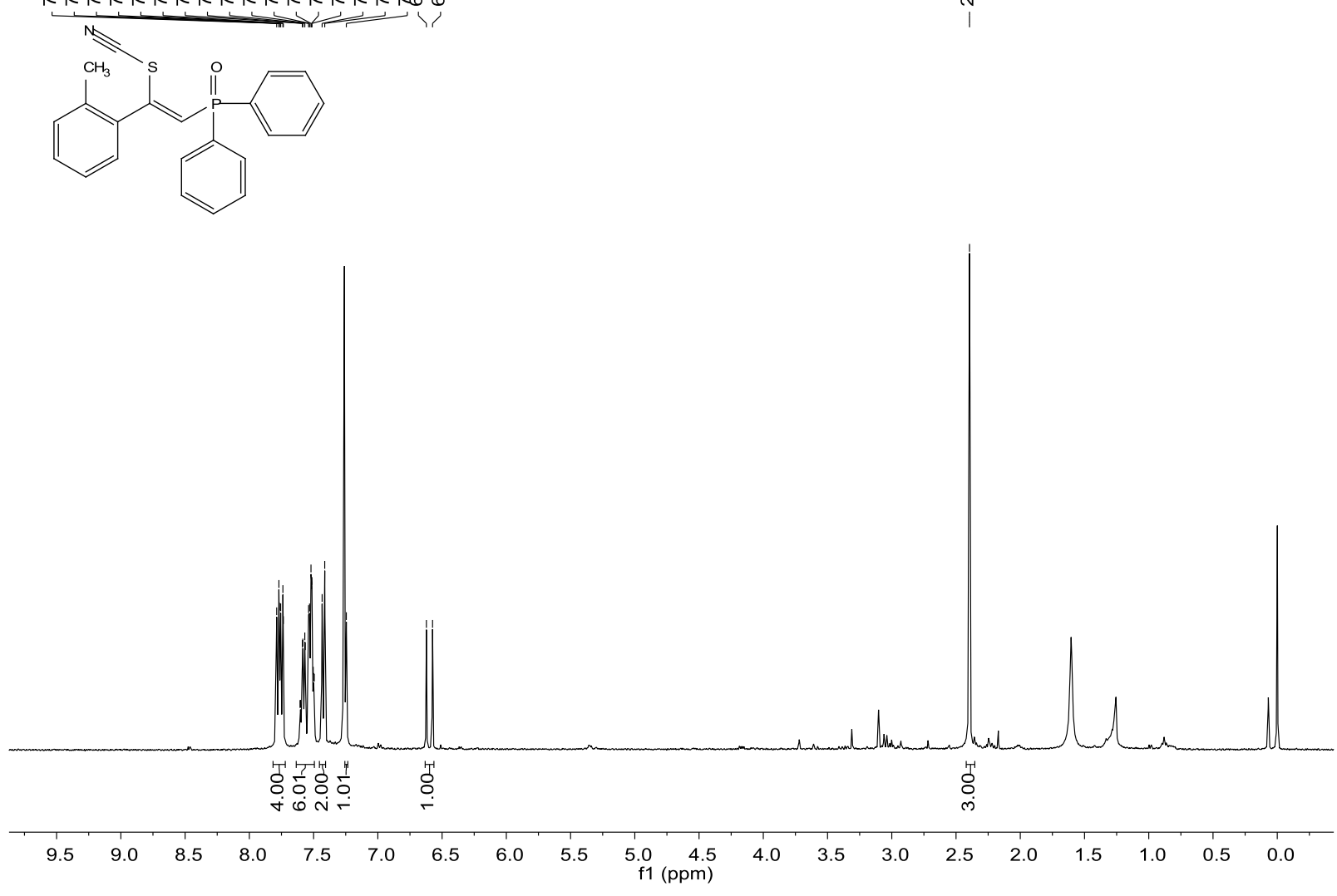
${ }^{31} \mathrm{P}$ NMR Spectrum of $7 \mathrm{~b}\left(\mathrm{CDCl}_{3}, 162 \mathrm{MHz}\right)$

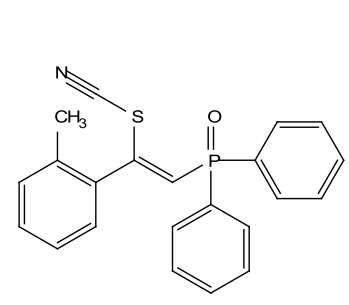

$\stackrel{\text { N }}{\text { N }}$

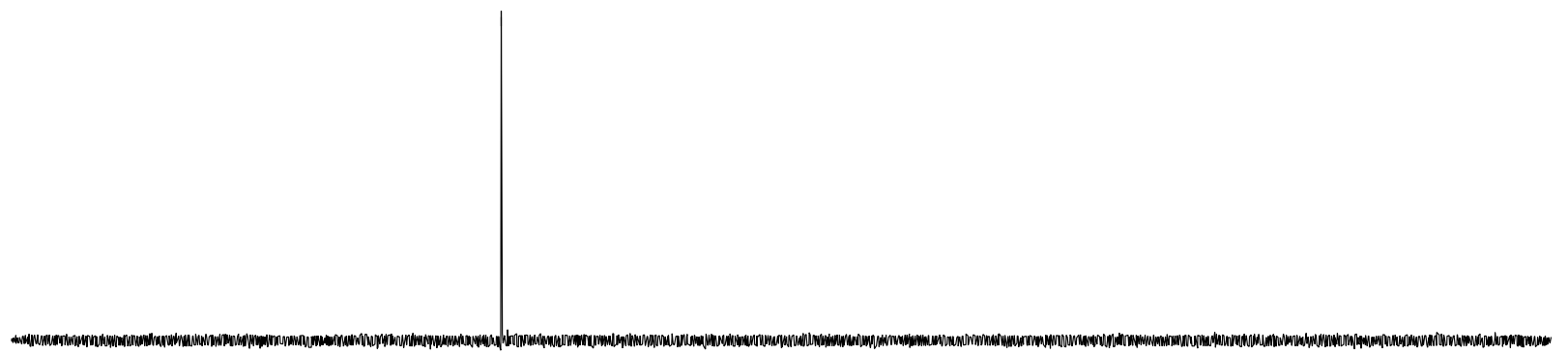

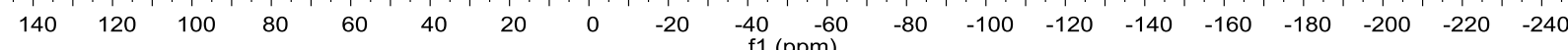

${ }^{13} \mathrm{C}$ NMR Spectrum of $7 \mathrm{~b}\left(\mathrm{CDCl}_{3}, 100 \mathrm{MHz}\right)$

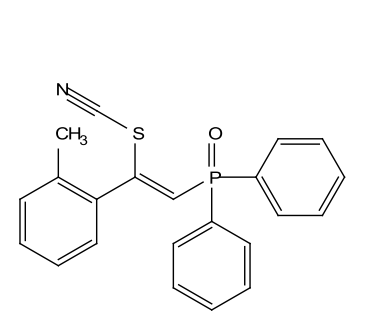

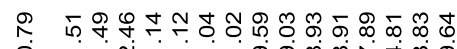

家

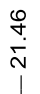

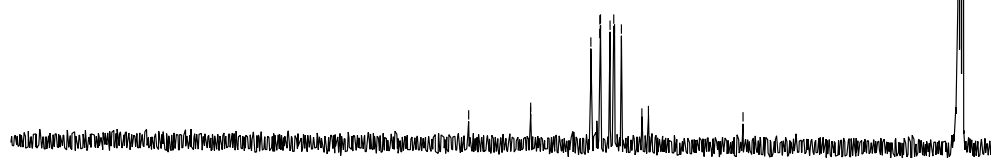

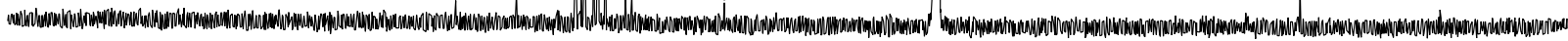

$\begin{array}{llllllllllllllllllllllll}210 & 200 & 190 & 180 & 170 & 160 & 150 & 140 & 130 & 120 & 110 & 100 & 90 & 80 & 70 & 60 & 50 & 40 & 30 & 20 & 10 & 0 & -10\end{array}$ 
${ }^{1} \mathrm{H}$ NMR Spectrum of $7 \mathrm{c}\left(\mathrm{CDCl}_{3}, 400 \mathrm{MHz}\right)$<smiles>Cc1ccc(/C(=C\P(=O)(c2ccccc2)c2ccccc2)SC#N)cc1</smiles>

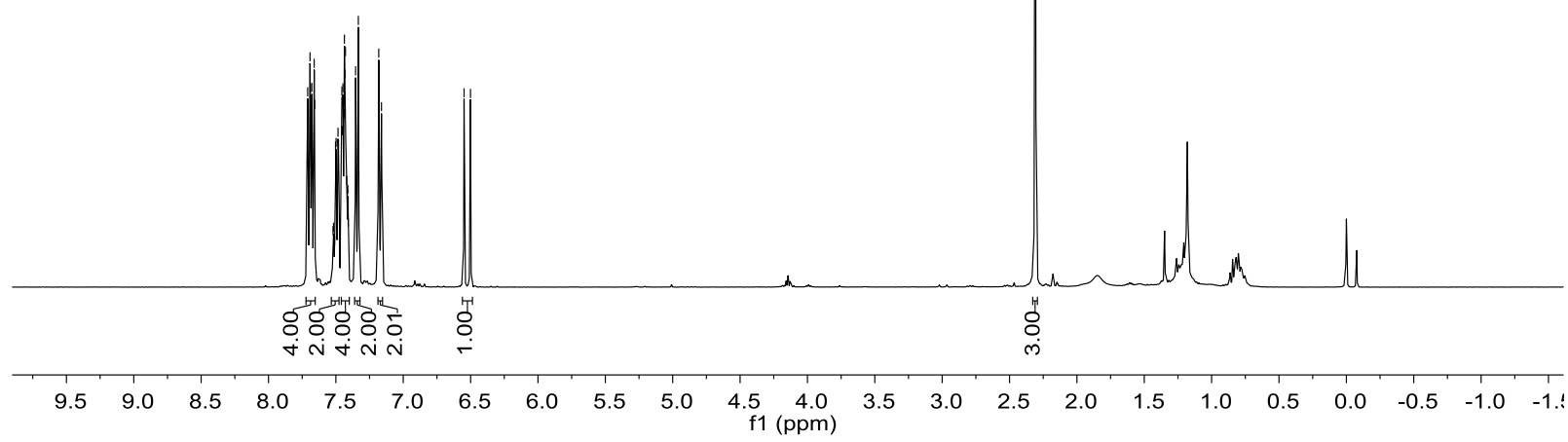

${ }^{31} \mathrm{P}$ NMR Spectrum of $7 \mathrm{c}\left(\mathrm{CDCl}_{3}, 162 \mathrm{MHz}\right)$<smiles>Cc1ccc(C(C#N)=CP(=O)(c2ccccc2)c2ccccc2)cc1</smiles>

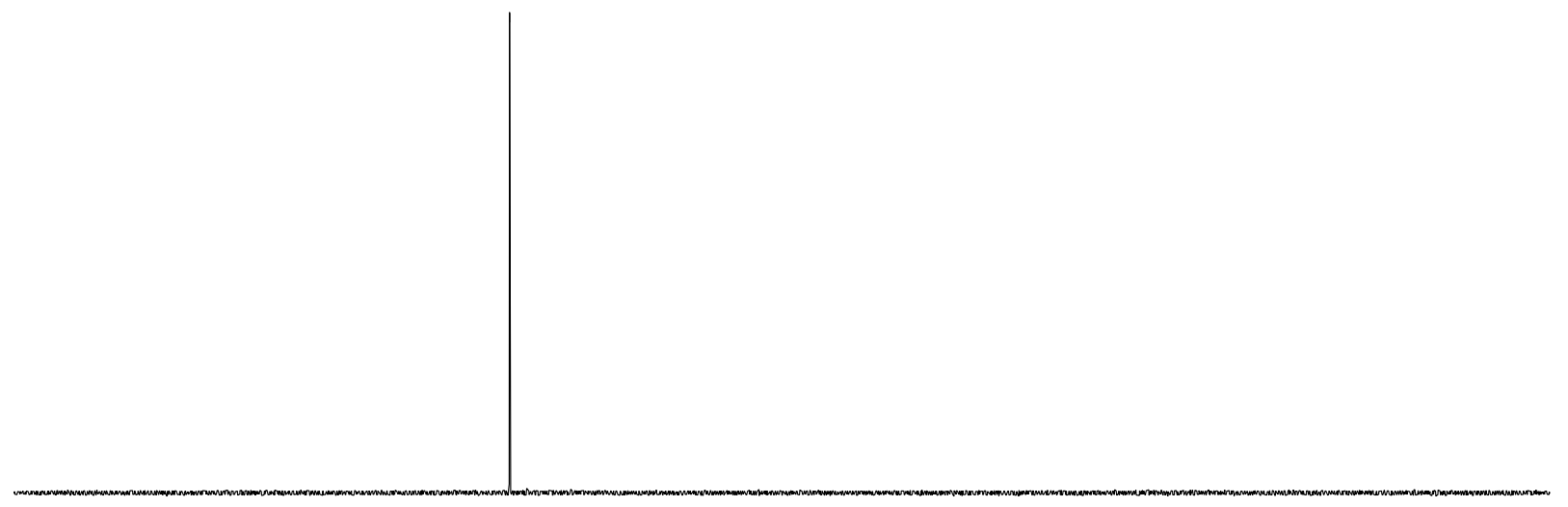

$\begin{array}{lllllllllllllllllllll}150 & 130 & 110 & 90 & 70 & 50 & 30 & 10 & -10 & -30 & \begin{array}{l}-50 \\ -50\end{array} & -70 & -90 & -110 & -130 & -150 & -170 & -190 & -210 & -230 & -25\end{array}$ 
${ }^{13} \mathrm{C}$ NMR Spectrum of $7 \mathrm{c}\left(\mathrm{CDCl}_{3}, 100 \mathrm{MHz}\right)$

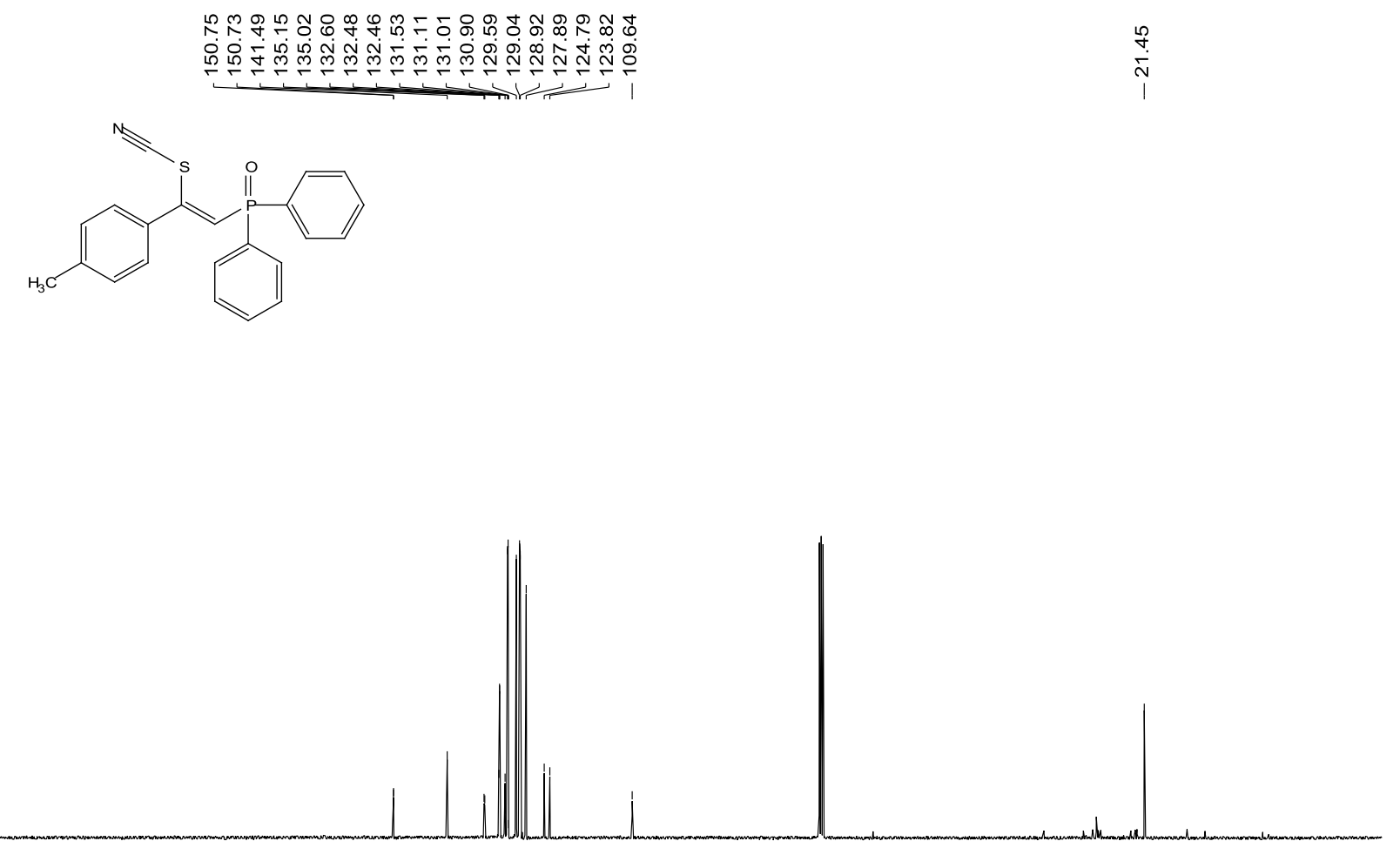

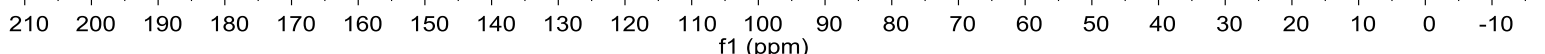

${ }^{1} \mathrm{H}$ NMR Spectrum of 7d (DMSO-d $\left.d_{6}, 400 \mathrm{MHz}\right)$

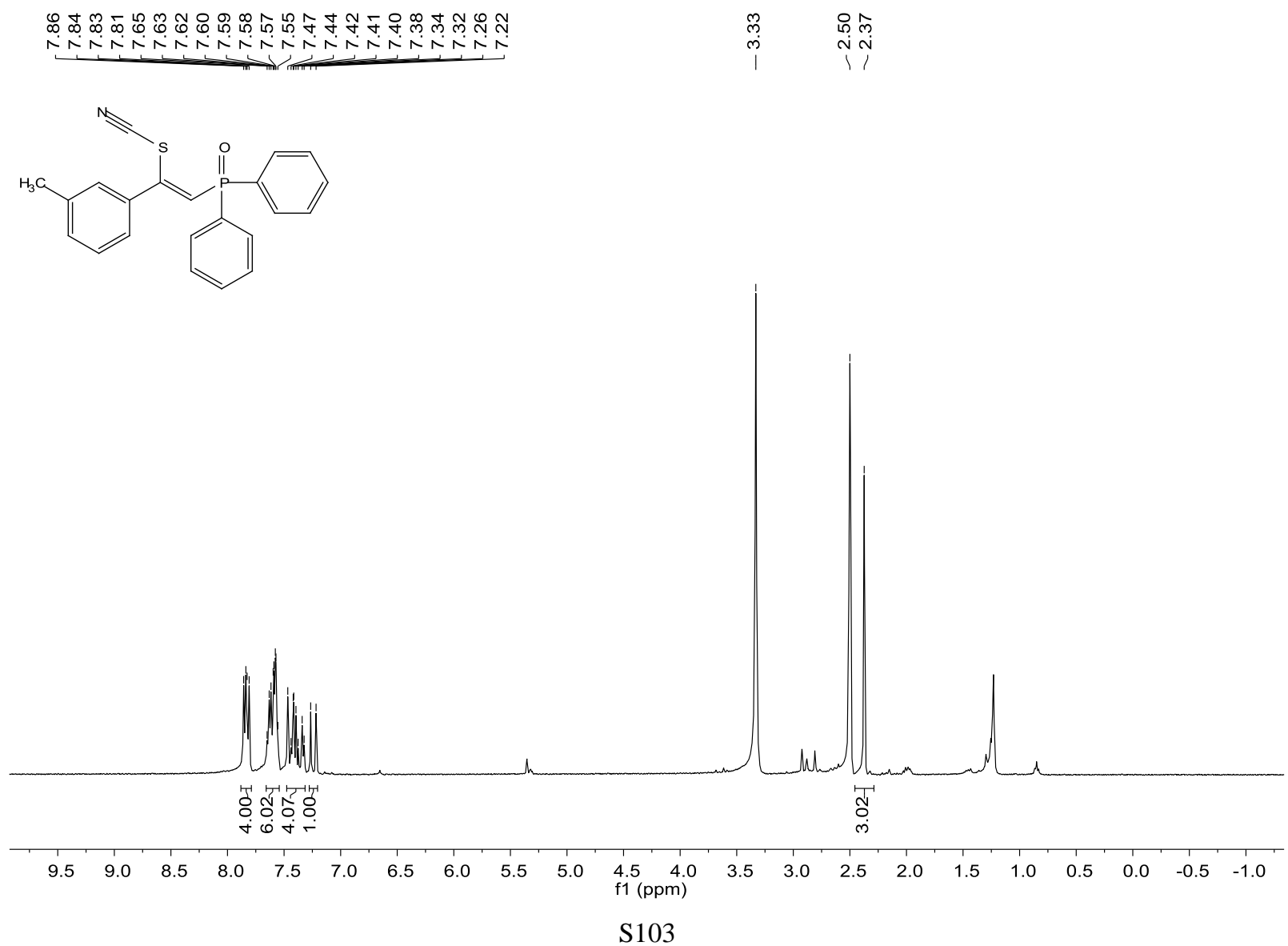


${ }^{31}$ P NMR Spectrum of 7d (DMSO- $d_{6}, 162 \mathrm{MHz}$ )<smiles>Cc1cccc(/C(=C/P(=O)(c2ccccc2)c2ccccc2)SC#N)c1</smiles>

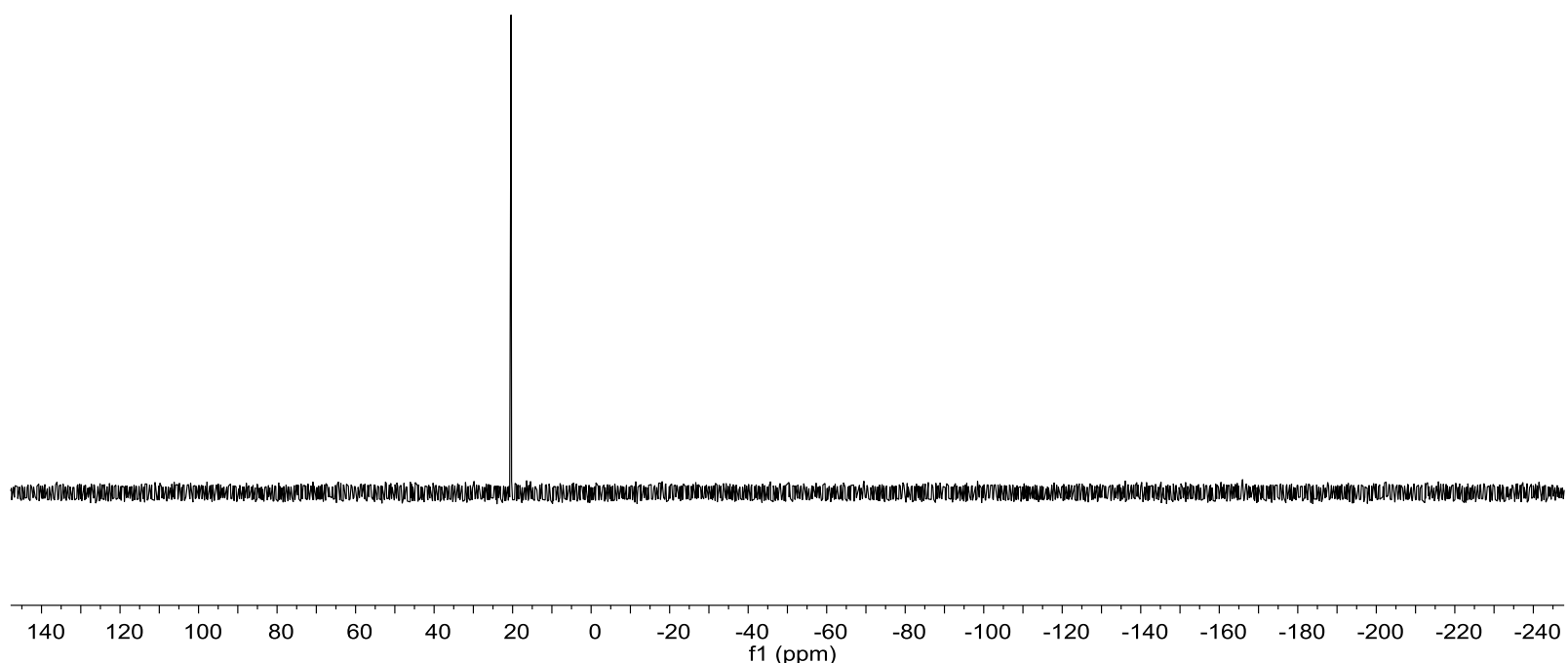

${ }^{13} \mathrm{C}$ NMR Spectrum of 7d (DMSO- $\left.d_{6}, 100 \mathrm{MHz}\right)$
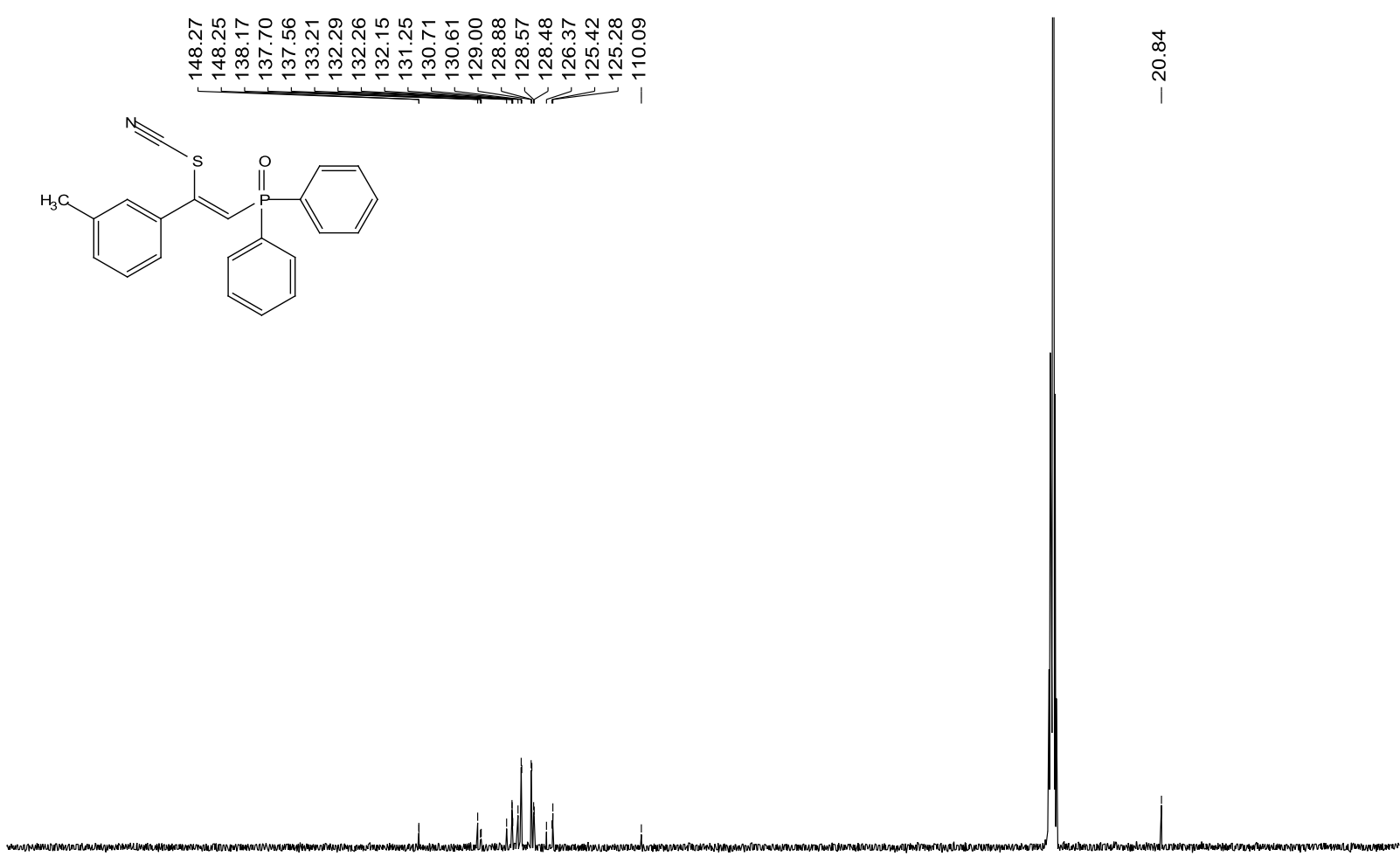

$\begin{array}{lllllllllllllllllllllll}210 & 200 & 190 & 180 & 170 & 160 & 150 & 140 & 130 & 120 & 110 \begin{array}{c}100 \\ \mathrm{f} 1(\mathrm{ppm})\end{array} & 90 & 80 & 70 & 60 & 50 & 40 & 30 & 20 & 10 & 0 & -10\end{array}$ 
${ }^{1} \mathrm{H}$ NMR Spectrum of $7 \mathrm{e}\left(\mathrm{CDCl}_{3}, 400 \mathrm{MHz}\right)$

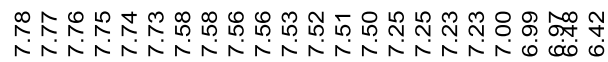

ले

(1)

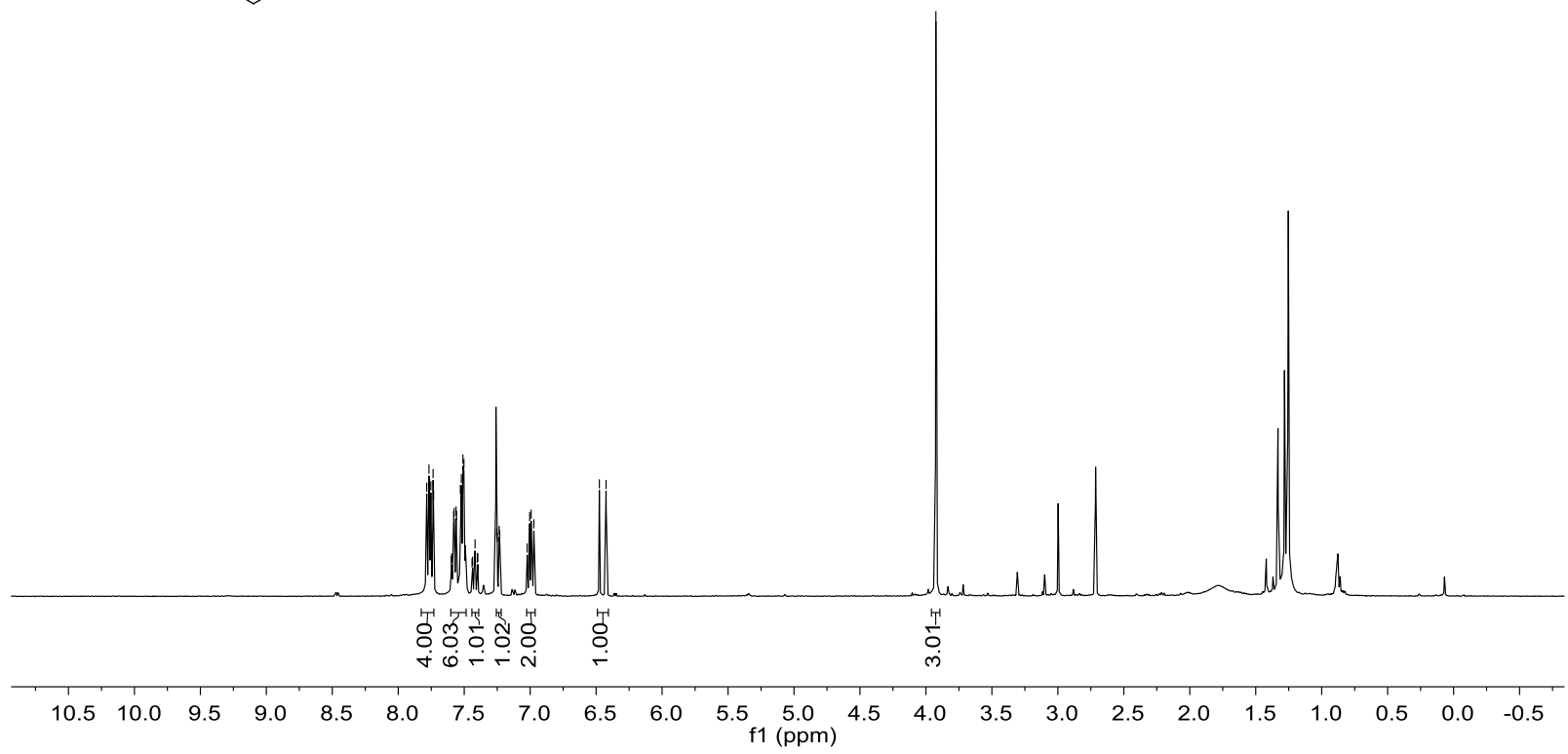

${ }^{31} \mathrm{P}$ NMR Spectrum of $7 \mathrm{e}\left(\mathrm{CDCl}_{3}, 162 \mathrm{MHz}\right)$

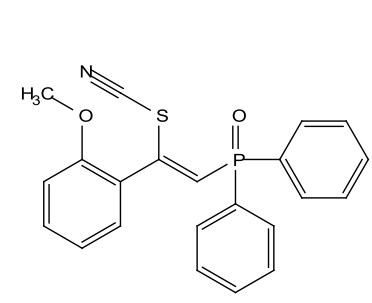

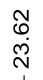

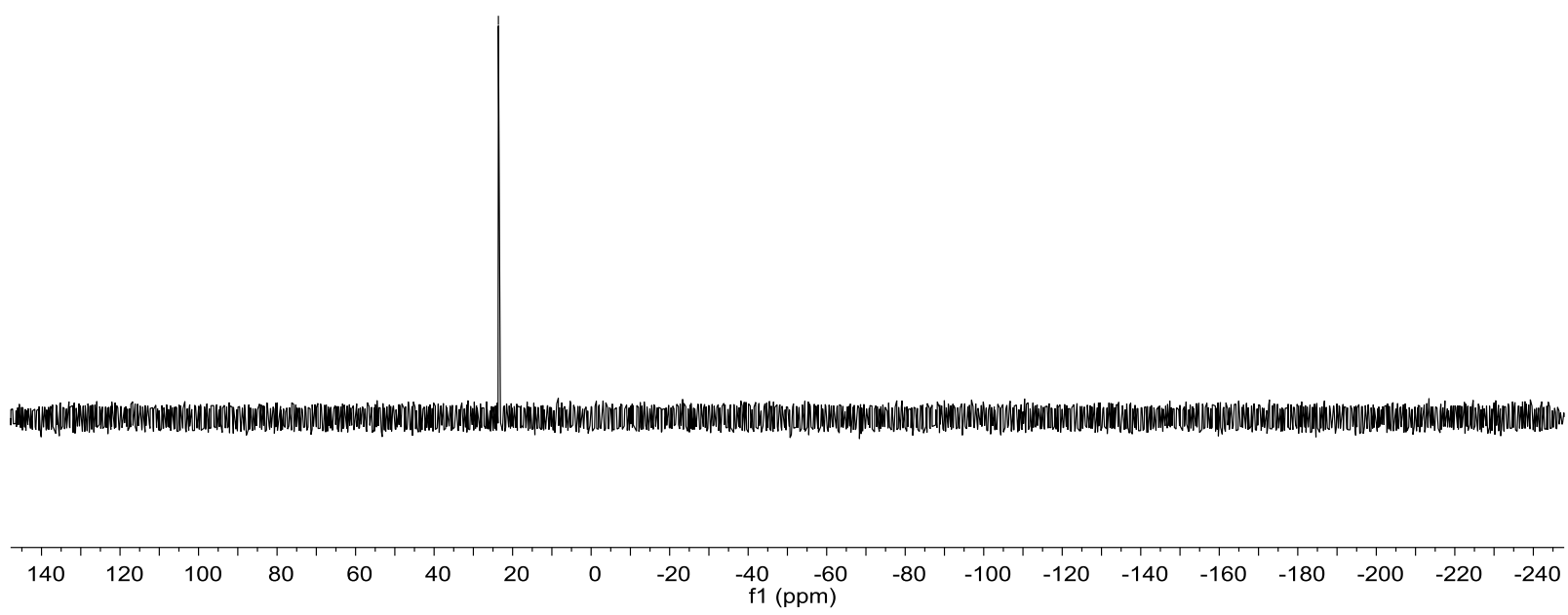


${ }^{13} \mathrm{C}$ NMR Spectrum of $7 \mathrm{e}\left(\mathrm{CDCl}_{3}, 100 \mathrm{MHz}\right)$

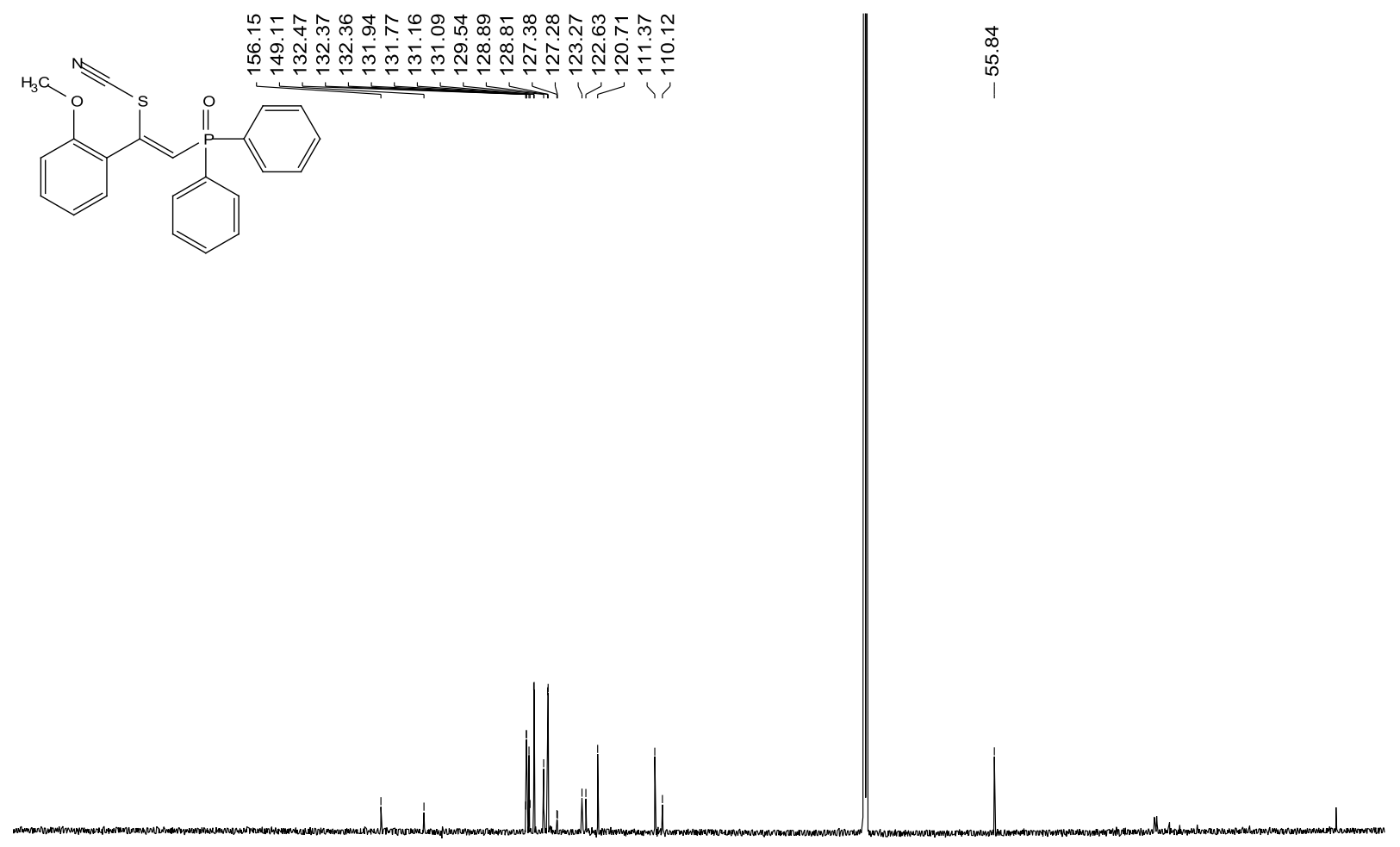

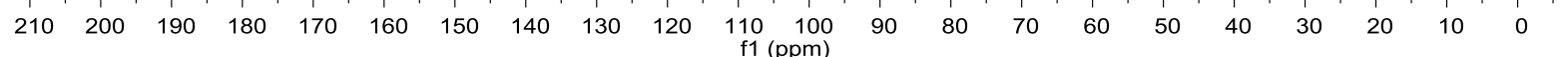

${ }^{1} \mathrm{H}$ NMR Spectrum of $7 \mathrm{f}\left(\mathrm{CDCl}_{3}, 400 \mathrm{MHz}\right)$

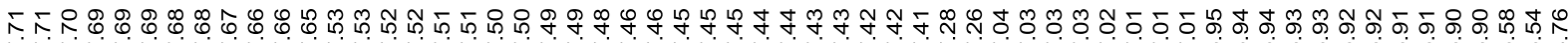

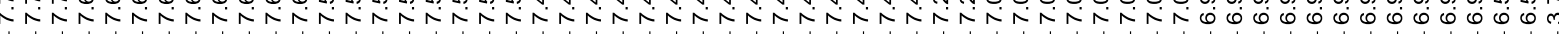<smiles>COc1cccc(/C(=C/P(=O)(c2ccccc2)c2ccccc2)SC#N)c1</smiles>

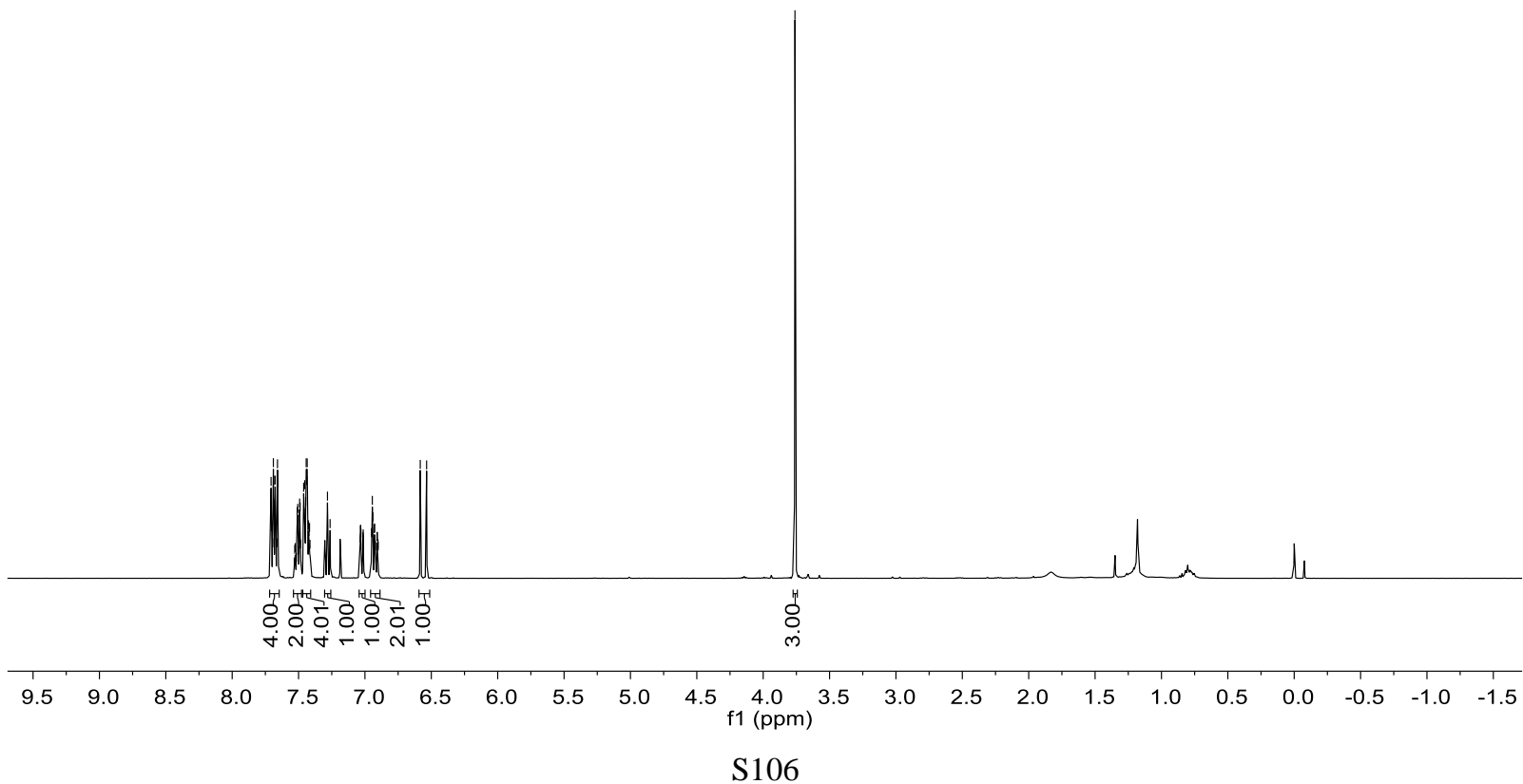


${ }^{31}$ P NMR Spectrum of $7 \mathrm{f}\left(\mathrm{CDCl}_{3}, 162 \mathrm{MHz}\right)$
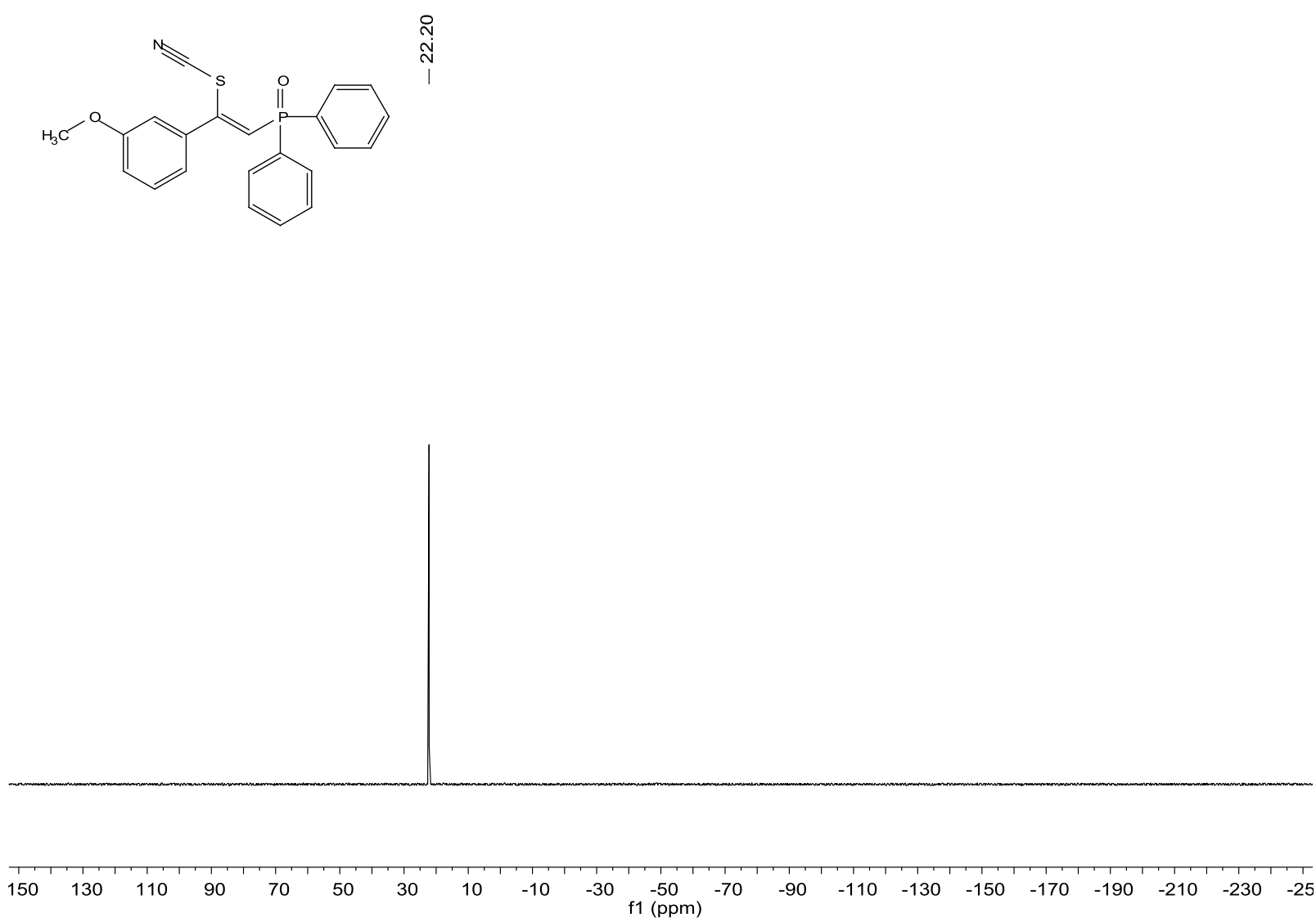

${ }^{13} \mathrm{C}$ NMR Spectrum of $7 \mathrm{f}\left(\mathrm{CDCl}_{3}, 100 \mathrm{MHz}\right)$
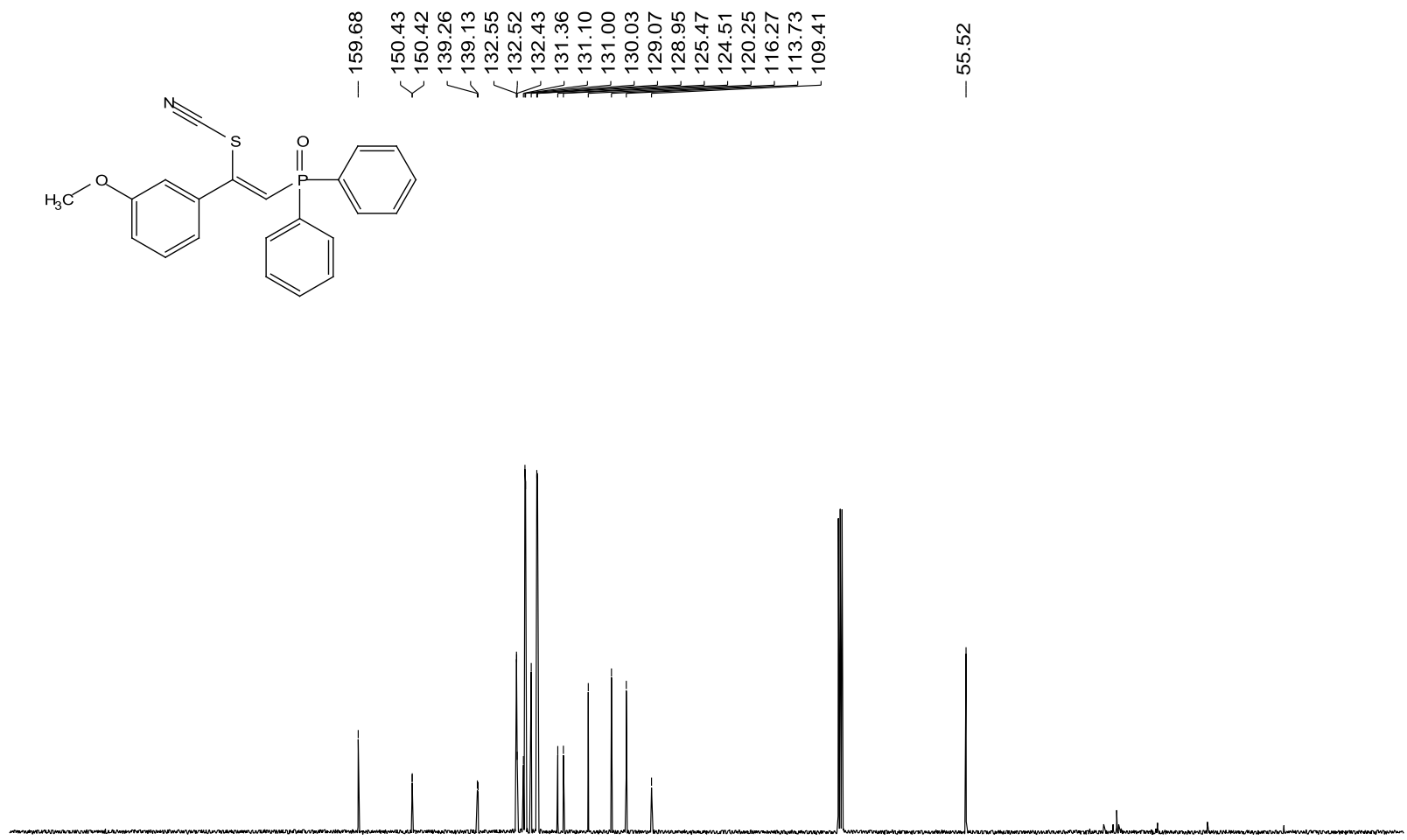

$\begin{array}{lllllllllllllllllllllllllllllll}210 & 200 & 190 & 180 & 170 & 160 & 150 & 140 & 130 & 120 & 110 & 100 & 90 & 80 & 70 & 60 & 50 & 40 & 30 & 20 & 10 & 0 & -10\end{array}$ 
${ }^{1} \mathrm{H}$ NMR Spectrum of $7 \mathrm{~g}\left(\mathrm{CDCl}_{3}, 400 \mathrm{MHz}\right)$

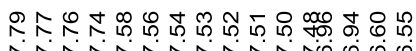

ticisisisingo,

品

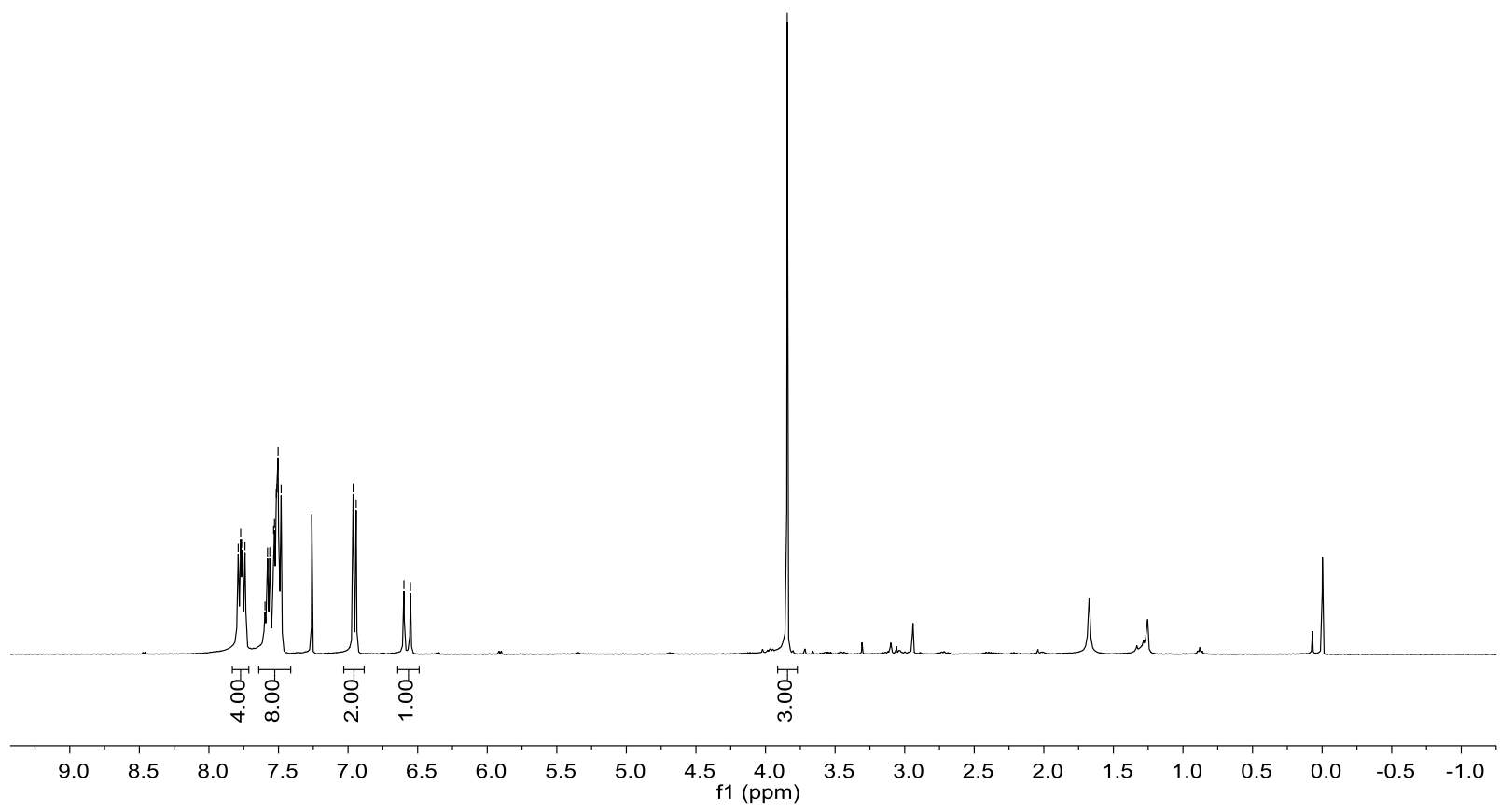

${ }^{31} \mathrm{P}$ NMR Spectrum of $7 \mathrm{~g}\left(\mathrm{CDCl}_{3}, 162 \mathrm{MHz}\right)$<smiles>COc1ccc(/C(=C/P(=O)(c2ccccc2)c2ccccc2)SC#N)cc1</smiles>

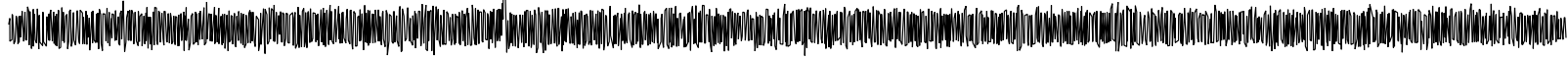

$\begin{array}{lllllllllllllllllllll}140 & 120 & 100 & 80 & 60 & 40 & 20 & 0 & -20 & -40 & -60 & -80 & -100 & -120 & -140 & -160 & -180 & -200 & -220 & -240\end{array}$

S108 
${ }^{13} \mathrm{C}$ NMR Spectrum of $7 \mathrm{~g}\left(\mathrm{CDCl}_{3}, 100 \mathrm{MHz}\right)$

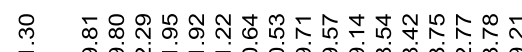

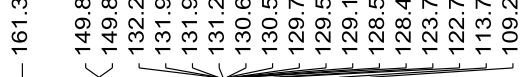

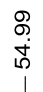

(1)

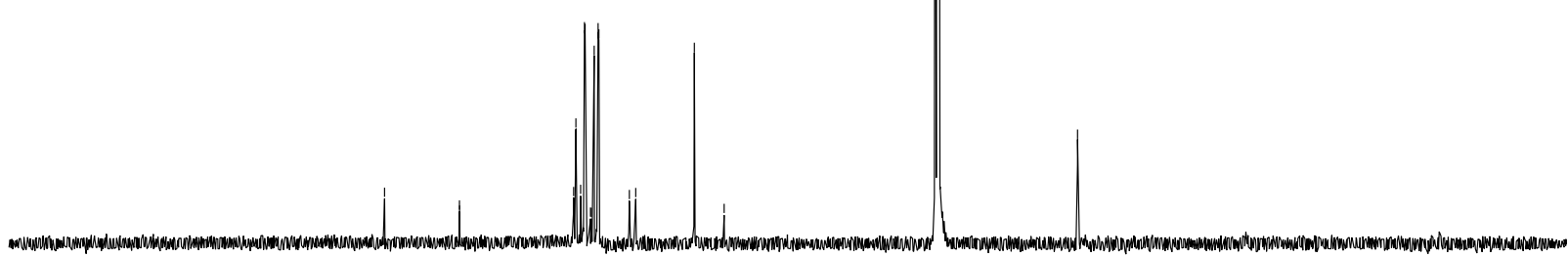

$\begin{array}{lllllllllllllllllllllllllllllll}210 & 200 & 190 & 180 & 170 & 160 & 150 & 140 & 130 & 120 & 110 & 100 & 90 & 80 & 70 & 60 & 50 & 40 & 30 & 20 & 10 & 0 & -10\end{array}$

${ }^{1} \mathrm{H}$ NMR Spectrum of $7 \mathrm{~h}\left(\mathrm{CDCl}_{3}, 400 \mathrm{MHz}\right)$

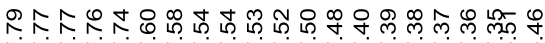

-
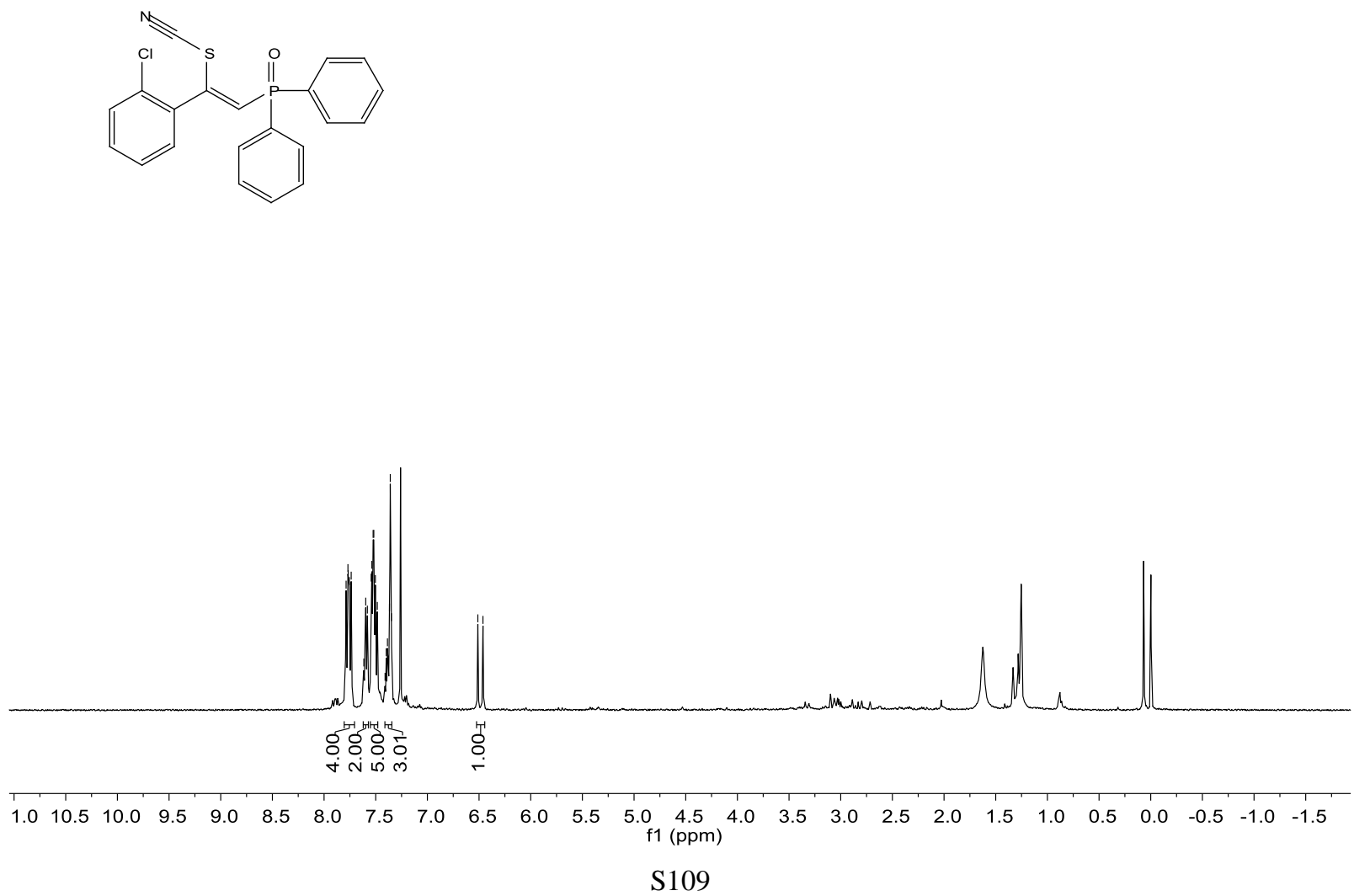
${ }^{31} \mathrm{P}$ NMR Spectrum of $7 \mathrm{~h}\left(\mathrm{CDCl}_{3}, 162 \mathrm{MHz}\right)$

(1)

$\stackrel{\infty}{\stackrel{\infty}{\dot{j}}}$

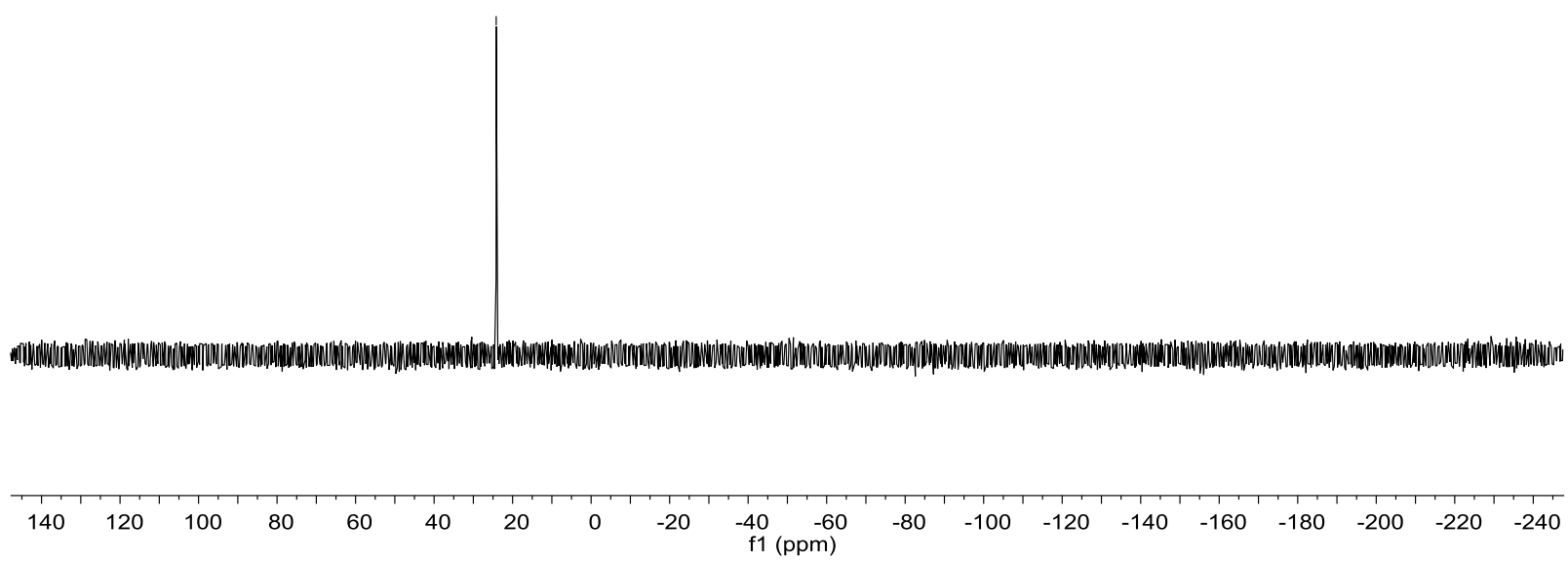

${ }^{13} \mathrm{C}$ NMR Spectrum of $7 \mathrm{~h}\left(\mathrm{CDCl}_{3}, 100 \mathrm{MHz}\right)$
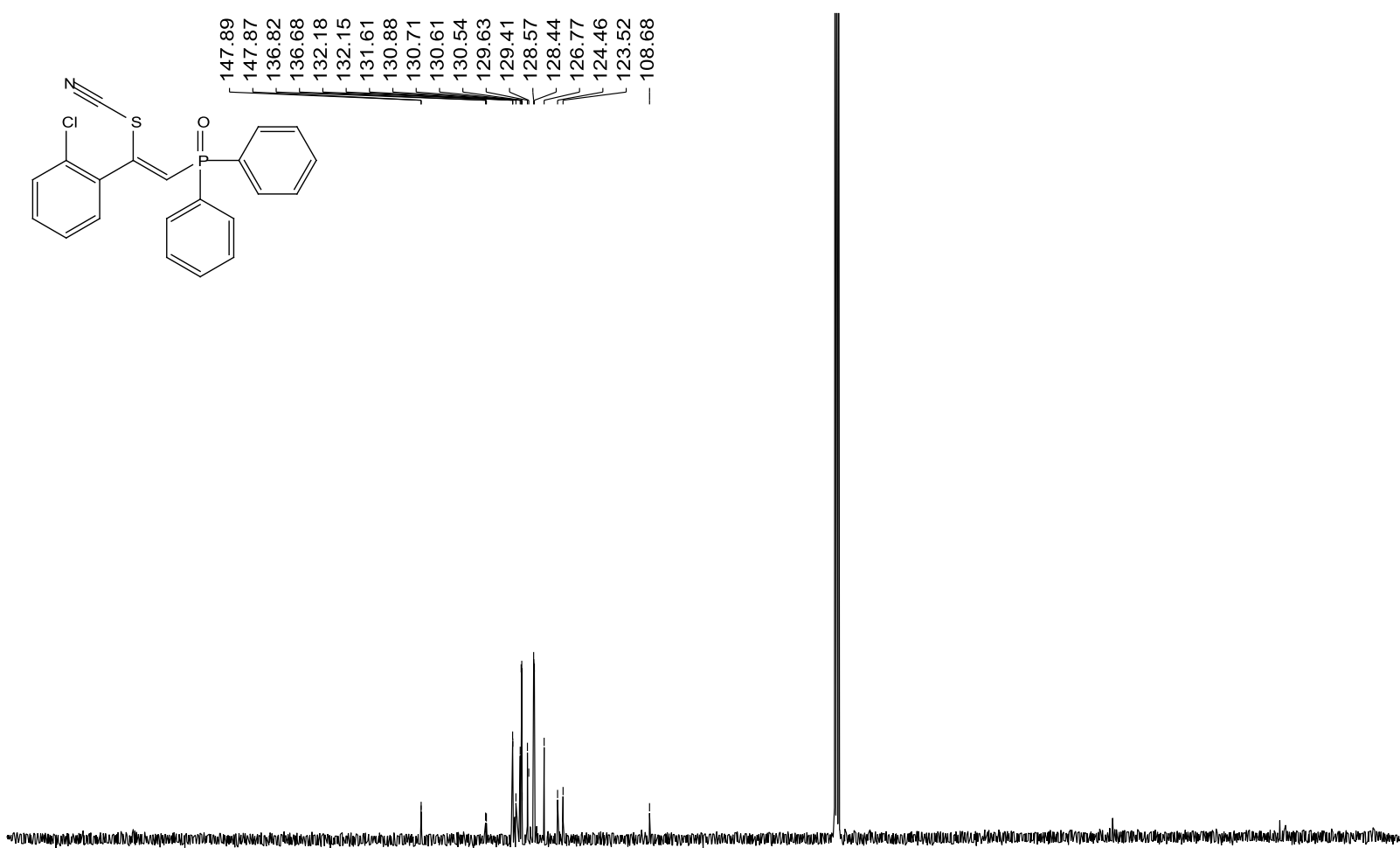

$\begin{array}{llllllllllllllllllllll}210 & 200 & 190 & 180 & 170 & 160 & 150 & 140 & 130 & 120 & \begin{array}{c}110 \\ \mathrm{f} 1(\mathrm{ppm})\end{array} & 90 & 80 & 70 & 60 & 50 & 40 & 30 & 20 & 10 & 0 & -10\end{array}$ 
${ }^{1} \mathrm{H}$ NMR Spectrum of 7i (DMSO- $\left.d_{6}, 400 \mathrm{MHz}\right)$

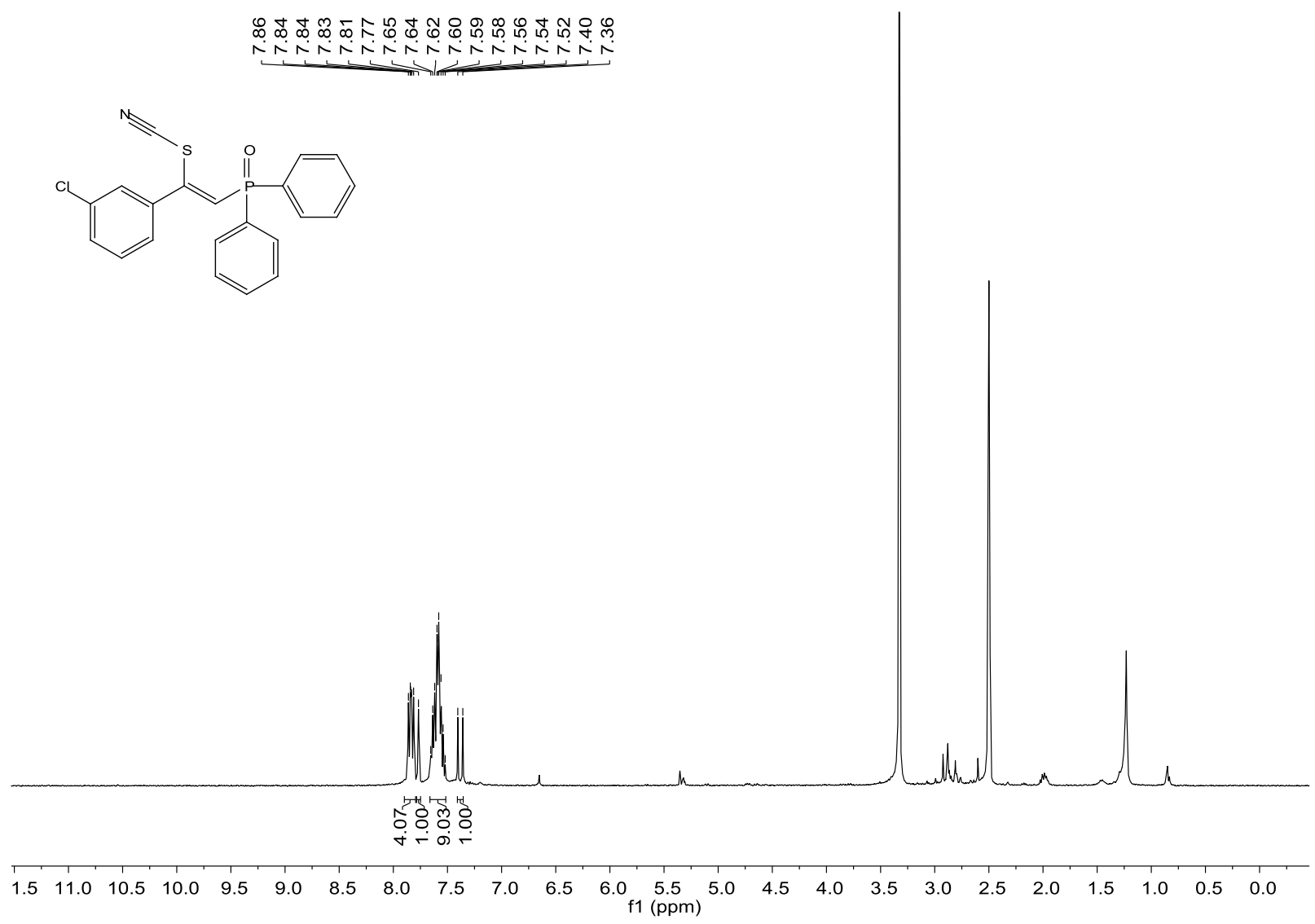

${ }^{31}$ P NMR Spectrum of 7i (DMSO- $d_{6}, 162 \mathrm{MHz}$ )

$\stackrel{\infty}{\sim}$<smiles>N#CS/C(=C\P(=O)(c1ccccc1)c1ccccc1)c1cccc(Cl)c1</smiles>

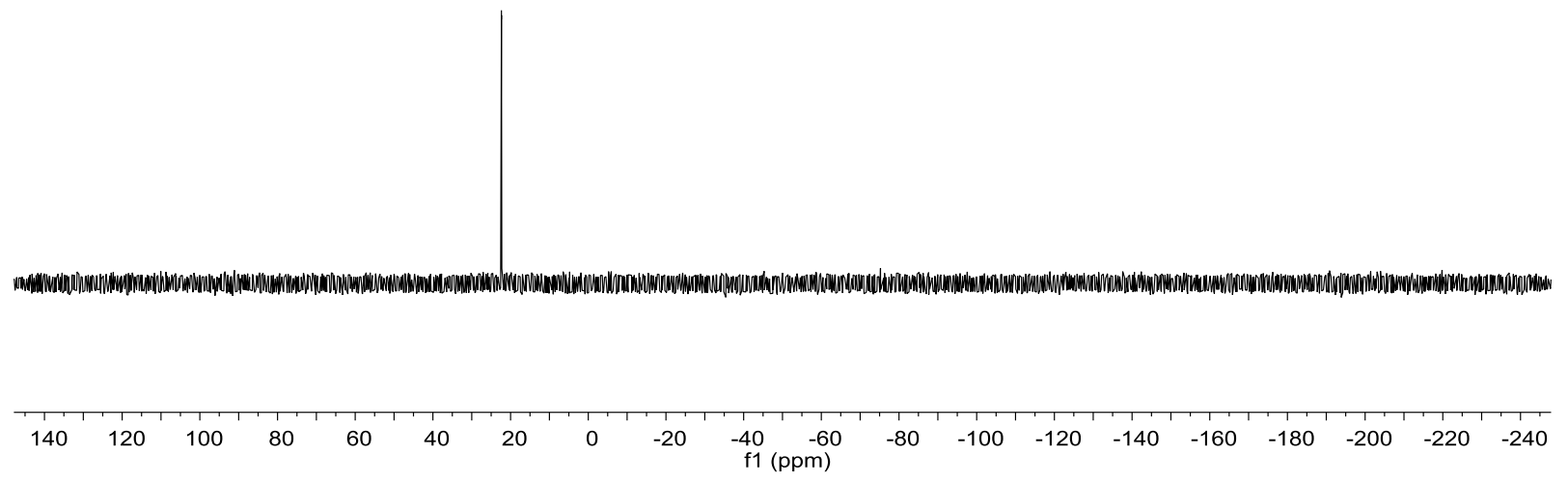


${ }^{13} \mathrm{C}$ NMR Spectrum of 7i (DMSO-d, $\left.100 \mathrm{MHz}\right)$
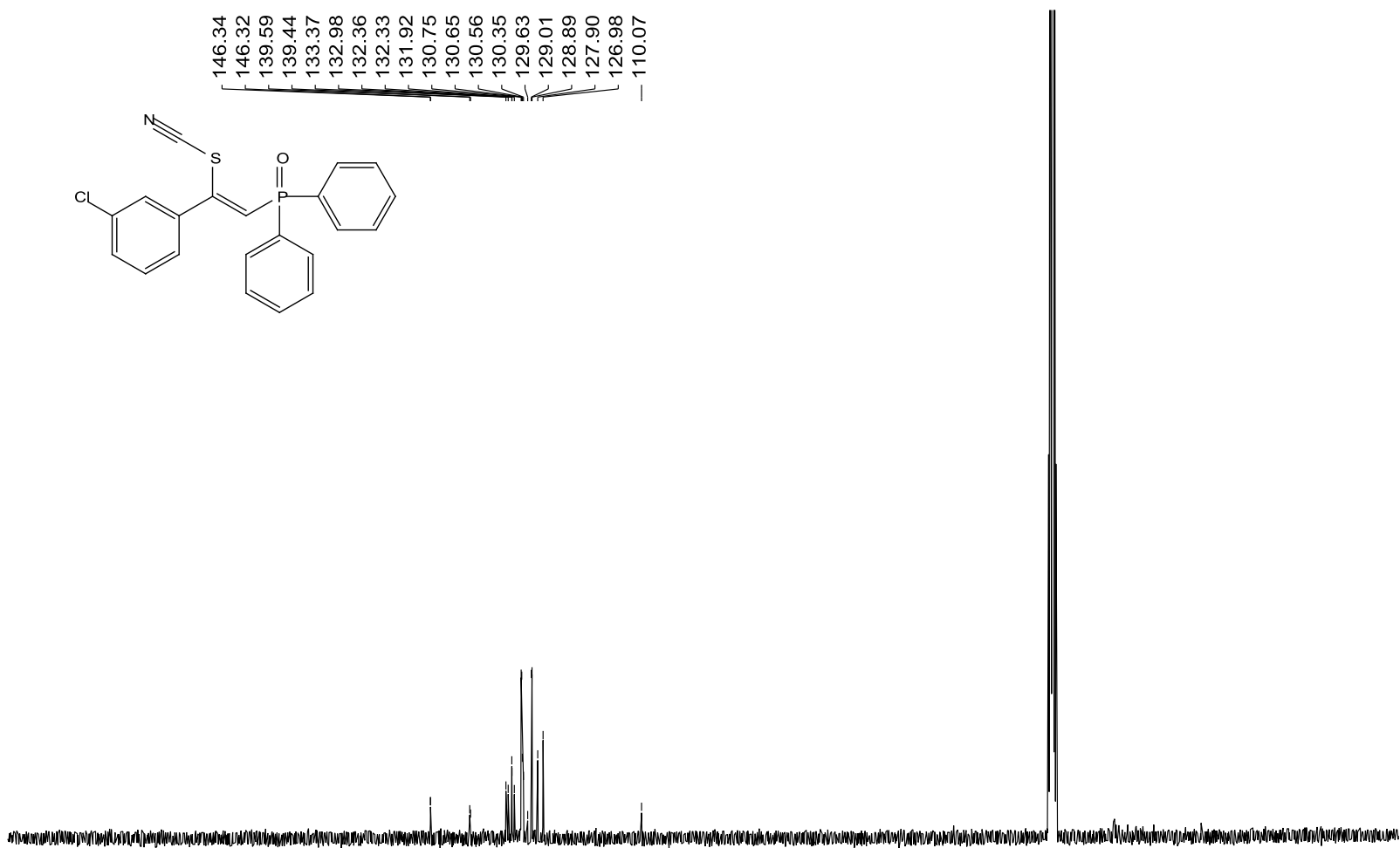

$\begin{array}{lllllllllllllllllllllll}210 & 200 & 190 & 180 & 170 & 160 & 150 & 140 & 130 & 120 & 110 & 100 & 90 & 80 & 70 & 60 & 50 & 40 & 30 & 20 & 10 & 0 & -10\end{array}$

${ }^{1} \mathrm{H}$ NMR Spectrum of $7 \mathbf{j}\left(\mathrm{CDCl}_{3}, 400 \mathrm{MHz}\right)$

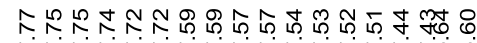

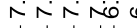
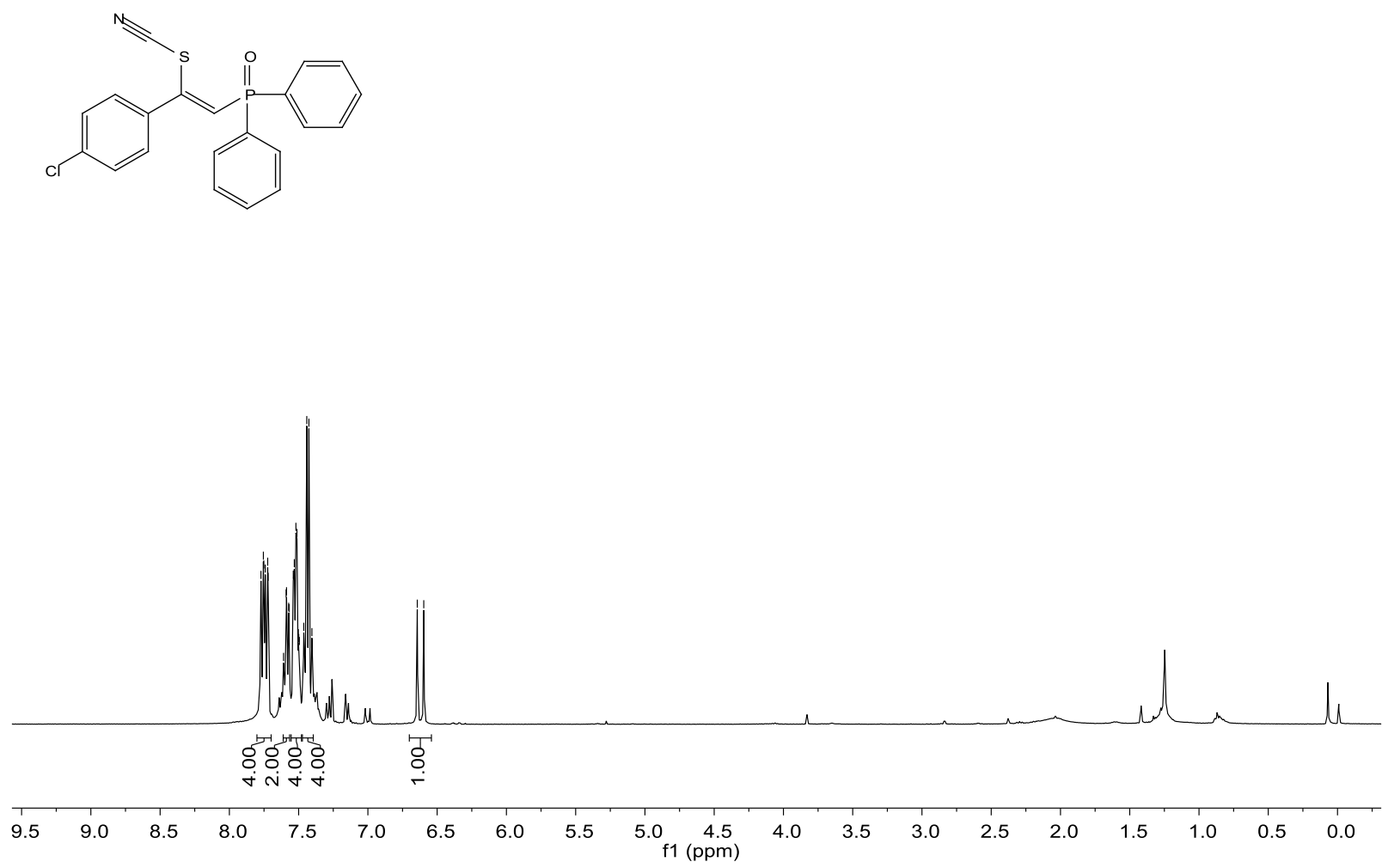
${ }^{31} \mathrm{P}$ NMR Spectrum of $7 \mathbf{j}\left(\mathrm{CDCl}_{3}, 162 \mathrm{MHz}\right)$
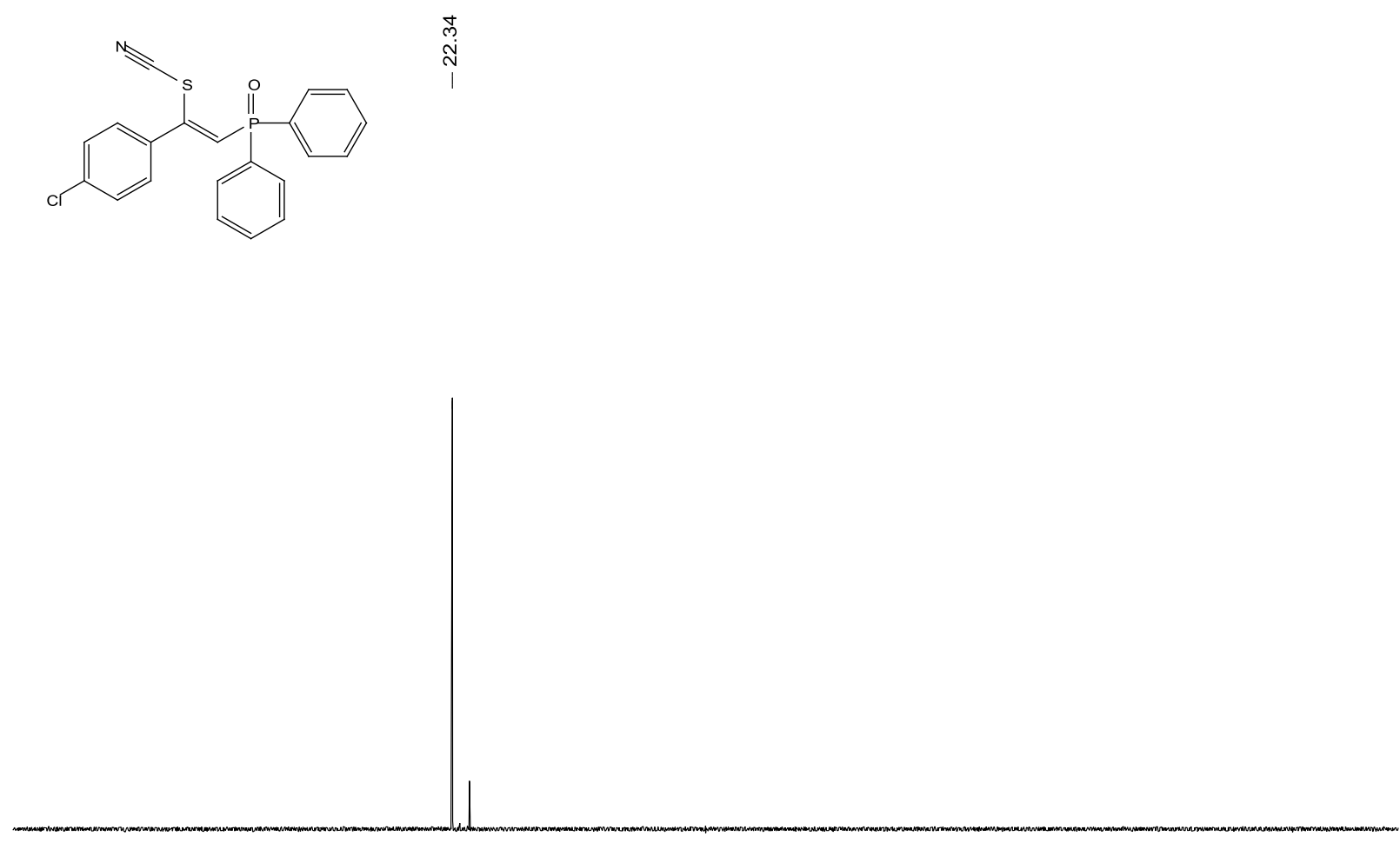

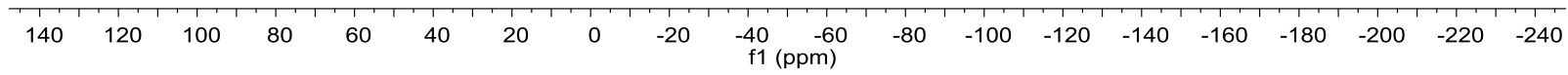

${ }^{13} \mathrm{C}$ NMR Spectrum of $7 \mathbf{j}\left(\mathrm{CDCl}_{3}, 100 \mathrm{MHz}\right)$
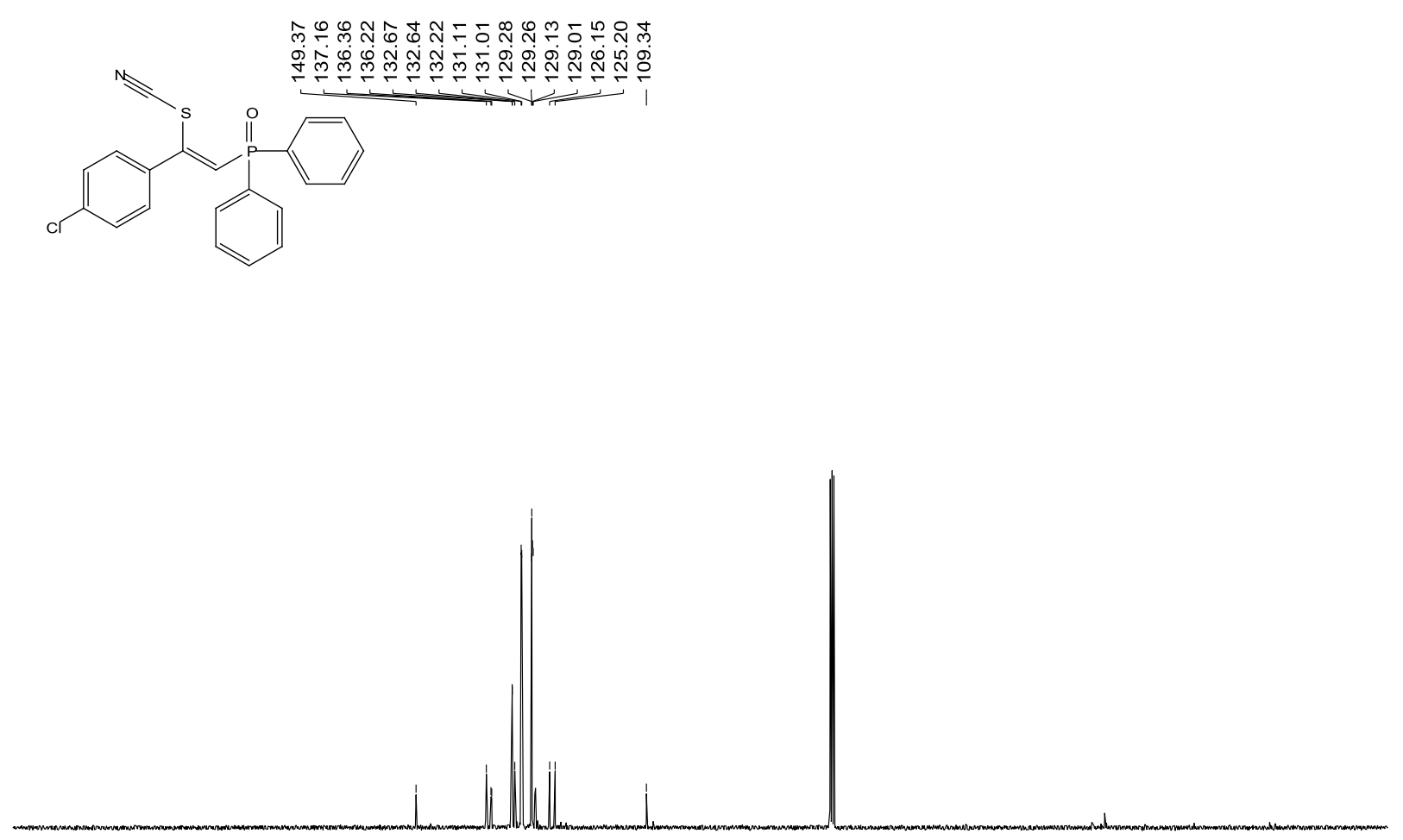

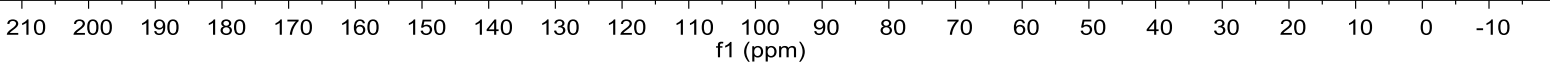


${ }^{1} \mathrm{H}$ NMR Spectrum of $7 \mathrm{k}\left(\mathrm{CDCl}_{3}, 400 \mathrm{MHz}\right)$

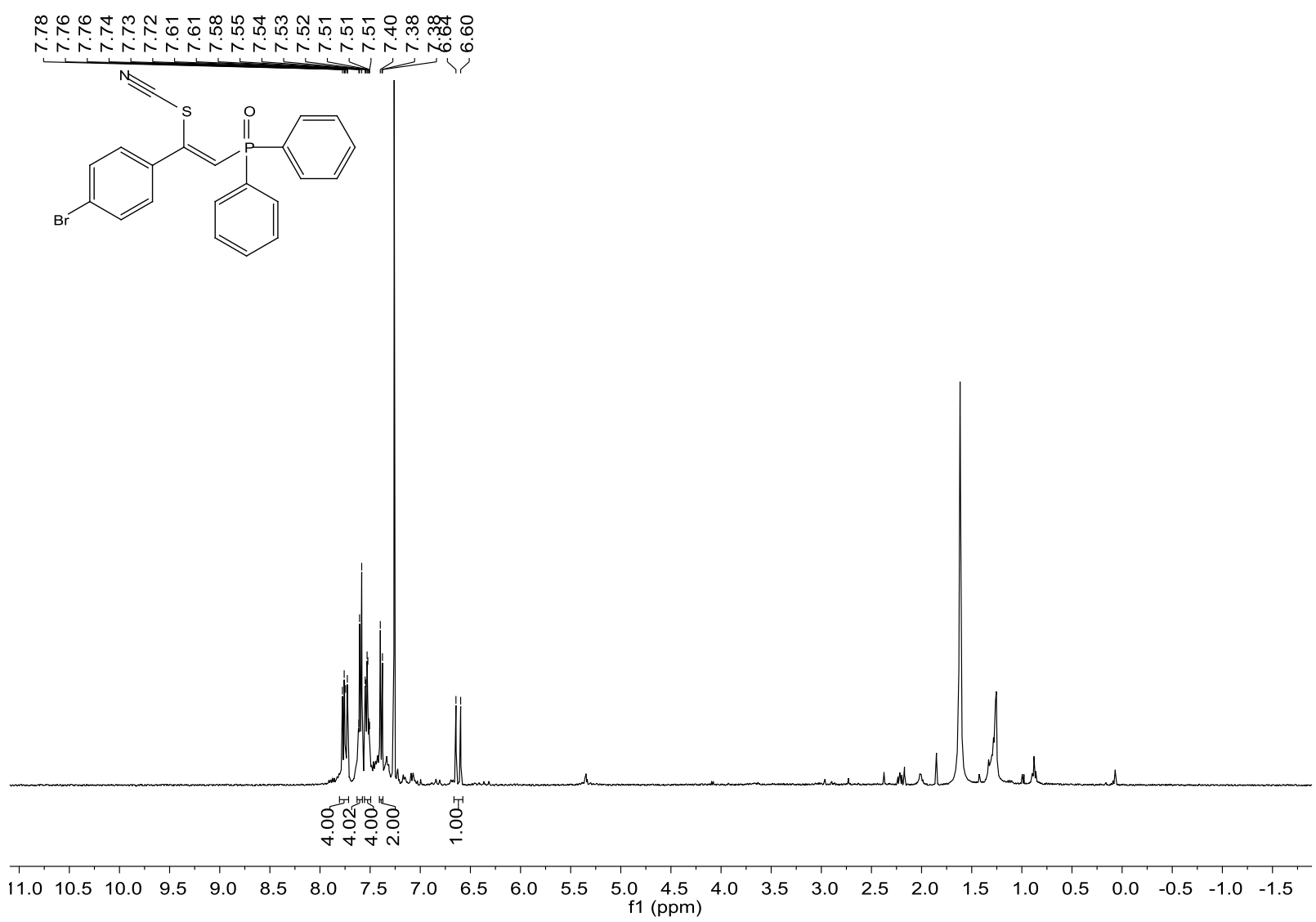

${ }^{31} \mathrm{P}$ NMR Spectrum of $7 \mathrm{k}\left(\mathrm{CDCl}_{3}, 162 \mathrm{MHz}\right)$

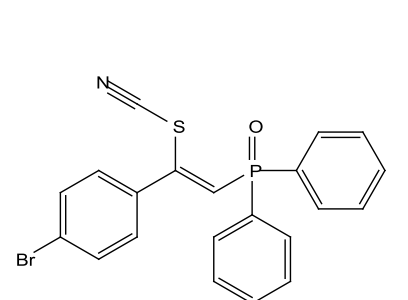

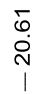

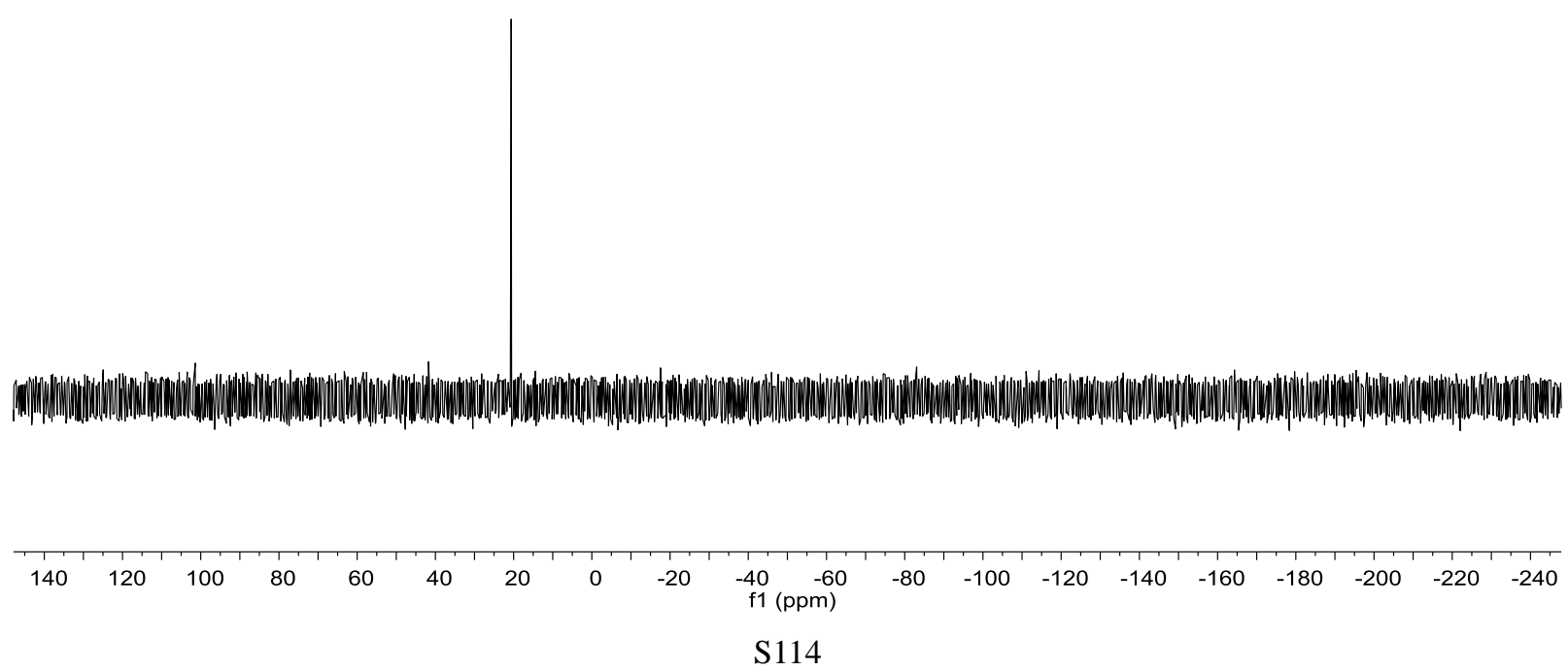


${ }^{13} \mathrm{C}$ NMR Spectrum of $7 \mathrm{k}\left(\mathrm{CDCl}_{3}, 100 \mathrm{MHz}\right)$

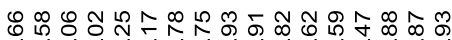

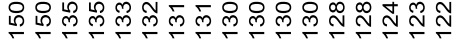
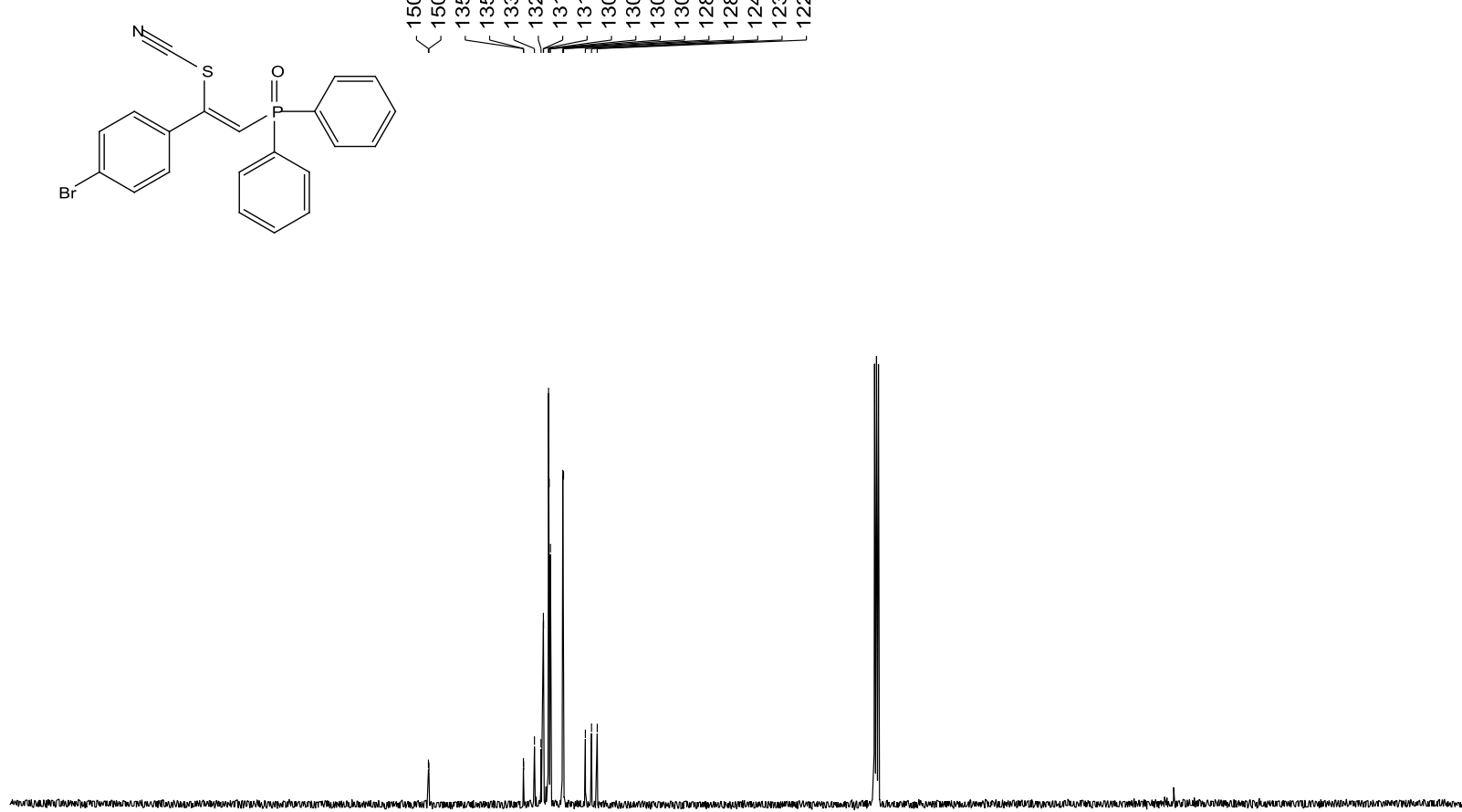

$\begin{array}{lllllllllllllllllllllll}210 & 200 & 190 & 180 & 170 & 160 & 150 & 140 & 130 & 120 & 110 & 100 & 90 & 80 & 70 & 60 & 50 & 40 & 30 & 20 & 10 & 0 & -10\end{array}$

${ }^{1} \mathrm{H}$ NMR Spectrum of $71\left(\mathrm{CDCl}_{3}, 400 \mathrm{MHz}\right)$

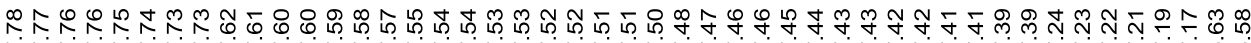

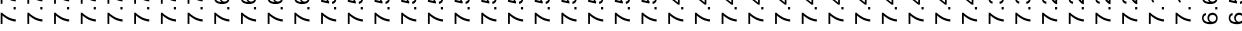

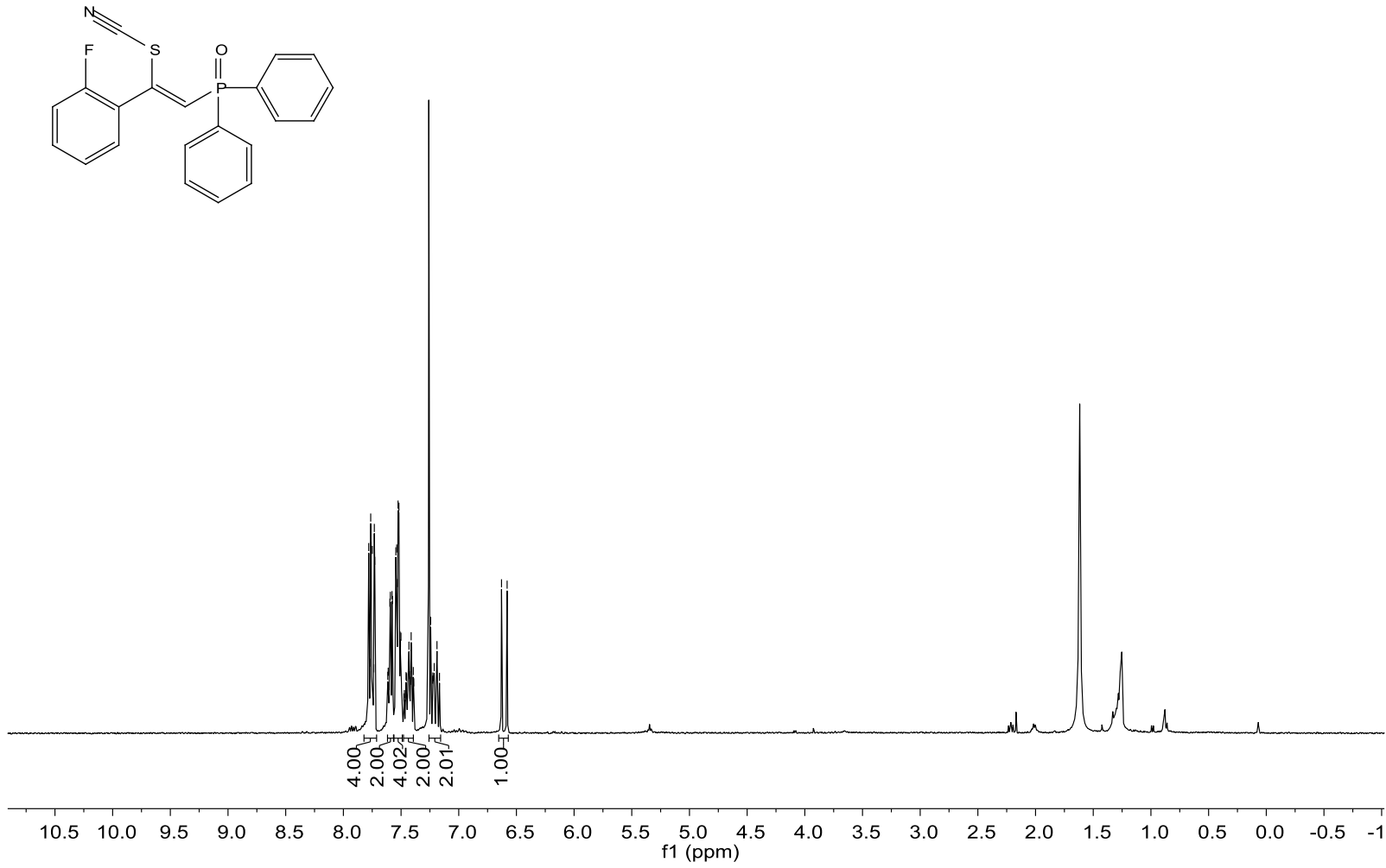




\section{${ }^{31} \mathrm{P}$ NMR Spectrum of $71\left(\mathrm{CDCl}_{3}, 162 \mathrm{MHz}\right)$}

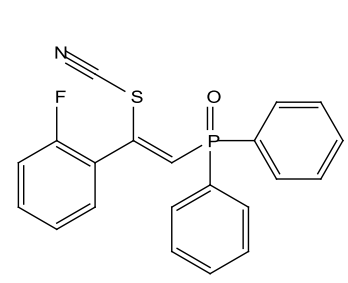

$\stackrel{\substack{\infty \\ \sim}}{\text { । }}$
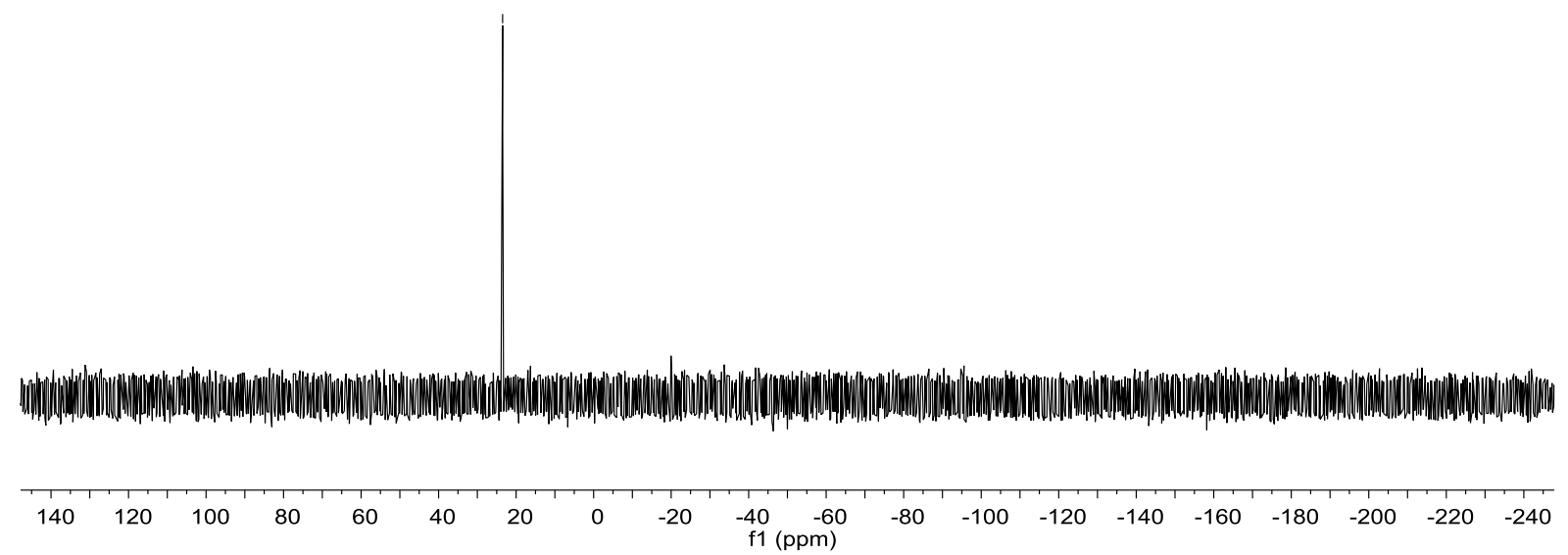

${ }^{13} \mathrm{C}$ NMR Spectrum of $7 \mathrm{l}\left(\mathrm{CDCl}_{3}, 100 \mathrm{MHz}\right)$
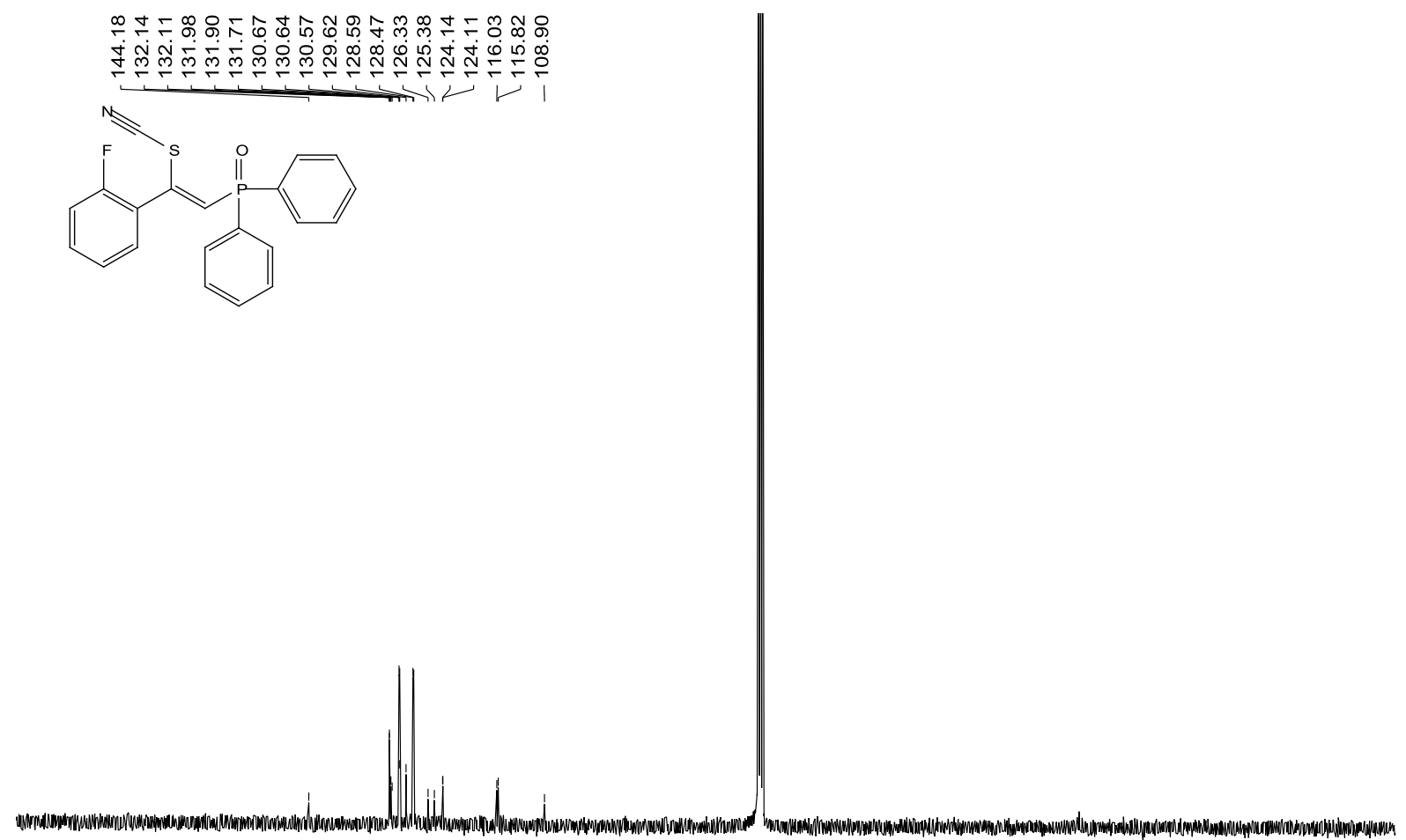

$\begin{array}{llllllllll}180 & 170 & 160 & 150 & 140 & 130 & 120 & 110 & 100 & 90 \\ \mathrm{f} 1(\mathrm{ppm})\end{array}$ 
${ }^{1} \mathrm{H}$ NMR Spectrum of $7 \mathrm{~m}\left(\mathrm{CDCl}_{3}, 400 \mathrm{MHz}\right)$
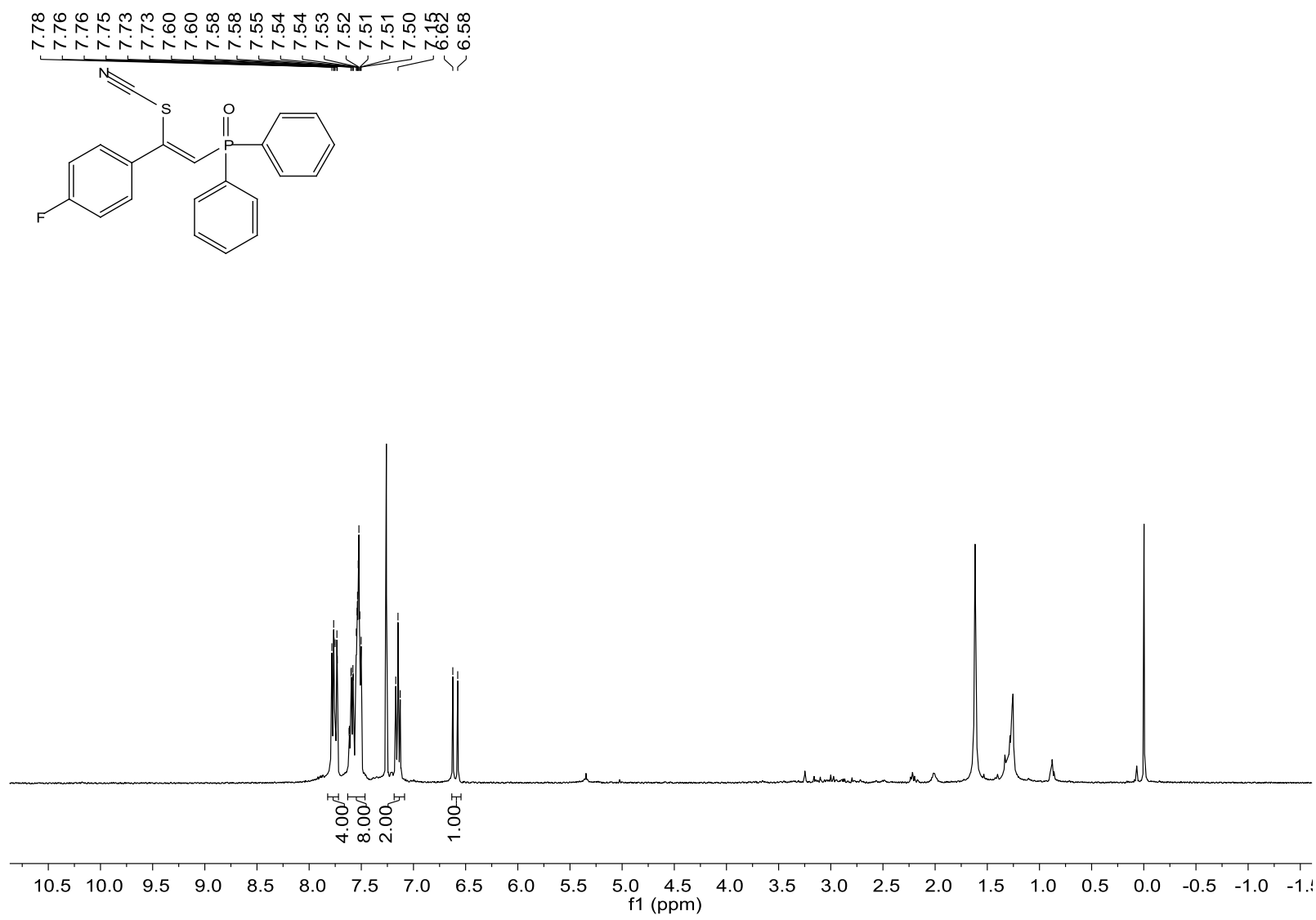

${ }^{31} \mathrm{P}$ NMR Spectrum of $7 \mathrm{~m}\left(\mathrm{CDCl}_{3}, 162 \mathrm{MHz}\right)$<smiles>N#CS/C(=C\P(=O)(c1ccccc1)c1ccccc1)c1ccc(F)cc1</smiles> 
${ }^{13} \mathrm{C}$ NMR Spectrum of $7 \mathrm{~m}\left(\mathrm{CDCl}_{3}, 100 \mathrm{MHz}\right)$
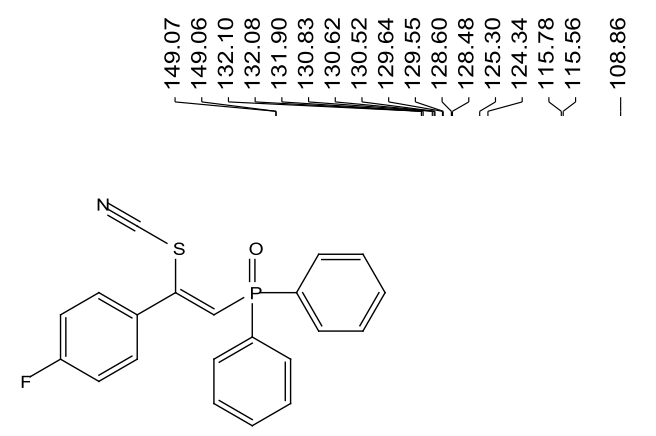
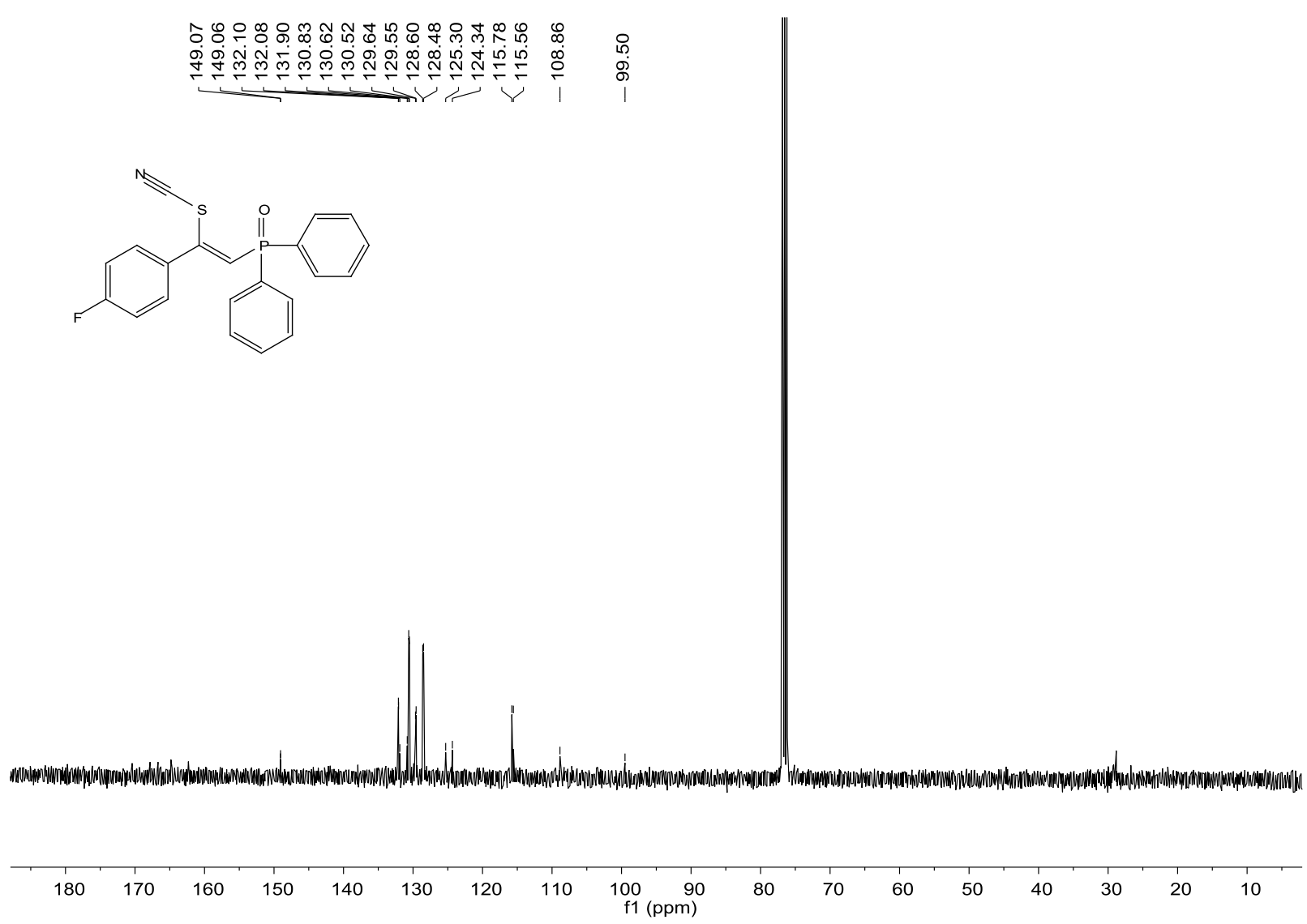

${ }^{1} \mathrm{H}$ NMR Spectrum of $7 \mathrm{n}\left(\mathrm{CDCl}_{3}, 400 \mathrm{MHz}\right)$

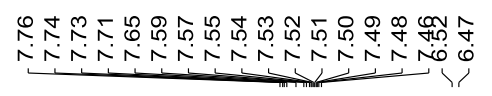
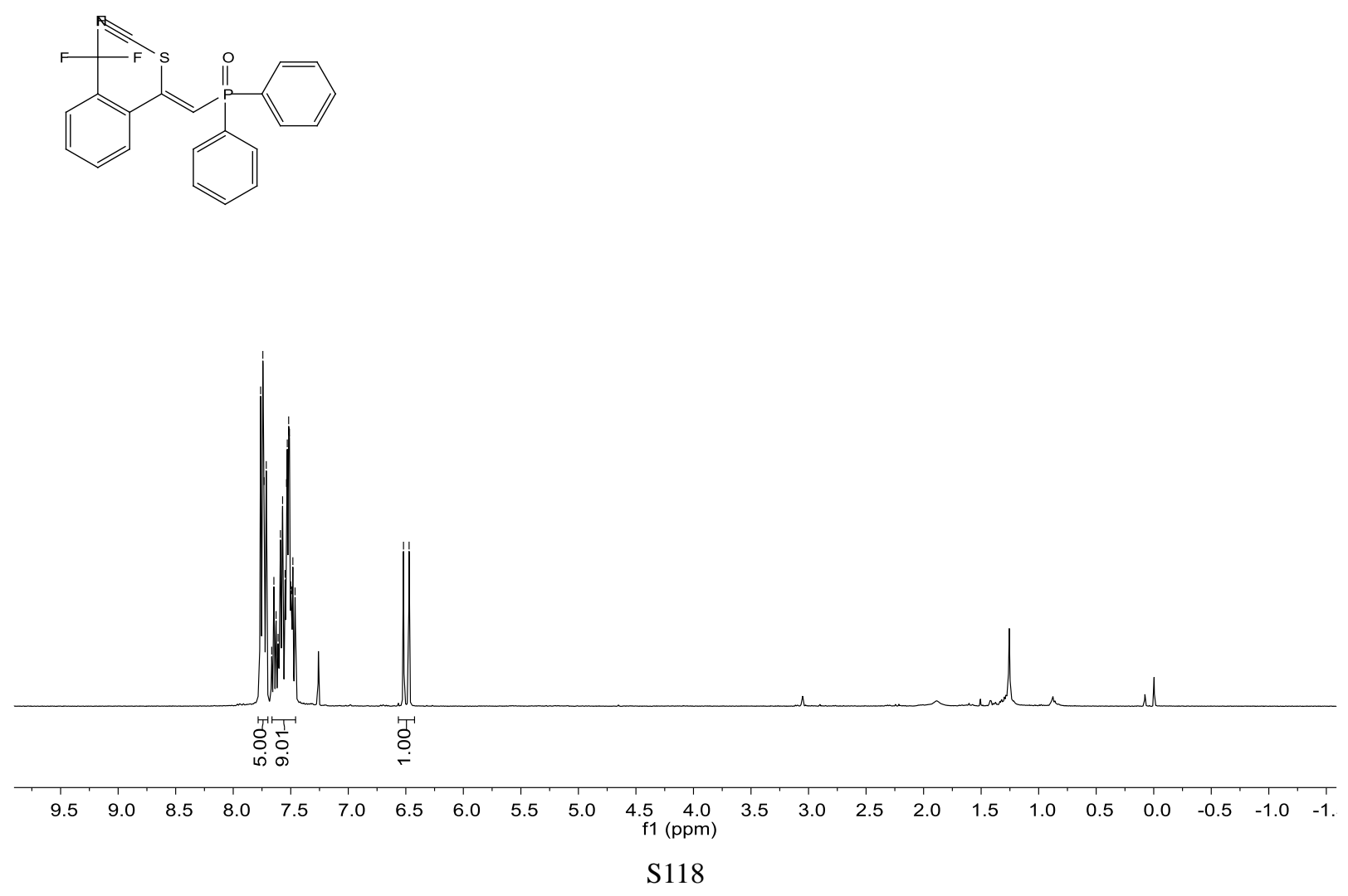
${ }^{31} \mathrm{P}$ NMR Spectrum of $7 \mathrm{n}\left(\mathrm{CDCl}_{3}, 162 \mathrm{MHz}\right)$

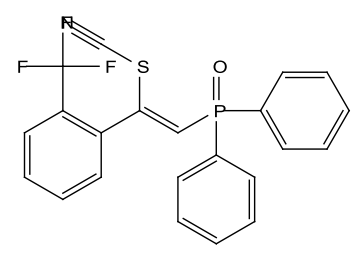

$\stackrel{\stackrel{n}{\sim}}{\stackrel{\sim}{N}}$

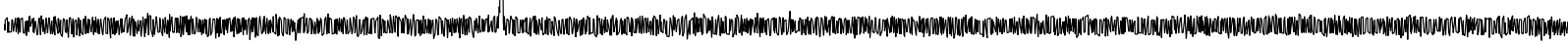

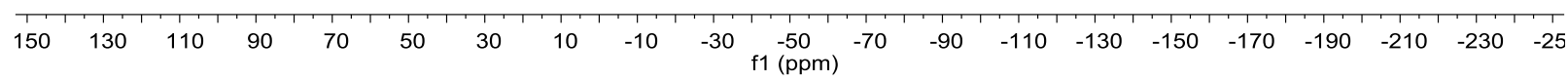

${ }^{13} \mathrm{C}$ NMR Spectrum of $7 \mathrm{n}\left(\mathrm{CDCl}_{3}, 100 \mathrm{MHz}\right)$

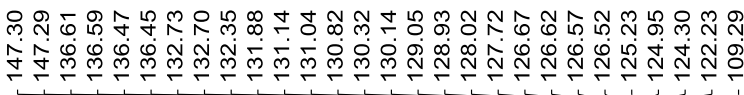
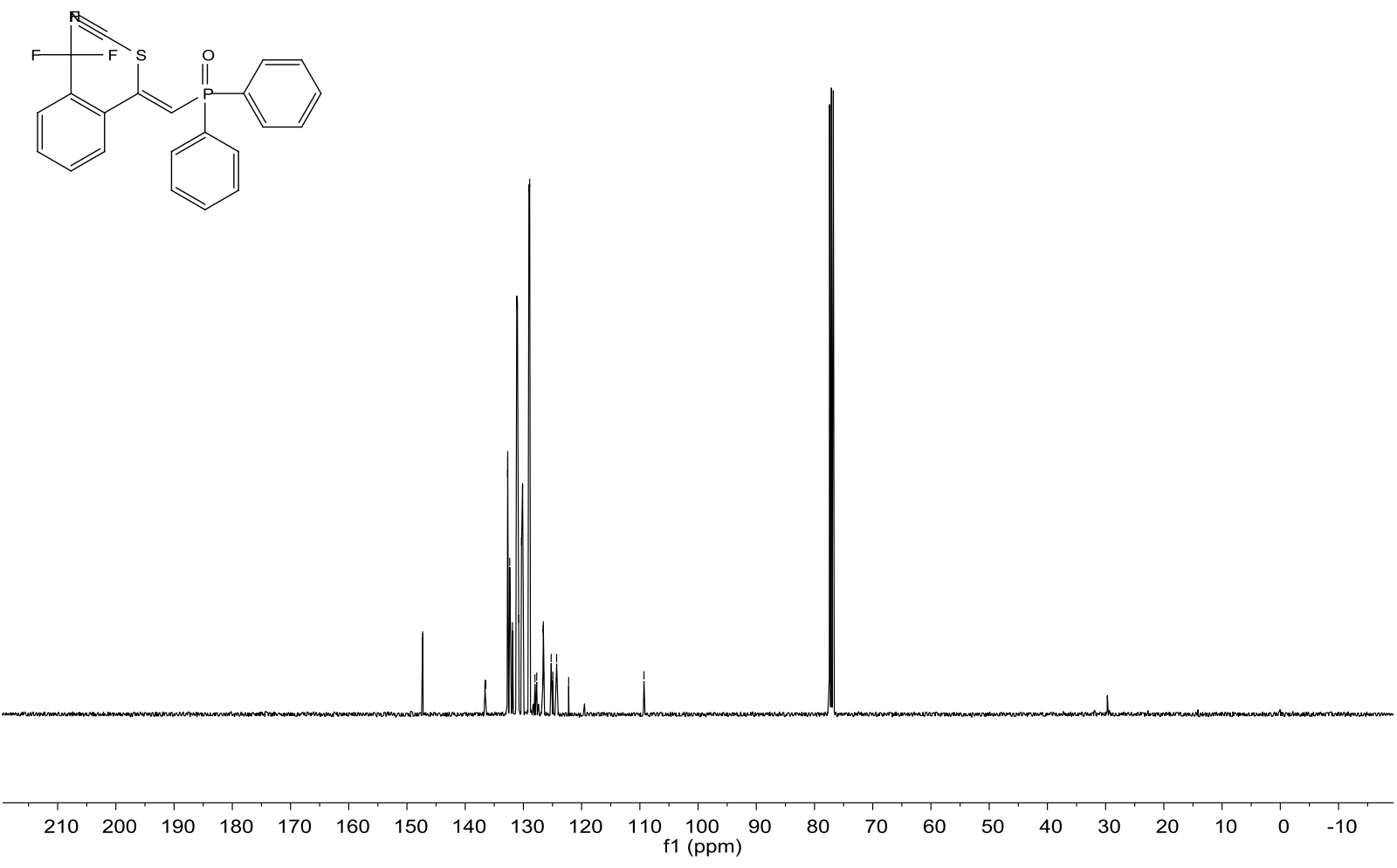

S119 
${ }^{1} \mathrm{H}$ NMR Spectrum of $7 \mathrm{o}\left(\mathrm{CDCl}_{3}, 400 \mathrm{MHz}\right)$
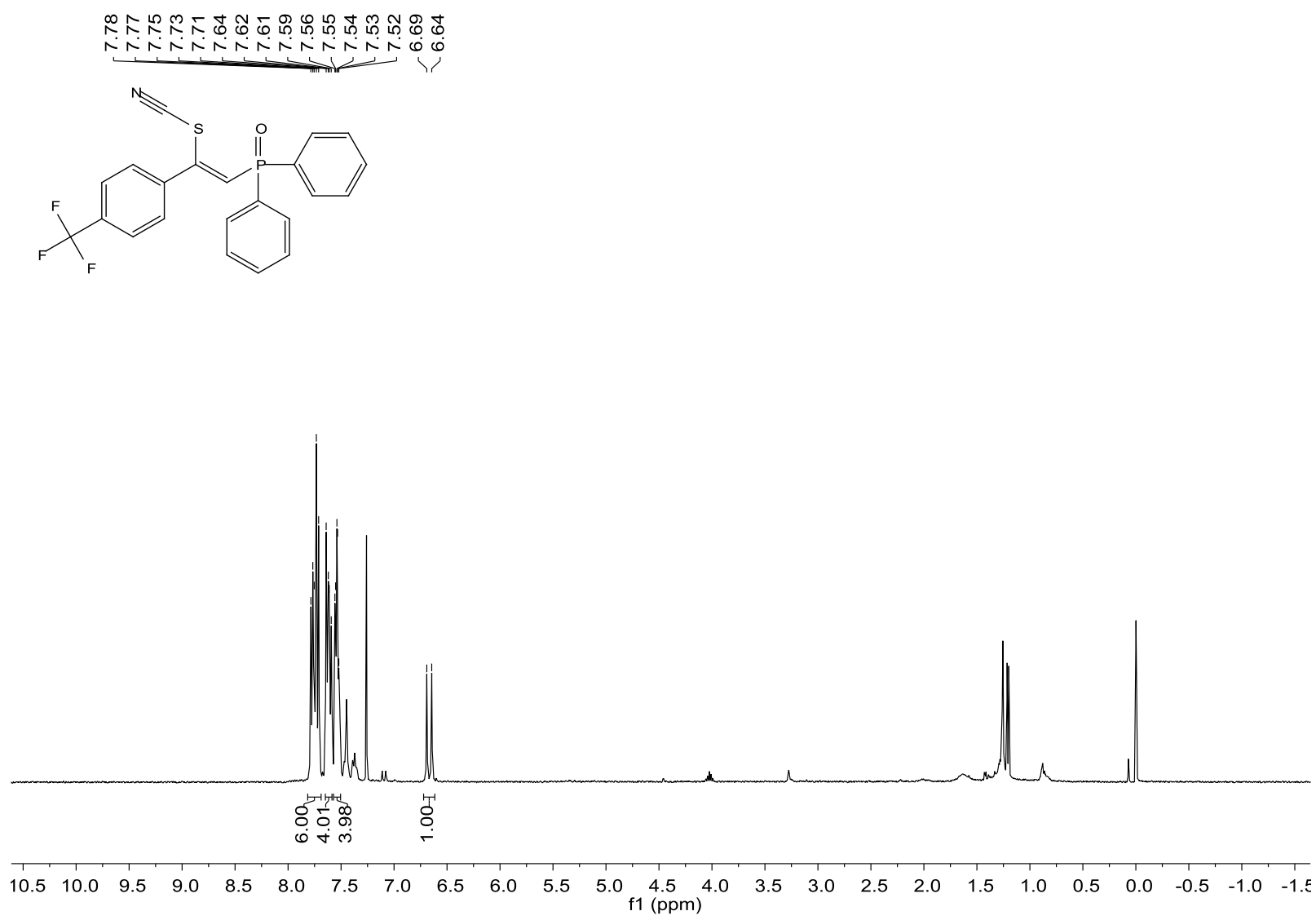

${ }^{31}$ P NMR Spectrum of $7 \mathrm{o}\left(\mathrm{CDCl}_{3}, 162 \mathrm{MHz}\right)$
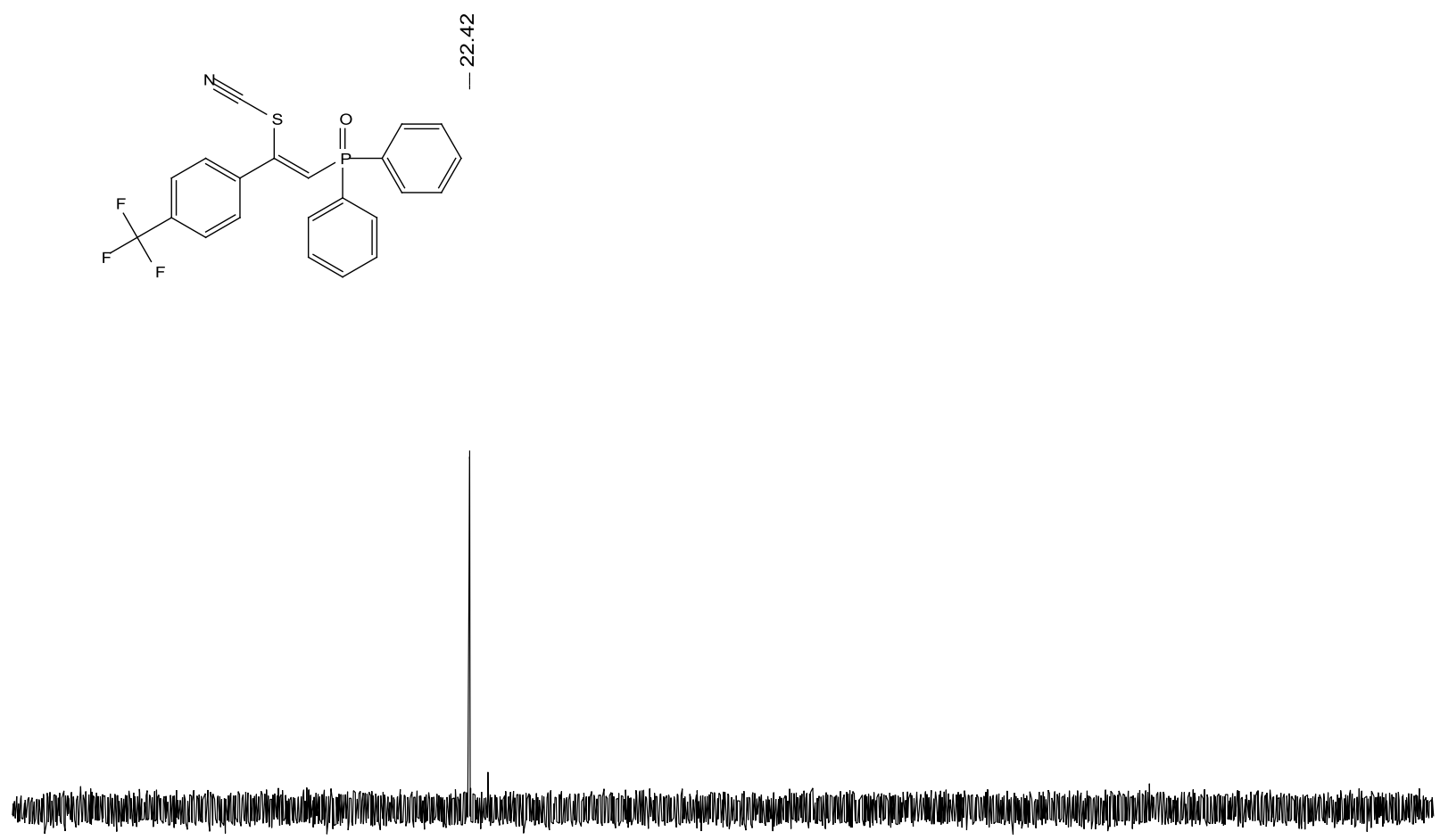

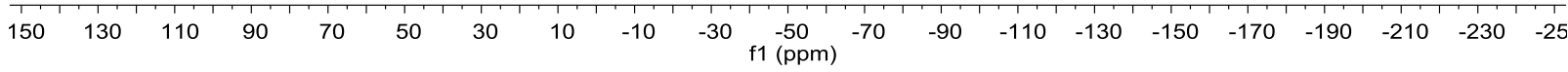


${ }^{13} \mathrm{C}$ NMR Spectrum of $7 \mathrm{o}\left(\mathrm{CDCl}_{3}, 100 \mathrm{MHz}\right)$

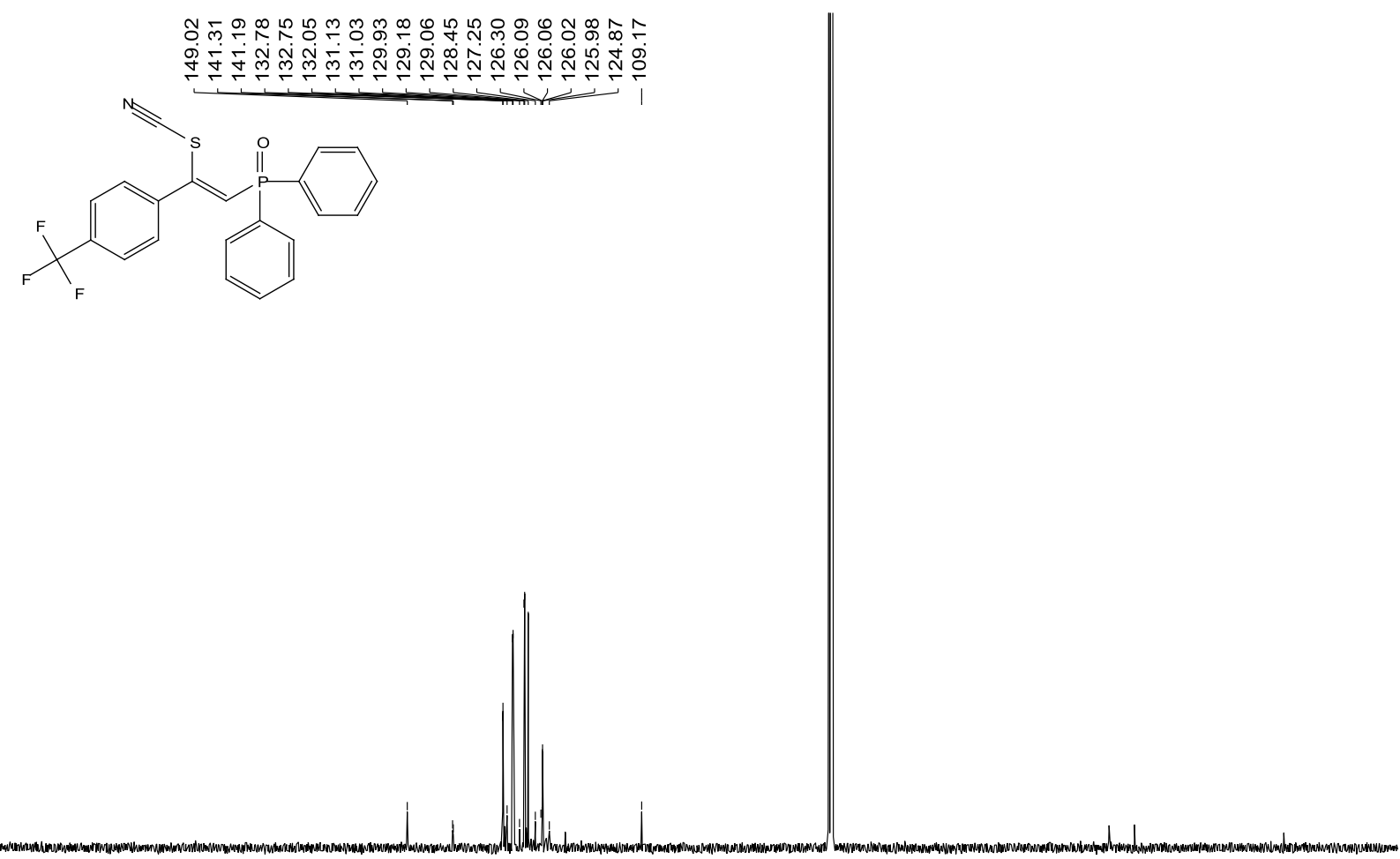

$\begin{array}{llllllllllllllllllllllll}210 & 200 & 190 & 180 & 170 & 160 & 150 & 140 & 130 & 120 & 110 & 100 & 90 & 80 & 70 & 60 & 50 & 40 & 30 & 20 & 10 & 0 & -10\end{array}$

${ }^{1} \mathrm{H}$ NMR Spectrum of $7 \mathrm{p}\left(\mathrm{CDCl}_{3}, 400 \mathrm{MHz}\right)$
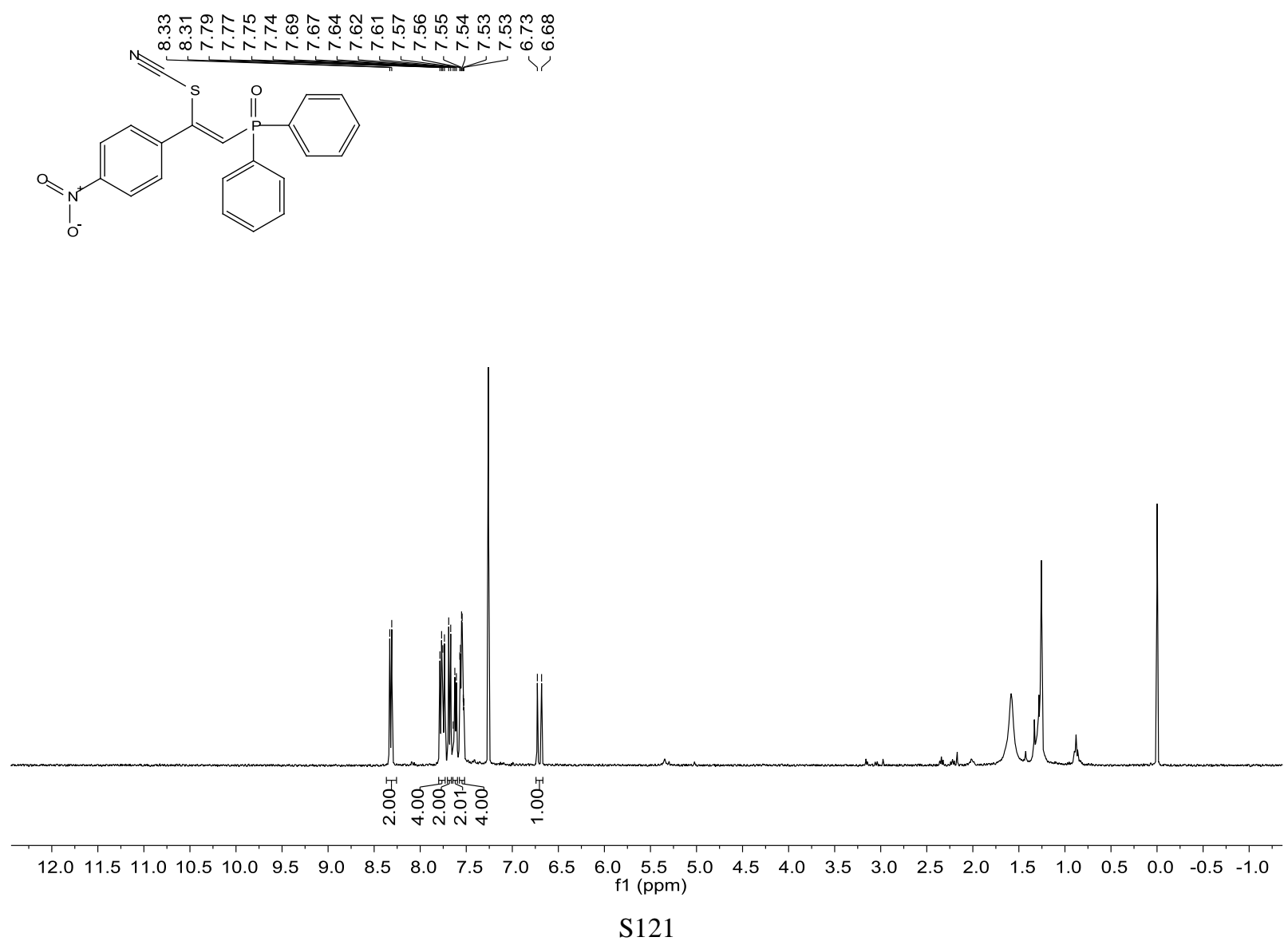
${ }^{31} \mathrm{P}$ NMR Spectrum of $7 \mathrm{p}\left(\mathrm{CDCl}_{3}, 162 \mathrm{MHz}\right)$<smiles>N#CS/C(=C/C(=O)c1ccccc1)c1ccc([N+](=O)[O-])cc1</smiles>

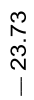

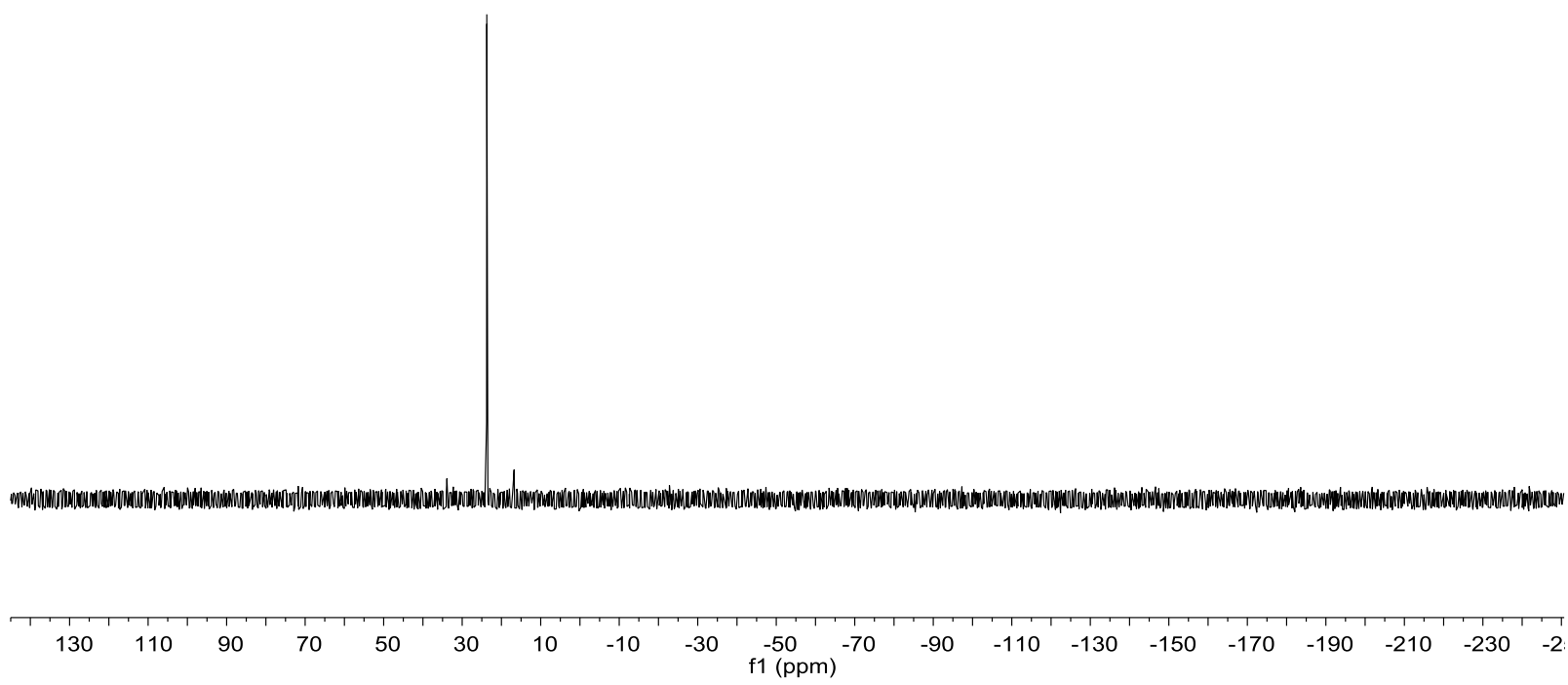

${ }^{13} \mathrm{C}$ NMR Spectrum of $7 \mathrm{p}\left(\mathrm{CDCl}_{3}, 100 \mathrm{MHz}\right)$
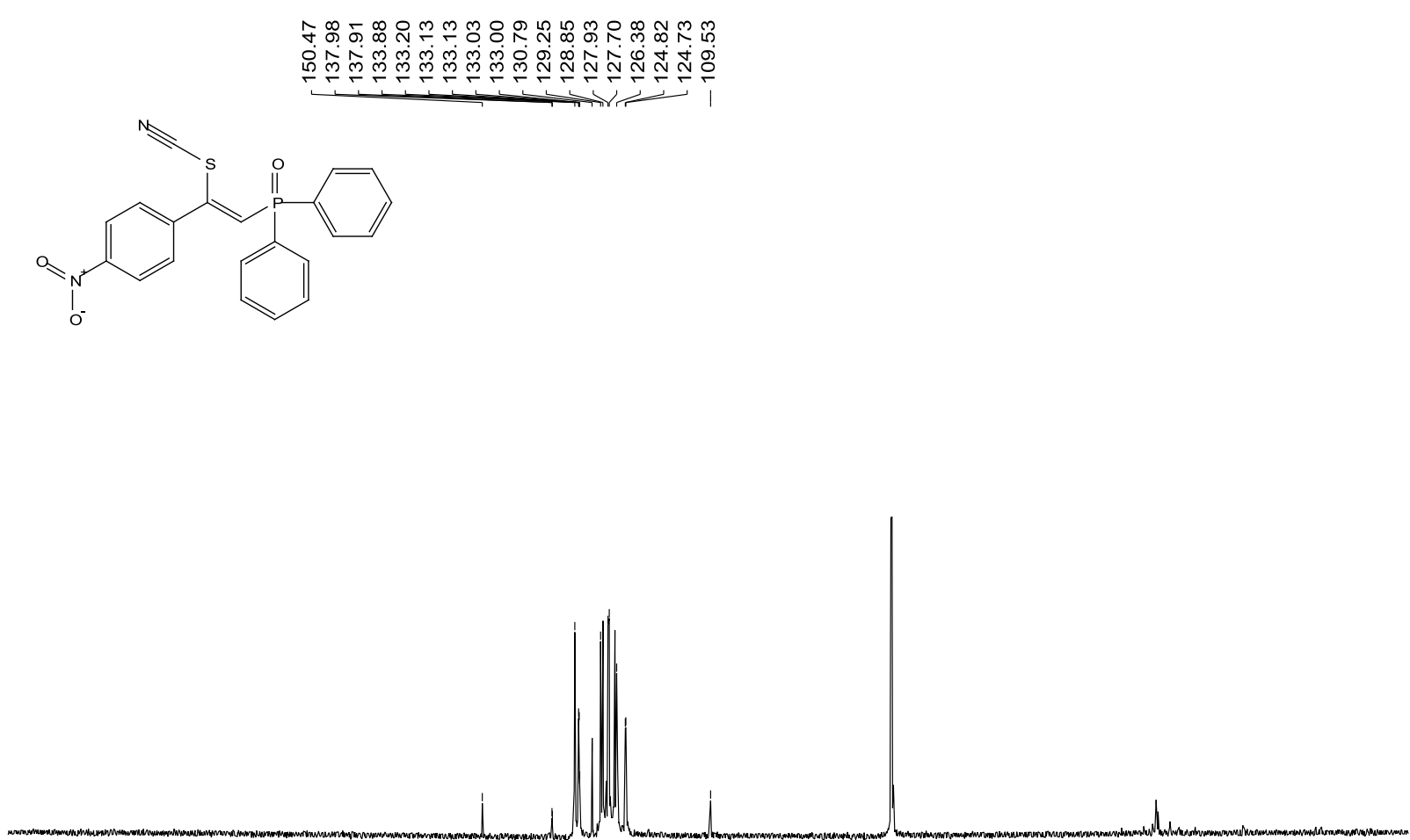

$\begin{array}{llllllllllllllllllllllll}230 & 220 & 210 & 200 & 190 & 180 & 170 & 160 & 150 & 140 & 130 & 120 & \begin{array}{c}110 \\ \mathrm{f} 1(\mathrm{ppm})\end{array} & 90 & 80 & 70 & 60 & 50 & 40 & 30 & 20 & 10 & 0 & -10\end{array}$ 
${ }^{1} \mathrm{H}$ NMR Spectrum of $7 \mathrm{q}\left(\mathrm{CDCl}_{3}, 400 \mathrm{MHz}\right)$

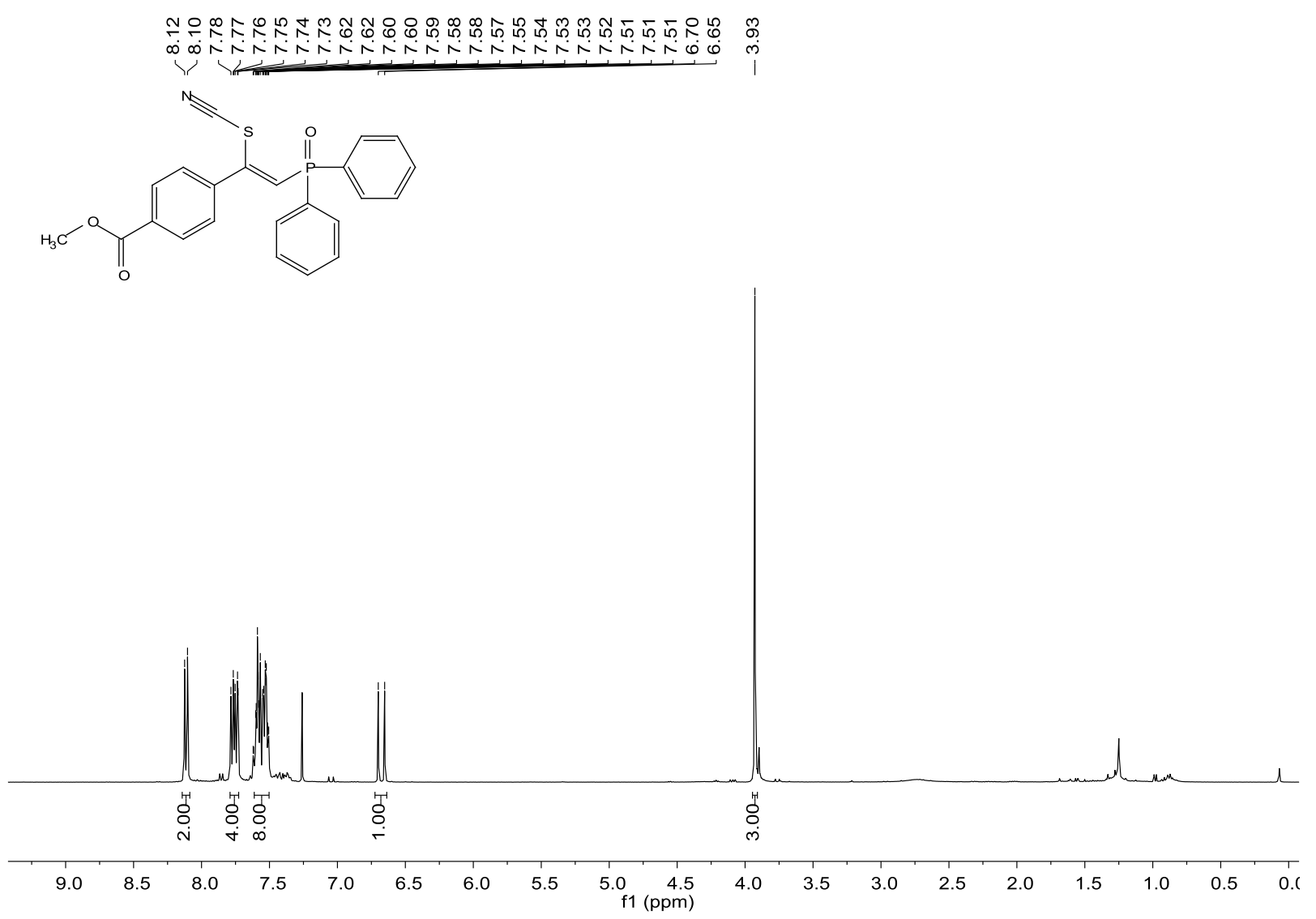

${ }^{31}$ P NMR Spectrum of $7 q\left(\mathrm{CDCl}_{3}, 162 \mathrm{MHz}\right)$<smiles>COC(=O)c1ccc(/C(=C/P(=O)(c2ccccc2)c2ccccc2)Sc2ccccc2)cc1</smiles>

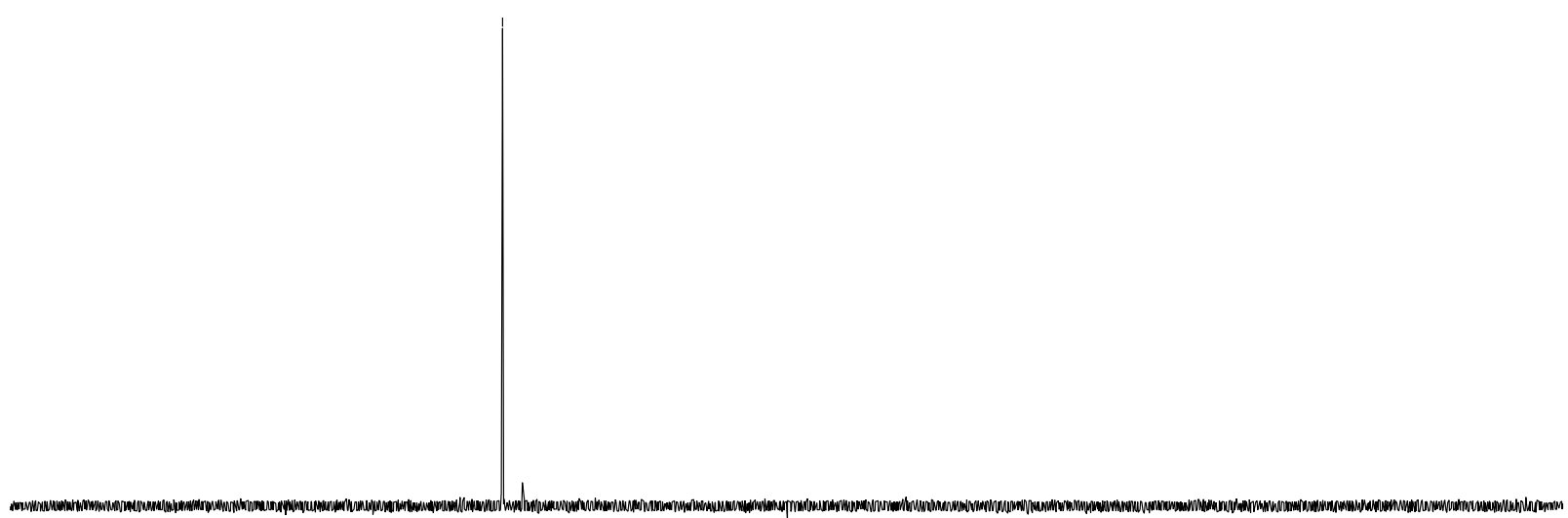

$\begin{array}{lllllllllllllllllllll}140 & 120 & 100 & 80 & 60 & 40 & 20 & 0 & -20 & -40 & -60 & -80 & -100 & -120 & -140 & -160 & -180 & -200 & -220 & -240\end{array}$ 
${ }^{13} \mathrm{C}$ NMR Spectrum of $7 \mathrm{q}\left(\mathrm{CDCl}_{3}, 100 \mathrm{MHz}\right)$
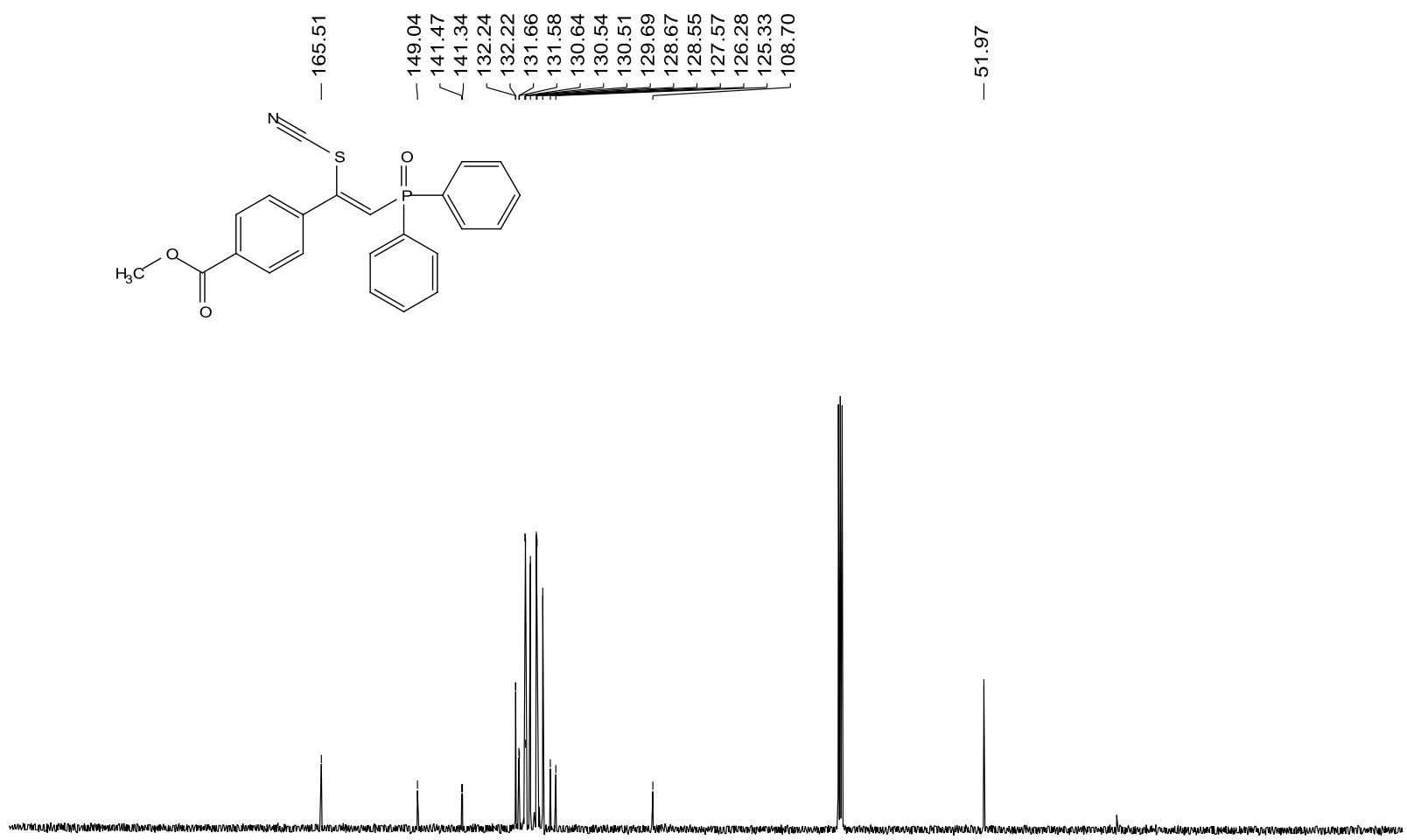

$\begin{array}{llllllllllllllllllllllllllllll}210 & 200 & 190 & 180 & 170 & 160 & 150 & 140 & 130 & 120 & 110 & 100 & 90 & 80 & 70 & 60 & 50 & 40 & 30 & 20 & 10 & 0 & -10\end{array}$

${ }^{1} \mathrm{H}$ NMR Spectrum of $7 \mathbf{r}\left(\mathrm{CDCl}_{3}, 400 \mathrm{MHz}\right)$

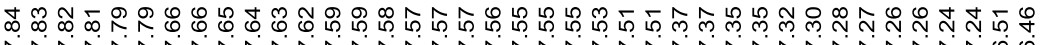

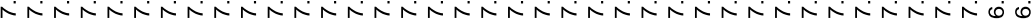
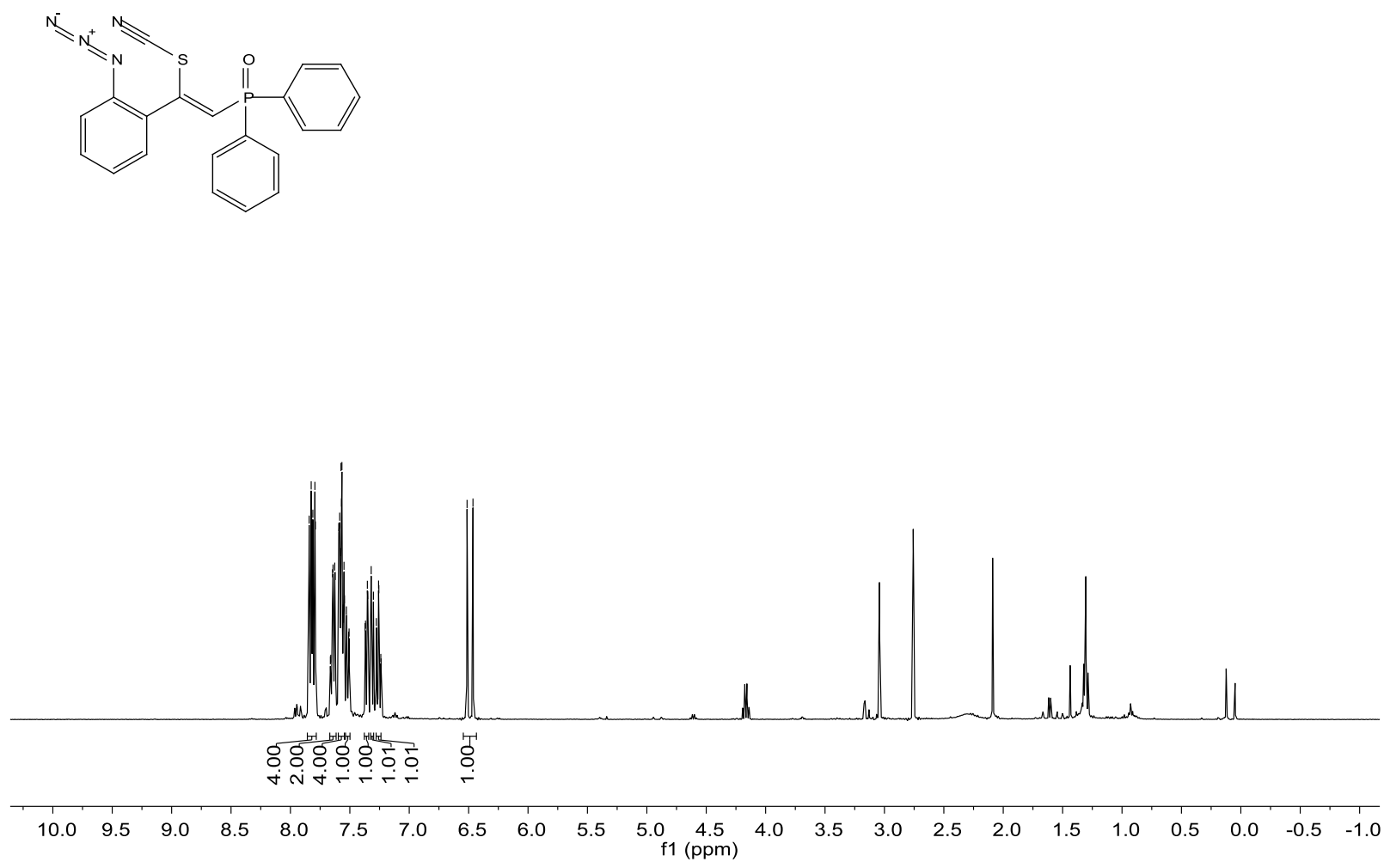
${ }^{31} \mathrm{P}$ NMR Spectrum of $7 \mathrm{r}\left(\mathrm{CDCl}_{3}, 162 \mathrm{MHz}\right)$

คุ

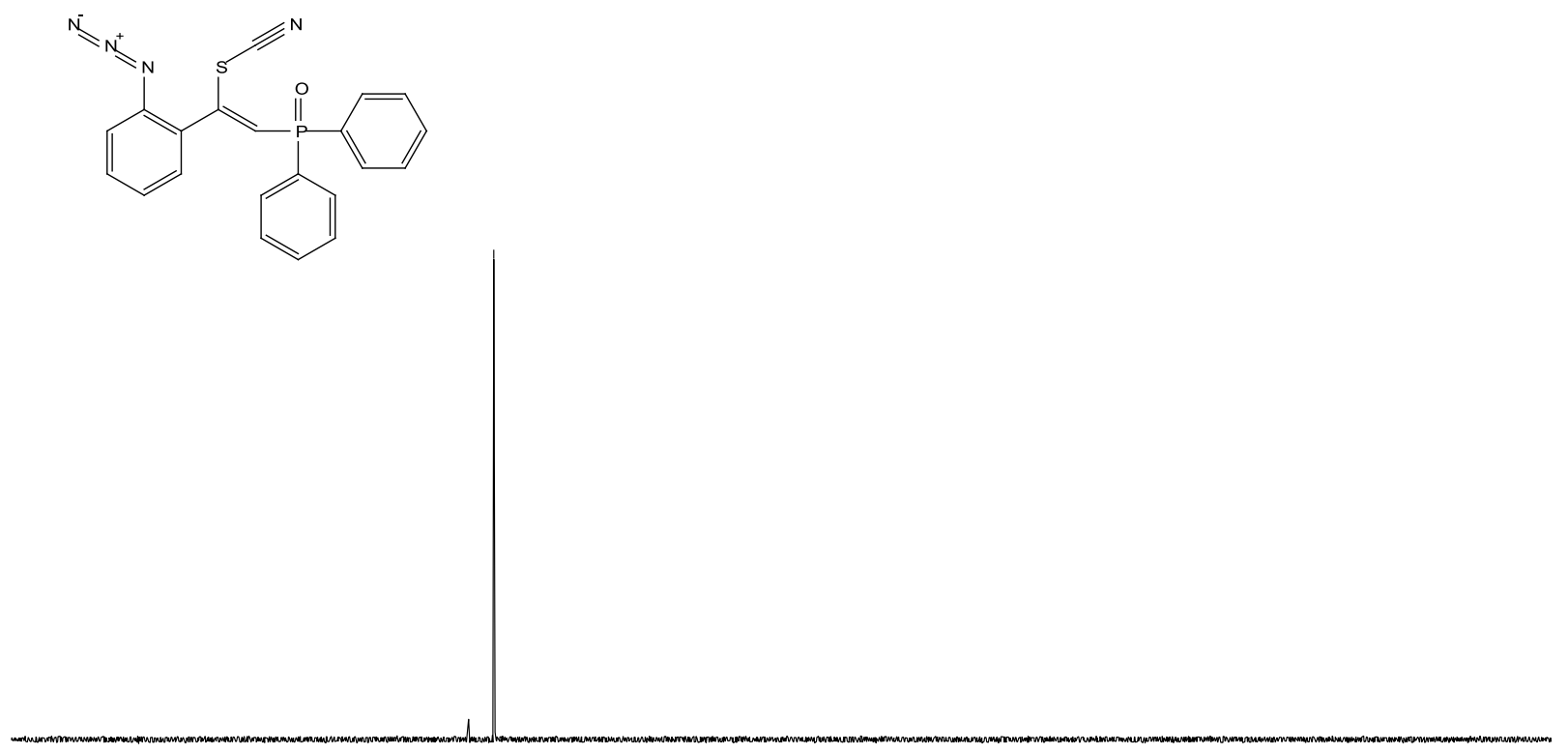

$\begin{array}{lllllllllllllllllllllllllll}140 & 120 & 100 & 80 & 60 & 40 & 20 & 0 & -20 & -40 & -60 & -80 & -100 & -120 & -140 & -160 & -180 & -200 & -220 & -240\end{array}$

${ }^{13} \mathrm{C}$ NMR Spectrum of $7 \mathrm{r}\left(\mathrm{CDCl}_{3}, 100 \mathrm{MHz}\right)$

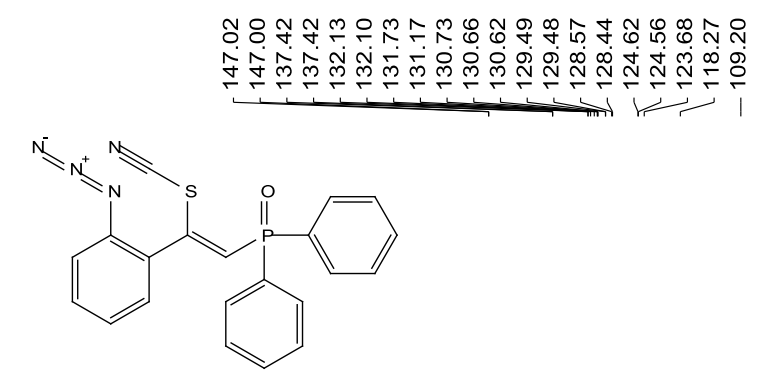

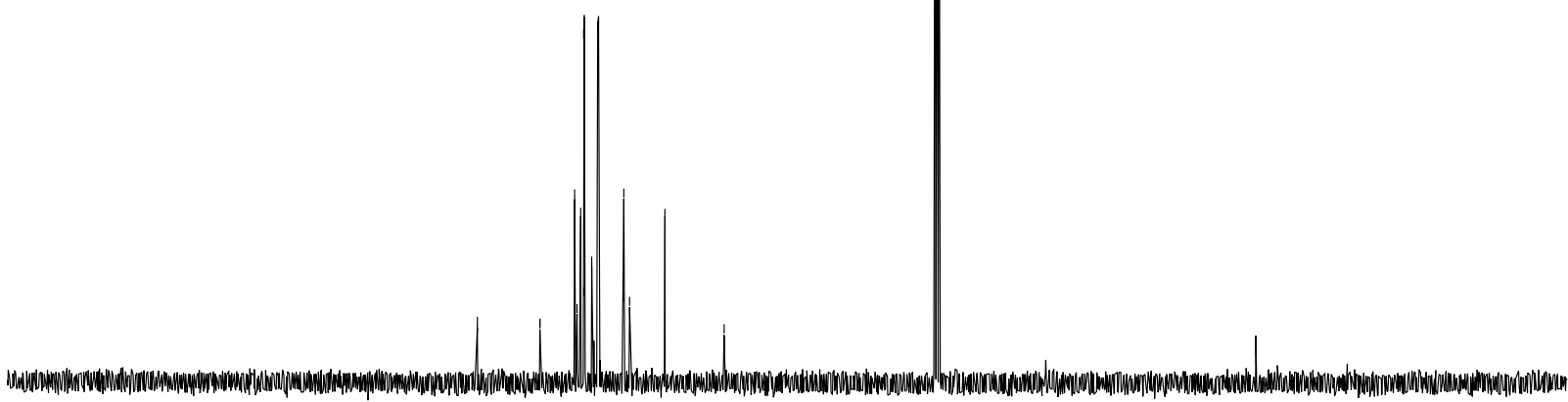

$\begin{array}{lllllllllllllllllllllll}210 & 200 & 190 & 180 & 170 & 160 & 150 & 140 & 130 & 120 & 110 & 100 & 90 & 80 & 70 & 60 & 50 & 40 & 30 & 20 & 10 & 0 & -10\end{array}$ 
${ }^{1} \mathrm{H}$ NMR Spectrum of $7 \mathrm{~s}\left(\mathrm{CDCl}_{3}, 400 \mathrm{MHz}\right)$

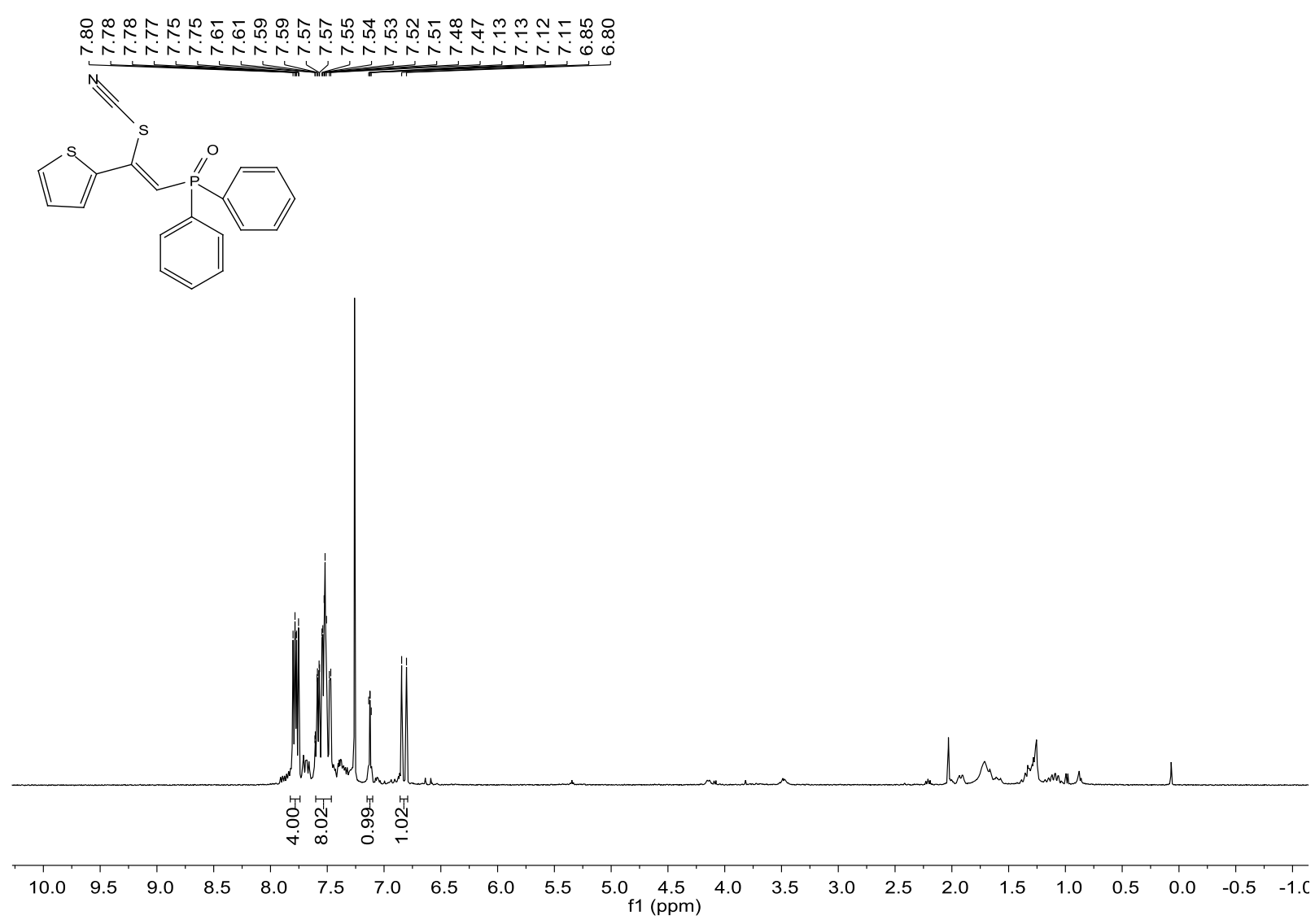

${ }^{31} \mathrm{P}$ NMR Spectrum of $7 \mathrm{~s}\left(\mathrm{CDCl}_{3}, 162 \mathrm{MHz}\right)$

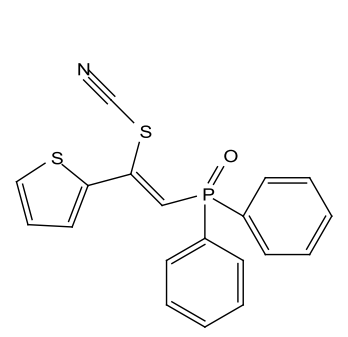

$\frac{10}{\grave{1}}$

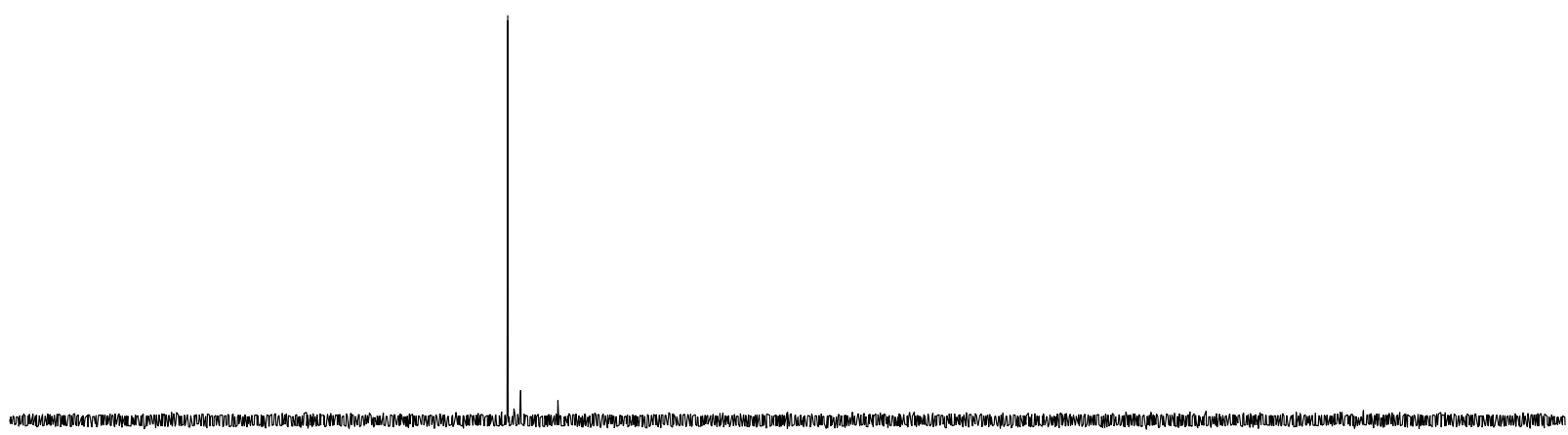

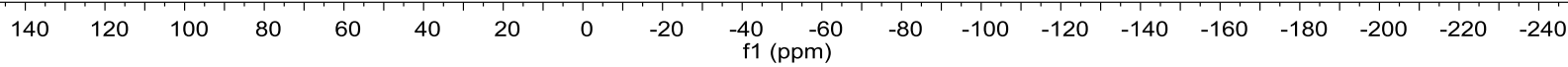


${ }^{13} \mathrm{C}$ NMR Spectrum of $7 \mathrm{~s}\left(\mathrm{CDCl}_{3}, 100 \mathrm{MHz}\right)$
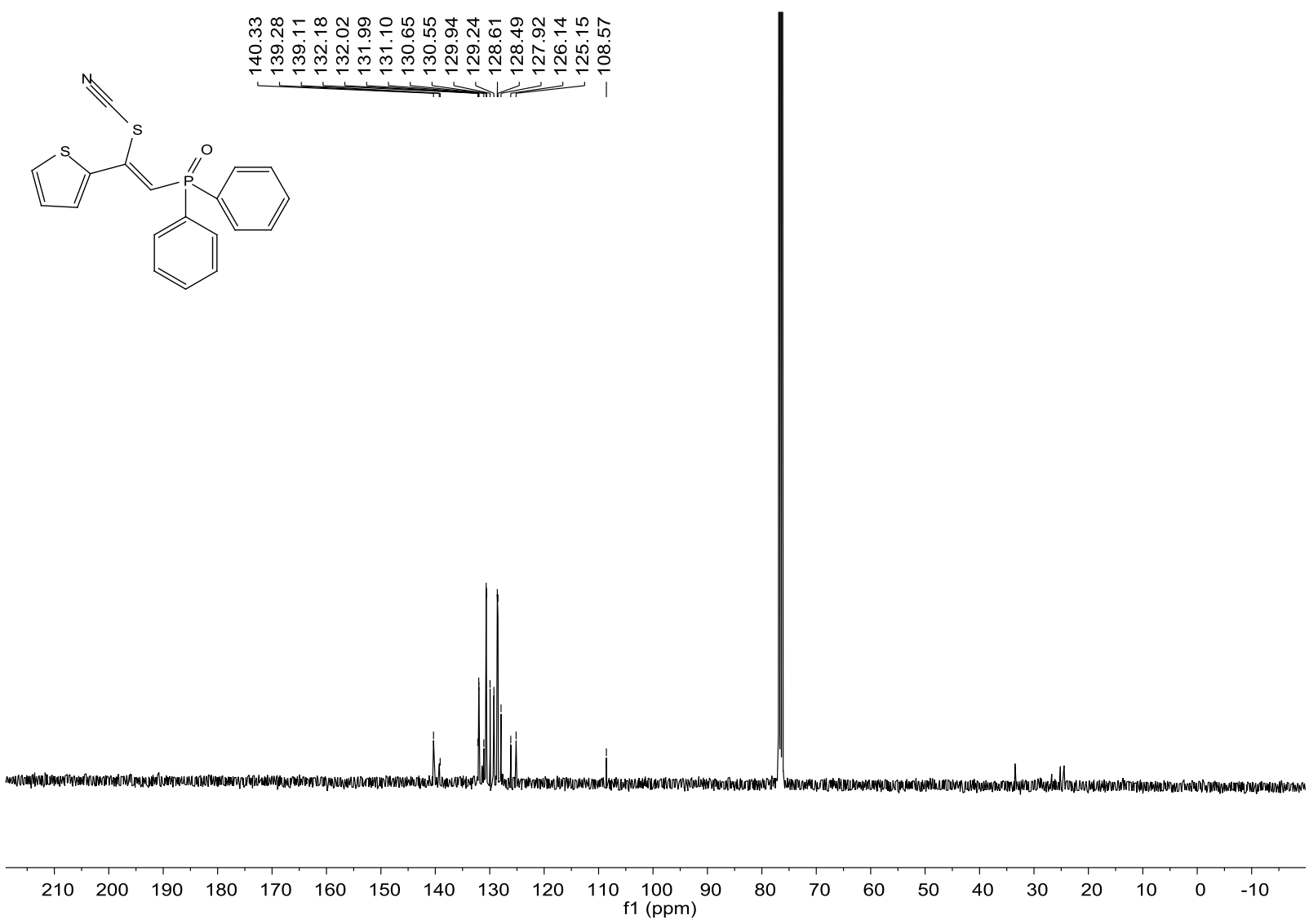

${ }^{1} \mathrm{H}$ NMR Spectrum of $7 \mathrm{t}$ (DMSO- $\left.d_{6}, 400 \mathrm{MHz}\right)$

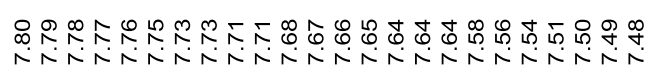

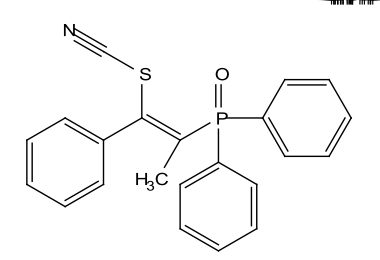

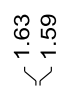

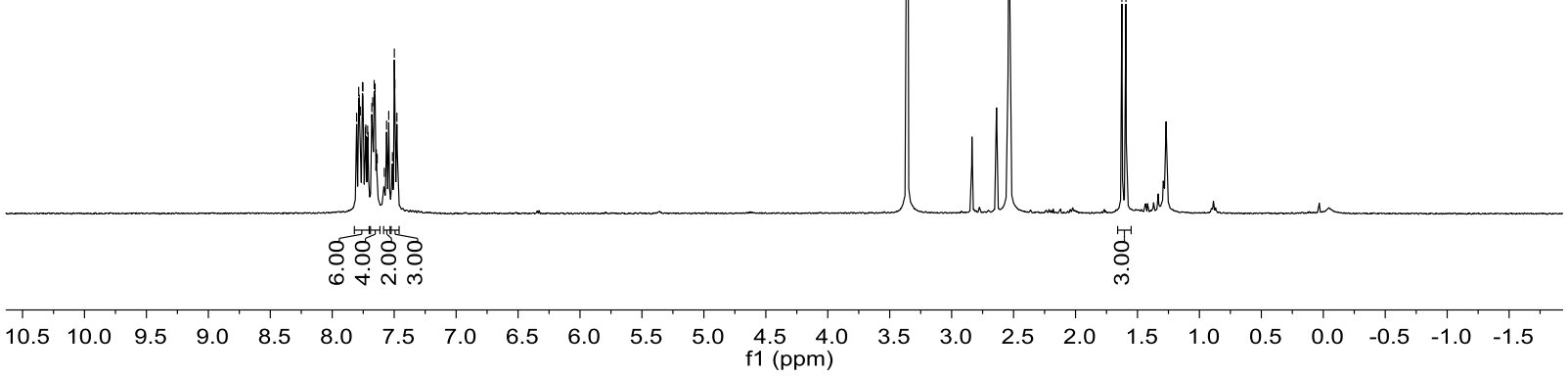

S127 
${ }^{31}$ P NMR Spectrum of $7 \mathrm{t}\left(\mathrm{DMSO}-d_{6}, 162 \mathrm{MHz}\right)$

$\frac{m}{m}$
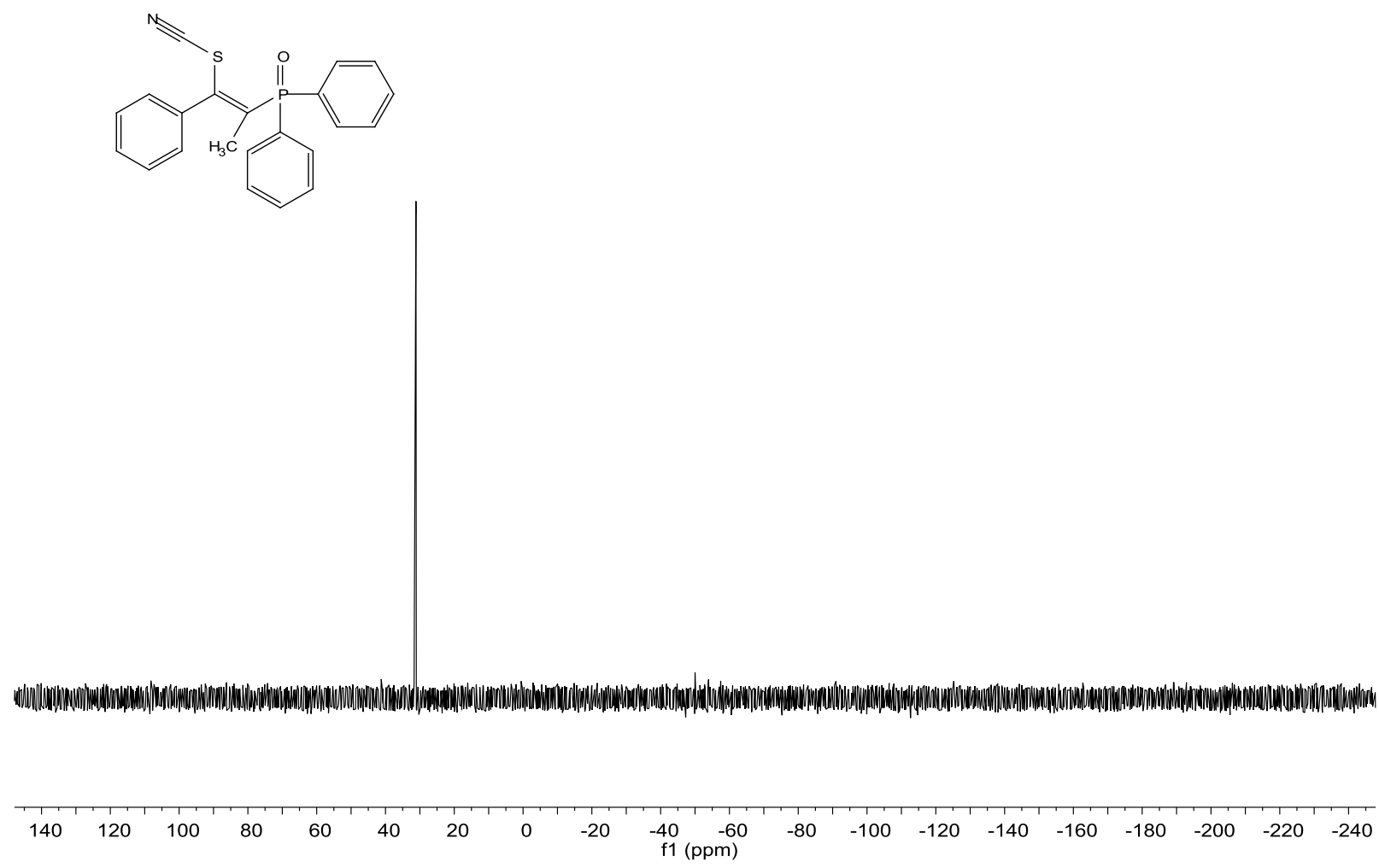

${ }^{13} \mathrm{C}$ NMR Spectrum of $7 \mathrm{t}\left(\mathrm{DMSO}-d_{6}, 100 \mathrm{MHz}\right)$
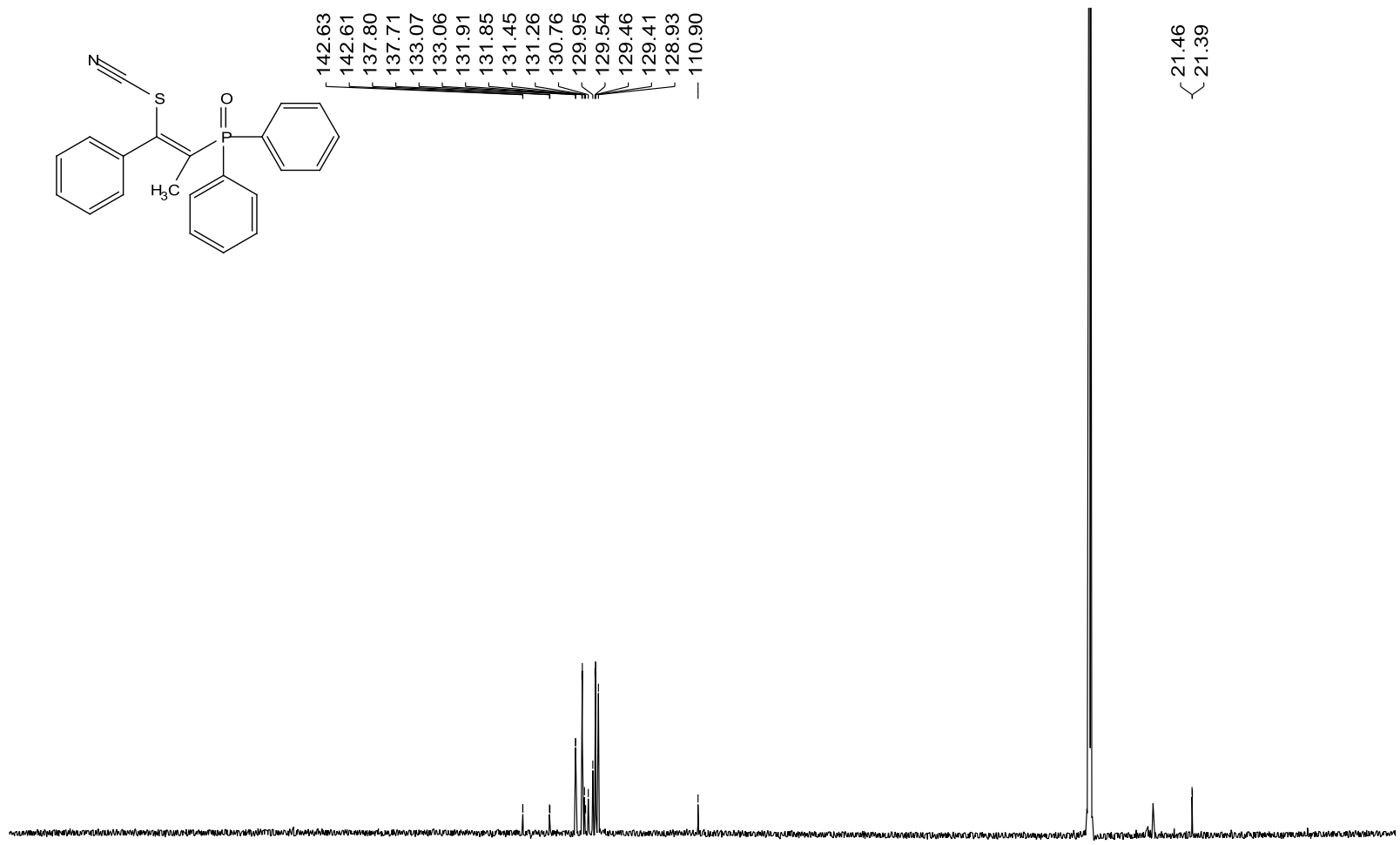

$\begin{array}{llllllllllllllllllllllllllllllllll}230 & 220 & 210 & 200 & 190 & 180 & 170 & 160 & 150 & 140 & 130 & 120 & 110 & 100 & 90 & 80 & 70 & 60 & 50 & 40 & 30 & 20 & 10 & 0 & -10\end{array}$ 
${ }^{1} \mathrm{H}$ NMR Spectrum of $7 \mathrm{u}\left(\mathrm{CDCl}_{3}, 400 \mathrm{MHz}\right)$

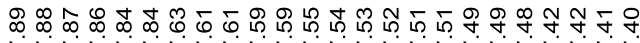

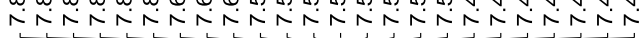<smiles>CC(=O)C(=C(SC#N)c1ccccc1)P(=O)(c1ccccc1)c1ccccc1</smiles>

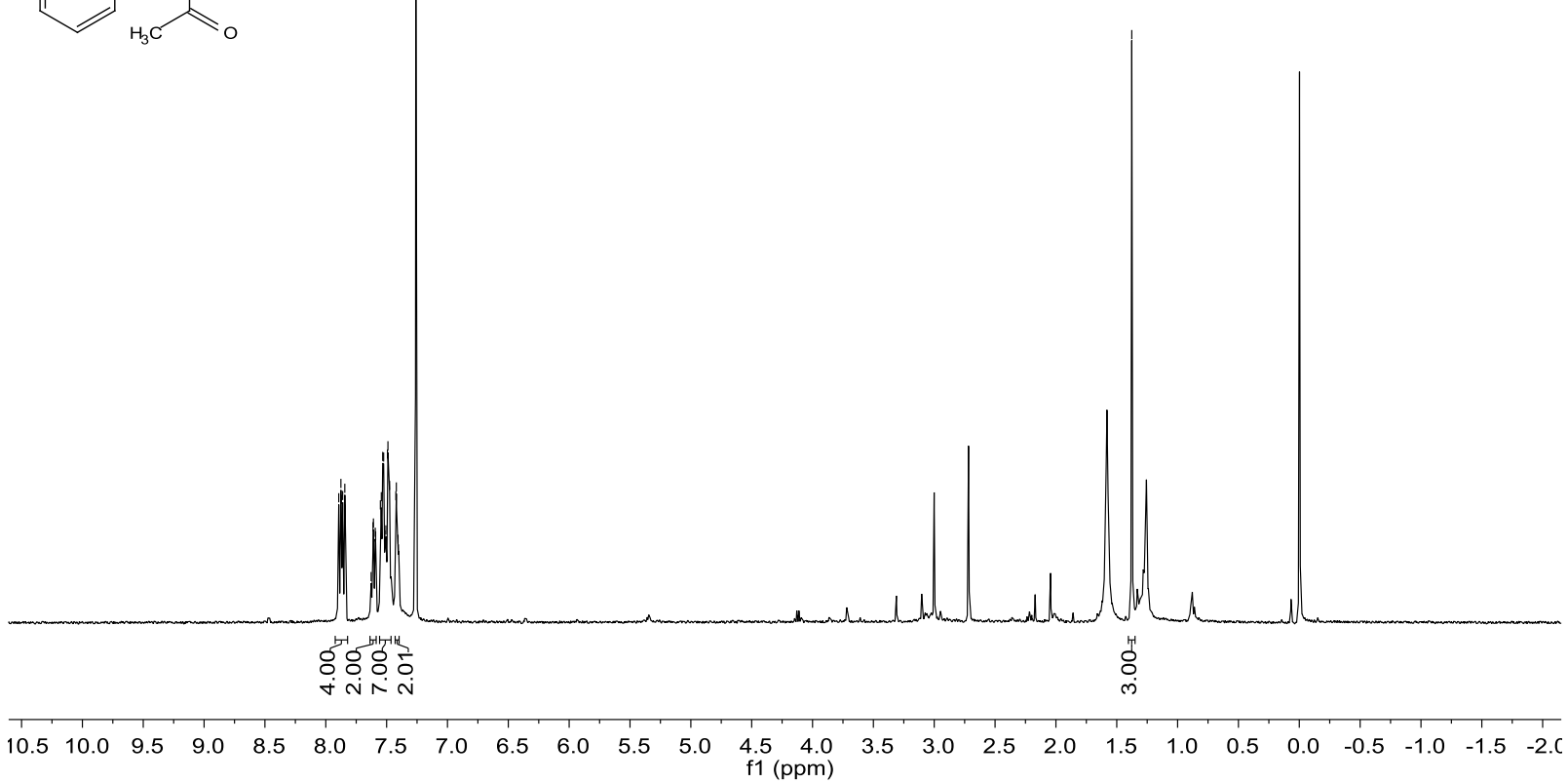

${ }^{31} \mathrm{P}$ NMR Spectrum of $7 \mathrm{u}\left(\mathrm{CDCl}_{3}, 162 \mathrm{MHz}\right)$

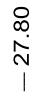<smiles>CC(=O)/C(=C(\SC#N)P(=O)(c1ccccc1)c1ccccc1)c1ccccc1</smiles>

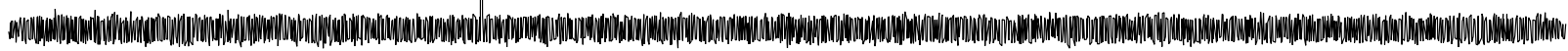

$\begin{array}{llllllllllllllllllll}140 & 120 & 100 & 80 & 60 & 40 & 20 & 0 & -20 & -40 & -60 & -80 & -100 & -120 & -140 & -160 & -180 & -200 & -220 & -240\end{array}$


${ }^{13} \mathrm{C}$ NMR Spectrum of $7 \mathrm{u}\left(\mathrm{CDCl}_{3}, 100 \mathrm{MHz}\right)$

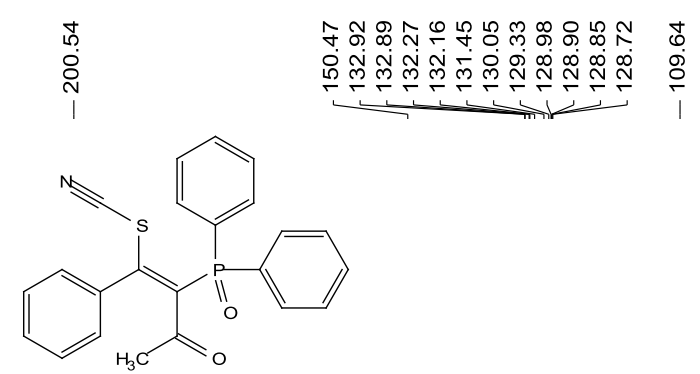

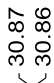

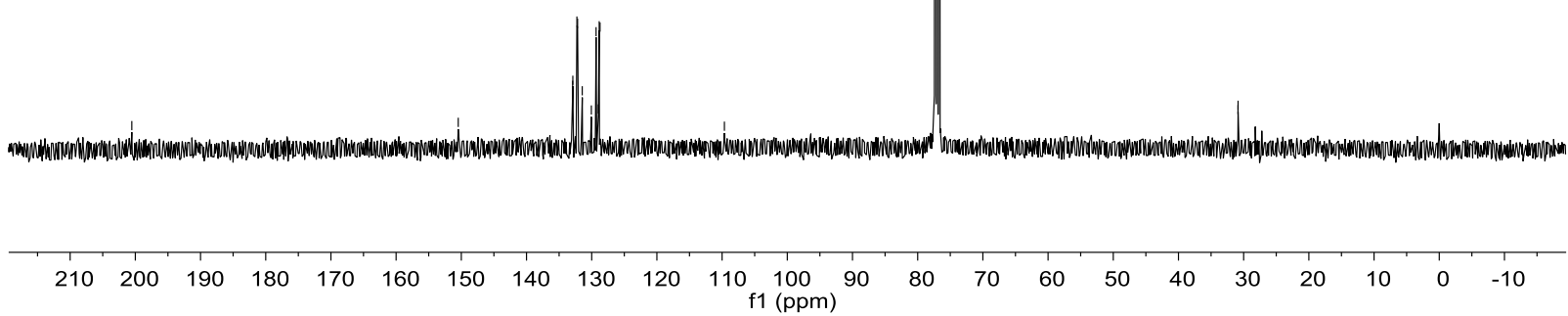

${ }^{1} \mathrm{H}$ NMR Spectrum of $7 \mathrm{v}\left(\mathrm{CDCl}_{3}, 400 \mathrm{MHz}\right)$

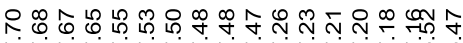

-

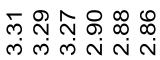

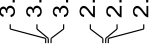
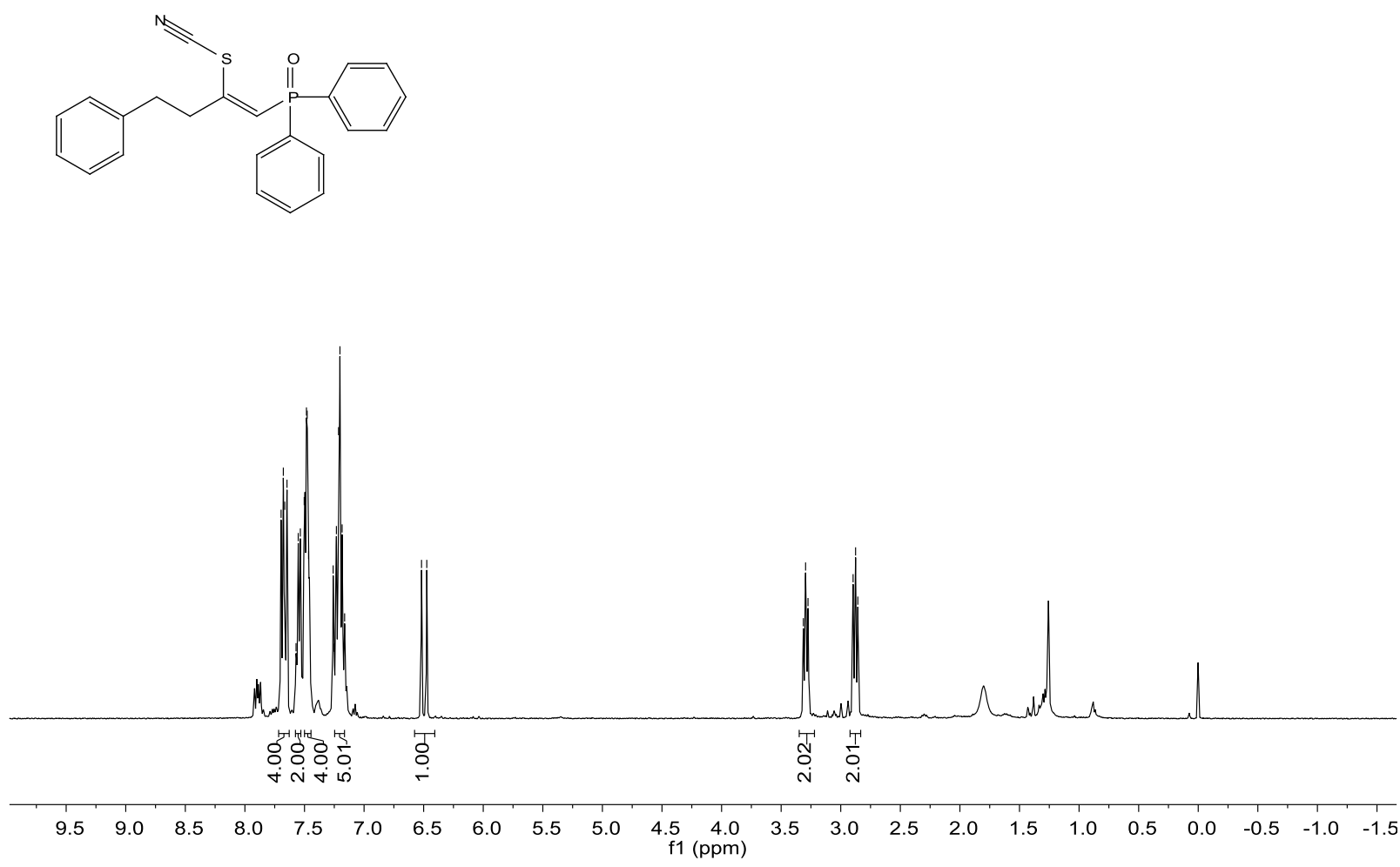

S130 
${ }^{31} \mathrm{P}$ NMR Spectrum of $7 \mathrm{v}\left(\mathrm{CDCl}_{3}, 162 \mathrm{MHz}\right)$

$\stackrel{\substack{n \\ \stackrel{2}{N}}}{1}$
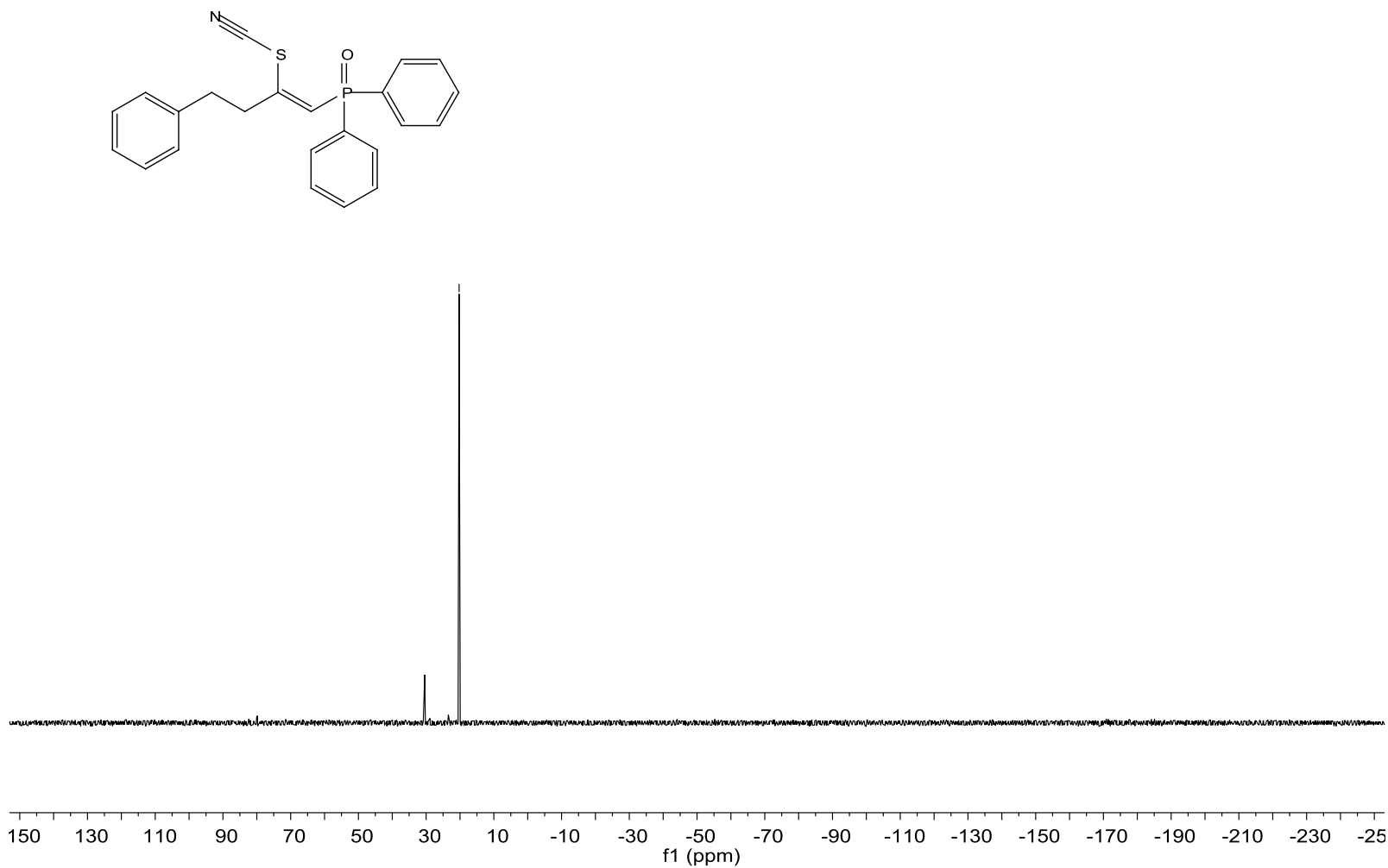

${ }^{13} \mathrm{C}$ NMR Spectrum of $7 \mathrm{v}\left(\mathrm{CDCl}_{3}, 100 \mathrm{MHz}\right)$

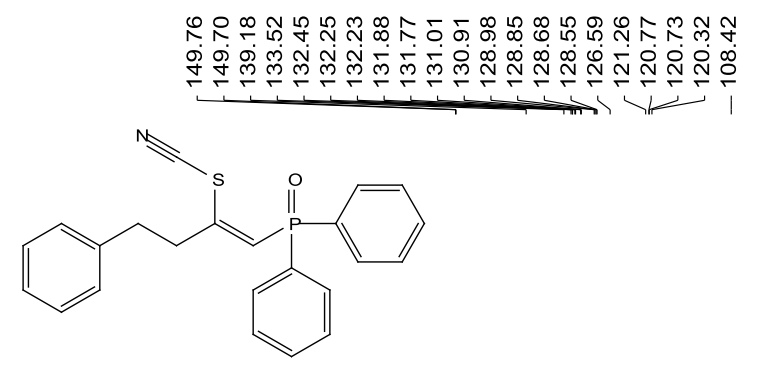

씨요요듀

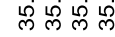

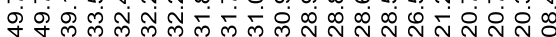

牙

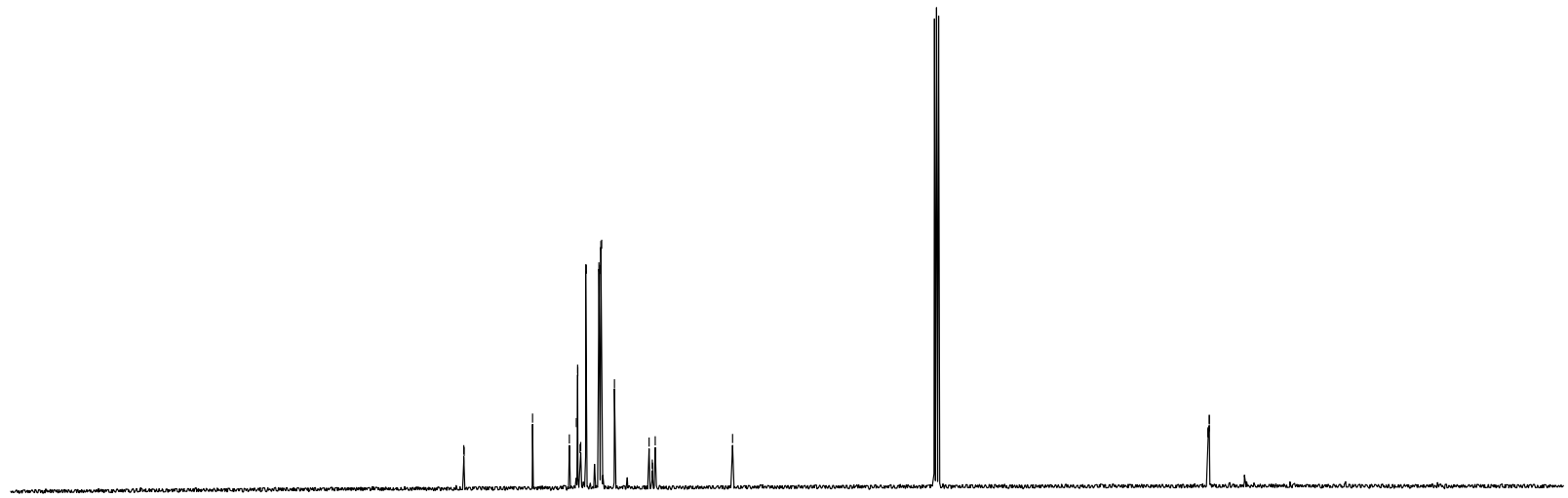

$\begin{array}{llllllllllllllllllllllllllll}210 & 200 & 190 & 180 & 170 & 160 & 150 & 140 & 130 & 120 & 110 & 100 & 90 & 80 & 70 & 60 & 50 & 40 & 30 & 20 & 10 & 0 & -10\end{array}$ 
${ }^{1} \mathrm{H}$ NMR Spectrum of $7 \mathrm{w}\left(\mathrm{CDCl}_{3}, 400 \mathrm{MHz}\right)$

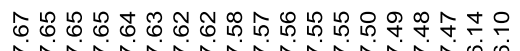

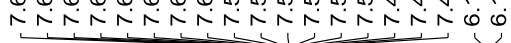

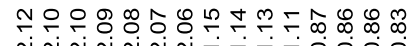

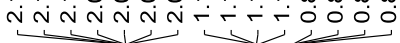
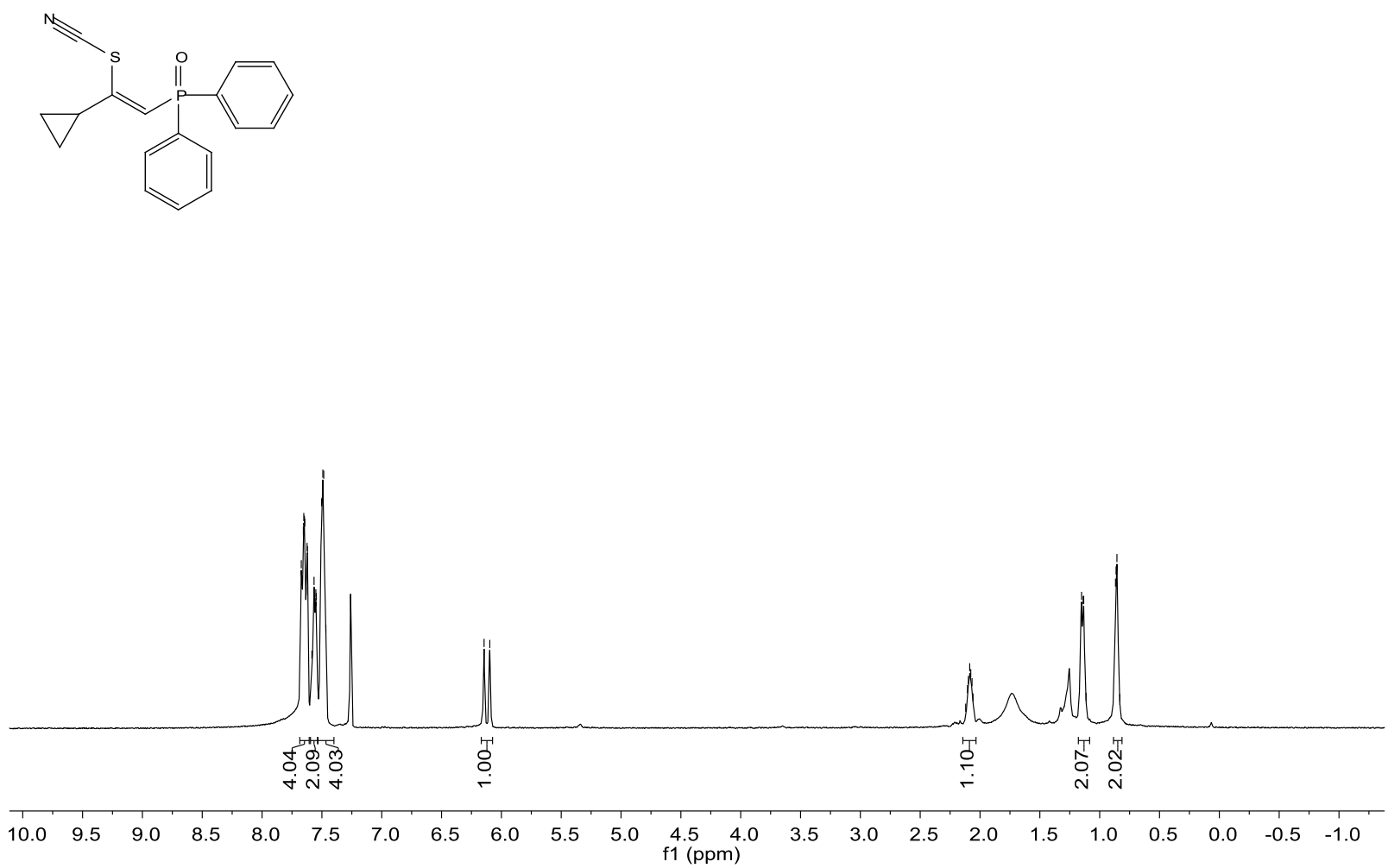

${ }^{31} \mathrm{P}$ NMR Spectrum of $7 \mathrm{w}\left(\mathrm{CDCl}_{3}, 162 \mathrm{MHz}\right)$

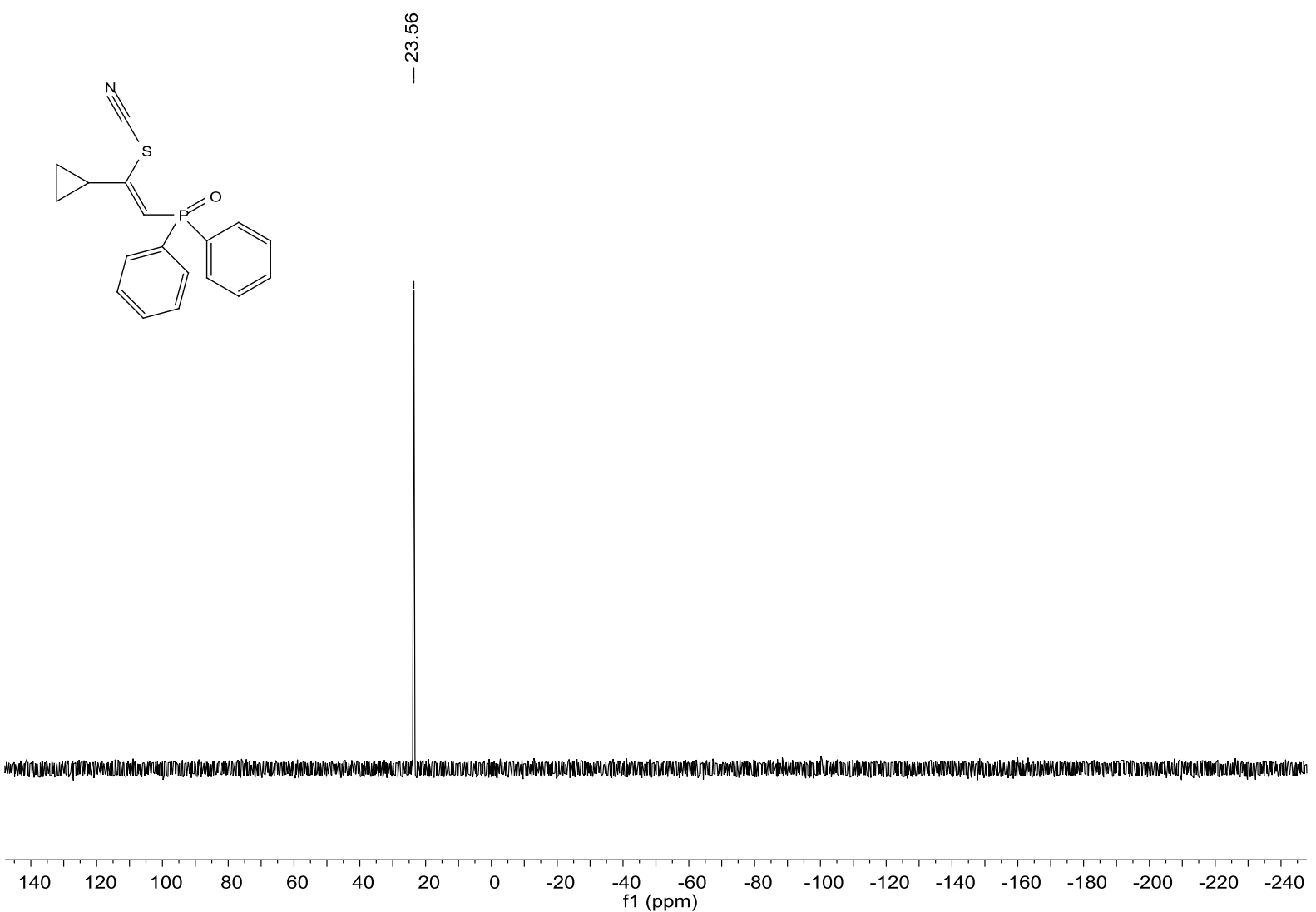


${ }^{13} \mathrm{C}$ NMR Spectrum of $7 \mathrm{w}\left(\mathrm{CDCl}_{3}, 100 \mathrm{MHz}\right)$

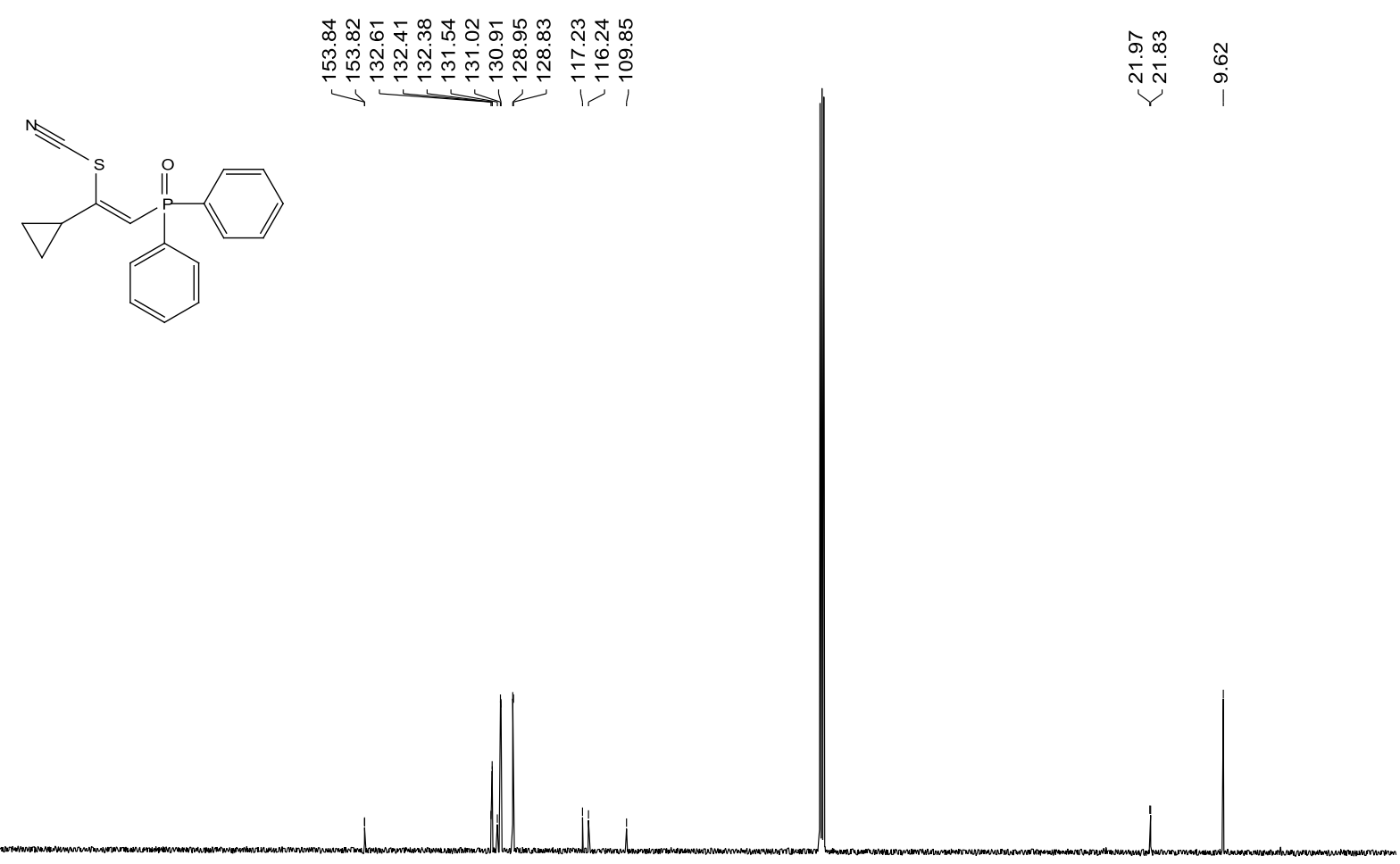

$\begin{array}{lllllllllllllllllllllllllllll}210 & 200 & 190 & 180 & 170 & 160 & 150 & 140 & 130 & 120 & 110 & 100 & 90 & 80 & 70 & 60 & 50 & 40 & 30 & 20 & 10 & 0 & -10\end{array}$

${ }^{1} \mathrm{H}$ NMR Spectrum of $7 \mathrm{x}^{\prime}\left(\mathrm{CDCl}_{3}, 400 \mathrm{MHz}\right)$

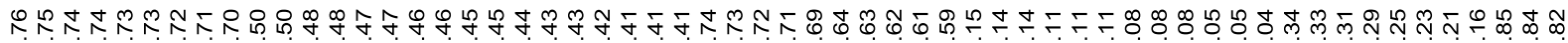

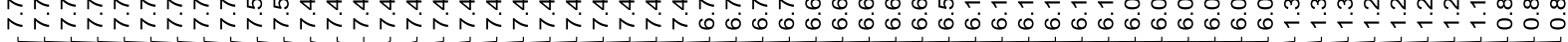
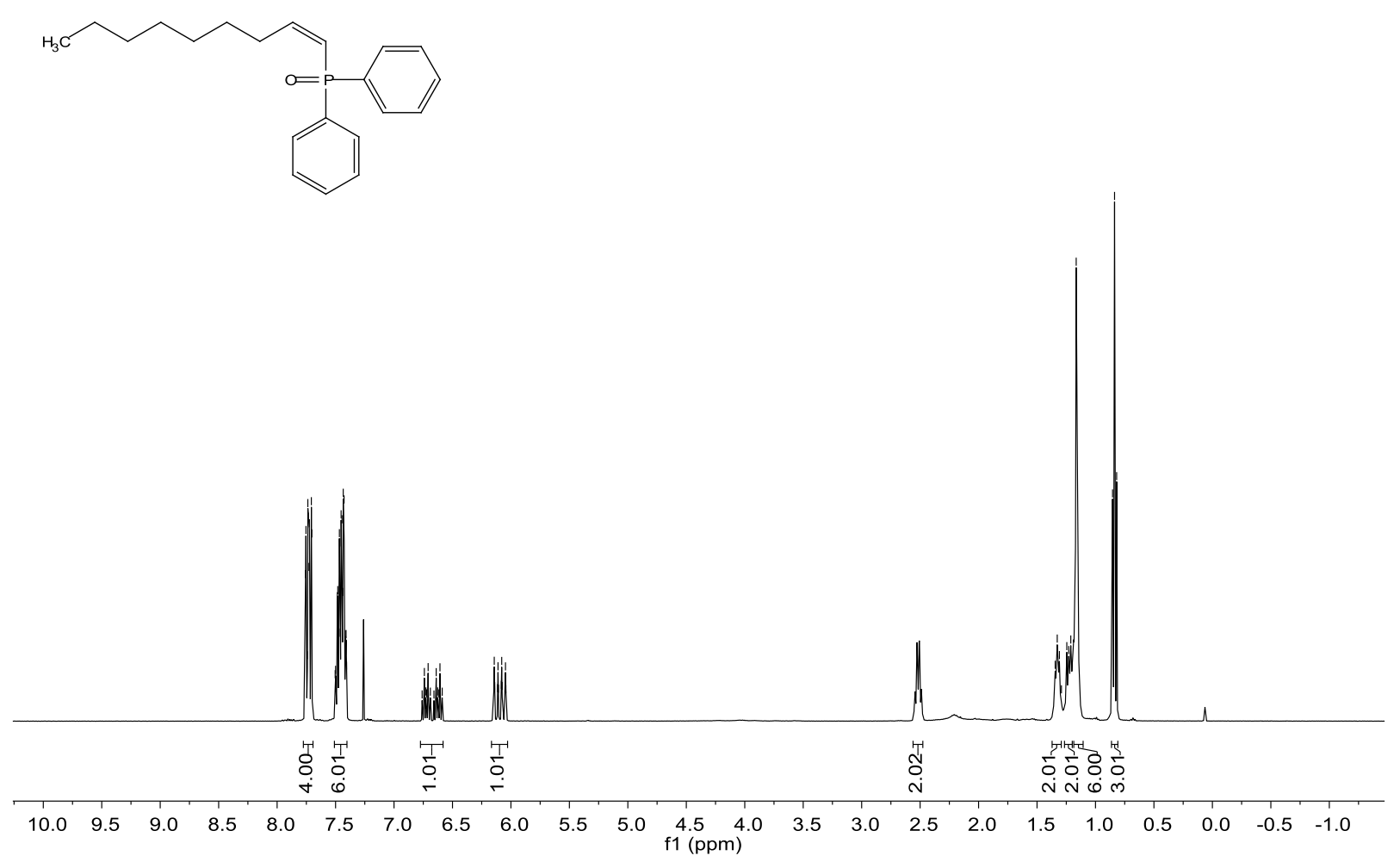
${ }^{31} \mathrm{P}$ NMR Spectrum of $7 \mathrm{x}^{\prime}\left(\mathrm{CDCl}_{3}, 162 \mathrm{MHz}\right)$

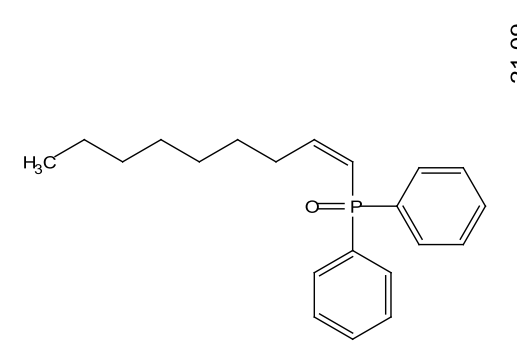

$\frac{8}{\stackrel{2}{1}}$

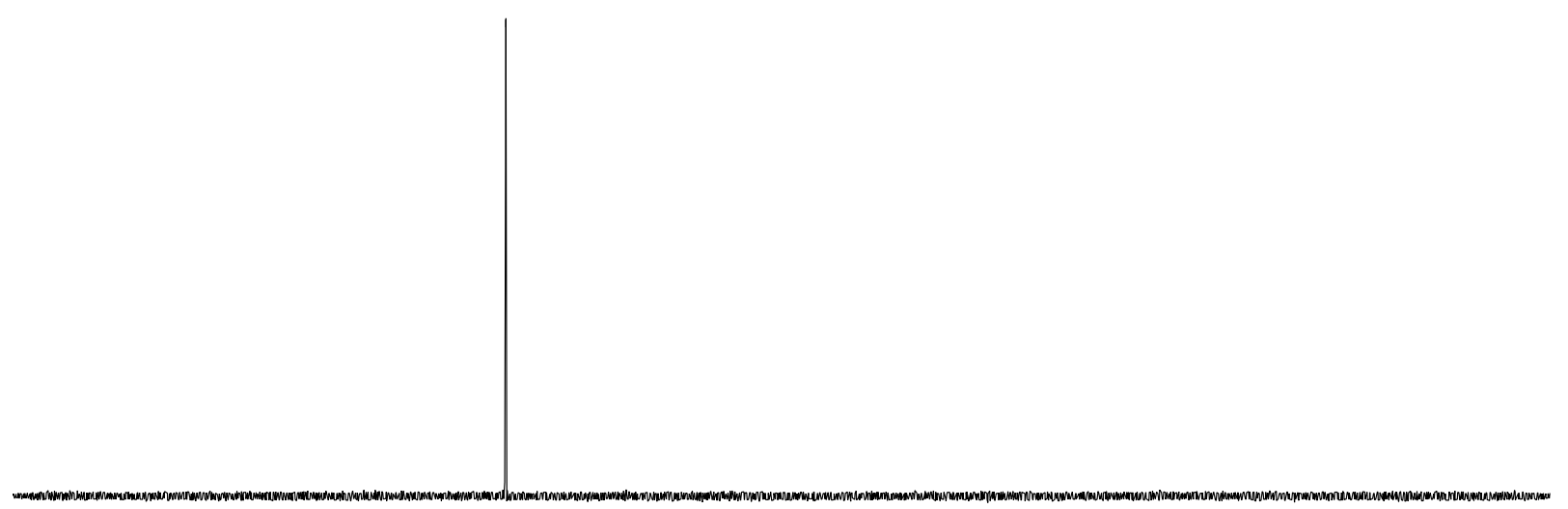

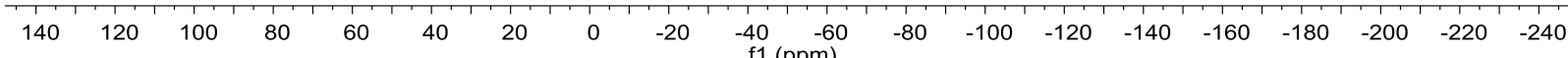

${ }^{13} \mathrm{C}$ NMR Spectrum of $7 \mathrm{x}^{\prime}\left(\mathrm{CDCl}_{3}, 100 \mathrm{MHz}\right)$
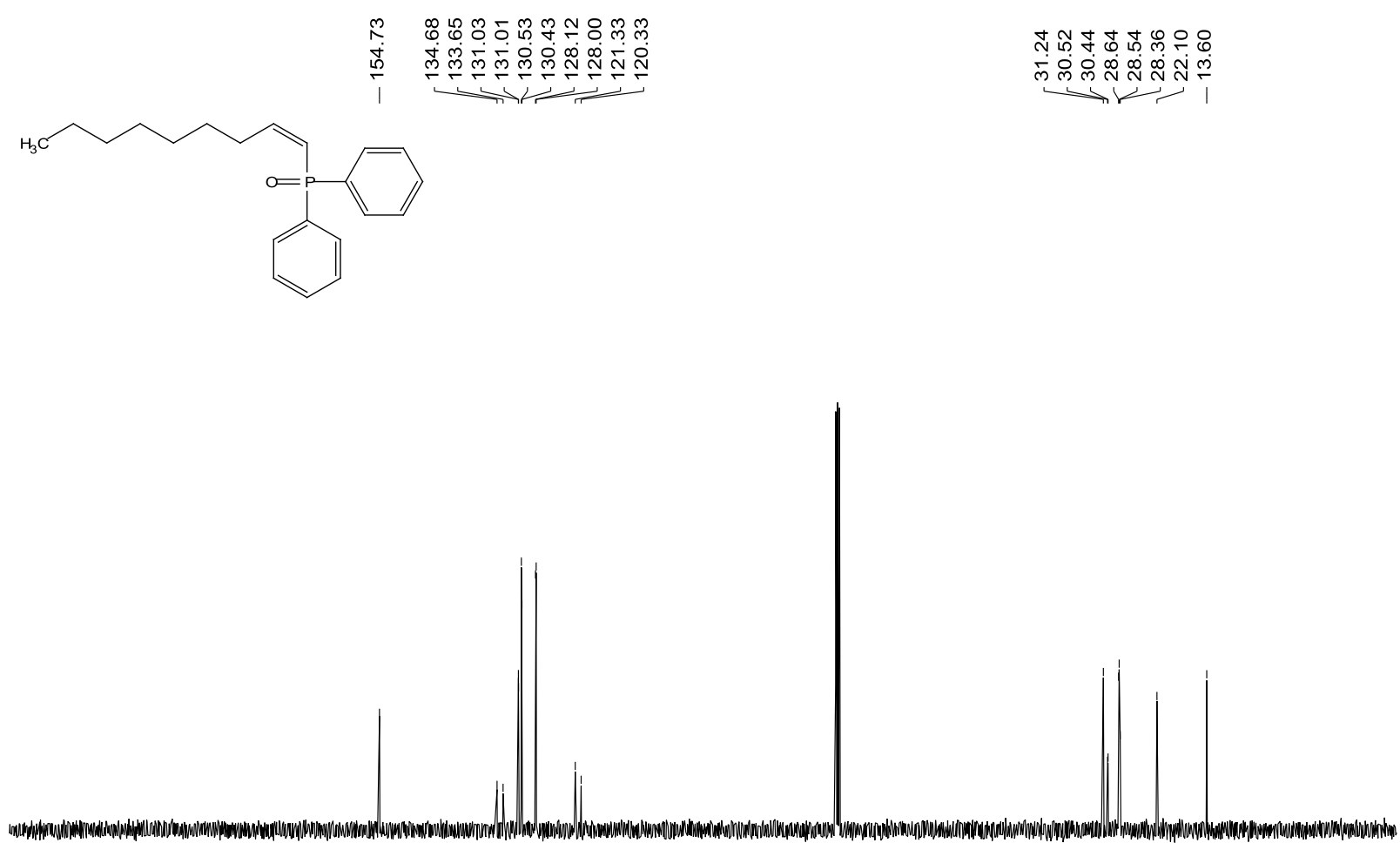

$\begin{array}{lllllllllllllllllllllll}210 & 200 & 190 & 180 & 170 & 160 & 150 & 140 & 130 & 120 & 110 \begin{array}{c}100 \\ \mathrm{f} 1(\mathrm{ppm})\end{array} & 90 & 80 & 70 & 60 & 50 & 40 & 30 & 20 & 10 & 0 & -10\end{array}$ 
${ }^{1} \mathrm{H}$ NMR Spectrum of $7 \mathrm{y}^{\prime}\left(\mathrm{CDCl}_{3}, 400 \mathrm{MHz}\right)$

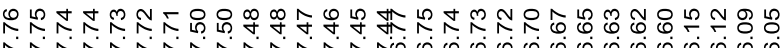

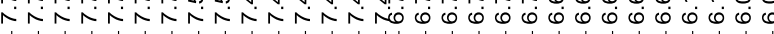

负

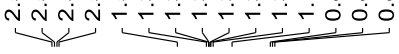

(l)

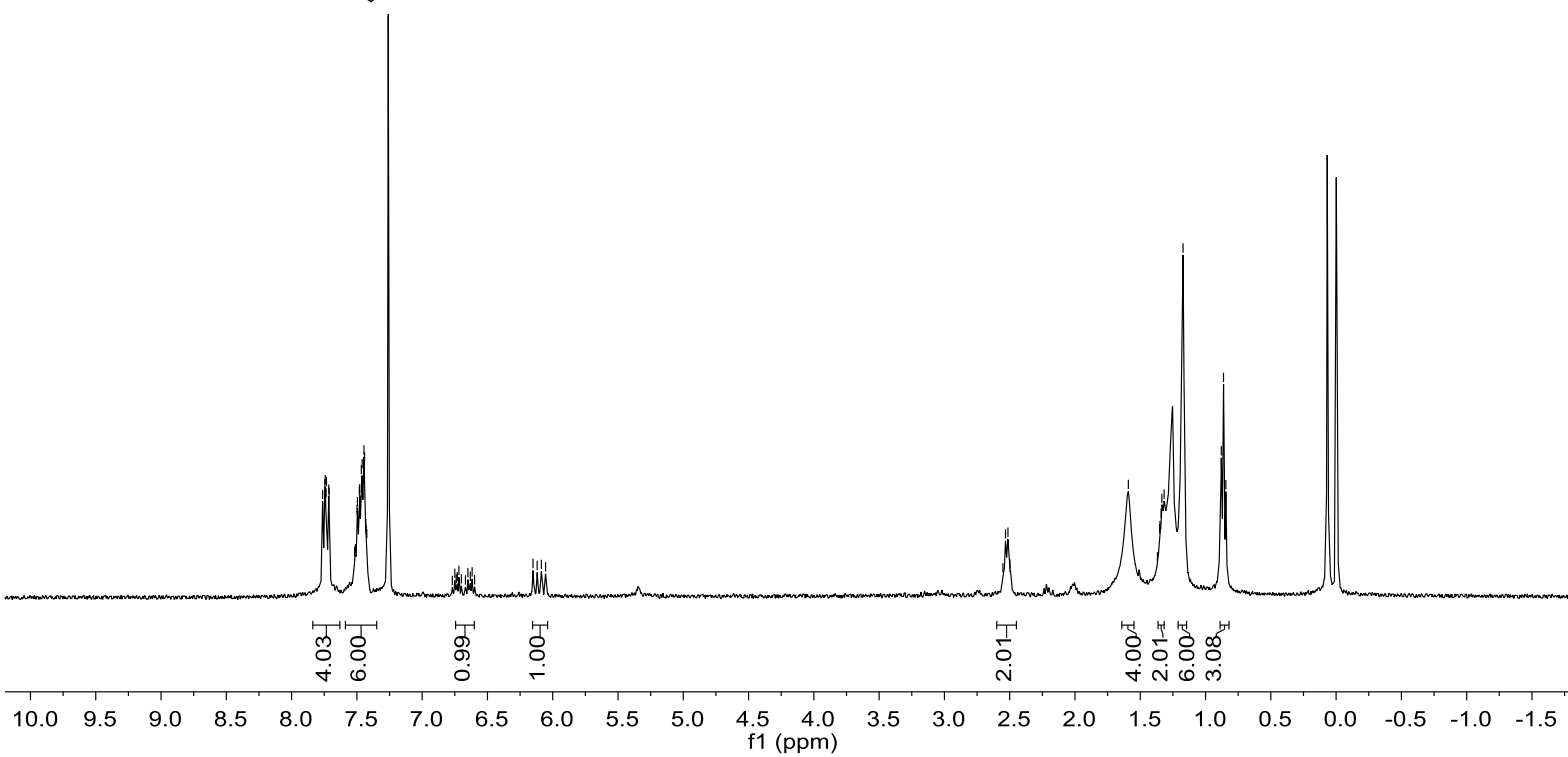

${ }^{31} \mathrm{P}$ NMR Spectrum of $7 \mathrm{y}^{\prime}\left(\mathrm{CDCl}_{3}, 162 \mathrm{MHz}\right)$

ڤ̊
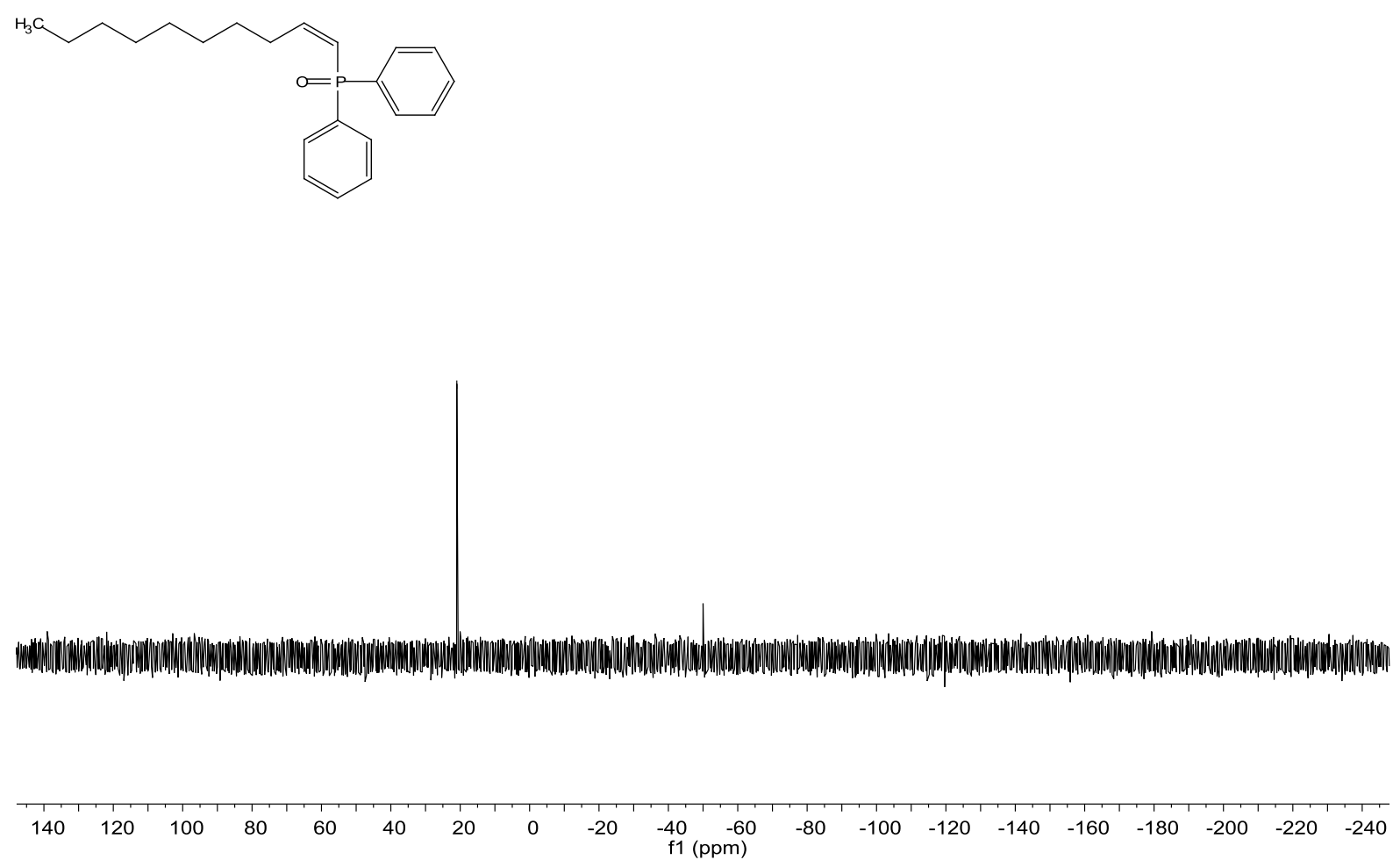


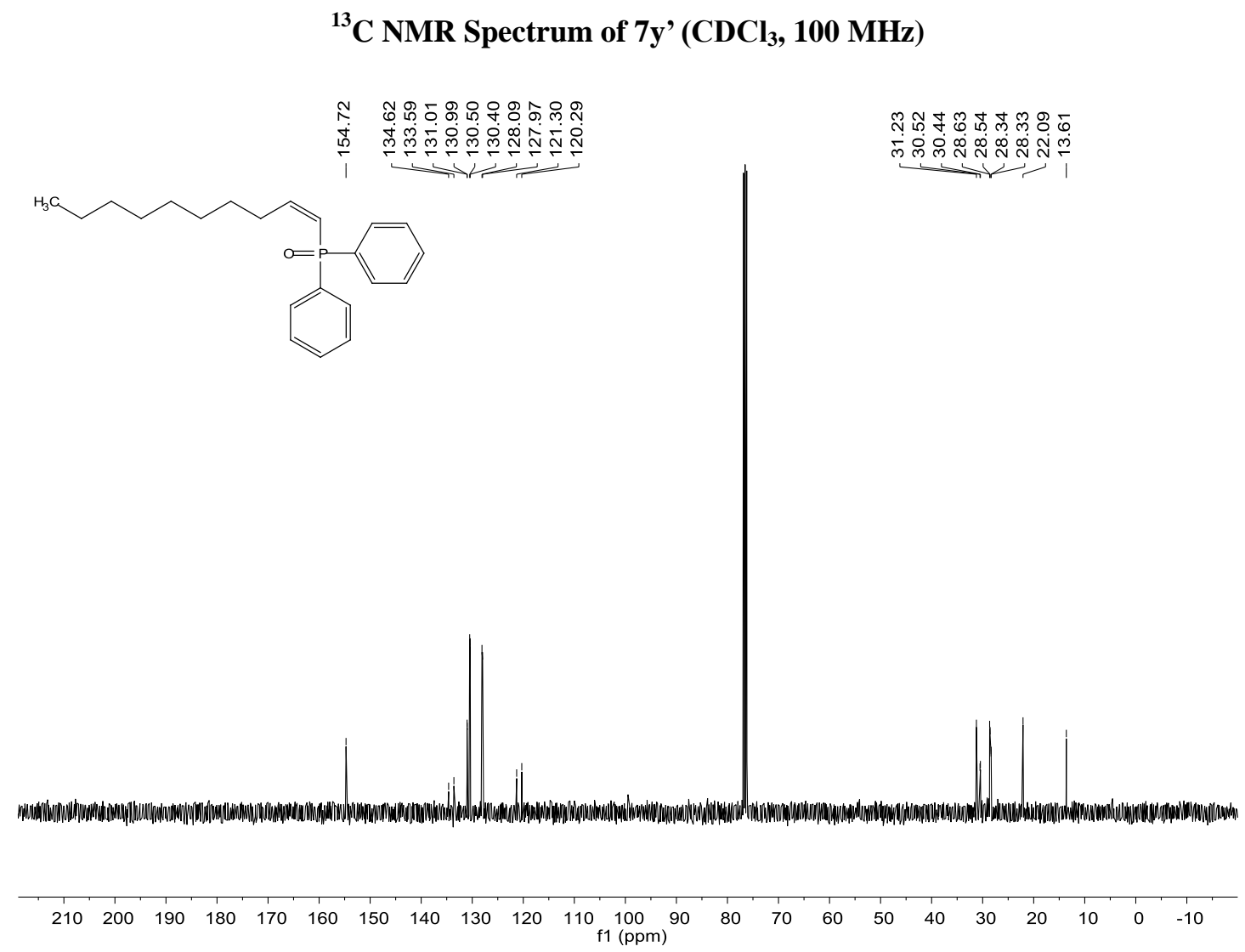

10-3 NMR spectra for chlorophosphorylation products 9

${ }^{1} \mathrm{H}$ NMR Spectrum of $9 \mathrm{a}\left(\mathrm{CDCl}_{3}, 400 \mathrm{MHz}\right)$

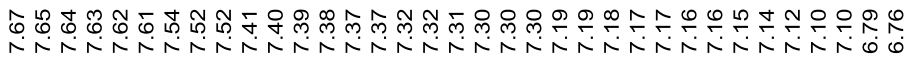
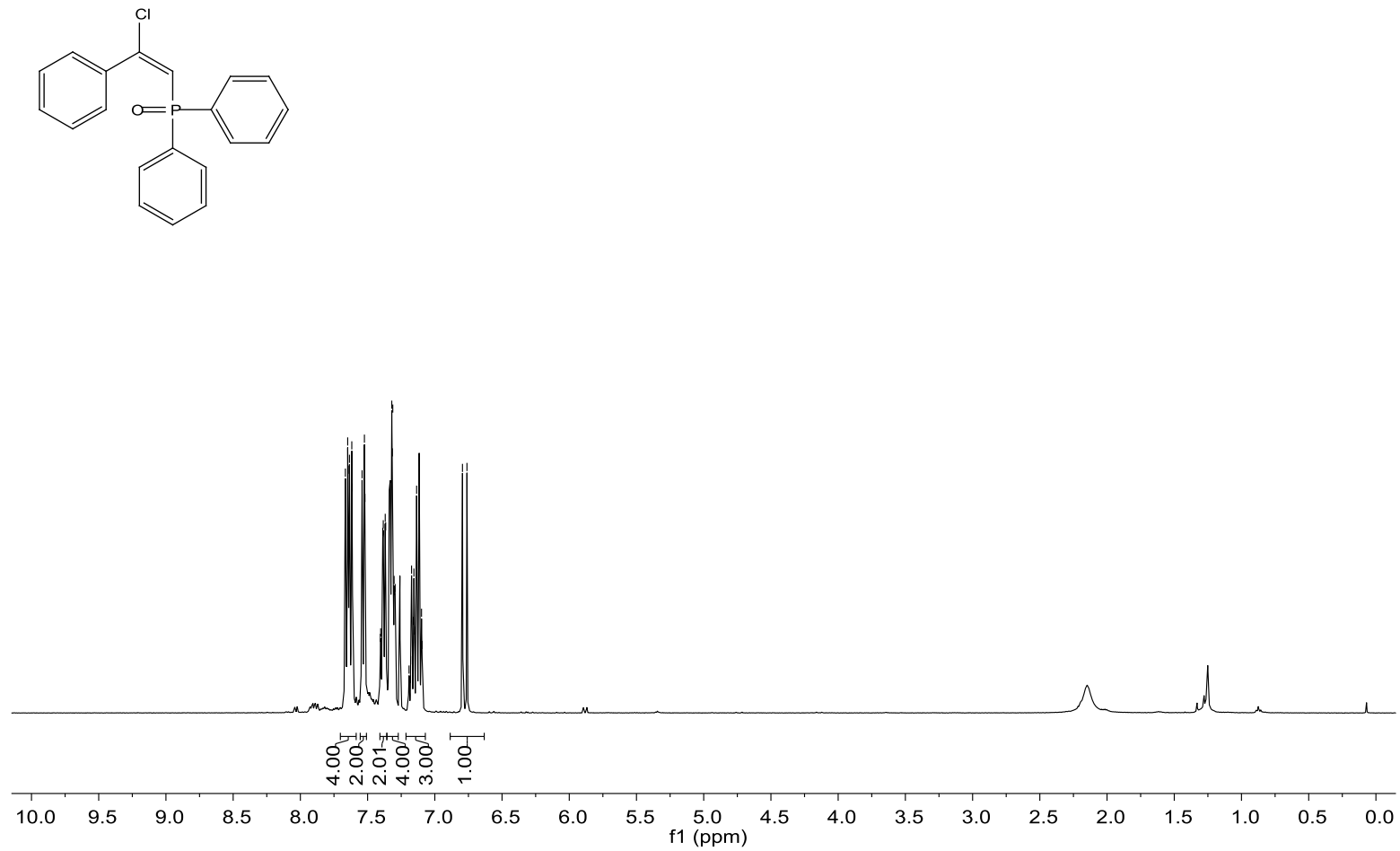
${ }^{31} \mathrm{P}$ NMR Spectrum of $9 \mathrm{a}\left(\mathrm{CDCl}_{3}, 162 \mathrm{MHz}\right)$

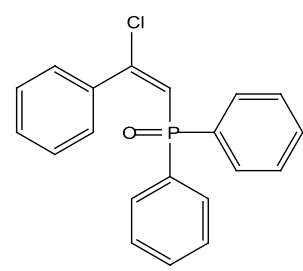

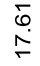

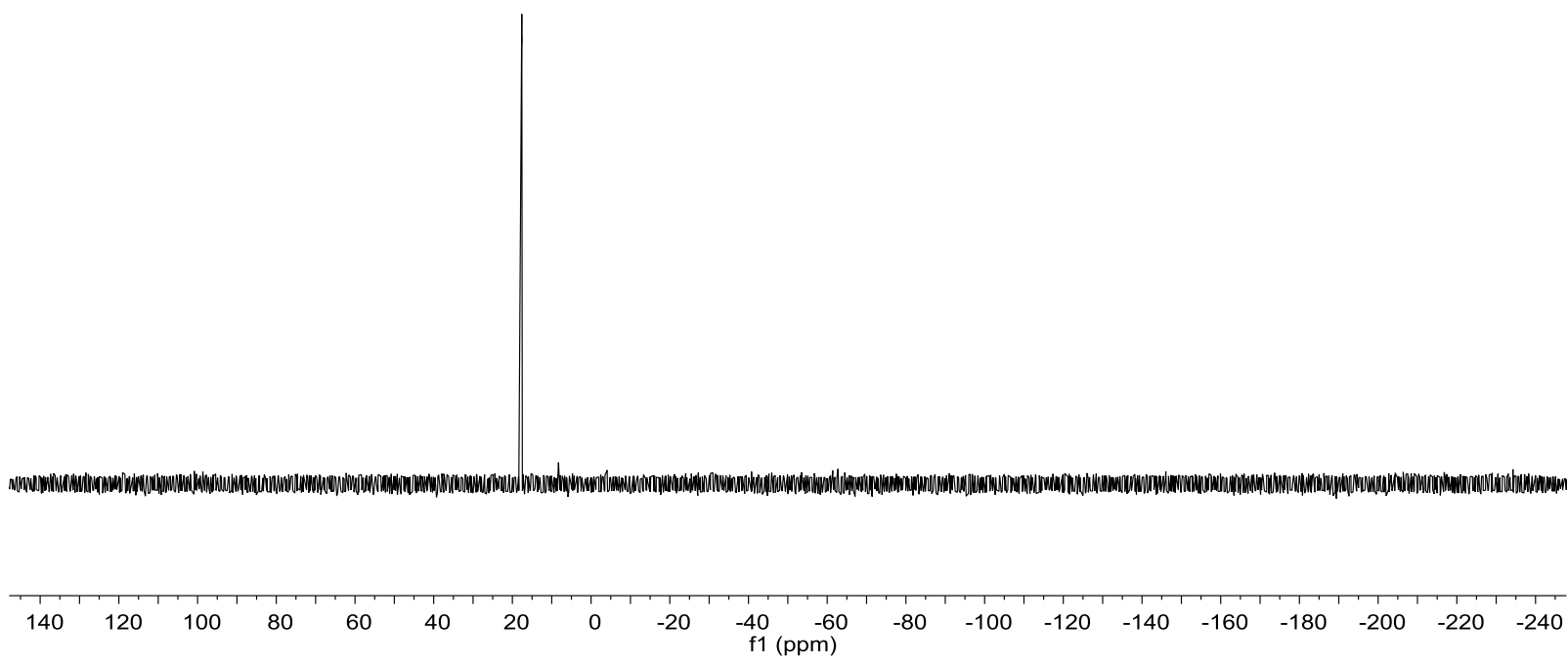

${ }^{13} \mathrm{C}$ NMR Spectrum of 9a $\left(\mathrm{CDCl}_{3}, 100 \mathrm{MHz}\right)$

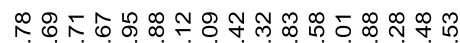

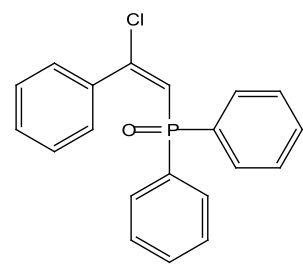

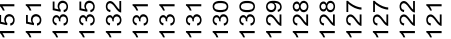

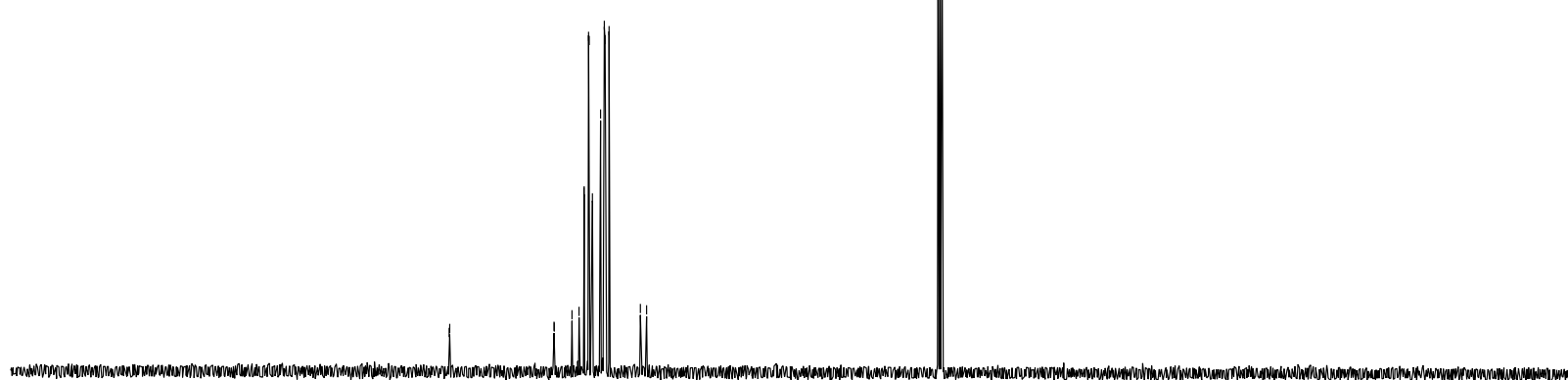

$\begin{array}{llllllllllllllllllllllll}210 & 200 & 190 & 180 & 170 & 160 & 150 & 140 & 130 & 120 & 110 & 100 & 90 & 80 & 70 & 60 & 50 & 40 & 30 & 20 & 10 & 0 & -10 & \end{array}$ 
${ }^{1} \mathrm{H}$ NMR Spectrum of $9 \mathrm{~b}\left(\mathrm{CDCl}_{3}, 400 \mathrm{MHz}\right)$

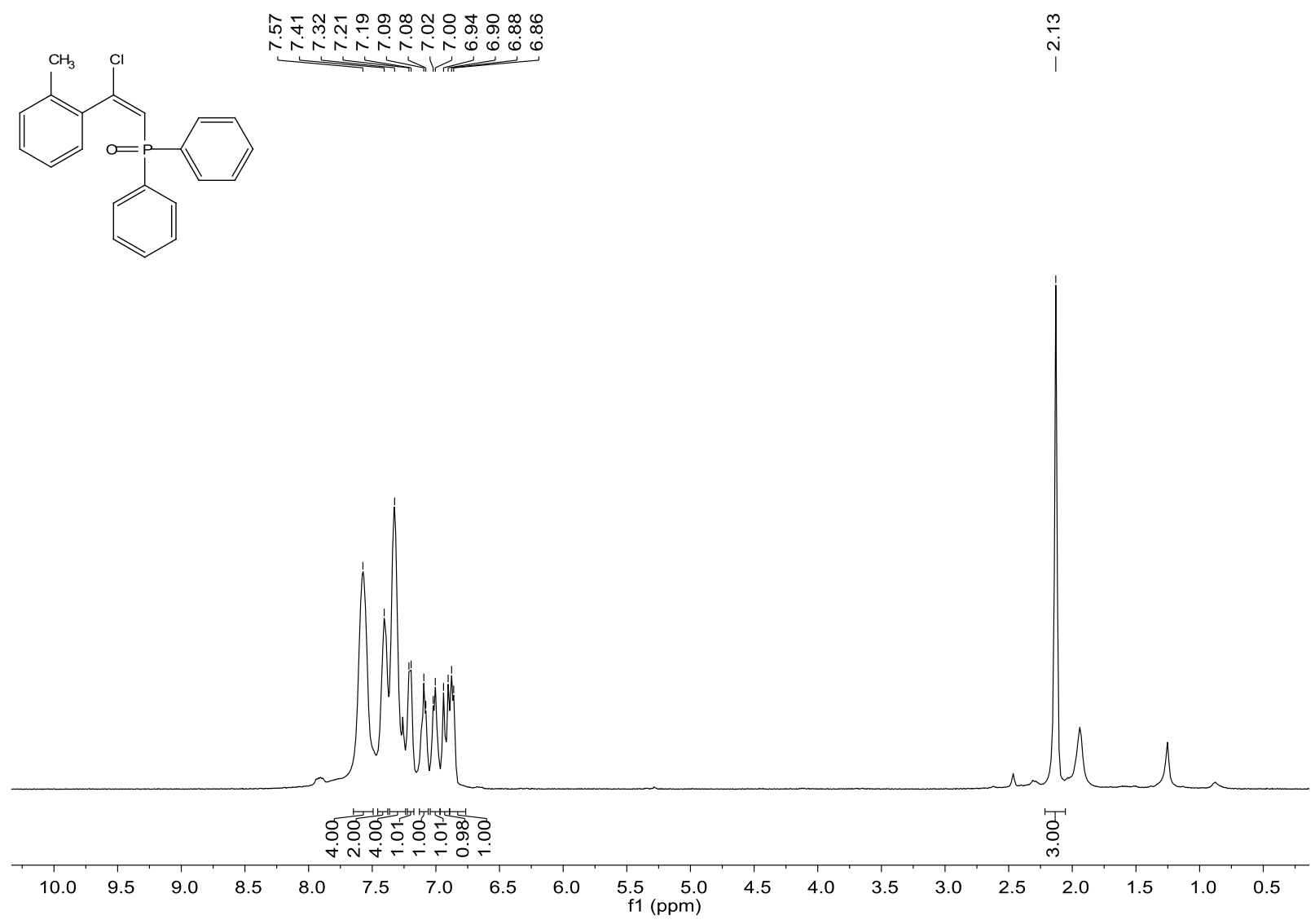

${ }^{31} \mathrm{P}$ NMR Spectrum of $9 \mathrm{~b}\left(\mathrm{CDCl}_{3}, 162 \mathrm{MHz}\right)$

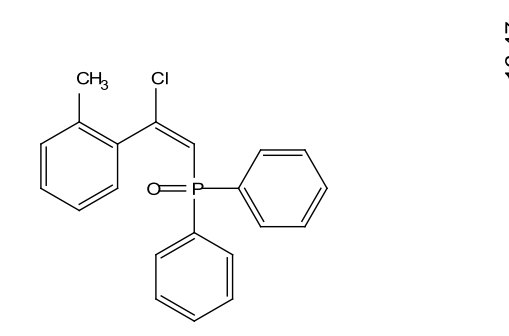

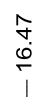

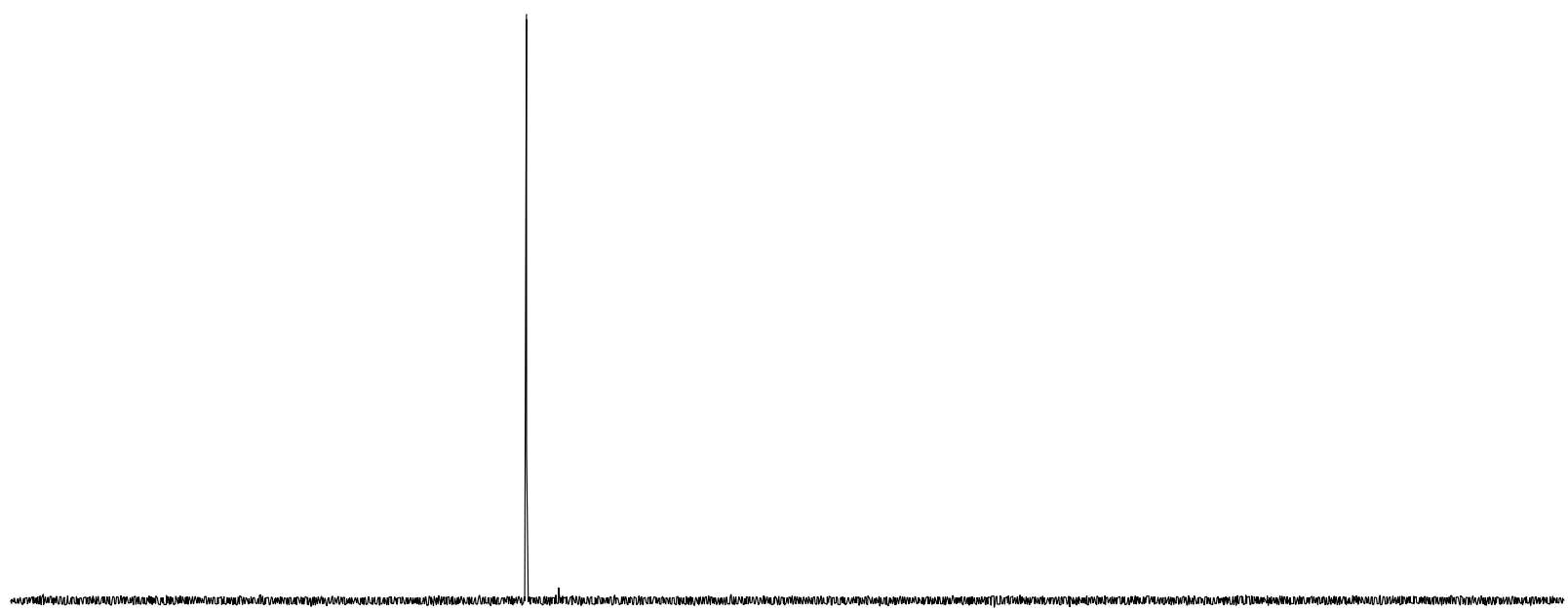

$\begin{array}{lllllllllllllllllllll}140 & 120 & 100 & 80 & 60 & 40 & 20 & 0 & -20 & -40 & -60 & -80 & -100 & -120 & -140 & -160 & -180 & -200 & -220 & -240\end{array}$ 
${ }^{13} \mathrm{C}$ NMR Spectrum of $9 \mathrm{~b}\left(\mathrm{CDCl}_{3}, 100 \mathrm{MHz}\right)$

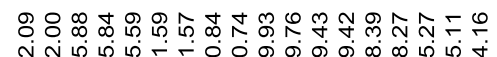

议
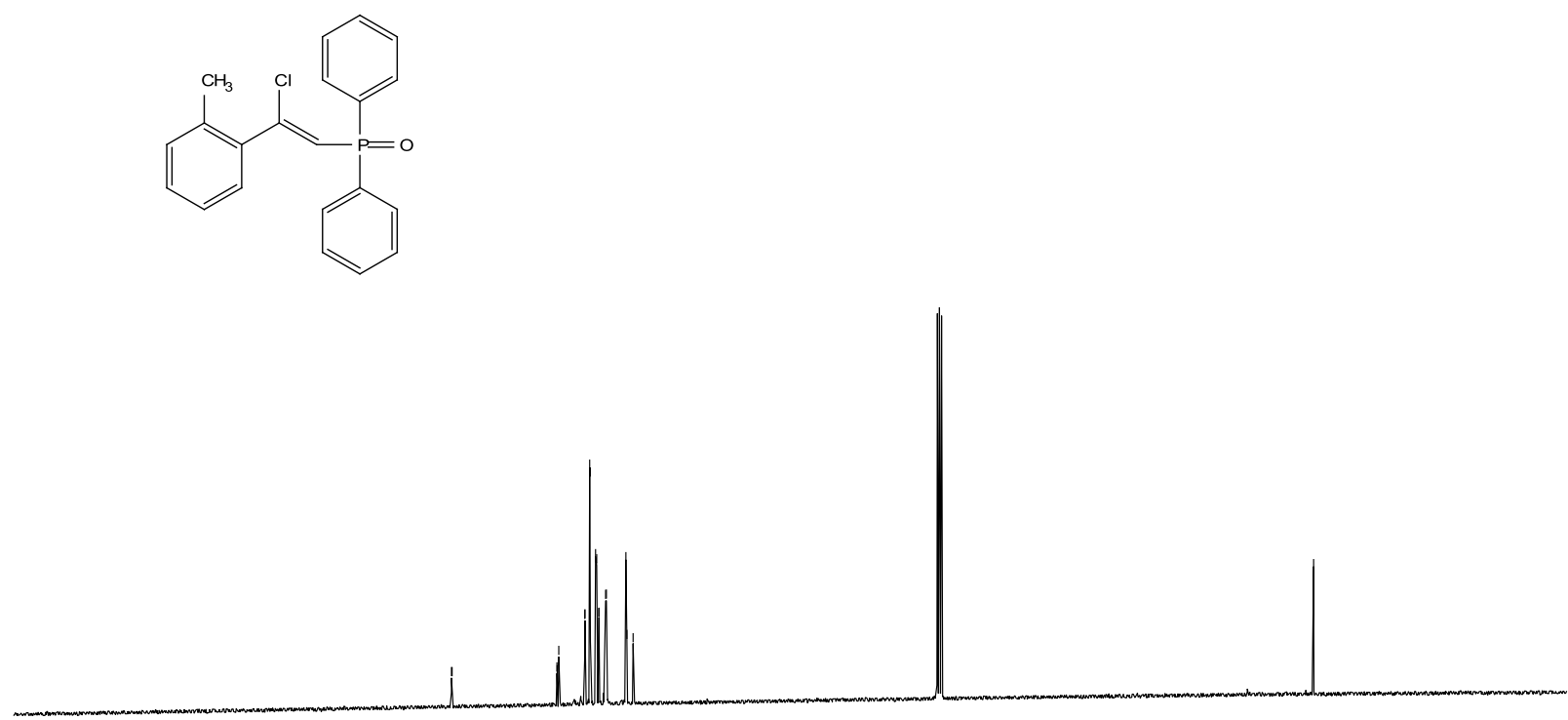

$\begin{array}{llllllllllllllllllllllllll}210 & 200 & 190 & 180 & 170 & 160 & 150 & 140 & 130 & 120 & 110 & 100 & 90 & 80 & 70 & 60 & 50 & 40 & 30 & 20 & 10 & 0 & -10\end{array}$

${ }^{1} \mathrm{H}$ NMR Spectrum of $9 \mathrm{c}\left(\mathrm{CDCl}_{3}, 400 \mathrm{MHz}\right)$

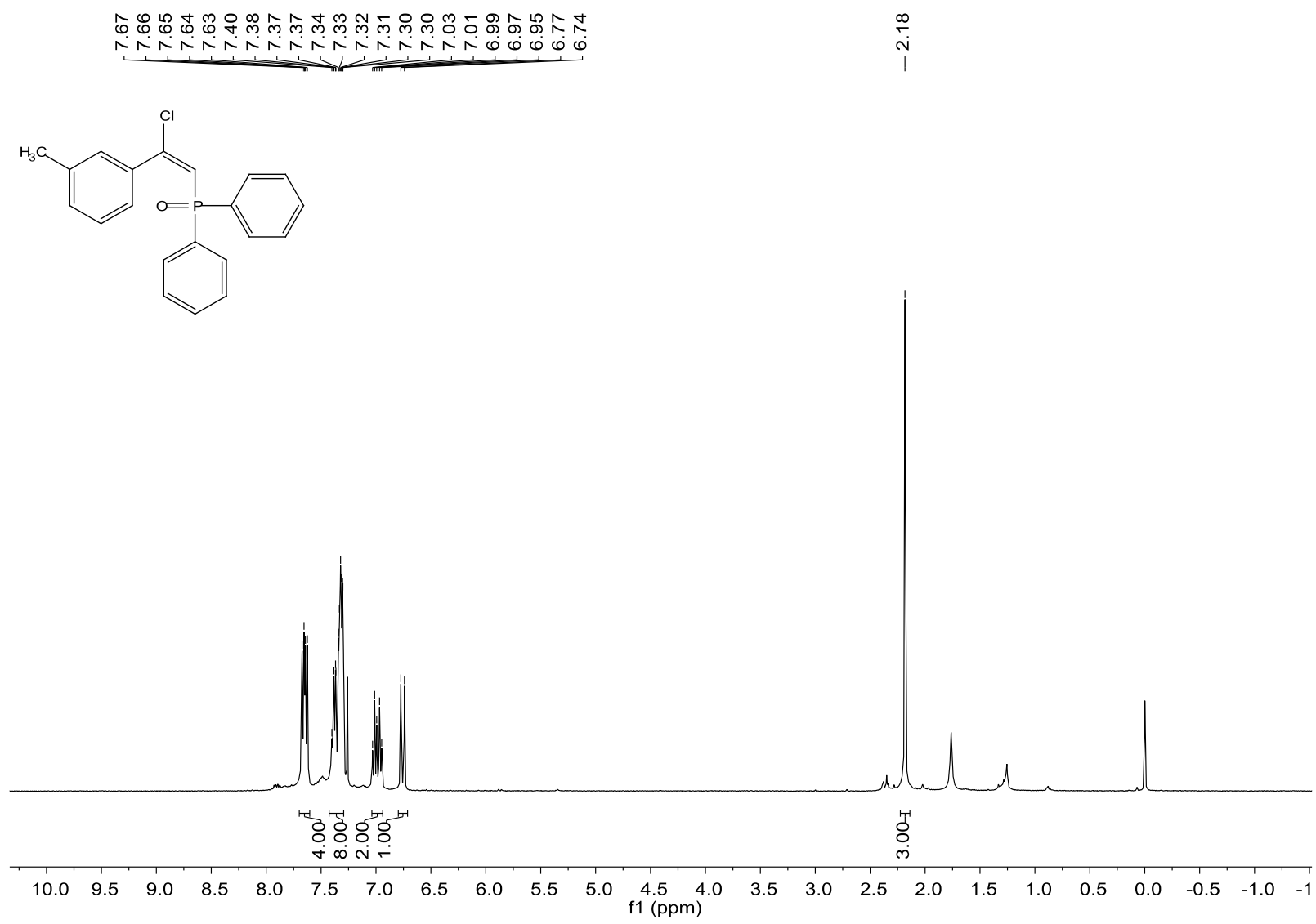

S139 
${ }^{31} \mathrm{P}$ NMR Spectrum of $9 \mathrm{c}\left(\mathrm{CDCl}_{3}, 162 \mathrm{MHz}\right)$

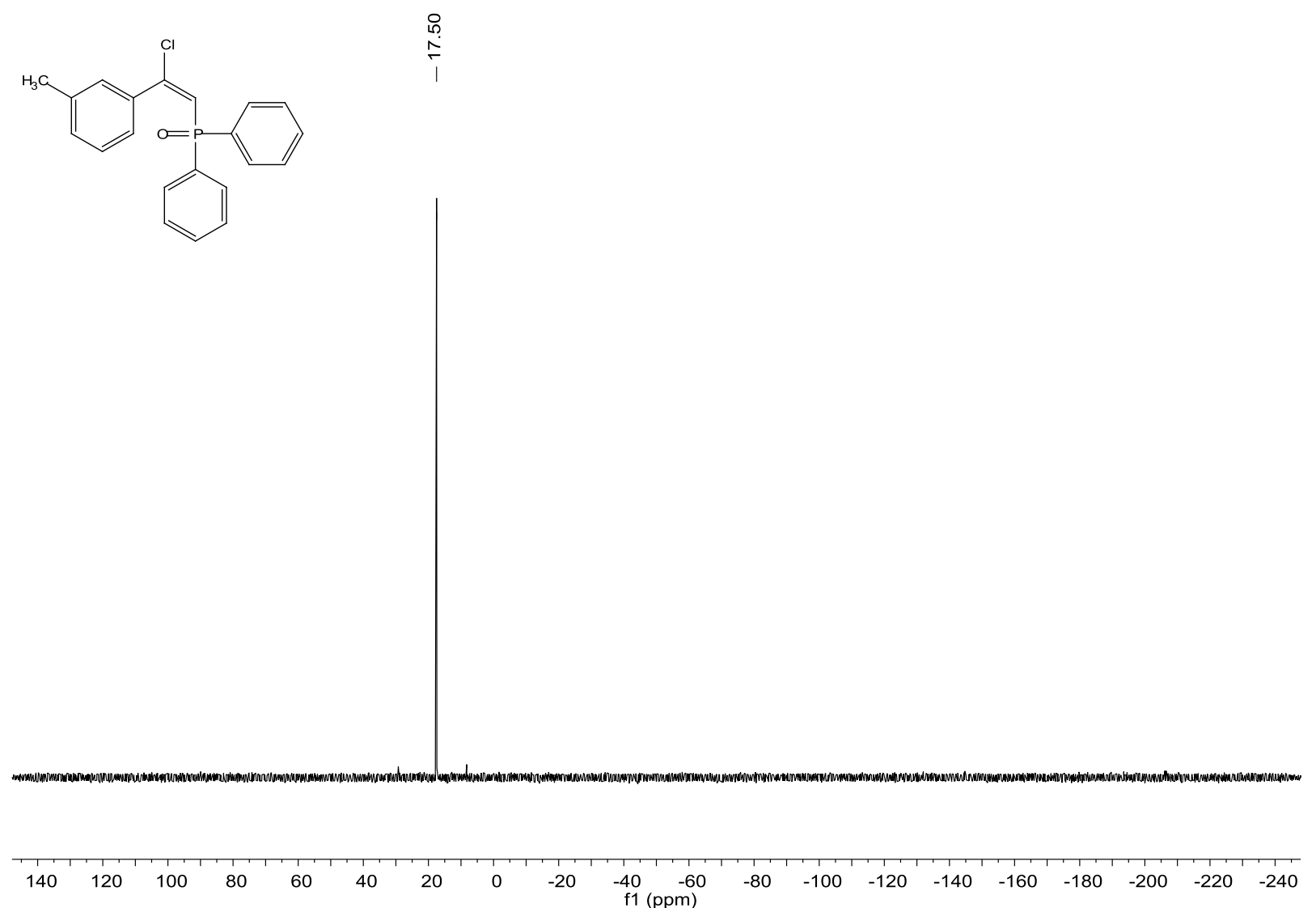

${ }^{13} \mathrm{C}$ NMR Spectrum of $9 \mathrm{c}\left(\mathrm{CDCl}_{3}, 100 \mathrm{MHz}\right)$

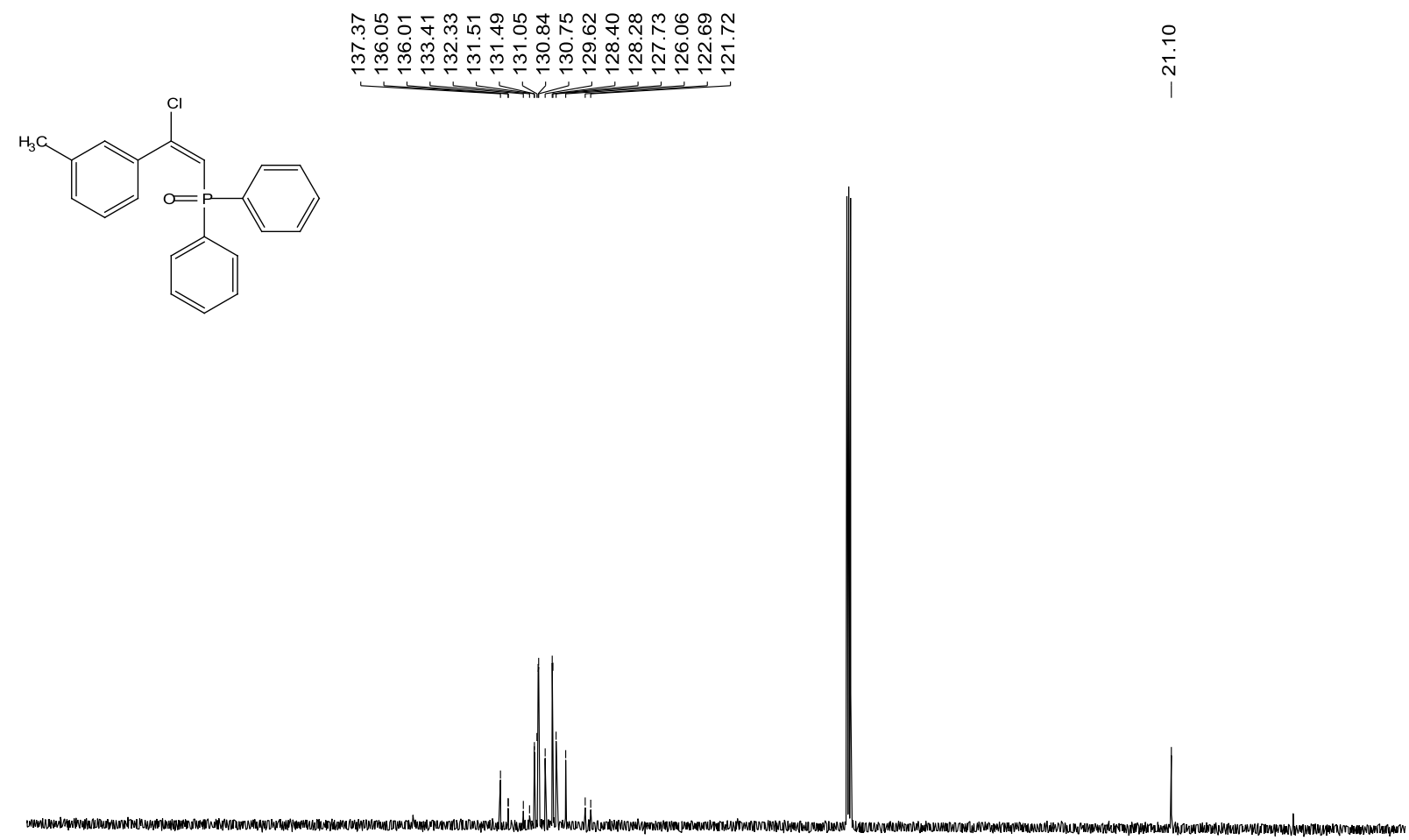

$\begin{array}{lllllllllllllllllllllll}210 & 200 & 190 & 180 & 170 & 160 & 150 & 140 & 130 & 120 & 110 & 100 & 90 & 80 & 70 & 60 & 50 & 40 & 30 & 20 & 10 & 0 & -10\end{array}$ 
${ }^{1} \mathrm{H}$ NMR Spectrum of $9 \mathrm{~d}\left(\mathrm{CDCl}_{3}, 400 \mathrm{MHz}\right)$

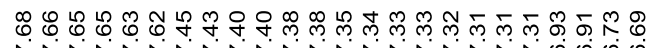

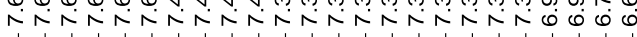

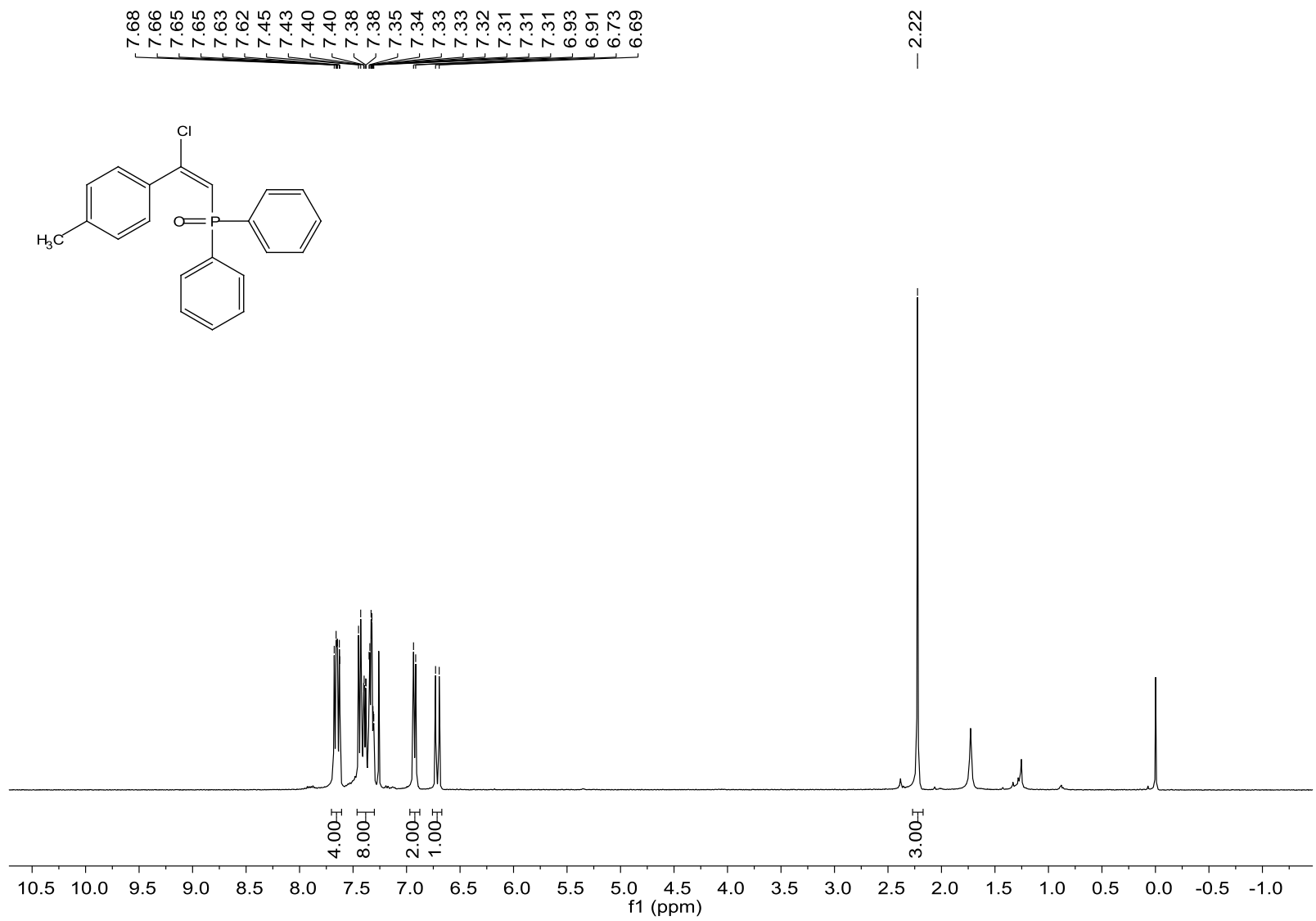

${ }^{31} \mathrm{P}$ NMR Spectrum of 9d $\left(\mathrm{CDCl}_{3}, 162 \mathrm{MHz}\right)$

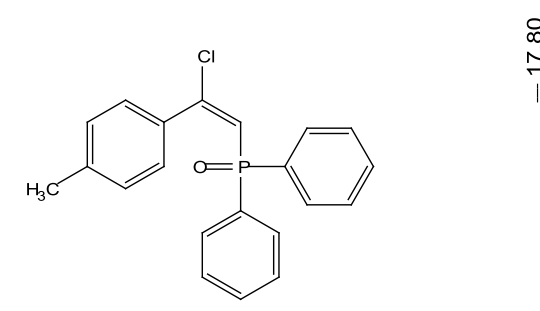

$\stackrel{\stackrel{\infty}{\infty}}{\stackrel{\infty}{\perp}}$

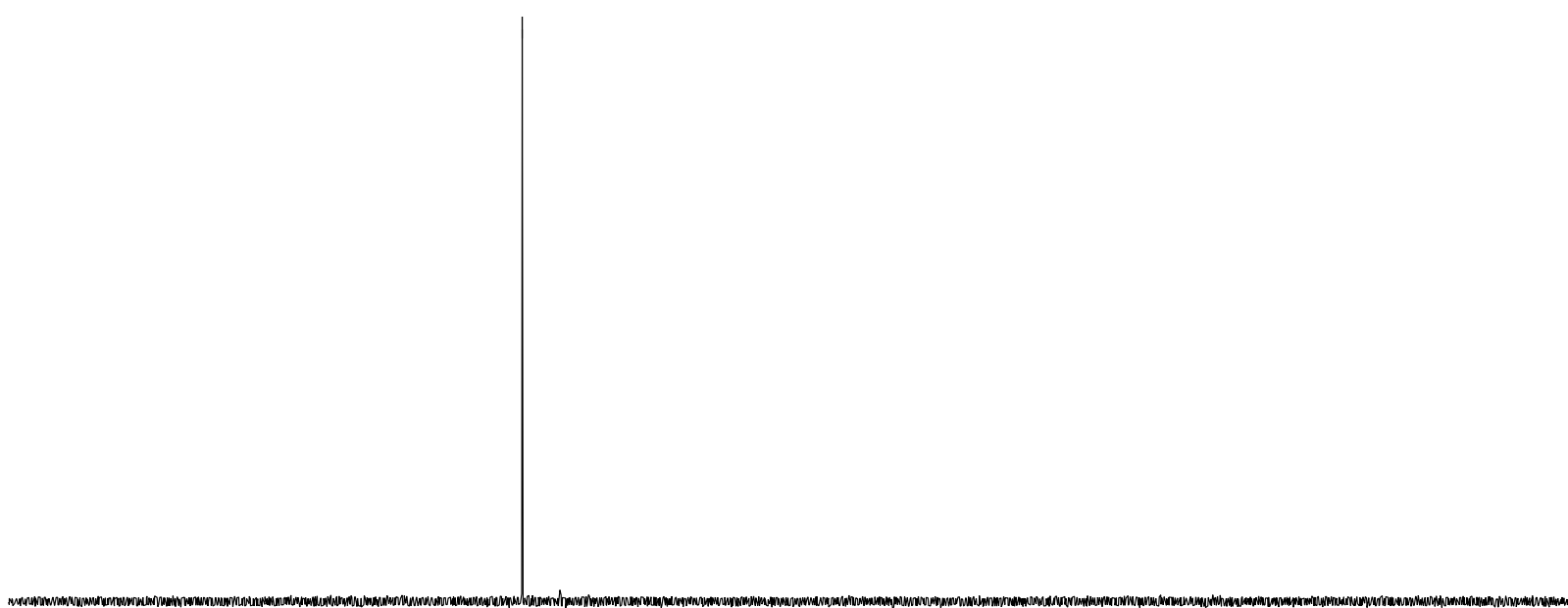

$\begin{array}{llllllllllllllllllllllllll}140 & 120 & 100 & 80 & 60 & 40 & 20 & 0 & -20 & -40 & -60 & -80 & -100 & -120 & -140 & -160 & -180 & -200 & -220 & -240\end{array}$ 
${ }^{13} \mathrm{C}$ NMR Spectrum of $9 \mathrm{~d}\left(\mathrm{CDCl}_{3}, 100 \mathrm{MHz}\right)$
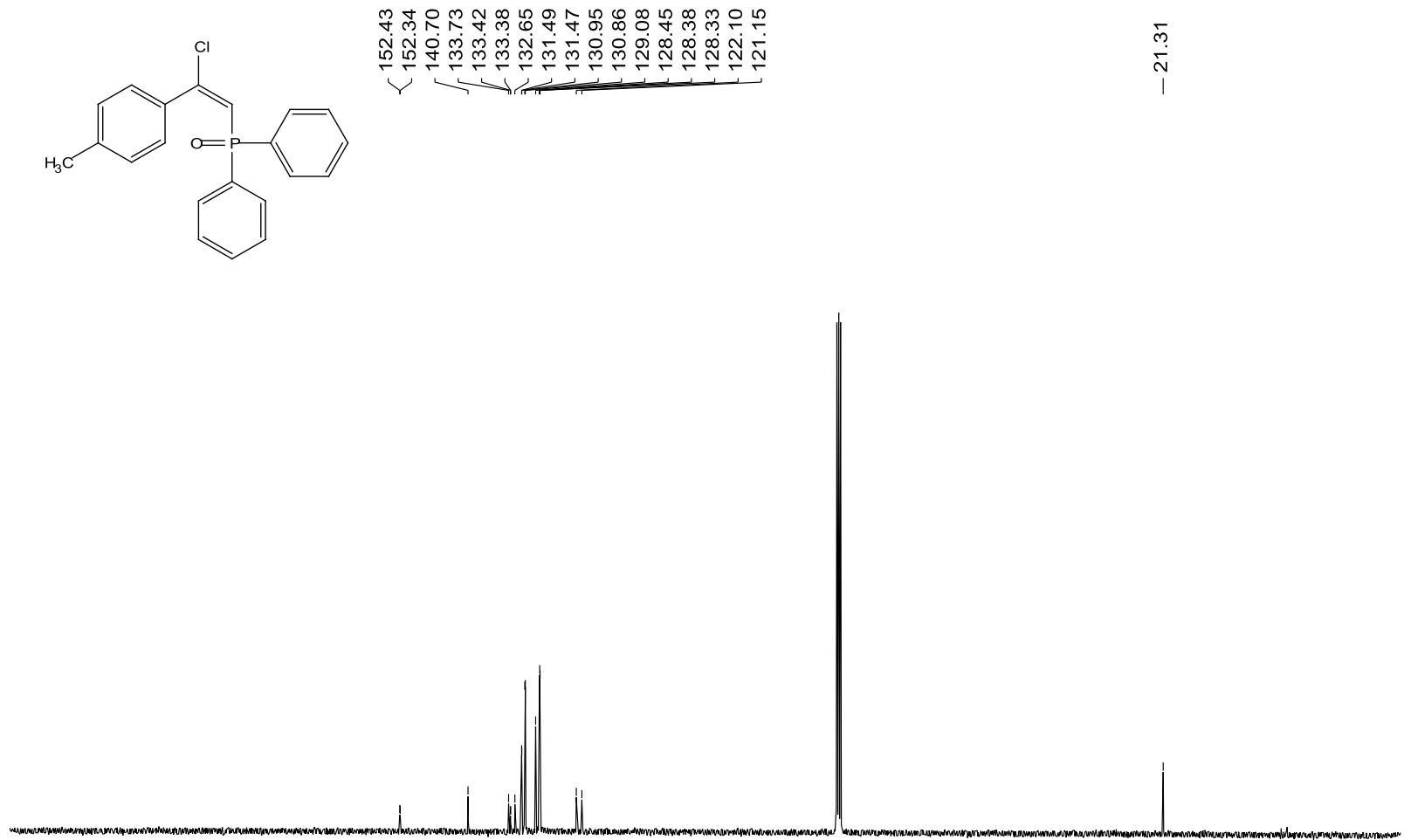

$\begin{array}{lllllllllllllllllllllll}210 & 200 & 190 & 180 & 170 & 160 & 150 & 140 & 130 & 120 & 110 & 100 & 90 & 80 & 70 & 60 & 50 & 40 & 30 & 20 & 10 & 0 & -10\end{array}$

${ }^{1} \mathrm{H}$ NMR Spectrum of $9 \mathrm{e}$ and $9 \mathrm{e}^{9}(3: 1)\left(\mathrm{CDCl}_{3}, 400 \mathrm{MHz}\right)$

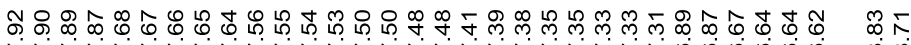

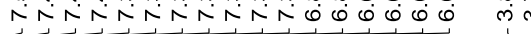<smiles>COc1ccc(C#CP(=O)(c2ccccc2)c2ccccc2)cc1</smiles>

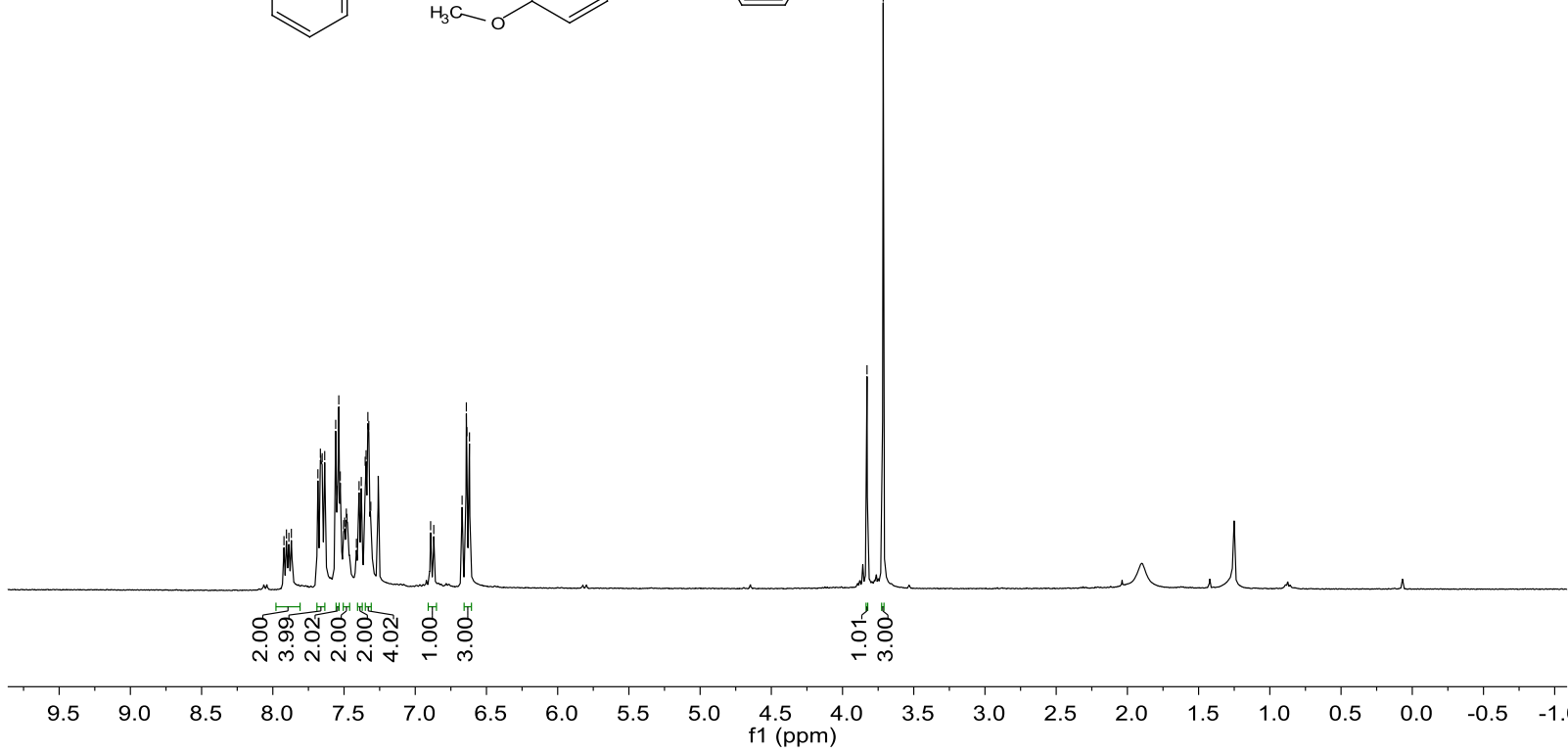


${ }^{31}$ P NMR Spectrum of 9e and 9e' (3:1) $\left(\mathrm{CDCl}_{3}, 162 \mathrm{MHz}\right)$

$\begin{array}{cc}\bar{r} & \substack{n \\ \infty} \\ 1 & 0 \\ 1 & 1\end{array}$
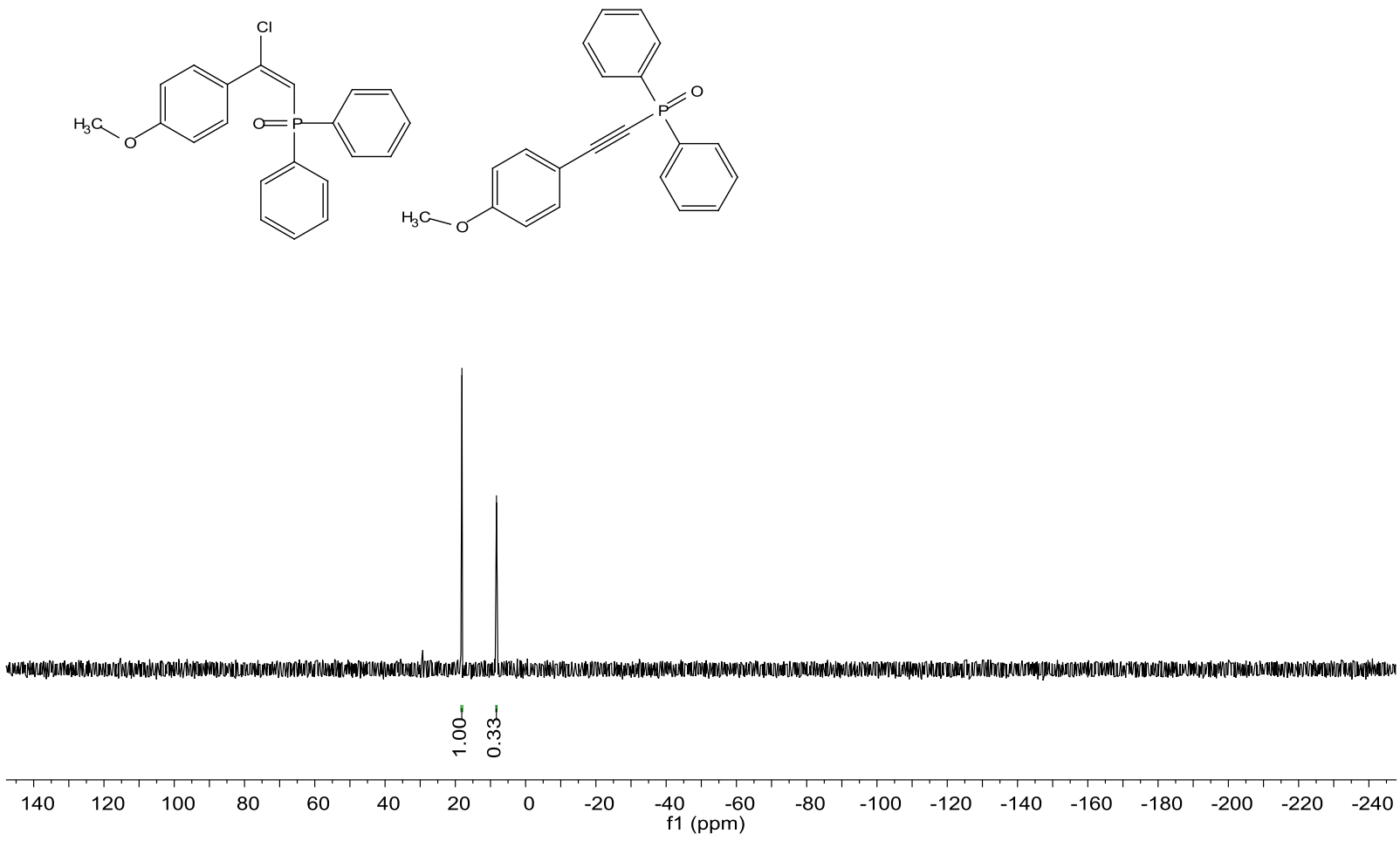

${ }^{13} \mathrm{C}$ NMR Spectrum of 9e and 9e' (3:1) $\left(\mathrm{CDCl}_{3}, 100 \mathrm{MHz}\right)$
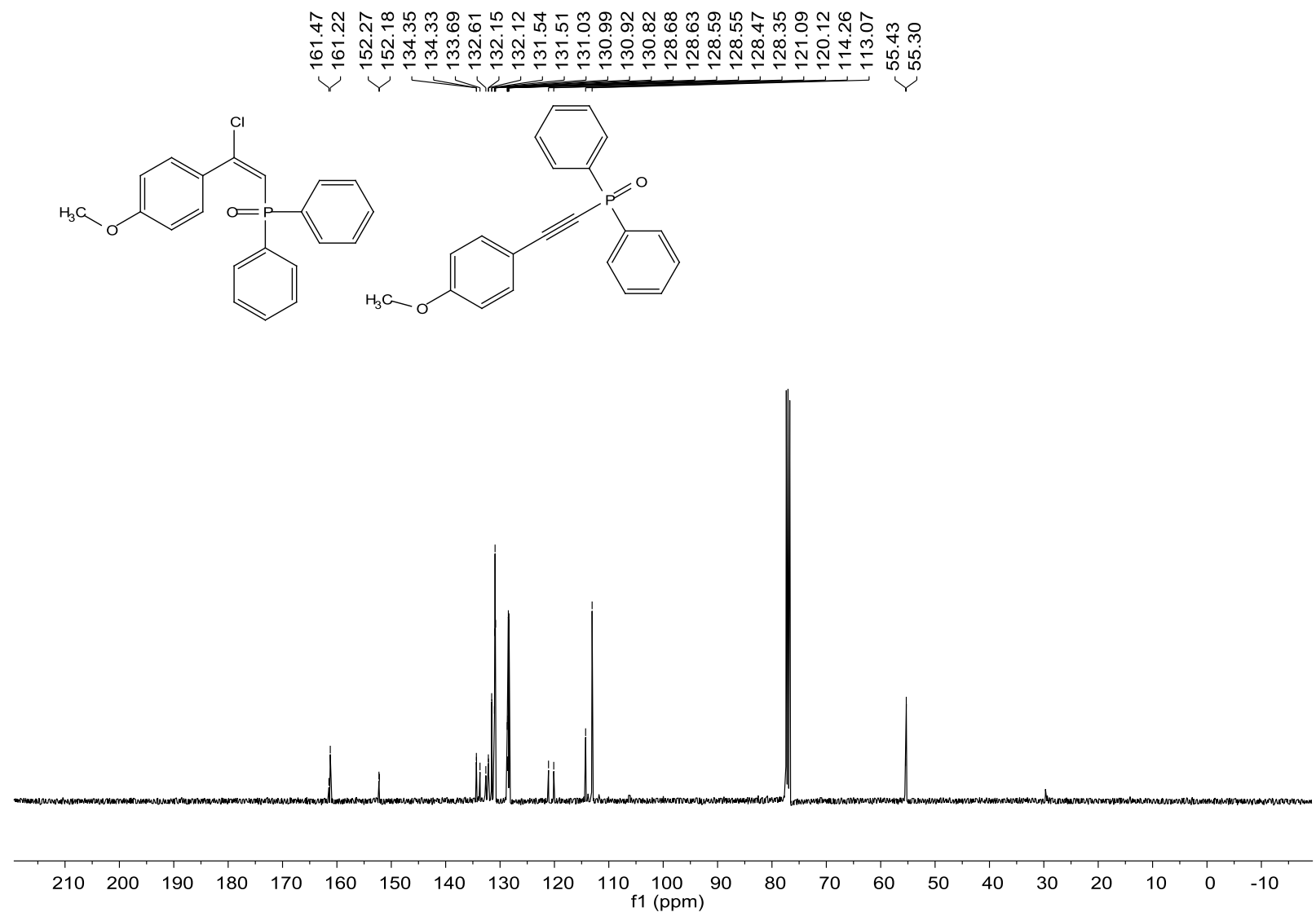

S143 
${ }^{1} \mathrm{H}$ NMR Spectrum of $9 \mathrm{f}\left(\mathrm{CDCl}_{3}, 400 \mathrm{MHz}\right)$

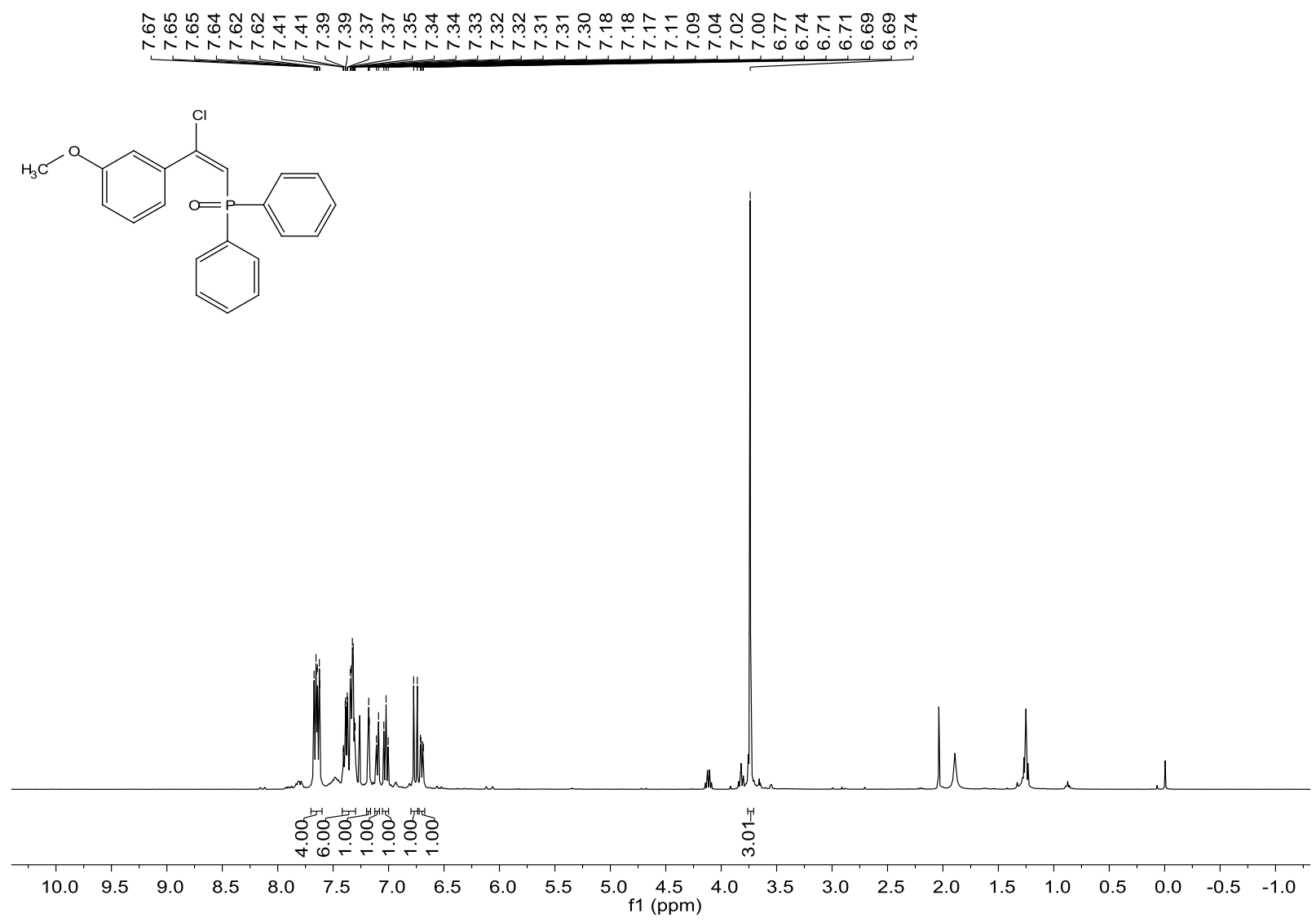

${ }^{31}$ P NMR Spectrum of $9 f\left(\mathrm{CDCl}_{3}, 162 \mathrm{MHz}\right)$<smiles>COc1cccc(/C(Cl)=C/P(=O)(c2ccccc2)c2ccccc2)c1</smiles>

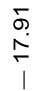

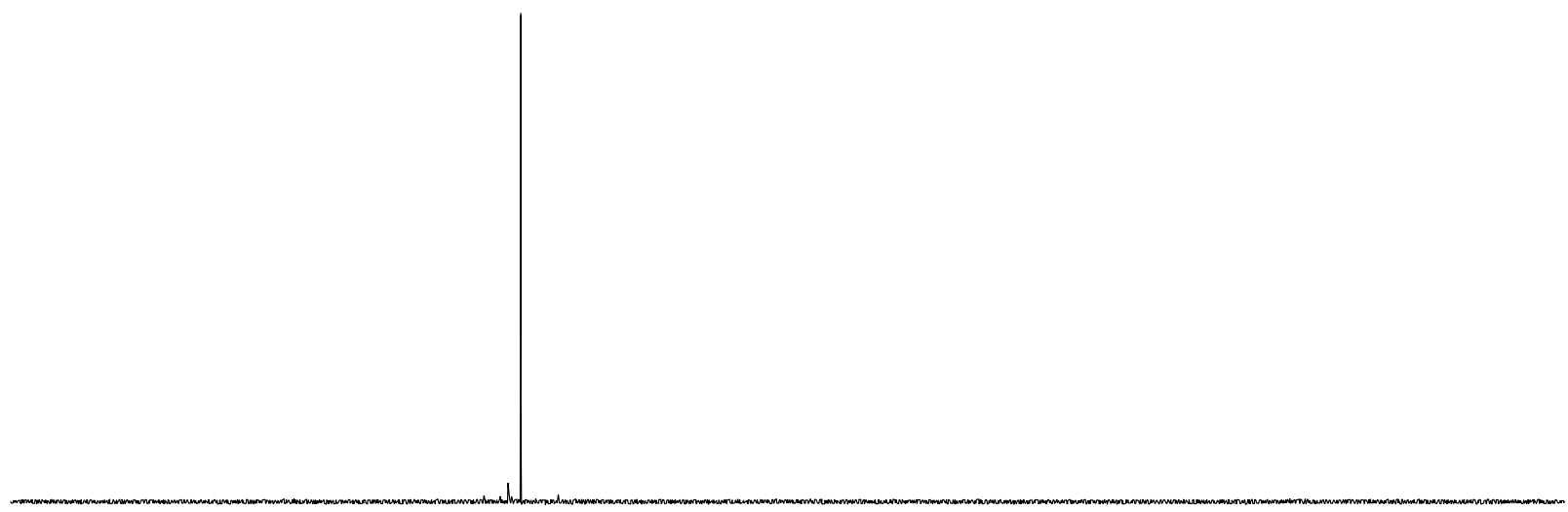

$\begin{array}{llllllllll}140 & 120 & 100 & 80 & 60 & 40 & 20 & 0 & -20 & -40 \begin{array}{r}-60 \\ \mathrm{f} 1(\mathrm{ppm})\end{array}\end{array}$ 
${ }^{13} \mathrm{C}$ NMR Spectrum of $9 f\left(\mathrm{CDCl}_{3}, 100 \mathrm{MHz}\right)$

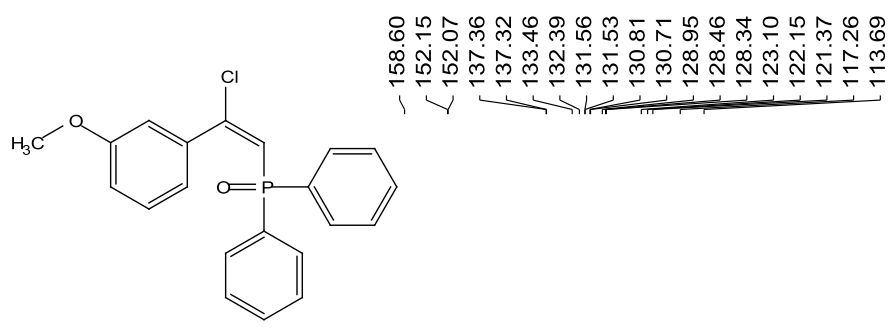

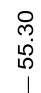
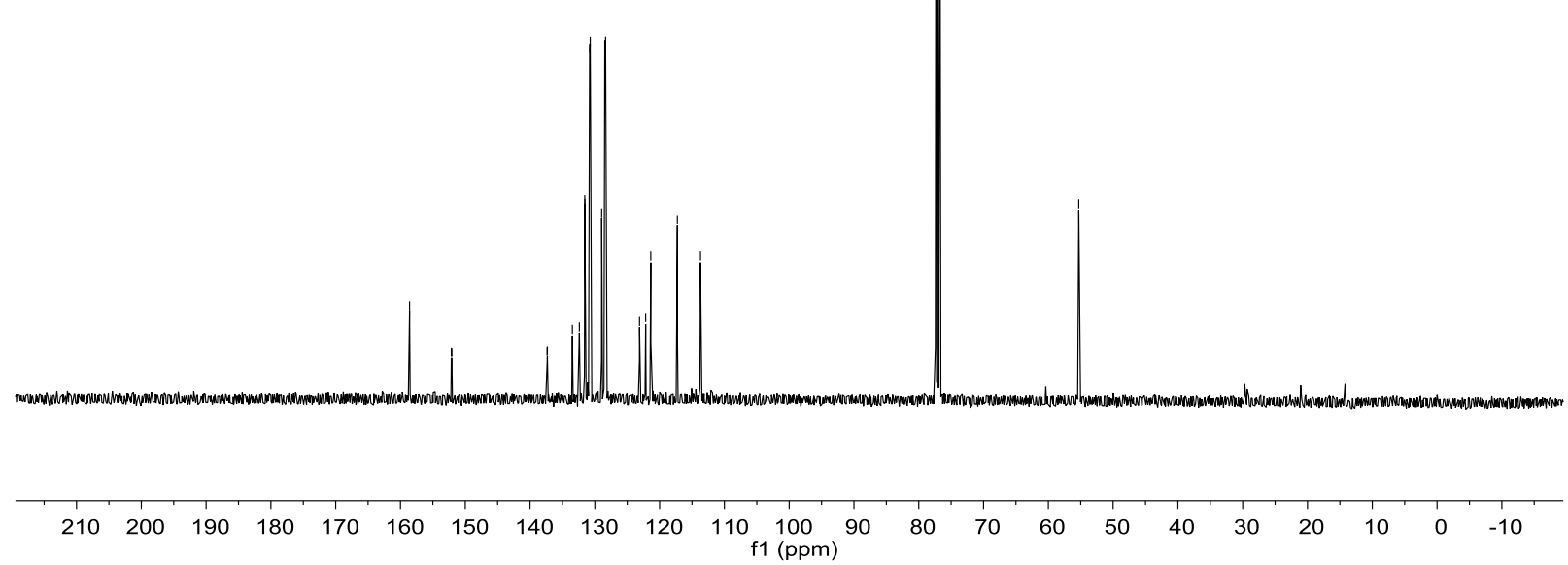

${ }^{1} \mathrm{H}$ NMR Spectrum of $9 \mathrm{~g}\left(\mathrm{CDCl}_{3}, 400 \mathrm{MHz}\right)$

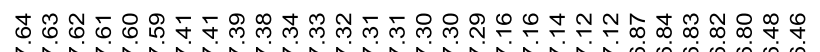
$\sqrt{3}$

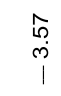

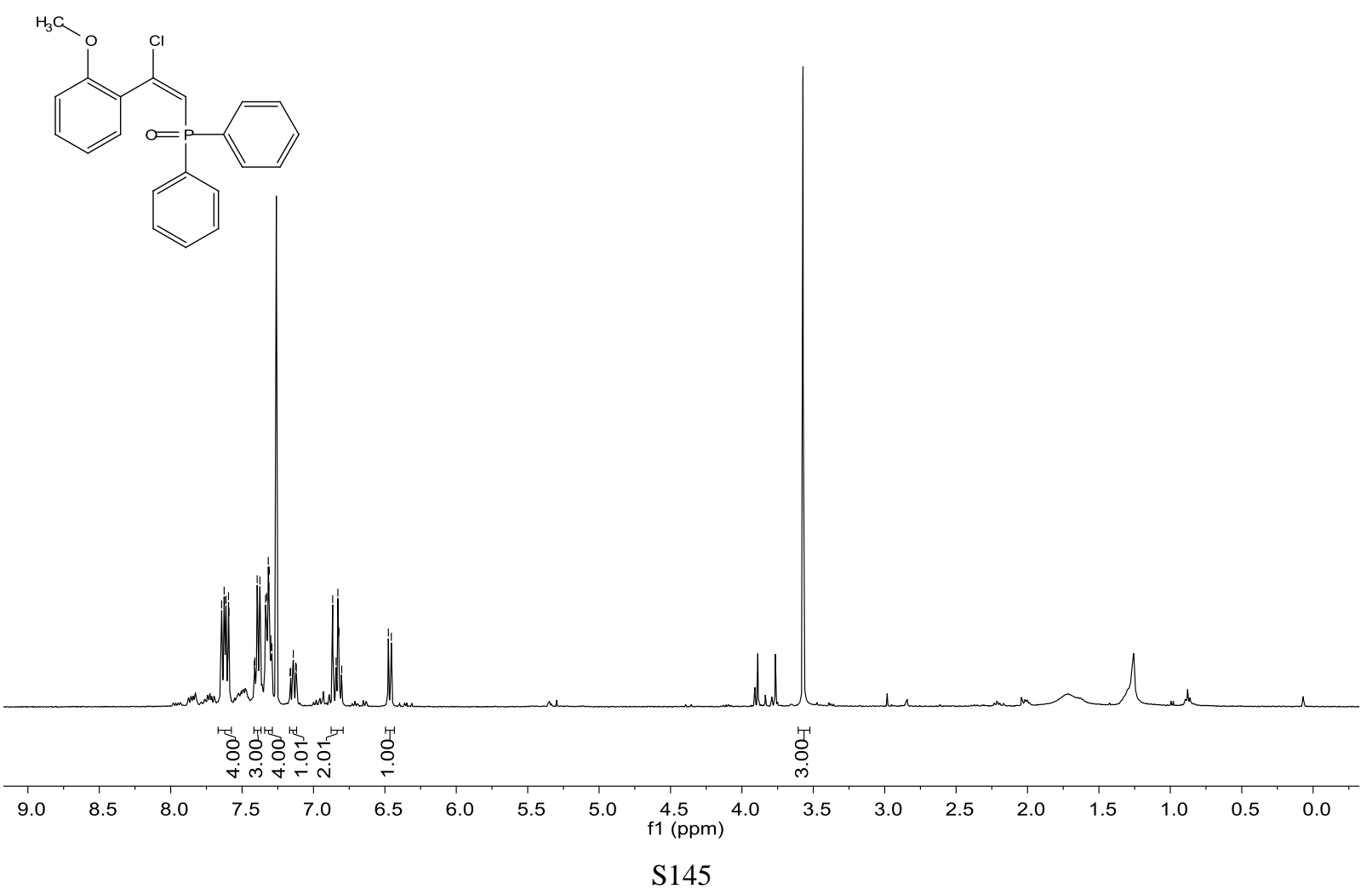


${ }^{31} \mathrm{P}$ NMR Spectrum of $9 \mathrm{~g}\left(\mathrm{CDCl}_{3}, 162 \mathrm{MHz}\right)$

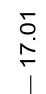

(1)

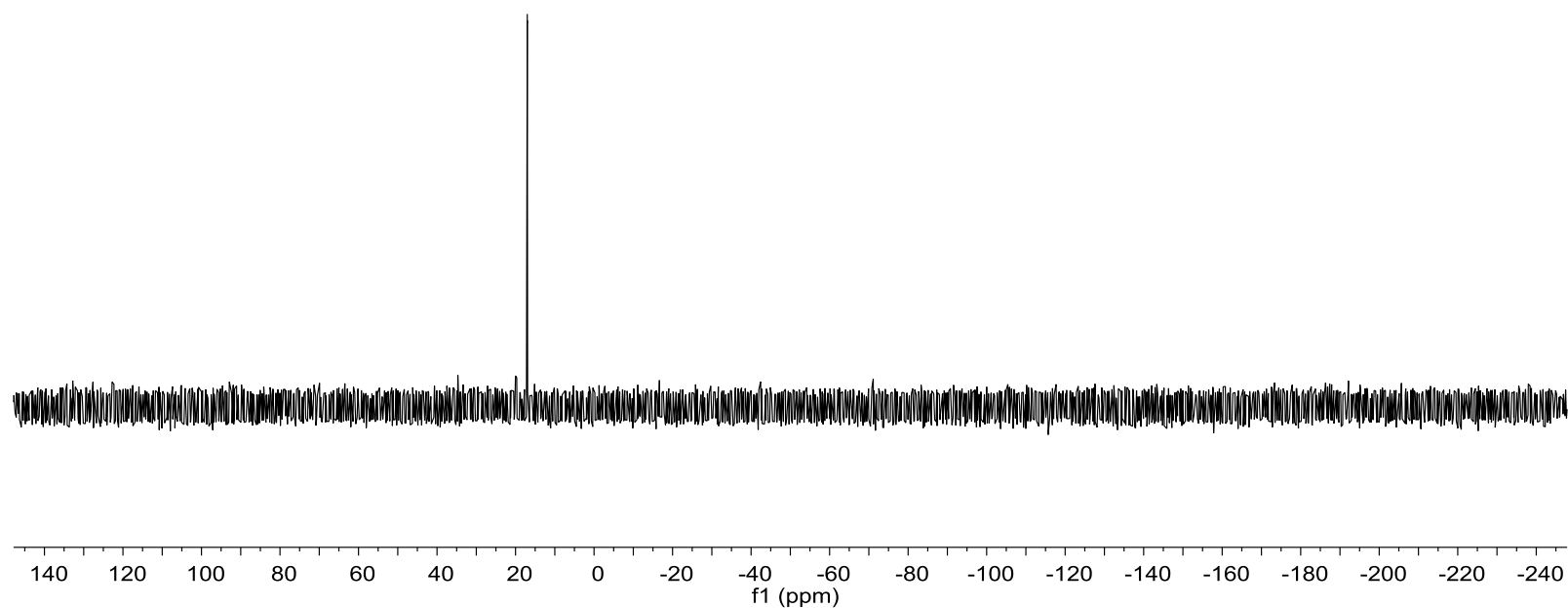

${ }^{13} \mathrm{C}$ NMR Spectrum of $9 \mathrm{~g}\left(\mathrm{CDCl}_{3}, 100 \mathrm{MHz}\right)$

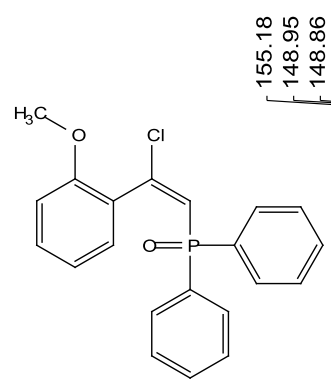

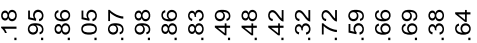

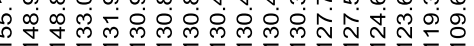

(1)

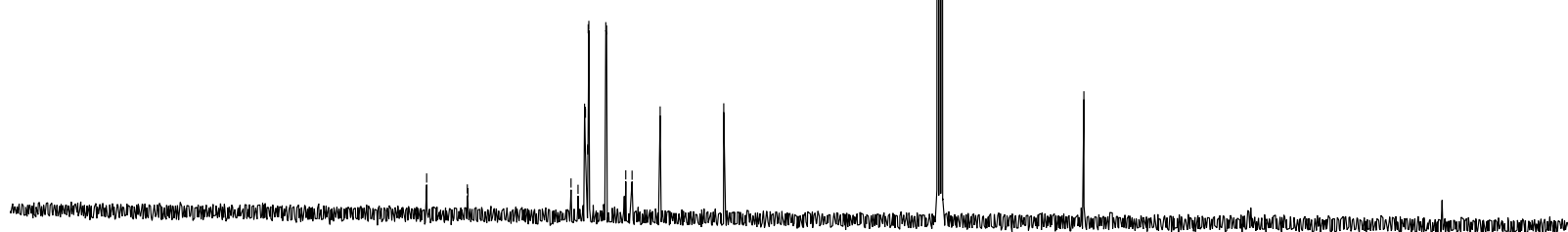

$\begin{array}{llllllllllllllllllllllll}210 & 200 & 190 & 180 & 170 & 160 & 150 & 140 & 130 & 120 & 110 \begin{array}{c}100 \\ \mathrm{f} 1(\mathrm{ppm})\end{array} & 90 & 80 & 70 & 60 & 50 & 40 & 30 & 20 & 10 & 0 & -10\end{array}$ 
${ }^{1} \mathrm{H}$ NMR Spectrum of $9 \mathrm{~h}\left(\mathrm{CDCl}_{3}, 400 \mathrm{MHz}\right)$

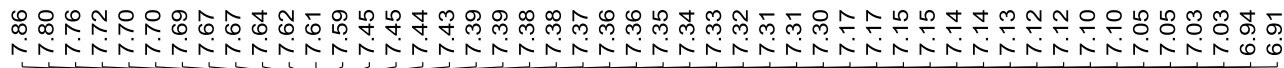<smiles>O=P(/C=C(/Cl)c1ccccc1Cl)(c1ccccc1)c1ccccc1</smiles>

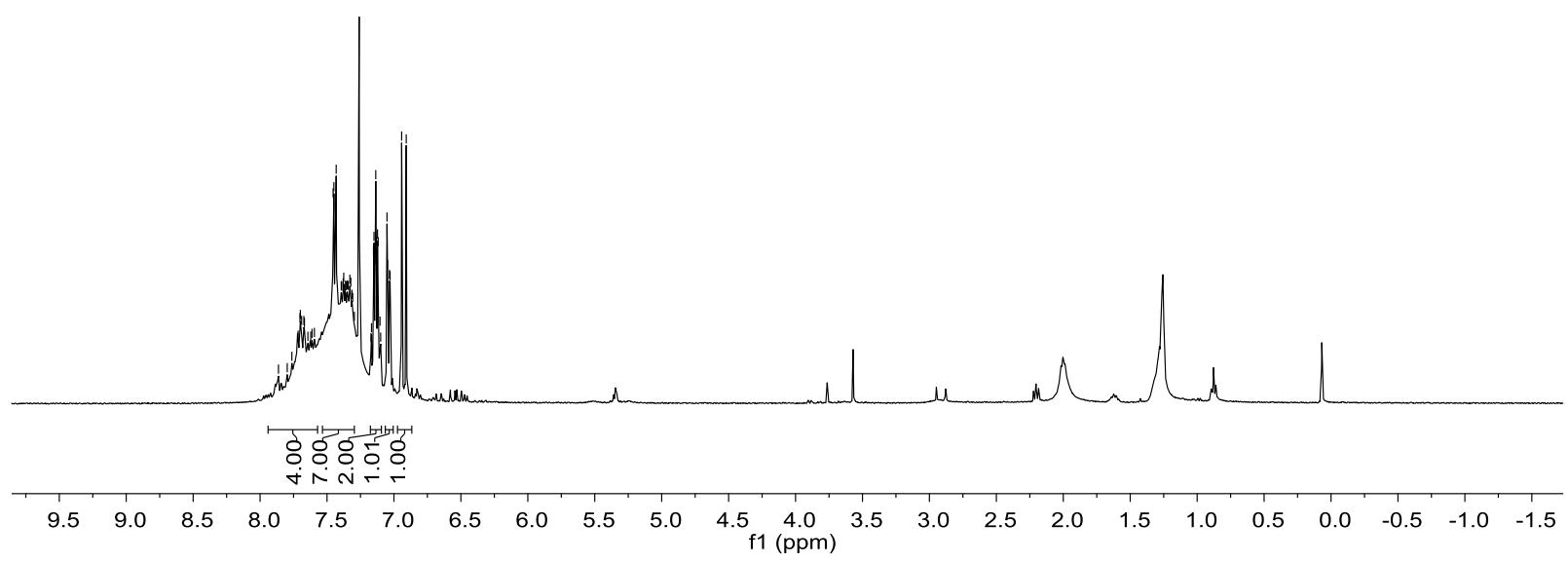

${ }^{31} \mathrm{P}$ NMR Spectrum of $9 \mathrm{~h}\left(\mathrm{CDCl}_{3}, 162 \mathrm{MHz}\right)$

$\stackrel{\circ}{\stackrel{0}{\Gamma}}$<smiles>O=P(/C=C(/Cl)c1ccccc1Cl)(c1ccccc1)c1ccccc1</smiles>

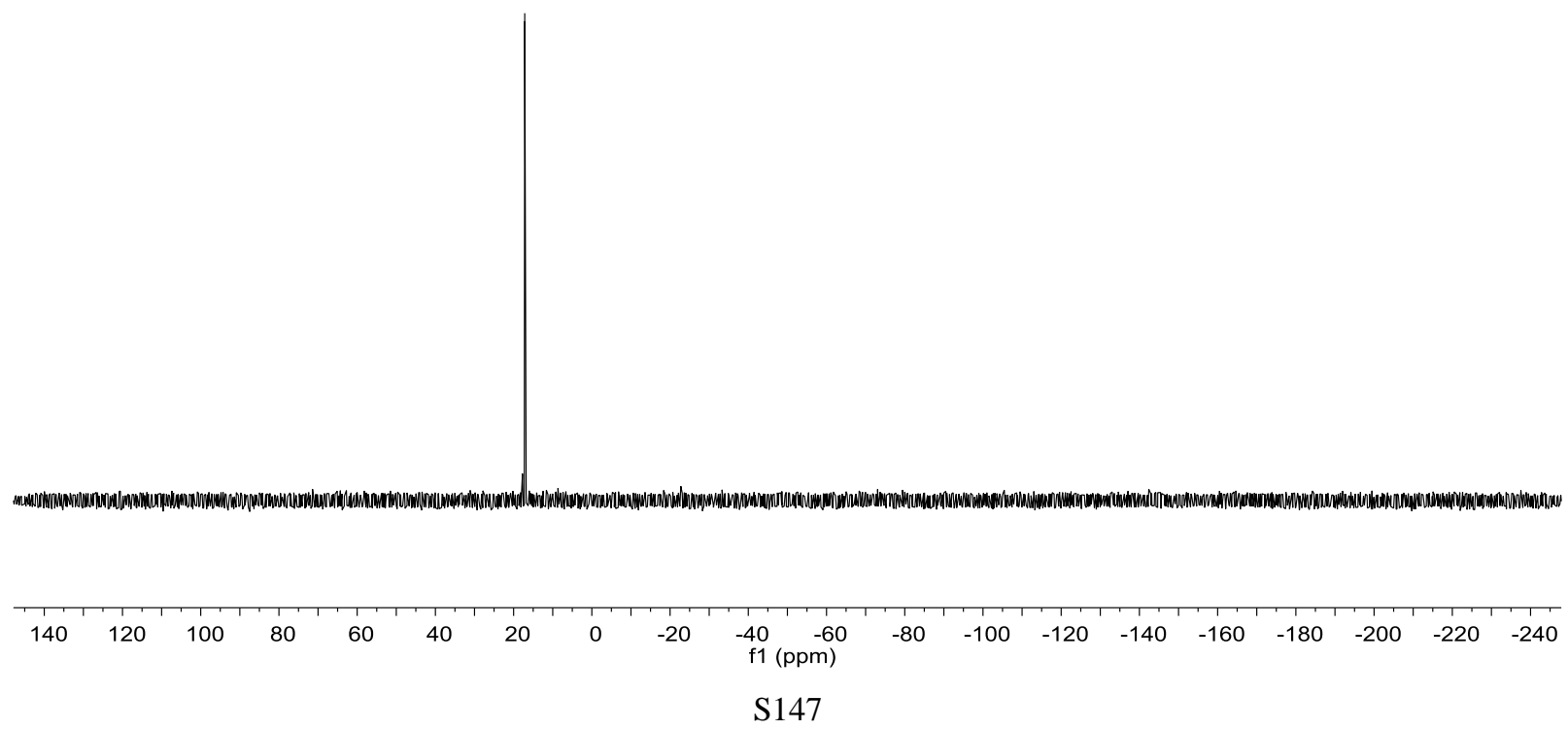


${ }^{31} \mathrm{C}$ NMR Spectrum of $9 \mathrm{~h}\left(\mathrm{CDCl}_{3}, 100 \mathrm{MHz}\right)$

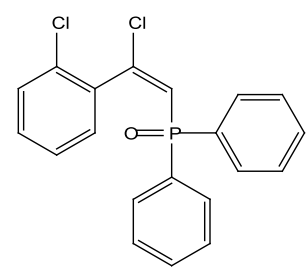

क्ष

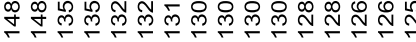

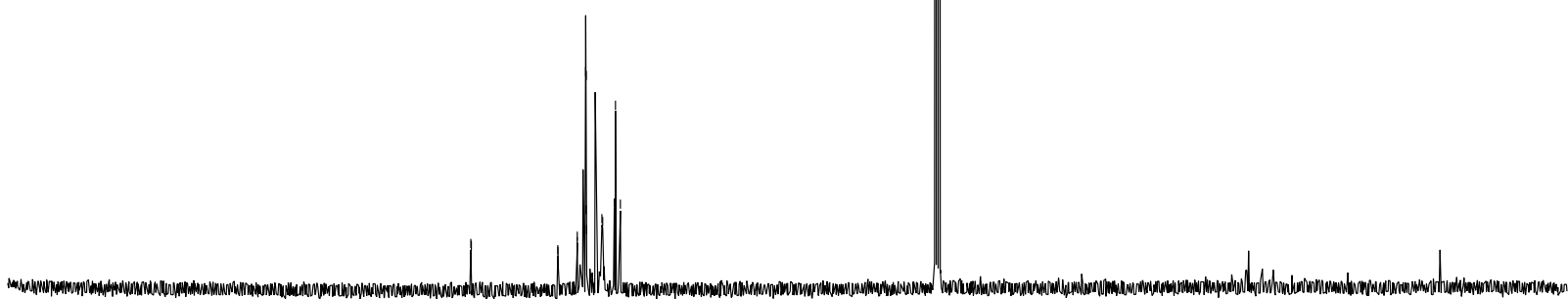

$\begin{array}{lllllllllllllllllllllll}210 & 200 & 190 & 180 & 170 & 160 & 150 & 140 & 130 & 120 & 110 & 100 & 90 & 80 & 70 & 60 & 50 & 40 & 30 & 20 & 10 & 0 & -10\end{array}$

${ }^{1} \mathrm{H}$ NMR Spectrum of $9 \mathrm{i}\left(\mathrm{CDCl}_{3}, 400 \mathrm{MHz}\right)$

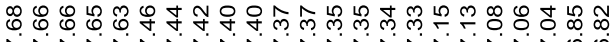

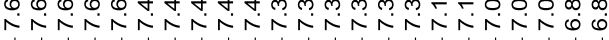
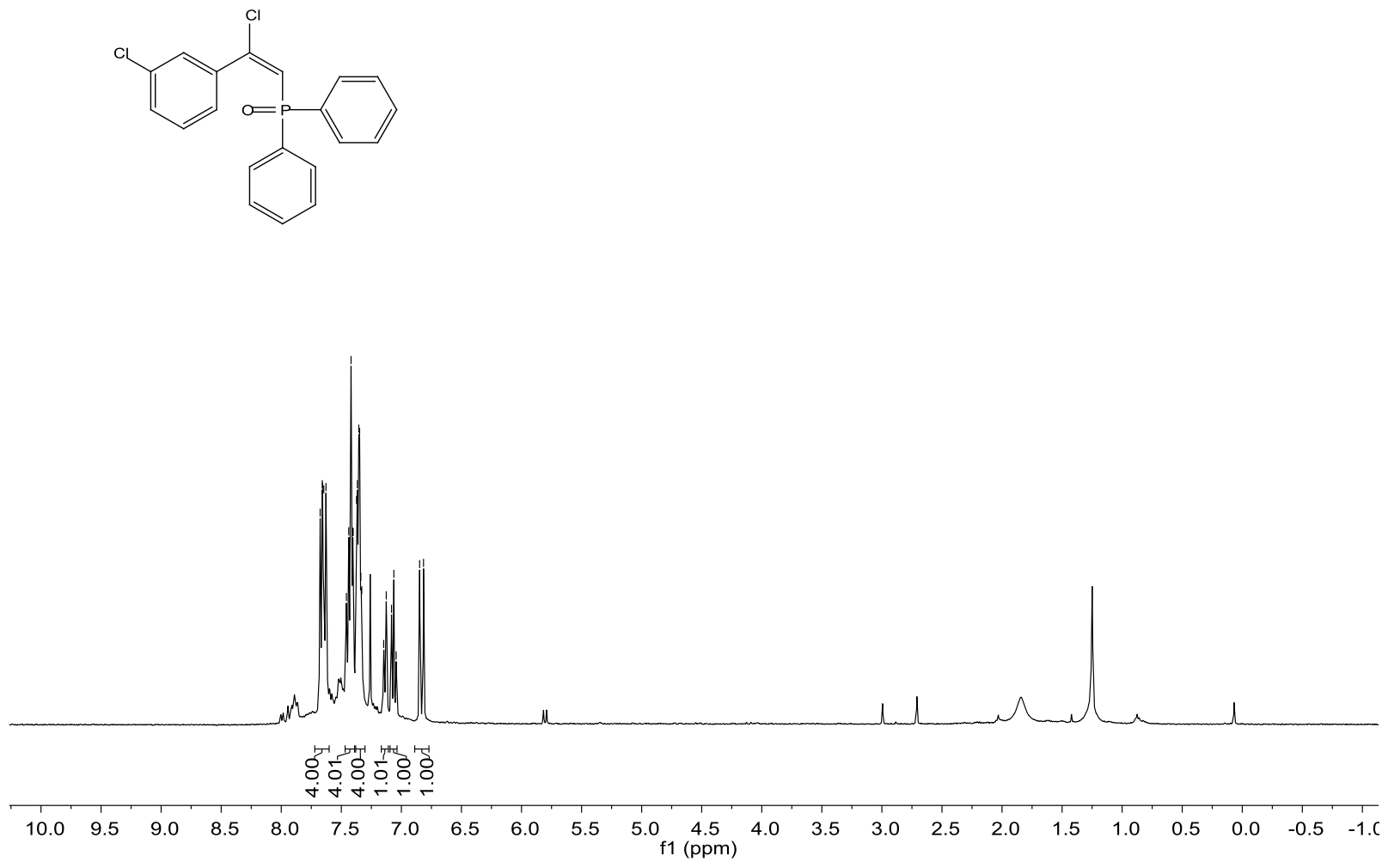
${ }^{31} \mathrm{P}$ NMR Spectrum of $9 \mathrm{i}\left(\mathrm{CDCl}_{3}, 162 \mathrm{MHz}\right)$

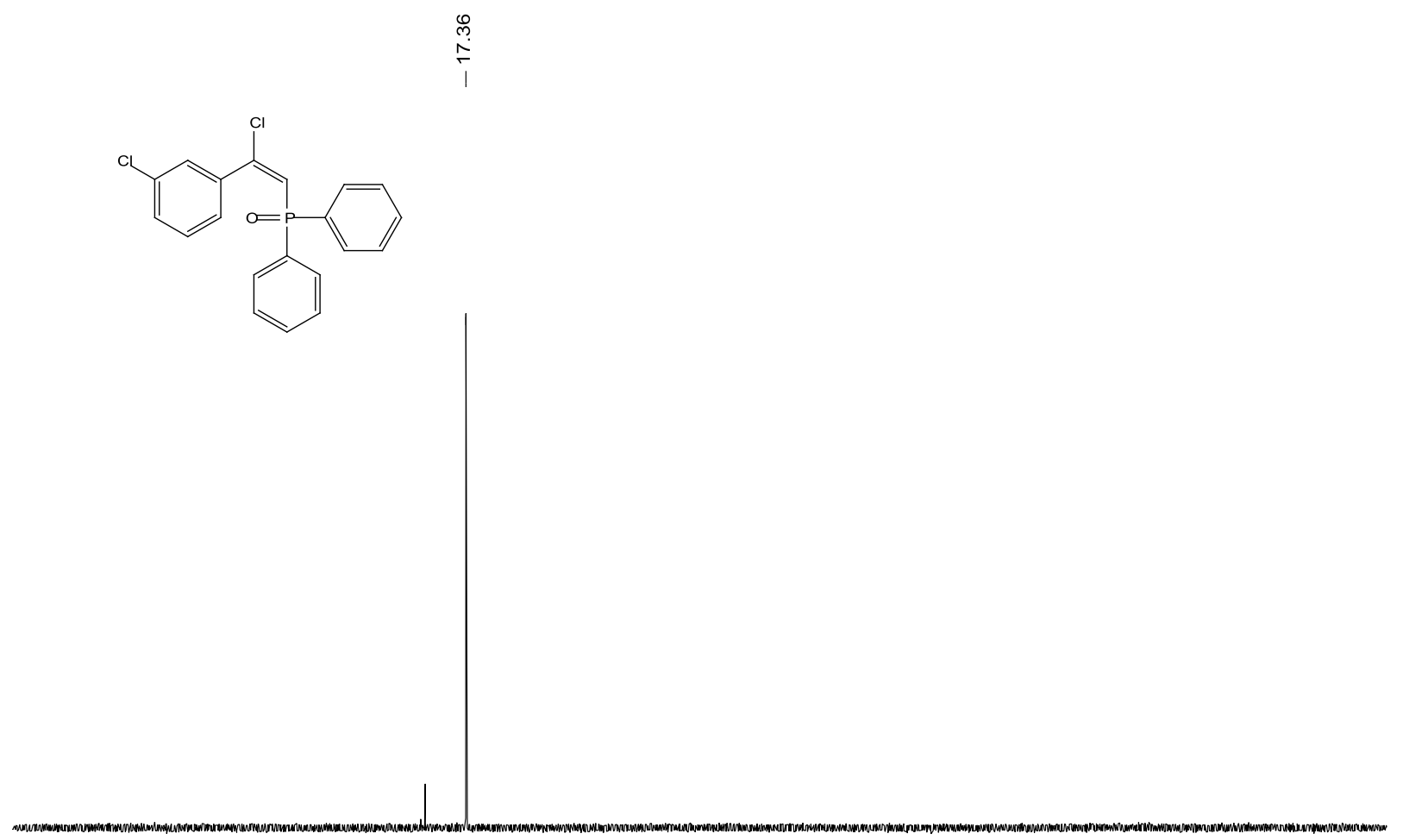

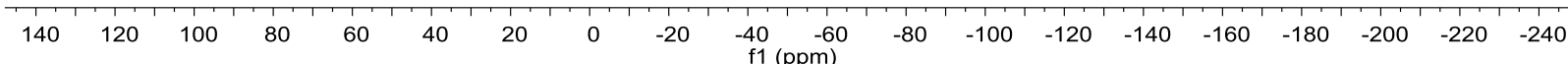

${ }^{13} \mathrm{C}$ NMR Spectrum of $9 \mathrm{i}\left(\mathrm{CDCl}_{3}, 100 \mathrm{MHz}\right)$

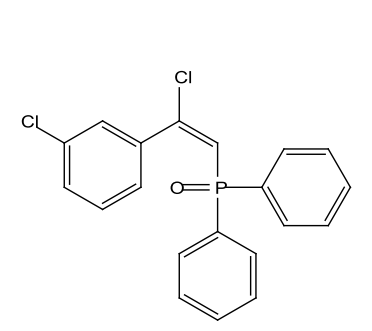

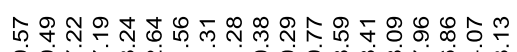

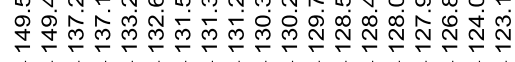

$\|$

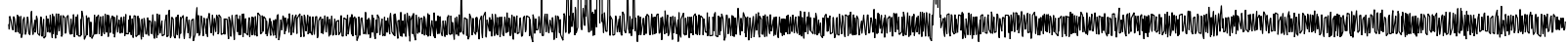

$\begin{array}{lllllllllllllllllllllll}210 & 200 & 190 & 180 & 170 & 160 & 150 & 140 & 130 & 120 & 110 & 100 & 90 & 80 & 70 & 60 & 50 & 40 & 30 & 20 & 10 & 0 & -10\end{array}$ 
${ }^{1} \mathrm{H}$ NMR Spectrum of $9 \mathrm{j}\left(\mathrm{CDCl}_{3}, 400 \mathrm{MHz}\right)$

œ

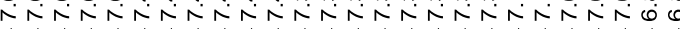<smiles>O=P(C=C(Cl)c1ccc(Cl)cc1)(c1ccccc1)c1ccccc1</smiles>

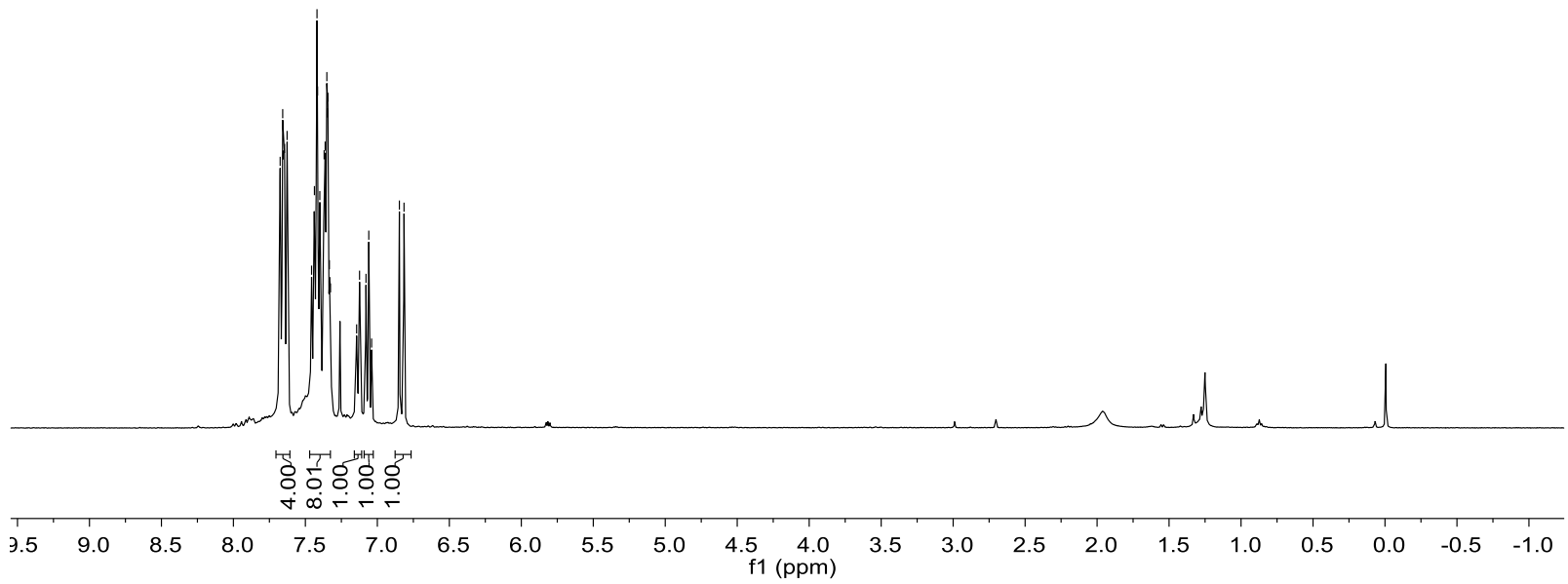

${ }^{31}$ P NMR Spectrum of $9 \mathrm{j}\left(\mathrm{CDCl}_{3}, 162 \mathrm{MHz}\right)$

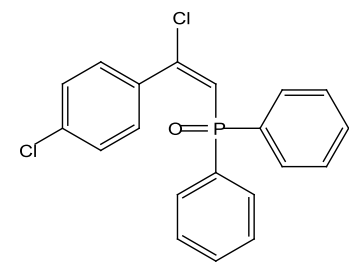

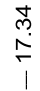

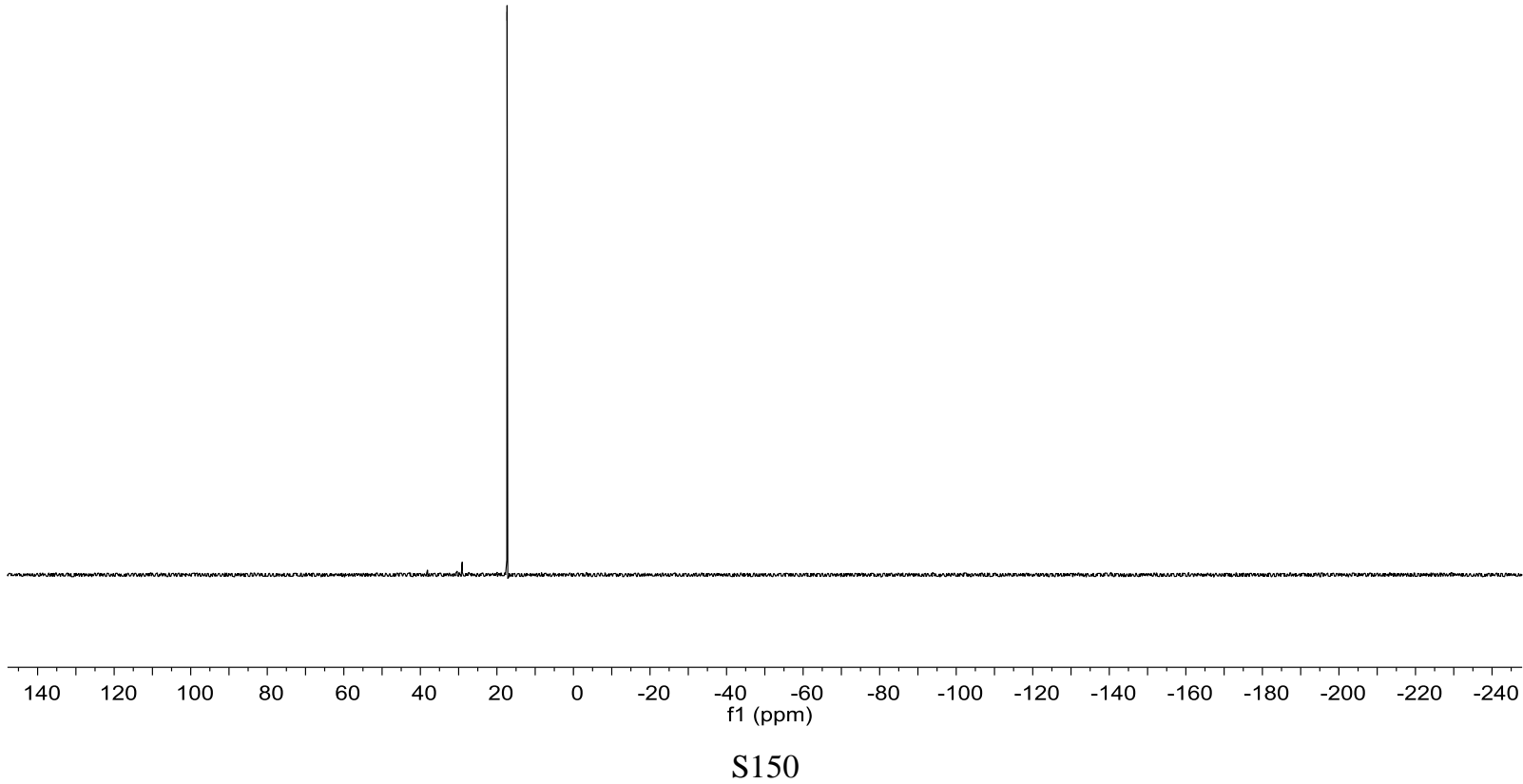




\section{${ }^{13} \mathrm{C}$ NMR Spectrum of $9 \mathrm{j}\left(\mathrm{CDCl}_{3}, 100 \mathrm{MHz}\right)$}
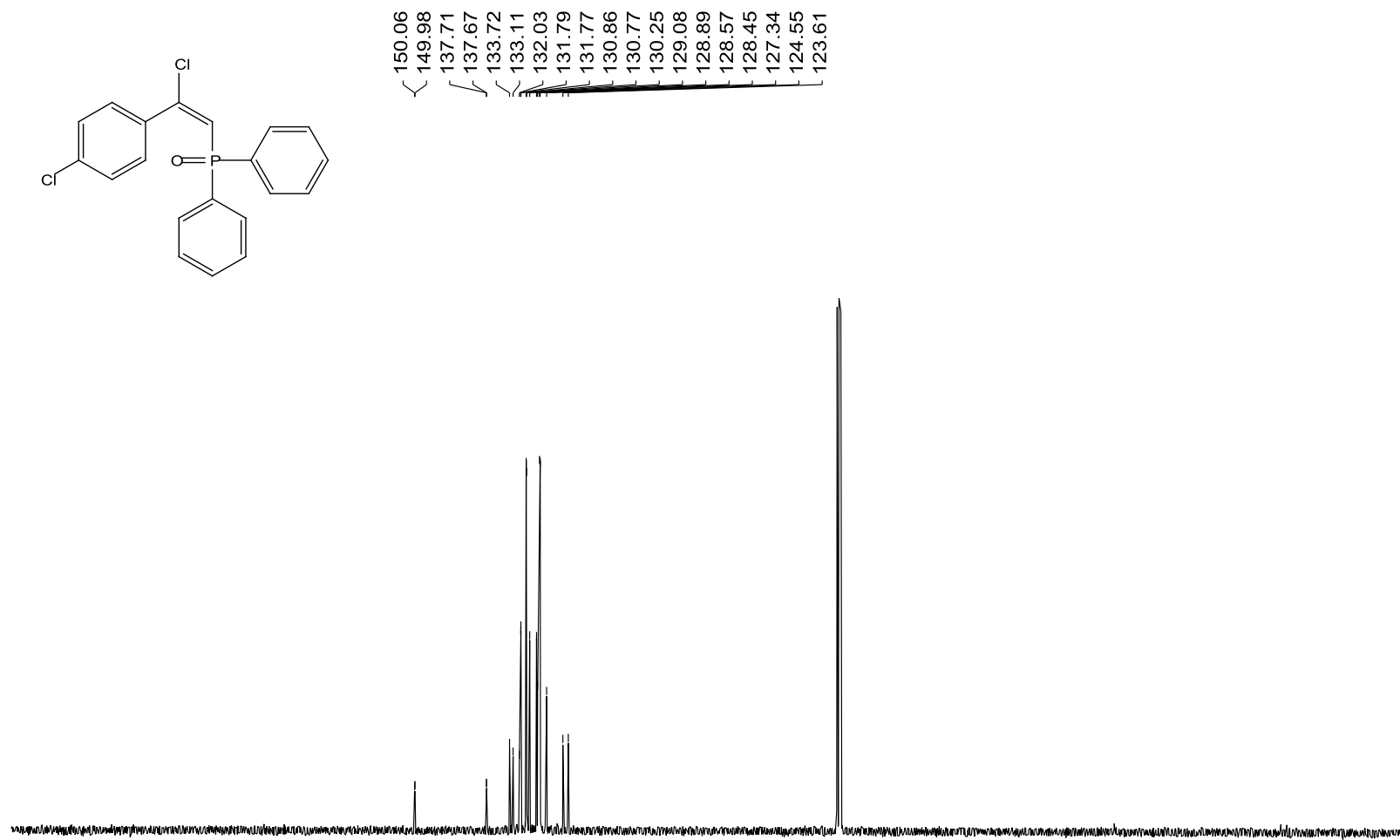

$\begin{array}{lllllllllllllllllllllll}210 & 200 & 190 & 180 & 170 & 160 & 150 & 140 & 130 & 120 & 110 & 100 & 90 & 80 & 70 & 60 & 50 & 40 & 30 & 20 & 10 & 0 & -10\end{array}$

${ }^{1} \mathrm{H}$ NMR Spectrum of $9 \mathrm{k}\left(\mathrm{CDCl}_{3}, 400 \mathrm{MHz}\right)$

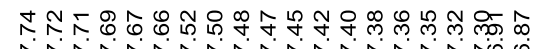

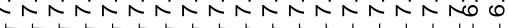

(1)

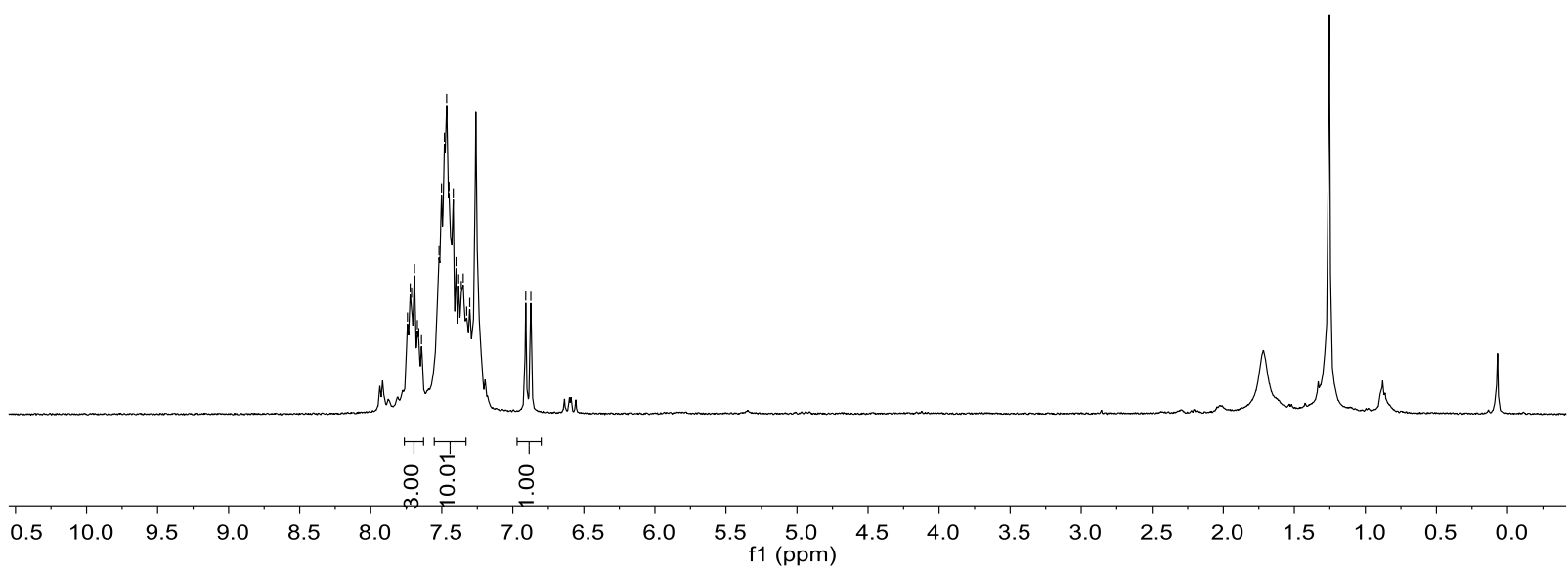


${ }^{31} \mathrm{P}$ NMR Spectrum of 9k $\left(\mathrm{CDCl}_{3}, 162 \mathrm{MHz}\right)$

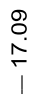

(1)

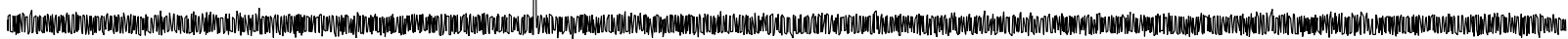

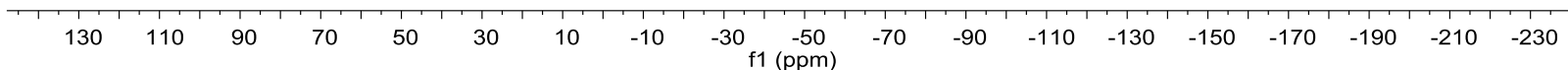

${ }^{13} \mathrm{C}$ NMR Spectrum of $9 \mathrm{k}\left(\mathrm{CDCl}_{3}, 100 \mathrm{MHz}\right)$

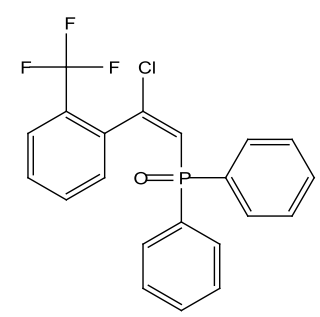

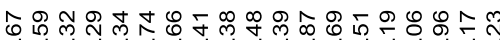

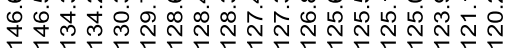

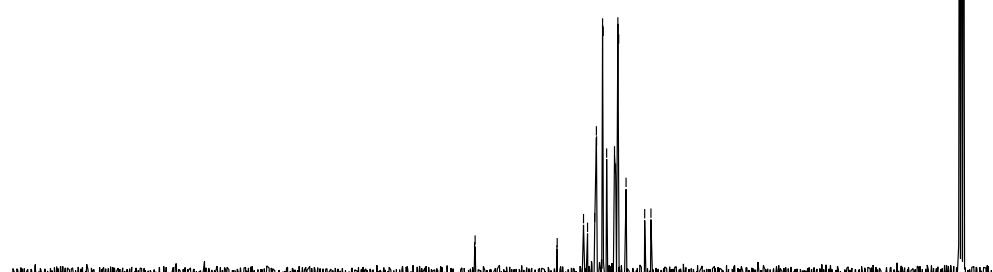

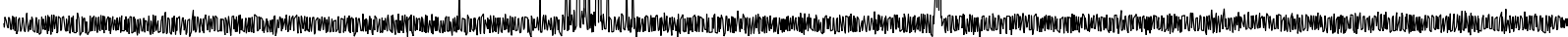

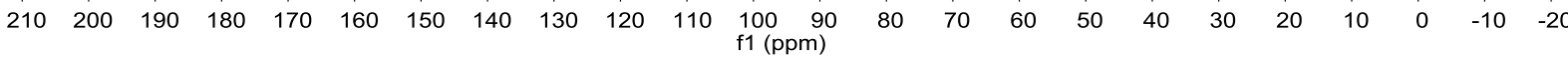


${ }^{1} \mathrm{H}$ NMR Spectrum of $91\left(\mathrm{CDCl}_{3}, 400 \mathrm{MHz}\right)$

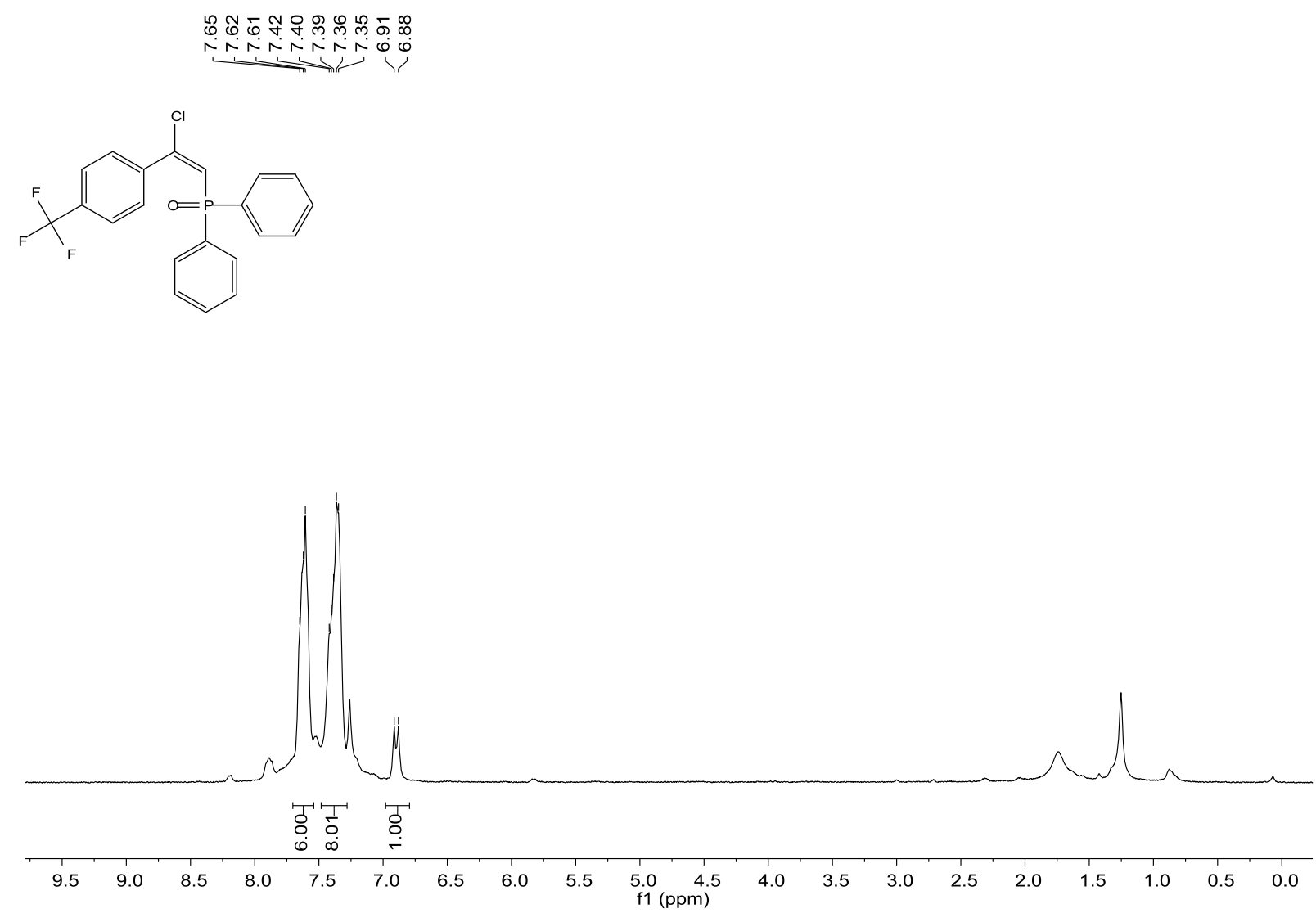

${ }^{31} \mathrm{P}$ NMR Spectrum of $91\left(\mathrm{CDCl}_{3}, 162 \mathrm{MHz}\right)$

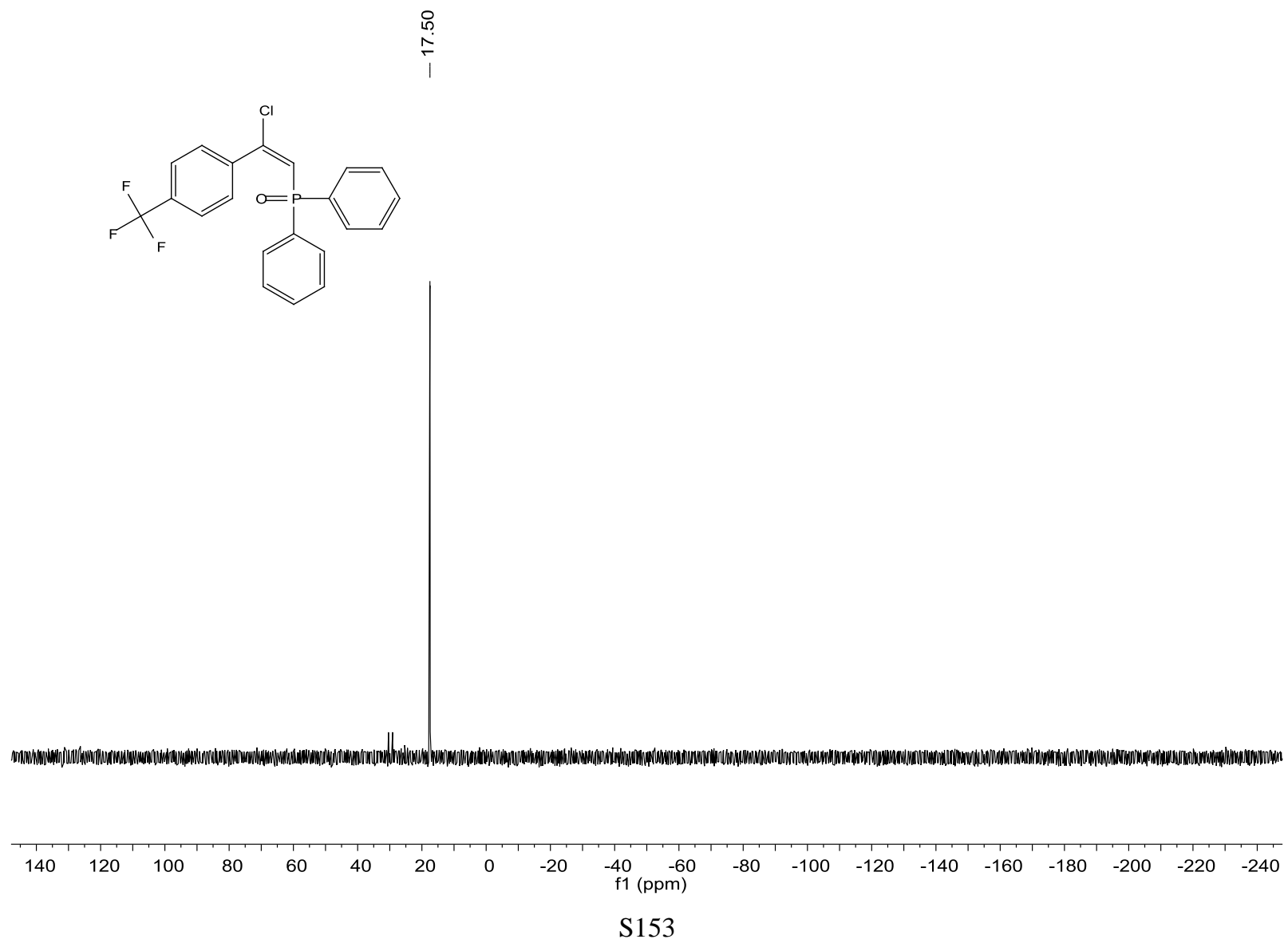




\section{${ }^{13} \mathrm{C}$ NMR Spectrum of $91\left(\mathrm{CDCl}_{3}, 100 \mathrm{MHz}\right)$}

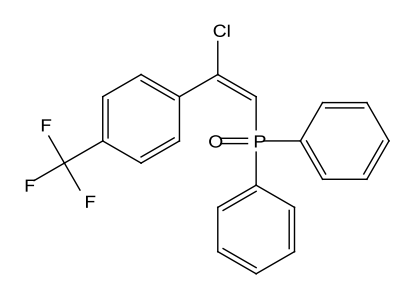

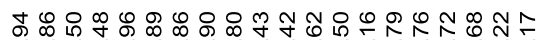

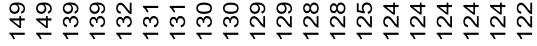

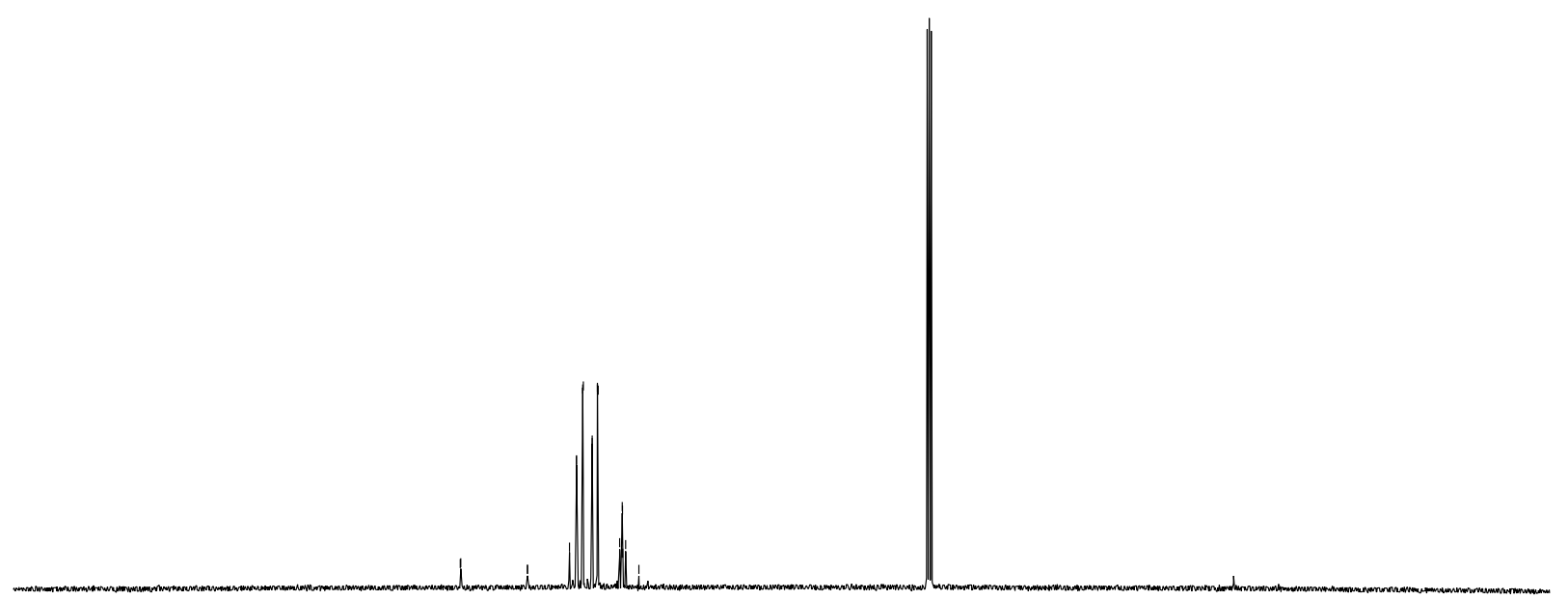

$\begin{array}{llllllllllllllllllllllllll}210 & 200 & 190 & 180 & 170 & 160 & 150 & 140 & 130 & 120 & 110 & 100 & 90 & 80 & 70 & 60 & 50 & 40 & 30 & 20 & 10 & 0 & -10\end{array}$

${ }^{1} \mathrm{H}$ NMR Spectrum of 9m $\left(\mathrm{CDCl}_{3}, 400 \mathrm{MHz}\right)$

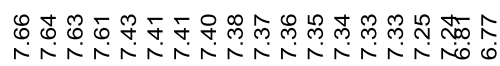

(c)

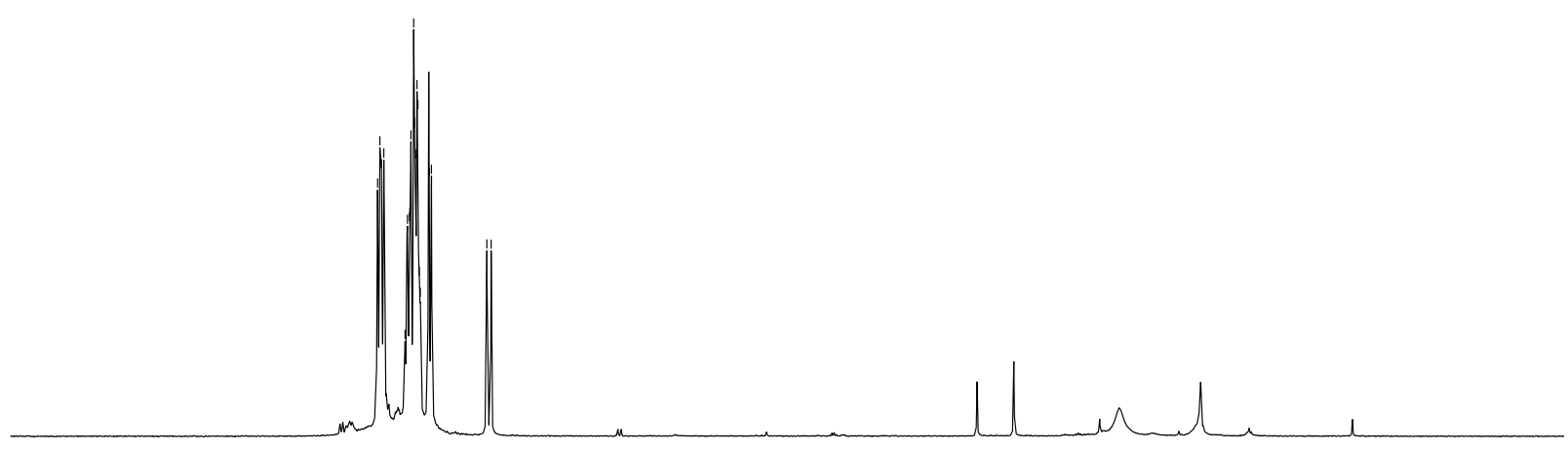

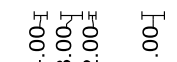

\begin{tabular}{lllllllllllllllllllllllllll}
\hline .5 & 10.0 & 9.5 & 9.0 & 8.5 & 8.0 & 7.5 & 7.0 & 6.5 & 6.0 & 5.5 & 5.0 & $\begin{array}{c}4.5 \\
\mathrm{f} 1\end{array}(\mathrm{ppm})$ & 4.0 & 3.5 & 3.0 & 2.5 & 2.0 & 1.5 & 1.0 & 0.5 & 0.0 & -0.5 & -1.0 & -1.
\end{tabular} 
${ }^{31} \mathrm{P}$ NMR Spectrum of $9 \mathrm{~m}\left(\mathrm{CDCl}_{3}, 162 \mathrm{MHz}\right)$
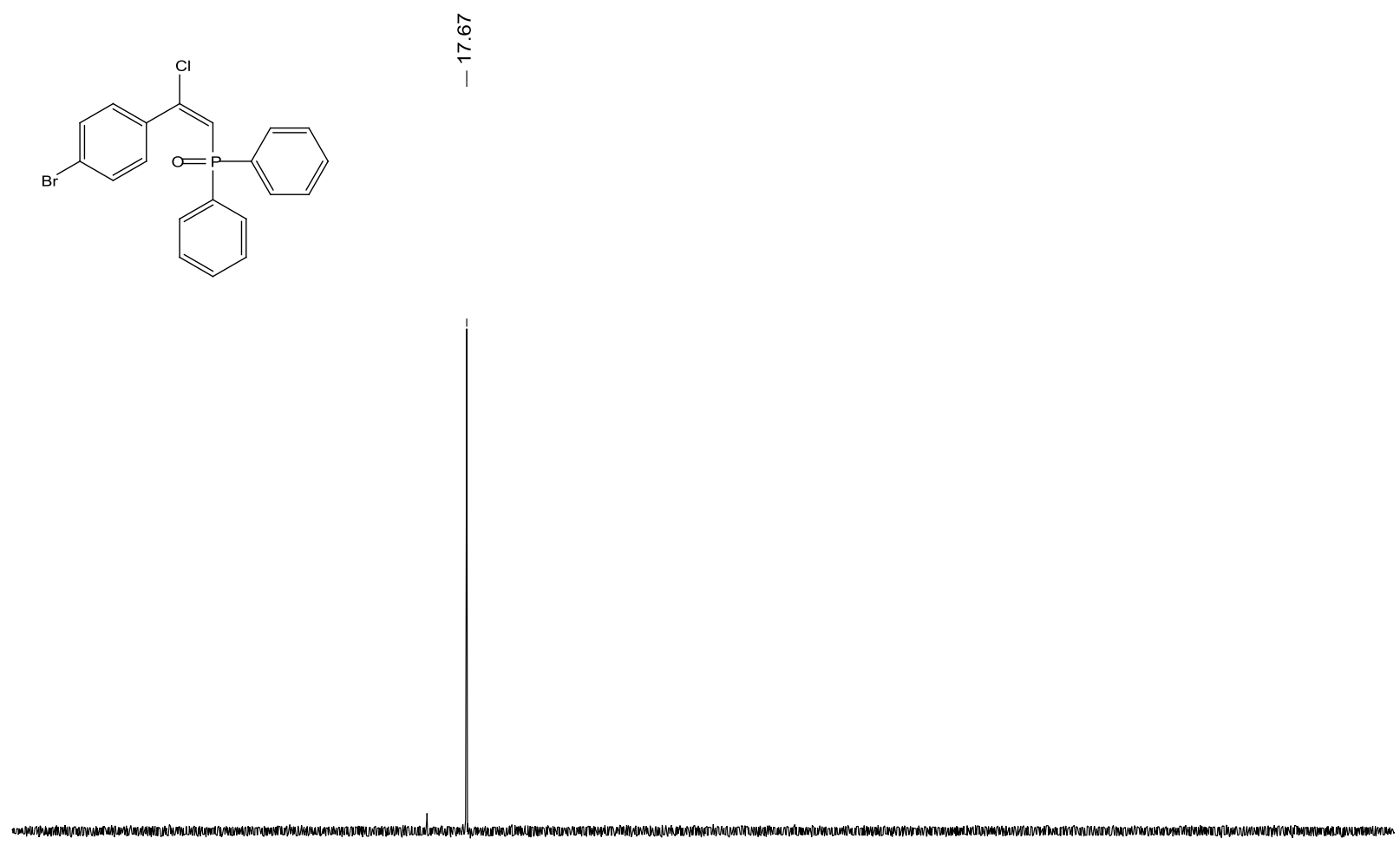

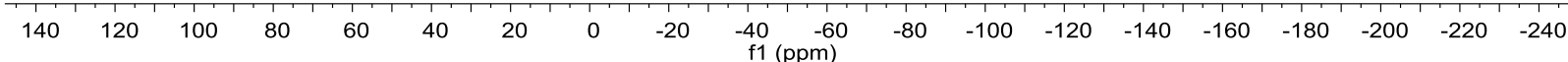

${ }^{13} \mathrm{C}$ NMR Spectrum of $9 \mathrm{~m}\left(\mathrm{CDCl}_{3}, 100 \mathrm{MHz}\right)$

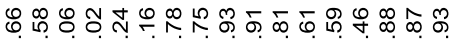

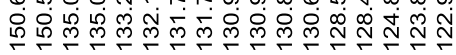
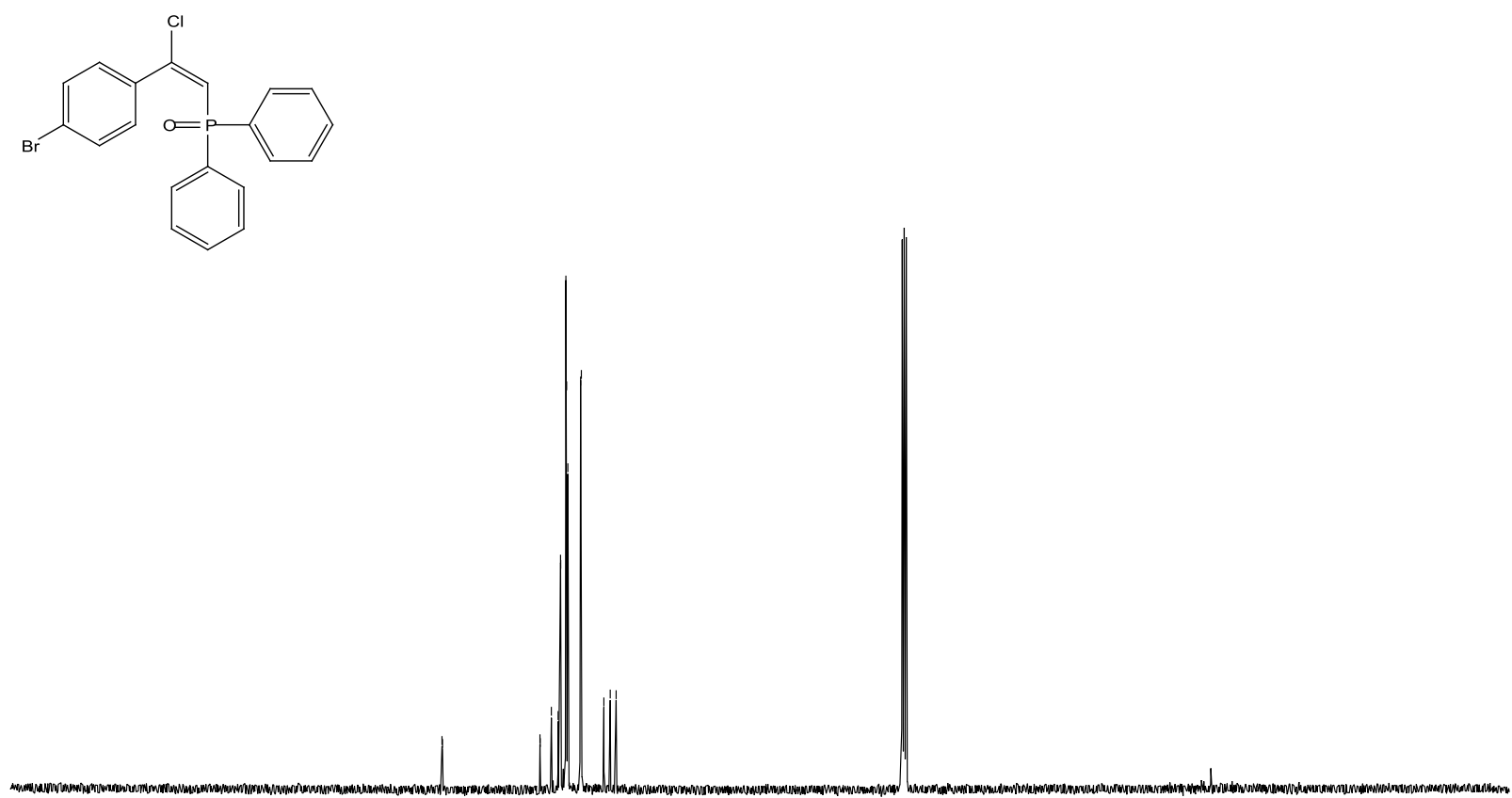

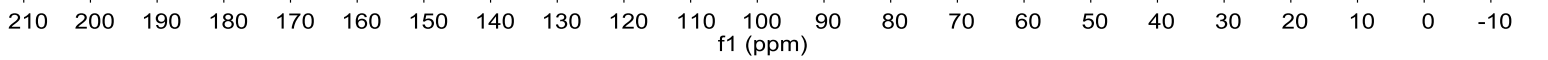


${ }^{1} \mathrm{H}$ NMR Spectrum of 9n $\left(\mathrm{CDCl}_{3}, 400 \mathrm{MHz}\right)$

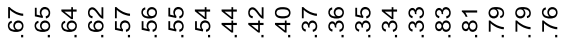

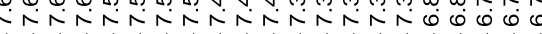

(c)

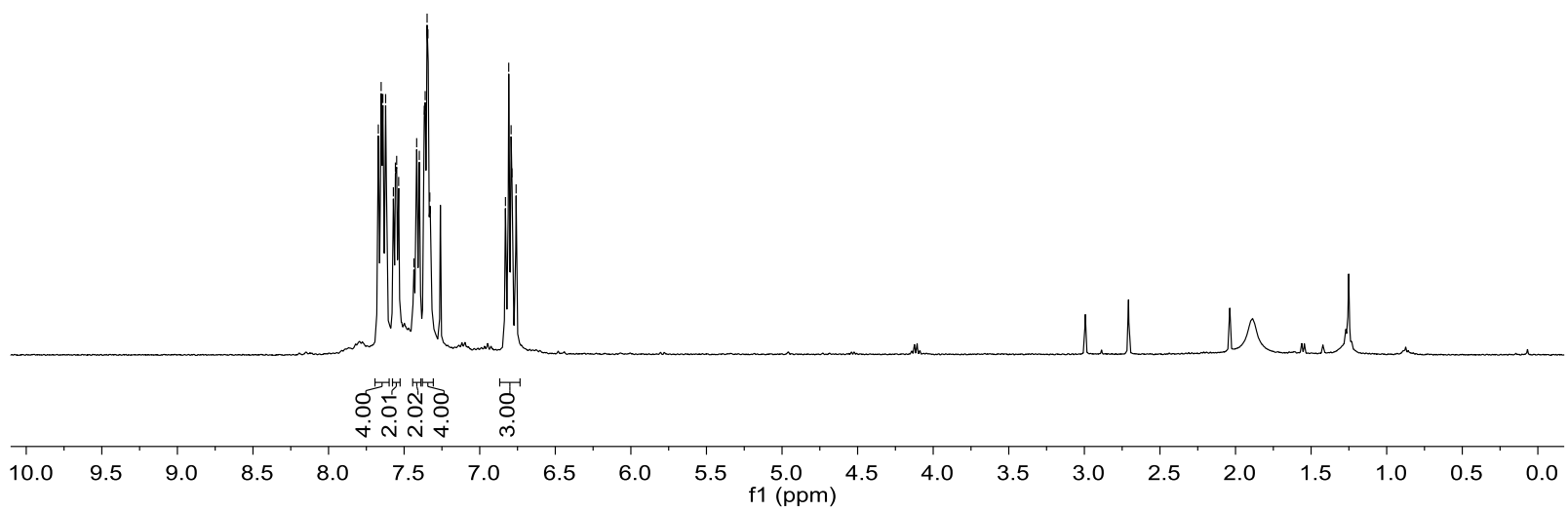

${ }^{31} \mathrm{P}$ NMR Spectrum of 9n $\left(\mathrm{CDCl}_{3}, 162 \mathrm{MHz}\right)$

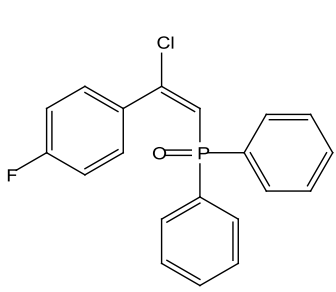

$\stackrel{0}{\stackrel{N}{i}}$

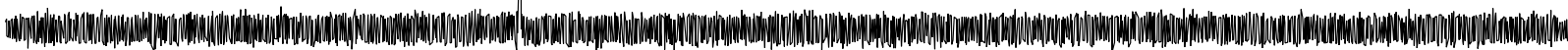

$\begin{array}{lllllllllllllllllllll}140 & 120 & 100 & 80 & 60 & 40 & 20 & 0 & -20 & -40 & -60 & -80 & -100 & -120 & -140 & -160 & -180 & -200 & -220 & -240\end{array}$


${ }^{13} \mathrm{C}$ NMR Spectrum of 9n $\left(\mathrm{CDCl}_{3}, 100 \mathrm{MHz}\right)$
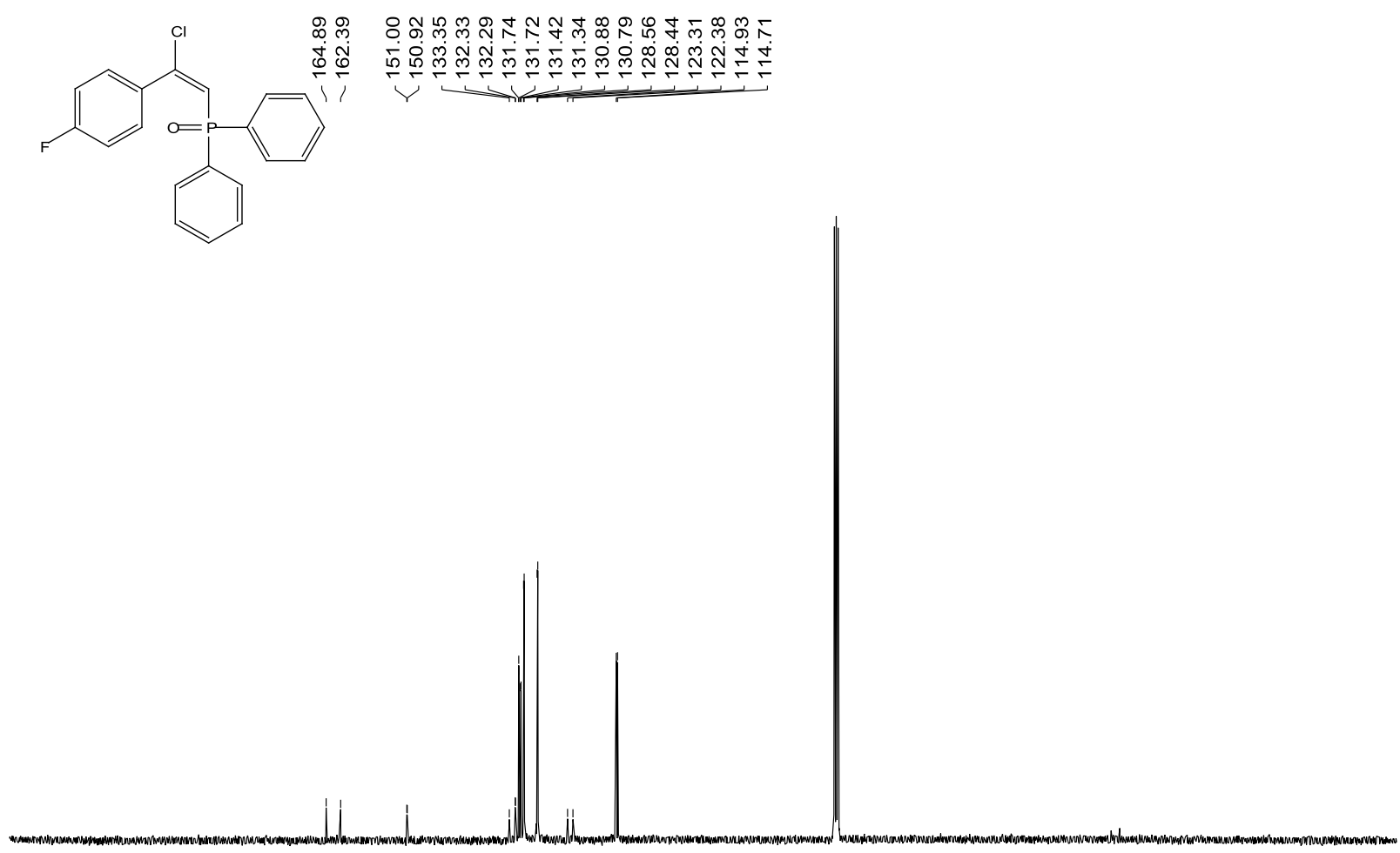

$\begin{array}{llllllllllllllllllllllllll}210 & 200 & 190 & 180 & 170 & 160 & 150 & 140 & 130 & 120 & 110 & 100 & 90 & 80 & 70 & 60 & 50 & 40 & 30 & 20 & 10 & 0 & -10\end{array}$

${ }^{1} \mathrm{H}$ NMR Spectrum of $90\left(\mathrm{CDCl}_{3}, 400 \mathrm{MHz}\right)$
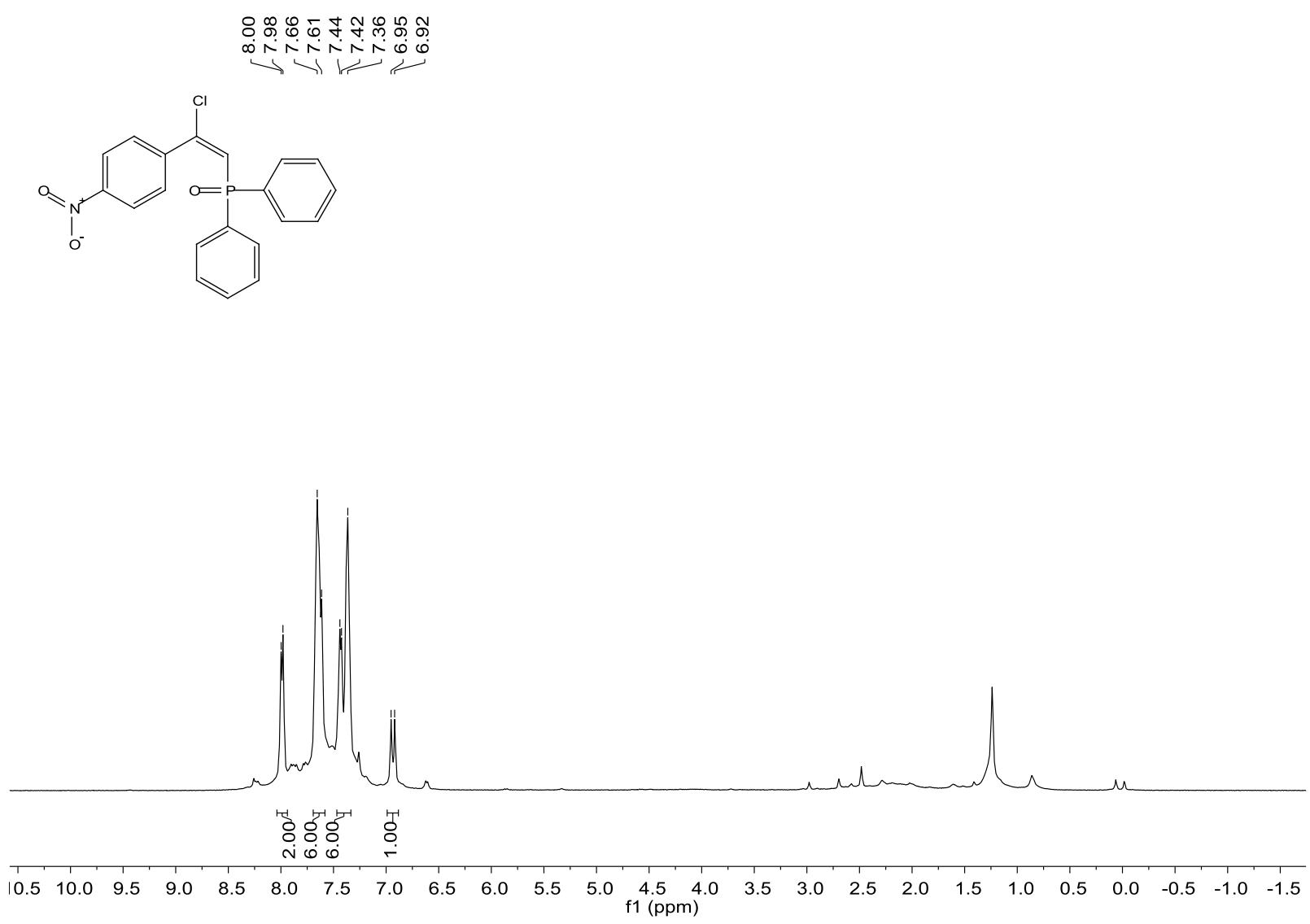
${ }^{31}$ P NMR Spectrum of $90\left(\mathrm{CDCl}_{3}, 162 \mathrm{MHz}\right)$

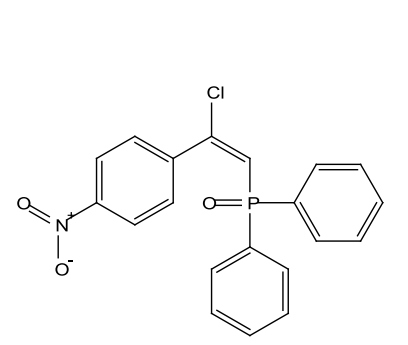

$\stackrel{\substack{\stackrel{\rho}{R} \\ \Gamma}}{\Gamma}$

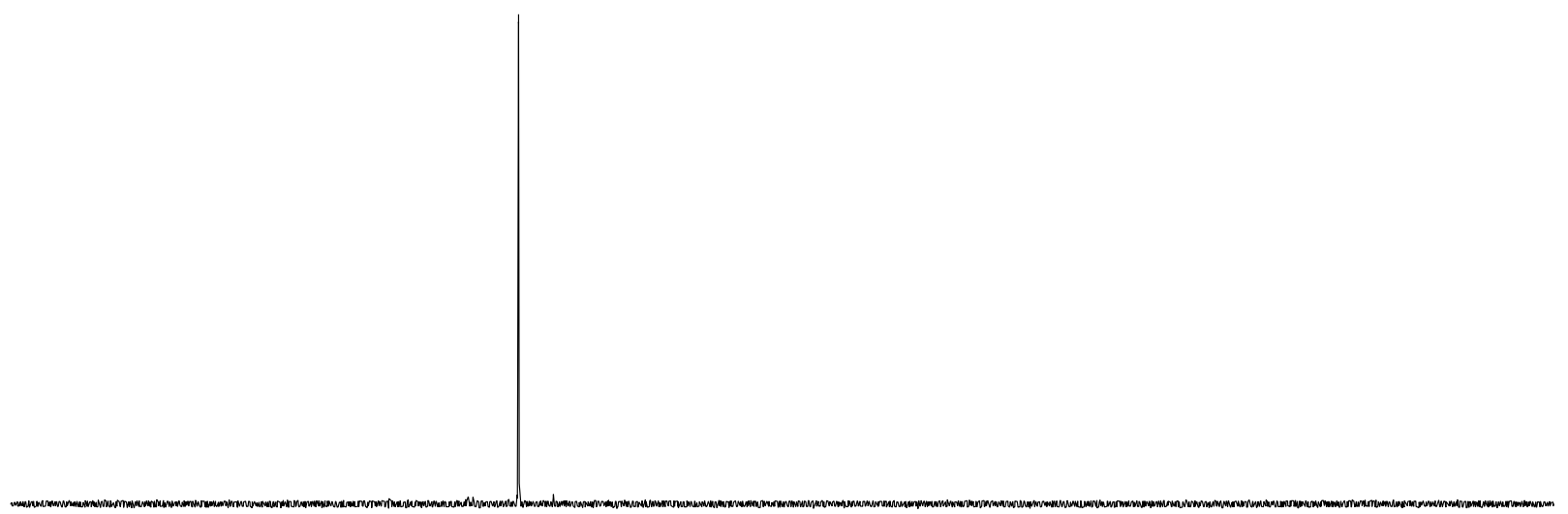

$\begin{array}{llllllllllllllllllll}140 & 120 & 100 & 80 & 60 & 40 & 20 & 0 & -20 & -40 & -60 & -80 & -100 & -120 & -140 & -160 & -180 & -200 & -220 & -240\end{array}$

${ }^{13} \mathrm{C}$ NMR Spectrum of $90\left(\mathrm{CDCl}_{3}, 100 \mathrm{MHz}\right)$
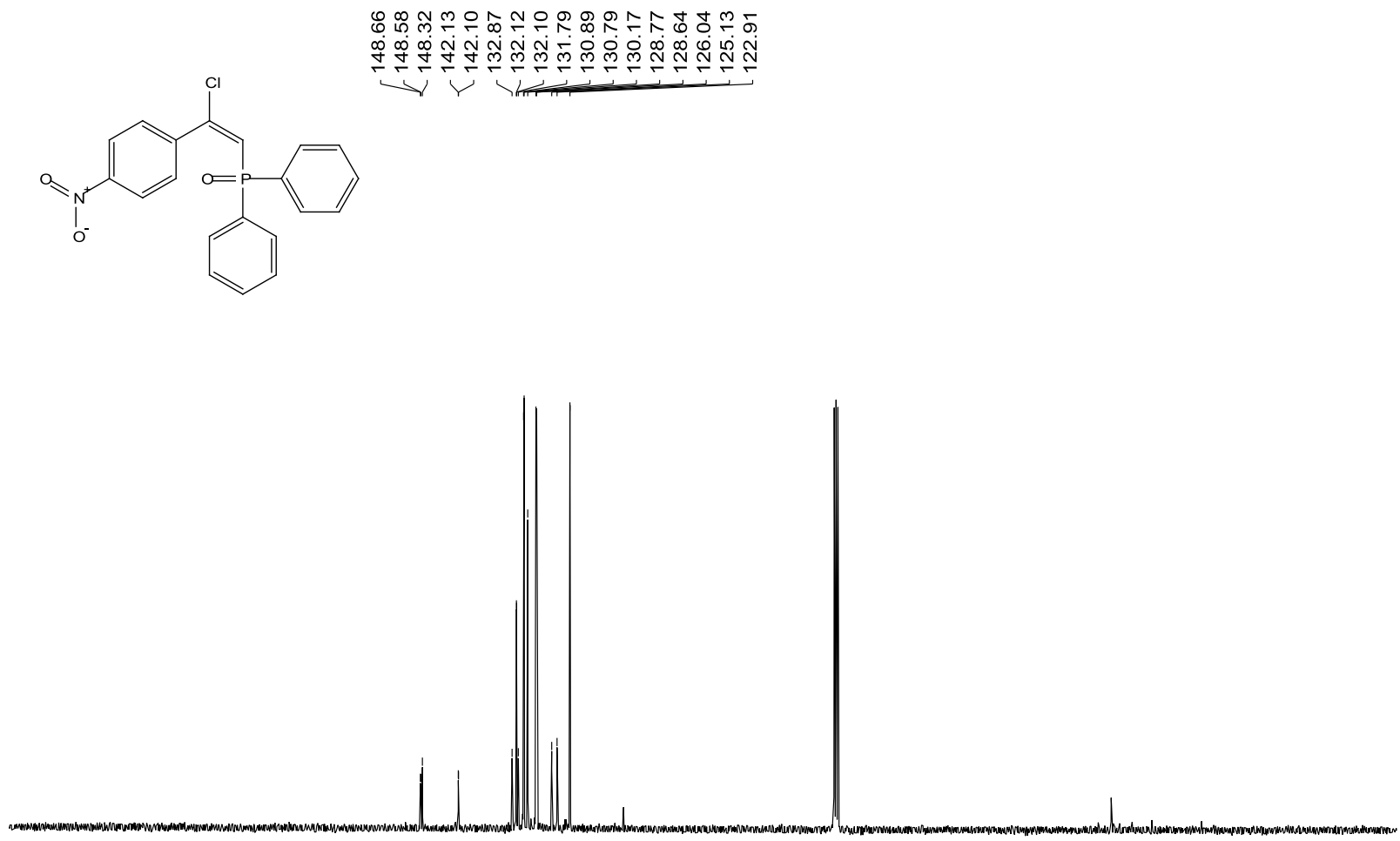

$\begin{array}{llllllllllllllllllllll}210 & 200 & 190 & 180 & 170 & 160 & 150 & 140 & 130 & 120 & 110 \begin{array}{c}100 \\ \mathrm{f} 1(\mathrm{ppm})\end{array} & 90 & 80 & 70 & 60 & 50 & 40 & 30 & 20 & 10 & 0 & -10\end{array}$ 
${ }^{1} \mathrm{H}$ NMR Spectrum of $9 \mathrm{p}\left(\mathrm{CDCl}_{3}, 400 \mathrm{MHz}\right)$

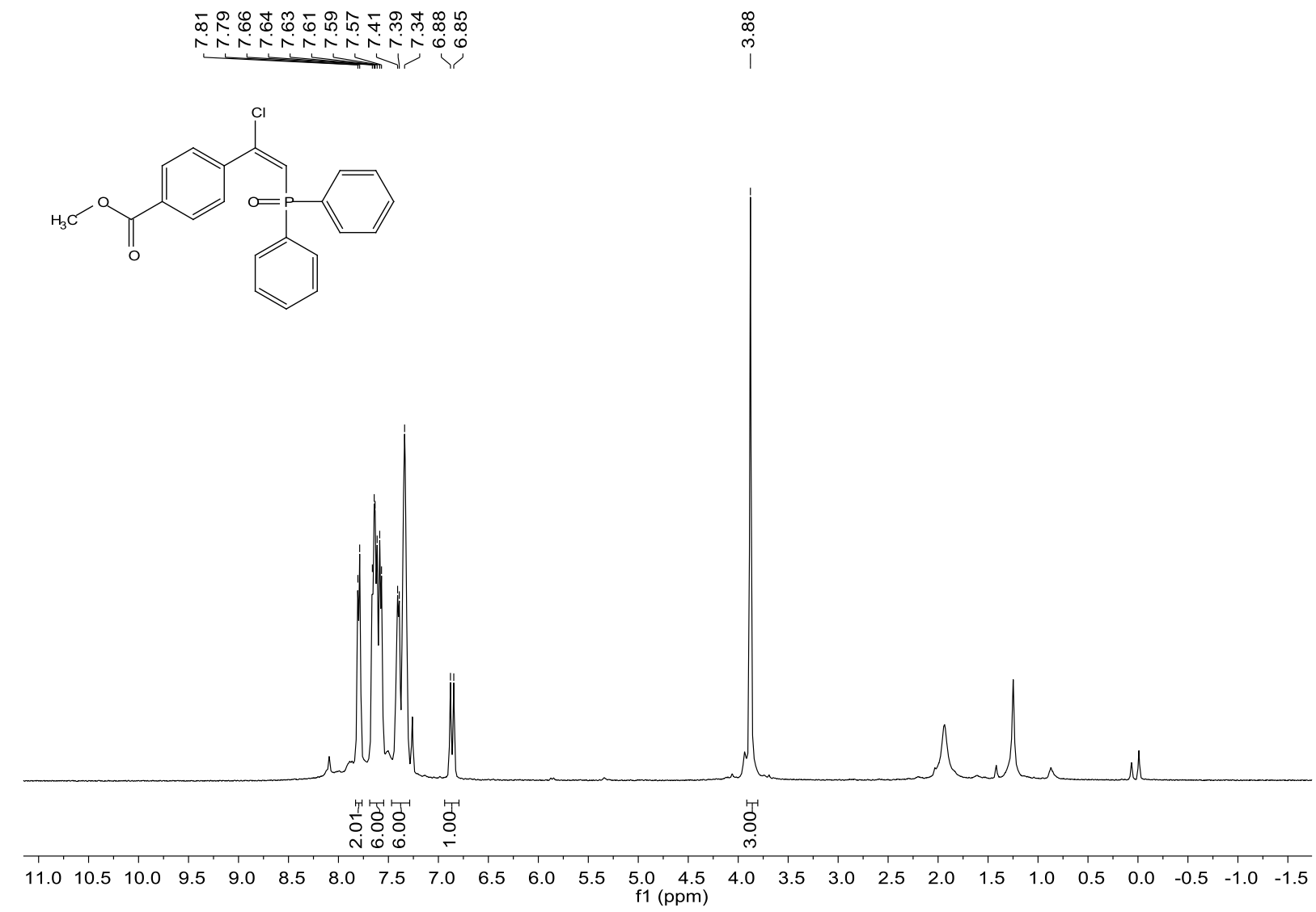

${ }^{31} \mathrm{P}$ NMR Spectrum of $9 \mathrm{p}\left(\mathrm{CDCl}_{3}, 162 \mathrm{MHz}\right)$
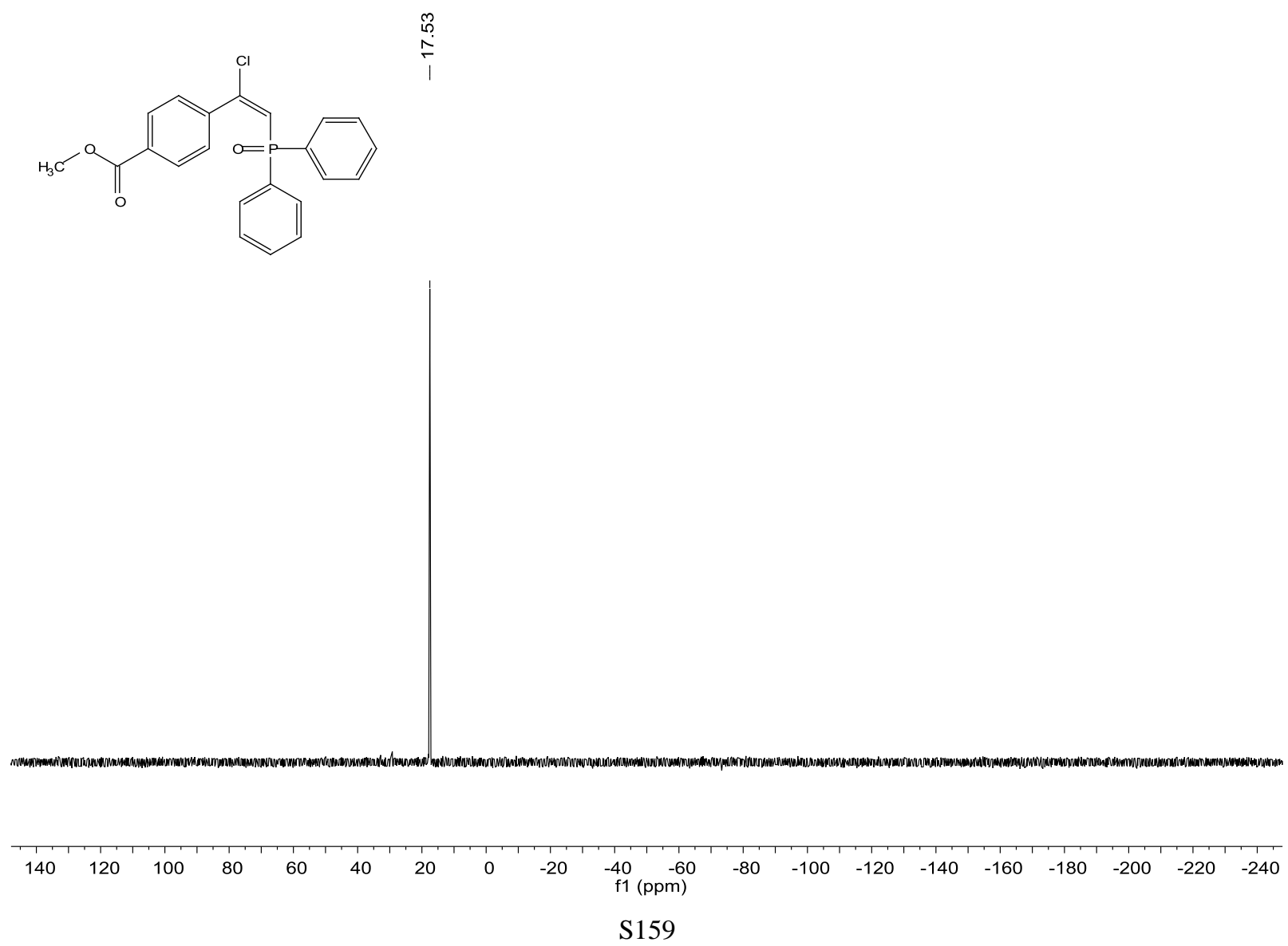
${ }^{13} \mathrm{C}$ NMR Spectrum of 9p $\left(\mathrm{CDCl}_{3}, 100 \mathrm{MHz}\right)$

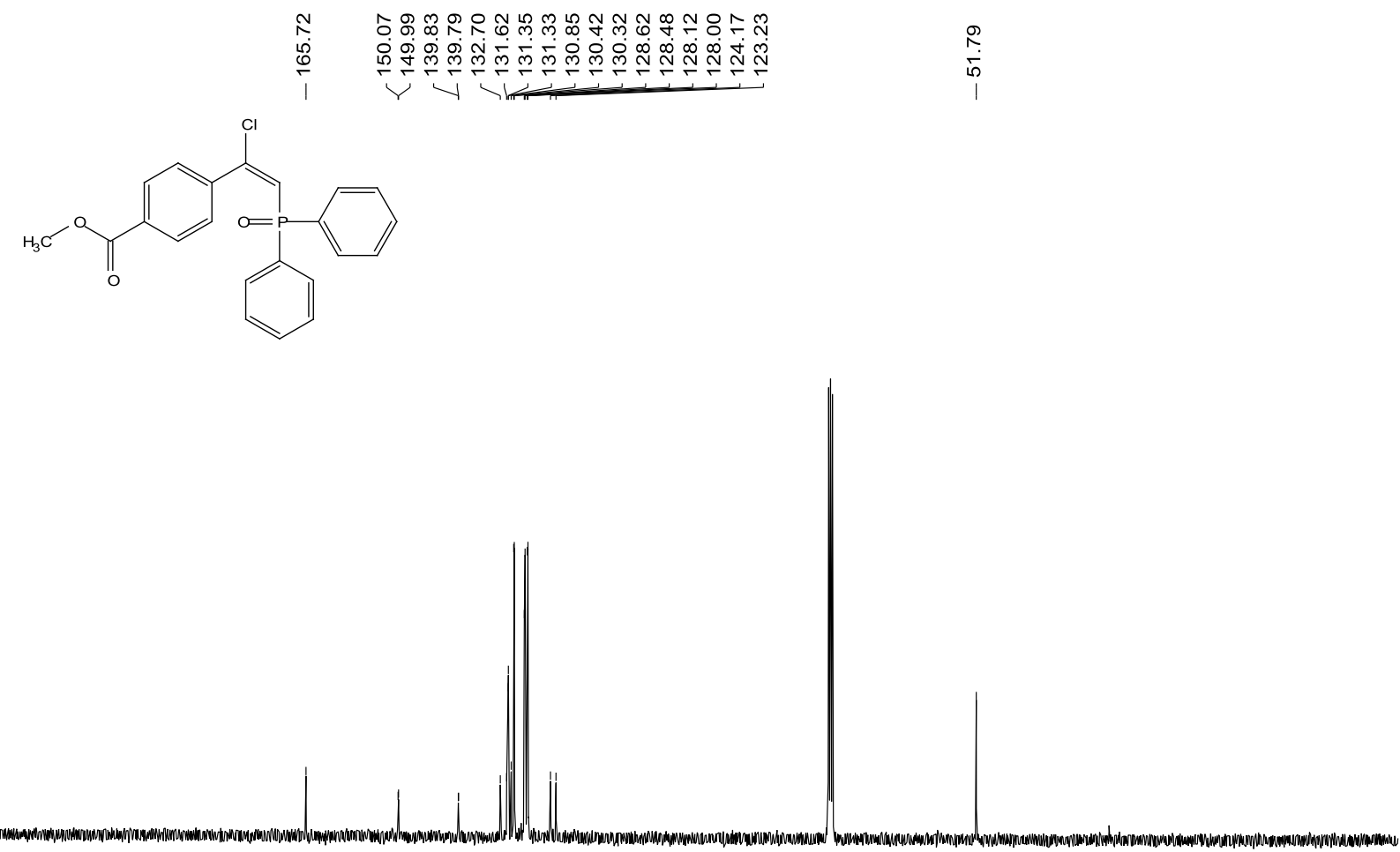

$\begin{array}{lllllllllllllllllllllll}210 & 200 & 190 & 180 & 170 & 160 & 150 & 140 & 130 & 120 & 110 & 100 & 90 & 80 & 70 & 60 & 50 & 40 & 30 & 20 & 10 & 0 & -10\end{array}$

${ }^{1} \mathrm{H}$ NMR Spectrum of $9 \mathrm{q}\left(\mathrm{CDCl}_{3}, 400 \mathrm{MHz}\right)$

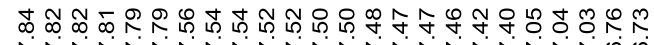

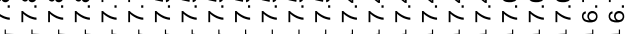
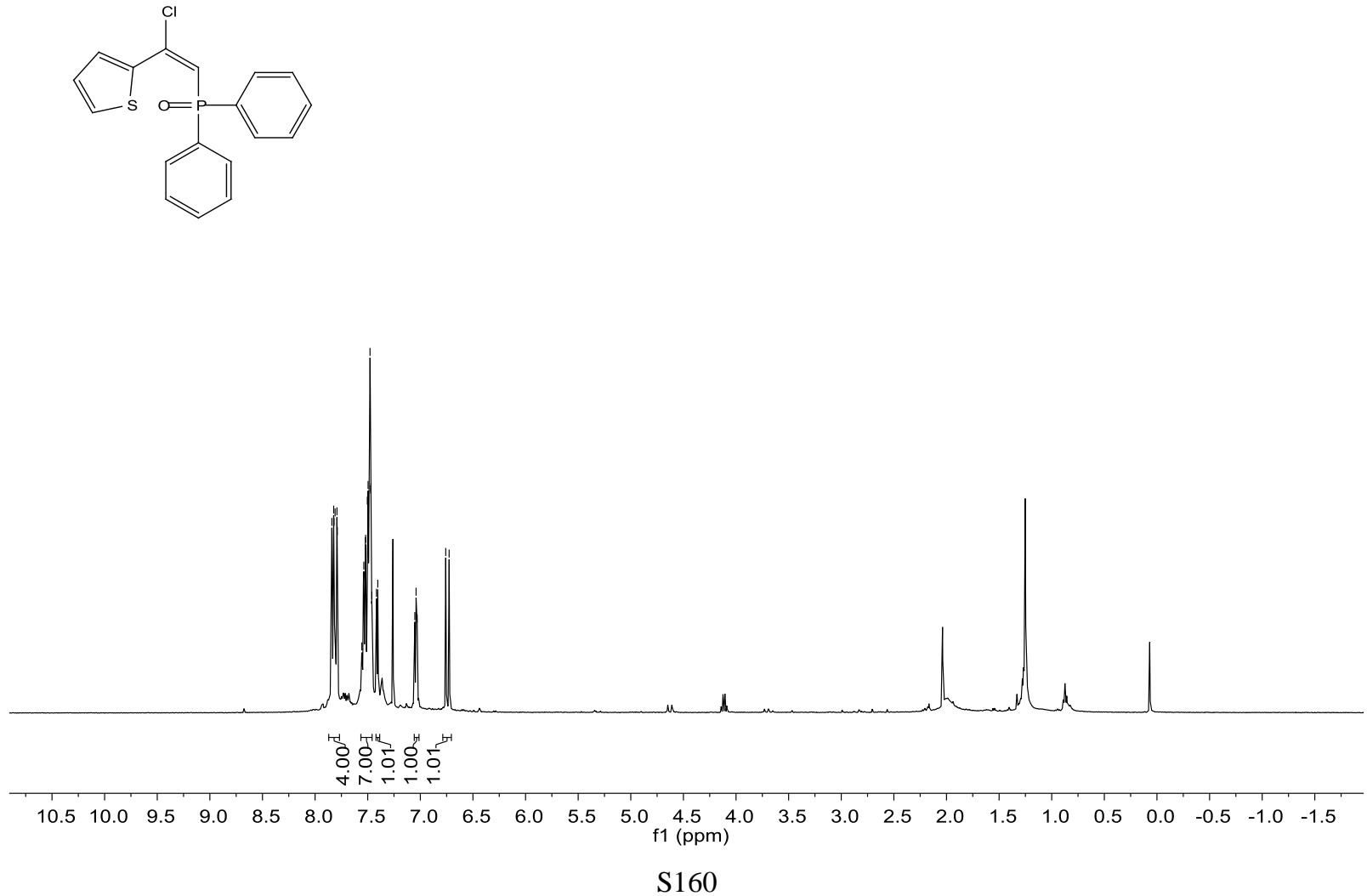
${ }^{31} \mathrm{P}$ NMR Spectrum of 9q $\left(\mathrm{CDCl}_{3}, 162 \mathrm{MHz}\right)$
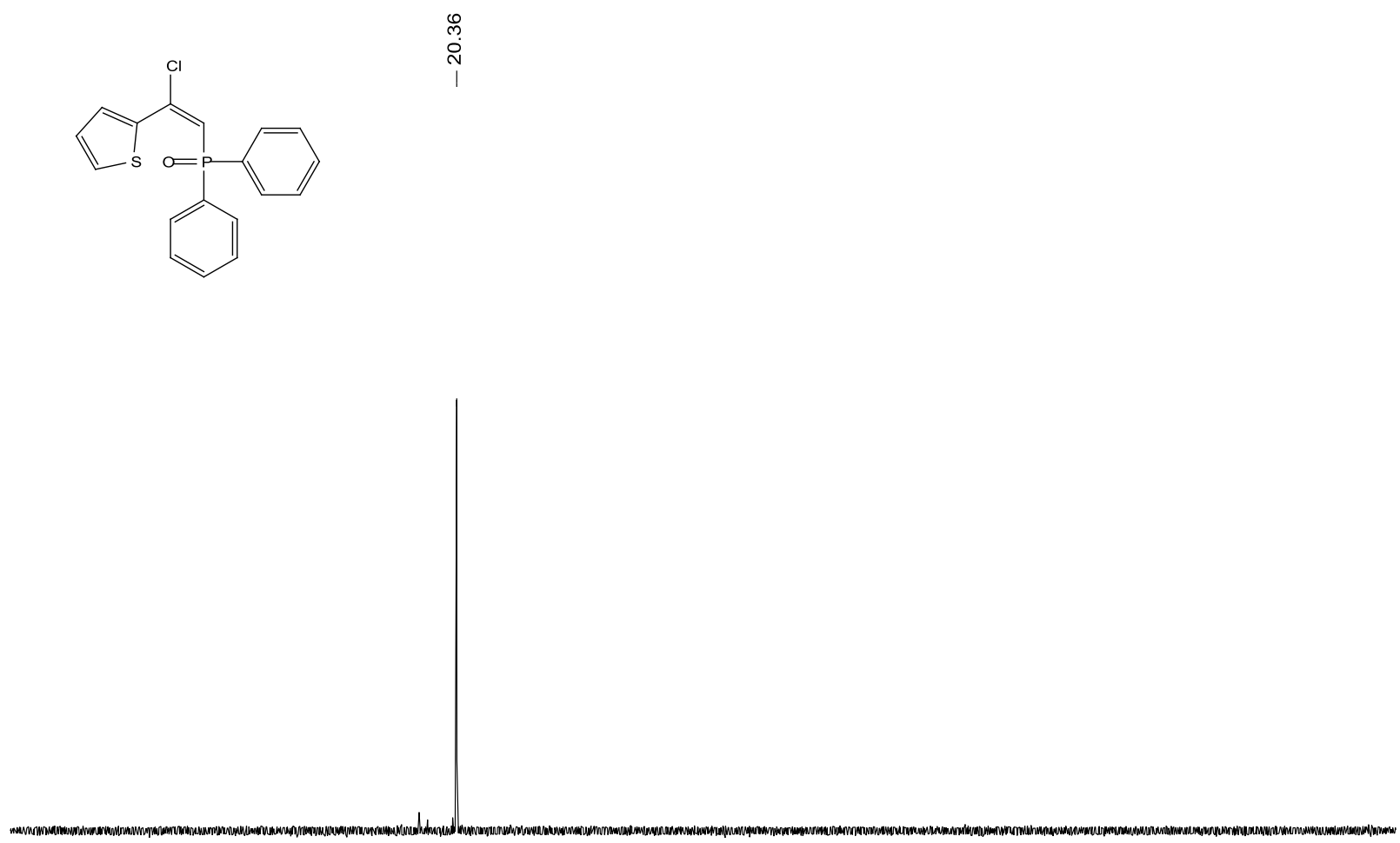

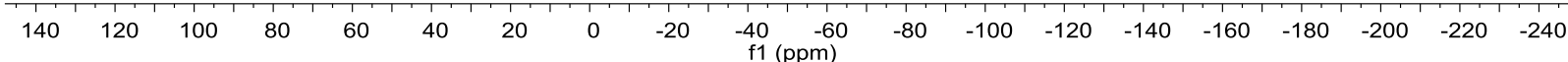

${ }^{13} \mathrm{C}$ NMR Spectrum of 9q $\left(\mathrm{CDCl}_{3}, 100 \mathrm{MHz}\right)$
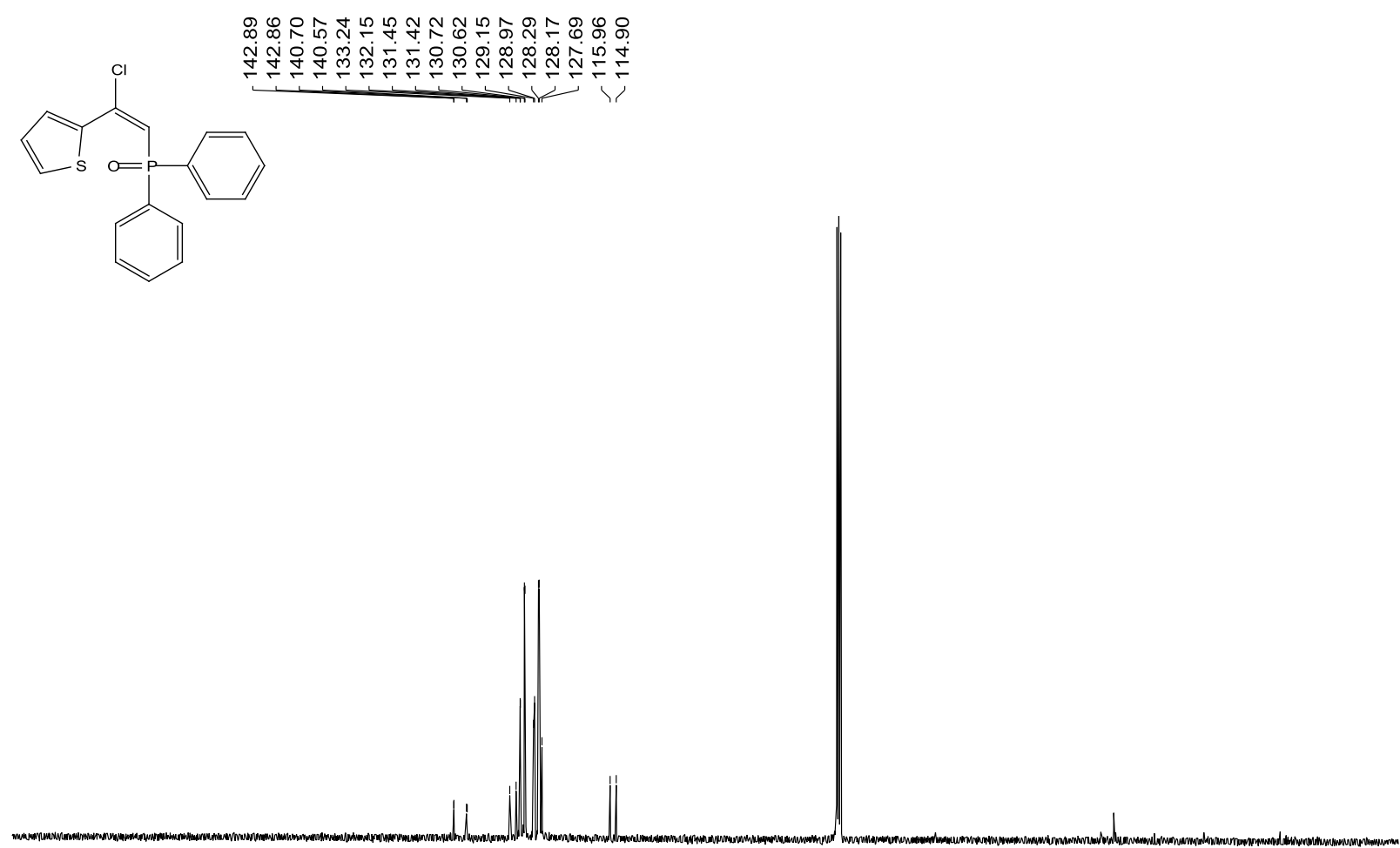

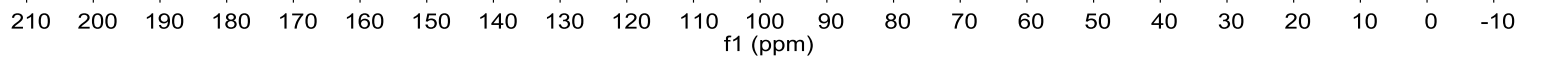


${ }^{1} \mathrm{H}$ NMR Spectrum of $9 \mathrm{r}\left(\mathrm{CDCl}_{3}, 400 \mathrm{MHz}\right)$<smiles>O=P(C(O)=C(Cl)c1ccccc1)(c1ccccc1)c1ccccc1</smiles>

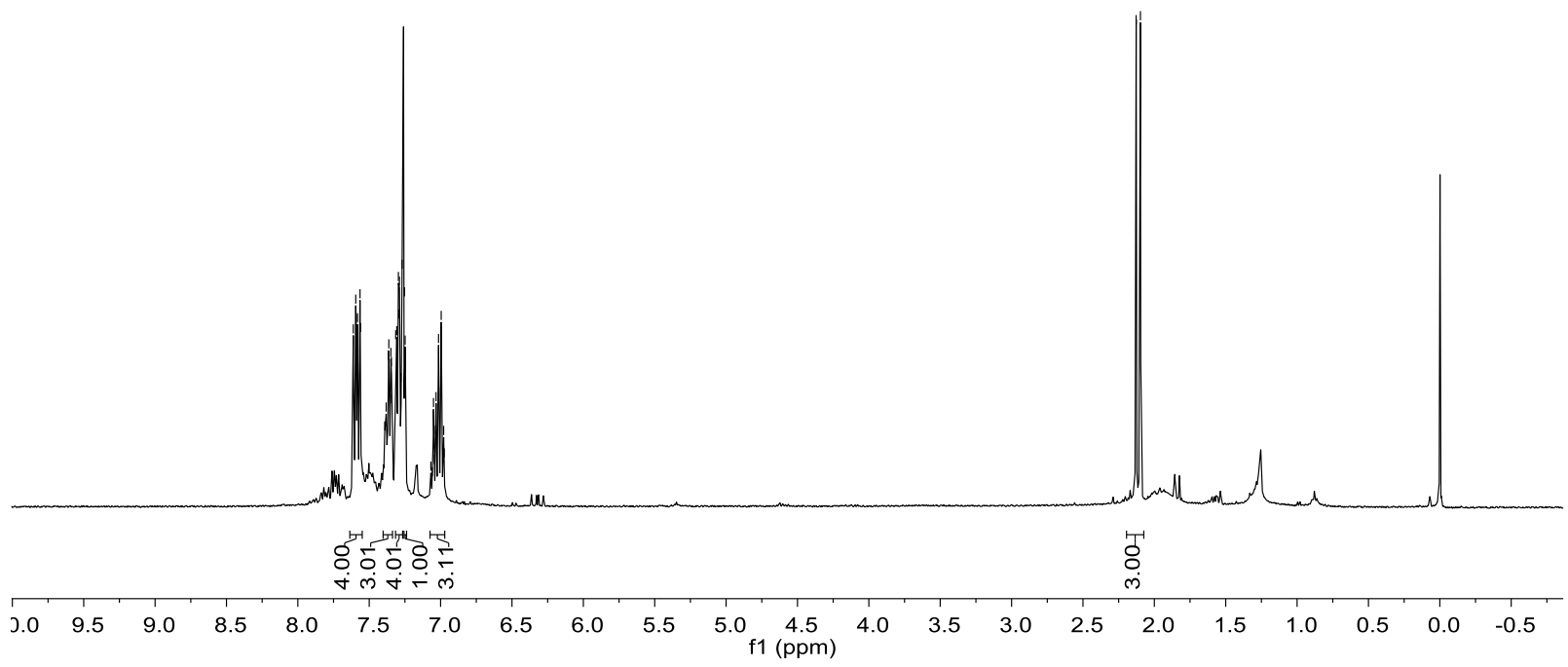

${ }^{31}$ P NMR Spectrum of 9r $\left(\mathrm{CDCl}_{3}, 162 \mathrm{MHz}\right)$

$\underset{\substack{N \\ \text { i }}}{1}$<smiles>C/C(=C(/Cl)c1ccccc1)P(=O)(c1ccccc1)c1ccccc1</smiles>

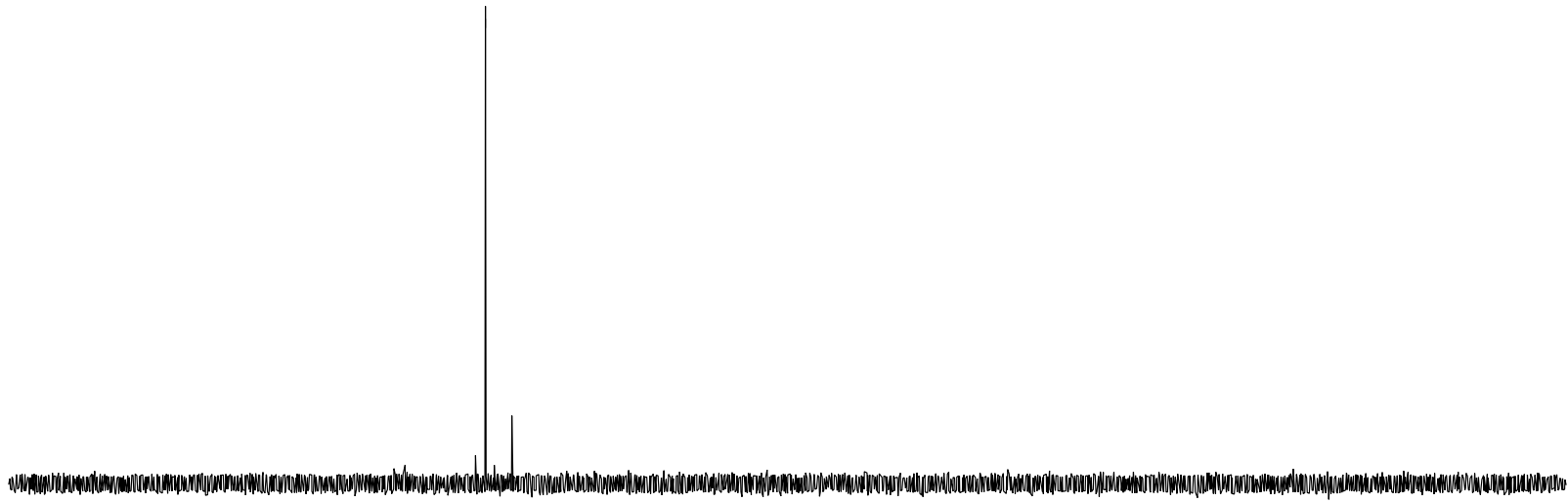

$\begin{array}{llllllllllllllllllll}140 & 120 & 100 & 80 & 60 & 40 & 20 & 0 & -20 & -40 & -60 & -80 & -100 & -120 & -140 & -160 & -180 & -200 & -220 & -240\end{array}$ 
${ }^{13} \mathrm{C}$ NMR Spectrum of $9 \mathrm{r}\left(\mathrm{CDCl}_{3}, 100 \mathrm{MHz}\right)$

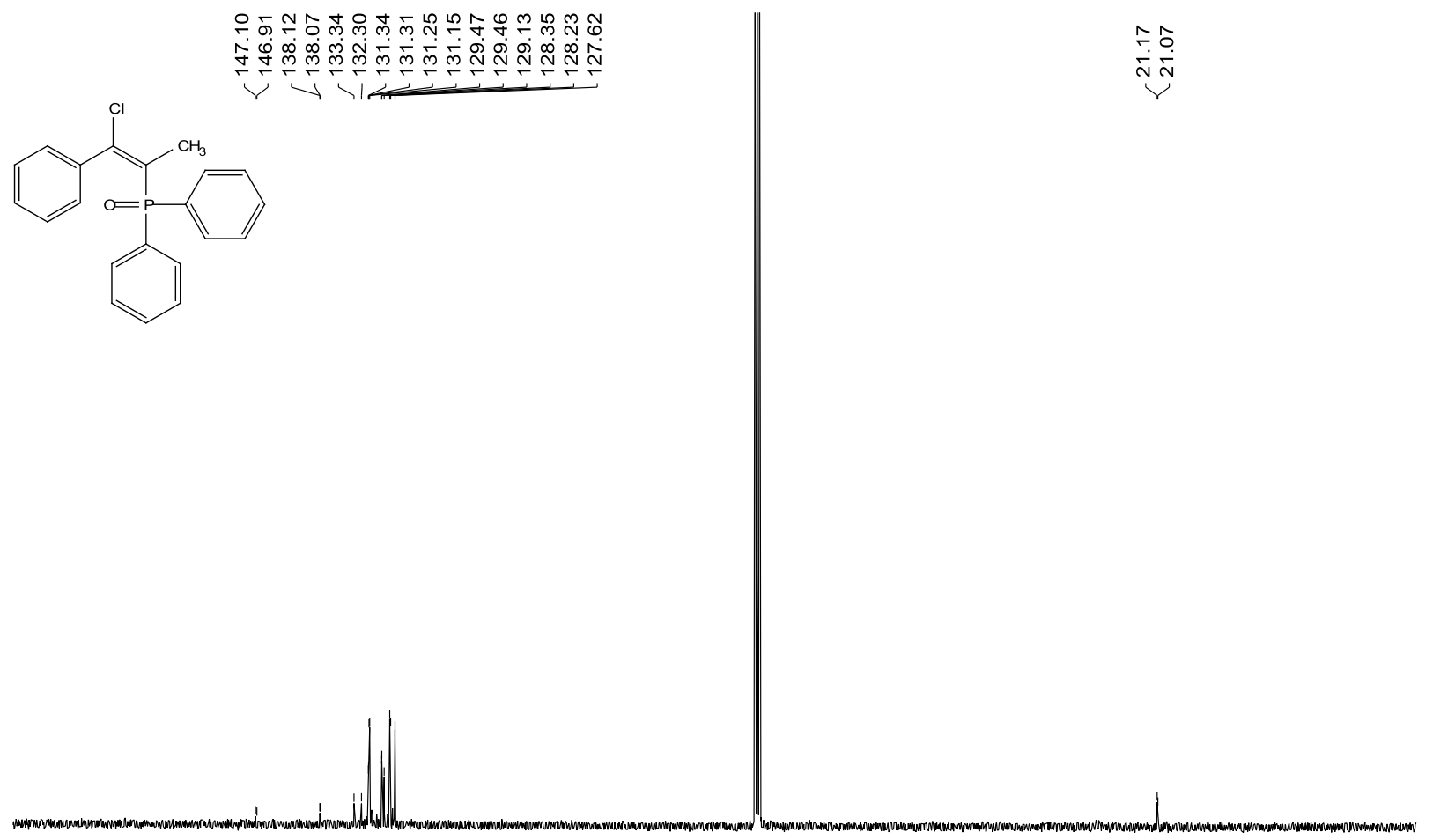

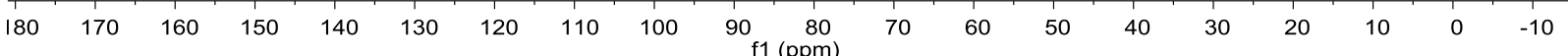

${ }^{1} \mathrm{H}$ NMR Spectrum of $9 \mathrm{~s}\left(\mathrm{CDCl}_{3}, 400 \mathrm{MHz}\right)$

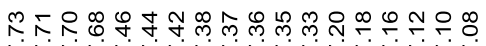

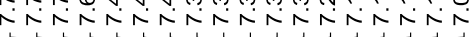<smiles>O=C(O)C(=O)c1ccccc1C(=O)C(Cl)c1ccccc1</smiles>

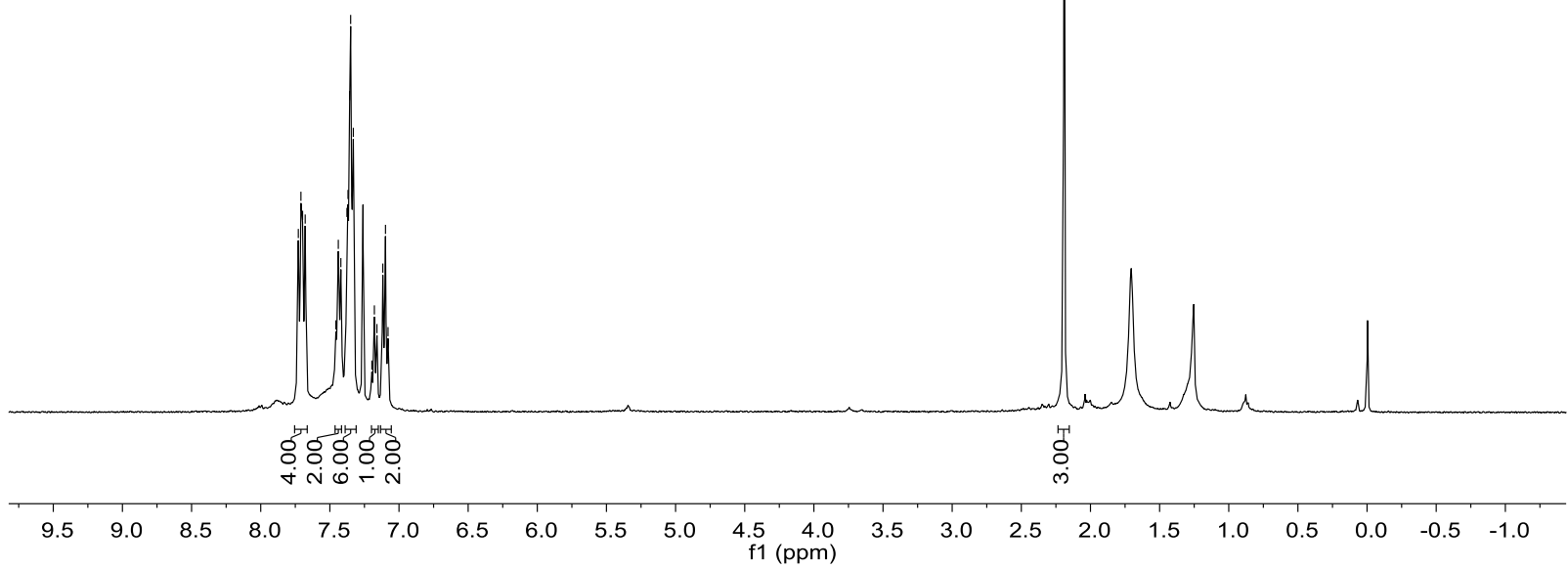


${ }^{31} \mathrm{P}$ NMR Spectrum of $9 \mathrm{~S}\left(\mathrm{CDCl}_{3}, 162 \mathrm{MHz}\right)$<smiles>O=C(O)C(=C(Cl)c1ccccc1)P(c1ccccc1)c1ccccc1</smiles>

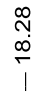

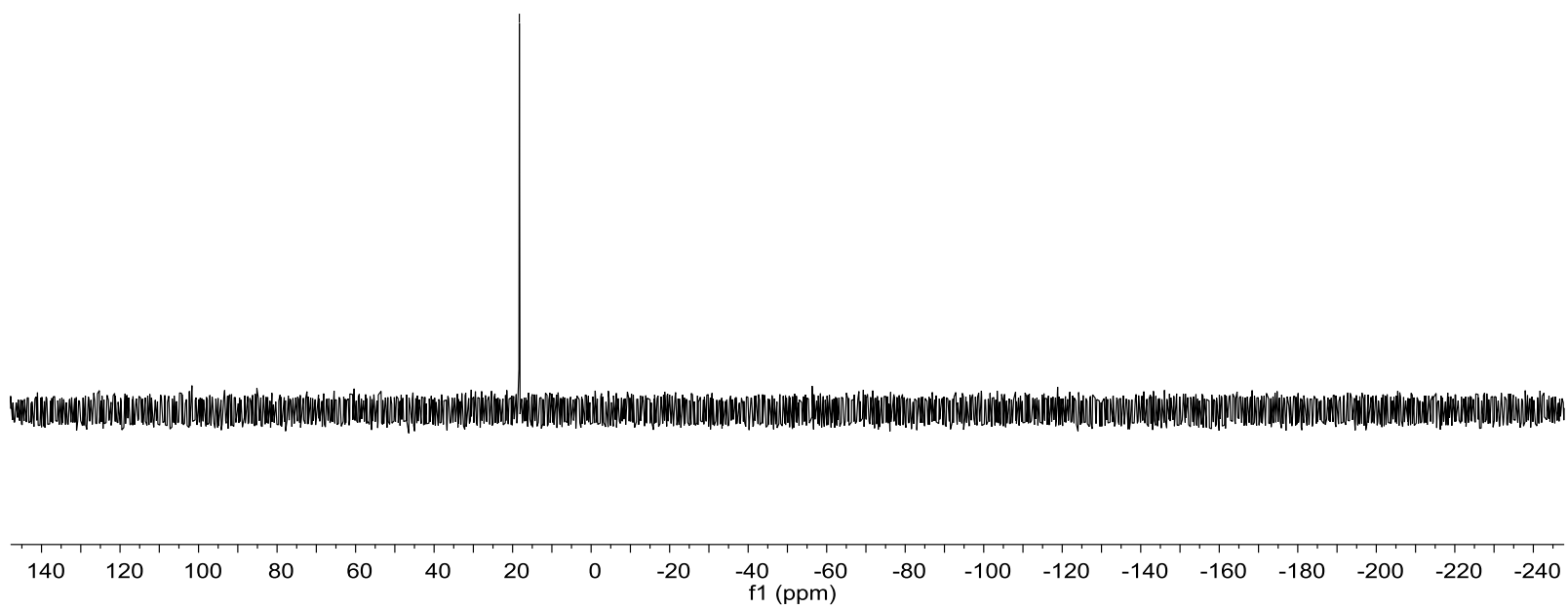

${ }^{13} \mathrm{C}$ NMR Spectrum of 9s $\left(\mathrm{CDCl}_{3}, 100 \mathrm{MHz}\right)$
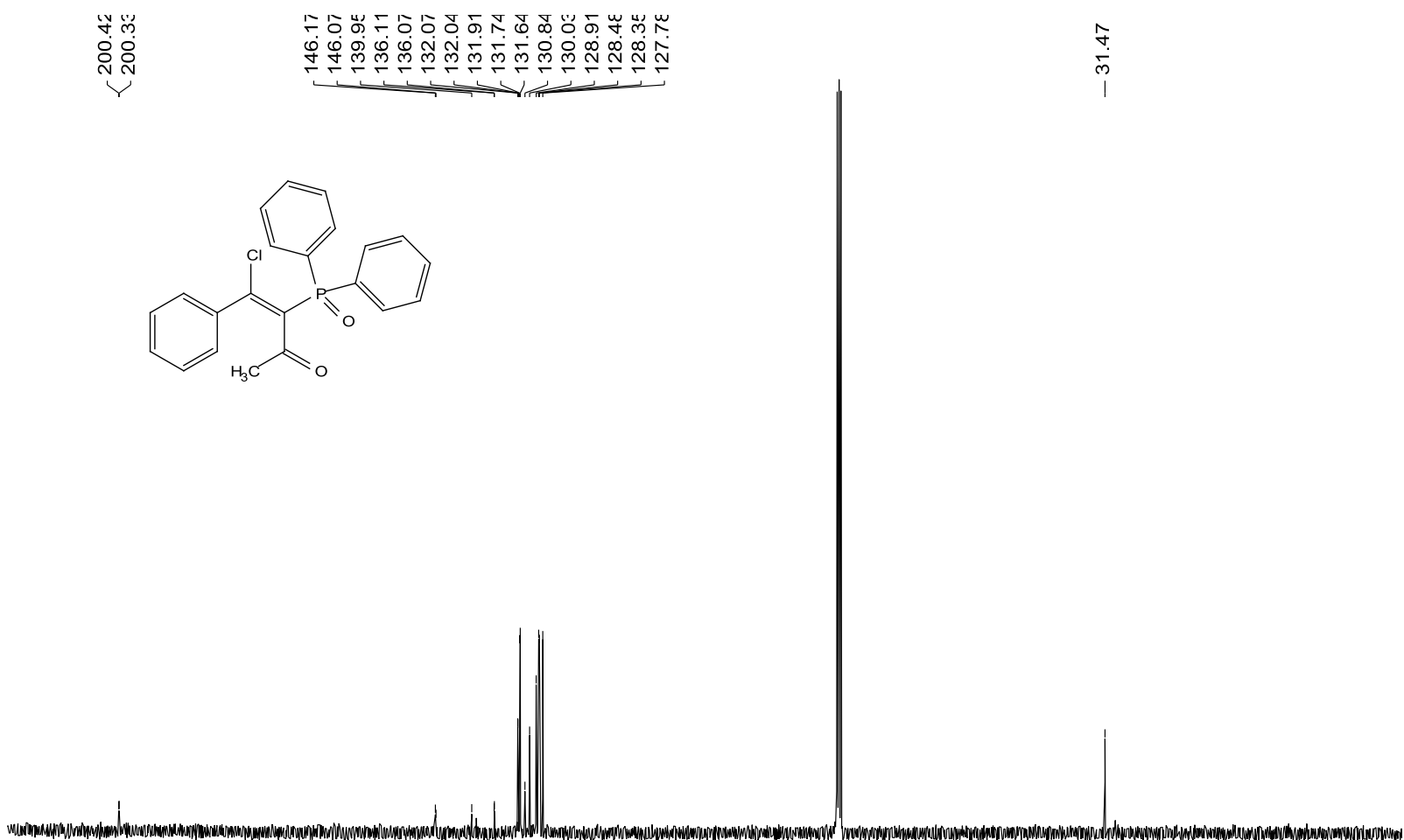

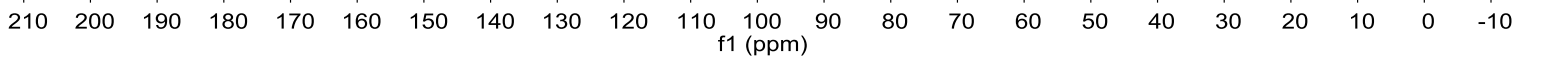


${ }^{1} \mathrm{H}$ NMR Spectrum of $10\left(\mathrm{CDCl}_{3}, 400 \mathrm{MHz}\right)$

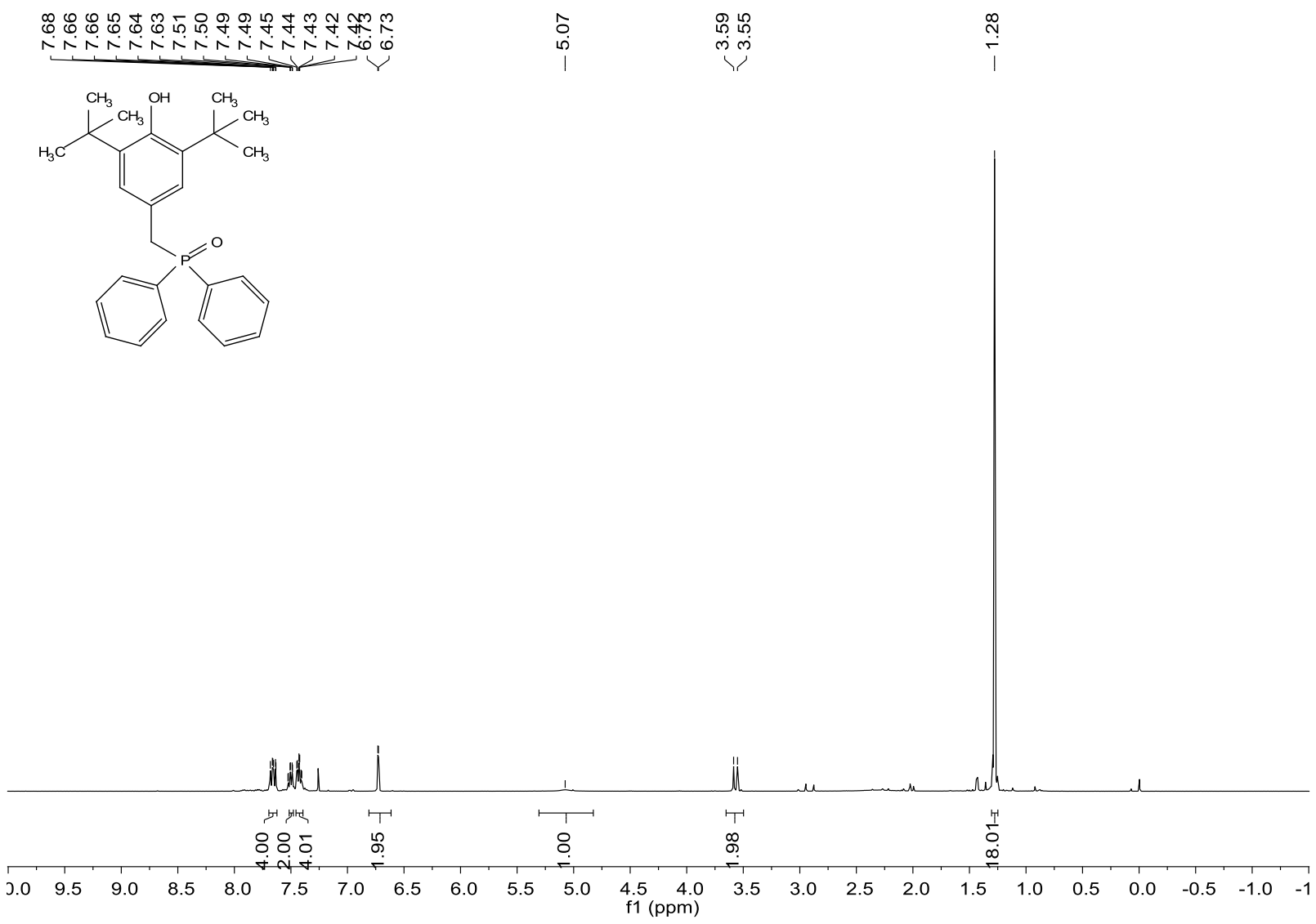

${ }^{31} \mathrm{P}$ NMR Spectrum of $10\left(\mathrm{CDCl}_{3}, 162 \mathrm{MHz}\right)$

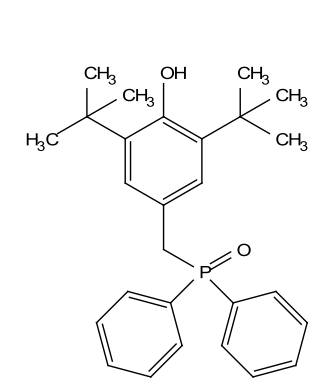

ঙ্ণ

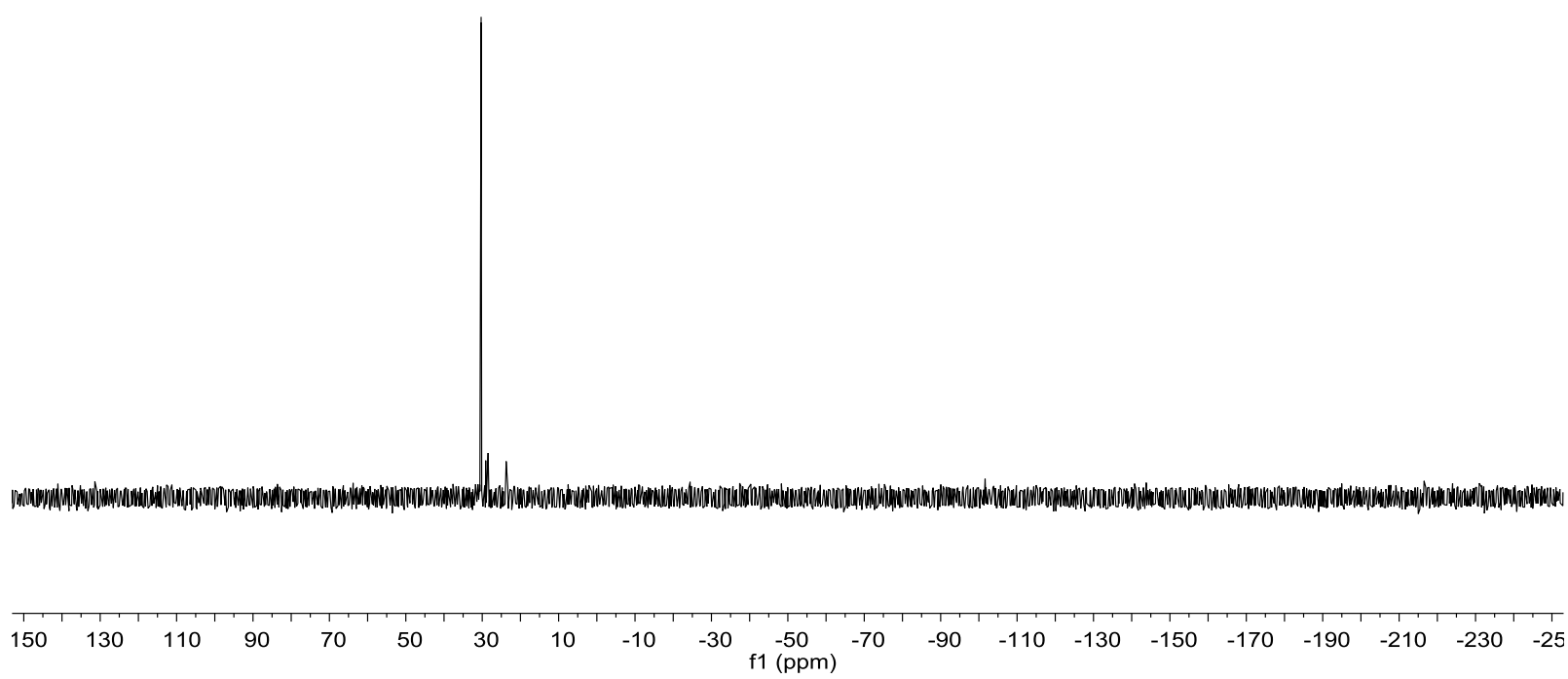




\section{${ }^{13} \mathrm{C}$ NMR Spectrum of $10\left(\mathrm{CDCl}_{3}, 100 \mathrm{MHz}\right)$}

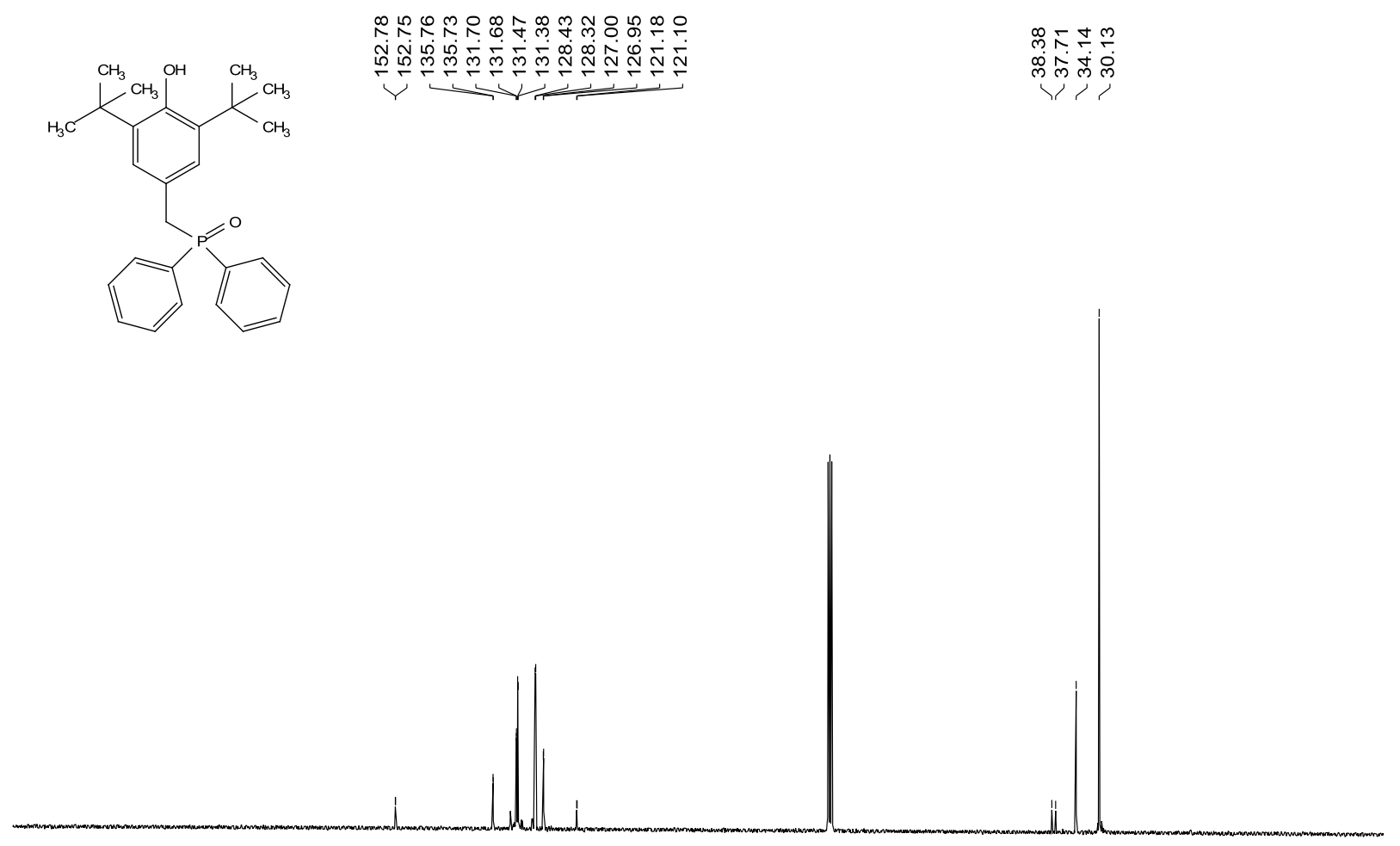

$\begin{array}{lllllllllllllllllllllllll}210 & 200 & 190 & 180 & 170 & 160 & 150 & 140 & 130 & 120 & 110 & 100 & 90 & 80 & 70 & 60 & 50 & 40 & 30 & 20 & 10 & 0 & -10\end{array}$

${ }^{1} \mathrm{H}$ NMR Spectrum of $11\left(\mathrm{CDCl}_{3}, 400 \mathrm{MHz}\right)$

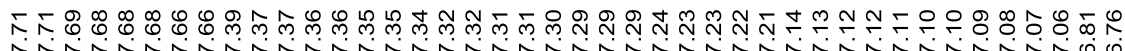

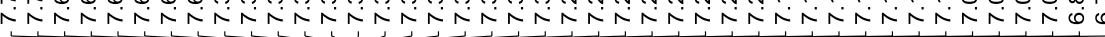

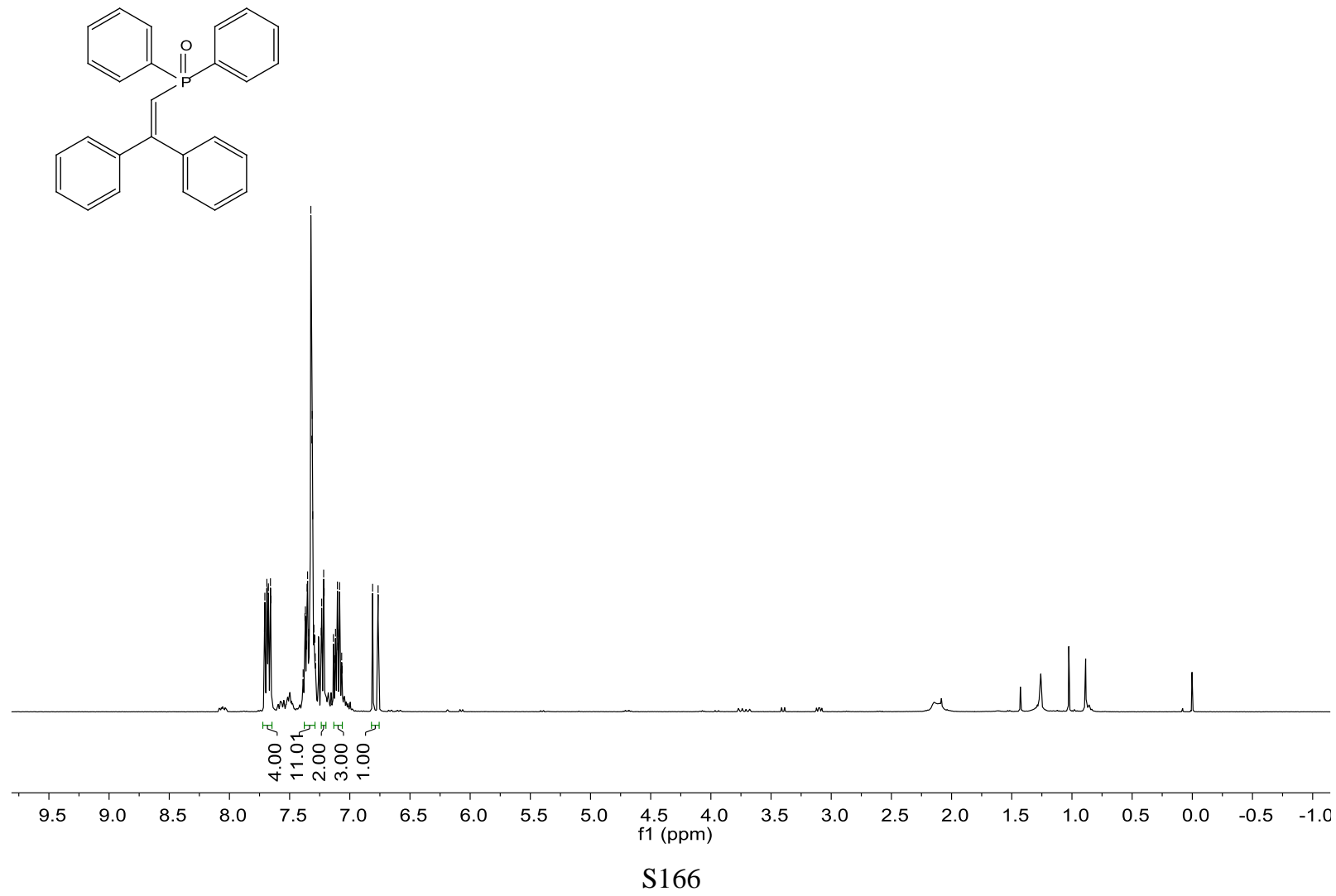


${ }^{31} \mathrm{P}$ NMR Spectrum of $11\left(\mathrm{CDCl}_{3}, 162 \mathrm{MHz}\right)$

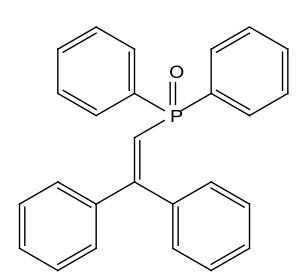

$\underset{\substack{\hat{N} \\ \infty}}{\infty}$

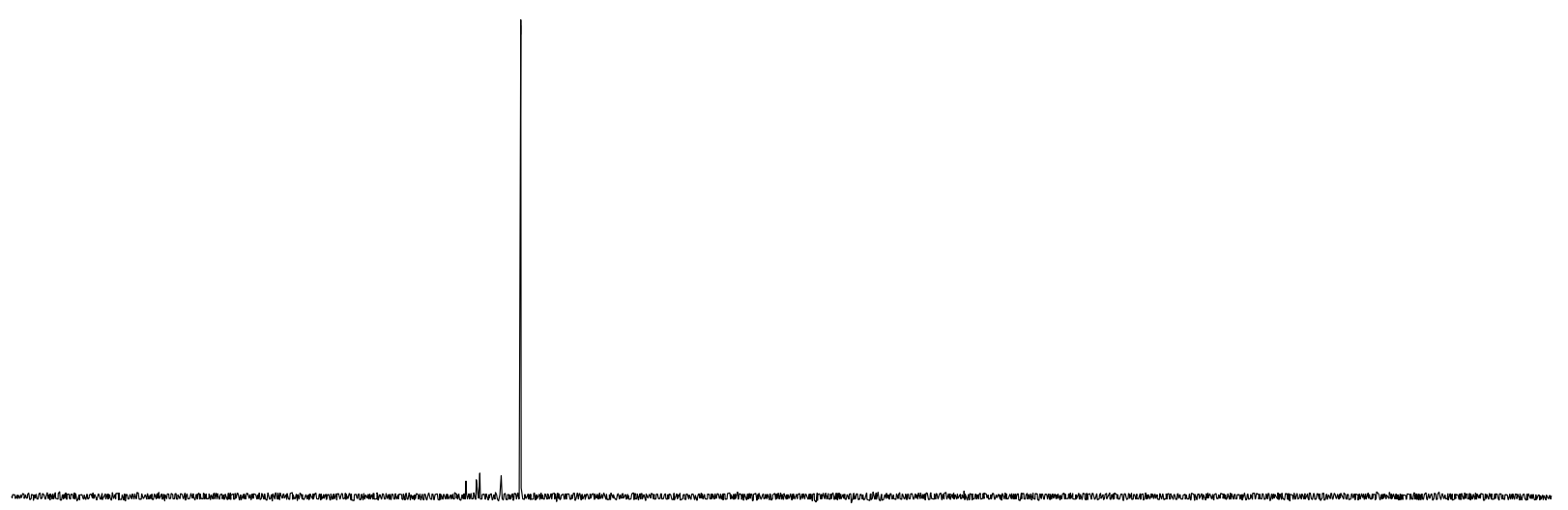

$\begin{array}{lllllllllllllllllllllllllll}150 & 130 & 110 & 90 & 70 & 50 & 30 & 10 & -10 & -30 & -50 & -70 & -90 & -110 & -130 & -150 & -170 & -190 & -210 & -230 & -25\end{array}$

${ }^{13} \mathrm{C}$ NMR Spectrum of $11\left(\mathrm{CDCl}_{3}, 100 \mathrm{MHz}\right)$

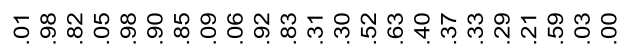

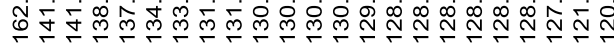
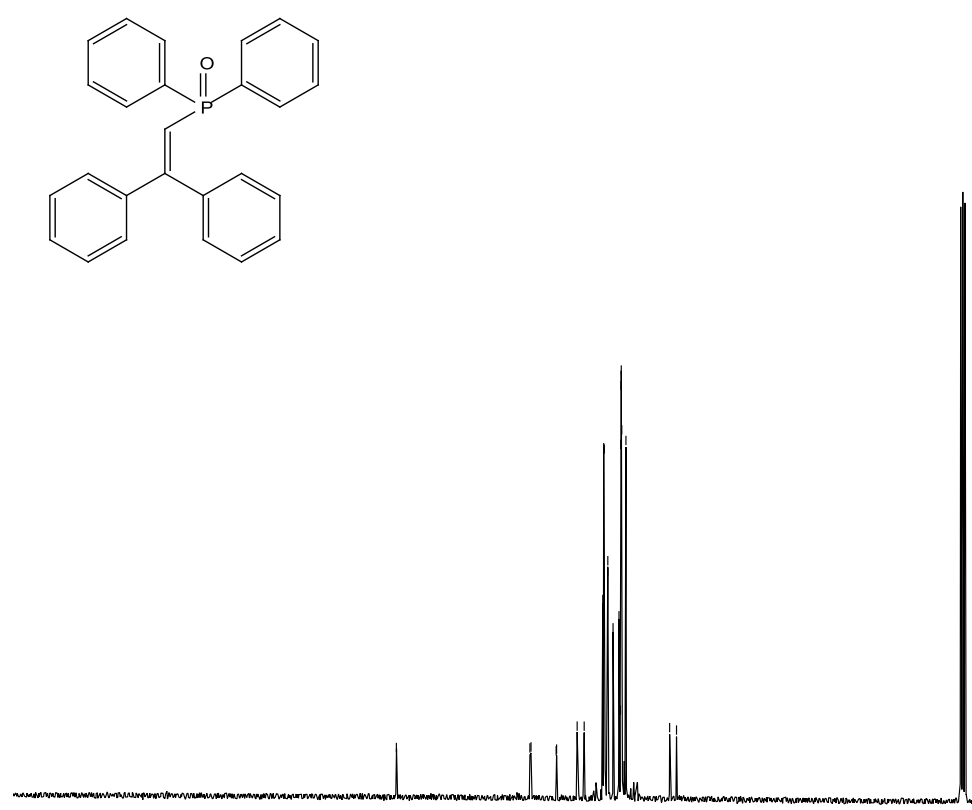

$\begin{array}{llllllllllllllllllllllll}210 & 200 & 190 & 180 & 170 & 160 & 150 & 140 & 130 & 120 & 110 & 100 & 90 & 80 & 70 & 60 & 50 & 40 & 30 & 20 & 10 & 0 & -10\end{array}$ 
<smiles>O=P(C=C(Br)c1ccccc1)(c1ccccc1)c1ccccc1</smiles>

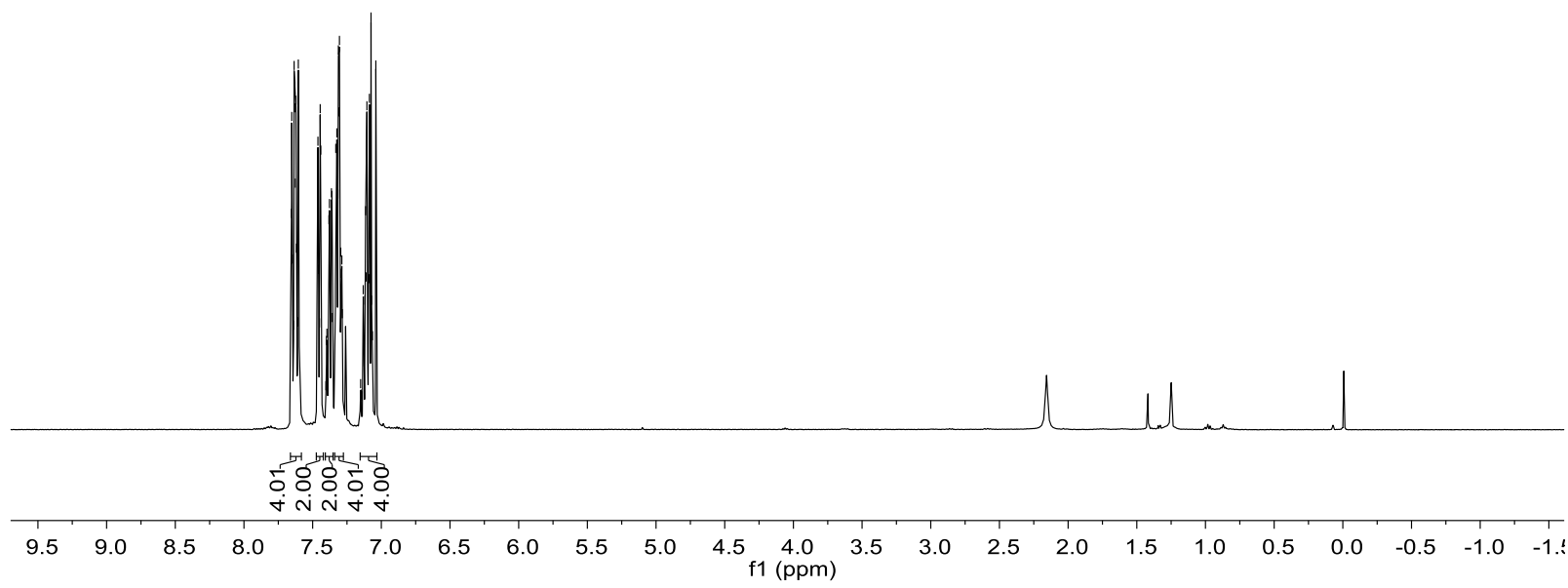

${ }^{31}$ P NMR Spectrum of $12\left(\mathrm{CDCl}_{3}, 162 \mathrm{MHz}\right)$

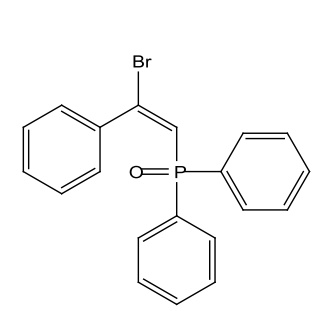

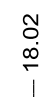

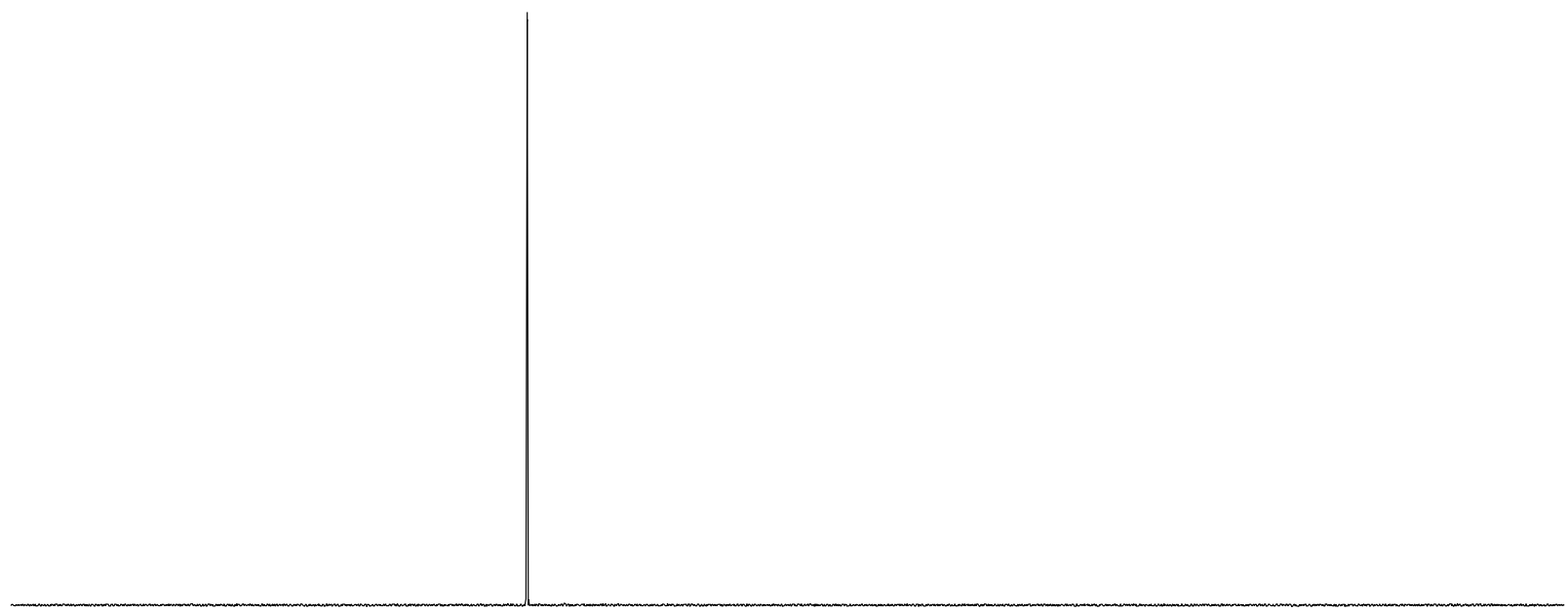

$\begin{array}{llllllllllllllllllllll}150 & 130 & 110 & 90 & 70 & 50 & 30 & 10 & -10 & -30 & -50 & -70 & -90 & -110 & -130 & -150 & -170 & -190 & -210 & -230 & -25\end{array}$ 
${ }^{13} \mathrm{C}$ NMR Spectrum of $12\left(\mathrm{CDCl}_{3}, 100 \mathrm{MHz}\right)$
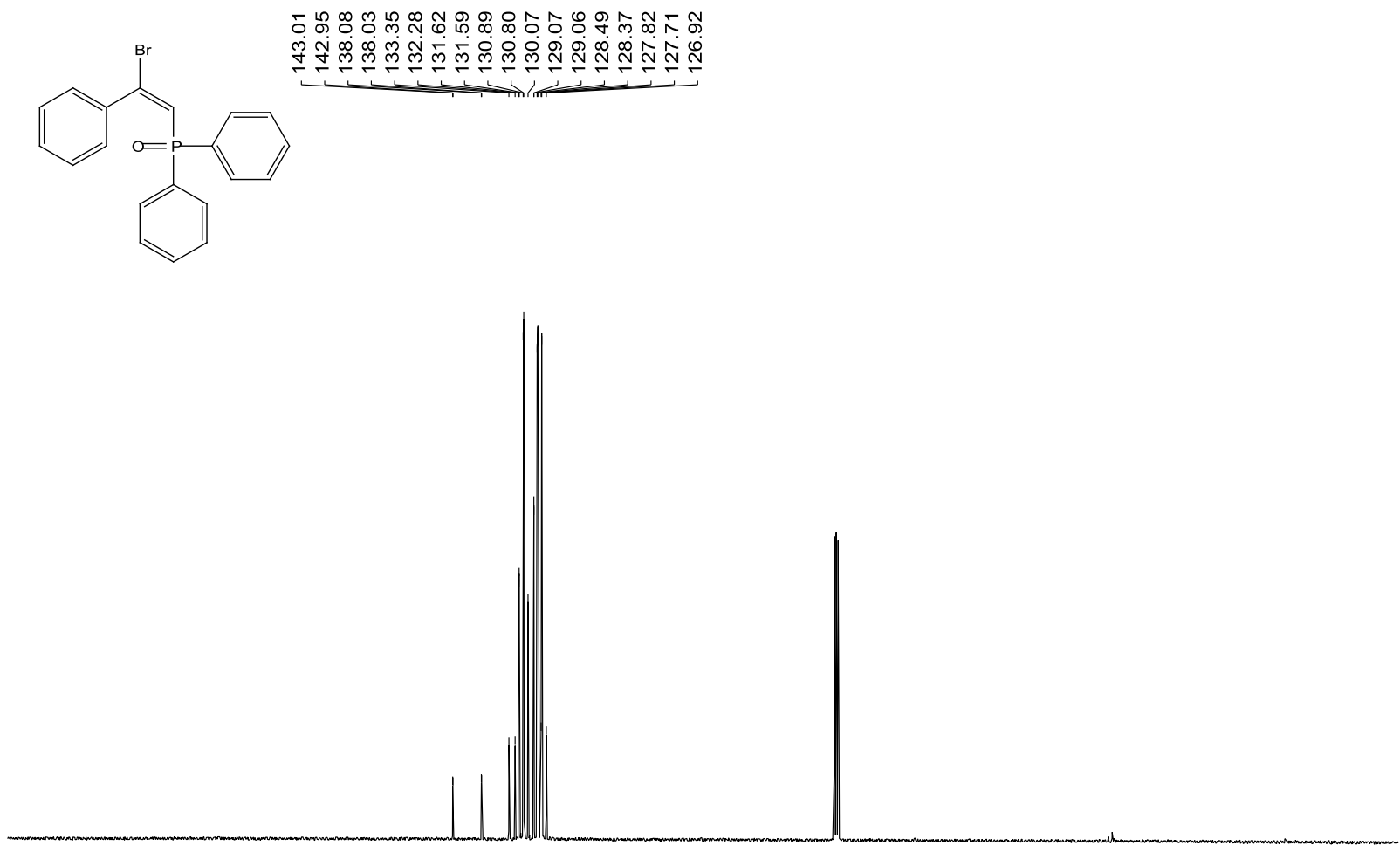

$\begin{array}{lllllllllllllllllllllllll}210 & 200 & 190 & 180 & 170 & 160 & 150 & 140 & 130 & 120 & 110 & 100 & 90 & 80 & 70 & 60 & 50 & 40 & 30 & 20 & 10 & 0 & -10\end{array}$

${ }^{1} \mathrm{H}$ NMR Spectrum of 13 (DMSO-d $\left.6,400 \mathrm{MHz}\right)$

⿹

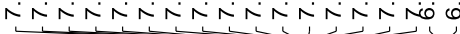

กे

(c)

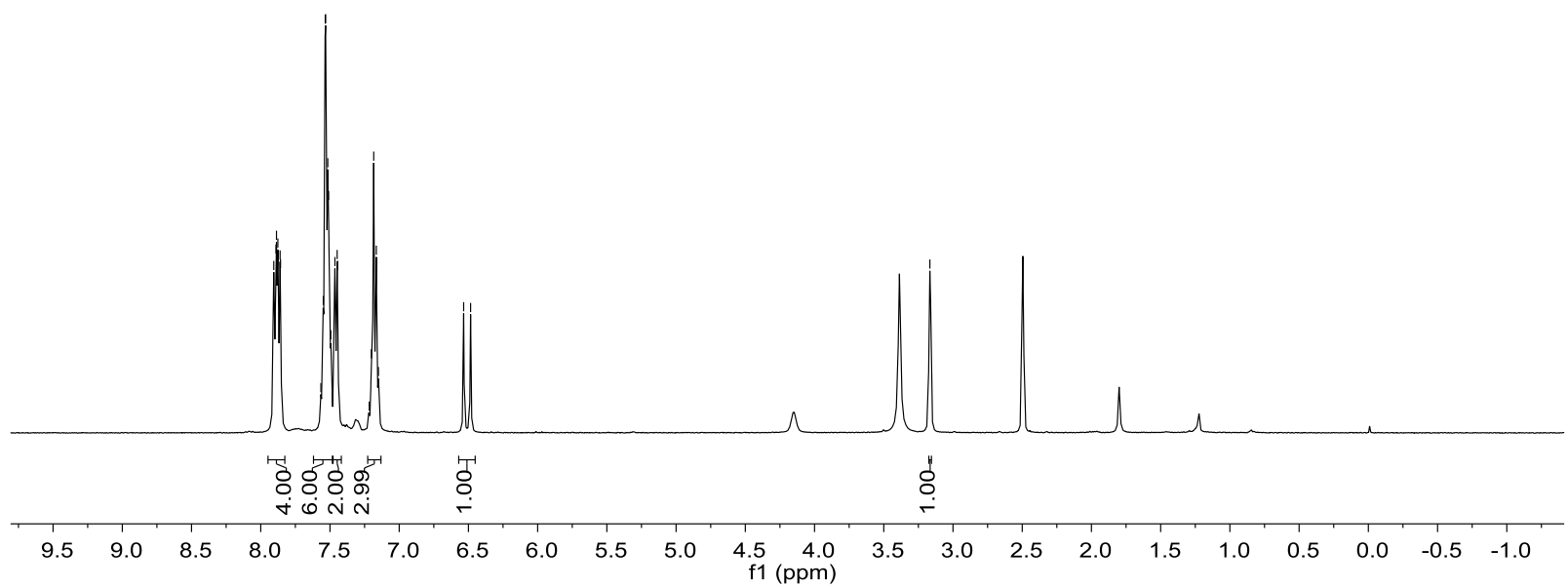

S169 
${ }^{31}$ P NMR Spectrum of 13 (DMSO-d $\left.d_{6}, 162 \mathrm{MHz}\right)$

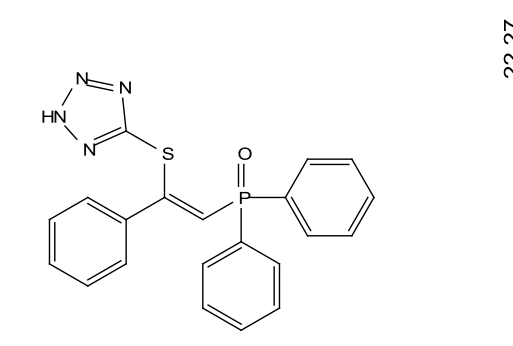

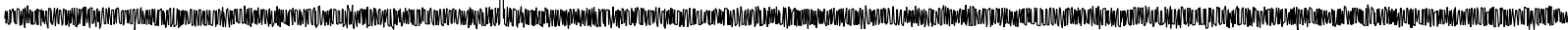

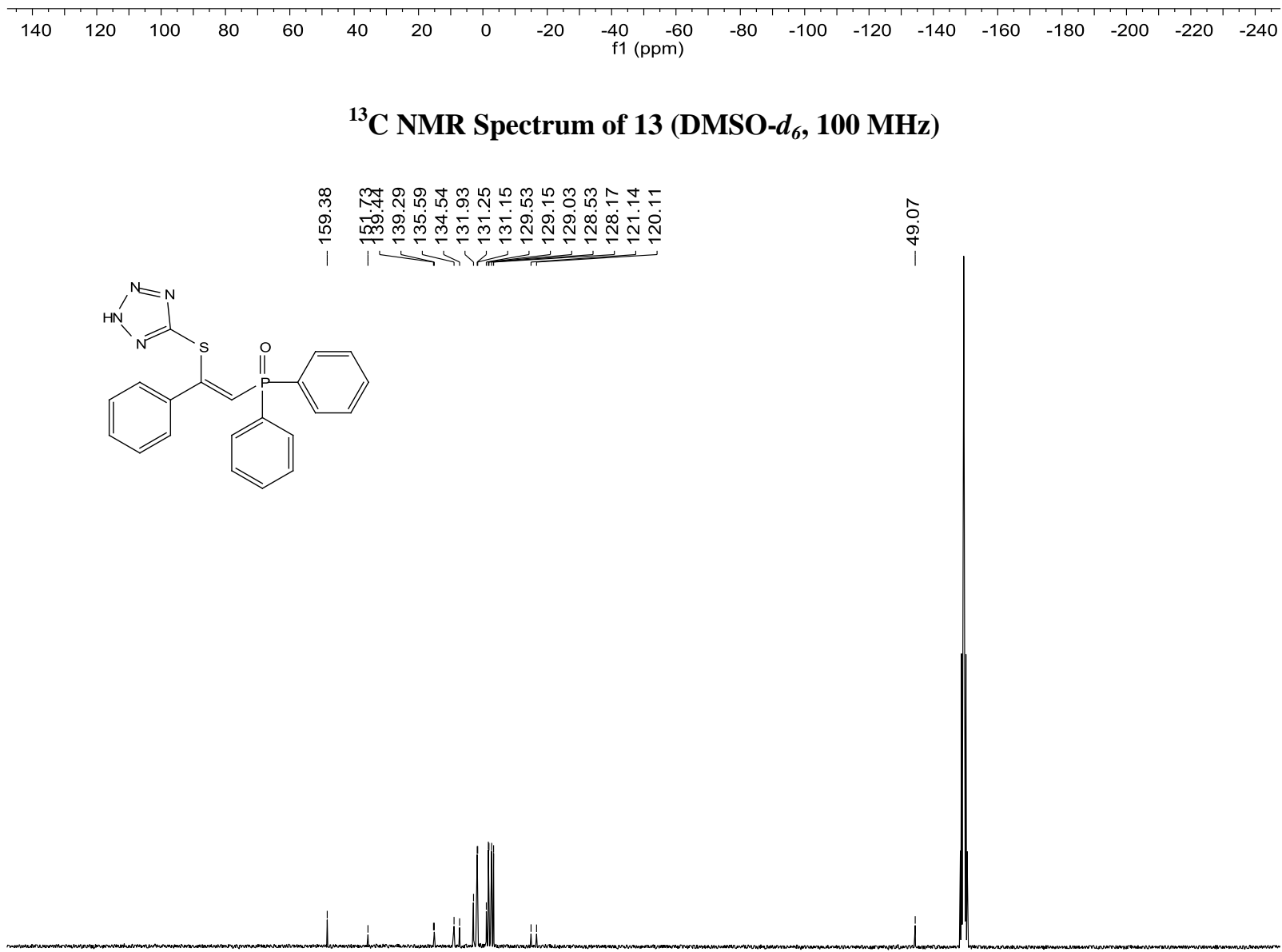

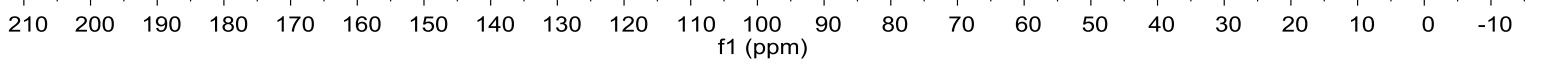




\section{${ }^{1} \mathrm{H}$ NMR Spectrum of $14\left(\mathrm{CDCl}_{3}, 400 \mathrm{MHz}\right)$}

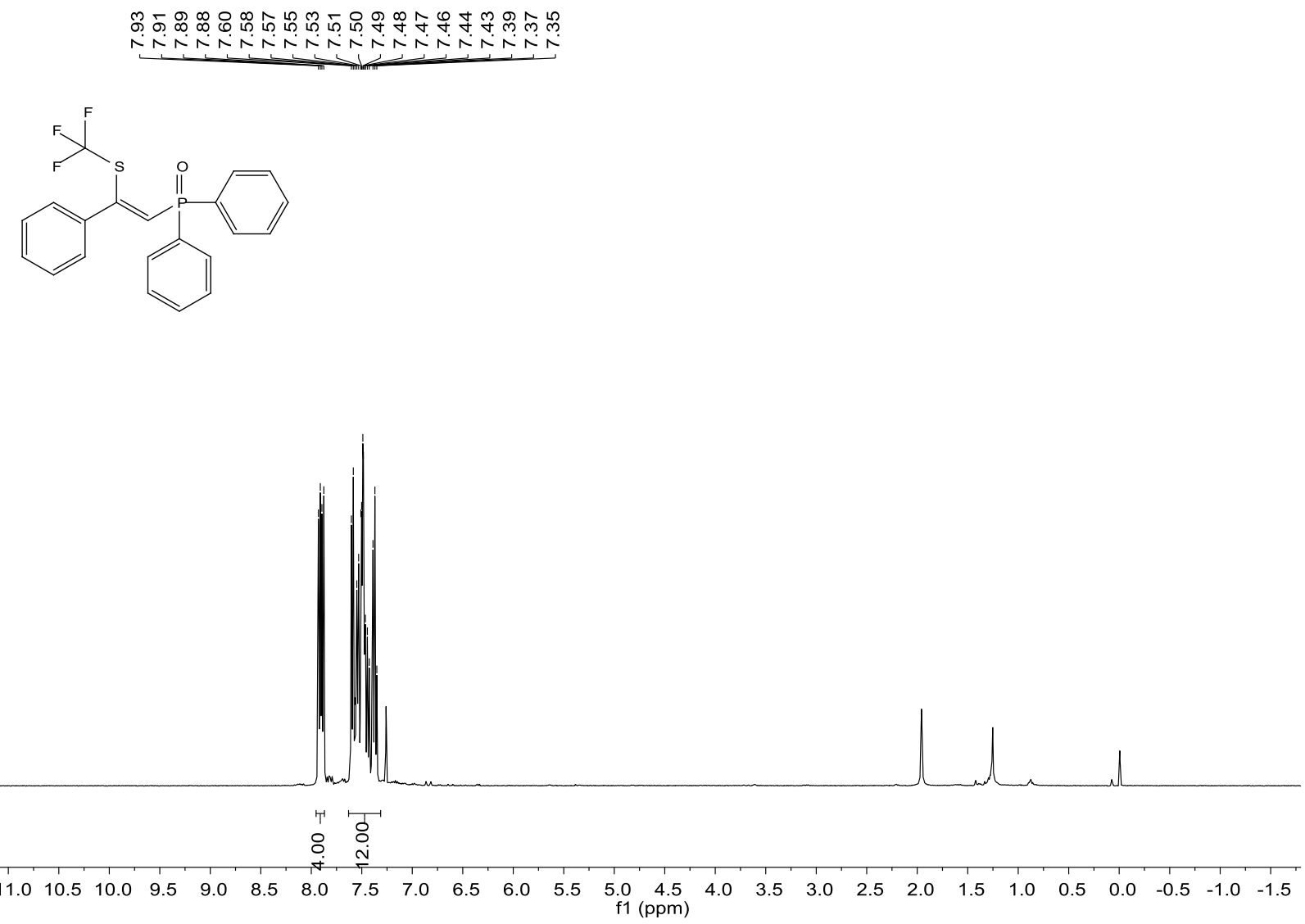

${ }^{31} \mathrm{P}$ NMR Spectrum of $14\left(\mathrm{CDCl}_{3}, 162 \mathrm{MHz}\right)$

$\stackrel{\substack{\infty \\ \infty}}{\infty}$<smiles>O=P(C=C(SC(F)(F)F)c1ccccc1)(c1ccccc1)c1ccccc1</smiles>

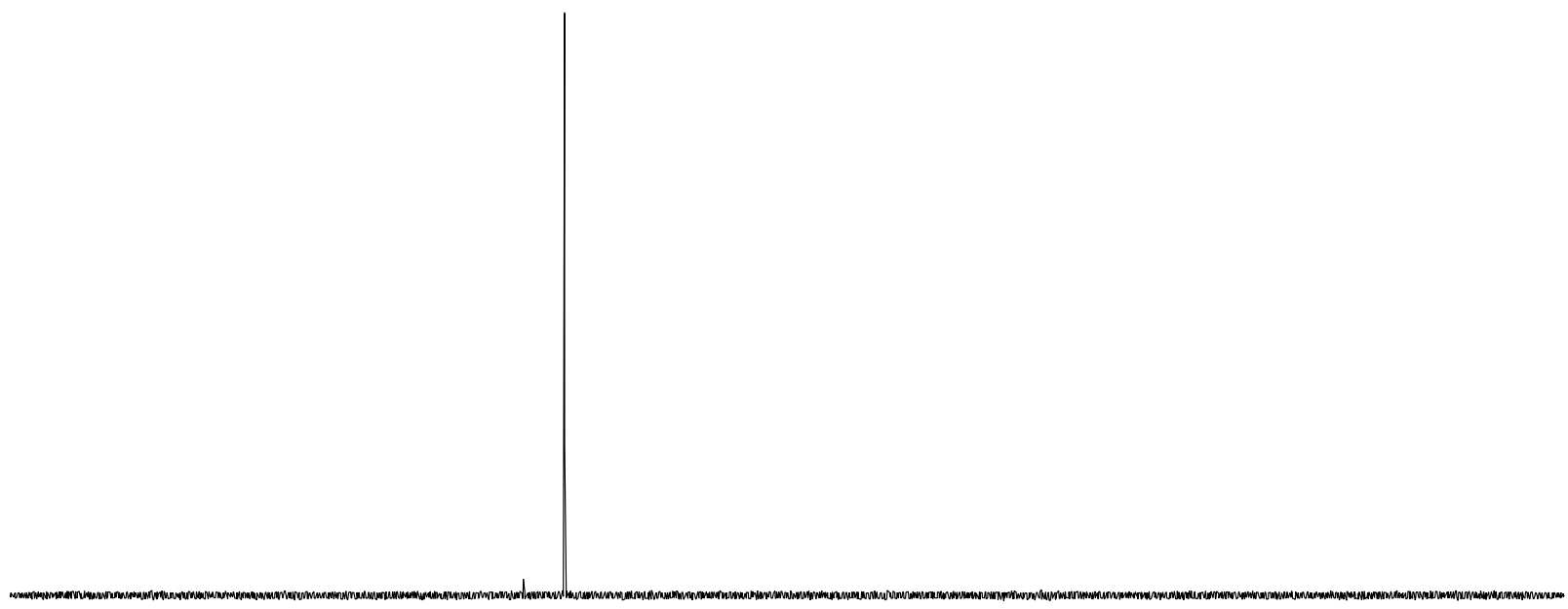

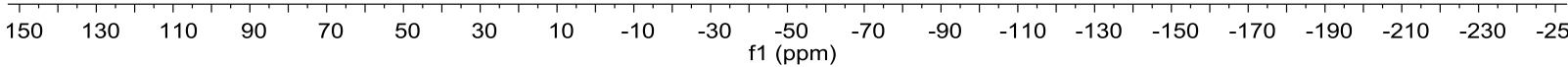


${ }^{19}$ F NMR Spectrum of $14\left(\mathrm{CDCl}_{3}, 376 \mathrm{MHz}\right)$

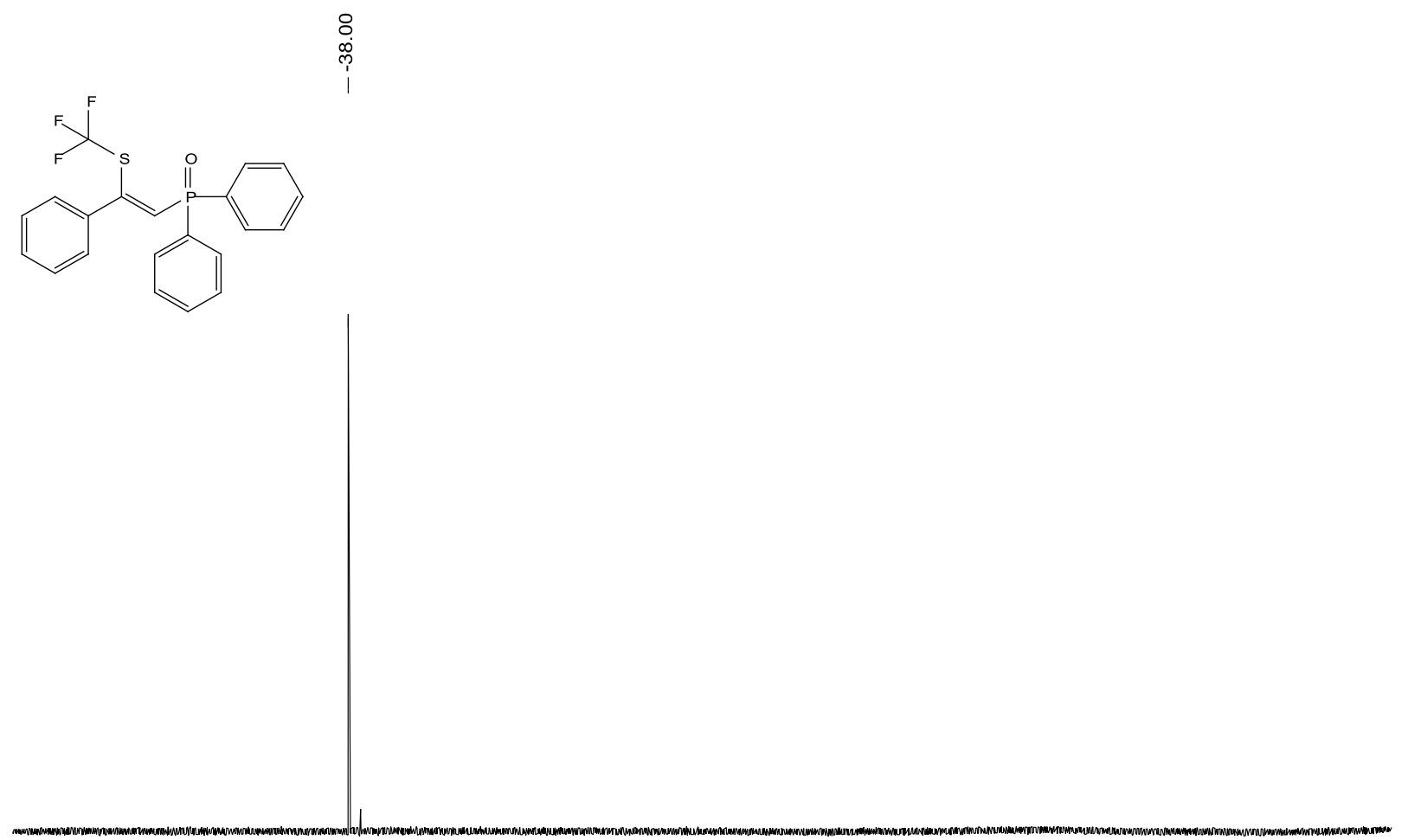

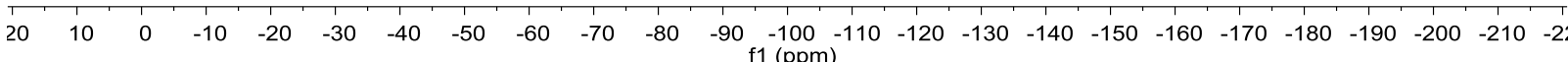

${ }^{13} \mathrm{C}$ NMR Spectrum of $14\left(\mathrm{CDCl}_{3}, 100 \mathrm{MHz}\right)$
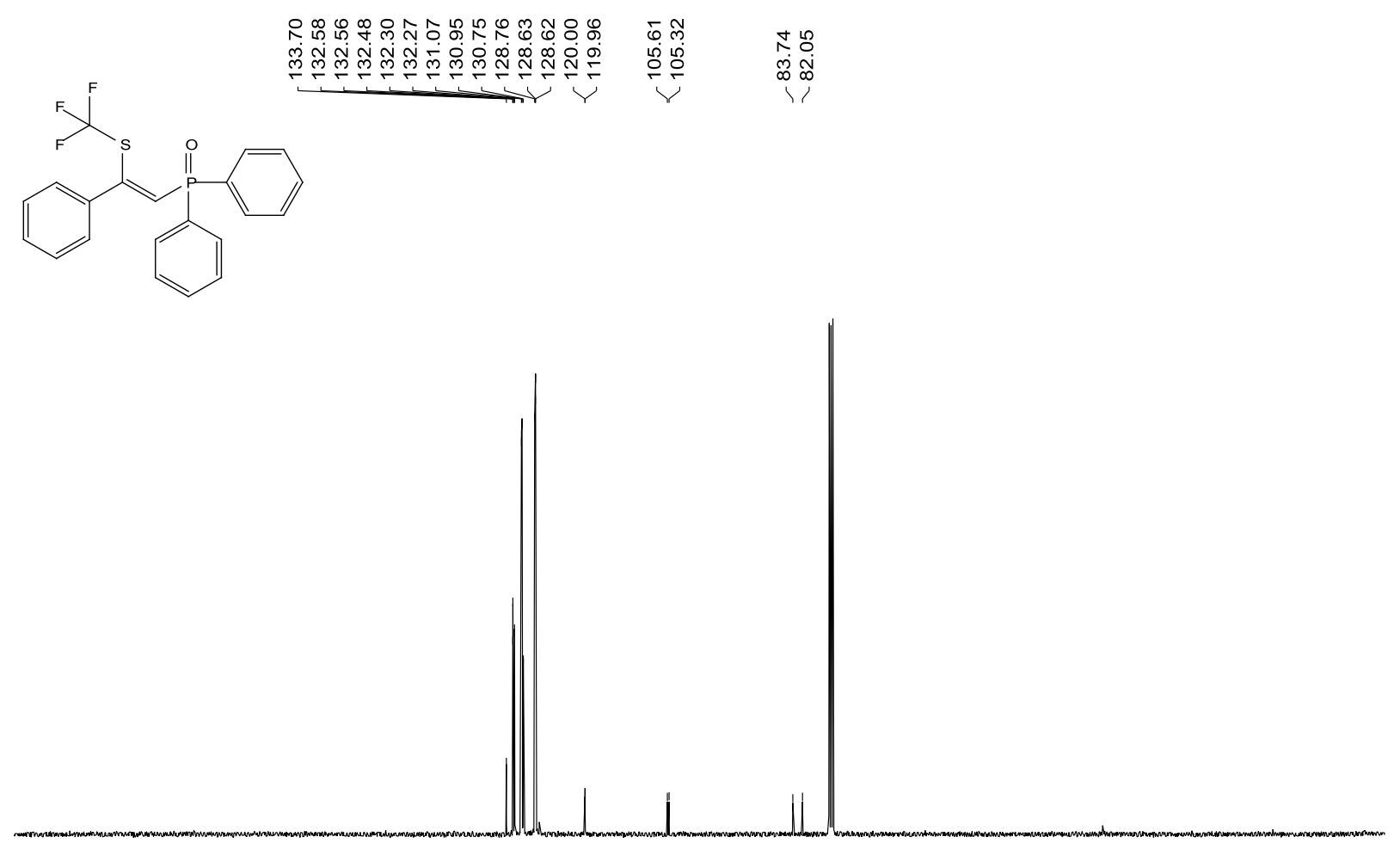

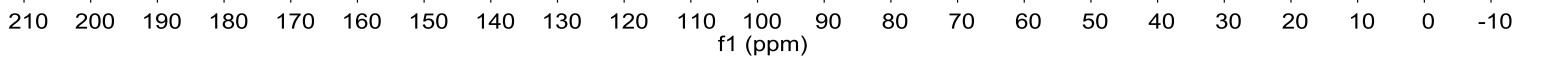




\section{${ }^{1} \mathrm{H}$ NMR Spectrum of $15\left(\mathrm{CDCl}_{3}, 400 \mathrm{MHz}\right)$}

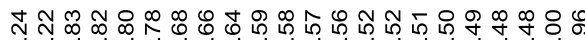

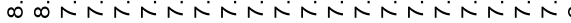
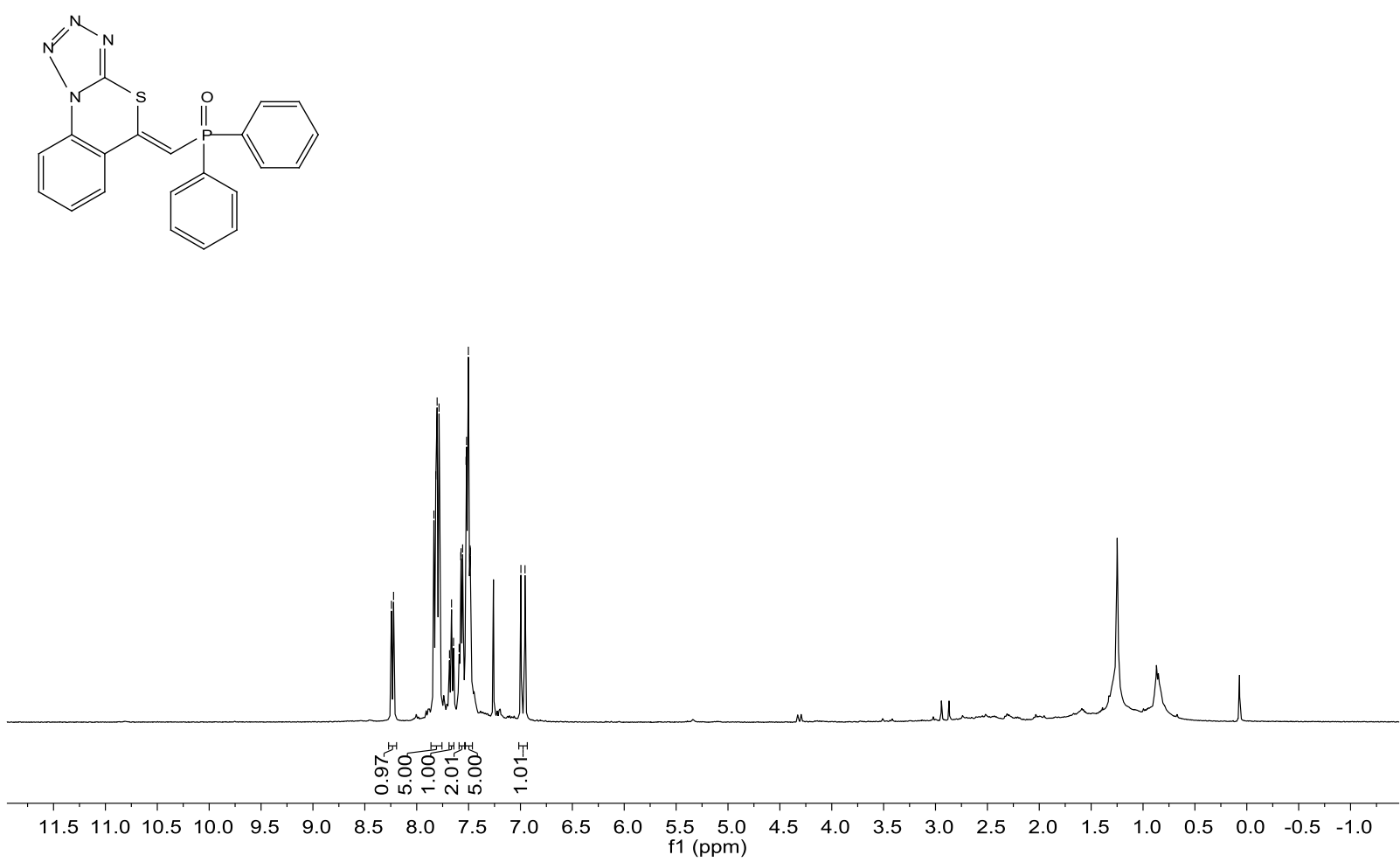

${ }^{31}$ P NMR Spectrum of $15\left(\mathrm{CDCl}_{3}, 162 \mathrm{MHz}\right)$

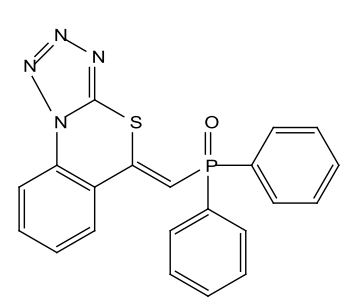

$\stackrel{\infty}{\stackrel{\infty}{N}}$

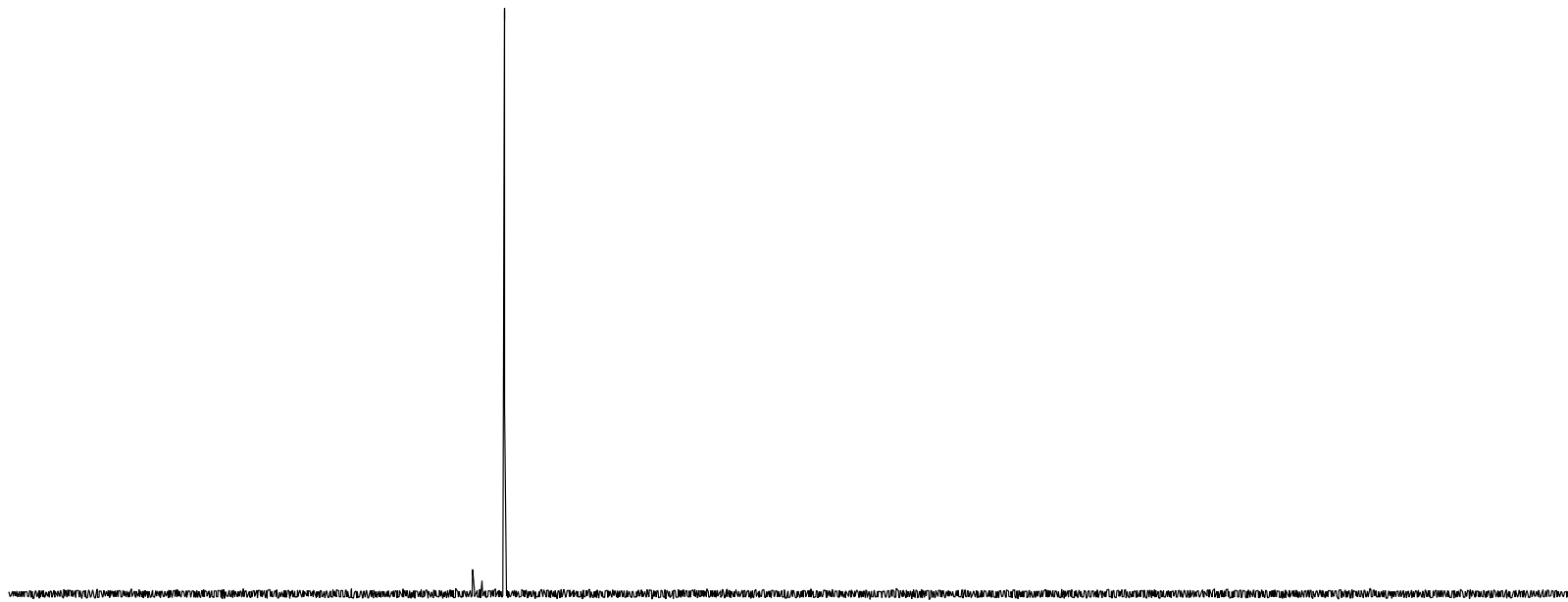

$\begin{array}{llllllllllllllllllll}140 & 120 & 100 & 80 & 60 & 40 & 20 & 0 & -20 & -40 & -60 & -80 & -100 & -120 & -140 & -160 & -180 & -200 & -220 & -240\end{array}$ 
${ }^{13} \mathrm{C}$ NMR Spectrum of $15\left(\mathrm{CDCl}_{3}, 100 \mathrm{MHz}\right)$

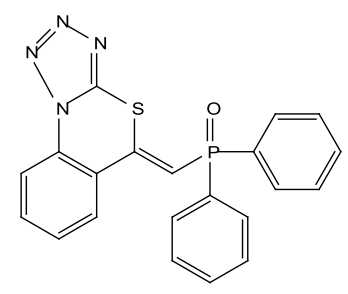

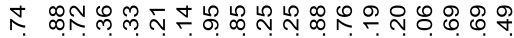

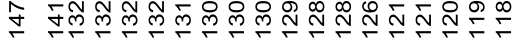

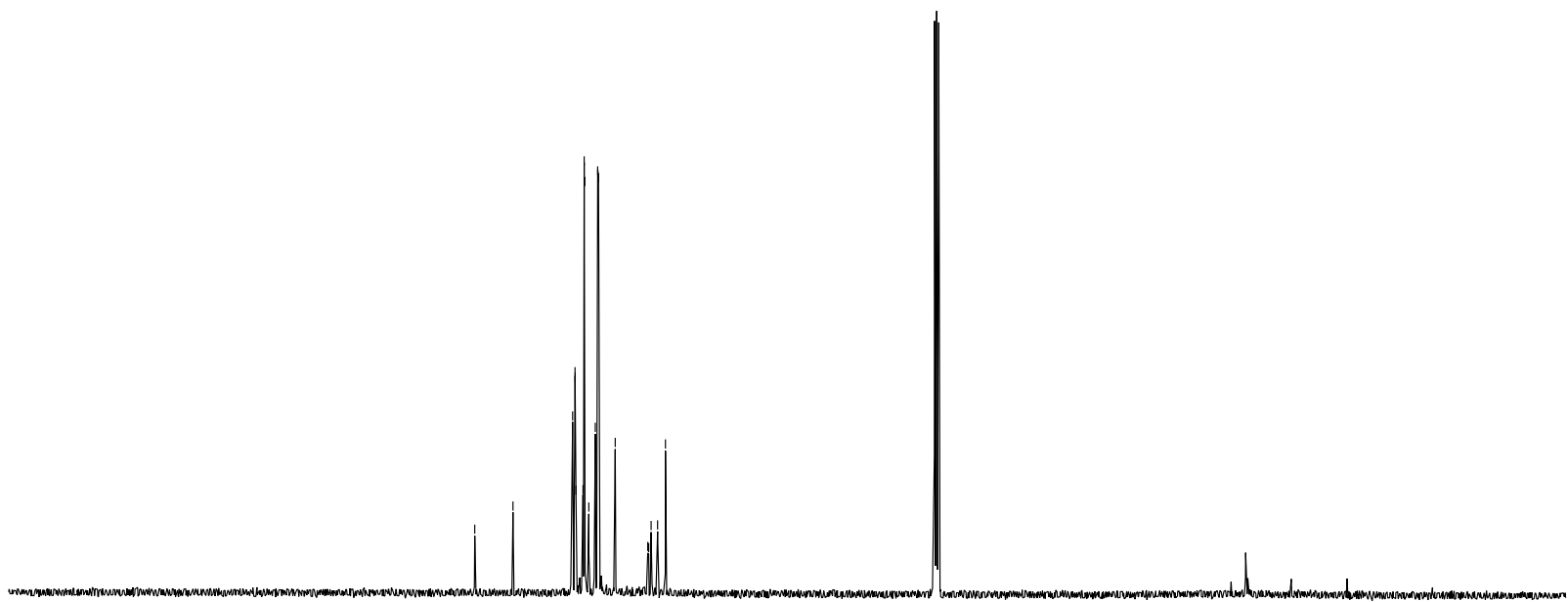

$\begin{array}{lllllllllllllllllllllll}210 & 200 & 190 & 180 & 170 & 160 & 150 & 140 & 130 & 120 & 110 & 100 & 90 & 80 & 70 & 60 & 50 & 40 & 30 & 20 & 10 & 0 & -10\end{array}$ 